WSRC-TR-2002-00459, Rev 0

SRT-RPP-2002-00221

\title{
PILOT SCALE PRECIPITATION TEST OF AN-107 SIMULANT WITH CAUSTIC ADJUSTMENT AT $20{ }^{\circ} \mathrm{C}(\mathrm{U})$
}

OCTOBER 31, 2002

Engineering Development Laboratory, Engineered Equipment \& Systems Department, Savannah River Technology Center

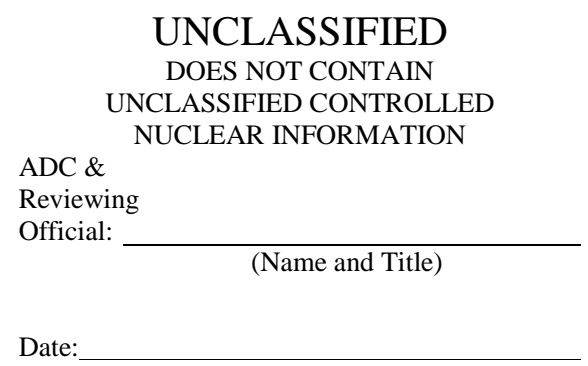

ADDITIONAL APPROVAL:

Official:

R\&T Manager, RPP Hanford

Date:

Westinghouse Savannah River Company

Savannah River Site

Aiken, SC 29808

Prepared for the U.S. Department of Energy Under Contract Number DE-AC09-96SR18500

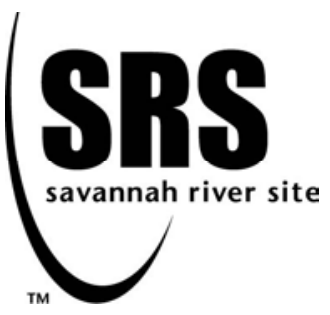


This document was prepared in conjunction with work accomplished under Contract No. DE-AC09-96SR18500 with the U. S. Department of Energy.

\section{DISCLAIMER}

This report was prepared as an account of work sponsored by an agency of the United States Government. Neither the United States Government nor any agency thereof, nor any of their employees, makes any warranty, express or implied, or assumes any legal liability or responsibility for the accuracy, completeness, or usefulness of any information, apparatus, product or process disclosed, or represents that its use would not infringe privately owned rights. Reference herein to any specific commercial product, process or service by trade name, trademark, manufacturer, or otherwise does not necessarily constitute or imply its endorsement, recommendation, or favoring by the United States Government or any agency thereof. The views and opinions of authors expressed herein do not necessarily state or reflect those of the United States Government or any agency thereof.

This report has been reproduced directly from the best available copy.

Available for sale to the public, in paper, from: U.S. Department of Commerce, National Technical Information Service, 5285 Port Royal Road, Springfield, VA 22161, phone: (800) 553-6847, fax: (703) 605-6900

email: orders@ntis.fedworld.gov

online ordering: http://www.ntis.gov/help/index.asp

Available electronically at http://www.osti.gov/bridge

Available for a processing fee to U.S. Department of Energy and its contractors, in paper, from: U.S. Department of Energy, Office of Scientific and Technical Information, P.O. Box 62, Oak Ridge, TN 37831-0062,

phone: (865)576-8401,

fax: (865)576-5728

email: $\underline{\text { reports@ adonis.osti.gov }}$ 
Key Words:

River Protection Project, Crossflow Filter, Envelope C Waste,

Hanford Tank 241-AN-107

\title{
Retention: Permanent
}

Key WTP R\&T References:

Test Plan SRT-RPP-2000-00050, Rev. 0

Test Scoping Statement S-51

\section{PILOT SCALE PRECIPITATION TEST OF AN-107 SIMULANT WITH CAUSTIC ADJUSTMENT AT $20^{\circ} \mathrm{C}(\mathrm{U})$}

\author{
Michael R. Williams, 786-5A \\ Timothy J. Steeper, 786-5A
}

Issue Date: October 31, 2002

Westinghouse Savannah River Company

Savannah River Site

Aiken, SC 29808

Prepared for the U.S. Department of Energy Under Contract Number DE-AC09-96SR18500

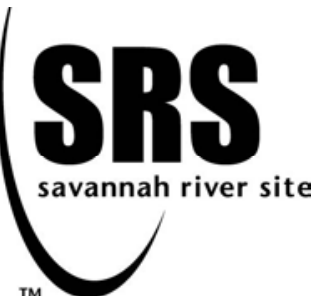


WSRC-TR-2002-00459, Rev. 0

SRT-RPP-2002-00221

\section{REVIEWS AND APPROVALS}

Michael R. Williams, Task Lead, EDS/EES/SRTC

Date

Timothy J Steeper, Data Analysis \& Report Writer, EDS/EES/SRTC

Date

James C. Marra, Pretreatment Manager, RPP

Date

Hector N. Guerrero, Technical Reviewer, EDS/EES/SRTC

Date

Reid A. Peterson, RPP-WTP Customer Acceptance

Date

\section{Completeness of Testing}

This report describes the results of work and testing specified by SRT-2000-00050. The work and any associated testing followed established quality assurance requirements and was conducted as authorized. The descriptions provided in this test report are an accurate account of both the conduct of the work and the data collected. Results required by the test plan are reported. Also reported are any unusual or anomalous occurrences that are different from starting hypotheses. The test results and this report have been reviewed and verified. 


\section{TABLE OF CONTENTS}

LIST OF FIGURES ......................................................................................................................................IV

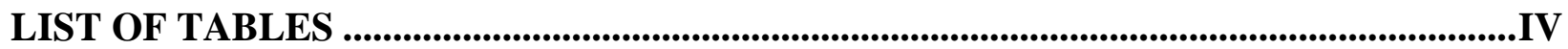

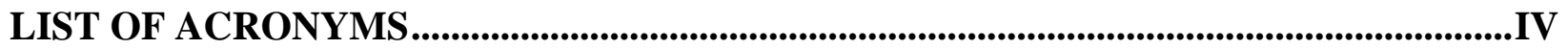

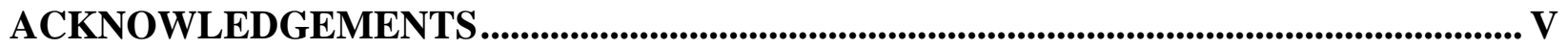

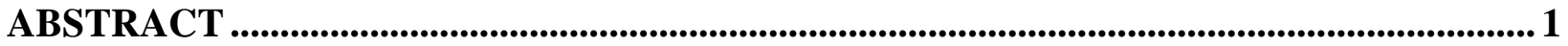

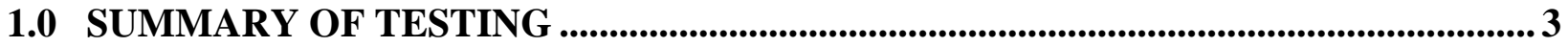

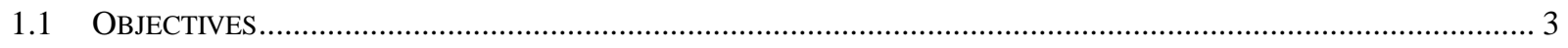

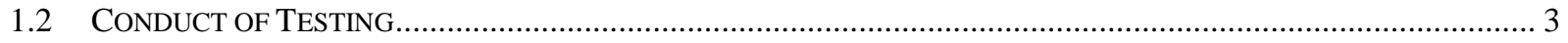

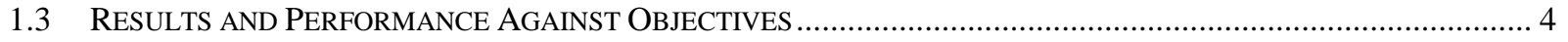

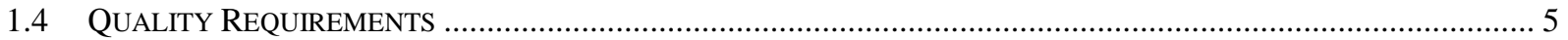

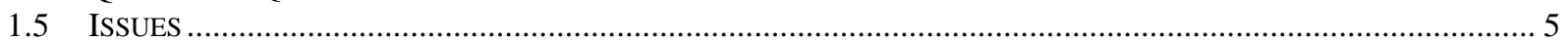

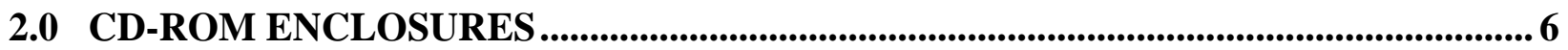

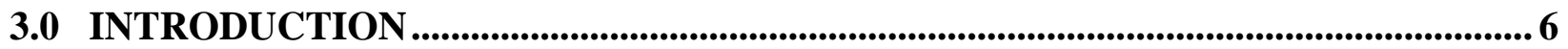

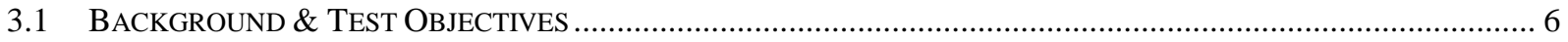

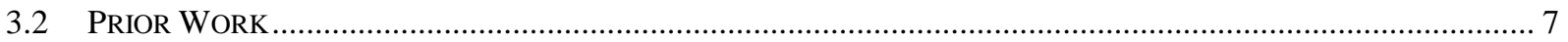

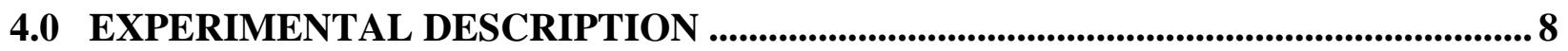

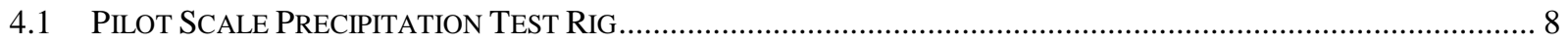

4.2 Pilot SCALE CROSSFLOW FILTER TEST RIG DESCRIPTION ................................................................. 10

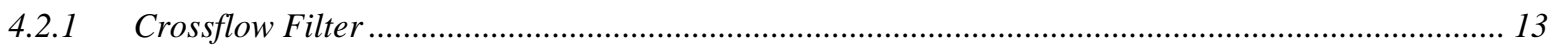

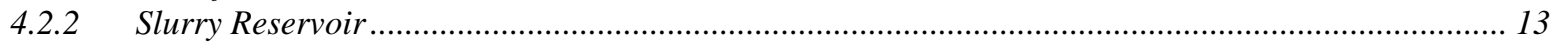

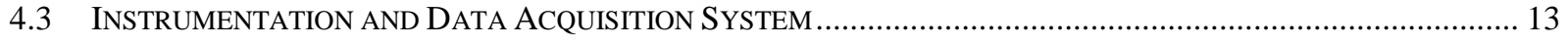

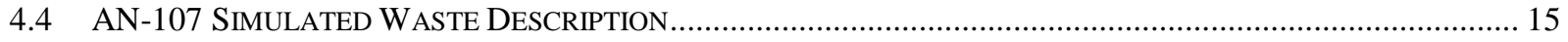

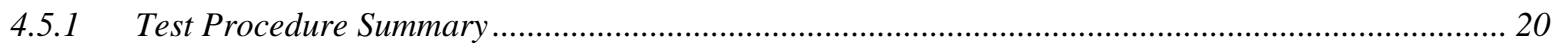

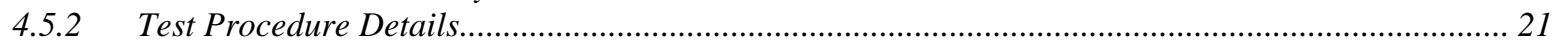

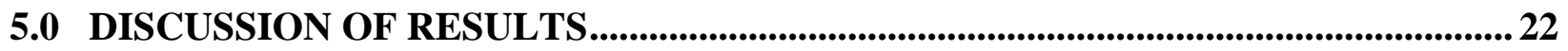

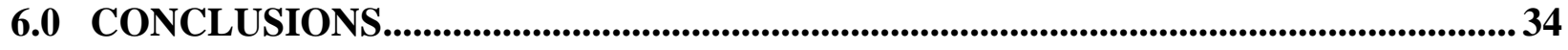

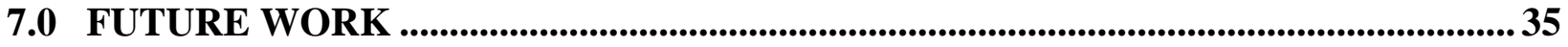

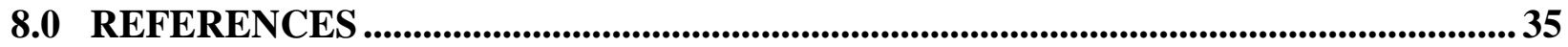

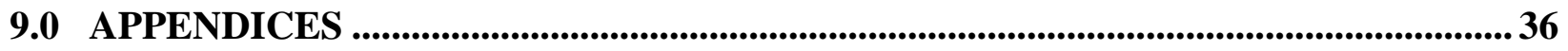




\section{LIST OF FIGURES}

Figure 4-1 Pilot SCALE Precipitation TeSt SetUP .................................................................................. 10

FigURE 4-2 PiLot SCALE CROSSFLow FILTER TEST RIG................................................................................ 12

FiguRE 5-1 LATHANIDE ELEMENTS IN BATCH \#2 PRECIPITATED LIQUID............................................................. 25

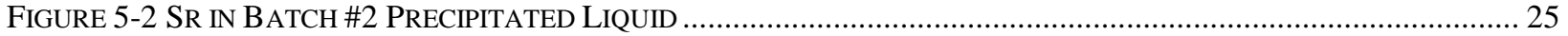

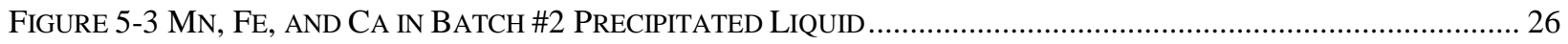

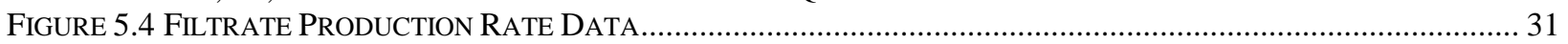

Figure 5-5 Crossflow Filter Filtrate Production with AN-107 PreciPitated Simulant SLURRy EARLy

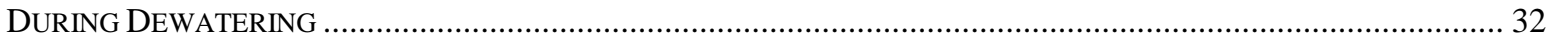

Figure 5-6 Crossflow Filter Filtrate Production with AN-107 Precipitated Simulant Slurry at the

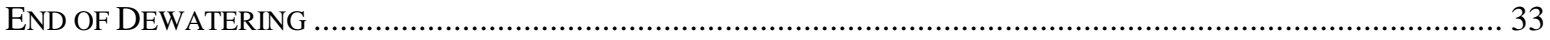

Figure 5-7 Crossflow Filter FluX With Clear Water Prior to BatCh \#2 Processing ................................ 33

Figure 5-8 Crossflow Filter FluX with Clear Water After BAtCh \#2 Processing .................................... 34

\section{LIST OF TABLES}

TABle 4-1 PSP DAS Channel List For Pilot Scale PreciPitation TeSt Facility ............................................ 13

TABLE 4-2 XFLOW DAS CHANNEL LIST FOR CROSSFLOW FILTER TEST RIG ........................................................... 14

TABLE 4-3 BATCH \#2 RECIPE BEFORE PRECIPITATING REAGENTS ADDED ................................................................. 17

TABLE 5-1 CONCENTRATION OF SELECTED ELEMENTS IN BATCH \#2 LIQUID SAMPLES .............................................. 24

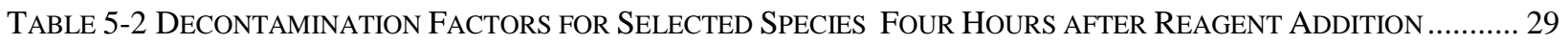

TABLE 5-3 CRossflow Filter OPERATIONS DATA WITH AN-107 SimUlant ........................................................... 30

\section{LIST OF ACRONYMS}

ADS Analytical Development Section

AN-102 Refers to waste in Hanford tank 241-AN-102. This waste is one of the Envelop C type wastes.

AN-107 Refers to waste in Hanford tank 241-AN-102. This waste is another of the Envelop C type wastes.

DIF Deionized and Filtered (0.2 micron) Water

EDL Engineering Development Laboratory, SRTC, WSRC

FBRM Focused Beam Reflectance Measurement

HLW High Level Waste

LAW Low Activity Waste

RCRA Resource Conservation and Recovery Act of 1976

RPP River Protection Project

SRTC Savannah River Technology Center

TMP Transmembrane Pressure (the average pressure drop across the thickness of the filter medium - perpendicular to the slurry flow.)

TRU Transuranic

WGI Washington Group International

WSRC Westinghouse Savannah River Company

WTP Waste Treatment and Immobilization Plant 


\section{ACKNOWLEDGEMENTS}

The authors would like to thank the RPP Team for their patience while we analyzed the data and prepared this report. We would like to thank all the support personnel of the Engineering Development Lab of SRTC. Michael Armstrong contributed in a major way to the successful operation of the test apparatus. Support from the other Engineering Development Laboratory personnel: Vern Bush, Andy Foreman, Mark Fowley, Gean Bridgmon, Jimmy Mills, was instrumental in keeping this test going and maintaining the readiness of the test rig, from instrument calibration to last minute changes that were needed due to unforeseen difficulties. The Lab Supervisor, Susan Hatcher, took every opportunity to reinforce safety on the job during all aspects of the testing, and was a tremendous help in handling chemical purchases disposing of wastes. We would also like to thank Charles Nash, Chris Bannochie, Bill Wilmarth, Bill King and John Steimke for their help in planning and technically reviewing this test. The management assistance of both Dan Burns and Steve Wach contributed invaluably to both procurement and execution of the plan for this test. Thanks to Hector Guerrero and Chris Bannochie for reviewing this report. 


\section{ABSTRACT}

This report discusses the results of the coupled operation of the Pilot Scale Precipitation Test Facility and Crossflow Test Facility conducted for the Hanford River Protection Project. These test facilities were constructed and operated by the Engineering Development Laboratory of the Savannah River Technology Center, Westinghouse Savannah River Company. The Pilot Scale Precipitation Test Facility coupled with the Crossflow Test Facility mimics the Hanford Waste Treatment and Immobilization Plant Pretreatment Ultrafiltration System. This system will remove entrained and precipitated solids, complexed strontium ( $\mathrm{Sr}$ ), and transuranic elements (TRU) from an Envelope $\mathrm{C}$ waste stream. Envelope $\mathrm{C}$ waste streams include the contents of Hanford waste tanks 241-AN-107 and 241-AN-102.

This report deals specifically with a non-radioactive AN-107 simulant and precipitating reagents of strontium nitrate and sodium permanganate to determine decontamination factors and operability of the process coupled with crossflow filter operation. The experiment precipitated a 634-liter batch of AN-107 simulant with caustic adjustment (Batch \#2) at a reaction temperature of $20{ }^{\circ} \mathrm{C}$. The results of the Batch \#1 test, with similar conditions except no caustic was added and the reaction temperature was $50{ }^{\circ} \mathrm{C}$, were reported previously (20). The processing of other batches for the study of the precipitation reaction will be the subject of future reports.

The liquid, solid and gaseous products of the reaction sampled over a period of eight hours were analyzed to determine the concentration of elements removed from the initial feed as solids by the process. About $85 \%$ of the reduction in concentration of lanthanides in the AN-107 simulant occurred within 7.5 minutes of the completion of the addition of the precipitating reagents. Lanthanide elements cerium, lanthanum, and neodymium in the simulated waste were used as non-radioactive surrogates for the radioactive actinide elements (including plutonium, americium, and uranium) in the real waste.

To show the removal efficiency, the initial mass of the element in the feed is divided by the final mass in the treated solution; this measure is called the decontamination factor. For the precipitation reaction in Batch \#2, the decontamination factors four hours after reagent addition were 9.4 for cerium, 11.1 for lanthanum, and 7.5 for neodymium. These decontamination factors demonstrate that the precipitation reaction is effective in reducing the concentration of lanthanide surrogates for TRU elements and supports the conclusion that application of this process to the real waste would meet the immobilization regulatory requirements.

The decontamination factor for strontium after four hours, based on the relative concentrations alone, was 0.02 . For the radioactive strontium in real waste, the addition of non-radioactive strontium nitrate prior to precipitation serves to make the radioactive strontium only a small percentage of the total strontium. The removal of radioactive strontium would include an isotopic dilution factor as well as the conventional DF. For a non-radioactive simulant, as used in this experiment, it is not possible to evaluate this dilution factor. The maximum possible isotopic dilution factor can be calculated based on the relative amounts of strontium in the feed and the reagents; for the process used in this experiment this maximum isotopic dilution factor is 1834. If complete isotopic mixing throughout the simulant is assumed prior to filtration, then 
this process should result in a decontamination factor for strontium-90 in real waste of $(0.02)^{*}(1834)=37$. This DF is significantly higher than required to meet the immobilization regulatory requirements.

Four hours after reagent addition was completed some of the contents of the precipitation tank were transferred to the Crossflow Test Facility and filtered under various conditions. Filtration production in about $3 \mathrm{wt} \%$ slurry varied between $0.026 \mathrm{gpm} / \mathrm{ft}^{2}$ at an axial velocity of $10 \mathrm{ft} / \mathrm{sec}$ and transmembrane pressure of $50 \mathrm{psid}$, and $0.038 \mathrm{gpm} / \mathrm{ft}^{2}$ at a slurry velocity of $18 \mathrm{ft} / \mathrm{sec}$ and transmembrane pressure of 45 psid. (There was no test specification detailing a filtration test matrix for this work, and the approved test plan simply stated that crossflow filtration would be performed to demonstrate filterability. Hindsight shows that the filtration conditions chosen by the task leader were not the best to obtain definitive data in the ranges matching current planned plant operation.)

After the filtration data was collected with dilute slurry, the slurry was dewatered continuously for several days. The inventory in the crossflow filter slurry loop was maintained between 70 and 90 liters by transferring from the precipitation tank whenever necessary. The filtrate production rate after about 10 hours of dewatering was about $0.033 \mathrm{gpm} / \mathrm{ft}^{2}$ of filter area at a slurry loop velocity of $16 \mathrm{ft} / \mathrm{sec}$ and a transmembrane pressure of $45 \mathrm{psid}$. Dewatering continued until the slurry volume was reduced from 764 liters to about 70 . The filtrate production rate was about $0.018 \mathrm{gpm} / \mathrm{ft}^{2}$ at $\mathrm{V}=15.4 \mathrm{ft} / \mathrm{sec}$ and TMP $=49.0 \mathrm{psid}$ with the concentrated slurry. (Note: all flowrates are corrected to a filtration temperature of $25^{\circ} \mathrm{C}$.)

The filtrate collected from slurry precipitated with this process appeared to be fairly stable. After several months of storage some solids were formed, but there were very little solids formed in filtrate allowed to stand a day or two. 


\subsection{SUMMARY OF TESTING}

\section{$1.1 \quad$ Objectives}

The Task Plan (1) states the objectives of this Pilot Scale Test Program as follows:

- Design, fabricate and install in building 786-A Pilot Scale components for the RPP Ultrafiltration System capable of processing envelope C waste simulant batches of about 650 liters through the precipitation reaction and coupled crossflow filter operations under varying reaction conditions.

- Precipitate and filter a series of batches at varying conditions to examine the effect of feed material, reaction temperature, degree of mixing, and amount and timing of reagent addition. The pilot scale work was intended to provide data that could be compared to the very small, well mixed actual waste precipitation experiments, and to the slightly larger actual waste and simulant filtration studies.

- Specifically, test Batch \#2 (AN-107 waste simulant with caustic addition) under the following reaction conditions:

1. Reaction Temperature of $20 \pm 2^{\circ} \mathrm{C}$,

2. Maximum mechanical agitator speed to ensure uniform mixing in Precipitate Tank,

3. Addition of Sodium Hydroxide to make a $1 \mathrm{M}$ increase in the $\mathrm{OH}^{-}$concentration,

4. Addition of $1 \mathrm{M}$ Strontium Nitrate to reach a $0.075 \mathrm{M}$ concentration in the treated slurry, followed by the addition of $1 \mathrm{M}$ Sodium Permanganate to reach a $0.05 \mathrm{M}$ concentration in the treated slurry (most of the strontium and manganese will form solids during the precipitation reaction),

5. Ten minutes time between reagent additions,

6. Reagent addition at a rate of 8 liters per minute,

7. Four hours reaction time before filtration in the Crossflow Test Rig.

- Analyze liquid, solid and Precipitate Tank vapor samples to determine:

1. Decontamination factors (DF) for strontium and each of the lanthanide surrogates added,

2. If volatile/flammable gases are produced by the precipitation reaction,

3. Filterability of the precipitated waste simulant slurry,

4. If post-filtration precipitation occurs.

\section{$1.2 \quad$ Conduct of Testing}

The size of Batch \#2 at 634 liters is approximately 1/300 scale of the full size RPP Ultrafiltration Feed Vessel batch to be precipitated by the Waste Treatment and Immobilization Plant Pretreatment Ultrafiltration System. The plant plans to use pulse jet mixers that will not require maintenance for the life of the plant, but also may not provide the vigorous mixing provided by a mechanical agitator. Since it is not possible to conduct the small real waste experiments with pulse jet mixing, they were done under well mixed conditions. A mechanical agitator was mounted vertically inside the baffled Pilot Scale Precipitate Tank to provide uniform and vigorous mixing for Batch \#2. A future batch will be run with with a pulse jet mixer to help evaluate the effect of mixing on the precipitation. The AN-107 simulant used in Batch \#2 was a 
non-radioactive recipe of metal salts, organic compounds, entrained solids, and lanthanide surrogates (15).

The AN-107 simulant in the Precipitate Tank was maintained at the reaction temperature of 20 ${ }^{\circ} \mathrm{C}$ with the maximum agitation possible without splashing or vortex formation. Nineteen molar (50 wt\%) sodium hydroxide (caustic) was added in sufficient quantity to raise the free hydroxide concentration to $1 \mathrm{M}$ in the resultant mixture. One molar strontium nitrate was added at eight liters/minute, followed ten minutes later by the addition of one molar sodium permanganate at eight liters/min. Sufficient reagents were added to increase the concentration in the precipitation tank mathematically to $0.075 \mathrm{M}$ in strontium and $0.05 \mathrm{M}$ in manganese. Several one-liter slurry samples were taken throughout an eight-hour period. Vapor samples were taken six inches above the liquid surface during the reaction. These samples were analyzed to determine the concentration of analytes in the liquids, solids and gases produced by the precipitate reaction. Filterability of the precipitated slurry was determined by measuring the filtrate production rates while operating the crossflow filter under varying conditions of slurry flow and filter transmembrane pressure. One-liter filtrate samples were taken from the Filtrate Loop on the Crossflow Filter Test Rig to be analyzed by Lasentec particle analysis for evidence of postfiltration solids formation.

\subsection{Results and Performance Against Objectives}

The Pilot Scale Precipitation Test Facility established the test conditions and processed the AN107 simulant to produce the samples required to determine the DF values for strontium and each of the lanthanide surrogates and to determine if volatile gases were produced by the precipitation reaction. The Crossflow Filter was operated at various flows and filter pressures to demonstrate filterability and to obtain samples for evaluation of post-filtration solid formation.

The solids filtered out of one-liter samples of the precipitation tank contents increased from 4.0 grams before precipitation to about 45 grams after precipitation, about an 11 -fold increase. The crossflow filter was able to dewater the slurry at the rate of $0.033 \mathrm{gpm} / \mathrm{ft}^{2}$ of filter area with a 16 $\mathrm{ft} / \mathrm{sec}$ axial velocity and $45 \mathrm{psid}$ transmembrane pressure when the solids content was about 3 $\mathrm{wt} \%$. The filtrate production dropped off to about $0.018 \mathrm{gpm} / \mathrm{ft}^{2}$ with a $15.4 \mathrm{ft} / \mathrm{sec}$ axial velocity and 49 psid transmembrane pressure after the slurry had been concentrated from 762 liters to about 70 liters.

The analysis of samples taken before and after the precipitation reaction of the AN-107 simulant in Batch \#2 indicate a significant reduction in the amount of dissolved transuranic surrogate elements in the solution. The addition of a substantial amount of aqueous strontium during the processing raised the slurry up to the saturation point, but most of the added strontium was converted to a solid that could be filtered out. Prior work by Nash et al. (11) indicates that complete isotopic mixing occurs even though the strontium carbonate precipitates very rapidly. This implies a very rapid dynamic equilibrium allowing the strontium atoms in solution and the precipitated strontium atoms to exchange. This is the desired result since the removal of radioactive strontium is accomplished by isotopic dilution with the non-radioactive strontium added during processing. 
These results from the Sr/TRU precipitation of AN-107 simulant in Batch \#2 are within the range achieved with actual AN-102 waste. The Sr/TRU removal system performed as expected to remove aqueous strontium and transuranic surrogate elements as solids from the AN-107 simulant. The addition of strong caustic to increase the simulant concentration by $1 \mathrm{M} \mathrm{NaOH}$ before the addition of precipitating reagents reduced the removal of strontium slightly but increased the removal of cerium and lanthanum as compared to the Batch \#1 results (20).

Vapor samples were also collected and analyzed for volatile gases evolving near the liquid surface. No trace of volatile gases was found.

Filtration was started about three hours after all reagents were added. Samples of filtrate were collected at various times during the filtration of the slurry and allowed to stand for about 20 hours before being examined with a particle size analyzer. The filtrate collected from slurry precipitated with this process appeared to be fairly stable. After several months of storage some solids were formed, but there were very few solids formed in filtrate allowed to stand a day or two. The purpose of the particle size analysis was to determine if post-filtration solid formation occurred or not. Since the shape of the particles was unknown and no standard for calibration existed, the analysis could not provide quantitative data regarding wt $\%$ solids.

\subsection{Quality Assurance Requirements}

A Test Specification was not written specifically for the pilot precipitation work. The approved Task Technical and Quality Assurance Plan (1) specified the WSRC QA requirements that would be applied. These requirements were followed.

\section{$1.5 \quad$ Issues}

\subsubsection{Excess EDTA in simulant}

The calculations used in the formulation sheet submitted to the vendor for preparation of the AN107 supernatant simulant presumed use of disodium ethylenediaminetetraacetic (EDTA) acid dihydrate, but the chemical formula specified on the sheet did not include the two waters of hydration. The compound in question is only available as the dihydrate. The vendor made the appropriate adjustment to use the dihydrate material based upon the specified chemical formula and hence the shipped material appeared to meet the requested recipe, but it actually contained $12 \%$ additional EDTA. The error was an internal one made when the simulant recipe sheet was prepared, it should have included the two waters of hydration in the reagent formula because this is what was used in the calculations and included in the approved recipe.

The vendor was given a recipe to follow that included the mass and order of addition of each reagent in the simulant recipe, including the chemical formula of the reagent to use. It did not include the molecular weights of the reagents used in the calculations.

The simulant received was not analyzed because it represented only a portion of the final recipe. The remaining reagents and entrained solids were added to the simulant in the precipitate reactor prior to the start of the pilot run. Following completion of the additions, a final simulant sample was taken and submitted for analysis. Since the EDTA method is one of the more difficult 
analyses, it was not received for some time following the pilot experiments. This analysis is only good to $\pm 10 \%$, thus the preparation error was within the combined experimental and reagent purity uncertainty $\left(\mathrm{Na}_{2}\right.$ EDTA $2 \mathrm{H}_{2} \mathrm{O}$ is not a primary standard material) and would not have been detected had the measurement been received prior to the start of precipitation.

The increased level of EDTA would result in greater Sr solubility in the simulant and potentially a lower decontamination factor. Since the EDTA measurements in the actual waste samples used to prepare the simulant recipe differ by $\pm 20 \%$ (one standard deviation), the uncertainty in what the actual value should be exceeds the discrepancy found in the simulant preparation. The experimentally calculated Sr DF exceeds the process requirements, so the impact appears to be negligible.

\subsection{CD-ROM ENCLOSURES}

No CD-ROM enclosures are planned for this report.

\subsection{INTRODUCTION}

\subsection{Background \& Test Objectives}

The River Protection Project (RPP) is an effort by DOE to process approximately 190 million curies in 54 million gallons of highly radioactive and mixed hazardous waste stored in underground storage tanks at the Hanford site. The tank waste includes solids (sludge), liquids (supernate), and salt cake (dried salts that will dissolve in water forming supernate). The waste tanks will be remediated through treatment and immobilization to protect the environment and meet regulatory requirements. DOE determined that the preferred alternative to remediate the Hanford Tank Waste is to:

- Pretreat the waste to separate it into two fractions, Low-Activity Waste (LAW) and HighLevel Waste (HLW);

- Immobilize the LAW for on-site disposal; and

- Immobilize the HLW for ultimate disposal in a National repository.

LAW is a mixed, characteristic, and listed waste regulated under the Resource Conservation and Recovery Act of 1976 (RCRA), and must meet certain treatment standards and performance standards for on-site disposal of the waste form. LAW is comprised of the tank waste liquids (and dissolved salt cake) containing the bulk of the tank waste chemicals and certain radionuclides (e.g., cesium, technetium, strontium and transuranics) that must be at least partially removed prior to immobilizing the waste. The Sr/TRU is precipitated by the addition of strontium nitrate and sodium permanganate. The Sr/TRU precipitate and entrained solids are collected by filtration with the concentrated product routed to high level waste (HLW) for vitrification.

HLW is comprised of the long half-life elements contained in the tank waste solids and high activity radionuclides separated from the LAW fraction. HLW is a mixed, characteristic, and listed waste regulated under RCRA, and must meet specific treatment and performance standards for storage and repository disposal of the final waste form. 
The scope of SRTC pilot scale precipitation testing per Test Scoping Statement S-51 is to demonstrate operability and determine throughput for the Sr/TRU precipitation process by investigating the precipitation process with strontium nitrate and sodium permanganate on a significantly larger scale than has been done previously. Eight batches of simulated waste were orginally scheduled for processing through the Pilot Scale Precipitation Test Facility and coupled Crossflow Filter Test Rig under various process parameters including those of temperature, reagent addition rate, and mixing rates. Non-radioactive simulants of Hanford tank AN-102 and AN-107 wastes will be used. Results from this testing will be compared to bench-scale testing previously performed with real and simulated wastes. This report deals solely with Batch \#2 which utilizes AN-107 simulant.

\subsection{Prior Work}

Previous bench scale work by Nash et al. (10) used AN-107 simulant with additional caustic added and precipitated with $0.075 \mathrm{M}$ strontium nitrate and $0.04 \mathrm{M}$ sodium permanganate to produce decontamination factors of 91 for strontium, 2.8 for cerium, $>4.2$ for lanthanum, and 3.4 for neodymium.

Previous Sr/TRU precipitation reactions performed by Nash et al. (11) on 16.5 liters of Tank 241-AN-102 "Large C" supernatant liquid containing entrained solids indicated that strontium levels are reduced by simple isotopic dilution and strontium decontamination factors can be predicted a priori. Strontium decontamination factors ranging from 40 to 50 were achieved in this study. Transuranic elements including plutonium, curium, and americium were reported to have decontamination factors between 2.9 and 12.4. Some discoloration of the high sodium filtrate bottles was seen after several days, which could be attributed to trace amounts of postfiltration solid formation. Free solids were observed in the lower sodium content filtrate from washing operations after the filtrate was allowed to stand for two days.

A 1.2 liter "Small" Envelope C sample from Tank 241-AN-102 was caustic adjusted, and strontium and permanganate precipitated, as reported by Nash et al (13) to produce a filtrate product decontamination factor of 30 for Sr-90, 9.2 for Am-241, and 7.2 for Cm-244.

Previous pilot scale precipitation of an AN-107 simulant by Duignan (17) reported filtrate production rates of: $0.03 \mathrm{gpm} / \mathrm{ft}^{2}$ at $8.9 \mathrm{ft} / \mathrm{sec}$ axial velocity and $32.1 \mathrm{psid}$ transmembrane pressure, $0.04 \mathrm{gpm} / \mathrm{ft}^{2}$ at $12.3 \mathrm{ft} / \mathrm{sec}$ and $51.1 \mathrm{psid}$, and $0.07 \mathrm{gpm} / \mathrm{ft}^{2}$ at $15.4 \mathrm{ft} / \mathrm{sec}$ and $30.0 \mathrm{psid}$. He found the filtrate flux was strongly affected by the slurry velocity but only weakly by the transmembrane pressure in the 30 to 55 psid range. During dewatering of his slurry, the filtrate rate gradually decreased from about $0.07 \mathrm{gpm} / \mathrm{ft}^{2}$ at $2 \mathrm{wt} \%$ to $0.01 \mathrm{gpm} / \mathrm{ft}^{2}$ at $22 \mathrm{wt} \%$ insoluble solids. Although not the major focus of Duignan's work, decontamination factors were calculated as follows: 69 for Sr, 20 for $\mathrm{La}, 22$ for $\mathrm{Fe}, 2$ for $\mathrm{Cu}$ and $\mathrm{Ca}$, and approximately 1 for $\mathrm{P}$, $\mathrm{S}, \mathrm{Ni}$, and $\mathrm{Al}$.

Hallen et al (18) treated a 1.4 liter sample of diluted actual AN-107 tank waste by adding sodium hydroxide, strontium nitrate, and sodium permanganate. Target concentrations for the final treated waste were $6.0 \mathrm{M}$ sodium, $1.0 \mathrm{M}$ hydroxide, $0.075 \mathrm{M}$ strontium, and $0.05 \mathrm{M}$ permanganate. The waste was thoroughly mixed between each reagent addition. After adding the permanganate, the waste was mixed for 30 minutes at ambient conditions, then heated for 4 
hours at $50{ }^{\circ} \mathrm{C}$. Filtration was carried out in a Cell Unit Filtration (CUF) system equipped with a $0.1-\mu \mathrm{m}$ filter element. Their filtrate sample number DF-11 had decontamination factors of 82 for Sr-90, 28 for Am-241, and 22 for Cm-242. Their composite filtration sample DF-20 supernate had decontamination factors of 78 for Sr-90, 38 for Am-241, 32 for Cm-242, and 24 for $\mathrm{Pu}-239$. They reported that $74 \%$ of the neodymium was removed based on their DF-20 filtrate composite sample. Filtration was first conducted with the filtrate returned to the slurry feed tank to maintain a constant concentration. The plot of the first CUF run at $12.2 \mathrm{ft} / \mathrm{sec}$ axial velocity and 49 psid transmembrane pressure showed the filtrate flux starting at about 0.07 $\mathrm{gpm} / \mathrm{ft}^{2}$, rapidly dropping to $0.037 \mathrm{gpm} / \mathrm{ft}^{2}$ within 10 minutes, then gradually falling off to about $0.025 \mathrm{gpm} / \mathrm{ft}^{2}$ after an hour of filtration. Although they were unable to operate at $15.2 \mathrm{ft} / \mathrm{sec}$ and 50 psid because of pump wear, they concluded there would be little improvement in filtrate flux by increasing the velocity from $12.2 \mathrm{ft} / \mathrm{sec}$ to $15.2 \mathrm{ft} / \mathrm{sec}$ based on the flux increases at lower velocities. During dewatering, the flux averaged $0.021 \mathrm{gpm} / \mathrm{ft}^{2}$.

Steeper and Williams (20) reported on the Batch \#1 precipitation of 647 liters of AN-107 simulant without caustic adjustment at $50{ }^{\circ} \mathrm{C}$ with an addition of $1 \mathrm{M}$ strontium nitrate followed 10 minutes later by an addition of $1 \mathrm{M}$ sodium permanagate. Sufficient reagents were added to bring the final concentrations in the resultant slurry to $0.075 \mathrm{M}$ strontium and $0.05 \mathrm{M}$ manganese. Decontamination factors four hours after reagent addition were 5.2 for cerium, 8.4 for lanthanum, and 7.1 for neodymium. If complete isotopic mixing were assumed, the process would result in a decontamination factor of 1000 for strontium. Crossflow filtration achieved fluxes as high as $0.05 \mathrm{gpm} / \mathrm{ft}^{2}$ with tube axial velocity of $20.8 \mathrm{ft} / \mathrm{sec}$ and a transmembrane pressure of $29.2 \mathrm{psid}$. During initial dewatering, a steady flux of $0.02 \mathrm{gpm} / \mathrm{ft}^{2}$ was obtained at a velocity of about $16 \mathrm{ft} / \mathrm{sec}$ and a transmembrane pressure of about $42 \mathrm{psid}$.

\subsection{EXPERIMENTAL DESCRIPTION}

\subsection{Pilot Scale Precipitation Test Rig}

Figure 4-1 is a schematic of the Pilot Scale Precipitation Test Rig assembled in the rear of the Engineering Development Laboratory, Building 786-A. The Precipitation Test Rig was fabricated utilizing PVC and CPVC plastic pipe with tubing made of stainless steel and polypropylene. A 938 liter polypropylene Precipitate Tank with baffles is provided for the addition of the precipitating reagents. (As discussed previously, the plant is planning to precipitate in an unbaffled tank with pulse jet mixers. Our baffled tank is intended to enhance mixing to match smaller scale studies. A later batch is planned with a pulse jet mixer to evaluate the mixing issue.) The pilot scale 650 liter batch approximates a 1/300 scale of the full size batch, 48383 gallons per Bergman (16), precipitated in the Ultrafiltration Feed Vessel by the WTP Ultrafiltration System. The temperature of the batch can be controlled by pumping the Precipitate Tank contents through a recirculation loop containing an electric heater and a chilled water heat exchanger. A vertically mounted Lightnin agitator with 12 inch diameter A-310 style impeller can provide vigorous uniform mixing of the simulant in the Precipitate Tank without splashing. Both the agitator and recirculation pump have speed controllers to vary the mixing conditions.

An individual addition system including a tank, recirculation pump, and flowmeter is provided for each of the reagents. The sodium permanganate tank is opaque because the reagent is light sensitive. Typically, the reagents are mixed in the tanks by recirculating the solution at the 
desired flow rate, and then the aqueous solution is valved over to the precipitation tank for addition. The strontium nitrate is added first and allowed to mix throughout the tank before adding the sodium permanganate. For Batch \#2 the strontium nitrate was allowed to mix in the precipitation tank for 10 minutes prior to adding the sodium permanganate. The sodium permanganate was not standardized, but was prepared from new, sealed regent stock immediately prior to use.

A sample of the precipitation tank contents can be drawn off with a pump connected to the main recirculation loop. The take-off from the recirculation line is a thin wall, small diameter tube about a foot long installed concentrically inside the recirculation pipe and pointing into the flow. The tube and recirculation pipe are sized to have identical flow velocities at a recirculation flowrate of $9.64 \mathrm{gpm}$ and a sample rate of one liter every 20 seconds. Typically, flow is established through the sample pump and returned to the recirculation loop to ensure the line is full and flushed with fresh material. The flow is then quickly valved to the collection container, then valved back to the recirculation loop when approximately one liter of sample is collected. Samples are dead-end filtered to separate the solids from the liquid portion. The solid samples are not washed before drying at $100{ }^{\circ} \mathrm{C}$ for at least eight hours or longer until the solids freely crumble.

The Precipitation Test Rig is made up of a precipitation tank and several flow loops as detailed below. It is connected to the Crossflow Test Rig via the V41 valve.

1. The Precipitation Tank Recirculation Loop includes the 938-liter Precipitation Tank with an agitator, recirculation pump, chilled water heat exchanger, and an electric heater. This loop is the primary source of AN-107 simulant samples and is used to maintain the temperature as specified in the task plan Test Matrix.

2. The Liquid Sample Loop includes a pump that takes suction from and discharges to the Precipitation Tank Recirculation Loop permitting samples to be drawn from a three-way valve.

3. The Strontium Nitrate Reagent Loop includes an 80-liter polypropylene reagent tank for the $1 \mathrm{M}$ strontium nitrate, a centrifugal pump, flowmeter, and a three-way valve permitting recycle for mixing, or transfer of the reagent to the Precipitation Tank.

4. The Sodium Permanganate Reagent Loop includes a covered 80-liter stainless steel reagent tank for the 1M sodium permanganate, a centrifugal pump, and a three-way valve permitting recycle for mixing, or transfer of the reagent to the Precipitation Tank.

5. The Vapor Sampling Loop includes a diaphragm pump connected to a tube through the Plexiglas Precipitation Tank cover to within six inches of the liquid surface. The discharge of the vapor sampling pump is connected to a three-way valve with tubing leading to a Tedlar vapor sample storage bag or back to the to Precipitation Tank vapor space. 


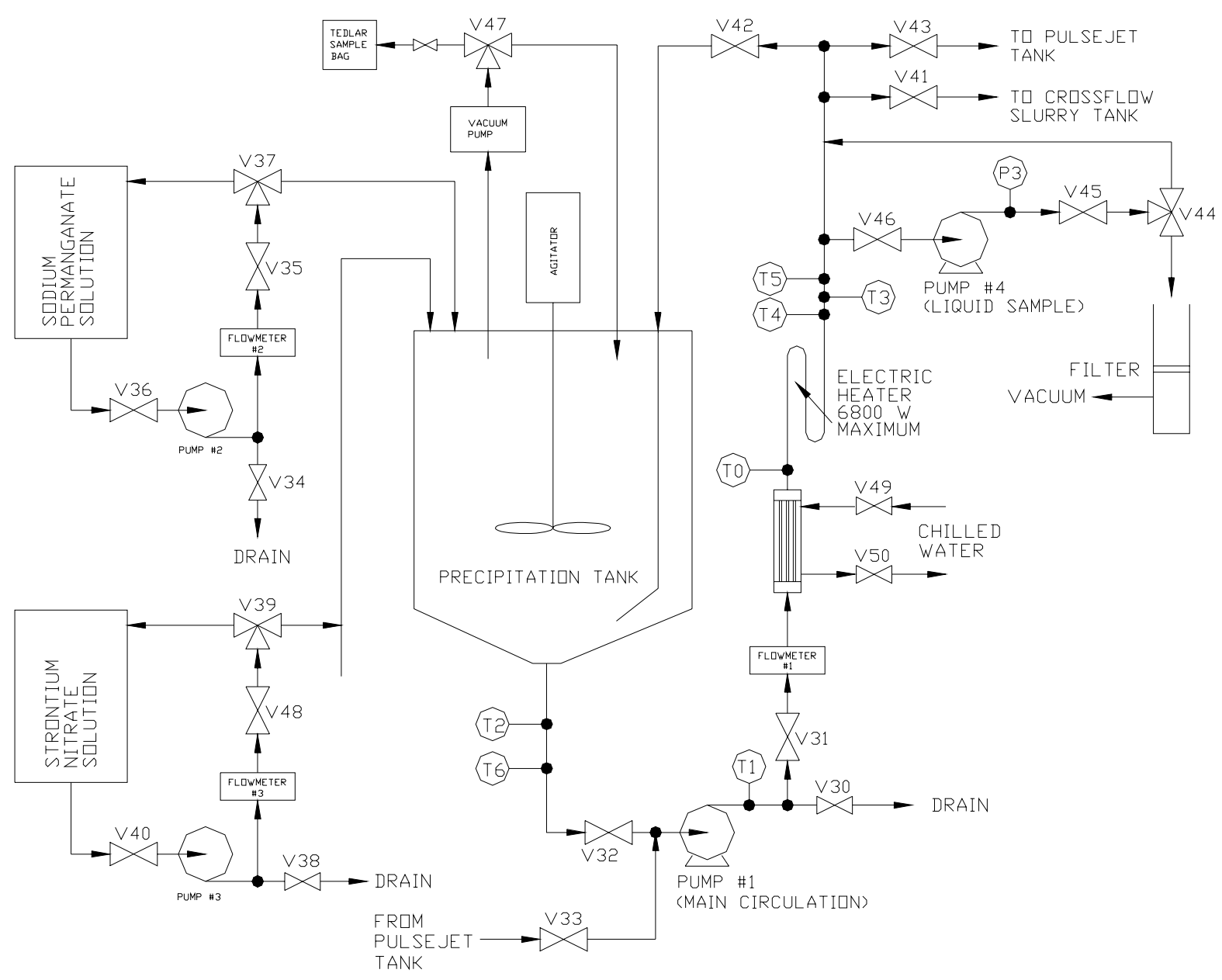

Figure 4-1 Pilot Scale Precipitation Test Setup

\subsection{Pilot Scale Crossflow Filter Test Rig Description}

Figure 4-2 is a schematic of the Pilot Scale Crossflow Filter Test Rig located in the rear of the Engineering Development Laboratory, Building 786-A. (Note: for this experiment only two slurry pumps were used instead of the three slurry pumps shown.) The Crossflow Filter Test Rig was fabricated utilizing PVC and CPVC plastic pipe to connect the slurry pumps and the Slurry Reservoir, and 300 series stainless steel for the remainder of the Slurry, Filtration, and Cleaning Loops. The rig is approximately 25 feet tall and is serviced by a two-level mezzanine. The Crossflow Test Rig is made up of three basic flow loops:

1. Slurry Loop, which contains two 3-hp centrifugal pumps, flowmeter, throttle valve, and crossflow filter. This loop serves as the primary flow path for circulating slurries. This loop has an internal volume of approximately 20 liters, excluding the Slurry Reservoir.

2. Filtrate Loop, which begins at the filter housing and allows the separated filtrate liquid to flow up through the backpulse piston before returning to the top of the slurry loop to close the circuit. This loop has an internal volume of approximately six liters. Note that this loop 
has a three-way valve that can be positioned to draw off the filtrate to a collection tank rather than returning it to the slurry loop. This option is used during dewatering to concentrate the slurry.

3. Cleaning Loop, which enables cleaning of the crossflow filter in place without having to remove the slurry from the test rig. This loop has an internal volume of approximately 15 liters and contains a three hp centrifugal pump.

Two other flow circuits that are subsections of the other loops are the backpulse and the bypass loops:

1. The backpulse loop is part of the filtrate loop and functions to reverse the flow of filtrate back through the filter. A pulse forces filtrate back through the seven filter elements in order to knock off built-up slurry cake on the inside diameter of the porous tubes. (An air driven backpulse piston assembly controls the amount of filtrate used for a backpulse. The piston delivers a constant pressure pulse of $0.036 \mathrm{gal} / \mathrm{ft}^{2}$ of filter tube inside surface area. This pulse is sufficient to generate a significant improvement in filtrate flux immediately following a backpulse generated by opening the V15 valve. The filtrate flow is interrupted for only about ten seconds during the backpulse operation. The actual backpulse has a duration of only a few seconds.)

2. The bypass loop is part of the slurry loop and routes part of the flow through valve V6 back to the reservoir. This loop is used to better control the slurry flow during slurry pump startup, improve mixing, and ensure the slurry is well-mixed when the flow through the filter needs to be stopped.

To circulate slurries and liquids, the Crossflow Filter Test Rig was equipped with three stainless steel centrifugal pumps: two for the slurry loop and one for the cleaning loop. The Slurry Loop pump motors were manually controlled by a Leeson Speedmaster Variable Frequency Drive connected to a 208-volt three-phase power supply, providing excellent control of the flow of fluid from the pump discharge. Data correlating frequency input to Slurry Loop pumps versus flow achieved was recorded in a Laboratory Notebook (4). 


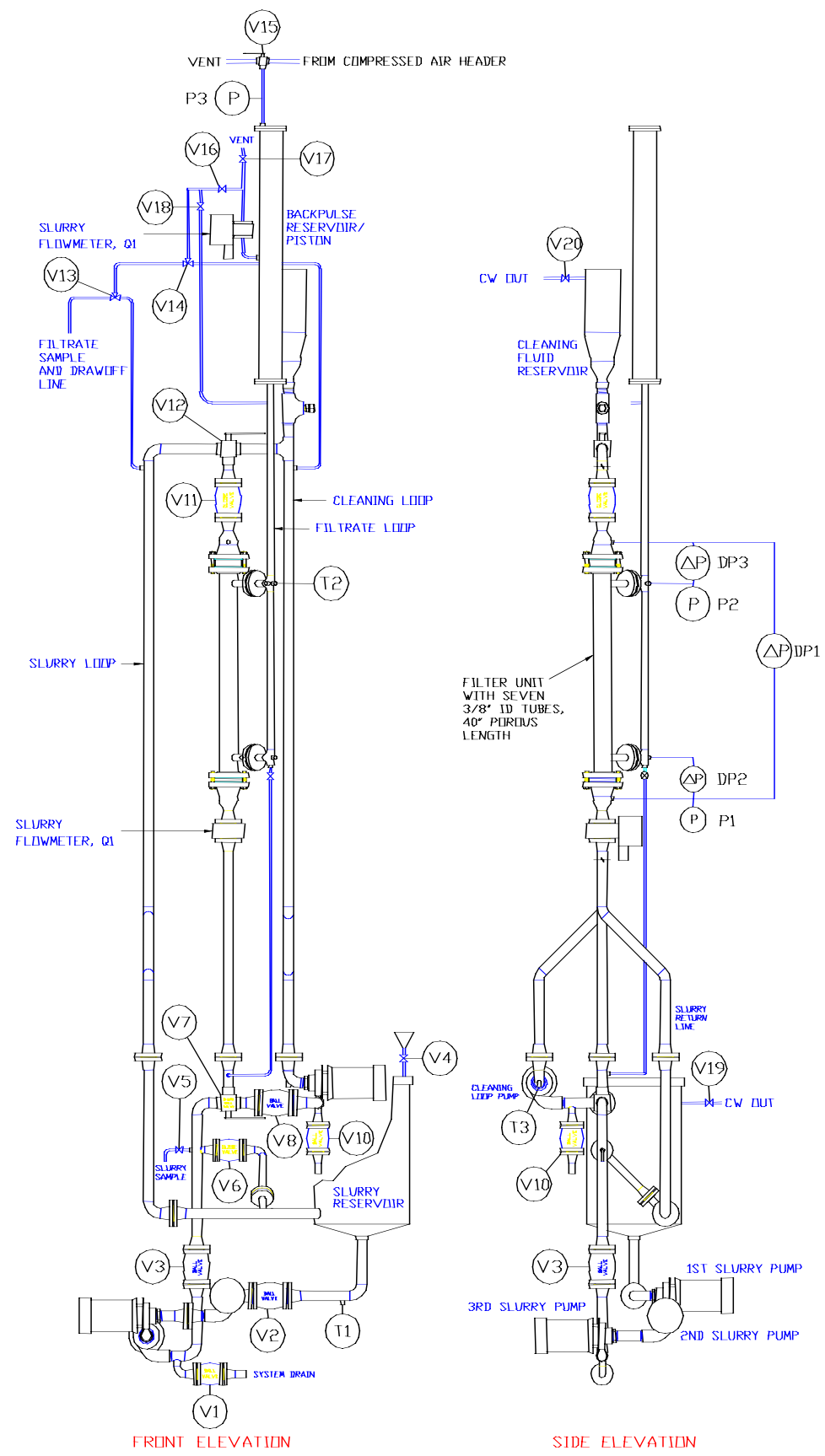

Figure 4-2 Pilot Scale Crossflow Filter Test Rig 


\subsubsection{Crossflow Filter}

The crossflow filter is the primary component in the Crossflow Test Rig used to establish filterability of the precipitate and associated operational characteristics under various flow conditions. Successful past operating experience (9), similarity to the planned RPP filter, and availability dictated the utilization of an existing Mott crossflow filter. The specifications for the filter unit are:

Material: $\quad 316$ stainless steel (sintered metal)

Porosity: nominal rated 0.1 micron

Length: $\quad 40$ inches

Diameter: $\quad$ 3/8-inch ID, $1 / 2$-inch OD

Number of tubes: $\quad 7$

This filter is thoroughly described in Reference 9.

\subsubsection{Slurry Reservoir}

The slurry reservoir is a 110-liter plastic tank that receives feed from the RPP Pilot Precipitation System via the V41 valve. Direct addition to the slurry reservoir is made via the funnel attached to the V4 valve. The precipitated waste simulant in the slurry reservoir was kept well mixed utilizing the slurry pumps in the slurry loop drawing from the bottom of the tank.

\subsection{Instrumentation and Data Acquisition System}

The measurement equipment used to collect data during the precipitation reaction included that recorded by the Pilot Scale Precipitation Data Acquisition System (PSP DAS) listed in Table 4-1 and other instruments not connected to the PSP DAS.

Table 4-1 PSP DAS Channel List for Pilot Scale Precipitation Test Facility

\begin{tabular}{|c|c|c|c|c|c|}
\hline CHAN & INSTRUMENT LABEL & $\begin{array}{l}\text { INSTRUMENT } \\
\text { LOCATION }\end{array}$ & RANGE & UNCERTAINTY & M\&TE \\
\hline 0 & HX Outlet TC-T0 & HX OUTLET & 0 to $100^{\circ} \mathrm{C}$ & $+1.1^{\circ} \mathrm{C}$ & TR-02953 \\
\hline 1 & Recirc Pump Outlet TC-T1 & PUMP DISCH & 0 to $100^{\circ} \mathrm{C}$ & $+1.2^{\circ} \mathrm{C}$ & TR-02948 \\
\hline 2 & Tank Bottom TC-T2 & TANK & 0 to $100^{\circ} \mathrm{C}$ & $\pm 1.3^{\circ} \mathrm{C}$ & TR-02947 \\
\hline 3 & Heater Outlet TC-T3 & HEATER OUT & 0 to $100^{\circ} \mathrm{C}$ & $\pm 0.9^{\circ} \mathrm{C}$ & TR-02955 \\
\hline 6 & Recirc Pump Flow & RECIRC FLOW & $0-50 \mathrm{gpm}$ & $\pm 0.2 \mathrm{gpm}$ & TR-03661 \\
\hline 7 & $\mathrm{NaMnO}_{4}$ Tank Flow & REAGENT FLOW1 & $0-5 \mathrm{gpm}$ & $\pm 0.3 \mathrm{gpm}$ & TR-03563 \\
\hline 8 & $\mathrm{Sr}\left(\mathrm{NO}_{3}\right)_{2}$ Tank Flow & REAGENT FLOW2 & $0-6.2 \mathrm{gpm}$ & $\pm 0.02 \mathrm{gpm}$ & TR-03670 \\
\hline 9 & Ammeter & $\begin{array}{l}\text { TEMPERATURE } \\
\text { CONTROLLER }\end{array}$ & $0-200 \mathrm{amps}$ & * & $3-1982$ \\
\hline 10 & Voltmeter & $\begin{array}{l}\text { TEMPERATURE } \\
\text { CONTROLLER }\end{array}$ & $0-200$ volts & * & $3-1981$ \\
\hline
\end{tabular}

* Uncertainty not determined. The ammeter and voltmeter information were recorded in the raw PSP DAS data simply to show when the heater was on or off. 
The uncertainty introduced through the use of the 16-bit data acquisition system (DAS) was insignificant $(<0.1 \%$ reading $)$ and was not included in the values above.

Instruments not connected to the PSP DAS, but used during the experiment include the following:

A specific gravity hydrometer to measure the ratio of the density of the slurry sample compared to the density of pure water at $60{ }^{\circ} \mathrm{F}\left(15.6{ }^{\circ} \mathrm{C}\right)$ with a range of 1.000 to $1.600 \mathrm{~g} / \mathrm{mL}$ and an accuracy of $\pm 0.005 \mathrm{~g} / \mathrm{mL}$

A variable frequency drive (VFD) for the agitator with a range of 60 to 550 with a 5\% accuracy

A VFD for the Precipitation Tank Recirculation Pump with indication from 0 to $60 \mathrm{~Hz}$ and an accuracy of $0.010 \mathrm{~Hz}$. A PID control loop on the DAS takes input from magnetic flow meter TR03661 and outputs a signal to the VFD to control flow in the range of 0 to $50 \mathrm{gpm}$.

A Temperature Controller connected to a $6 \mathrm{~kW}$ heater and two Type E thermocouples with an accuracy of $\pm 2.0^{\circ} \mathrm{C}$

The measurement equipment used to collect data during filtration included that recorded by the Crossflow Data Acquisition System (Xflow DAS) listed in Table 4-2 and other instruments not connected to the Xflow DAS.

Table 4-2 Xflow DAS Channel List for Crossflow Filter Test Rig

\begin{tabular}{|c|l|l|l|l|l|}
\hline CHAN & INSTRUMENT & INSTRUMENT & RANGE & UNCER- & M\&TE \\
\hline & LABEL & LOCATION & & TAINTY & NUMBER \\
\hline D I/O & V-15 Solenoid & Solenoid Control & Open or closed & $*$ & Solenoid \\
\hline 0 & Filtrate TC -T2 & FLTRT $\left({ }^{\circ} \mathrm{C}\right)$ & 0 to $100{ }^{\circ} \mathrm{C}$ & $\pm 1.0{ }^{\circ} \mathrm{C}$ & TR-02927 \\
\hline 1 & $\begin{array}{l}\text { Cleaning Loop TC- } \\
\text { T3 }\end{array}$ & CL LOOP $\left({ }^{\circ} \mathrm{C}\right)$ & 0 to $100{ }^{\circ} \mathrm{C}$ & $\pm 1.2{ }^{\circ} \mathrm{C}$ & TR-02930 \\
\hline 2 & Slurry Loop TC-T1 & SL LOOP $\left({ }^{\circ} \mathrm{C}\right)$ & 0 to $100{ }^{\circ} \mathrm{C}$ & $\pm 1.4{ }^{\circ} \mathrm{C}$ & TR-02929 \\
\hline 3 & $\begin{array}{l}\text { Upper Ambient TC- } \\
\text { T4 }\end{array}$ & UP AMB $\left({ }^{\circ} \mathrm{C}\right)$ & 0 to $100{ }^{\circ} \mathrm{C}$ & $\pm 1.3{ }^{\circ} \mathrm{C}$ & TR-02925 \\
\hline 4 & $\begin{array}{l}\text { Bottom Ambient } \\
\text { TC-T5 }\end{array}$ & BOT AMB $\left({ }^{\circ} \mathrm{C}\right)$ & 0 to $100{ }^{\circ} \mathrm{C}$ & $\pm 1.4{ }^{\circ} \mathrm{C}$ & TR-02926 \\
\hline 6 & Bottom DP-DP2 & BOT DP $(\mathrm{psid})$ & $0-100 \mathrm{psid}$ & $\pm 0.1 \mathrm{psid}$ & TR-00532 \\
\hline 7 & Filter Pressure-P1 & FLTR $(\mathrm{psig})$ & $0-100 \mathrm{psig}$ & $\pm 0.1 \mathrm{psig}$ & TR-02917 \\
\hline 8 & Filter DP-DP1 & FLTR DP $(\mathrm{psid})$ & $0-26 \mathrm{psid}$ & $\pm 0.03 \mathrm{psid}$ & TR-03495 \\
\hline 9 & Top DP-DP3 & TOP DP $(\mathrm{psid})$ & $0-100 \mathrm{psid}$ & $\pm 0.8 \mathrm{psid}$ & TR-03115 \\
\hline 10 & Filtrate Pressure-P2 & FLTRATE $(\mathrm{psig})$ & $0-91 \mathrm{psig}$ & $\pm 0.2 \mathrm{psig}$ & TR-03109 \\
\hline 11 & Piston Pressure-P3 & PISTON $(\mathrm{psig})$ & $0-151 \mathrm{psig}$ & $\pm 0.3 \mathrm{psig}$ & TR-02145 \\
\hline 12 & Filter Flow-Q2 & FLTR FLOW $(\mathrm{gpm})$ & $0-1.21 \mathrm{gpm}$ & $\pm 0.01 \mathrm{gpm}$ & TR-20353 \\
\hline 13 & Slurry Flow-Q1 & SL FLOW $(\mathrm{gpm})$ & $0-100 \mathrm{gpm}$ & $\pm 0.4 \mathrm{gpm}$ & TR-20350 \\
\hline 14 & HI Filter Flow-Q3 & FLTR FLOW $(\mathrm{gpm})$ & $0-5 \mathrm{gpm}$ & $\pm 0.01 \mathrm{gpm}$ & TR-03562 \\
\hline
\end{tabular}

* Uncertainty not applicable as the solenoid valve is either open or closed with a value of " 1 " or “ 0 " respectively. 
Instruments not connected to the Xflow DAS, but used during the experiment include the following:

A Type $\mathrm{J}$ thermocouple with accuracy of $1.1{ }^{\circ} \mathrm{C}$ used for the Sample Drying Oven with local indication, and

A VFD for each Slurry Pump manually controlled from 0 to $60 \mathrm{~Hz}$ with an accuracy of $0.010 \mathrm{~Hz}$.

The uncertainties in the instrument readings were based on multiple point calibrations with reference standards. Calibration sheets are included in the task logbook (4).

The velocity through the crossflow filter tubes is calculated based on the slurry loop flow measured by magnetic flowmeter Q1 divided by the cross-sectional area of seven tubes with 3/8" nominal ID. The uncertainty in this calculated value is the combination of the instrument uncertainty and the uncertainty in the flow area. A typical flow in the slurry loop exceeds 10 gpm. The uncertainty in the instrument is therefore 3.6\% or less. An accurate measurement of the average inside diameter of the filtrate tubes was imposible since it may vary down the length of each filter tube and may vary from tube to tube. Even measuring the diameter at the filter tube ends is difficult because of the weldments to the tube sheets. For these reasons, the uncertainty in flow area will be based on the manufacturer's stated tolerances. For a Mott 3/8" tube the diameter tolerances are stated to be +0.025 inch and -0.005 inch. The diameter of the filter tubes can presumably vary anywhere between those tolerances; therefore, for this task the diameter uncertainty will be taken as $+/-0.015$ inch, or $4 \%$ of the nominal diameter. The combined uncertainty is $\left(3.6 \% \%^{\wedge} 2+4.0 \% \wedge 2\right)^{\wedge} 1 / 2=5.4 \%$.

The transmembrane pressure is calculated by averaging the differential pressure between the slurry side and the filtrate side at the top (DP3) and bottom (DP2) of the filter. The uncertainty for these instruments at about $40 \mathrm{psid}$ is $0.74 \mathrm{psid}$ or about $1.8 \%$. The combined uncertainty would be about $(1.8 \% \wedge 2+1.8 \% \wedge 2)^{\wedge} 1 / 2=2.5 \%$.

The filtrate flow is calculated based on either magnetic flowmeters Q2 or Q3, depending on the range of flow, divided by the inside surface area of the filtrate tubes. A typical flow rate is 0.5 gpm. The instrument uncertainty is about $0.014 \mathrm{gpm}$, or $2.8 \%$. The uncertainty of the inside diameter of the filter tubes has already been addressed above. The uncertainty of the length of the filter tubes was estimated from in-house measurements as $1 / 8$ inch, or $0.3 \%$ of the nominal 40 " length. The combined uncertainty is about $\left(2.8 \% \wedge 2+4.0 \%^{\wedge} 2+0.3 \% \wedge 2\right)^{\wedge} 1 / 2=4.9 \%$.

\subsection{AN-107 Simulated Waste Description}

A 634-liter volume of Envelope C Tank AN-107 simulant was used for the initial feed of Batch \#2. The non-radioactive AN-107 simulant recipe was chemically similar to the radioactive waste characterized in Hanford Tank AN-107 and included transition metals, complexing agents, other organic compounds and entrained solids. An approved recipe from Eibling and Nash (15) was used per the Task Plan (1). Optima Chemical was selected as the supernate vendor to supply 1300 liters of AN-107 simulant supernate (see Table 4-3). 
The partial simulant obtained from Optima contained 12\% more sodium EDTA than desired. (See section 1.5 .3 for a more complete explanation.) Small deviations in the water content caused the volume to be about $1.5 \%$ less than specified resulting in correspondingly higher density and chemical concentrations.

Entrained solids $(0.5 \mathrm{w} \%)$ and sodium perrhenate were added to the purchased partial supernate simulant in the Precipitate Tank at the EDL to complete the AN-107 simulant.

Surrogates for the transuranic compounds were cerium nitrate, lanthanum nitrate, and neodymium nitrate. These surrogates were selected in sufficient quantity to be comparable to those selected by Nash et al. (10) for bench scale studies. This selection assumes that the lanthanides react chemically in the same manner as the actinides. Sodium perrhenate was added as a surrogate for technetium in small quantity, based on the work of Darab and Smith (14), so that filtrate produced could later be used to demonstrate the effectiveness of cesium and technetium removal by ion exchange. 
Table 4-3 Batch \#2 Recipe before Precipitating Reagents added

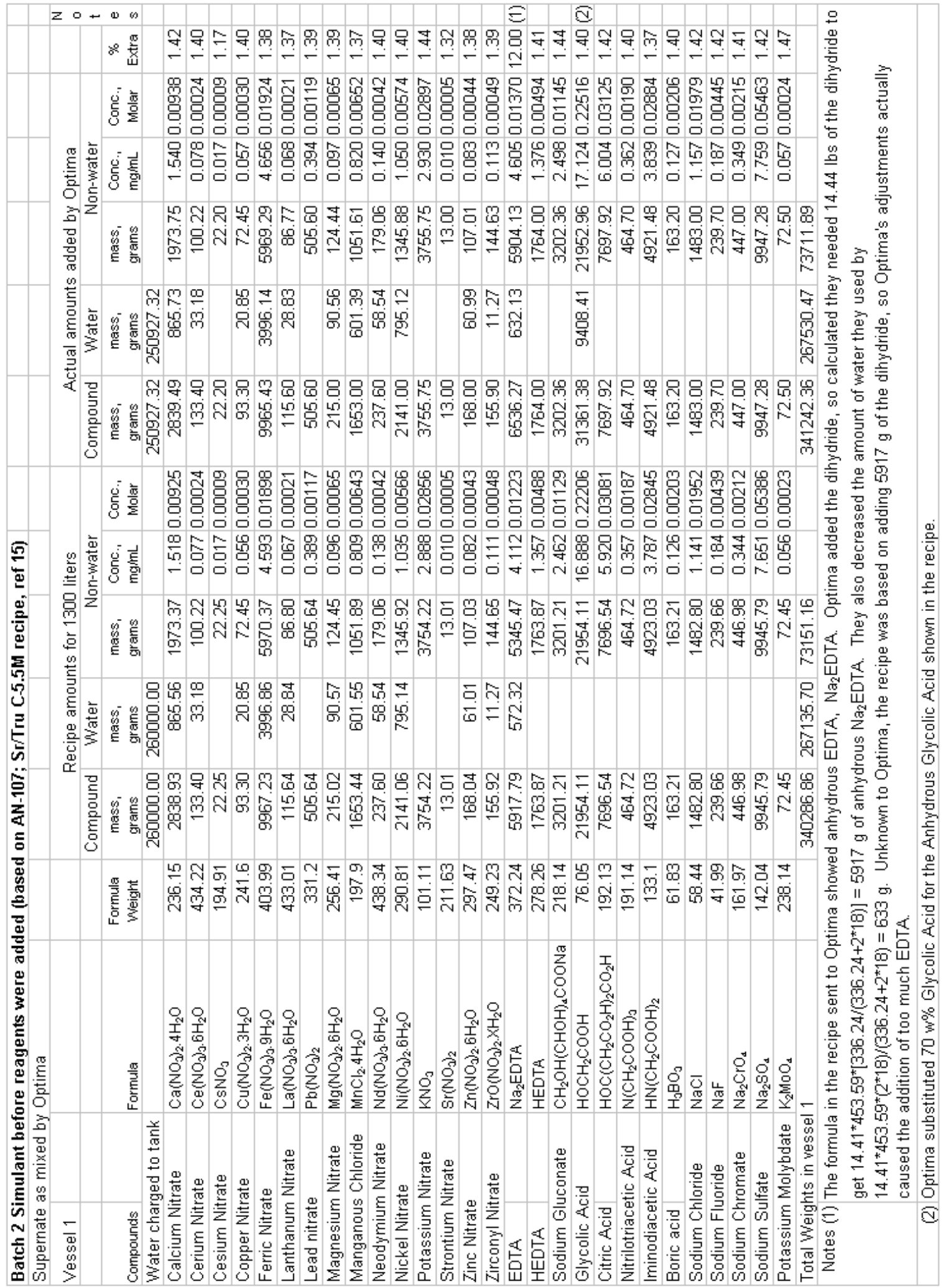


WSRC-TR-2002-00459, Rev. 0

SRT-RPP-2002-00221

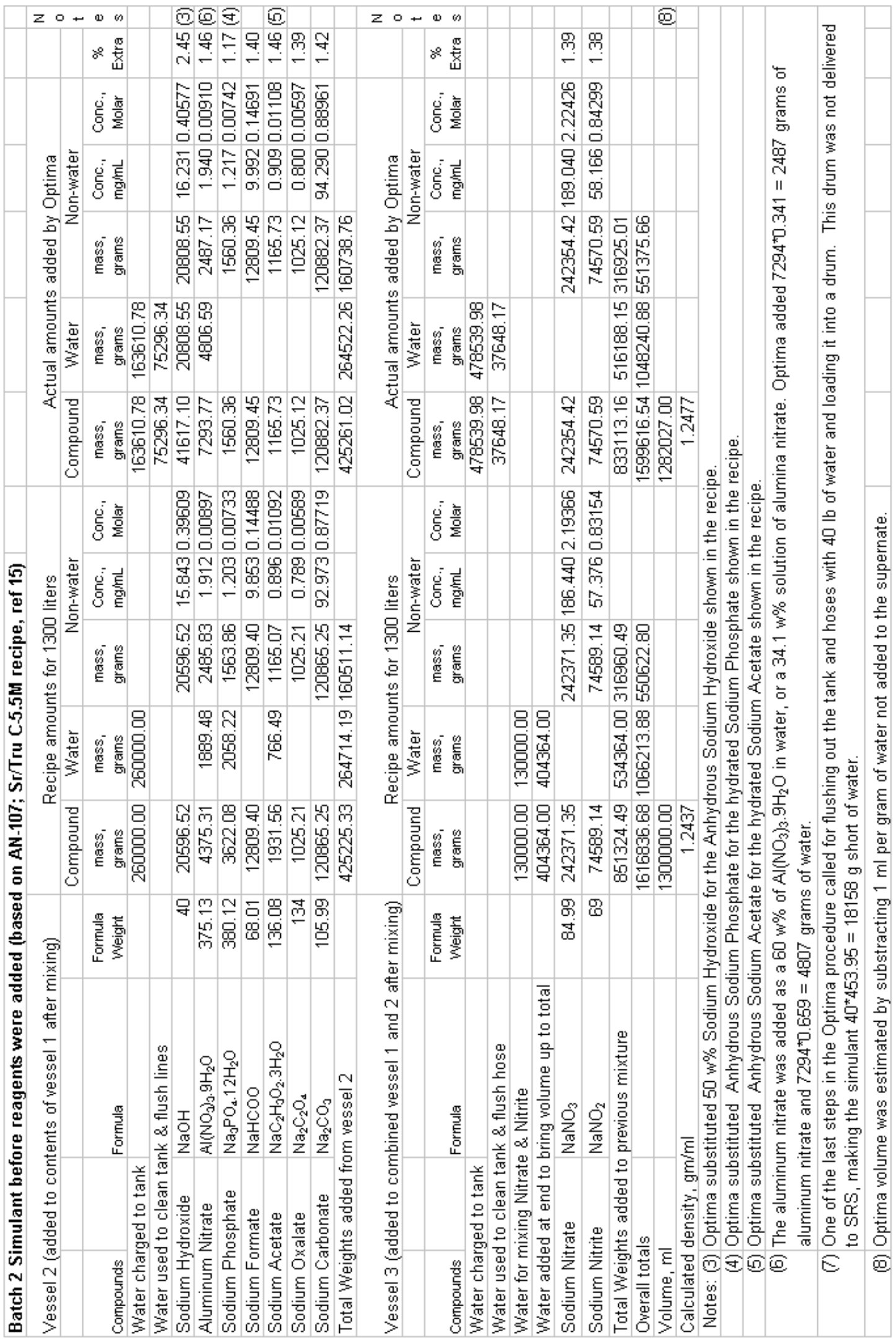




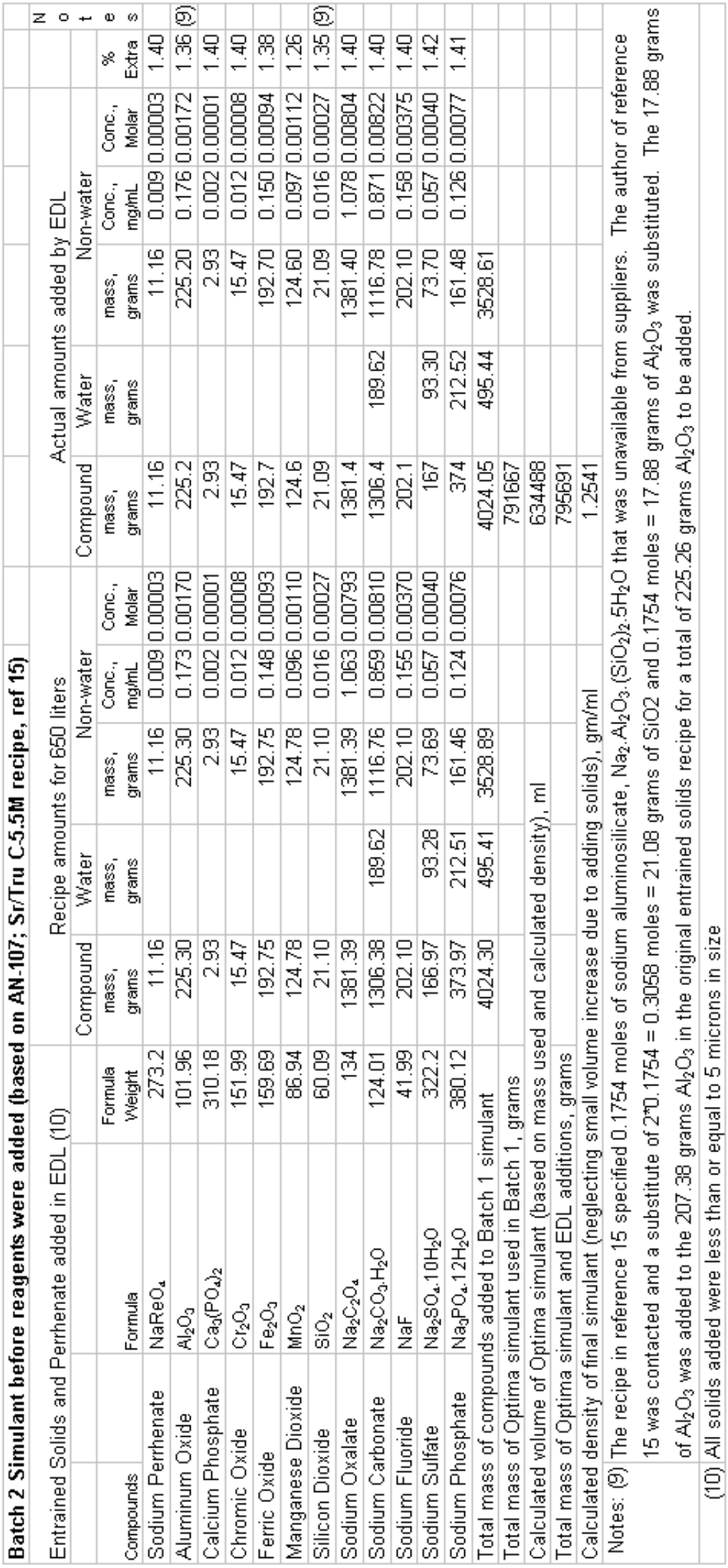




\subsubsection{Test Procedure Summary}

\begin{tabular}{|c|c|c|c|}
\hline Time & $\begin{array}{l}\text { M/D, } \\
2001 \\
\text { UOS }\end{array}$ & Action & Condition/Comment \\
\hline $12: 45$ & $10 / 11$ & $\begin{array}{l}\text { Filled Precipitate Tank w/AN-107 simulant supernate, } \\
\text { solids and Sodium Perrhenate }\end{array}$ & $\begin{array}{l}\text { Well mixed at } 20^{\circ} \mathrm{C} \text { and Task Plan Conditions } \\
\text { for Batch \#2 }\end{array}$ \\
\hline 08:54 & $10 / 23$ & $\begin{array}{l}\text { Started caustic addition of } 34 \text { liters at } 19 \mathrm{M} \mathrm{NaOH} \text { to } \\
\text { raise } \mathrm{NaOH} \text { concentration by } 1 \mathrm{M} \text {. }\end{array}$ & $\begin{array}{l}\text { Test Conditions for one hour before caustic } \\
\text { addition include Recirculation Pump flow } 9.64 \\
\text { gpm, Agitator } 327.6 \mathrm{rpm} \text {, TCs } 20^{\circ} \mathrm{C} \text {. }\end{array}$ \\
\hline $09: 15$ & $10 / 23$ & $\begin{array}{l}\text { Caustic adjustment completed and first Slurry Sample } \\
(1 \mathrm{~L}+1 \mathrm{~S}) \text { taken }\end{array}$ & $\begin{array}{l}\text { Sample vacuum filtered and solids before } \\
\text { reagent addition completed. }\end{array}$ \\
\hline 09:25 & $10 / 23$ & Started strontium nitrate addition & Addition complete at $09: 32$ \\
\hline $09: 43$ & $10 / 23$ & Started sodium permanganate addition & Addition complete at 09:47 \\
\hline 09:47 & $10 / 23$ & First vapor sample $1 \mathrm{G}$ completed & Sample to ADS for analysis. \\
\hline 09:54 & $10 / 23$ & $\begin{array}{l}\text { Second Slurry Sample }(2 \mathrm{~L}+2 \mathrm{~S}) \text { taken at } 7.5 \text { minutes } \\
\text { after reagent addition completed }\end{array}$ & Sample vacuum filtered and solids dried \\
\hline 09:57 & $10 / 23$ & Second vapor sample $2 \mathrm{G}$ completed & Sample to ADS for Analysis \\
\hline 10:03 & $10 / 23$ & $\begin{array}{l}\text { Third Slurry Sample }(3 \mathrm{~L}+3 \mathrm{~S}) \text { taken at } 15 \text { minutes after } \\
\text { reagent addition completed }\end{array}$ & Sample vacuum filtered and solids dried \\
\hline $10: 18$ & $10 / 23$ & $\begin{array}{l}\text { Fourth Slurry Sample }(4 \mathrm{~L}+4 \mathrm{~S}) \text { taken at } 30 \text { minutes } \\
\text { after reagent addition completed }\end{array}$ & Sample vacuum filtered and solids dried \\
\hline $10: 48$ & $10 / 23$ & $\begin{array}{l}\text { Fifth Slurry Sample }(5 \mathrm{~L}+5 \mathrm{~S}) \text { taken at } 60 \text { minutes after } \\
\text { reagent addition completed }\end{array}$ & Sample vacuum filtered and solids dried \\
\hline 11:00 & $10 / 23$ & Third vapor sample $3 \mathrm{G}$ completed & Sample to ADS for Analysis \\
\hline $11: 47$ & $10 / 23$ & $\begin{array}{l}\text { Sixth Slurry Sample }(6 \mathrm{~L}+6 \mathrm{~S}) \text { taken at } 120 \text { minutes } \\
\text { after reagent addition completed }\end{array}$ & Sample vacuum filtered and solids dried \\
\hline $12: 45$ & $10 / 23$ & $\begin{array}{l}\text { Seventh Slurry Sample }(7 \mathrm{~L}+7 \mathrm{~S}) \text { taken at } 180 \text { minutes } \\
\text { after reagent addition completed }\end{array}$ & Sample vacuum filtered and solids dried \\
\hline $12: 55$ & $10 / 23$ & Fourth vapor sample $4 \mathrm{G}$ completed & Sample to ADS for Analysis \\
\hline $13: 00$ & $10 / 23$ & First filtrate sample (PF2-1) taken for Lasentec. & $\begin{array}{l}3.2 \text { hours reaction time after reagent addition } \\
\text { completed }\end{array}$ \\
\hline $13: 44$ & $10 / 23$ & $\begin{array}{l}\text { Eighth Slurry Sample }(8 \mathrm{~L}+8 \mathrm{~S}) \text { taken at } 240 \text { minutes } \\
\text { after reagent addition completed }\end{array}$ & Sample vacuum filtered and solids dried \\
\hline $14: 44$ & $10 / 23$ & $\begin{array}{l}\text { Filled Crossflow Filter Slurry Reservoir and started } \\
\text { filtering }\end{array}$ & $\mathrm{V}=10.25 \mathrm{ft} / \mathrm{sec}, \mathrm{TMP}=48.74 \mathrm{psid}$ \\
\hline $15: 44$ & $10 / 23$ & $\begin{array}{l}\text { Ninth Slurry Sample }(9 \mathrm{~L}+9 \mathrm{~S}) \text { taken at } 360 \text { minutes } \\
\text { after reagent addition completed }\end{array}$ & Sample vacuum filtered and solids dried \\
\hline $17: 44$ & $10 / 23$ & $\begin{array}{l}\text { Tenth Slurry Sample }(10 \mathrm{~L}+10 \mathrm{~S}) \text { taken at } 480 \text { minutes } \\
\text { after reagent addition completed. }\end{array}$ & Sample vacuum filtered and solids dried. \\
\hline $00: 15$ & $10 / 24$ & Took second filtrate sample (PF2-2) for Lasentec. & $\begin{array}{l}14.5 \text { hours reaction time after reagent addition } \\
\text { completed }\end{array}$ \\
\hline $07: 30$ & $10 / 24$ & Took third filtrate sample (PF2-3) for Lasentec. & $\begin{array}{l}21.7 \text { hours reaction time after reagent addition } \\
\text { completed }\end{array}$ \\
\hline $16: 00$ & $10 / 24$ & Took fourth filtrate sample (PF2-4) for Lasentec. & $\begin{array}{l}30.2 \text { hours reaction time after reagent addition } \\
\text { completed }\end{array}$ \\
\hline 00:05 & $10 / 25$ & Took fifth filtrate sample (PF2-5) for Lasentec. & $\begin{array}{l}38.3 \text { hours reaction time after reagent addition } \\
\text { completed }\end{array}$ \\
\hline $07: 30$ & $10 / 25$ & Took sixth filtrate sample (PF2-6) for Lasentec. & $\begin{array}{l}45.7 \text { hours reaction time after reagent addition } \\
\text { completed }\end{array}$ \\
\hline $14: 00$ & $11 / 13$ & $\begin{array}{l}\text { Completed pulling a } 100 \mathrm{ml} \text { ADS Sample } 172182 \text { from } \\
\text { AN-107 Slurry Drum Batch \#2 }\end{array}$ & $\begin{array}{l}\text { Sample submitted to ADS for Microtrac } \\
\text { Analysis of slurry solids }\end{array}$ \\
\hline $11: 30$ & $11 / 20$ & $\begin{array}{l}\text { Completed pulling several liters of Coliwasa samples } \\
\text { from the Batch } \# 2 \text { Filtrate in drums } \# 2 \text { and } \# 3 \text { in } \\
\text { storage for ADS Sample } 172483 \text { solids analysis }\end{array}$ & $\begin{array}{l}\text { The samples collected were vacuum filtered } \\
\text { and dried to produce approximately one gram } \\
\text { of solids for XRD, ICP-MS and ICP-ES } \\
\text { analysis. }\end{array}$ \\
\hline $11: 00$ & $\begin{array}{l}01 / 22 \\
2002\end{array}$ & $\begin{array}{l}\text { Completed pulling a one liter liquid sample from AN- } \\
107 \text { Batch \#2 Filtrate Drum for Analysis by the Mobile } \\
\text { Lab }\end{array}$ & $\begin{array}{l}\text { ICP-ES elemental analysis performed for } \\
\text { comparison with ADS } 170454\end{array}$ \\
\hline
\end{tabular}




\subsubsection{Test Procedure Details}

Details of the test execution can be found in the Test Procedure (7) and the accompanying Operational Instructions (8), which are also listed in the Laboratory Notebook (4). AN-107 supernate simulant (634 liters) at $5.5 \mathrm{M}$ sodium concentration and 1.248 specific gravity, remaining metal complexants, and entrained solids were added to a cleaned and empty Precipitate Tank. The measured $\mathrm{pH}$ of Batch \#2 was 10 with a free $\mathrm{OH}^{-}$concentration of $0.04 \mathrm{M}$. (Note: this free $\mathrm{OH}^{-}$measurement was made with an early method using titration to a fixed $\mathrm{pH}$. This early method has since been shown to be fairly inaccurate when applied to the complicated RPP simulants.) The PSP DAS data file in Appendix B was started, the Recirculation Pump was set to provide a flow of $9.64 \mathrm{gpm}$ in the Recirculation Loop, and the Temperature Controller set at 20 ${ }^{\circ} \mathrm{C}$. The agitator speed was set at $327 \mathrm{rpm}$ to produce vigorous mixing, and the precipitate tank contents were maintained for one hour at $20{ }^{\circ} \mathrm{C}$ prior to reagent addition.

Thirty four liters of $50 \mathrm{w} \%$ (19M) caustic was added to the tank and allowed to mix thoroughly. The Liquid Sample Loop flow was established with the liquid sample pump discharge throttled to provide a one-liter sample in 20 seconds. The first one-liter slurry sample of Batch \#2 was collected. The sample was poured into a 0.2-micron nylon filter cup for vacuum filtering to separate the liquid and solids. The solids were not washed before drying at $100{ }^{\circ} \mathrm{C}$ for at least eight hours or longer until the solids freely crumbled. After the solids in the filter cup were dried, they were removed, placed in sample vials and weighed prior to transport to ADS for analysis. The analytical results are provided in Appendix A.

A 56.3-liter batch of 1.0 M strontium nitrate solution in DIF water was mixed in the PPE Reagent Tank and a 38.1-liter batch of $1.0 \mathrm{M}$ sodium permanganate solution in DIF water was mixed in the SS Reagent Tank. (These volumes of the reagents were sufficient to bring the strontium concentration to $0.075 \mathrm{M}$ and the manganese concentration to $0.05 \mathrm{M}$ in the resultant slurry.) The reagent throttle valves were adjusted to establish a recycle of $2.1 \mathrm{gpm}$ ( 8 liters $/ \mathrm{min}$ ). The strontium nitrate was valved to the precipitation tank first, followed by the sodium permanganate eleven minutes later. Immediately following the sodium permanganate addition, the first vapor sample was taken and transported to ADS for analysis. Subsequently, slurry and vapor samples were obtained from the Precipitate System as required by the Test Procedure (7). The results of all ADS analyses are provided in Appendix A.

About 90 liters of the precipitation tank contents were pumped to the Crossflow Filter Slurry Reservoir. The chiller was then started to maintain the slurry at about $25^{\circ} \mathrm{C}$. The Xflow DAS file in Appendix $\mathrm{C}$ was started, and the slurry pumps were started to fill the Slurry and Filtrate Loops. Filtration data was taken for various slurry flows up to $18 \mathrm{ft} / \mathrm{sec}$ and transmembrane pressures up to 50 psid. The slurry flows and transmembrane pressure were varied by adjusting the slurry pump VFD and adjusting V3 and V11 valves controlling the inlet and outlet of the crossflow filter. The crossflow slurry samples were taken from the V5 valve on the Slurry Loop. Filtrate from the filter was collected in polydrums.

Filtration was conducted continuously until the 762 liter batch was concentrated to about 70 liters. The inventory in the crossflow filter slurry loop was maintained between 70 and 90 liters by transferring slurry from the precipitation tank whenever necessary. The contents of the 
precipitation tank were agitated vigorously prior to transfers and the precipitation recirculation pump was then operated to transfer material to the crossflow filter slurry reservoir.

About three hours after reagent addition was completed, a one-liter filtrate sample was collected in a dark gray container for Lasentec particle analysis and observation for post-filtration precipitation. Five more one-liter filtrate samples were taken for Lasentec analysis at later times. In addition to samples taken for Lasentec analysis, two more samples of filtrate were subsequently taken from the filtrate drum about three weeks after the test to characterize postfiltration solids. ADS sample 172182 consisting of $100 \mathrm{~mL}$ of slurry was obtained from the concentrated slurry drum for Microtrac analysis of particle size distribution. Coliwasa (Composite Liquid Waste Samplers) were used repeatedly to obtain representative samples from the filtrate drum. These samples were combined, filtered through a 0.2-micron nylon filter cup, and the solids dried for analysis (sample 172484). Results are provided in Appendix A.

A backpulse was performed prior to shutdown to drain both test rigs. The slurry contents of the crossflow rig were drained into a drum labeled as Batch \#2 slurry. Cleaning operations on the test rigs commenced in accordance with Operating Instructions (8). After cleaning, a DIF water run was made to check the effectiveness of the cleaning operation.

\subsection{DISCUSSION OF RESULTS}

The solids filtered out of one-liter samples of the precipitation tank contents increased from 4.0 grams before precipitation to about 45 grams after precipitation, about a 11-fold increase. Based on a filtrate density of 1.24, the maximum the insoluble solids content after precipitation could have been is $45 \mathrm{gms} /(1240+45) \mathrm{gms} * 100 \%=3.5 \mathrm{w} \%$. Since some of the solids collected were actually dissolved salts that were left behind when the interstitial liquid was evaporated, the actual insoluble solids content is less than this maximum.

The effectiveness of the AN-107 simulant Sr/TRU precipitation reaction with $0.075 \mathrm{M}$ strontium nitrate and $0.05 \mathrm{M}$ sodium permanganate after addition of $1 \mathrm{M}$ additional caustic was determined by the reduction in concentration of strontium and non-radioactive surrogate TRU elements Ce, $\mathrm{La}$, and $\mathrm{Nd}$ in the slurry liquid. Nash et al. (11) report that the $\mathrm{Sr} / \mathrm{TRU}$ reaction with radioactive Large C waste from Hanford Tanks AN-107 and AN-102 reduces strontium-90 by isotopic dilution with non-radioactive strontium nitrate addition through precipitation of strontium carbonate, with permanganate precipitating lanthanides (and TRU elements). Permanganate destroys organics that form soluble complexes with TRU elements. The AN-107 simulant initially added into the Precipitate Tank by the recipe contained non-radioactive lanthanum, neodymium, and cerium as surrogates for the TRU elements along with a very small quantity of strontium. The concentrations of these elements in the liquid as a function of time after reagent addition were determined by ADS as shown in Appendix A. In order to make an accurate comparison, the concentrations after reagent additions need to be corrected for the effect of dilution by the reagents. To make this correction, all concentrations after reagents were added have been multiplied by the appropriate mass dilution factor MD: 
$\mathrm{MD}=\frac{(\text { mass of simulant }+ \text { mass of all additions) }}{\text { (mass of simulant) }}$

After just the caustic addition the mass dilution factor is $\mathrm{MD}_{\mathrm{NaOH}}=(795691$ grams simulant + 51860 grams caustic $) /(795691$ grams simulant $)=1.07$. After all reagents are added the mass dilution factor is $\mathrm{MD}_{\mathrm{All}}$ Reagents $=(795691$ grams +51860 grams caustic +65275 grams strontium nitrate +42660 grams sodium permanganate $) /(795691$ grams simulant $)=1.20$.

Table 5-1 reports the ADS analysis of the Batch \#2 liquid with concentrations corrected for dilution due to reagent addition. EDL sample $1 \mathrm{~L}$ is the liquid portion of the slurry sample collected from the precipitation tank after caustic addition but prior to adding the reagents. EDL samples $2 \mathrm{~L}$ through $8 \mathrm{~L}$ are the liquid portions of the precipitation tank samples collected from one-eighth to four hours after the reagent additions were completed. Since the initial Batch \#2 simulant was made up essentially identically to the initial Batch \#1 simulant, a sample was not collected. The values reported as EDL sample 0L are actually the analytical results from the initial simulant sample of the Batch \#1 experiment. After three hours of reaction time, a portion of the precipitation tank contents was transferred to the Crossflow Filter Rig. EDL samples 9L and 10L are the liquid portions of the slurry samples collected from the Crossflow Filter Rig six and eight hours after the reagent additions were completed. (The amount of concentration due to filtering was insignificant at the time these samples were collected.) There is also a substantial step change in strontium concentration when the strontium nitrate is added. The rapid addition and reaction was not caught by any sample; however, by calculation, the dilution-corrected concentration for strontium would approach $6200 \mu \mathrm{g} / \mathrm{gm}$ if the strontium remained in solution. 
WSRC-TR-2002-00459, Rev. 0 SRT-RPP-2002-00221

Table 5-1 Concentration of Selected Elements in Batch \#2 Liquid Samples

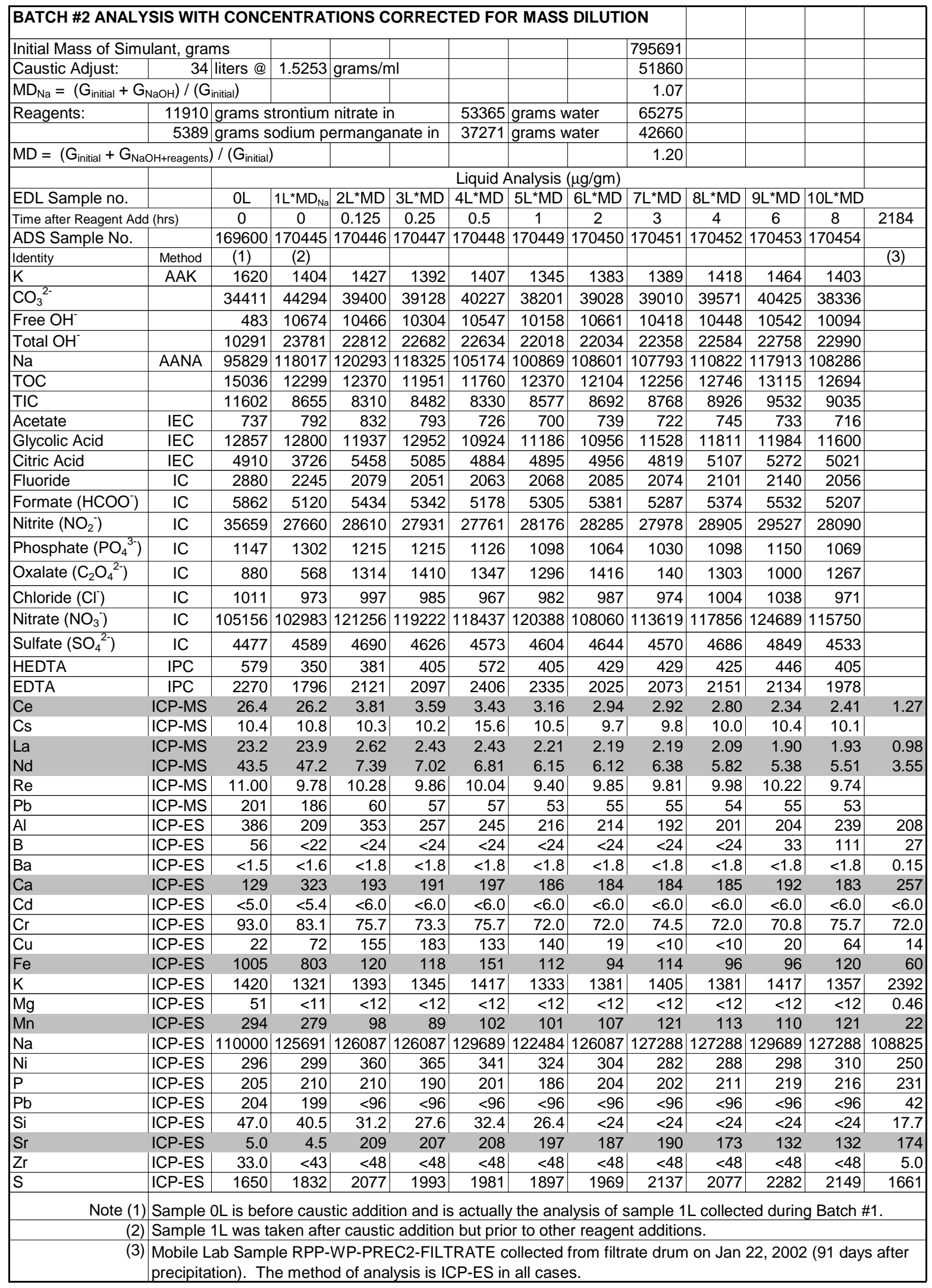


The concentrations of selected elements of interest are shown in the following plots. As mentioned previously, no sample captured the rapid transient concentration of the reagents during addition. No attempt has been made to show these concentrations in the plots.

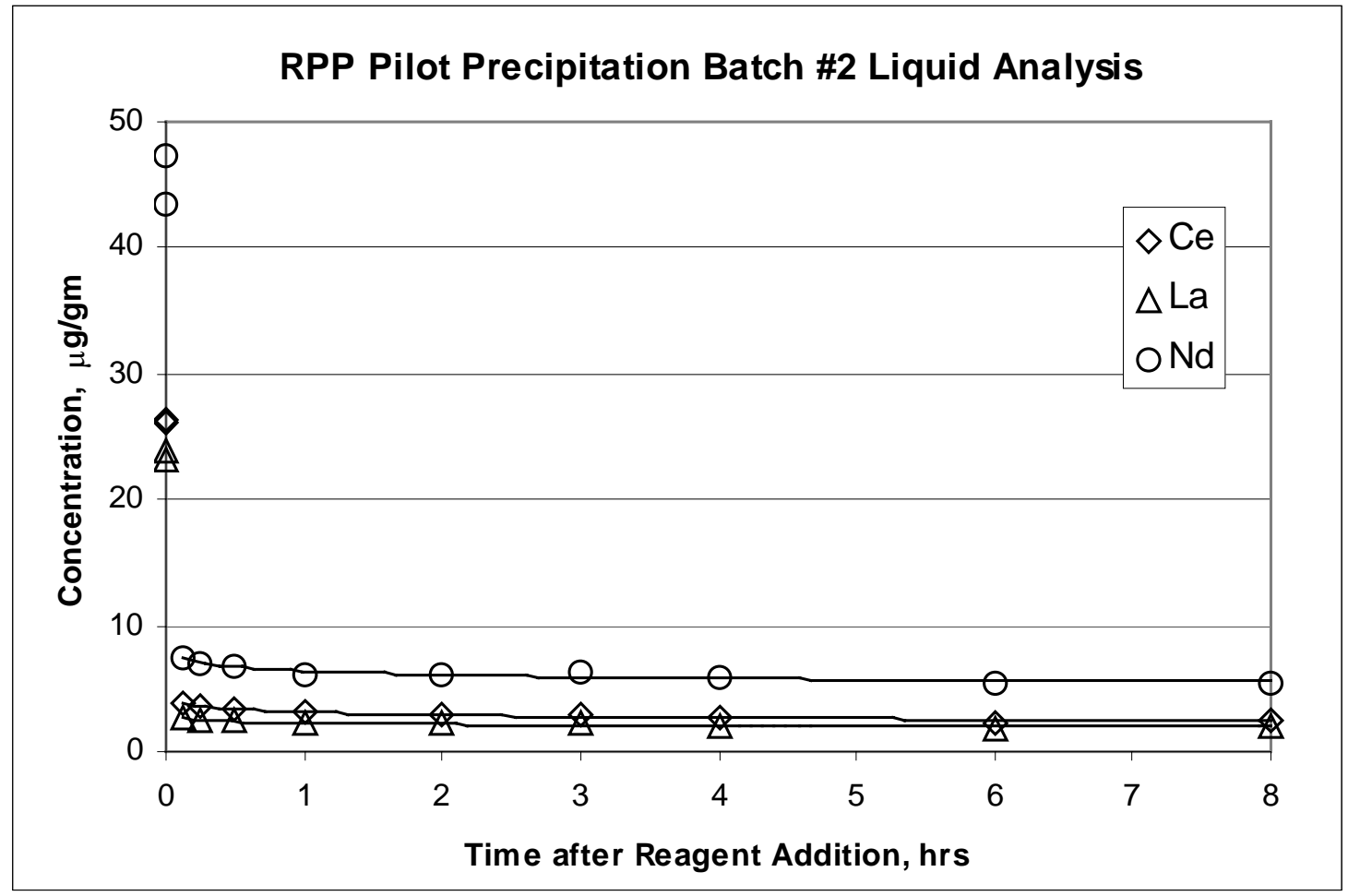

Figure 5-1 Lathanide Elements in Batch \#2 Precipitated Liquid

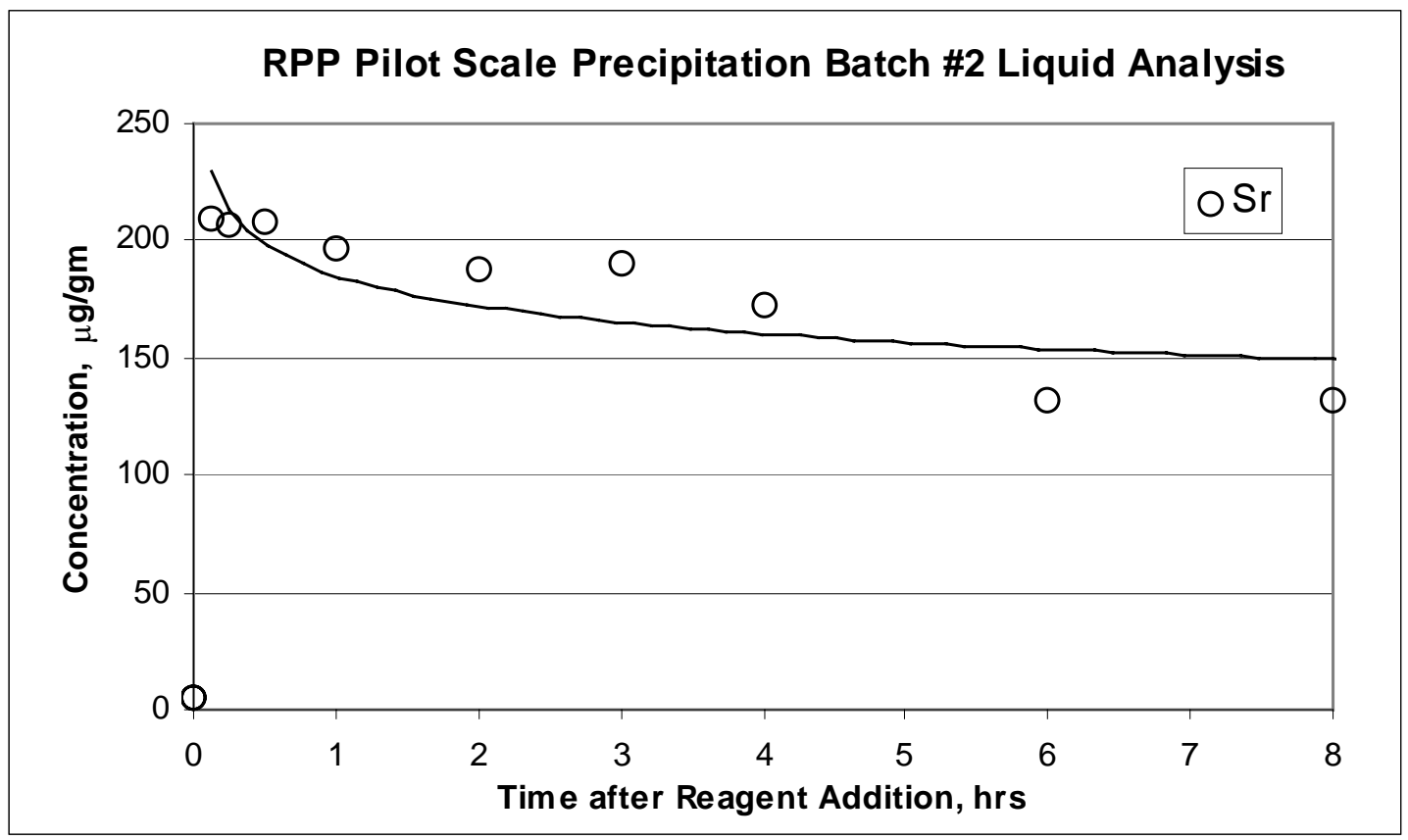

Figure 5-2 Sr in Batch \#2 Precipitated Liquid 


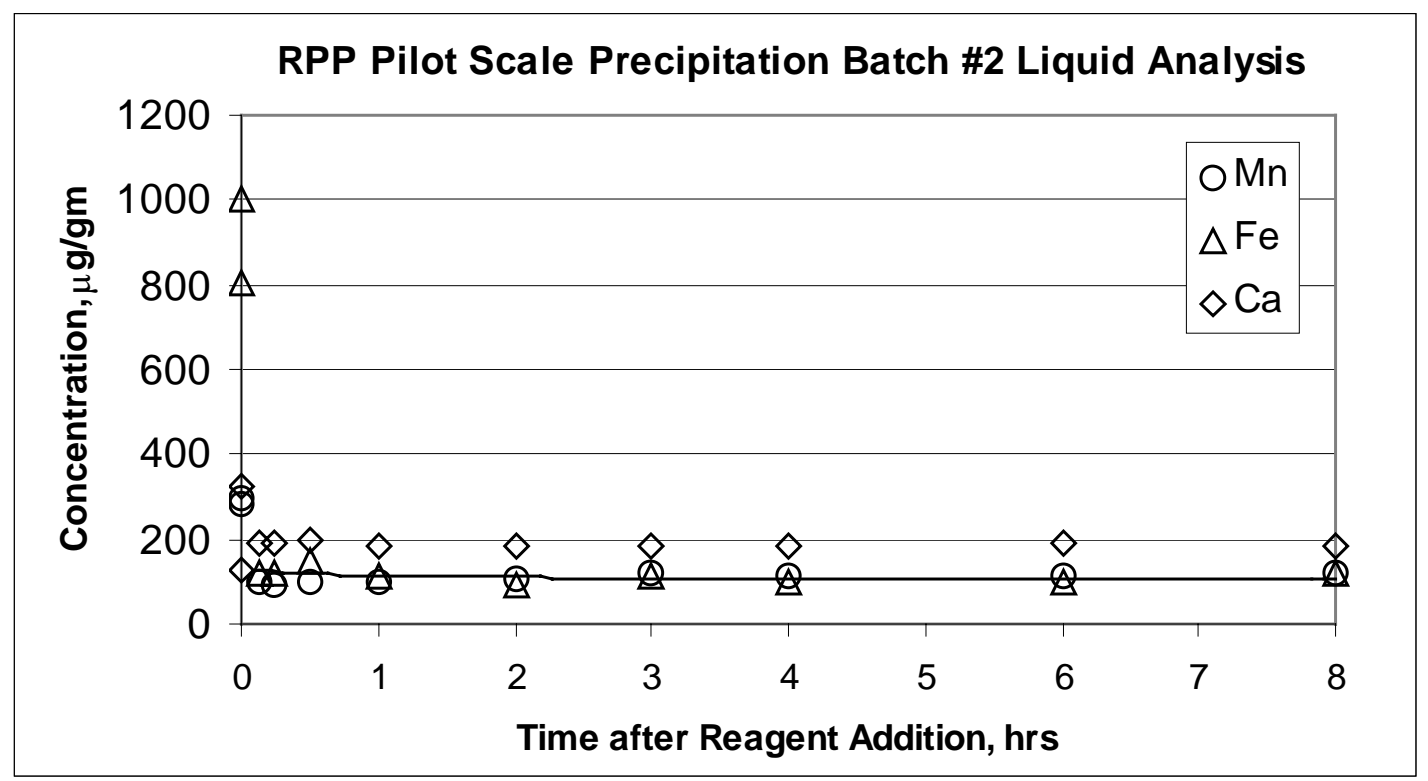

Figure 5-3 Mn, Fe, and Ca in Batch \#2 Precipitated Liquid

It can be seen that about $75 \%$ of the reductions in concentration occur within the first 7.5 minutes after reagent addition, yet the concentrations continue to change for at least 8 hours. The slow, gradual change after the initial fast precipitation reaction is likely due to adsorption onto the solids or substitution of elements in microcrystals rather than continued precipitation from the liquid.

Since the amount of final glass product that has to be made depends on the amount of sodium added to the waste during processing as well as the amount originally in the waste, it is useful to introduce a waste sodium factor defined as:

$\mathrm{WNaC}=\frac{(\text { grams of } \mathrm{Na} \text { in simulant }+ \text { all grams of } \mathrm{Na} \text { added })}{(\text { grams Na in simulant })}$

For the process used in Batch $\# 2, \mathrm{WNaC}=(83156$ grams $+14907+877$ grams $) /(83156$ grams $)$ $=1.19$. The reagents added nineteen percent of the sodium in the treated liquid.

As a common measure of the removal efficiency, the initial amount of the element in the feed is divided by the final amount in the treated liquid; this measure is called the decontamination factor (DF).

DF for element "A" $=\frac{\text { (grams of element "A" in simulant) }}{\text { (grams of element "A" in treated liquid) }}$

$$
=\frac{\text { (mass concentration of element " } \mathrm{A} \text { " in simulant) }}{\text { (mass concentration of element "A" in treated liquid)(MD) }}
$$


The decontamination factor was calculated for the TRU surrogates $\mathrm{La}, \mathrm{Ce}$, and $\mathrm{Nd}$ four hours after addition of reagents. The DFs for Batch \#2 were determined to be 11.1 for La, 9.4 for Ce, and 7.5 for $\mathrm{Nd}$. The DFs obtained for Batch \#2 compared to Batch \#1 (20) were about $80 \%$ higher for $\mathrm{Ce}, 30 \%$ higher for $\mathrm{La}$, and about the same for $\mathrm{Nd}$.

Wilmarth (19) has developed the following approximate correlations between La and Nd DFs and Am DF: $\mathrm{DF}_{\mathrm{Nd}}=0.62 \mathrm{DF}_{\mathrm{Am}}+0.44$ and $\mathrm{DF}_{\mathrm{La}}=0.51 \mathrm{DF}_{\mathrm{Am}}+1.1$. Using these equations the Am DF would be estimated to be in the range 11 to 20. There is at yet no correlation for $\mathrm{Pu}$ and Cm DFs.

In a similar precipitation study on a small sample of diluted real AN-107 waste, Hallen et al. (18) reported $74 \%$ of the neodymium was removed implying a $(1 /(1-0.74))=3.9 \mathrm{DF}$ for $\mathrm{Nd}$. They reported DFs of 22 for Cm-242 and 28 for Am-241. In a similar precipitation study, but with actual AN-102 waste instead of AN-107, Nash et al. (13) reported DFs of 7.2 for Cm-244 and 9.2 for Am-241.

The strontium DF based upon the relative liquid concentrations only was calculated as 0.02 . This calculation used the recipe value of strontium in the simulant rather than the ADS values. Typical small levels of impurities in some of the chemicals added in large amounts could easily increase the strontium in the simulant from the theoretical 2.69 grams to 4 or 5 grams. Since such a large amount of strontium is added as a reagent and precipitated out, the concentration in the treated liquid should have no relation to the amount originally in the simulant. But in real waste, the small amount of strontium in the feed would be strontium-90. Since the 2.69 grams of strontium in the simulant represents the "strontium-90", that is the value that should be used in calculating the DF. The Sr/TRU precipitation studies by Nash et al. (11) identified strontium-90 levels reduced by simple isotopic dilution. The Batch \#2 experiment used a non-radioactive simulant, so the isotopic dilution effect cannot be experimentally determined. The maximum possible isotopic dilution factor can be calculated as shown below for the process used with Batch \#2:

Isotopic Dilution Factor $=$

(Initial Grams of Strontium in simulant plus Strontium added as Precipitating Reagent)

(Initial Grams of Strontium in simulant)

$=(2.69 \mathrm{gm}+4931 \mathrm{gm}) / 2.69 \mathrm{gm}$

$=1834$

Assuming complete isotopic mixing, the DF for radioactive strontium in real waste treated with this process would be the product of the DF based upon the relative liquid concentrations only and the Isotopic Dilution Factor, or DF $=(0.02) *(1834)=37$. This DF is significantly higher than required to meet the immobilization regulatory requirements. Previous bench scale experiments measured strontium decontamination factors in the range 30 to 100. This contrasts with the Batch \#1 (20) result of 1000. (The reader is cautioned that very large DFs can be misleading. A DF of 100 represents removal of $99 \%$ of an element; a DF of 1000 represents removal of $99.9 \%$, only an additional $0.9 \%$.) The most likely significant difference between this experiment and the Batch \#1 experiment is addition of caustic prior to precipitation. The difference in caustic level will cause a difference in strontium solubility. Higher strontium 
solubility will result in more strontium remaining in the liquid and passing through the filter into the filtrate.

X-ray diffraction results (reported in Appendix A) show the presence of $\mathrm{Sr}$ in strontianite and a $\mathrm{Na}, \mathrm{Sr}$, Silicate. The Batch \#1 (20) experiment found a Ca, Sr, Mn oxide in addition to the other two, but this material was not identified in the Batch \#2 solid samples. Although the analysis was not quantitative, most of the strontium is expected to be in the strontianite, $\mathrm{SrCO}_{3}$.

The behavior of highly radioactive elements technetium-99 and cesium-137 are also of considerable interest in waste treatment processes. The concentrations of Tc and Cs in the liquid as reported by Nash et al. (11) were unaffected by the Sr/TRU reaction. Based upon the work of Darab and Smith (14), rhenium was selected as a non-radioactive surrogate for Tc-99 in the Batch \#2 simulant. Rhenium was added as sodium perrhenate. Inspection of the table of dilution corrected concentration shows the rhenium to be unaffected. Cs-132 is a non-radioactive isotope chemically identical to Cs-137 and would be expected to behave the same. Again, inspection of the table of dilution corrected concentration shows the cesium to be unaffected. These results indicate the technetium and cesium in the real waste would be unaffected by the precipitation process used on Batch \#2.

Table 5-1 can be examined to determine some of the other changes in the waste simulant after the reaction. A portion of the carbonate was consumed which would be expected in precipitating the strontium. Other carbon species included in the TIC/TOC analysis did not show significant changes. Analytes in the liquid that decreased significantly in concentration included lead, calcium, iron, magnesium, and boron. In the Batch \#2 experiment the iron removal matched the results observed previously by Nash et al. (11), the Batch \#1 experiment did not remove as much. Higher iron removal would be expected in a solution with higher hydroxide concentrations. There was also a larger fraction of the lead removed in the Batch \#2 experiment compared to the Batch \#1 experiment (about $75 \%$ vs. about $50 \%$ ).

Calculations of DFs for other components in the simulant four hours after the reagents were added are shown below. (The calculated DFs should be viewed in conjunction with Table 5.1, as scatter in the analytical results is not readily apparent with a single point calculation such as this.) 


\section{Table 5-2 Decontamination Factors for Selected Species Four Hours after Reagent Addition}

\begin{tabular}{|c|c|c|c|c|c|c|c|c|}
\hline \multicolumn{9}{|c|}{ Batch \#2 Decontamination Factors } \\
\hline \multicolumn{3}{|c|}{ Initial Mass of Simulant, grams } & & & & & & 795691 \\
\hline Caustic Adjust: & 34 & liters @ & 1.5253 & \multicolumn{2}{|c|}{ grams $/ \mathrm{ml}$} & & & 51860 \\
\hline \multicolumn{3}{|c|}{$M D_{N a}=\left(G_{\text {initial }}+G_{N a O H}\right) /\left(G_{\text {initial }}\right)$} & & & & & & 1.07 \\
\hline \multirow[t]{2}{*}{ Reagents: } & \multicolumn{5}{|c|}{11910 grams strontium nitrate in } & \multirow{2}{*}{\multicolumn{2}{|c|}{$\begin{array}{l}53365 \text { grams water } \\
37271 \text { grams water }\end{array}$}} & 65275 \\
\hline & \multicolumn{5}{|c|}{5389 grams sodium permanganate in } & & & 42660 \\
\hline \multicolumn{6}{|c|}{$M D=$ Mass Dilution factor $=\left(G_{\text {initial }}+G_{\text {reagents }}\right) /\left(G_{\text {initial }}\right)$} & & & 1.20 \\
\hline & & $\begin{array}{c}\text { Recipe } \\
(\mathrm{R}) \\
(\mu \mathrm{g} / \mathrm{gm})\end{array}$ & $\begin{array}{c}\text { EDL } \\
\text { Sample } \\
\text { OL } \\
(\mu \mathrm{g} / \mathrm{gm})\end{array}$ & $\begin{array}{c}\text { EDL } \\
\text { Sample } \\
8 \mathrm{~L} \\
(\mu \mathrm{g} / \mathrm{gm})\end{array}$ & $\begin{array}{c}\text { Dilution } \\
\text { corrected } \\
\text { Conc } \\
(8 \mathrm{~L})^{\star} \mathrm{MD} \\
(\mu \mathrm{g} / \mathrm{gm})\end{array}$ & $\begin{array}{c}\text { Analysis } \\
\text { based } \\
D F= \\
1 \mathrm{~L} /\left(8 \mathrm{~L}^{*} \mathrm{MD}\right) \\
(\mu \mathrm{g} / \mathrm{gm})\end{array}$ & \begin{tabular}{|c|} 
Recipe \\
based \\
$\mathrm{DF}=$ \\
$\mathrm{R} /\left(8 \mathrm{~L}^{*} \mathrm{MD}\right)$ \\
$(\mu \mathrm{g} / \mathrm{gm})$
\end{tabular} & \\
\hline ADS Sample No. & & & 169600 & 170452 & & & & \\
\hline Compound & Method & & & & & & & \\
\hline Acetate & IEC & & 737 & 620 & 745 & 0.99 & & \\
\hline Glycolic Acid & IEC & & 12857 & 9836 & 11811 & 1.09 & & \\
\hline Citric Acid & IEC & & 4910 & 4253 & 5107 & 0.96 & & \\
\hline Fluoride & IC & & 2880 & 1750 & 2101 & 1.37 & & \\
\hline Formate $\left(\mathrm{HCOO}^{-}\right)$ & IC & & 5862 & 4475 & 5374 & 1.09 & & \\
\hline Nitrite $\left(\mathrm{NO}_{2}^{-}\right)$ & IC & & 35659 & 24071 & 28905 & 1.23 & & \\
\hline Phosphate $\left(\mathrm{PO}_{4}^{3-}\right)$ & IC & & 1147 & 914 & 1098 & 1.05 & & \\
\hline Oxalate $\left(\mathrm{C}_{2} \mathrm{O}_{4}{ }^{2-}\right)$ & IC & & 880 & 1085 & 1303 & 0.68 & & \\
\hline Chloride $\left(\mathrm{Cl}^{-}\right)$ & IC & & 1011 & 836 & 1004 & 1.01 & & \\
\hline Nitrate $\left(\mathrm{NO}_{3}^{-}\right)$ & IC & & 105156 & 98146 & 117856 & 0.89 & & \\
\hline Sulfate $\left(\mathrm{SO}_{4}{ }^{2-}\right)$ & IC & & 4477 & 3902 & 4686 & 0.96 & & \\
\hline HEDTA & ICP & 1097 & 579 & 354 & 425 & 1.36 & 2.58 & \\
\hline EDTA & ICP & 3170 & 2270 & 1791 & 2151 & 1.06 & 1.47 & \\
\hline $\mathrm{Ce}$ & ICP-MS & 26.8 & 26.4 & 2.33 & 3 & 9.44 & 9.57 & \\
\hline Cs & ICP-MS & 9.4 & 10.4 & 8.3 & 10 & 1.04 & 0.94 & \\
\hline $\mathrm{La}$ & ICP-MS & 23.1 & 23.2 & 1.74 & 2 & 11.10 & 11.04 & \\
\hline $\mathrm{Nd}$ & ICP-MS & 48.6 & 43.5 & 4.85 & 6 & 7.47 & 8.35 & \\
\hline $\operatorname{Re}$ & ICP-MS & 9.56 & 11 & 8.31 & 10 & 1.10 & 0.96 & \\
\hline $\mathrm{Pb}$ & ICP-MS & 197 & 201 & 44.9 & 54 & 3.73 & 3.65 & \\
\hline $\mathrm{Al}$ & ICP-ES & 196 & 386 & 167 & 201 & 1.92 & 0.98 & \\
\hline $\mathrm{Ca}$ & ICP-ES & 300 & 129 & 154 & 185 & 0.70 & 1.62 & \\
\hline $\mathrm{Cr}$ & ICP-ES & 89.3 & 93 & 60 & 72 & 1.29 & 1.24 & \\
\hline $\mathrm{Fe}$ & ICP-ES & 857 & 1005 & 80 & 96 & 10.46 & 8.92 & \\
\hline $\mathrm{K}$ & ICP-ES & 911 & 1420 & 1150 & 1381 & 1.03 & 0.66 & \\
\hline $\mathrm{Mn}$ & ICP-ES & 285 & 294 & 94 & 113 & 2.60 & 2.53 & \\
\hline $\mathrm{Na}$ & ICP-ES & 111132 & 110000 & 106000 & 127288 & 0.86 & 0.87 & \\
\hline $\mathrm{Ni}$ & ICP-ES & 269 & 296 & 240 & 288 & 1.03 & 0.93 & \\
\hline $\mathrm{P}$ & ICP-ES & 222 & 205 & 176 & 211 & 0.97 & 1.05 & \\
\hline $\mathrm{Sr}$ & ICP-ES & 3.35 & 5 & 144 & 173 & 0.03 & 0.02 & \\
\hline$S$ & ICP-ES & 1396 & 1650 & 1730 & 2077 & 0.79 & 0.67 & \\
\hline
\end{tabular}


The Crossflow Filter Test Rig was operated successfully with different slurry flows and filter pressures with the following feeds:

- AN-107 precipitated simulant slurry

- DIF water after cleaning to confirm that the cleaning performed was adequate to restore filter function

The results of filtration tests with the AN-107 precipitated simulant slurry are shown below. The velocity shown is based on seven tubes with a nominal ID of 3/8 inch and the slurry loop (SL) flow measured with a magnetic flowmeter. Differential pressures are measured between the filter slurry headers and filtrate ports at both the top and bottom ends of the filter. The transmembrane pressure (TMP) is the calculated average of these two differential pressure measurements. The filtrate flow (flux rate) is the total flow from the filtrate housing (measured by a magnetic flowmeter in gpm) divided by the $2.29 \mathrm{ft}^{2}$ inside surface area of the filter.

The amount of solids collected ranged from 40 to 50 (average 45) grams per liter in the samples collected after precipitation. At least a few grams of that were sodium salts left behind when the sample was dead-end filtered. Assuming 41 grams/liter with a filtrate of density 1.24, there were $41 /(1240+41) *(100)=3.2 \mathrm{w} \%$ solids when filtration was started.

Table 5-3 Crossflow Filter Operations Data with AN-107 Simulant

\begin{tabular}{|c|c|c|}
\hline $\begin{array}{c}\text { Velocity } \\
(\mathrm{ft} / \mathrm{sec})\end{array}$ & $\begin{array}{c}\text { TMP } \\
(\mathrm{psi})\end{array}$ & $\begin{array}{c}\text { Filtrate Flow } \\
\left(\mathrm{gpm} / \mathrm{ft}^{2}\right)\end{array}$ \\
\hline 9.8 & 51.2 & 0.026 \\
\hline 13.2 & 45.2 & 0.035 \\
\hline 17.4 & 44.2 & 0.038 \\
\hline 18.3 & 21.1 & 0.028 \\
\hline
\end{tabular}

The filtrate flowrates shown above were corrected to a filtration temperature of $25{ }^{\circ} \mathrm{C}$ by multiplying by the correction factor $\mathrm{CF}=\mathrm{e}^{[(2500)(1 /(273+\text { Slurry Temperature) }-1 / 298)]}$. Filtration temperatures ranged from 24.9 to $27.4{ }^{\circ} \mathrm{C}$, with corresponding correction factors from 1.00 to 0.93. The uncorrected raw data collected during filtration is shown in Appendix C.

A Test Specification was not provided for the pilot precipitation work. The approved Task Plan simply stated that the crossflow filter would be used to demonstrate filterability of the slurry, and a filtration matrix was not specified. In hindsight the filtration conditions chosen by the task leader were not maintained long enough to obtain definitive data in the ranges best matching planned plant operation. The limited amount of data shown in the plot below had to be extrapolated appreciably to provide the values reported in the table above. 


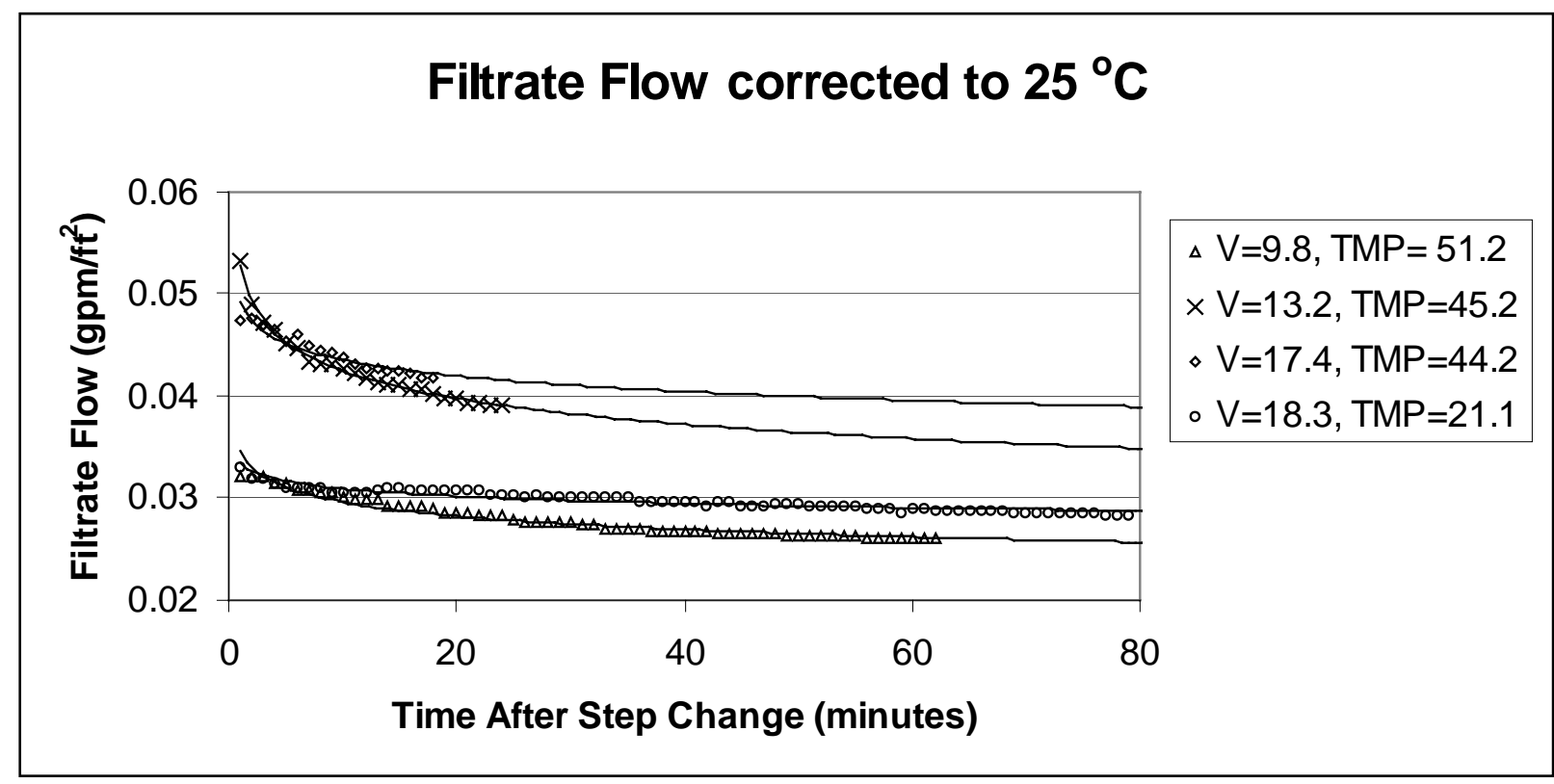

Figure 5.4 Filtrate Production Rate Data

These filtrate rates are similar to those reported by Duignan (17), especially considering the insoluble solids content of approximately $3 \%$ in this study was higher than the $2 \%$ in his study. The slightly higher sodium concentration (6.0 M vs. 5.5 M) may also have had some effect. He measured a flitrate flow of $0.03 \mathrm{gpm} / \mathrm{ft}^{2}$ at an axial velocity $(\mathrm{V})=8.9 \mathrm{ft} / \mathrm{sec}$ and transmembrane pressure $(\mathrm{TMP})=32.1$ psid. The lower value of $0.026 \mathrm{gpm} / \mathrm{ft}^{2}$ at $\mathrm{V}=9.8$ and $\mathrm{TMP}=51.2$ measured in this experiment would be reasonable due to the effect of higher solids. This study measured a flux of about $0.035 \mathrm{gpm} / \mathrm{ft}^{2}$ at $\mathrm{V}=13.2 \mathrm{ft} / \mathrm{sec}$ and TMP $=45.2 \mathrm{psid}$, comparable to Duignan's $0.04 \mathrm{gpm} / \mathrm{ft}^{2}$ at $\mathrm{V}=12.3 \mathrm{ft} / \mathrm{sec}$ and $\mathrm{TMP}=51.1$. The $0.038 \mathrm{gpm} / \mathrm{ft}^{2}$ flux at $\mathrm{V}=17.4$ and $\mathrm{TMP}=44.2$ is lower than expected compared to his $0.52 \mathrm{gpm} / \mathrm{ft}^{2}$ flux at the lower velocity of 15.3 $\mathrm{ft} / \mathrm{sec}$ and lower TMP of $29.6 \mathrm{psid}$

The filtrate fluxes found in this study were slightly higher than obtained in the study of Hallen et al. (18) under similar but not exactly the same conditions. Their study noted the same observation that the flux dropped rapidly in the first 10 minutes after starting filtration then fell gradually for the next 50 minutes. The fluxes reported in their work are averages over the period from 10 minutes to 60 minute after starting filtration; they were: $0.019 \mathrm{gpm} / \mathrm{ft}^{2}$ at $9.0 \mathrm{ft} / \mathrm{sec}$ and $50 \mathrm{psid}, 0.025 \mathrm{gpm} / \mathrm{ft}^{2}$ at $12.2 \mathrm{ft} / \mathrm{sec}$ and $30 \mathrm{psid}$, and $0.024 \mathrm{gpm} / \mathrm{ft}^{2}$ at $13.1 \mathrm{ft} / \mathrm{sec}$ and $49 \mathrm{psid}$.

In general, the fluxes obtained in the Batch \#2 experiment during the abbreviated filtration matrix were similar to the fluxes measured in the Batch \#1 experiment (20).

After collecting the abbreviated filter performance data, filtration was continuous until the volume of slurry was reduced to about 70 liters. Although filter fluxes of about $0.04 \mathrm{gpm} / \mathrm{ft}^{2}$ were achieved during the filter performance testing, long-term operation at the high axial flowhigh transmembrane pressure conditions was not possible due to heat from the pump work. (Additional heat removal capacity will be added to the rig.) Sustained operation at $25{ }^{\circ} \mathrm{C}$ could be maintained with the filter tube velocity set at about $16 \mathrm{ft} / \mathrm{sec}$ and the transmembrane pressure set at about 45 psid. The filtrate production achieved with AN-107 precipitated simulant under 
these conditions and with infrequent backpulsing was about $0.03 \mathrm{gpm} / \mathrm{ft}^{2}$. This initial rate was higher than the $0.02 \mathrm{gpm} / \mathrm{ft}^{2}$ measured under similar conditions in the Batch \#1 experiment (20). Figure 5-5 shows a typical set of data taken early during dewatering. Figure 5-6 shows typical data taken after the slurry volume was reduced from 764 liters to about 70 liters. The flux with the concentrated slurry dropped to about $0.018 \mathrm{gpm} / \mathrm{ft}^{2}$. Hallen et al. (18) measured 0.021 $\mathrm{gpm} / \mathrm{ft}^{2}$ average flux during dewatering with somewhat lower velocity of $11.6 \mathrm{ft} / \mathrm{sec}$ velocity and slightly higher transmembrane pressure of 48 psid.

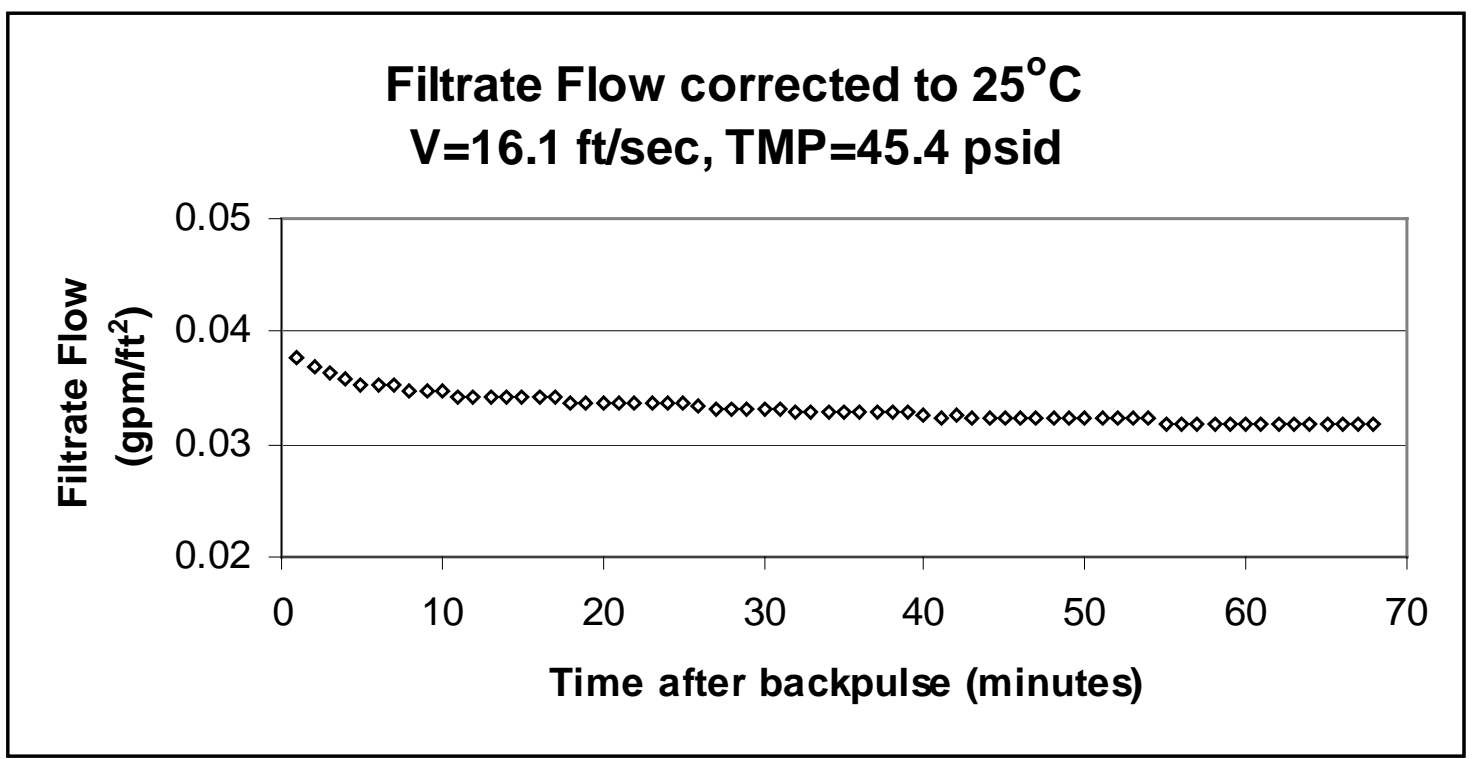

Figure 5-5 Crossflow Filter Filtrate Production with AN-107 Precipitated Simulant Slurry Early During Dewatering

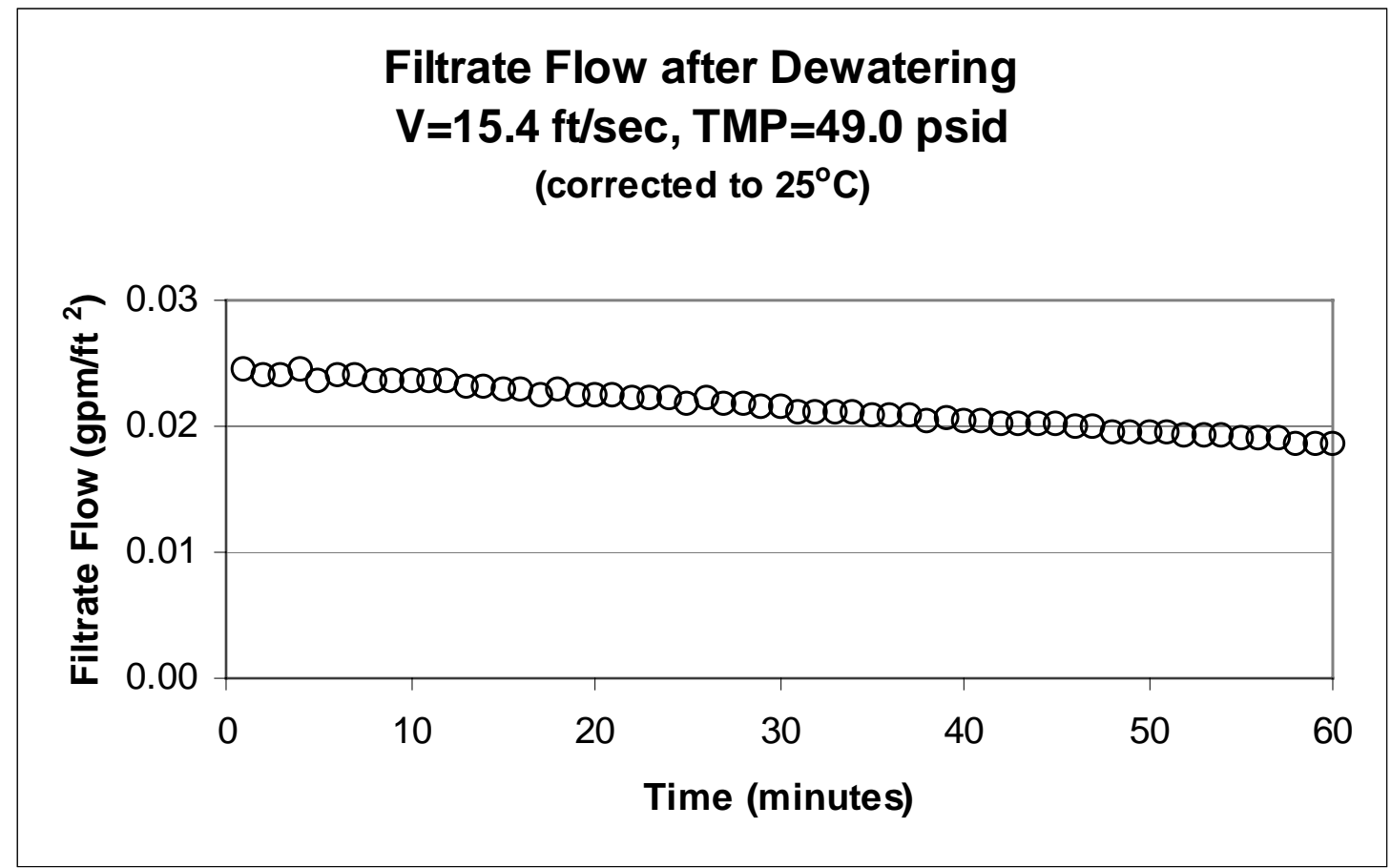


Figure 5-6 Crossflow Filter Filtrate Production with AN-107 Precipitated Simulant Slurry at the End of Dewatering

The filtrate collected from slurry precipitated with this process appeared to be fairly stable. After several months of storage some solids were formed, but there were very little solids formed in filtrate allowed to stand a day or two. This result is in marked contrast to the results of the Batch \#1 experiment (20), where filtrate collected within the first eight hours after precipitation formed significant amounts of solids when allowed to stand for 20 hours.

A sample of the slurry collected in a drum was taken several weeks after filtration and sent for particle size analysis. The Microtrac analysis showed most of the particles to be between 0.8 and 10 microns, with a median of about 2 microns. (Note: Microtrac does not measure below 0.08 microns.) A plot of the data is included in Appendix A.

A set of DIF water runs was made after cleaning the filter to compare with the water runs made after cleaning the filter after the Batch \#1 experiment. In hindsight the runs were not maintained at constant conditions long enough to obtain definitive fluxes. The available data for two sets of runs at similar conditions before and after Batch $\# 2$ are plotted below. All of the raw data collected during the DIF water runs is included in Appendix C.

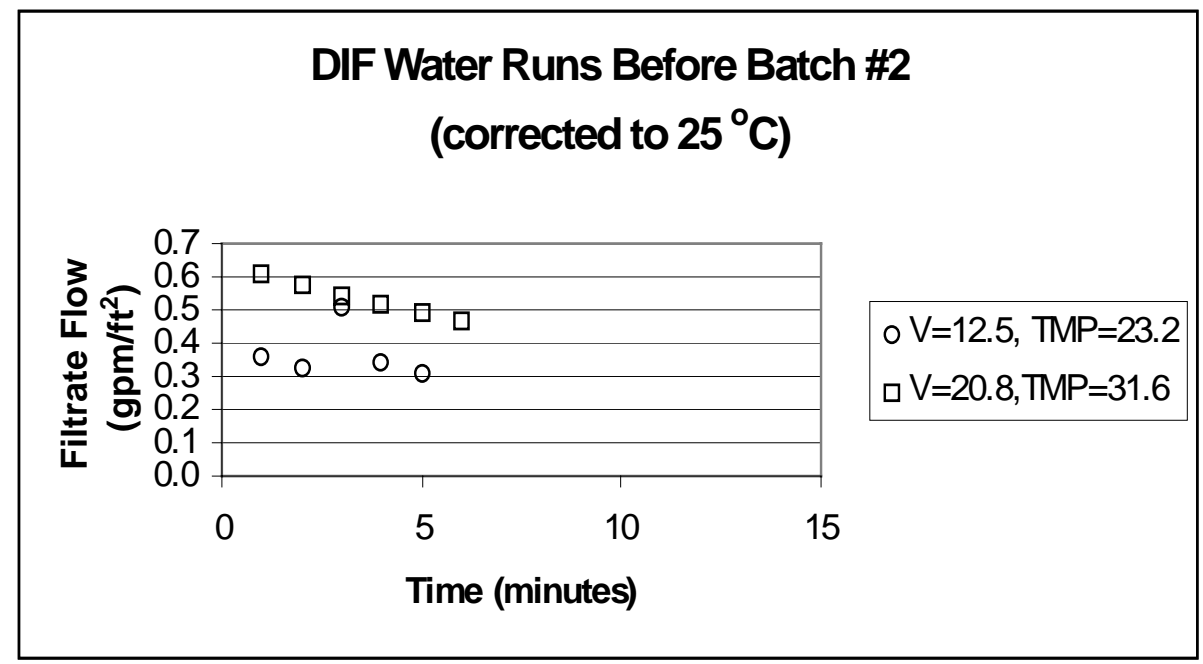

Figure 5-7 Crossflow Filter Flux with Clear Water Prior to Batch \#2 Processing 


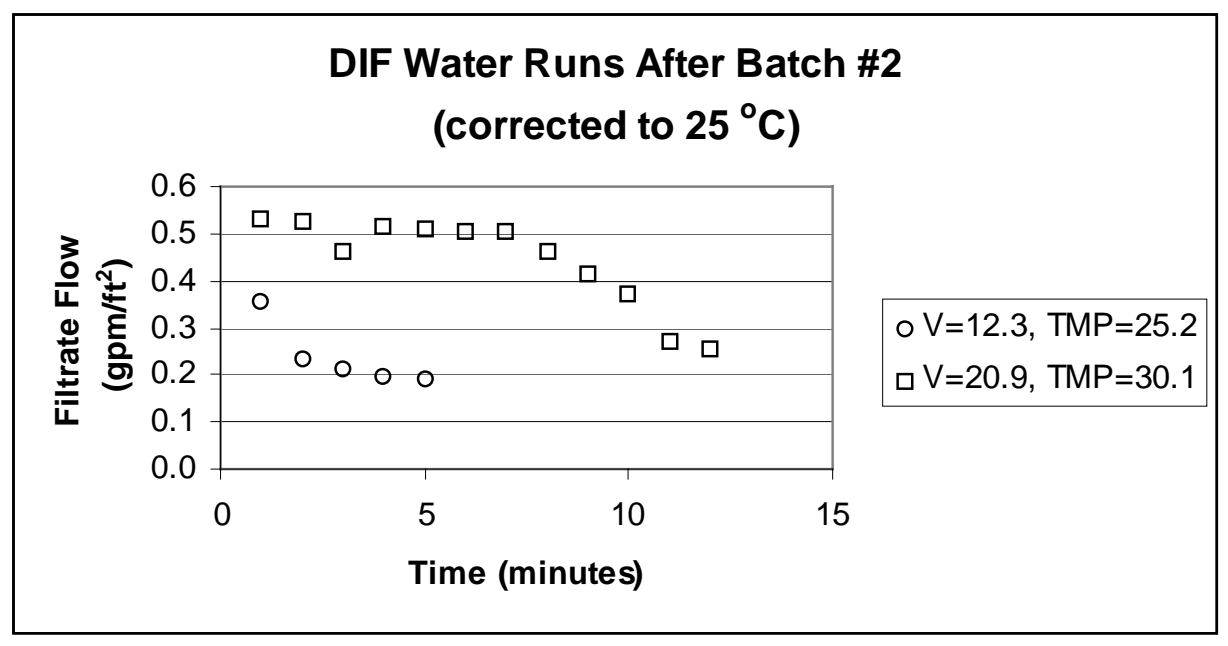

Figure 5-8 Crossflow Filter Flux with Clear Water After Batch \#2 Processing

\subsection{CONCLUSIONS}

- The decontamination factors four hours after completion of reagent addition were 9.4 for cerium, 11.1 for lanthanum, and 7.5 for neodymium. A recently developed correlation between the non-radioactive lanthanides surrogates $\mathrm{La}$ and $\mathrm{Nd}$ and the radioactive americium would indicate the DF for americium should be in the range 11 to 20 , which is adequate to meet regulatory requirements.

- The decontamination factor for strontium four hours after completion of reagent addition was 0.02. The effect of isotopic dilution of strontium could not be determined experimentally since only non-radioactive strontium was used; the calculated maximum possible isotopic dilution factor was 1834. If complete isotopic dilution before filtering is assumed, the decontamination factor for radioactive strontium in real waste treated by this process would be $0.02 * 1834=37$, more than adequate to meet regulatory requirements

- The concentrations of Re and $\mathrm{Cs}$ in the Batch \#2 liquid were unaffected by the Sr/TRU precipitation.

- About $85 \%$ of the reduction in concentration of the lanthanides and strontium occurs within the first 7.5 minutes after reagent addition is complete.

- Coupled operation of the Crossflow Filter Test Rig with the Precipitation Test Rig was successfully demonstrated. Filtrate production rates varied between $0.026 \mathrm{gpm} / \mathrm{ft}^{2}$ at 9.8 $\mathrm{ft} / \mathrm{sec}$ axial velocity and $51.2 \mathrm{psid}$ transmembrane pressure, and $0.038 \mathrm{gpm} / \mathrm{ft}^{2}$ at $17.4 \mathrm{ft} / \mathrm{sec}$ axial velocity and 44.2 psid transmembrane pressure.

- The filtrate collected from slurry precipitated with this process appeared to be fairly stable. After several months of storage some solids were formed, but there were very little solids formed in filtrate allowed to stand a day or two.

- No volatile/flammable off gases were produced. 


\subsection{FUTURE WORK}

The vigorous mixing capability of the mechanical agitator in the Pilot Scale Precipitate Tank may not be duplicated by the current Pulse Jet Mixer design. Additional testing with a Pilot Scale Pulse Jet Mixer system is recommended to confirm scale-up of reaction kinetics without the use of mechanical agitation.

\subsection{REFERENCES}

1. Williams, M. R., "Task Technical and Quality Assurance Plan In Support of RPP-WTP Pilot-Scale Precipitation Testing," SRT-RPP-2000-00050, Rev. 0, approved on June 19, 2001.

2. Williams, M. R., Screening Process Review Report, approved on Jan. 3, 2001.

3. Williams, M. R., Environmental Evaluation Checklist, EEC Document No. TC-A-2000-073, Rev. 1, approved on January 23, 2001.

4. Williams, M. R., "RPP Job Folder 22806 Pilot Scale Precipitation Testing," Laboratory Notebook, Document No. WSRC-NB-2001-00078. [Construction, shakedown and Batch \#2 test activities are documented.]

5. Steeper, T. J., WSRC Drawing No. EES-22806-M6-001, "RPP-WTP Pilot Scale Precipitation Testing Test Rig Setup", Rev. B, May 5, 2001.

6. Steeper, T. J., WSRC Drawing No. EES-22699-M6-003, "RPP Part B2 Pilot Scale Crossflow Filter Tests Test Rig Assembly with 40" Filter and New Pumps", Rev. A, May 1, 2001.

7. Williams, M. R., "RPP-WTP Pilot Scale Precipitation Testing Procedure", EDS Field Procedure, Document No. FP-882, Rev. 0, Effective September 24, 2001.

8. Williams, M. R., Operational Instructions No. 1-4, starting from No. 1: "Determination of Cleanliness of the Pilot Scale Precipitation System and Crossflow Test Rig per FP-882", October 2, 2001 to No. 4: "Caustic Addition to 1M NaOH of Batch \#2 AN-107 simulant in the Pilot Scale Precipitation and additional Filtrate samples", October 15, 2001.

9. Duignan, M. R., "Final Report: Pilot-Scale Cross-flow Ultrafiltration Test Using a Hanford Site Tank 241-AN-105 Waste Simulant - Envelope A + Entrained Solids", BNF-003-980221, February 23, 2000.

10. Nash, C. A., Rosencrance, S. W., Walker, B. W., Wilmarth, B. R., "Investigation of Varied Strontium-Transuranic Precipitation Chemistries for Crossflow Filtration", BNF-003-980171, Revision 0, April 18, 2000.

11. Nash, C. A., Saito, H. H., Wilmarth, B. R., "Strontium-Transuranic Precipitation and Crossflow Filtration of 241-AN-102 Large C", BNF-003-98-0317, Revision 0, December 5, 2000.

12. Benedict, M., Pigford, T. H., Levi, H. W., "Nuclear Chemical Engineering", Second Edition, McGraw-Hill Book Company, New York, 1981, pp. 407-420.

13. Nash, C. A., Rosencrance, S. W., Wilmarth, B. R., "Entrained Solids, StrontiumTransuranic Precipitation and Crossflow Filtration of 241-AN-102 Large C", SRT-RPP2000-00003, Revision 0, August, 2000.

14. Darab, J. G. and Smith, P. A., "Chemistry of Technetium and Rhenium Species during LowLevel Radioactive Waste Vitrification”, Chem. Mater. 1996, 8, 1004-1021, March 5, 1996. 
15. Eibling, R. E. and Nash, C. A. "Hanford Waste Simulants Created to Support the Research and Development on the River Protection Project - Waste Treatment Plant", SRT-RPP-200000017, Revision 0, February 2001.

16. Bergmann, L. M. "WTP Material Balance and Process Flowsheet Bases, Requirements, and Results”, 24590-WPT-RPT-ENG-01-004, Rev. 0, October 15, 2001.

17. Duignan, M. R., "Final Report: Pilot-Scale Cross-flow Ultrafiltration Test Using a Hanford Site Tank 241-AN-107 Waste Simulant - Envelope C + Entrained Solids + StrontiumTransuranic Precipitation”, BNF-003-98-0226, Rev. 0, March 24, 2000.

18. Hallen, R. T., Bredt, P. R., Brooks, K. P., and Jagoda, L. K. "Combined Entrained Solids and Sr/TRU Removal from AN-107 Diluted Feed”, BNFL-RPT-027, Rev. 0, August 2000.

19. W. R. Wilmarth, V. H. Dukes, J. T. Mills, F. F. Fondeur, C. C. DiPrete and D. P. DiPrete, "Optimization Study for Strontium and Actinide Removal from 241-AN-107 Supernate," WSRC-TR-2002-00258, September 18, 2002.

20. Williams, Michael R. and Steeper, Timothy J., "Pilot Scale Precipitation Test of AN-107 Simulant Without Caustic Adjustment at $50{ }^{\circ} \mathrm{C}$ ", WSRC-TR-2002-00122, Rev. 0, SRT-RPP2002-00064, Rev. 0, August 19, 2002.

\subsection{APPENDICES}

1. Appendix A - Analytical Data

2. Appendix B - Experimental Data: Precipitation Test Rig Operations Data

3. Appendix C - Experimental Data: Crossflow Test Rig Operations Data 


\section{Appendix A}

\section{Analytical Data}

\section{Appendix Contents}

- Liquid, Solid, and Gas Sample Analyses

- Microtrac Data (Particle Size Distribution of solids collected from concentrated slurry drum 3 weeks after filtration.)

- Lasentec Data

Special Notes:

- Each Solid Sample was divided into four segments for various dissolutions and analyses except the post-filtration solids which were not subdivided due to their small quantity.

- < values indicate below detection limits.

- The simulant samples $0 \mathrm{~L}$ and $0 \mathrm{~S}$ are actually the analysis of the simulant sample collected during the Batch \#1 experiment prior to adding any reagents. The mixing procedure for Batch \#1 and \#2 were identical, so an additional sample was not thought to be necessary.

- The simulant sample that was separated into the 1L liquid and 1S solid samples was actually taken about an hour minutes before reagent addition. This sample is considered the "0" time sample for comparison purposes.

- The post-filtration solid sample 172483 was obtained using a coliwasa from the filtrate drum on 11/20/01, which is 26 days after the filtering was completed on 10/25/01. The filtrate was filtered and the solids dried. Analyses performed on this sample included XRD, ICPMS, and ICP-ES.

- SVOC Analysis of Solid Samples was performed on ADS 300170474 through ADS 300170483, which are also identified as 170474 through 1170483 in the data pages.

- VOC Gas Analysis was performed on ADS 3-170441 through ADS 3-170454 and ADS 3-170474 through ADS 3-170483, which are also identified as 170441 through 170454, and 170474 through 170483 in the data sheets.

- A $100 \mathrm{ml}$ sample was obtained from the Batch \#2 Slurry drum in storage at 1400 hours on 11/13/01 as ADS sample 3-172182 for Microtrac-SRA150 analysis to determine the distribution of particle sizes in the precipitated slurry 21 days after the precipitation reaction. 
WSRC-TR-2002-00459, Rev. 0 SRT-RPP-2002-00221

\begin{tabular}{|c|c|c|c|c|c|c|c|c|c|c|c|c|c|c|}
\hline \multicolumn{4}{|c|}{ BATCH \#2 ANALYTICAL RESULTS } & & & & & & & & & & & \\
\hline & & & \multicolumn{12}{|c|}{ Liquid Analysis } \\
\hline EDL Sample No. & & & $\mathrm{OL}$ & $1 \mathrm{~L}$ & $2 \mathrm{~L}$ & $3 \mathrm{~L}$ & $4 \mathrm{~L}$ & $5 \mathrm{~L}$ & $6 \mathrm{~L}$ & $7 \mathrm{~L}$ & $8 \mathrm{~L}$ & $9 \mathrm{~L}$ & $10 \mathrm{~L}$ & $\begin{array}{l}\text { Post- } \\
\text { Filtration }\end{array}$ \\
\hline \multicolumn{3}{|c|}{ Time after Reagent Add (hrs) } & 0 & 0 & 0.125 & 0.25 & 0.5 & 1 & 2 & 3 & 4 & 6 & 8 & 2184 \\
\hline ADS Sample No. & & & 169600 & 170445 & 170446 & 170447 & 170448 & 170449 & 170450 & 170451 & 170452 & 170453 & 170454 & (3) \\
\hline Identity & Method & Units & (1) & (2) & & & & & & & & & & \\
\hline $\mathrm{K}$ & AAK & $\mu \mathrm{g} / \mathrm{gm}$ & 1620 & 1318 & 1188 & 1159 & 1172 & 1120 & 1152 & 1157 & 1181 & 1219 & 1168 & \\
\hline $\mathrm{AlO}_{2}^{-}$ & & molar & 0.0156 & $<0.02$ & $<0.02$ & $<0.02$ & $<0.02$ & $<0.02$ & $<0.02$ & $<0.02$ & $<0.02$ & $<0.02$ & $<0.02$ & \\
\hline $\mathrm{CO}_{3}{ }^{2-}$ & & molar & 0.7032 & 0.8585 & 0.6668 & 0.6622 & 0.6808 & 0.6465 & 0.6605 & 0.6602 & 0.675 & 0.695 & 0.6488 & \\
\hline Free $\mathrm{OH}^{-}$ & & molar & 0.036 & 0.7545 & 0.646 & 0.636 & 0.651 & 0.627 & 0.658 & 0.643 & 0.65 & 0.661 & 0.623 & \\
\hline Total $\mathrm{OH}^{-}$ & & molar & 0.767 & 1.681 & 1.408 & 1.4 & 1.397 & 1.359 & 1.36 & 1.38 & 1.405 & 1.427 & 1.419 & \\
\hline $\mathrm{Na}$ & AANA & $\mu \mathrm{g} / \mathrm{gm}$ & 95829 & 110796 & 100175 & 98536 & 87585 & 84000 & 90439 & 89766 & 92288 & 98193 & 90176 & \\
\hline Specific Gravity & & & 1.267 & 1.280 & 1.260 & 1.260 & 1.260 & 1.260 & 1.260 & 1.260 & 1.270 & 1.280 & 1.260 & $\begin{array}{ll}0 \\
\end{array}$ \\
\hline $\mathrm{pH}$ & & & 10.2 & 12.34 & 12.38 & 12.38 & 12.32 & 12.37 & 12.32 & 12.31 & 12.29 & 12.27 & 12.28 & \\
\hline Total Carbon & & $\mu \mathrm{g} / \mathrm{ml}$ & 33800 & 25400 & 21600 & 21400 & 20100 & 22000 & 21800 & 22000 & 23000 & 24200 & 22800 & \\
\hline TOC & & $\mu \mathrm{g} / \mathrm{ml}$ & 19050 & 14780 & 12980 & 12540 & 12340 & 12980 & 12700 & 12860 & 13480 & 13980 & 13320 & \\
\hline TIC & & $\mu \mathrm{g} / \mathrm{ml}$ & 14700 & 10400 & 8720 & 8900 & 8740 & 9000 & 9120 & 9200 & 9440 & 10160 & 9480 & \\
\hline Acetate & IEC & $\mathrm{mg} / \mathrm{kg}$ & 737 & 744 & 693 & 660 & 605 & 583 & 615 & 601 & 620 & 610 & 596 & \\
\hline Glycolic Acid & IEC & $\mathrm{mg} / \mathrm{kg}$ & 12857 & 12017 & 9941 & 10786 & 9097 & 9315 & 9124 & 9600 & 9836 & 9980 & 9660 & \\
\hline Citric Acid & IEC & $\mathrm{mg} / \mathrm{kg}$ & 4910 & 3498 & 4545 & 4235 & 4067 & 4076 & 4127 & 4013 & 4253 & 4390 & 4181 & \\
\hline Succinic acid & IEC & $\mathrm{mg} / \mathrm{kg}$ & $\mathrm{N} / \mathrm{A}$ & $\mathrm{N} / \mathrm{A}$ & $\mathrm{N} / \mathrm{A}$ & $\mathrm{N} / \mathrm{A}$ & $\mathrm{N} / \mathrm{A}$ & $\mathrm{N} / \mathrm{A}$ & $\mathrm{N} / \mathrm{A}$ & $\mathrm{N} / \mathrm{A}$ & $\mathrm{N} / \mathrm{A}$ & $\mathrm{N} / \mathrm{A}$ & $\mathrm{N} / \mathrm{A}$ & \\
\hline $\mathrm{D}_{2} \mathrm{EHPA}$ & IEC & $\mathrm{mg} / \mathrm{kg}$ & $\mathrm{N} / \mathrm{A}$ & $\mathrm{N} / \mathrm{A}$ & $\mathrm{N} / \mathrm{A}$ & $\mathrm{N} / \mathrm{A}$ & $\mathrm{N} / \mathrm{A}$ & $\mathrm{N} / \mathrm{A}$ & $\mathrm{N} / \mathrm{A}$ & $\mathrm{N} / \mathrm{A}$ & $\mathrm{N} / \mathrm{A}$ & $\mathrm{N} / \mathrm{A}$ & $\mathrm{N} / \mathrm{A}$ & \\
\hline Gluconate & IEC & $\mathrm{mg} / \mathrm{kg}$ & $\mathrm{N} / \mathrm{A}$ & $\mathrm{N} / \mathrm{A}$ & $\mathrm{N} / \mathrm{A}$ & $\mathrm{N} / \mathrm{A}$ & $\mathrm{N} / \mathrm{A}$ & $\mathrm{N} / \mathrm{A}$ & $\mathrm{N} / \mathrm{A}$ & $\mathrm{N} / \mathrm{A}$ & $\mathrm{N} / \mathrm{A}$ & $\mathrm{N} / \mathrm{A}$ & $\mathrm{N} / \mathrm{A}$ & \\
\hline NTA & IEC & $\mathrm{mg} / \mathrm{kg}$ & $\mathrm{N} / \mathrm{A}$ & $\mathrm{N} / \mathrm{A}$ & $\mathrm{N} / \mathrm{A}$ & $\mathrm{N} / \mathrm{A}$ & $\mathrm{N} / \mathrm{A}$ & $\mathrm{N} / \mathrm{A}$ & $\mathrm{N} / \mathrm{A}$ & $\mathrm{N} / \mathrm{A}$ & $\mathrm{N} / \mathrm{A}$ & $\mathrm{N} / \mathrm{A}$ & $\mathrm{N} / \mathrm{A}$ & \\
\hline IDA & IEC & $\mathrm{mg} / \mathrm{kg}$ & $\mathrm{N} / \mathrm{A}$ & $\mathrm{N} / \mathrm{A}$ & $\mathrm{N} / \mathrm{A}$ & $\mathrm{N} / \mathrm{A}$ & $\mathrm{N} / \mathrm{A}$ & $\mathrm{N} / \mathrm{A}$ & $\mathrm{N} / \mathrm{A}$ & $\mathrm{N} / \mathrm{A}$ & $\mathrm{N} / \mathrm{A}$ & $\mathrm{N} / \mathrm{A}$ & $\mathrm{N} / \mathrm{A}$ & \\
\hline Fluoride & $\mathrm{IC}$ & $\mu \mathrm{g} / \mathrm{gm}$ & 2880 & 2108 & 1731 & 1708 & 1718 & 1722 & 1736 & 1727 & 1750 & 1782 & 1712 & \\
\hline Formate $\left(\mathrm{HCOO}^{\circ}\right)$ & IC & $\mu \mathrm{g} / \mathrm{gm}$ & 5862 & 4807 & 4525 & 4449 & 4312 & 4418 & 4481 & 4403 & 4475 & 4607 & 4336 & \\
\hline Nitrite $\left(\mathrm{NO}_{2}{ }^{-}\right)$ & IC & $\mu \mathrm{g} / \mathrm{gm}$ & 35659 & 25968 & 23825 & 23260 & 23118 & 23464 & 23555 & 23299 & 24071 & 24589 & 23392 & \\
\hline Phosphate $\left(\mathrm{PO}_{4}{ }^{3-}\right)$ & IC & $\mu \mathrm{g} / \mathrm{gm}$ & 1147 & 1222 & 1012 & 1012 & 938 & 914 & 886 & 858 & 914 & 958 & 890 & \\
\hline Oxalate $\left(\mathrm{C}_{2} \mathrm{O}_{4}{ }^{2-}\right)$ & IC & $\mu \mathrm{g} / \mathrm{gm}$ & 880 & 533 & 1094 & 1174 & 1122 & 1079 & 1179 & 117 & 1085 & 833 & 1055 & \\
\hline Chloride $\left(\mathrm{Cl}^{-}\right)$ & IC & $\mu \mathrm{g} / \mathrm{gm}$ & 1011 & 913 & 830 & 820 & 805 & 818 & 822 & 811 & 836 & 864 & 809 & \\
\hline Nitrate $\left(\mathrm{NO}_{3}^{-}\right)$ & IC & $\mu \mathrm{g} / \mathrm{gm}$ & 105156 & 96682 & 100977 & 99283 & 98630 & 100254 & 89988 & 94617 & 98146 & 103836 & 96392 & \\
\hline Sulfate $\left(\mathrm{SO}_{4}{ }^{2-}\right)$ & IC & $\mu \mathrm{g} / \mathrm{gm}$ & 4477 & 4308 & 3906 & 3852 & 3808 & 3834 & 3867 & 3806 & 3902 & 4038 & 3775 & \\
\hline HEDTA & IPC & $\mathrm{mg} / \mathrm{l}$ & 734 & 420 & 400 & 425 & 600 & 425 & 450 & 450 & 450 & 475 & 425 & \\
\hline EDTA & IPC & $\mathrm{mg} / \mathrm{l}$ & 2876 & 2158 & 2225 & 2200 & 2525 & 2450 & 2125 & 2175 & 2275 & 2275 & 2075 & \\
\hline $\mathrm{Ce}$ & ICP-MS & $\mu \mathrm{g} / \mathrm{gm}$ & 26.4 & 24.6 & 3.17 & 2.99 & 2.86 & 2.63 & 2.45 & 2.43 & 2.33 & 1.95 & 2.01 & 1. \\
\hline Cs & ICP-MS & $\mu \mathrm{g} / \mathrm{gm}$ & 10.40 & 10.10 & 8.58 & 8.52 & 13.00 & 8.73 & 8.06 & 8.12 & 8.30 & 8.67 & 8.38 & \\
\hline $\mathrm{La}$ & ICP-MS & $\mu \mathrm{g} / \mathrm{gm}$ & 23.2 & 22.4 & 2.18 & 2.02 & 2.02 & 1.84 & 1.82 & 1.82 & 1.74 & 1.58 & 1.61 & 0.8 \\
\hline $\mathrm{Nd}$ & ICP-MS & $\mu \mathrm{g} / \mathrm{gm}$ & 43.5 & 44.3 & 6.15 & 5.85 & 5.67 & 5.12 & 5.1 & 5.31 & 4.85 & 4.48 & 4.59 & 2. \\
\hline $\operatorname{Re}$ & ICP-MS & $\mu \mathrm{g} / \mathrm{gm}$ & 11 & 9.18 & 56 & 8.21 & 36 & 7.83 & 8.2 & 8.17 & 31 & 8.51 & 8.11 & \\
\hline $\mathrm{Pb}$ & ICP-MS & $\mu \mathrm{g} / \mathrm{gm}$ & 201 & 5 & & 4 & 7.7 & 44 & 45.6 & 5.8 & .9 & 45.8 & 44 & \\
\hline Al & ICP-ES & $\mu \mathrm{g} / \mathrm{gm}$ & 386 & 196 & 94 & 214 & 204 & 180 & 178 & 160 & 167 & 170 & 199 & 17 \\
\hline B & ICP-ES & $\mu \mathrm{g} / \mathrm{gm}$ & 56 & $<20$ & 20 & $<20$ & $<20$ & $<20$ & $<20$ & $<20$ & $<20$ & 33 & 111 & 1 \\
\hline $\mathrm{Ba}$ & ICP-ES & $\mu \mathrm{g} / \mathrm{gm}$ & $<1.5$ & $<1.5$ & 1.5 & $<1.5$ & $<1.5$ & $<1.5$ & $<1.5$ & $<1.5$ & $<1.5$ & $<1.5$ & $<1.5$ & 0. \\
\hline $\mathrm{Ca}$ & ICP-ES & $\mu \mathrm{g} / \mathrm{gm}$ & 129 & 303 & 161 & 159 & 164 & 155 & 153 & 153 & 154 & 160 & 152 & 2 \\
\hline $\mathrm{Cd}$ & ICP-ES & $\mu \mathrm{g} / \mathrm{gm}$ & $<5.0$ & $<5.0$ & $<5.0$ & $<5.0$ & $<5.0$ & $<5.0$ & $<5.0$ & $<5.0$ & $<5.0$ & $<5.0$ & $<5.0$ & $<0.0$ \\
\hline $\mathrm{Cr}$ & ICP-ES & $\mu \mathrm{g} / \mathrm{gm}$ & 93 & 78 & 63 & 61 & 63 & 60 & 60 & 62 & 60 & 59 & 63 & 3 \\
\hline $\mathrm{Cu}$ & ICP-ES & $\mu \mathrm{g} / \mathrm{gm}$ & 22 & 68 & 129 & 152 & 111 & 117 & 16 & $<10$ & $<10$ & 17 & 53 & \\
\hline $\mathrm{Fe}$ & ICP-ES & $\mu \mathrm{g} / \mathrm{gm}$ & 005 & 754 & 00 & 98 & 126 & 93 & 78 & 95 & 80 & 80 & 100 & \\
\hline $\mathrm{K}$ & ICP-ES & $\mu \mathrm{g} / \mathrm{gm}$ & 1420 & 1240 & 1160 & 1120 & 1180 & 1110 & 1150 & 1170 & 1150 & 1180 & 1130 & 199 \\
\hline $\mathrm{Mg}$ & ICP-ES & $\mu \mathrm{g} / \mathrm{gm}$ & 51 & $<10$ & $<10$ & $<10$ & $<10$ & $<10$ & $<10$ & $<10$ & $<10$ & $<10$ & $<10$ & 0.3 \\
\hline $\mathrm{Mn}$ & ICP-ES & $\mu \mathrm{g} / \mathrm{gm}$ & 294 & 262 & 82 & 74 & 85 & 84 & 89 & 101 & 94 & 92 & 101 & \\
\hline Mo & ICP-ES & $\mu \mathrm{g} / \mathrm{gm}$ & & & & & & & & & & & & \\
\hline $\mathrm{Na}$ & ICP-ES & $\mu \mathrm{g} / \mathrm{gm}$ & 0000 & 118000 & 105000 & 105000 & 108000 & 102000 & 105000 & 106000 & 106000 & 108000 & 106000 & 906 \\
\hline $\mathrm{Ni}$ & ICP-ES & $\mu \mathrm{g} / \mathrm{gm}$ & 296 & 281 & 300 & 304 & 284 & 270 & 253 & 235 & 240 & 248 & 258 & 2 \\
\hline $\mathrm{P}$ & ICP-ES & $\mu \mathrm{g} / \mathrm{gm}$ & 205 & 197 & 175 & 158 & 167 & 155 & 170 & 168 & 176 & 182 & 180 & 15 \\
\hline $\mathrm{Pb}$ & ICP-ES & $\mu \mathrm{g} / \mathrm{gm}$ & 204 & 187 & $<80$ & $<80$ & $<80$ & $<80$ & $<80$ & $<80$ & $<80$ & $<80$ & $<80$ & (3) \\
\hline $\mathrm{Si}$ & ICP-ES & $\mu \mathrm{g} / \mathrm{gm}$ & 47 & 38 & 26 & 23 & 27 & 22 & $<20$ & $<20$ & $<20$ & $<20$ & $<20$ & \\
\hline Sr & ICP-ES & $\mu \mathrm{g} / \mathrm{gm}$ & 5 & 4.2 & 174 & 172 & 173 & 164 & 156 & 158 & 144 & 110 & 110 & 1 \\
\hline $\mathrm{Zn}$ & ICP-ES & $\mu \mathrm{g} / \mathrm{gm}$ & & & & & & & & & & & & 19.0 \\
\hline $\mathrm{Zr}$ & ICP-ES & $\mu \mathrm{g} / \mathrm{gm}$ & 33 & $<40$ & $<40$ & $<40$ & $<40$ & $<40$ & $<40$ & $<40$ & $<40$ & $<40$ & $<40$ & 4. \\
\hline$S$ & ICP-ES & $\mu \mathrm{g} / \mathrm{gm}$ & 1650 & 1720 & 1730 & 1660 & 1650 & 1580 & 1640 & 1780 & 1730 & 1900 & 1790 & 138 \\
\hline & Sampl & is & re cau & addi & anc & ctu & he a & is & $\mathrm{npl}$ & coll & & $D_{0}$ & & \\
\hline & & & & & & & 0 & $\mathrm{a}$ & all & & & & & \\
\hline & Mob & ion). & $\mathrm{e} \mathrm{R}$ & - & $C_{2-1}$ & $\{\mathrm{~A} \mid$ & IIecte & from & te d & on & 2,2 & (91 & $s$ afte & \\
\hline & & & & & & & & & & & & & & \\
\hline
\end{tabular}


WSRC-TR-2002-00459, Rev. 0 SRT-RPP-2002-00221

\begin{tabular}{|c|c|c|c|c|c|c|c|c|c|c|c|c|c|c|}
\hline & & & & & & & & & & & & \multirow[b]{3}{*}{$\begin{array}{c}\text { Post- } \\
\text { Filtration }\end{array}$} \\
\hline \multicolumn{3}{|c|}{ BATCH \#2 ANALYTICAL RESULTS } & \multicolumn{11}{|c|}{ Solids Analysis } & \\
\hline EDL Sample No. & & & OS & 1S & $2 S$ & $3 S$ & $4 \mathrm{~S}$ & $5 S$ & $6 \mathrm{~S}$ & $7 S$ & $8 \mathrm{~S}$ & 9S & $10 \mathrm{~S}$ & \\
\hline \multicolumn{2}{|c|}{ Time after Reagent Add (hrs) } & & 0 & 0 & 0.125 & 0.25 & 0.5 & 1 & 2 & 3 & 4 & 6 & 8 & 674 \\
\hline Identity & Units & Method & & & & & & & & & & & & \\
\hline Quantity collected & grams & & 3.98 & 5.27 & 44.26 & 40.46 & 43.51 & 50.78 & 47.06 & 45.51 & 41.71 & 67.36 & 84.78 & \\
\hline ADS Sample No. & & & 169764 & 170431 & 170432 & 170433 & 170434 & 170435 & 170436 & 170437 & 170438 & 170439 & 170440 & 172484 \\
\hline \multicolumn{12}{|c|}{ Pretreatment: $0.25 \mathrm{gm}$ of solids disolved in aquaregia $\left.(9 \mathrm{ml} \mathrm{HCl}+3 \mathrm{ml} \mathrm{HNO})_{3}\right)$ then diluted with water to $250 \mathrm{ml}$} & & & \\
\hline $\mathrm{Cd}$ & $\mu \mathrm{g} / \mathrm{gm}$ & ICP-MS & $<1$ & $<4$ & $<4$ & $<4$ & $<4$ & $<4$ & $<4$ & $<4$ & $<4$ & $<4$ & $<4$ & $<2$ \\
\hline La & $\mu \mathrm{g} / \mathrm{gm}$ & SP-MS & 528 & 150 & 383 & 512 & 486 & 529 & 557 & 535 & 597 & 618 & 492 & 12.9 \\
\hline $\mathrm{Ce}$ & $\mu \mathrm{g} / \mathrm{gm}$ & CP-MS & 843 & 170 & 422 & 567 & 550 & 583 & 624 & 606 & 671 & 703 & 560 & 22.7 \\
\hline $\mathrm{Nd}$ & $\mu \mathrm{g} / \mathrm{gm}$ & CP-MS & 1720 & 291 & 778 & 1040 & 993 & 1060 & 1140 & 1080 & 1220 & 1260 & 1010 & 32.2 \\
\hline $\mathrm{Re}$ & $\mu \mathrm{g} / \mathrm{gm}$ & ICP-MS & 2.1 & 14.5 & 12.7 & 18.2 & 19.8 & 20.6 & 17.7 & 18.3 & 15.2 & 15.6 & 20.8 & 9.35 \\
\hline $\mathrm{Pb}$ & $\mu \mathrm{g} / \mathrm{gm}$ & ICP-MS & 413 & 1080 & 2520 & 3410 & 3230 & 3410 & 3660 & 3490 & 3910 & 4090 & 3230 & 479 \\
\hline $\mathrm{Al}$ & $\mu \mathrm{g} / \mathrm{gm}$ & ICP-ES & 30100 & 5030 & 547 & 549 & 473 & 638 & 851 & 720 & 1010 & 1410 & 649 & 3220 \\
\hline$B$ & $\mu \mathrm{g} / \mathrm{gm}$ & ICP-ES & 910 & $<250$ & $<250$ & $<250$ & $<250$ & $<250$ & $<250$ & $<250$ & $<250$ & $<250$ & $<250$ & 3450 \\
\hline $\mathrm{Ba}$ & $\mu \mathrm{g} / \mathrm{gm}$ & ICP-ES & 21 & 22 & 1270 & 1050 & 989 & 1070 & 1170 & 1110 & 1220 & 1240 & 989 & 2540 \\
\hline $\mathrm{Ca}$ & $\mu \mathrm{g} / \mathrm{gm}$ & ICP-ES & 87900 & 3720 & 4680 & 3800 & 3710 & 4180 & 4500 & 4140 & 4470 & 4960 & 3960 & 1650 \\
\hline $\mathrm{Cd}$ & $\mu \mathrm{g} / \mathrm{gm}$ & ICP-ES & $<15$ & $<20$ & $<20$ & $<20$ & $<20$ & $<20$ & $<20$ & $<20$ & $<20$ & $<20$ & $<20$ & $<4.0$ \\
\hline $\mathrm{Cr}$ & $\mu \mathrm{g} / \mathrm{gm}$ & ICP-ES & 925 & 1780 & 525 & 488 & 522 & 457 & 528 & 462 & 553 & 701 & 446 & 163 \\
\hline $\mathrm{Fe}$ & $\mu \mathrm{g} / \mathrm{gm}$ & ICP-ES & 48600 & 58500 & 26700 & 22400 & 21300 & 22900 & 24500 & 23500 & 26000 & 27000 & 21400 & 3410 \\
\hline $\mathrm{Mg}$ & $\mu \mathrm{g} / \mathrm{gm}$ & ICP-ES & 2880 & 12400 & 1410 & 1150 & 1130 & 1190 & 1280 & 1240 & 1320 & 1390 & 1090 & 201 \\
\hline $\mathrm{Mn}$ & $\mu \mathrm{g} / \mathrm{gm}$ & ICP-ES & 26000 & 33900 & 75300 & 61500 & 58600 & 64100 & 70500 & 65200 & 71200 & 72500 & 58300 & 12100 \\
\hline $\mathrm{Na}$ & $\mu \mathrm{g} / \mathrm{gm}$ & ICP-ES & 165000 & 204000 & 141000 & 182000 & 183000 & 198000 & 187000 & 174000 & 149000 & 166000 & 204000 & 111000 \\
\hline $\mathrm{Ni}$ & $\mu \mathrm{g} / \mathrm{gm}$ & ICP-ES & $<70$ & 243 & 247 & 306 & 318 & 417 & 376 & 304 & 227 & 322 & 408 & 189 \\
\hline$P$ & $\mu \mathrm{g} / \mathrm{gm}$ & ICP-ES & 1140 & $<750$ & $<750$ & $<750$ & $<750$ & $<750$ & $<750$ & $<750$ & $<750$ & $<750$ & $<750$ & 184 \\
\hline $\mathrm{Pb}$ & $\mu \mathrm{g} / \mathrm{gm}$ & ICP-ES & $<700$ & 983 & 3730 & 3240 & 3170 & 3290 & 3710 & 3330 & 3740 & 3960 & 3180 & 492 \\
\hline $\mathrm{Si}$ & $\mu \mathrm{g} / \mathrm{gm}$ & ICP-ES & 2250 & 1120 & 274 & 427 & 145 & 485 & 490 & 233 & 259 & 423 & 378 & 225 \\
\hline $\mathrm{Sr}$ & $\mu \mathrm{g} / \mathrm{gm}$ & ICP-ES & 743 & 66 & 77000 & 146000 & 1000 & 52000 & 167000 & 54000 & 9000 & 4000 & 40000 & 459 \\
\hline $\mathrm{Zr}$ & $\mu \mathrm{g} / \mathrm{gm}$ & ICP-ES & 378 & 265 & 709 & 610 & 544 & 627 & 657 & 652 & 703 & 729 & 591 & 16 \\
\hline $\mathrm{K}$ & $\mu \mathrm{g} / \mathrm{gm}$ & ICP-ES & $<500$ & 1040 & 1400 & 1800 & 1880 & 2420 & 2010 & 1720 & 1620 & 1680 & 2200 & 3940 \\
\hline S & $\mu \mathrm{g} / \mathrm{gm}$ & ICP-ES & 1190 & 1150 & 1280 & 1880 & 1740 & 2000 & 1950 & 1760 & 1490 & 1900 & 2230 & 773 \\
\hline $\mathrm{Nd}$ & $\mu \mathrm{g} / \mathrm{gm}$ & ICP-ES & 1071 & $<300$ & 1180 & 972 & 960 & 921 & 1040 & 959 & 1150 & 1210 & 1040 & $<100$ \\
\hline $\mathrm{Cu}$ & $\mu \mathrm{g} / \mathrm{gm}$ & ICP-ES & $<50$ & 58 & 110 & 85 & 76 & 94 & 96 & 106 & 96 & 99 & 103 & 18 \\
\hline$Z n$ & $\mu \mathrm{g} / \mathrm{gm}$ & ICP-ES & & & & & & & & & & & & 3740 \\
\hline ADS Sample No. & & & 169774 & 170421 & 170422 & 170423 & 170424 & 170425 & 170426 & 170427 & 170428 & 170429 & 170430 & \\
\hline \multicolumn{13}{|c|}{ Pretreatment: 0.25 gram of solids fused with $1.5 \mathrm{gm} \mathrm{Na} \mathrm{O}_{2}+1.0 \mathrm{gm} \mathrm{NaOH}$, disolved in $25 \mathrm{ml}$ of HCL, and diluted with water to $250 \mathrm{ml}$} & & \\
\hline $\mathrm{Al}$ & $\mu \mathrm{g} / \mathrm{gm}$ & ICP-ES & 28300 & 46400 & 4600 & 4700 & 5100 & 5200 & 5100 & 5000 & 4800 & 5200 & 5000 & \\
\hline$B$ & $\mu \mathrm{g} / \mathrm{gm}$ & ICP-ES & 247 & $<250$ & $<250$ & $<250$ & $<250$ & $<250$ & $<250$ & $<250$ & $<250$ & $<250$ & $<250$ & \\
\hline $\mathrm{Ba}$ & $\mu \mathrm{g} / \mathrm{gm}$ & ICP-ES & 50 & 89 & 1132 & 1104 & 1269 & 1126 & 1130 & 1258 & 1188 & 1224 & 1136 & \\
\hline $\mathrm{Ca}$ & $\mu \mathrm{g} / \mathrm{gm}$ & ICP-ES & 86100 & 4862 & 5034 & 5398 & 5718 & 6346 & 6774 & 5780 & 6488 & 5823 & 5573 & \\
\hline $\mathrm{Cd}$ & $\mu \mathrm{g} / \mathrm{gm}$ & ICP-ES & 36 & $<20$ & $<20$ & $<20$ & $<20$ & $<20$ & $<20$ & $<20$ & $<20$ & $<20$ & $<20$ & \\
\hline $\mathrm{Cr}$ & $\mu \mathrm{g} / \mathrm{gm}$ & ICP-ES & 2161 & 5515 & 820 & 854 & 880 & 895 & 824 & 859 & 919 & 991 & 845 & \\
\hline $\mathrm{Fe}$ & $\mu \mathrm{g} / \mathrm{gm}$ & ICP-ES & 26600 & 64600 & 24000 & 23300 & 26800 & 23100 & 23500 & 26500 & 25400 & 26200 & 24200 & \\
\hline $\mathrm{Mg}$ & $\mu \mathrm{g} / \mathrm{gm}$ & ICP-ES & 1430 & 14000 & 1190 & 1230 & 1350 & 1320 & 1250 & 1410 & 1340 & 1330 & 1130 & \\
\hline $\mathrm{Mn}$ & $\mu \mathrm{g} / \mathrm{gm}$ & ICP-ES & 16800 & 37600 & 67200 & 64900 & 75600 & 63600 & 64900 & 74300 & 70700 & 71700 & 65900 & \\
\hline $\mathrm{Ni}$ & $\mu \mathrm{g} / \mathrm{gm}$ & ICP-ES & 270 & 343 & 282 & 363 & 316 & 437 & 417 & 297 & 294 & 380 & 450 & \\
\hline $\mathrm{P}$ & $\mu \mathrm{g} / \mathrm{gm}$ & ICP-ES & 681 & $<700$ & $<700$ & $<700$ & $<700$ & $<700$ & $<700$ & $<700$ & $<700$ & $<700$ & $<700$ & \\
\hline $\mathrm{Si}$ & $\mu \mathrm{g} / \mathrm{gm}$ & ICP-ES & 7090 & 7860 & 1160 & 1130 & 1300 & 1030 & 1140 & 1100 & 1100 & 1030 & 1050 & \\
\hline $\mathrm{Sr}$ & $\mu \mathrm{g} / \mathrm{gm}$ & ICP-ES & 810 & 7210 & 145000 & 150000 & 164000 & 152000 & 144000 & 167000 & 158000 & 151000 & 138000 & \\
\hline$Z n$ & $\mu \mathrm{g} / \mathrm{gm}$ & ICP-ES & & $<400$ & $<400$ & $<400$ & $<400$ & $<400$ & $<400$ & $<400$ & $<400$ & $<400$ & $<400$ & \\
\hline $\mathrm{K}$ & $\mu \mathrm{g} / \mathrm{gm}$ & ICP-ES & 1460 & 595 & 1940 & 2010 & 1700 & 1900 & 2100 & 1610 & 1260 & 1910 & 2920 & \\
\hline S & $\mu \mathrm{g} / \mathrm{gm}$ & ICP-ES & 1950 & 909 & 1170 & 1500 & 1020 & 1420 & 1440 & 1020 & 1120 & 1580 & 1370 & \\
\hline $\mathrm{Nd}$ & $\mu \mathrm{g} / \mathrm{gm}$ & ICP-ES & 778 & 528 & 1410 & 1290 & 1440 & 1280 & 1310 & 1350 & 1370 & 1560 & 1160 & \\
\hline ADS Sample No. & & & 169754 & 170374 & 170375 & 170376 & 170377 & 170378 & 170379 & 170380 & 170381 & 170382 & 170383 & \\
\hline \multicolumn{14}{|c|}{ Pretreatment: 0.25 gram of solids fused with $1.5 \mathrm{gm} \mathrm{Na} \mathrm{O}_{2}+1.0 \mathrm{gm} \mathrm{NaOH}$, then disolved by and diluted with water to $250 \mathrm{ml}$} & \\
\hline \begin{tabular}{|l} 
Fluoride \\
\end{tabular} & $\mu \mathrm{g} / \mathrm{ml}$ & ICA & $<198$ & 869 & 130 & 306 & 207 & 246 & 384 & 282 & 172 & 280 & 225 & \\
\hline Formate (HCOO') & $\mu \mathrm{g} / \mathrm{ml}$ & ICA & $<989$ & 4009 & 16743 & 14039 & 12542 & 17328 & 20668 & 11221 & 15455 & 15850 & 12241 & \\
\hline Nitrite $\left(\mathrm{NO}_{2}^{-}\right)$ & $\mu \mathrm{g} / \mathrm{ml}$ & ICA & $<989$ & 16081 & 23743 & 35446 & 28874 & 37136 & 44908 & 33768 & 23819 & 35380 & 31862 & \\
\hline Phosphate $\left(\mathrm{PO}_{4}^{3-}\right)$ & $\mu \mathrm{g} / \mathrm{ml}$ & ICA & $<989$ & $<88$ & $<94$ & 150 & $<93$ & 180 & 226 & $<93$ & $<99$ & 140 & 152 & \\
\hline Oxalate $\left(\mathrm{C}_{2} \mathrm{O}_{4}{ }^{2-}\right)$ & $\mu \mathrm{g} / \mathrm{ml}$ & ICA & $<989$ & 226039 & 36806 & 42505 & 42992 & 45231 & 49436 & 47859 & 44101 & 48548 & 45106 & \\
\hline Chloride $\left(\mathrm{Cl}^{-}\right)$ & $\mu \mathrm{g} / \mathrm{ml}$ & ICA & 989 & 639 & 1068 & 1490 & 1174 & 1720 & 1940 & 1289 & 986 & 1551 & 1358 & \\
\hline Nitrate $\left(\mathrm{NO}_{3}{ }^{-}\right)$ & $\mu \mathrm{g} / \mathrm{ml}$ & ICA & 2966 & 63840 & 92427 & 153814 & 116599 & 140153 & 175323 & 142136 & 109304 & 144817 & 126780 & \\
\hline Sulfate $\left(\mathrm{SO}_{4}{ }^{2-}\right)$ & $\mu \mathrm{g} / \mathrm{ml}$ & ICA & 2966 & 2598 & 3521 & 8505 & 5683 & 4373 & 5509 & 5251 & 4635 & 4759 & 5701 & \\
\hline
\end{tabular}


WSRC-TR-2002-00459, Rev. 0 SRT-RPP-2002-00221

\begin{tabular}{|c|c|c|c|c|c|c|c|c|c|c|c|c|c|c|}
\hline \multicolumn{15}{|c|}{ BATCH \#2 ANALYTICAL RESULTS } \\
\hline & & & \multirow[b]{2}{*}{ OS } & \multirow[b]{2}{*}{ 1S } & \multirow[b]{2}{*}{$2 S$} & \multirow[b]{2}{*}{ 3S } & \multirow[b]{2}{*}{ 4S } & \multicolumn{2}{|c|}{ Solids Analysis } & \multirow[b]{2}{*}{$7 S$} & \multirow[b]{2}{*}{$8 \mathrm{~S}$} & \multirow[b]{2}{*}{ 9S } & \multirow[b]{2}{*}{$10 \mathrm{~S}$} & \multirow[b]{2}{*}{$\begin{array}{c}\text { Post- } \\
\text { Filtration }\end{array}$} \\
\hline EDL Sample No. & & & & & & & & $5 S$ & $6 S$ & & & & & \\
\hline \multicolumn{2}{|c|}{ Time after Reagent Add (hrs) } & & 0 & 0 & 0.125 & 0.25 & 0.5 & 1 & 2 & 3 & 4 & 6 & 8 & 674 \\
\hline Identity & Units & Method & & & & & & & & & & & & \\
\hline ADS Sample No. & & & 169784 & 170474 & 170475 & 170476 & 170477 & 170478 & 170479 & 170480 & 170481 & 170482 & 170483 & 172484 \\
\hline \multicolumn{15}{|l|}{ Solids microwave dried } \\
\hline Total Carbon & $\mu \mathrm{g} / \mathrm{ml}$ & & 39996 & 102600 & 35800 & 58600 & 38800 & 45400 & 37800 & 40800 & 21400 & 48800 & 48600 & \\
\hline TOC & $\mu \mathrm{g} / \mathrm{ml}$ & & 16362 & 92000 & 22800 & 39000 & 24000 & 30800 & 23600 & 25000 & 15180 & 31800 & 31800 & \\
\hline TIC & $\mu \mathrm{g} / \mathrm{ml}$ & & 23634 & 10400 & 12960 & 19500 & 14800 & 14400 & 16000 & 15960 & 6340 & 16840 & 16600 & \\
\hline Fluoride & $\mu \mathrm{g} / \mathrm{ml}$ & & 147 & 842 & 157 & 239 & 222 & 277 & 470 & 255 & 179 & 285 & 198 & \\
\hline Formate $\left(\mathrm{HCOO}^{-}\right)$ & $\mu \mathrm{g} / \mathrm{ml}$ & & 170 & 4410 & 15859 & 19150 & 14471 & 18436 & 16662 & 11298 & 16634 & 14766 & 13064 & \\
\hline Nitrite $\left(\mathrm{NO}_{2}^{-}\right)$ & $\mu \mathrm{g} / \mathrm{ml}$ & & 169 & 15823 & 21879 & 37332 & 32929 & 36607 & 38391 & 34951 & 26248 & 27271 & 30652 & \\
\hline Phosphate $\left(\mathrm{PO}_{4}{ }^{3-}\right)$ & $\mu \mathrm{g} / \mathrm{ml}$ & & 81 & $<92$ & $<95$ & 136 & $<95$ & 188 & 262 & 84 & $<95$ & 104 & 142 & \\
\hline Oxalate $\left(\mathrm{C}_{2} \mathrm{O}_{4}{ }^{2-}\right)$ & $\mu \mathrm{g} / \mathrm{ml}$ & & 31269 & 198880 & 39924 & 43363 & 48354 & 43951 & 40128 & 57243 & 39756 & 27271 & 49182 & \\
\hline Chloride $\left(\mathrm{Cl}^{-}\right)$ & $\mu \mathrm{g} / \mathrm{ml}$ & & 5533 & 878 & 902 & 1610 & 1173 & 1637 & 1538 & 1288 & 1037 & 1199 & 1267 & \\
\hline Nitrate $\left(\mathrm{NO}_{3}{ }^{-}\right)$ & $\mu \mathrm{g} / \mathrm{ml}$ & & 897 & 72872 & 94864 & 152580 & 120504 & 155045 & 192328 & 135608 & 110163 & 128537 & 121881 & \\
\hline Sulfate $\left(\mathrm{SO}_{4}{ }^{2-}\right)$ & $\mu \mathrm{g} / \mathrm{ml}$ & & 278 & 2807 & 4558 & 4983 & 6493 & 5191 & 9302 & 7838 & 4349 & 5050 & 4845 & \\
\hline $\mathrm{Al}_{2} \mathrm{O}_{3}$ (corundrum) & & XRD & 74-1081 & $46-1212$ & & & & & & & & & & \\
\hline $\mathrm{MnO}_{2}$ (pyrolusite) & & XRD & 24-0735 & 24-0735 & & & & & & & & & & \\
\hline $\mathrm{Fe}_{2} \mathrm{O}_{3}$ (Hermatite) & & XRD & 72-0469 & $86-2368$ & & & & & & & & & & \\
\hline \multicolumn{2}{|l|}{$\mathrm{C}_{2} \mathrm{CaO}_{4} ! \mathrm{H}_{2} \mathrm{O}$ (Whewelite $)$} & XRD & $20-0231$ & & & & & & & & & & & \\
\hline $\mathrm{CaCO}_{3}$ (calcite) & & XRD & 86-0174 & & & & & & & & & & & \\
\hline $\mathrm{SiO}_{2}$ (Quartz) & & XRD & $46-1045$ & $46-1045$ & & & & & & & & & & \\
\hline \multicolumn{2}{|c|}{$\mathrm{Mn}\left(\mathrm{SO}_{3}\right) \mathrm{H}_{2} \mathrm{O}$ (Mang Sulf Hyd) } & XRD & 82-0764 & & & & & & & & & & & \\
\hline $\mathrm{C}_{2} \mathrm{Na}_{2} \mathrm{O}_{4}$ (Natroxalate) & & XRD & $20-1149$ & $20-1149$ & $49-1816$ & $49-1816$ & 49-1816 & 49-1816 & $49-1816$ & $49-1816$ & $49-1816$ & 49-1816 & $49-1816$ & \\
\hline \multicolumn{2}{|l|}{$\mathrm{NaNO}_{3}$ (Sodium Nitrate) } & XRD & & 72-0025 & $72-0027$ & 72-0027 & $72-0027$ & 72-0027 & 72-0027 & $72-0027$ & 72-0027 & 72-0027 & $72-0027$ & \\
\hline $\mathrm{SrCO}_{3}($ Strontianite $)$ & & XRD & & & $84-1778$ & $84-1778$ & 84-1778 & 84-1778 & $84-1778$ & $84-1778$ & $84-1778$ & 84-1778 & $84-1778$ & \\
\hline \multicolumn{2}{|c|}{$\mathrm{Na}_{4} \mathrm{Sr}\left(\mathrm{SiO}_{3}\right)_{3}-\mathrm{Na}, \mathrm{Sr}$, Silicate } & XRD & & & 06-0392 & 06-0392 & 06-0392 & 06-0392 & 06-0392 & 06-0392 & 06-0392 & 06-0392 & 06-0392 & \\
\hline \multicolumn{2}{|c|}{$\mathrm{NiMn}_{2} \mathrm{O}_{3}(\mathrm{OH})_{4} ! \mathrm{H}_{2} \mathrm{O}$ (Asbolane) } & XRD & & & & & & & & & & & & $42-1319$ \\
\hline
\end{tabular}


WSRC-TR-2002-00459, Rev. 0 SRT-RPP-2002-00221

SRT-ADS-02-0031

ADS Number

300170474

300170475

300170476

300170477

300170478

300170479

300170480

300170481

300170482

300170483
Customer ID

RPP-WTP-PREC2-1S

RPP-WTP-PREC2-2S

RPP-WTP-PREC2-3S

RPP-WTP-PREC2-4S

RPP-WTP-PREC2-5S

RPP-WTP-PREC2-6S

RPP-WTP-PREC2-7S

RPP-WTP-PREC2-8S

RPP-WTP-PREC2-9S

RPP-WTP-PREC2-10S

Results

Ten samples were submitted for semivolatile organic compound (SVOC) analysis. The only SVOC analytes that were detected were phthalates, as well as adipate and maleate, commonly used as commercial plasticizers, as shown in the table below. The method detection limit (MDL) for the samples in this study was $1 \mathrm{mg} / \mathrm{kg}$.

Sample ID

RPP-WTP-PREC2-1S

RPP-WTP-PREC2-2S

RPP-WTP-PREC2-3S

RPP-WTP-PREC2-4S

RPP-WTP-PREC2-5S

RPP-WTP-PREC2-6S

RPP-WTP-PREC2-7S

RPP-WTP-PREC2-8S

RPP-WTP-PREC2-9S

RPP-WTP-PREC2-10S

DEP = Diethylphthalate

DBP = Dibutylphthalate

DOA = Diisooctyadipate

$\mathrm{DOM}=$ Diisooctylmaleate

Experimental

The liquid samples were extracted with methylene chloride and analyzed.

Gas Chromatography / Mass Spectrometry (GC/MS) analysis was employed to identify organic compounds in the sample. Analysis were carried out in building 773-A, laboratory B-123. It should be noted that ADS is not certified by DHEC for NPDES discharge compliance monitoring. Analytical separations were carried out on a Hewlett Packard 6890 gas chromatograph, equipped with a $30 \mathrm{~m}$ DB-5 column, with 0.25 mm diameter and 0.25 um film thickness. Quantitation was performed using a Hewlett Packard 5973 mass selective detector. The mass spectrometer tuning was confirmed within 24 hours prior to each measurement using perfluorotributylamine. 
WSRC-TR-2002-00459, Rev. 0 SRT-RPP-2002-00221

GC/MS Analysis Results-VOC

solid, liquid, gas samples no detectable VOC analytes

SRT-ADS-01-0537

Sample ID

ADS Number

3-170441

3-170442

3-170443

3-170444

3-170445

3-170446

3-170447

3-170448

3-170449

3-170450

3-170451

3-170452

3-170453

3-170454

3-170474

3-170475

3-170476

3-170477

3-170478

3-170479

3-170480

3-170481

3-170482

3-170483

Results

Ten solid, ten liquid, and four gas samples were submitted for volatile organic compounds (VOC) analysis.

The samples did not contain any detectable VOC analytes, and the limits of detection for this study are tabulated below.

Sample Matrix

MDL

Gas

Liquid (Aqueous)

Solid

$0.2 \mathrm{ppmv}$

$1 \mathrm{ug} / \mathrm{L}$

$10 \mathrm{ug} / \mathrm{kg}$

Experimental

Solid, liquid, and gas samples were analyzed using purge and trap Gas Chromatography / Mass Spectrometry (GC/MS).

$\mathrm{GC} / \mathrm{MS}$ analysis was employed to identify organic compounds in the samples. Analyses were carried out in building 773-A, laboratory B-159. It should be noted that ADS is not certified by DHEC for NPDES discharge compliance monitoring.

Samples were concentrated using a Tekmar 2016 Purge and Trap concentrator, using a three stage $(10 \mathrm{~cm}$ Carbopack B / $6 \mathrm{~cm}$

Carboxen $1000 / 1 \mathrm{~cm}$ Carboxen 1001) trap. Analytical separations were carried out on a Hewlett Packard 6890 gas chromatograph, equipped with a $20 \mathrm{~m}$ DB-624 column, with $0.18 \mathrm{~mm}$ diameter and 1.0 um film thickness.

Quantification was performed using a Hewlett Packard 5973 mass selective detector.

The mass spectrometer tuning was confirmed within 24 hours prior to each measurement using perfluorotributylamine.

Some VOC samples for this study were analyzed on the following instrument.

Volatile organic analyses were performed by Gas Chromatography - Mass Spectrometry (GC-MS), using the ADS method 2656 (Contract Laboratory Program SOW 7-93 for Volatile Organics).

Samples were concentrated using an OI Analytical model 4460A Dynamic Headspace concentrator (Purge and Trap), using a three stage (10 cm Carbopack B / $6 \mathrm{~cm}$ Carboxen $1000 / 1 \mathrm{~cm}$ Carboxen 1001) trap.

Separation was performed with a Hewlett Packard 5890 series II gas chromatograph on a $105 \mathrm{~m} \times 0.32 \mathrm{~mm}$ VOCOL glass capillary column with 3 um film thickness. Quantitation was performed with a Hewlett Packard model 5971 quadrupole mass spectrometer. Internal standard and recovery surrogate compounds were added as specified in the Contract Laboratory Program for volatile organics (SOW 7-93). The mass spectrometer tuning was confirmed within 12 hours prior to each measurement using 4-bromofluorobenzene.

Tuning verification was performed against CLP tuning requirements, specifically to optimize CLP requirements for high mass sensitivity. $50 / 95$ ratios which are between $8 \%-15 \%$, may require appropriate flagging if used for other purposes. 
WSRC-TR-2002-00459, Rev. 0

SRT-RPP-2002-00221

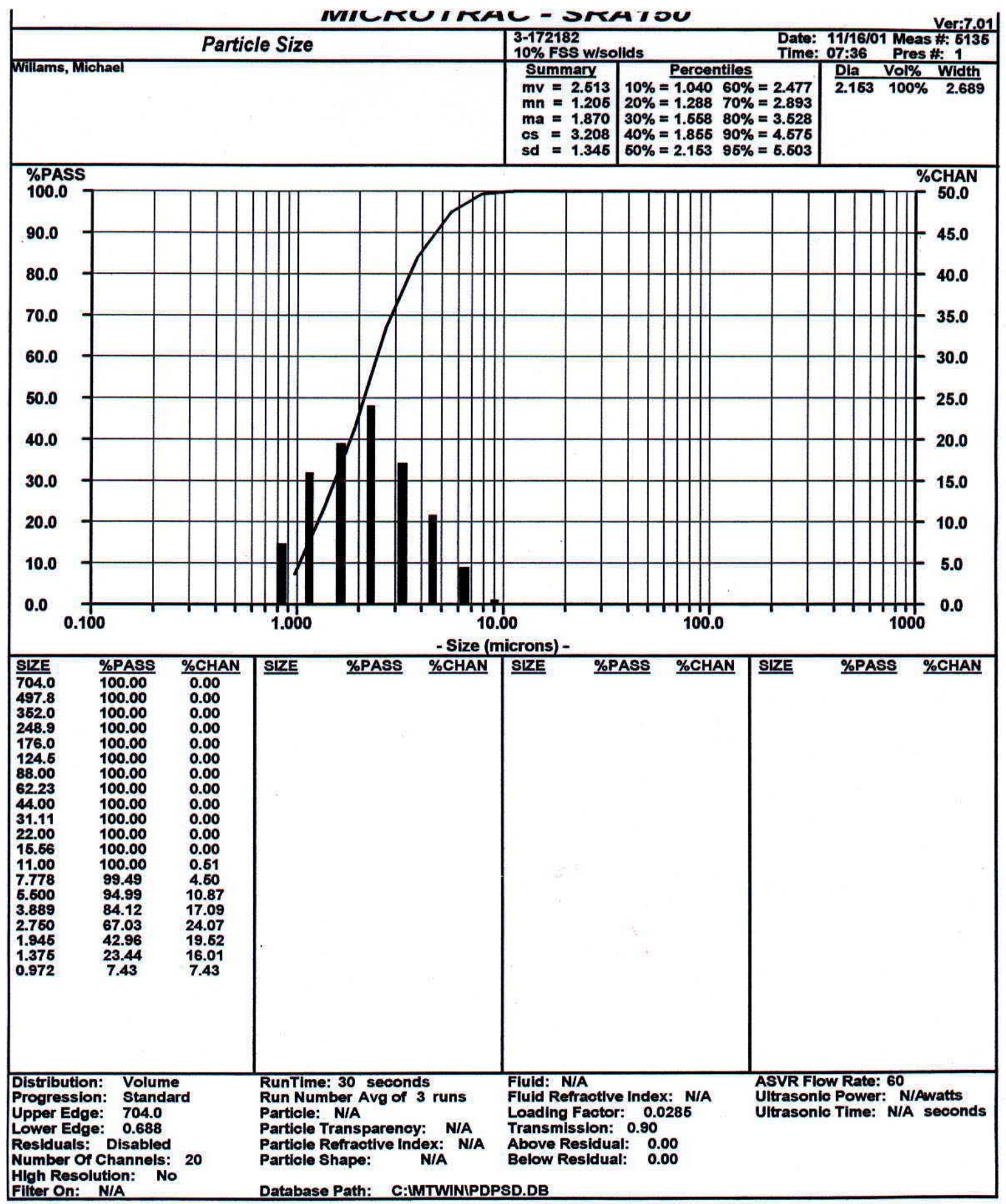




\section{Lasentec Chord Length Data for Pilot-Scale Precipitation Run \#2 Post Filtration Samples}

Hanford AN-107 simulant samples were received from Engineering Development Laboratory personnel for analysis of post filtration solids. The samples were isolated at various times after the completion of precipitate reagent additions (on 10-23-01 at 9:47 hours) as indicated in Table 1 below. Each sample was immediately filtered through a 0.45 micron disposable Nalgene Nylon filter unit to yield approximately $1 \mathrm{~L}$ of filtrate. The samples were stored in $1 \mathrm{~L}$ widemouth, amber polypropylene bottles to minimize interactions with light during storage. This bottle type allowed for Lasentec chord length analysis without removing the sample from the storage container. Samples were initially analyzed with the Lasentec within 24 hours of the filtration to determine whether any solids were present.

The Lasentec FBRM is a laser-based technique, which utilizes backscattered signal from particles within the detector measurement zone to obtain chord length data for a population of particles. The FBRM is a highly sensitive technique due to the fact that it measures backscattered laser intensity from individual particles within the sample. In addition, the method requires no sample preparation and is suitable for in-process analysis. These are significant advantages over traditional methods for the analysis of suspended solids in liquid media. The FBRM method requires that the particles be passed across the probe surface. This is generally achieved by placing the probe within a flowing liquid stream or (in the case of individual samples) by stirring the liquid using an appropriately designed and positioned impeller blade. A particle chord length is defined as the diameter of the particle as it is presented to the detector. For a given non-spherical particle, the particle may be presented to the detector in a number of orientations and a number of unique chords may be measured. Since the AN-107 simulant composition is complex, post-filtration solids may contain a mixture of particles with different compositions and morphologies (shapes). This adds to the complexity of the measured chord length distribution. In addition, as particle counts increase, the FBRM response may not be linear and the data cannot be considered to be highly quantitative unless suitable standards can be prepared and a calibration curve generated. Nonetheless, general comparisons of particle counts can sometimes be made between samples of the same type.

Table 1. AN-107 Filtrate Sample Isolation Times

\begin{tabular}{|c|c|c|}
\hline $\begin{array}{c}\text { Sample } \\
\text { ID }\end{array}$ & $\begin{array}{c}\text { Sample } \\
\text { Collection } \\
\text { Date/Time }\end{array}$ & $\begin{array}{c}\text { Reaction } \\
\text { Time Before } \\
\text { Filtration (hr) }\end{array}$ \\
\hline PF2-1 & $10-23-01 / 13: 00$ & 3.2 \\
\hline PF2-2 & $10-24-01 / 00: 15$ & 14.5 \\
\hline PF2-3 & $10-24-01 / 07: 30$ & 21.7 \\
\hline PF2-4 & $10-24-01 / 16: 00$ & 30.2 \\
\hline PF2-5 & $10-25-01 / 00: 05$ & 38.3 \\
\hline PF2-6 & $10-25-01 / 07: 30$ & 45.7 \\
\hline
\end{tabular}

Figure 1 shows the chord length data obtained for selected samples from pilot scale precipitation test \#2 after filtration. Table 2 provides the total chord length counts measured per second, the mean chord length for each sample, and the time that after filtration that each sample was 
analyzed. Sample PF2-2 was analyzed on two successive days (PF2-2-A and -B). Measurable solids were observed for samples PF2-5 and -6, although the total counts did not exceed 65 for either sample. The counts observed for samples PF2-2-A, -3, and -4 were below normal instrumental background levels (typically around 15 counts per second). Note that the counts per second provided in Table 2 cannot be directly related to weight $\%$ solids in the samples, since this measurement was not conducted. Visible solids could not be observed in any of these samples and it is unlikely that the total mass of solid material was high enough to be isolated and measured accurately. Significantly higher counts were observed for sample PF2-2-B

approximately 23 hours after the initial analysis of this sample (PF2-2-A) although the total solid content was still very low and no solids were visually observed. The analysis of the -2-B sample does provide an idea of the formation time scale and size of the initial precipitate.

Table 2. Chord Length Data Obtained Following Filtration

\begin{tabular}{|l|c|c|c|}
\hline Sample & Total Counts/sec & $\begin{array}{c}\text { Mean Chord } \\
\text { Length }(\mu \mathrm{m})\end{array}$ & $\begin{array}{c}\text { Measurement } \\
\text { Time After } \\
\text { Filtration (hr) }\end{array}$ \\
\hline PF2-1 & Not measured & Not measured & Not measured \\
\hline PF2-2-A & 9 & 139 & 18.8 \\
\hline PF2-2-B & 187 & 21 & 41.6 \\
\hline PF2-3 & 8 & 145 & 11.6 \\
\hline PF2-4 & 9 & 141 & 2.9 \\
\hline PF2-5 & 59 & 29 & 17.7 \\
\hline PF2-6 & 62 & 28 & 10.1 \\
\hline
\end{tabular}

Figure 1. Pilot Scale Run \#2 Chord Length Data After Filtration (Chord Length vs. \%/Channel)

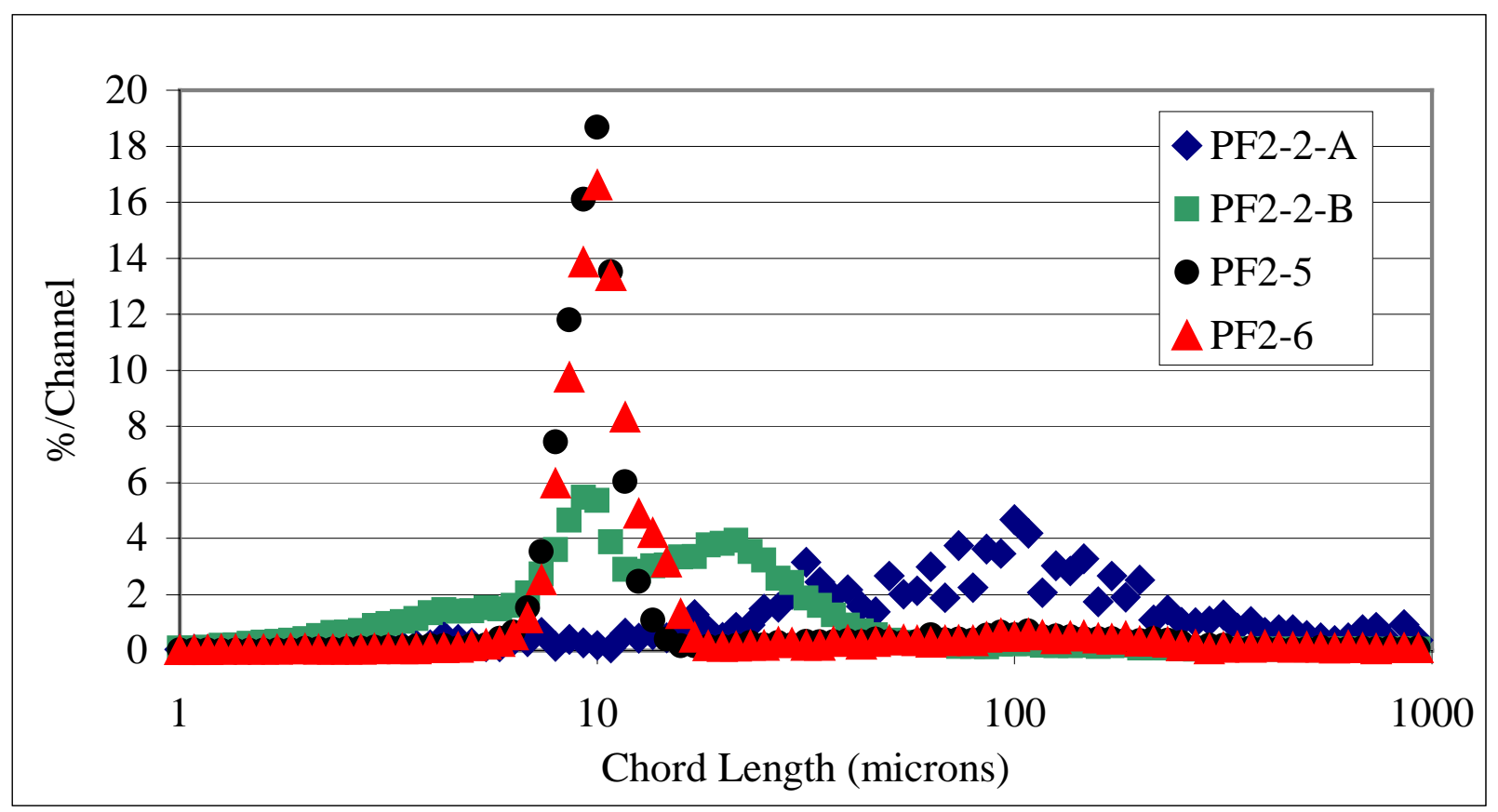

The samples were stored after analysis for nearly 5 months at ambient temperature in the amber bottles. The air headspace above the samples was $<10 \%$ of the total volume. Table 3 provides 
the chord length data obtained for the samples after storage. The samples were all analyzed on 3-6-02. Visible solids were observed for all samples on the container bottoms and sides. Approximately $75 \%$ of the solids were removed from the container walls by shaking the bottles. The total counts and mean chord lengths for all measured samples were similar (average counts: 2040; average mean chord length: $27 \mu \mathrm{m}$ ). The chord length distribution shown in Figure 2 for sample PF2-2 is typical of the distributions observed for the other samples.

Table 3. Chord Length Data Obtained After Storage for Several Months

\begin{tabular}{|l|c|c|}
\hline Sample & $\begin{array}{c}\text { Total } \\
\text { Counts/sec }\end{array}$ & $\begin{array}{c}\text { Mean Chord } \\
\text { Length }(\mu \mathrm{m})\end{array}$ \\
\hline PF2-1 & 2013 & 33 \\
\hline PF2-2 & 1972 & 28 \\
\hline PF2-3 & 2030 & 23 \\
\hline PF2-4 & 2256 & 24 \\
\hline PF2-5 & 1931 & 27 \\
\hline PF2-6 & Not measured & Not measured \\
\hline
\end{tabular}

Figure 2. Pilot Scale Run \#2 Chord Length Data for Sample PF2-2 After Storage (Chord Length vs. \%/channel)

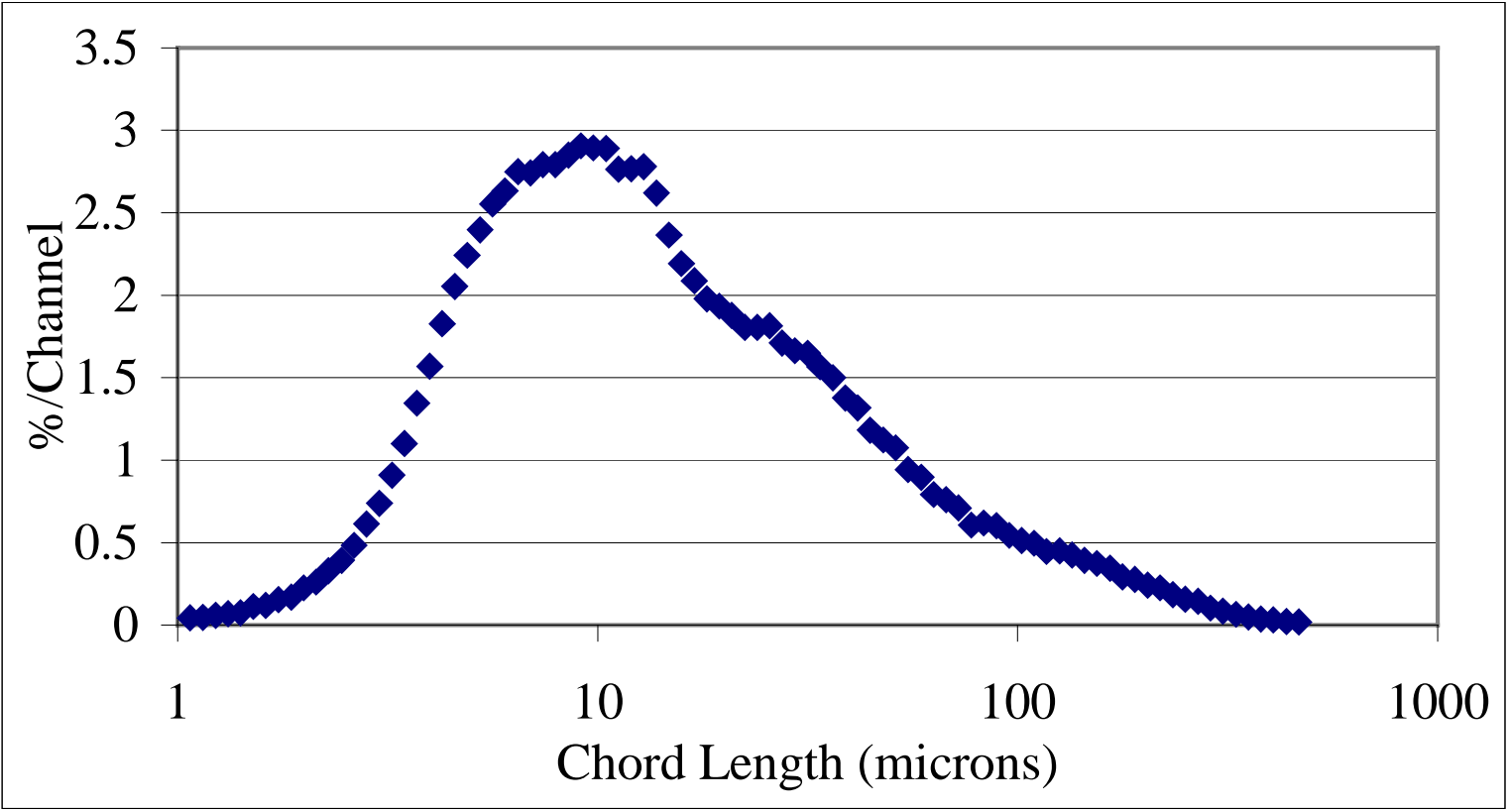




\section{Appendix B}

\section{Experimental Data: Precipitation Test Rig Operations Data}

\section{Appendix Contents}

Nomenclature for Data Sheets

- HX Outlet $\left({ }^{\circ} \mathrm{C}\right) \mathrm{TC}-\mathrm{T} 0$, Temperature of the liquid at the outlet of the Heat Exchanger for the Precipitate Tank

- REC PP Outlet $\left({ }^{\circ} \mathrm{C}\right) \mathrm{TC}-\mathrm{T} 1$, Temperature of the liquid at the discharge of the Recirculation Pump for the Precipitate Tank

- TK BOT $\left({ }^{\circ} \mathrm{C}\right) \mathrm{TC}-\mathrm{T} 2$, Temperature of the liquid at the bottom of the Precipitate Tank

- HT OUT $\left({ }^{\circ} \mathrm{C}\right) \mathrm{TC}-\mathrm{T} 3$, Temperature of the liquid at the outlet of the Heater

- Mn Flow (gpm), Flow of $1 \mathrm{M} \mathrm{NaMnO}$ r reagent through recirculation loop for mixing, or directed to precipitation tank

- Galigher Flow (gpm), Flow of liquid through the recirculation pump to precipitation tank

- Current to HX (amps), Power to heater for precipitation tank

- Voltage to HX (volts), Voltage to heater for precipitation tank

- Sr Flow (gpm), Flow of $1 \mathrm{M} \operatorname{Sr}\left(\mathrm{NO}_{3}\right)_{2}$ reagent through recirculation loop for mixing, or directed to precipitation tank 
WSRC-TR-2002-00459, Rev. 0 SRT-RPP-2002-00221

\begin{tabular}{|c|c|c|c|c|c|c|c|c|c|c|}
\hline \multicolumn{11}{|c|}{ PREC2_102301_0623 } \\
\hline DATE & TIME & $\begin{array}{c}\mathrm{HX} \\
\text { Outlet } \\
\left({ }^{\circ} \mathrm{C}\right) \\
\mathrm{TC} 0\end{array}$ & $\mid \begin{array}{c}\text { REC } \\
\text { PP } \\
\text { OUT } \\
\left({ }^{\circ} \mathrm{C}\right) \\
\text { TC1 }\end{array}$ & $\begin{array}{c}\text { TK } \\
\text { BOT } \\
\left({ }^{\circ} \mathrm{C}\right) \\
\text { TC2 }\end{array}$ & $\begin{array}{c}\text { HX } \\
\text { OUT } \\
\left({ }^{\circ} \mathrm{C}\right) \\
\text { TC3 }\end{array}$ & \begin{tabular}{|c|} 
Mn \\
Flow \\
(gpm)
\end{tabular} & \begin{tabular}{|c|} 
Galigher \\
Flow \\
$(\mathrm{gpm})$
\end{tabular} & $\begin{array}{l}\text { Current } \\
\text { to } \mathrm{HX}\end{array}$ & $\begin{array}{c}\text { Voltage } \\
\text { to } \mathrm{HX}\end{array}$ & $\begin{array}{c}\mathrm{Sr} \\
\text { Flow } \\
(\mathrm{gpm})\end{array}$ \\
\hline $10 / 23 / 01$ & $6: 24$ & 20.6 & 20.6 & 20.8 & 20.6 & 0.0 & -0.1 & 0 & 0 & 0.0 \\
\hline $10 / 23 / 01$ & $6: 25$ & 20.6 & 20.6 & 20.8 & 20.6 & 0.0 & -0.1 & 0 & 0 & 0.0 \\
\hline $10 / 23 / 01$ & $6: 26$ & 20.6 & 20.6 & 20.8 & 20.6 & 0.0 & -0.1 & 0 & 0 & 0.0 \\
\hline $10 / 23 / 01$ & $6: 27$ & 20.6 & 20.6 & 20.8 & 20.7 & 0.0 & -0.1 & 0 & 0 & 0.0 \\
\hline $10 / 23 / 01$ & $6: 28$ & 20.6 & 20.6 & 20.9 & 20.6 & 0.0 & -0.1 & 65 & 79 & 0.0 \\
\hline $10 / 23 / 01$ & $6: 29$ & 20.6 & 20.6 & 20.9 & 20.7 & 0.0 & -0.1 & 65 & 79 & 0.0 \\
\hline $10 / 23 / 01$ & $6: 30$ & 20.6 & 20.6 & 20.9 & 20.7 & 0.1 & -0.1 & 65 & 79 & 0.0 \\
\hline $10 / 23 / 01$ & $6: 31$ & 20.6 & 20.6 & 20.9 & 20.7 & 0.0 & -0.1 & 65 & 79 & 0.0 \\
\hline $10 / 23 / 01$ & 6:32 & 20.6 & 20.6 & 20.9 & 20.7 & 0.0 & -0.1 & 65 & 79 & 0.0 \\
\hline $10 / 23 / 01$ & $6: 33$ & 20.6 & 20.6 & 20.9 & 20.7 & 0.0 & -0.1 & 65 & 79 & 0.0 \\
\hline $10 / 23 / 01$ & $6: 34$ & 20.6 & 20.6 & 20.9 & 20.7 & 0.2 & -0.1 & 65 & 79 & 0.0 \\
\hline $10 / 23 / 01$ & $6: 35$ & 20.6 & 20.6 & 20.9 & 20.7 & 0.2 & -0.1 & 65 & 79 & 0.0 \\
\hline $10 / 23 / 01$ & $6: 36$ & 20.6 & 20.6 & 20.9 & 20.7 & 0.0 & -0.1 & 65 & 79 & 0.0 \\
\hline $10 / 23 / 01$ & 6:37 & 20.6 & 20.6 & 20.9 & 20.7 & 0.0 & -0.1 & 65 & 79 & 0.0 \\
\hline $10 / 23 / 01$ & $6: 38$ & 20.6 & 20.6 & 20.8 & 20.7 & 0.0 & -0.1 & 65 & 79 & 0.0 \\
\hline $10 / 23 / 01$ & 6:39 & 21.6 & 21.6 & 21.7 & 21.6 & 0.0 & 9.6 & 65 & 79 & 0.0 \\
\hline $10 / 23 / 01$ & $6: 40$ & 21.6 & 21.6 & 21.7 & 21.6 & 0.0 & 9.6 & 65 & 79 & 0.0 \\
\hline $10 / 23 / 01$ & $6: 41$ & 21.7 & 21.6 & 21.7 & 21.7 & 0.0 & 9.7 & 65 & 79 & 0.0 \\
\hline $10 / 23 / 01$ & $6: 42$ & 21.7 & 21.7 & 21.7 & 21.7 & 0.0 & 9.6 & 65 & 79 & 0.0 \\
\hline $10 / 23 / 01$ & $6: 43$ & 20.9 & 21.7 & 21.7 & 20.9 & 0.1 & 9.6 & 65 & 79 & 0.0 \\
\hline $10 / 23 / 01$ & $6: 44$ & 21.3 & 21.6 & 21.7 & 21.2 & 0.3 & 9.6 & 65 & 79 & 0.0 \\
\hline $10 / 23 / 01$ & $6: 45$ & 20.6 & 21.6 & 21.7 & 20.5 & 0.2 & 9.7 & 65 & 79 & 0.0 \\
\hline $10 / 23 / 01$ & 6:46 & 21.1 & 21.6 & 21.6 & 21.1 & 0.2 & 9.6 & 65 & 79 & 0.0 \\
\hline $10 / 23 / 01$ & $6: 47$ & 20.6 & 21.6 & 21.6 & 20.6 & 0.0 & 9.7 & 65 & 79 & 0.0 \\
\hline $10 / 23 / 01$ & $6: 48$ & 21.1 & 21.5 & 21.6 & 21.0 & 0.0 & 9.6 & 65 & 79 & 0.0 \\
\hline $10 / 23 / 01$ & $6: 49$ & 20.6 & 21.5 & 21.6 & 20.6 & 0.0 & 9.7 & 65 & 79 & 0.0 \\
\hline $10 / 23 / 01$ & $6: 50$ & 21.0 & 21.5 & 21.5 & 21.0 & 0.0 & 9.6 & 65 & 79 & 0.0 \\
\hline $10 / 23 / 01$ & 6:51 & 20.5 & 21.5 & 21.5 & 20.6 & 0.1 & 9.6 & 65 & 79 & 0.0 \\
\hline $10 / 23 / 01$ & $6: 52$ & 21.0 & 21.4 & 21.5 & 20.9 & 0.0 & 9.6 & 65 & 79 & 0.0 \\
\hline $10 / 23 / 01$ & 6:53 & 20.9 & 21.4 & 21.5 & 20.9 & 0.0 & 9.6 & 65 & 79 & 0.0 \\
\hline $10 / 23 / 01$ & 6:54 & 20.8 & 21.4 & 21.4 & 20.7 & 0.0 & 9.6 & 65 & 79 & 0.0 \\
\hline $10 / 23 / 01$ & 6:55 & 21.0 & 21.4 & 21.4 & 21.0 & 0.0 & 9.7 & 65 & 79 & 0.0 \\
\hline $10 / 23 / 01$ & 6:56 & 20.7 & 21.3 & 21.4 & 20.6 & 0.0 & 9.6 & 65 & 79 & 0.0 \\
\hline $10 / 23 / 01$ & $6: 57$ & 21.0 & 21.3 & 21.4 & 20.9 & 0.0 & 9.6 & 65 & 79 & 0.0 \\
\hline $10 / 23 / 01$ & 6:58 & 20.5 & 21.3 & 21.4 & 20.4 & 0.0 & 9.7 & 65 & 79 & 0.0 \\
\hline $10 / 23 / 01$ & $6: 59$ & 20.9 & 21.3 & 21.3 & 20.9 & 0.0 & 9.7 & 65 & 79 & 0.0 \\
\hline $10 / 23 / 01$ & 7:00 & 20.3 & 21.3 & 21.3 & 20.3 & 0.1 & 9.7 & 65 & 79 & 0.0 \\
\hline $10 / 23 / 01$ & 7:01 & 21.1 & 21.5 & 21.5 & 21.0 & 5.0 & 9.6 & 65 & 79 & 0.0 \\
\hline $10 / 23 / 01$ & 7:02 & 21.0 & 21.7 & 21.7 & 20.9 & 5.0 & 9.6 & 65 & 79 & 0.0 \\
\hline $10 / 23 / 01$ & 7:03 & 21.4 & 21.8 & 21.8 & 21.1 & 5.0 & 9.6 & 65 & 79 & 0.0 \\
\hline $10 / 23 / 01$ & 7:04 & 21.6 & 21.8 & 21.8 & 21.3 & 5.0 & 9.7 & 65 & 79 & 0.0 \\
\hline $10 / 23 / 01$ & 7:05 & 21.3 & 21.8 & 21.8 & 20.8 & 5.0 & 9.6 & 65 & 79 & 0.0 \\
\hline $10 / 23 / 01$ & 7:06 & 21.6 & 21.8 & 21.7 & 21.2 & 5.0 & 9.6 & 65 & 79 & 0.0 \\
\hline $10 / 23 / 01$ & 7:07 & 21.0 & 21.8 & 21.7 & 20.6 & 5.0 & 9.5 & 65 & 79 & 0.0 \\
\hline $10 / 23 / 01$ & 7:08 & 21.5 & 21.7 & 21.7 & 21.1 & 5.0 & 9.6 & 65 & 79 & 0.0 \\
\hline $10 / 23 / 01$ & 7:09 & 21.0 & 21.7 & 21.7 & 20.7 & 5.0 & 9.7 & 65 & 79 & 0.0 \\
\hline $10 / 23 / 01$ & $7: 10$ & 21.4 & \begin{tabular}{|l|}
21.7 \\
\end{tabular} & 21.6 & 21.0 & 5.0 & 9.6 & 65 & 79 & 0.0 \\
\hline $10 / 23 / 01$ & 7:11 & 21.5 & 21.7 & 21.6 & 21.1 & 5.0 & 9.6 & 65 & 79 & 0.0 \\
\hline $10 / 23 / 01$ & 7:12 & 21.2 & \begin{tabular}{|l|} 
\\
21.7 \\
\end{tabular} & 21.6 & 20.7 & 5.0 & 9.6 & 65 & 79 & 0.0 \\
\hline $10 / 23 / 01$ & $7: 13$ & 21.5 & 21.6 & 21.6 & 21.0 & 5.0 & 9.7 & 65 & 79 & 0.0 \\
\hline $10 / 23 / 01$ & 7:14 & 20.9 & 21.6 & 21.6 & 20.4 & 5.0 & 9.6 & 65 & 79 & 0.0 \\
\hline $10 / 23 / 01$ & $7: 15$ & 21.4 & 21.6 & 21.6 & 20.9 & 5.0 & 9.7 & 65 & 79 & 0.0 \\
\hline $10 / 23 / 01$ & $7: 16$ & 21.1 & 21.6 & 21.6 & 20.8 & 5.0 & 9.6 & 65 & 79 & 0.0 \\
\hline $10 / 23 / 01$ & 7:17 & 21.3 & 21.6 & 21.5 & 20.8 & 5.0 & 9.7 & 65 & 79 & 0.0 \\
\hline $10 / 23 / 01$ & $7: 18$ & 21.5 & 21.6 & 21.5 & 21.0 & 5.0 & 9.7 & 65 & 79 & 0.0 \\
\hline $10 / 23 / 01$ & $7: 19$ & 21.1 & 21.5 & 21.5 & 20.5 & 5.0 & 9.7 & 65 & 79 & 0.0 \\
\hline $10 / 23 / 01$ & 7:20 & 21.4 & 21.5 & 21.5 & 20.9 & 5.0 & 9.6 & 65 & 79 & 0.0 \\
\hline $10 / 23 / 01$ & 7:21 & 20.7 & 21.5 & 21.5 & 20.4 & 5.0 & 9.6 & 65 & 79 & 0.0 \\
\hline $10 / 23 / 01$ & 7:22 & 21.3 & 21.5 & 21.4 & 20.8 & 5.0 & 9.7 & 65 & 79 & 0.0 \\
\hline $10 / 23 / 01$ & 7:23 & 21.4 & 21.5 & 21.4 & 21.0 & 5.0 & 9.6 & 65 & 79 & 0.0 \\
\hline
\end{tabular}

\begin{tabular}{|c|c|c|c|c|c|c|c|c|c|c|}
\hline \multicolumn{11}{|c|}{ PREC2_102301_0623 } \\
\hline DATE & TIME & \begin{tabular}{|c|}
$\mathrm{HX}$ \\
Outlet \\
$\left({ }^{\circ} \mathrm{C}\right)$ \\
$\mathrm{TC} 0$ \\
\end{tabular} & \begin{tabular}{|c|} 
REC \\
PP \\
OUT \\
$\left({ }^{\circ} \mathrm{C}\right)$ \\
TC1 \\
\end{tabular} & $\begin{array}{c}\text { TK } \\
\text { BOT } \\
\left({ }^{\circ} \mathrm{C}\right) \\
\text { TC2 } \\
\end{array}$ & $\begin{array}{c}\mathrm{HX} \\
\text { OUT } \\
\left({ }^{\circ} \mathrm{C}\right) \\
\text { TC3 }\end{array}$ & \begin{tabular}{|c|} 
Mn \\
Flow \\
(gpm) \\
\end{tabular} & \begin{tabular}{|c|} 
Galigher \\
Flow \\
(gpm) \\
\end{tabular} & $\begin{array}{c}\text { Current } \\
\text { to } \mathrm{HX}\end{array}$ & $\begin{array}{c}\text { Voltage } \\
\text { to } \mathrm{HX} \\
\end{array}$ & $\begin{array}{c}\text { Sr } \\
\text { Flow } \\
(\mathrm{gpm})\end{array}$ \\
\hline 10/23/01 & 7:24 & 21.1 & 21.5 & 21.4 & 20.5 & 5.0 & 9.7 & 65 & 79 & 0.0 \\
\hline $10 / 23 / 01$ & 7:25 & 21.4 & 21.4 & 21.4 & 20.9 & 5.0 & 9.7 & 65 & 79 & 0.0 \\
\hline 10/23/01 & $7: 26$ & 20.7 & 21.5 & 21.4 & 20.3 & 5.0 & 9.6 & 65 & 79 & 0.0 \\
\hline 10/23/01 & 7:27 & 21.2 & 21.4 & 21.4 & 20.8 & 5.0 & 9.7 & 65 & 79 & 0.0 \\
\hline 10/23/01 & 7:28 & 21.4 & 21.4 & 21.4 & 20.9 & 5.0 & 9.6 & 65 & 79 & 0.0 \\
\hline $10 / 23 / 01$ & $7: 29$ & 21.0 & 21.4 & 21.4 & 20.5 & 5.0 & 9.6 & 65 & 79 & 0.0 \\
\hline $10 / 23 / 01$ & 7:30 & 21.3 & 21.4 & 21.3 & 20.8 & 5.0 & 9.6 & 65 & 79 & 0.0 \\
\hline 10/23/01 & 7:31 & 20.7 & 21.4 & 21.3 & 20.3 & 5.0 & 9.7 & 65 & 79 & 0.0 \\
\hline $10 / 23 / 01$ & 7:32 & 21.2 & 21.3 & 21.3 & 20.7 & 5.0 & 9.6 & 65 & 79 & 0.0 \\
\hline 10/23/01 & 7:33 & 21.3 & 21.3 & 21.3 & 20.8 & 5.0 & 9.7 & 65 & 79 & 0.0 \\
\hline 10/23/01 & 7:34 & 20.9 & 21.3 & 21.3 & 20.3 & 5.0 & 9.7 & 65 & 79 & 0.0 \\
\hline 10/23/01 & 7:35 & 21.2 & 21.3 & 21.3 & 20.7 & 5.0 & 9.5 & 65 & 79 & 0.0 \\
\hline 10/23/01 & $7: 36$ & 20.8 & 21.3 & 21.3 & 20.5 & 5.0 & 9.6 & 65 & 79 & 0.0 \\
\hline 10/23/01 & 7:37 & 21.1 & \begin{tabular}{|l|}
21.3 \\
\end{tabular} & 21.3 & 20.6 & 5.0 & 9.6 & 65 & 79 & 0.0 \\
\hline $10 / 23 / 01$ & 7:38 & 21.3 & \begin{tabular}{|l|}
21.3 \\
\end{tabular} & 21.2 & 20.8 & 5.0 & 9.7 & 65 & 79 & 0.0 \\
\hline $10 / 23 / 01$ & 7:39 & 20.7 & 21.2 & 21.2 & 20.2 & 5.0 & 9.7 & 65 & 79 & 0.0 \\
\hline $10 / 23 / 01$ & 7:40 & 21.0 & 21.2 & 21.1 & 20.6 & 0.6 & 9.6 & 65 & 79 & 0.0 \\
\hline 10/23/01 & 7:41 & 20.5 & \begin{tabular}{|l|}
20.9 \\
\end{tabular} & 20.9 & 20.3 & 0.0 & 9.7 & 65 & 79 & 0.0 \\
\hline 10/23/01 & 7:42 & 20.4 & \begin{tabular}{|l|}
20.7 \\
\end{tabular} & 20.7 & 20.2 & 0.0 & 9.6 & 65 & 79 & 0.0 \\
\hline $10 / 23 / 01$ & 7:43 & 20.5 & \begin{tabular}{|l|} 
\\
\end{tabular} & 20.7 & 20.4 & 0.0 & 9.7 & 65 & 79 & 0.0 \\
\hline $10 / 23 / 01$ & 7:44 & 19.9 & 20.6 & 20.7 & 19.8 & 0.0 & 9.6 & 65 & 79 & 0.0 \\
\hline $10 / 23 / 01$ & 7:45 & 20.4 & 20.6 & 20.6 & 20.3 & 0.0 & 9.7 & 65 & 79 & 0.0 \\
\hline $10 / 23 / 01$ & 7:46 & 20.3 & 20.6 & 20.6 & 20.3 & 0.0 & 9.6 & 65 & 79 & 0.0 \\
\hline 10/23/01 & 7:47 & 20.2 & \begin{tabular}{|l|}
20.6 \\
\end{tabular} & 20.6 & 20.1 & 0.0 & 9.7 & 65 & 79 & 0.0 \\
\hline 10/23/01 & 7:48 & 20.4 & \begin{tabular}{|l|}
20.6 \\
\end{tabular} & 20.6 & 20.4 & 0.0 & 9.6 & 65 & 79 & 0.0 \\
\hline 10/23/01 & 7:49 & 19.8 & 20.6 & 20.6 & 19.7 & 0.0 & 9.6 & 65 & 79 & 0.0 \\
\hline 10/23/01 & 7:50 & 20.4 & \begin{tabular}{|l|}
20.6 \\
\end{tabular} & 20.6 & 20.3 & 5.0 & 9.7 & 65 & 79 & 0.0 \\
\hline $10 / 23 / 01$ & 7:51 & 20.9 & \begin{tabular}{|l|} 
\\
\end{tabular} & 21.0 & 20.7 & 5.0 & 9.6 & 65 & 79 & 0.0 \\
\hline 10/23/01 & 7:52 & 20.7 & 21.1 & 21.1 & 20.4 & 5.0 & 9.6 & 65 & 79 & 0.0 \\
\hline 10/23/01 & 7:53 & 21.0 & 21.1 & 21.1 & 20.7 & 5.0 & 9.7 & 65 & 79 & 0.0 \\
\hline 10/23/01 & 7:54 & 20.4 & \begin{tabular}{|l|}
21.0 \\
\end{tabular} & 21.0 & 20.2 & 2.1 & 9.6 & 65 & 79 & 0.0 \\
\hline 10/23/01 & 7:55 & 20.6 & \begin{tabular}{|l|} 
\\
\end{tabular} & 20.8 & 20.3 & 2.1 & 9.6 & 65 & 79 & 2.0 \\
\hline $10 / 23 / 01$ & 7:56 & 20.6 & \begin{tabular}{|l|}
20.7 \\
\end{tabular} & 20.7 & 20.4 & 2.1 & 9.6 & 65 & 79 & 2.1 \\
\hline 10/23/01 & 7:57 & 20.1 & 20.6 & 20.7 & 19.9 & 2.2 & 9.6 & 65 & 79 & 2.1 \\
\hline 10/23/01 & 7:58 & 20.4 & \begin{tabular}{|l|} 
\\
\end{tabular} & 20.6 & 20.3 & 2.1 & 9.6 & 65 & 79 & 2.1 \\
\hline 10/23/01 & 7:59 & 20.2 & \begin{tabular}{|l|}
20.6 \\
\end{tabular} & 20.6 & 20.3 & 2.1 & 9.6 & 65 & 79 & 2.1 \\
\hline 10/23/01 & 8:00 & 20.2 & 20.5 & 20.6 & 20.1 & 2.1 & 9.7 & 65 & 79 & 2.1 \\
\hline 10/23/01 & 8:01 & 20.4 & \begin{tabular}{|l|} 
\\
\end{tabular} & 20.6 & 20.3 & 2.1 & 9.6 & 65 & 79 & 2.1 \\
\hline 10/23/01 & 8:02 & 19.8 & 20.5 & 20.6 & 19.7 & 2.1 & 9.6 & 65 & 79 & 2.0 \\
\hline 10/23/01 & 8:03 & 20.3 & 20.5 & 20.6 & 20.2 & 2.1 & 9.6 & 65 & 79 & 2.0 \\
\hline $10 / 23 / 01$ & 8:04 & 20.5 & 20.5 & 20.6 & 20.4 & 2.1 & 9.7 & 65 & 79 & 2.0 \\
\hline 10/23/01 & 8:05 & 20.0 & 20.5 & 20.6 & 19.9 & 2.1 & 9.6 & 65 & 79 & 2.0 \\
\hline 10/23/01 & 8:06 & 20.3 & 20.5 & 20.5 & 20.3 & 2.1 & 9.6 & 65 & 79 & 2.0 \\
\hline $10 / 23 / 01$ & 8:07 & 20.0 & 20.5 & 20.5 & 20.1 & 2.1 & 9.7 & 65 & 79 & 2.0 \\
\hline $10 / 23 / 01$ & 8:08 & 20.2 & \begin{tabular}{|l|} 
\\
\end{tabular} & 20.5 & 20.1 & 2.1 & 9.7 & 65 & 79 & 2.0 \\
\hline $10 / 23 / 01$ & 8:09 & 20.4 & 20.5 & 20.5 & 20.3 & 2.1 & 9.6 & 65 & 79 & 2.0 \\
\hline $10 / 23 / 01$ & $8: 10$ & 19.8 & 20.5 & 20.5 & 19.7 & 2.1 & 9.6 & 65 & 79 & 2.0 \\
\hline 10/23/01 & 8:11 & 20.3 & \begin{tabular}{|l|} 
\\
\end{tabular} & 20.5 & 20.2 & 2.1 & 9.6 & 65 & 79 & 2.0 \\
\hline 10/23/01 & $8: 12$ & 20.4 & \begin{tabular}{|l|}
20.4 \\
\end{tabular} & 20.5 & 20.3 & 2.1 & 9.7 & 65 & 79 & 2.0 \\
\hline 10/23/01 & $8: 13$ & 20.0 & \begin{tabular}{|l|} 
\\
\end{tabular} & 20.5 & 19.9 & 2.1 & 9.5 & 65 & 79 & 2.0 \\
\hline $10 / 23 / 01$ & $8: 14$ & 20.3 & \begin{tabular}{|l|} 
\\
\end{tabular} & 20.5 & 20.2 & 2.1 & 9.7 & 65 & 79 & 2.0 \\
\hline 10/23/01 & $8: 15$ & 20.2 & \begin{tabular}{|l|}
20.4 \\
\end{tabular} & 20.5 & 20.2 & 2.1 & 9.5 & 65 & 79 & 2.0 \\
\hline 10/23/01 & $8: 16$ & 20.1 & \begin{tabular}{|l|} 
\\
\end{tabular} & 20.5 & 20.0 & 2.1 & 9.6 & 65 & 79 & 2.0 \\
\hline $10 / 23 / 01$ & $8: 17$ & 20.3 & \begin{tabular}{|l|}
20.4 \\
\end{tabular} & 20.5 & 20.2 & 2.1 & 9.6 & 65 & 79 & 2.0 \\
\hline $10 / 23 / 01$ & $8: 18$ & 19.7 & 20.4 & 20.5 & 19.7 & 2.1 & 9.6 & 65 & 79 & 2.0 \\
\hline 10/23/01 & $8: 19$ & 20.2 & \begin{tabular}{|l|} 
\\
\end{tabular} & 20.4 & 20.1 & 2.1 & 9.6 & 65 & 79 & 2.0 \\
\hline 10/23/01 & $8: 20$ & 20.4 & \begin{tabular}{|l|} 
\\
\end{tabular} & 20.4 & 20.3 & 2.1 & 9.7 & 65 & 79 & 2.0 \\
\hline $10 / 23 / 01$ & $8: 21$ & 19.8 & \begin{tabular}{|l|} 
\\
\end{tabular} & 20.4 & 19.7 & 2.1 & 9.6 & 65 & 79 & 2.0 \\
\hline $10 / 23 / 01$ & $8: 22$ & 20.2 & \begin{tabular}{|l|}
20.4 \\
\end{tabular} & 20.4 & 20.1 & 2.1 & 9.6 & 65 & 79 & 2.0 \\
\hline 10/23/01 & $8: 23$ & 20.3 & \begin{tabular}{|l|}
20.4 \\
\end{tabular} & 20.4 & 20.3 & 2.1 & 9.6 & 65 & 79 & 2.0 \\
\hline
\end{tabular}


WSRC-TR-2002-00459, Rev. 0 SRT-RPP-2002-00221

\begin{tabular}{|c|c|c|c|c|c|c|c|c|c|c|}
\hline \multicolumn{11}{|c|}{ PREC2_102301_0623 } \\
\hline DATE & TIME & $\begin{array}{c}\mathrm{HX} \\
\text { Outlet } \\
\left({ }^{\circ} \mathrm{C}\right) \\
\mathrm{TCO} \\
\end{array}$ & $\begin{array}{c}\text { REC } \\
\text { PP } \\
\text { OUT } \\
\left({ }^{\circ} \mathrm{C}\right) \\
\text { TC1 }\end{array}$ & $\begin{array}{c}\text { TK } \\
\text { BOT } \\
\left({ }^{\circ} \mathrm{C}\right) \\
\text { TC2 } \\
\end{array}$ & $\begin{array}{c}\mathrm{HX} \\
\text { OUT } \\
\left({ }^{\circ} \mathrm{C}\right) \\
\text { TC3 }\end{array}$ & $\begin{array}{c}\text { Mn } \\
\text { Flow } \\
(\mathrm{gpm})\end{array}$ & $\begin{array}{c}\text { Galigher } \\
\text { Flow } \\
\text { (gpm) }\end{array}$ & $\begin{array}{c}\text { Current } \\
\text { to } \mathrm{HX}\end{array}$ & $\begin{array}{c}\text { Voltage } \\
\text { to } \mathrm{HX}\end{array}$ & $\begin{array}{c}\mathrm{Sr} \\
\text { Flow } \\
(\mathrm{gpm})\end{array}$ \\
\hline $10 / 23 / 01$ & 8:24 & 20.0 & 20.4 & 20.4 & 19.9 & 2.1 & 9.6 & 65 & 79 & 2.0 \\
\hline $10 / 23 / 01$ & $8: 25$ & 20.3 & 20.4 & 20.4 & 20.2 & 2.1 & 9.6 & 65 & 79 & 2.0 \\
\hline $10 / 23 / 01$ & 8:26 & 19.8 & 20.4 & 20.4 & 19.9 & 2.1 & 9.6 & 65 & 79 & 2.0 \\
\hline $10 / 23 / 01$ & 8:27 & 20.1 & 20.4 & 20.4 & 20.0 & 2.1 & 9.7 & 65 & 79 & 2.0 \\
\hline 10/23/01 & 8:28 & 20.3 & 20.3 & 20.4 & 20.2 & 2.1 & 9.7 & 65 & 79 & 2.0 \\
\hline $10 / 23 / 01$ & 8:29 & 19.7 & 20.4 & 20.4 & 19.6 & 2.0 & 9.7 & 65 & 79 & 2.0 \\
\hline 10/23/01 & 8:30 & 20.2 & 20.3 & 20.4 & 20.1 & 2.1 & 9.7 & 65 & 79 & 2.0 \\
\hline $10 / 23 / 01$ & $8: 31$ & 20.3 & 20.3 & 20.4 & 20.2 & 2.0 & 9.6 & 65 & 79 & 2.0 \\
\hline $10 / 23 / 01$ & 8:32 & 19.9 & 20.3 & 20.4 & 19.8 & 2.1 & 9.6 & 65 & 79 & 2.0 \\
\hline $10 / 23 / 01$ & 8:33 & 20.2 & 20.3 & 20.4 & 20.1 & 2.0 & 9.6 & 65 & 79 & 2.0 \\
\hline $10 / 23 / 01$ & 8:34 & 19.9 & 20.3 & 20.4 & 20.0 & 2.0 & 9.8 & 65 & 79 & 2.0 \\
\hline 10/23/01 & 8:35 & 20.1 & 20.3 & 20.4 & 20.0 & 2.0 & 9.6 & 65 & 79 & 2.0 \\
\hline $10 / 23 / 01$ & 8:36 & 20.2 & 20.3 & 20.4 & 20.2 & 2.0 & 9.6 & 65 & 79 & 2.0 \\
\hline $10 / 23 / 01$ & 8:37 & 19.6 & 20.3 & 20.4 & 19.6 & 2.0 & 9.7 & 65 & 79 & 2.0 \\
\hline $10 / 23 / 01$ & 8:38 & 20.1 & 20.3 & 20.3 & 20.0 & 2.0 & 9.7 & 65 & 79 & 2.0 \\
\hline 10/23/01 & 8:39 & 20.3 & 20.3 & 20.3 & 20.2 & 2.0 & 9.7 & 65 & 79 & 2.0 \\
\hline $10 / 23 / 01$ & $8: 40$ & 19.8 & 20.3 & 20.3 & 19.7 & 2.1 & 9.7 & 65 & 79 & 2.0 \\
\hline $10 / 23 / 01$ & $8: 41$ & 20.1 & 20.3 & 20.3 & 20.1 & 2.0 & 9.6 & 65 & 79 & 2.0 \\
\hline $10 / 23 / 01$ & $8: 42$ & 20.2 & 20.3 & 20.3 & 20.2 & 2.1 & 9.6 & 65 & 79 & 2.0 \\
\hline 10/23/01 & $8: 43$ & 20.0 & 20.3 & 20.3 & 19.9 & 2.1 & 9.7 & 65 & 79 & 2.0 \\
\hline 10/23/01 & 8:44 & 20.2 & 20.3 & 20.3 & 20.1 & 2.1 & 9.7 & 65 & 79 & 2.0 \\
\hline $10 / 23 / 01$ & $8: 45$ & 19.6 & 20.3 & 20.3 & 19.6 & 2.0 & 9.6 & 65 & 79 & 2.0 \\
\hline $10 / 23 / 01$ & 8:46 & 20.0 & 20.2 & 20.3 & 20.0 & 2.1 & 9.6 & 65 & 79 & 2.0 \\
\hline 10/23/01 & $8: 47$ & 20.2 & 20.2 & 20.3 & 20.1 & 2.0 & 9.6 & 65 & 79 & 2.0 \\
\hline $10 / 23 / 01$ & $8: 48$ & 19.7 & 20.2 & 20.3 & 19.6 & 2.0 & 9.6 & 65 & 79 & 2.0 \\
\hline 10/23/01 & $8: 49$ & 20.1 & 20.2 & 20.3 & 20.0 & 2.0 & 9.7 & 65 & 79 & 2.0 \\
\hline $10 / 23 / 01$ & 8:50 & 20.2 & 20.2 & 20.3 & 20.2 & 2.0 & 9.6 & 65 & 79 & 2.0 \\
\hline $10 / 23 / 01$ & $8: 51$ & 19.9 & 20.2 & 20.3 & 19.8 & 2.1 & 9.6 & 65 & 79 & 2.0 \\
\hline $10 / 23 / 01$ & $8: 52$ & 20.1 & 20.2 & 20.3 & 20.0 & 2.1 & 9.6 & 65 & 79 & 2.0 \\
\hline $10 / 23 / 01$ & 8:53 & 19.6 & 20.2 & 20.3 & 19.7 & 2.1 & 9.7 & 65 & 79 & 2.0 \\
\hline $10 / 23 / 01$ & 8:54 & 20.0 & 20.2 & 20.3 & 19.9 & 2.1 & 9.8 & 65 & 79 & 2.0 \\
\hline 10/23/01 & 8:55 & 20.5 & 20.6 & 20.7 & 20.3 & 2.1 & 9.7 & 65 & 79 & 2.0 \\
\hline $10 / 23 / 01$ & 8:56 & 20.5 & 21.4 & 21.5 & 20.4 & 2.1 & 9.7 & 65 & 79 & 2.0 \\
\hline $10 / 23 / 01$ & $8: 57$ & 21.3 & 22.0 & 22.1 & 21.2 & 2.1 & 9.7 & 65 & 79 & 2.0 \\
\hline $10 / 23 / 01$ & 8:58 & 21.3 & 22.0 & 22.1 & 21.4 & 2.1 & 9.7 & 65 & 79 & 2.0 \\
\hline $10 / 23 / 01$ & 8:59 & 21.6 & 22.2 & 22.2 & 21.4 & 2.1 & 9.6 & 65 & 79 & 2.0 \\
\hline $10 / 23 / 01$ & 9:00 & 21.9 & 22.5 & 22.5 & 21.9 & 2.1 & 9.6 & 65 & 79 & 2.0 \\
\hline 10/23/01 & 9:01 & 21.8 & 22.7 & 22.8 & 21.6 & 2.1 & 9.7 & 65 & 79 & 2.0 \\
\hline $10 / 23 / 01$ & 9:02 & 22.4 & 22.9 & 23.0 & 22.3 & 2.1 & 9.6 & 65 & 79 & 2.0 \\
\hline $10 / 23 / 01$ & 9:03 & 22.2 & 23.3 & 23.4 & 22.1 & 2.1 & 9.7 & 65 & 79 & 2.0 \\
\hline $10 / 23 / 01$ & 9:04 & 22.7 & 23.4 & 23.5 & 22.6 & 2.1 & 9.6 & 65 & 79 & 2.0 \\
\hline $10 / 23 / 01$ & 9:05 & 22.6 & 23.8 & 23.9 & 22.5 & 2.1 & 9.6 & 65 & 79 & 2.0 \\
\hline $10 / 23 / 01$ & 9:06 & 23.3 & 24.0 & 24.1 & 23.2 & 2.1 & 9.6 & 65 & 79 & 2.0 \\
\hline $10 / 23 / 01$ & 9:07 & 23.2 & 24.3 & 24.7 & 23.0 & 2.1 & 9.6 & 65 & 79 & 2.0 \\
\hline 10/23/01 & 9:08 & 23.6 & 24.6 & 24.7 & 23.6 & 2.1 & 9.6 & 65 & 79 & 2.0 \\
\hline $10 / 23 / 01$ & 9:09 & 23.8 & 24.9 & 24.9 & 23.6 & 2.1 & 9.6 & 65 & 79 & 2.0 \\
\hline 10/23/01 & $9: 10$ & 23.7 & 25.2 & 25.2 & 23.7 & 2.1 & 9.7 & 65 & 79 & 2.0 \\
\hline $10 / 23 / 01$ & 9:11 & 24.3 & 25.4 & 25.5 & 24.2 & 2.1 & 9.6 & 65 & 79 & 2.0 \\
\hline $10 / 23 / 01$ & 9:12 & 23.7 & 25.4 & 25.4 & 23.7 & 2.1 & 9.6 & 65 & 79 & 2.0 \\
\hline $10 / 23 / 01$ & 9:13 & 24.3 & 25.3 & 25.3 & 24.3 & 2.1 & 9.7 & 65 & 79 & 2.0 \\
\hline 10/23/01 & 9:14 & 23.9 & 25.2 & 25.3 & 23.8 & 2.1 & 9.6 & 65 & 79 & 2.0 \\
\hline $10 / 23 / 01$ & $9: 15$ & 23.8 & 25.2 & 25.2 & 23.9 & 2.1 & 9.7 & 65 & 79 & 2.0 \\
\hline $10 / 23 / 01$ & $9: 16$ & 24.0 & 25.1 & 25.1 & 23.9 & 2.1 & 9.6 & 65 & 79 & 2.0 \\
\hline 10/23/01 & 9:17 & 23.4 & 25.0 & 25.1 & 23.5 & 2.1 & 9.7 & 65 & 79 & 2.0 \\
\hline $10 / 23 / 01$ & 9:18 & 24.1 & 25.0 & 25.0 & 24.0 & 2.1 & 9.7 & 65 & 79 & 2.0 \\
\hline $10 / 23 / 01$ & 9:19 & 23.5 & 24.9 & 24.9 & 23.4 & 2.1 & 9.6 & 65 & 79 & 2.0 \\
\hline $10 / 23 / 01$ & 9:20 & 24.0 & 24.9 & 24.9 & 23.9 & 2.1 & 9.7 & 65 & 79 & 2.0 \\
\hline $10 / 23 / 01$ & 9:21 & 23.7 & 24.8 & 24.8 & 23.5 & 2.1 & 9.6 & 65 & 79 & 2.0 \\
\hline $10 / 23 / 01$ & 9:22 & 23.5 & 24.8 & 24.8 & 23.5 & 2.1 & 9.7 & 65 & 79 & 2.0 \\
\hline $10 / 23 / 01$ & 9:23 & 23.7 & 24.7 & 24.7 & 23.6 & 2.1 & 9.7 & 65 & 79 & 2.0 \\
\hline
\end{tabular}

\begin{tabular}{|c|c|c|c|c|c|c|c|c|c|c|}
\hline \multicolumn{3}{|c|}{ PREC2_102301_0623 } & & & & & & & & \\
\hline DATE & TIME & $\begin{array}{c}\mathrm{HX} \\
\text { Outlet } \\
\left({ }^{\circ} \mathrm{C}\right) \\
\text { TCO }\end{array}$ & $\begin{array}{c}\text { REC } \\
\text { PP } \\
\text { OUT } \\
\left({ }^{\circ} \mathrm{C}\right) \\
\text { TC1 }\end{array}$ & \begin{tabular}{|c|} 
TK \\
BOT \\
$\left({ }^{\circ} \mathrm{C}\right)$ \\
TC2 \\
T
\end{tabular} & $\begin{array}{c}\text { HX } \\
\text { OUT } \\
\left({ }^{\circ} \mathrm{C}\right) \\
\text { TC3 }\end{array}$ & $\begin{array}{c}\text { Mn } \\
\text { Flow } \\
(\mathrm{gpm})\end{array}$ & $\begin{array}{c}\text { Galigher } \\
\text { Flow } \\
(\mathrm{gpm})\end{array}$ & $\begin{array}{c}\text { Current } \\
\text { to } \mathrm{HX}\end{array}$ & $\begin{array}{c}\text { Voltage } \\
\text { to } \mathrm{HX}\end{array}$ & $\begin{array}{c}\mathrm{Sr} \\
\text { Flow } \\
(\mathrm{gpm})\end{array}$ \\
\hline 10/23/01 & 9:24 & 23.2 & 24.7 & 24.7 & 23.2 & 2.1 & 9.7 & 65 & 79 & 2.0 \\
\hline $10 / 23 / 01$ & 9:25 & 23.7 & 24.6 & 24.6 & 23.6 & 2.1 & 9.6 & 65 & 79 & 2.0 \\
\hline $10 / 23 / 01$ & $9: 26$ & 23.2 & 24.6 & 24.6 & 23.1 & 2.1 & 9.7 & 65 & 79 & 2.0 \\
\hline $10 / 23 / 01$ & $9: 27$ & 23.6 & 24.5 & 24.5 & 23.6 & 2.1 & 9.6 & 65 & 79 & 2.0 \\
\hline $10 / 23 / 01$ & $9: 28$ & 23.3 & 24.5 & 24.5 & 23.2 & 2.1 & 9.6 & 65 & 79 & 2.0 \\
\hline $10 / 23 / 01$ & $9: 29$ & 23.3 & 24.4 & 24.4 & 23.3 & 2.1 & 9.6 & 65 & 79 & 2.0 \\
\hline 10/23/01 & 9:30 & 23.4 & 24.3 & 24.4 & 23.3 & 2.1 & 9.6 & 65 & 79 & 2.0 \\
\hline $10 / 23 / 01$ & 9:31 & 22.9 & 24.3 & 24.3 & 22.9 & 2.1 & 9.6 & 65 & 79 & 2.0 \\
\hline 10/23/01 & 9:32 & 23.4 & 24.2 & 24.3 & 23.3 & 2.1 & 9.6 & 65 & 79 & 2.0 \\
\hline $10 / 23 / 01$ & 9:33 & 22.8 & 24.2 & 24.2 & 22.7 & 2.1 & 9.7 & 65 & 79 & 2.0 \\
\hline $10 / 23 / 01$ & 9:34 & 23.4 & 24.1 & 24.2 & 23.3 & 2.1 & 9.7 & 65 & 79 & 2.0 \\
\hline $10 / 23 / 01$ & $9: 35$ & 22.8 & 24.1 & 24.1 & 22.7 & 2.1 & 9.7 & 65 & 79 & 2.0 \\
\hline 10/23/01 & 9:36 & 23.3 & 24.1 & 24.1 & 23.2 & 2.1 & 9.6 & 65 & 79 & 2.2 \\
\hline $10 / 23 / 01$ & 9:37 & 23.0 & 24.1 & 24.1 & 22.8 & 2.1 & 9.6 & 65 & 79 & 2.2 \\
\hline $10 / 23 / 01$ & 9:38 & 23.1 & 24.0 & 24.1 & 23.1 & 2.1 & 9.6 & 65 & 79 & 2.2 \\
\hline $10 / 23 / 01$ & 9:39 & 23.1 & 24.0 & 24.0 & 22.9 & 2.1 & 9.6 & 65 & 79 & 2.1 \\
\hline $10 / 23 / 01$ & 9:40 & 22.7 & 24.0 & 24.0 & 22.8 & 2.1 & 9.7 & 65 & 79 & 2.1 \\
\hline $10 / 23 / 01$ & $9: 41$ & 23.1 & 24.0 & 24.0 & 23.0 & 2.1 & 9.7 & 65 & 79 & 2.1 \\
\hline $10 / 23 / 01$ & 9:42 & 22.5 & 23.9 & 24.0 & 22.5 & 2.1 & 9.7 & 65 & 79 & 2.1 \\
\hline $10 / 23 / 01$ & 9:43 & 23.1 & 23.9 & 23.9 & 23.0 & 2.1 & 9.6 & 65 & 79 & 0.0 \\
\hline $10 / 23 / 01$ & 9:44 & 22.6 & 23.9 & 23.9 & 22.5 & 2.1 & 9.7 & 65 & 79 & 0.0 \\
\hline $10 / 23 / 01$ & 9:45 & 23.2 & 23.8 & 23.9 & 23.1 & 2.1 & 9.6 & 65 & 79 & 0.0 \\
\hline $10 / 23 / 01$ & 9:46 & 22.7 & 23.8 & 23.8 & 22.5 & 2.1 & 9.6 & 65 & 79 & 0.0 \\
\hline $10 / 23 / 01$ & 9:47 & 23.0 & 23.8 & 23.8 & 23.0 & 2.1 & 9.6 & 65 & 79 & 0.0 \\
\hline $10 / 23 / 01$ & 9:48 & 22.8 & 23.7 & 23.7 & 22.6 & 2.1 & 9.6 & 65 & 79 & 0.0 \\
\hline $10 / 23 / 01$ & 9:49 & 22.7 & 23.7 & 23.7 & 22.7 & 2.1 & 9.6 & 65 & 79 & 0.0 \\
\hline $10 / 23 / 01$ & 9:50 & 22.8 & 23.6 & 23.7 & 22.7 & 2.1 & 9.7 & 65 & 79 & 0.0 \\
\hline $10 / 23 / 01$ & 9:51 & 22.4 & 23.6 & 23.6 & 22.4 & 2.1 & 9.6 & 65 & 79 & 0.0 \\
\hline $10 / 23 / 01$ & 9:52 & 22.8 & 23.5 & 23.6 & 22.7 & 2.1 & 9.6 & 65 & 79 & 0.0 \\
\hline $10 / 23 / 01$ & 9:53 & 22.4 & 24.0 & 24.1 & 22.3 & 2.3 & 9.7 & 65 & 79 & 0.0 \\
\hline $10 / 23 / 01$ & 9:54 & 23.8 & 24.9 & 25.0 & 23.6 & 2.1 & 9.4 & 65 & 79 & 0.0 \\
\hline $10 / 23 / 01$ & 9:55 & 24.0 & 26.0 & 26.2 & 23.9 & 2.1 & 9.9 & 65 & 79 & 0.0 \\
\hline $10 / 23 / 01$ & 9:56 & 25.5 & 27.0 & 27.1 & 25.3 & 2.1 & 9.6 & 65 & 79 & 0.0 \\
\hline $10 / 23 / 01$ & 9:57 & 25.8 & 28.0 & 28.1 & 25.6 & 2.0 & 9.7 & 65 & 79 & 0.0 \\
\hline $10 / 23 / 01$ & 9:58 & 26.3 & 28.2 & 28.3 & 26.4 & 0.0 & 9.7 & 65 & 79 & 0.0 \\
\hline $10 / 23 / 01$ & 9:59 & 26.4 & 28.1 & 28.2 & 26.3 & 0.0 & 9.6 & 65 & 79 & 0.0 \\
\hline $10 / 23 / 01$ & $10: 00$ & 25.8 & 28.1 & 28.1 & 25.8 & 0.0 & 9.7 & 65 & 79 & 0.0 \\
\hline $10 / 23 / 01$ & $10: 01$ & 26.4 & 28.0 & 28.0 & 26.4 & 0.0 & 9.7 & 65 & 79 & 0.0 \\
\hline $10 / 23 / 01$ & $10: 02$ & 26.2 & 27.9 & 28.0 & 26.0 & 0.0 & 9.6 & 65 & 79 & 0.0 \\
\hline $10 / 23 / 01$ & $10: 03$ & 25.7 & 27.9 & 27.9 & 25.7 & 0.0 & 9.7 & 65 & 79 & 0.0 \\
\hline 10/23/01 & $10: 04$ & 26.3 & 27.8 & 27.9 & 26.3 & 0.0 & 9.7 & 65 & 79 & 0.0 \\
\hline $10 / 23 / 01$ & $10: 05$ & 25.9 & 27.7 & 27.8 & 25.8 & 0.0 & 9.6 & 65 & 79 & 0.0 \\
\hline $10 / 23 / 01$ & $10: 06$ & 25.6 & 27.7 & 27.7 & 25.6 & 0.0 & 9.7 & 65 & 79 & 0.0 \\
\hline $10 / 23 / 01$ & $10: 07$ & 26.1 & 27.6 & 27.7 & 26.1 & 0.0 & 9.6 & 65 & 79 & 0.0 \\
\hline $10 / 23 / 01$ & $10: 08$ & 25.6 & 27.5 & 27.6 & 25.5 & 0.0 & 9.6 & 65 & 79 & 0.0 \\
\hline $10 / 23 / 01$ & $10: 09$ & 25.5 & 27.5 & 27.5 & 25.7 & 0.0 & 9.6 & 65 & 79 & 0.0 \\
\hline $10 / 23 / 01$ & $10: 10$ & 25.9 & 27.4 & 27.4 & 25.8 & 0.0 & 9.6 & 65 & 79 & 0.0 \\
\hline $10 / 23 / 01$ & $10: 11$ & 25.3 & 27.3 & 27.4 & 25.3 & 0.0 & 3.0 & 65 & 79 & 0.0 \\
\hline $10 / 23 / 01$ & $10: 12$ & 25.7 & 27.3 & 27.3 & 25.8 & 0. & 9. & 65 & 79 & 0.0 \\
\hline $10 / 23 / 01$ & $10: 13$ & 25.6 & 27.2 & 27.2 & 25.5 & 0.0 & 9.7 & 65 & 79 & 0.0 \\
\hline $10 / 23 / 01$ & $10: 14$ & 25.1 & 27.1 & 27.2 & 25.1 & 0.0 & 9.6 & 65 & 79 & 0.0 \\
\hline $10 / 23 / 01$ & $10: 15$ & 25.7 & 27.0 & 27.1 & 25.6 & 0.0 & 9.7 & 65 & 79 & 0.0 \\
\hline $10 / 23 / 01$ & $10: 16$ & 25.2 & 27.0 & 27.0 & 25.1 & 0.0 & 9.6 & 65 & 79 & 0.0 \\
\hline $10 / 23 / 01$ & $10: 17$ & 25.0 & 26.9 & 26.9 & 25.1 & 0.0 & 9.7 & 65 & 79 & 0.0 \\
\hline $10 / 23 / 01$ & $10: 18$ & 25.4 & 26.8 & 26.9 & 25.4 & 0.0 & 9.6 & 65 & 79 & 0.0 \\
\hline $10 / 23 / 01$ & $10: 19$ & 24.9 & 26.8 & 26.8 & 24.8 & 0.0 & 9.6 & 65 & 79 & 0.0 \\
\hline $10 / 23 / 01$ & $10: 20$ & 25.2 & 26.7 & 26.8 & 25.3 & 0.0 & 9.7 & 65 & 79 & 0.0 \\
\hline $10 / 23 / 01$ & $10: 21$ & 25.2 & 26.6 & 26.7 & 25.1 & 0.0 & 9.6 & 65 & 79 & 0.0 \\
\hline $10 / 23 / 01$ & $10: 22$ & 24.7 & 26.6 & 26.6 & 24.7 & 0.0 & 9.7 & 65 & 79 & 0.0 \\
\hline $10 / 23 / 01$ & $10: 23$ & 25.3 & 26.5 & 26.6 & 25.2 & 0.0 & 9.6 & 65 & 79 & 0.0 \\
\hline
\end{tabular}


WSRC-TR-2002-00459, Rev. 0 SRT-RPP-2002-00221

\begin{tabular}{|c|c|c|c|c|c|c|c|c|c|c|}
\hline \multicolumn{11}{|c|}{ PREC2_102301_0623 } \\
\hline DATE & TIME & $\begin{array}{c}\mathrm{HX} \\
\text { Outlet } \\
\left({ }^{\circ} \mathrm{C}\right) \\
\mathrm{TC} 0\end{array}$ & \begin{tabular}{|l|} 
REC \\
PP \\
OUT \\
$\left({ }^{\circ} \mathrm{C}\right)$ \\
TC1
\end{tabular} & $\begin{array}{c}\text { TK } \\
\text { BOT } \\
\left({ }^{\circ} \mathrm{C}\right) \\
\text { TC2 }\end{array}$ & $\begin{array}{c}\text { HX } \\
\text { OUT } \\
\left({ }^{\circ} \mathrm{C}\right) \\
\text { TC3 }\end{array}$ & $\begin{array}{c}\text { Mn } \\
\text { Flow } \\
\text { (gpm) }\end{array}$ & \begin{tabular}{|c|} 
Galigher \\
Flow \\
(gpm)
\end{tabular} & $\begin{array}{c}\text { Current } \\
\text { to } \mathrm{HX}\end{array}$ & $\begin{array}{c}\text { Voltage } \\
\text { to } \mathrm{HX}\end{array}$ & $\begin{array}{c}\mathrm{Sr} \\
\text { Flow } \\
(\mathrm{gpm})\end{array}$ \\
\hline $10 / 23 / 01$ & 10:24 & 24.8 & 26.5 & 26.5 & 24.7 & 0.0 & 9.6 & 65 & 79 & 0.0 \\
\hline $10 / 23 / 01$ & 10:25 & 24.7 & 26.4 & 26.4 & 24.8 & 0.0 & 9.6 & 65 & 79 & 0.0 \\
\hline $10 / 23 / 01$ & $10: 26$ & 25.0 & 26.3 & 26.4 & 24.9 & 0.0 & 9.6 & 65 & 79 & 0.0 \\
\hline $10 / 23 / 01$ & 10:27 & 24.4 & 26.3 & 26.3 & 24.4 & 0.0 & 9.7 & 65 & 79 & 0.0 \\
\hline $10 / 23 / 01$ & 10:28 & 24.9 & 26.2 & 26.2 & 24.9 & 0.0 & 9.6 & 65 & 79 & 0.0 \\
\hline $10 / 23 / 01$ & 10:29 & 24.7 & 26.1 & 26.2 & 24.6 & 0.0 & 9.6 & 65 & 79 & 0.0 \\
\hline $10 / 23 / 01$ & 10:30 & 24.3 & 26.1 & 26.1 & 24.4 & 0.0 & 9.7 & 65 & 79 & 0.0 \\
\hline $10 / 23 / 01$ & 10:31 & 24.8 & 26.0 & 26.0 & 24.8 & 0.0 & 9.7 & 65 & 79 & 0.0 \\
\hline $10 / 23 / 01$ & 10:32 & 24.3 & 26.0 & 26.0 & 24.2 & 0.0 & 9.7 & 65 & 79 & 0.0 \\
\hline $10 / 23 / 01$ & 10:33 & 24.5 & 25.9 & 25.9 & 24.6 & 0.0 & 9.6 & 65 & 79 & 0.0 \\
\hline $10 / 23 / 01$ & 10:34 & 24.5 & 25.8 & 25.9 & 24.4 & 0.0 & 9.6 & 65 & 79 & 0.0 \\
\hline $10 / 23 / 01$ & 10:35 & 24.0 & 25.8 & 25.8 & 24.0 & 0.0 & 9.6 & 65 & 79 & 0.0 \\
\hline $10 / 23 / 01$ & 10:36 & 24.6 & 25.7 & 25.7 & 24.5 & 0.0 & 9.6 & 65 & 79 & 0.0 \\
\hline 10/23/01 & 10:37 & 24.1 & 25.6 & 25.7 & 24.0 & 0.0 & 9.6 & 65 & 79 & 0.0 \\
\hline $10 / 23 / 01$ & 10:38 & 24.2 & 25.6 & 25.6 & 24.3 & 0.0 & 9.7 & 65 & 79 & 0.0 \\
\hline 10/23/01 & 10:39 & 24.3 & 25.5 & 25.6 & 24.2 & 0.0 & 9.6 & 65 & 79 & 0.0 \\
\hline $10 / 23 / 01$ & 10:40 & 23.8 & 25.5 & 25.5 & 23.8 & 0.0 & 9.6 & 65 & 79 & 0.0 \\
\hline $10 / 23 / 01$ & 10:41 & 24.3 & 25.4 & 25.4 & 24.3 & 0.0 & 9.6 & 65 & 79 & 0.0 \\
\hline $10 / 23 / 01$ & 10:42 & 23.8 & 25.3 & 25.4 & 23.7 & 0.0 & 9.6 & 65 & 79 & 0.0 \\
\hline $10 / 23 / 01$ & $10: 43$ & 24.1 & 25.3 & 25.3 & 24.2 & 0.0 & 9.7 & 65 & 79 & 0.0 \\
\hline $10 / 23 / 01$ & 10:44 & 24.0 & 25.3 & 25.3 & 23.9 & 0.0 & 9.7 & 65 & 79 & 0.0 \\
\hline $10 / 23 / 01$ & 10:45 & 23.6 & 25.2 & 25.3 & 23.7 & 0.0 & 9.6 & 65 & 79 & 0.0 \\
\hline $10 / 23 / 01$ & 10:46 & 24.1 & 25.2 & 25.2 & 24.1 & 0.0 & 9.6 & 65 & 79 & 0.0 \\
\hline $10 / 23 / 01$ & 10:47 & 23.6 & 25.1 & 25.2 & 23.6 & 0.0 & 9.6 & 65 & 79 & 0.0 \\
\hline $10 / 23 / 01$ & $10: 48$ & 24.1 & 25.1 & 25.2 & 24.1 & 0.0 & 9.6 & 65 & 79 & 0.0 \\
\hline $10 / 23 / 01$ & 10:49 & 23.9 & 25.1 & 25.1 & 23.7 & 0.0 & 9.7 & 65 & 79 & 0.0 \\
\hline $10 / 23 / 01$ & 10:50 & 23.6 & 25.1 & 25.1 & 23.6 & 0.0 & 9.7 & 65 & 79 & 0.0 \\
\hline $10 / 23 / 01$ & 10:51 & 24.0 & 25.0 & 25.0 & 23.9 & 0.0 & 9.6 & 65 & 79 & 0.0 \\
\hline $10 / 23 / 01$ & 10:52 & 23.4 & 24.9 & 25.0 & 23.4 & 0.0 & 9.6 & 65 & 79 & 0.0 \\
\hline $10 / 23 / 01$ & 10:53 & 23.9 & 24.9 & 24.9 & 23.9 & 0.0 & 9.6 & 65 & 79 & 0.0 \\
\hline $10 / 23 / 01$ & 10:54 & 23.7 & 24.8 & 24.9 & 23.5 & 0.0 & 9.6 & 65 & 79 & 0.0 \\
\hline $10 / 23 / 01$ & 10:55 & 23.4 & 24.8 & 24.8 & 23.5 & 0.0 & 9.6 & 65 & 79 & 0.0 \\
\hline $10 / 23 / 01$ & 10:56 & 23.8 & 24.7 & 24.8 & 23.7 & 0.0 & 9.6 & 65 & 79 & 0.0 \\
\hline $10 / 23 / 01$ & 10:57 & 23.2 & 24.7 & 24.7 & 23.2 & 0.0 & 9.6 & 65 & 79 & 0.0 \\
\hline $10 / 23 / 01$ & 10:58 & 23.8 & 24.6 & 24.7 & 23.7 & 0.0 & 9.6 & 65 & 79 & 0.0 \\
\hline $10 / 23 / 01$ & 10:59 & 23.4 & 24.6 & 24.6 & 23.3 & 0.0 & 9.7 & 65 & 79 & 0.0 \\
\hline $10 / 23 / 01$ & 11:00 & 23.1 & 24.5 & 24.6 & 23.2 & 0.0 & 9.6 & 65 & 79 & 0.0 \\
\hline $10 / 23 / 01$ & 11:01 & 23.4 & 24.5 & 24.5 & 23.4 & 0.0 & 9.7 & 65 & 79 & 0.0 \\
\hline $10 / 23 / 01$ & 11:02 & 22.8 & 24.4 & 24.5 & 22.8 & 0.0 & 9.6 & 65 & 79 & 0.0 \\
\hline $10 / 23 / 01$ & 11:03 & 23.0 & 24.4 & 24.4 & 22.8 & 0.0 & 9.7 & 65 & 79 & 0.0 \\
\hline $10 / 23 / 01$ & 11:04 & 22.5 & 24.3 & 24.3 & 22.4 & 0.0 & 9.7 & 65 & 79 & 0.0 \\
\hline $10 / 23 / 01$ & 11:05 & 22.9 & 24.2 & 24.2 & 22.9 & 0.0 & 9.6 & 65 & 79 & 0.0 \\
\hline $10 / 23 / 01$ & 11:06 & 22.4 & 24.1 & 24.2 & 22.3 & 0.0 & 9.6 & 65 & 79 & 0.0 \\
\hline $10 / 23 / 01$ & 11:07 & 22.4 & 24.0 & 24.1 & 22.4 & 0.0 & 9.7 & 65 & 79 & 0.0 \\
\hline $10 / 23 / 01$ & $11: 08$ & 22.6 & 24.0 & 24.0 & 22.4 & 0.0 & 9.6 & 65 & 79 & 0.0 \\
\hline $10 / 23 / 01$ & 11:09 & 22.1 & 23.9 & 23.9 & 22.1 & 0.0 & 9.6 & 65 & 79 & 0.0 \\
\hline $10 / 23 / 01$ & 11:10 & 22.6 & 23.8 & 23.9 & 22.6 & 0.0 & 9.7 & 65 & 79 & 0.0 \\
\hline $10 / 23 / 01$ & 11:11 & 22.1 & 23.8 & 23.8 & 22.0 & 0.0 & 9.6 & 65 & 79 & 0.0 \\
\hline $10 / 23 / 01$ & 11:12 & 22.1 & 23.7 & 23.7 & 22.2 & 0.0 & 9.7 & 65 & 79 & 0.0 \\
\hline $10 / 23 / 01$ & $11: 13$ & 21.9 & 23.6 & 23.6 & 21.9 & 0.0 & 9.6 & 65 & 79 & 0.0 \\
\hline $10 / 23 / 01$ & 11:14 & 21.5 & 23.5 & 23.6 & 21.5 & 0.0 & 9.6 & 65 & 79 & 0.0 \\
\hline $10 / 23 / 01$ & 11:15 & 21.9 & 23.4 & 23.5 & 21.9 & 0.0 & 9.6 & 65 & 79 & 0.0 \\
\hline $10 / 23 / 01$ & $11: 16$ & 21.3 & 23.4 & 23.4 & 21.3 & 0.0 & 9.6 & 65 & 79 & 0.0 \\
\hline $10 / 23 / 01$ & 11:17 & 21.6 & 23.3 & 23.3 & 21.6 & 0.0 & 9.6 & 65 & 79 & 0.0 \\
\hline $10 / 23 / 01$ & 11:18 & 21.4 & 23.2 & 23.2 & 21.2 & 0.0 & 9.7 & 65 & 79 & 0.0 \\
\hline $10 / 23 / 01$ & 11:19 & 21.2 & 23.1 & 23.1 & 21.2 & 0.0 & 9.7 & 65 & 79 & 0.0 \\
\hline $10 / 23 / 01$ & 11:20 & 21.5 & 23.0 & 23.0 & 21.4 & 0.0 & 9.6 & 65 & 79 & 0.0 \\
\hline $10 / 23 / 01$ & 11:21 & 21.0 & 22.9 & 22.9 & 21.0 & 0.0 & 9.7 & 65 & 79 & 0.0 \\
\hline $10 / 23 / 01$ & 11:22 & 21.5 & 22.8 & 22.9 & 21.4 & 0.0 & 9.6 & 65 & 79 & 0.0 \\
\hline$/ 23 / 01$ & 11:23 & 20.8 & \begin{tabular}{|l|}
22.8 \\
\end{tabular} & 22.8 & 20.8 & 0.0 & 9.6 & 65 & 79 & 0.0 \\
\hline
\end{tabular}

\begin{tabular}{|c|c|c|c|c|c|c|c|c|c|c|}
\hline \multicolumn{3}{|c|}{ PREC2_102301_0623 } & \multirow[b]{2}{*}{$\begin{array}{c}\text { REC } \\
\text { PP } \\
\text { OUT } \\
\left({ }^{\circ} \mathrm{C}\right) \\
\text { TC1 } \\
\end{array}$} & \multirow[b]{2}{*}{\begin{tabular}{|c|} 
TK \\
BOT \\
$\left({ }^{\circ} \mathrm{C}\right)$ \\
TC2 \\
\end{tabular}} & \multirow[b]{2}{*}{\begin{tabular}{|c|}
$\mathrm{HX}$ \\
OUT \\
$\left({ }^{\circ} \mathrm{C}\right)$ \\
TC3
\end{tabular}} & \multirow[b]{2}{*}{\begin{tabular}{|c|} 
Mn \\
Flow \\
(gpm)
\end{tabular}} & \multirow[b]{2}{*}{$\begin{array}{c}\text { Galigher } \\
\text { Flow } \\
\text { (gpm) }\end{array}$} & \multirow[b]{2}{*}{$\begin{array}{c}\text { Current } \\
\text { to } \mathrm{HX}\end{array}$} & \multirow[b]{2}{*}{$\begin{array}{c}\text { Voltage } \\
\text { to } \mathrm{HX}\end{array}$} & \multirow[b]{2}{*}{\begin{tabular}{|c|}
$\mathrm{Sr}$ \\
Flow \\
$(\mathrm{gpm})$ \\
\end{tabular}} \\
\hline DATE & TIME & \begin{tabular}{|c|}
$\mathrm{HX}$ \\
Outlet \\
$\left({ }^{\circ} \mathrm{C}\right)$ \\
TCO \\
\end{tabular} & & & & & & & & \\
\hline $10 / 23 / 01$ & $12: 24$ & 18.5 & \begin{tabular}{|l|}
19.3 \\
\end{tabular} & 19.3 & 18.5 & 0.0 & 9.6 & 65 & 79 & 0.0 \\
\hline $10 / 23 / 01$ & 12:25 & 18.3 & \begin{tabular}{|l|}
19.3 \\
\end{tabular} & 19.3 & \begin{tabular}{|l|}
18.1 \\
\end{tabular} & 0.0 & 9.6 & 65 & 79 & 0.0 \\
\hline 10/23/01 & $12: 26$ & 18.1 & 19.2 & 19.3 & 18.1 & 0.0 & 9.7 & 65 & 79 & 0.0 \\
\hline $10 / 23 / 01$ & $12: 27$ & 18.4 & 19.2 & 19.2 & 18.3 & 0.0 & 9.6 & 65 & 79 & 0.0 \\
\hline 10/23/01 & $12: 28$ & 18.0 & 19.2 & 19.2 & 17.9 & 0.0 & 9.7 & 65 & 79 & 0.0 \\
\hline $10 / 23 / 01$ & $12: 29$ & 18.5 & \begin{tabular}{|l|}
19.1 \\
\end{tabular} & 19.1 & \begin{tabular}{|l|}
18.4 \\
\end{tabular} & 0.0 & 9.6 & 65 & 79 & 0.0 \\
\hline $10 / 23 / 01$ & $12: 30$ & 18.0 & 19.1 & 19.1 & 17.8 & 0.0 & 9.6 & 65 & 79 & 0.0 \\
\hline $10 / 23 / 01$ & 12:31 & 18.1 & 19.0 & 19.1 & 18.1 & 0.0 & 9.6 & 65 & 79 & 0.0 \\
\hline $10 / 23 / 01$ & 12:32 & 18.2 & 19.0 & 19.0 & 18.0 & 0.0 & 9.7 & 65 & 79 & 0.0 \\
\hline 10/23/01 & 12:33 & 17.8 & 19.0 & 19.0 & 17.8 & 0.0 & 9.6 & 85 & 79 & 0.0 \\
\hline $10 / 23 / 01$ & $12: 34$ & 18.3 & \begin{tabular}{|l|}
18.9 \\
\end{tabular} & 19.0 & \begin{tabular}{|l|}
18.2 \\
\end{tabular} & 0.0 & 9.7 & 82 & 79 & 0.0 \\
\hline $10 / 23 / 01$ & 12:35 & 17.8 & 18.9 & 18.9 & \begin{tabular}{|l|}
17.7 \\
\end{tabular} & 0.0 & 9.6 & 84 & 79 & 0.0 \\
\hline $10 / 23 / 01$ & $12: 36$ & 18.1 & 18.8 & 18.9 & 18.1 & 0.0 & 9.7 & 83 & 79 & 0.0 \\
\hline $10 / 23 / 01$ & 12:37 & 17.9 & \begin{tabular}{|l|}
18.8 \\
\end{tabular} & 18.8 & \begin{tabular}{|l|}
17.8 \\
\end{tabular} & 0.0 & 9.6 & 68 & 79 & 0.0 \\
\hline 10/23/01 & 12:38 & 18.4 & \begin{tabular}{|l|}
18.8 \\
\end{tabular} & 18.8 & \begin{tabular}{|l|}
18.2 \\
\end{tabular} & 0.0 & 9.7 & 79 & 79 & 0.0 \\
\hline $10 / 23 / 01$ & 12:39 & 18.6 & \begin{tabular}{|l|}
18.8 \\
\end{tabular} & \begin{tabular}{|l|}
18.8 \\
\end{tabular} & 18.5 & 0.0 & 9.6 & 87 & 79 & 0.0 \\
\hline $10 / 23 / 01$ & $12: 40$ & 18.2 & \begin{tabular}{|l|}
18.8 \\
\end{tabular} & 18.8 & \begin{tabular}{|l|}
18.1 \\
\end{tabular} & 0.0 & 9.6 & 82 & 79 & 0.0 \\
\hline $10 / 23 / 01$ & $12: 41$ & 18.7 & \begin{tabular}{|l|}
18.8 \\
\end{tabular} & 18.8 & \begin{tabular}{|l|}
18.6 \\
\end{tabular} & 0.0 & 9.7 & 95 & 79 & 0.0 \\
\hline 10/23/01 & 12:42 & 18.1 & 18.8 & 18.8 & 18.1 & 0.0 & 9.6 & 98 & 79 & 0.0 \\
\hline 10/23/01 & $12: 43$ & 18.7 & \begin{tabular}{|l|}
18.8 \\
\end{tabular} & 18.8 & \begin{tabular}{|l|}
18.6 \\
\end{tabular} & 0.0 & 9.6 & 80 & 79 & 0.0 \\
\hline $10 / 23 / 01$ & $12: 44$ & 18.1 & \begin{tabular}{|l|}
18.8 \\
\end{tabular} & \begin{tabular}{|l|}
18.8 \\
\end{tabular} & 18.1 & 0.0 & 9.6 & 101 & 79 & 0.0 \\
\hline 10/23/01 & $12: 45$ & 18.7 & \begin{tabular}{|l|}
18.8 \\
\end{tabular} & 18.8 & \begin{tabular}{|l|}
18.6 \\
\end{tabular} & 0.0 & 9.7 & 111 & 79 & 0.0 \\
\hline 10/23/01 & $12: 46$ & 18.1 & \begin{tabular}{|l|}
18.8 \\
\end{tabular} & 18.8 & \begin{tabular}{|l|}
18.1 \\
\end{tabular} & 0.0 & 9.7 & 101 & 79 & 0.0 \\
\hline 10/23/01 & $12: 47$ & 18.7 & \begin{tabular}{|l|}
18.8 \\
\end{tabular} & 18.8 & \begin{tabular}{|l|}
18.6 \\
\end{tabular} & 0.0 & 9.6 & 106 & 79 & 0.0 \\
\hline 10/23/01 & 12:48 & 18.2 & \begin{tabular}{|l|}
18.8 \\
\end{tabular} & 18.8 & \begin{tabular}{|l|}
18.1 \\
\end{tabular} & 0.0 & 9.6 & 112 & 79 & 0.0 \\
\hline $10 / 23 / 01$ & 12:49 & 18.7 & \begin{tabular}{|l|}
18.8 \\
\end{tabular} & 18.8 & 18.6 & 0.0 & 9.7 & 104 & 79 & 0.0 \\
\hline $10 / 23 / 01$ & 12:50 & 18.2 & \begin{tabular}{|l|}
18.8 \\
\end{tabular} & 18.8 & \begin{tabular}{|l|}
18.1 \\
\end{tabular} & 0.0 & 9.7 & 96 & 79 & 0.0 \\
\hline $10 / 23 / 01$ & 12:51 & 18.7 & \begin{tabular}{|l|} 
\\
\end{tabular} & 18.8 & 18.6 & 0.0 & 9.7 & 112 & 79 & 0.0 \\
\hline $10 / 23 / 01$ & 12:52 & 18.3 & \begin{tabular}{|l|}
18.8 \\
\end{tabular} & 18.8 & \begin{tabular}{|l|}
18.2 \\
\end{tabular} & 0.0 & 9.6 & 87 & 79 & 0.0 \\
\hline 10/23/01 & 12:53 & 18.7 & \begin{tabular}{|l|}
18.7 \\
\end{tabular} & 18.8 & \begin{tabular}{|l|}
18.6 \\
\end{tabular} & 0.0 & 9.7 & 118 & 79 & 0.0 \\
\hline 10/23/01 & 12:54 & 18.3 & \begin{tabular}{|l|}
18.7 \\
\end{tabular} & 18.8 & \begin{tabular}{|l|}
18.2 \\
\end{tabular} & 0.0 & 9.7 & 101 & 79 & 0.0 \\
\hline $10 / 23 / 01$ & 12:55 & 18.7 & \begin{tabular}{|l|}
18.7 \\
\end{tabular} & 18.8 & \begin{tabular}{|l|}
18.6 \\
\end{tabular} & 0.0 & 9.5 & 86 & 79 & 0.0 \\
\hline $10 / 23 / 01$ & 12:56 & 18.4 & \begin{tabular}{|l|}
18.7 \\
\end{tabular} & 18.8 & \begin{tabular}{|l|}
18.3 \\
\end{tabular} & 0.0 & 9.6 & 115 & 79 & 0.0 \\
\hline 10/23/01 & 12:57 & 18.6 & \begin{tabular}{|l|}
18.7 \\
\end{tabular} & 18.8 & \begin{tabular}{|l|}
18.6 \\
\end{tabular} & 0.0 & 9.7 & 104 & 79 & 0.0 \\
\hline $10 / 23 / 01$ & 12:58 & 18.5 & \begin{tabular}{|l|}
18.7 \\
\end{tabular} & 18.8 & \begin{tabular}{|l|}
18.3 \\
\end{tabular} & 0.0 & 9.6 & 103 & 79 & 0.0 \\
\hline $10 / 23 / 01$ & 12:59 & 18.4 & \begin{tabular}{|l|}
18.7 \\
\end{tabular} & 18.8 & \begin{tabular}{|l|}
18.4 \\
\end{tabular} & 0.0 & 9.7 & 92 & 79 & 0.0 \\
\hline $10 / 23 / 01$ & 13:00 & 18.5 & \begin{tabular}{|l|}
18.7 \\
\end{tabular} & 18.7 & \begin{tabular}{|l|}
18.4 \\
\end{tabular} & 0.0 & 9.6 & 116 & 79 & 0.0 \\
\hline 10/23/01 & 13:01 & 18.3 & \begin{tabular}{|l|}
18.7 \\
\end{tabular} & 18.8 & \begin{tabular}{|l|}
18.3 \\
\end{tabular} & 0.0 & 9.6 & 110 & 79 & 0.0 \\
\hline $10 / 23 / 01$ & 13:02 & 18.6 & \begin{tabular}{|l|}
18.7 \\
\end{tabular} & \begin{tabular}{|l|}
18.7 \\
\end{tabular} & \begin{tabular}{|l|}
18.4 \\
\end{tabular} & 0.0 & 9.6 & 104 & 79 & 0.0 \\
\hline $10 / 23 / 01$ & 13:03 & 18.2 & \begin{tabular}{|l|}
18.7 \\
\end{tabular} & \begin{tabular}{|l|}
18.7 \\
\end{tabular} & \begin{tabular}{|l|}
18.2 \\
\end{tabular} & 0.0 & 9.6 & 94 & 79 & 0.0 \\
\hline $10 / 23 / 01$ & 13:04 & 18.6 & \begin{tabular}{|l|}
18.7 \\
\end{tabular} & \begin{tabular}{|l|}
$\mid 18.7$ \\
\end{tabular} & 18.5 & 0.0 & 9.6 & 120 & 79 & 0.0 \\
\hline $10 / 23 / 01$ & 13:05 & 18.1 & \begin{tabular}{|l|}
18.7 \\
\end{tabular} & 18.7 & \begin{tabular}{|l|}
18.1 \\
\end{tabular} & 0.0 & 9.7 & 90 & 79 & 0.0 \\
\hline $10 / 23 / 01$ & 13:06 & 18.6 & \begin{tabular}{|l|} 
\\
\end{tabular} & \begin{tabular}{|l|}
18.7 \\
\end{tabular} & \begin{tabular}{|l|}
18.5 \\
\end{tabular} & 0.0 & 9.6 & 107 & 79 & 0.0 \\
\hline $10 / 23 / 01$ & 13:07 & 18.1 & \begin{tabular}{|l|}
18.7 \\
\end{tabular} & \begin{tabular}{|l|}
18.7 \\
\end{tabular} & \begin{tabular}{|l|}
18.0 \\
\end{tabular} & 0.0 & 9.7 & 118 & 79 & 0.0 \\
\hline $10 / 23 / 01$ & 13:08 & 18.6 & \begin{tabular}{|l|}
18.7 \\
\end{tabular} & \begin{tabular}{|l|}
18.7 \\
\end{tabular} & 18.5 & 0.0 & 9.7 & 92 & 79 & 0.0 \\
\hline $10 / 23 / 01$ & 13:09 & 18.1 & \begin{tabular}{|l|}
18.7 \\
\end{tabular} & \begin{tabular}{|l|}
$\mid 18.7$ \\
\end{tabular} & 18.0 & 0.0 & 9.7 & 103 & 79 & 0.0 \\
\hline $10 / 23 / 01$ & $13: 10$ & 18.6 & \begin{tabular}{|l|}
18.7 \\
\end{tabular} & \begin{tabular}{|l|}
18.7 \\
\end{tabular} & 18.5 & 0.0 & 9.6 & 98 & 79 & 0.0 \\
\hline $10 / 23 / 01$ & 13:11 & 18.2 & \begin{tabular}{|l|}
18.7 \\
\end{tabular} & \begin{tabular}{|l|}
18.7 \\
\end{tabular} & 18.1 & 0.0 & 9.7 & 94 & 79 & 0.0 \\
\hline $10 / 23 / 01$ & 13:12 & 18.7 & \begin{tabular}{|l|}
18.7 \\
\end{tabular} & 18.7 & 18.6 & 0.0 & 9.7 & 114 & 79 & 0.0 \\
\hline $10 / 23 / 01$ & $13: 13$ & 18.3 & \begin{tabular}{|l|}
18.7 \\
\end{tabular} & 18.7 & 18.1 & 0.0 & 9.7 & 103 & 79 & 0.0 \\
\hline $10 / 23 / 01$ & 13:14 & 18.7 & \begin{tabular}{|l|}
18.7 \\
\end{tabular} & \begin{tabular}{|l|}
18.7 \\
\end{tabular} & 18.6 & 0.0 & 9.6 & 121 & 79 & 0.0 \\
\hline $10 / 23 / 01$ & 13:15 & 18.3 & \begin{tabular}{|l|l|}
18.7 \\
\end{tabular} & \begin{tabular}{|l|}
18.7 \\
\end{tabular} & 18.2 & 0.0 & 9.6 & 134 & 79 & 0.0 \\
\hline $10 / 23 / 01$ & $13: 16$ & 18.6 & \begin{tabular}{|l|}
18.7 \\
\end{tabular} & \begin{tabular}{|l|}
18.7 \\
\end{tabular} & 18.6 & 0.0 & 9.6 & 96 & 79 & 0.0 \\
\hline $10 / 23 / 01$ & 13:17 & 18.4 & \begin{tabular}{|l|}
18.7 \\
\end{tabular} & \begin{tabular}{|l|}
$\mid 18.7$ \\
\end{tabular} & 18.3 & 0.0 & 9.6 & 110 & 79 & 0.0 \\
\hline $10 / 23 / 01$ & $13: 18$ & 18.4 & \begin{tabular}{|l|}
18.7 \\
\end{tabular} & 18.7 & 18.4 & 0.0 & 9.6 & 93 & 79 & 0.0 \\
\hline $10 / 23 / 01$ & 13:19 & 18.5 & \begin{tabular}{|l|}
18.7 \\
\end{tabular} & \begin{tabular}{|l|}
18.7 \\
\end{tabular} & 18.3 & 0.0 & 9.6 & 96 & 79 & 0.0 \\
\hline $10 / 23 / 01$ & $13: 20$ & 18.2 & \begin{tabular}{|l|}
18.7 \\
\end{tabular} & \begin{tabular}{|l|}
$\mid 18.7$ \\
\end{tabular} & 18.3 & 0.0 & 9.6 & 98 & 79 & 0.0 \\
\hline $10 / 23 / 01$ & 13:21 & 18.5 & \begin{tabular}{|l|}
18.7 \\
\end{tabular} & \begin{tabular}{|l|}
18.7 \\
\end{tabular} & 18.4 & 0.0 & 9.6 & 109 & 79 & 0.0 \\
\hline $10 / 23 / 01$ & $13: 22$ & 18.1 & \begin{tabular}{|l|}
18.7 \\
\end{tabular} & \begin{tabular}{|l|}
$\mid 18.7$ \\
\end{tabular} & 18.1 & 0.0 & 9.7 & 118 & 79 & 0.0 \\
\hline $10 / 23 / 01$ & 13:23 & 18.6 & \begin{tabular}{|l|}
18.7 \\
\end{tabular} & \begin{tabular}{|l|}
18.7 \\
\end{tabular} & 18.4 & 0.0 & 9.6 & 134 & 79 & 0.0 \\
\hline
\end{tabular}


WSRC-TR-2002-00459, Rev. 0 SRT-RPP-2002-00221

\begin{tabular}{|c|c|c|c|c|c|c|c|c|c|c|}
\hline \multicolumn{11}{|c|}{ PREC2_102301_0623 } \\
\hline DATE & TIME & $\begin{array}{c}\mathrm{HX} \\
\text { Outlet } \\
\left({ }^{\circ} \mathrm{C}\right) \\
\mathrm{TC} 0\end{array}$ & $\begin{array}{c}\text { REC } \\
\text { PP } \\
\text { OUT } \\
\left({ }^{\circ} \mathrm{C}\right) \\
\text { TC1 }\end{array}$ & $\begin{array}{c}\text { TK } \\
\text { BOT } \\
\left({ }^{\circ} \mathrm{C}\right) \\
\text { TC2 }\end{array}$ & $\begin{array}{c}\mathrm{HX} \\
\text { OUT } \\
\left({ }^{\circ} \mathrm{C}\right) \\
\text { TC3 }\end{array}$ & $\begin{array}{c}\mathrm{Mn} \\
\text { Flow } \\
(\mathrm{gpm})\end{array}$ & $\begin{array}{l}\text { Galigher } \\
\text { Flow } \\
\text { (gpm) }\end{array}$ & $\begin{array}{c}\text { Current } \\
\text { to } \mathrm{HX}\end{array}$ & $\begin{array}{c}\text { Voltage } \\
\text { to } \mathrm{HX}\end{array}$ & $\begin{array}{c}\mathrm{Sr} \\
\text { Flow } \\
\text { (gpm) }\end{array}$ \\
\hline $10 / 23 / 01$ & 13:24 & 18.1 & $|18.7|$ & 18.7 & 18.0 & 0.0 & 9.6 & 121 & 79 & 0. \\
\hline $10 / 23 / 01$ & $13: 25$ & 18.6 & \begin{tabular}{|l|}
18.7 \\
\end{tabular} & 18.7 & 18.5 & 0.0 & 9.7 & 97 & 79 & 0 \\
\hline $10 / 23 / 01$ & $13: 26$ & 18.1 & \begin{tabular}{|l|}
18.7 \\
\end{tabular} & 18.7 & 18.0 & 0.0 & 9.6 & 103 & 79 & 0 \\
\hline $10 / 23 / 01$ & 13:27 & 18.6 & 18.6 & 18.7 & 18.5 & 0.0 & 9.6 & 88 & 79 & 0 \\
\hline $10 / 23 / 01$ & 13:28 & 18.1 & \begin{tabular}{|l|}
18.7 \\
\end{tabular} & 18.7 & 18.0 & 0.0 & 9.7 & 120 & 79 & 0 \\
\hline $10 / 23 / 01$ & $13: 29$ & 18.6 & \begin{tabular}{|l|}
18.7 \\
\end{tabular} & 18.7 & 18.5 & 0.0 & 9.7 & 115 & 79 & 0 \\
\hline $10 / 23 / 01$ & 13:30 & 18.2 & \begin{tabular}{|l|}
$\mid 18.7$ \\
\end{tabular} & 18.7 & 18.0 & 0.0 & 9.7 & 122 & 79 & 0 \\
\hline $10 / 23 / 01$ & 13:31 & 18.7 & $\mid 18.7$ & 18.7 & 18.5 & 0.0 & 9.7 & 93 & 79 & \\
\hline $10 / 23 / 01$ & 13:32 & 18.3 & $|18.7|$ & 18.7 & 18.1 & 0.0 & 9.5 & 124 & 79 & 0. \\
\hline $10 / 23 / 01$ & $13: 33$ & 18.6 & 18.6 & 18.7 & 18.5 & 0.0 & 9.7 & 143 & 79 & 0. \\
\hline $10 / 23 / 01$ & $13: 34$ & 18.4 & 18.6 & 18.7 & 18.2 & 0.0 & 9.7 & 103 & 79 & 0 \\
\hline $10 / 23 / 01$ & $13: 35$ & 18.5 & $|18.7|$ & 18.7 & 18.5 & 0.0 & 9.6 & 130 & 79 & 0 \\
\hline $10 / 23 / 01$ & $13: 36$ & 18.4 & 18.6 & 18.7 & 18.3 & 0.0 & 9.6 & 130 & 79 & 0 \\
\hline $10 / 23 / 01$ & 13:37 & 18.3 & 18.6 & 18.7 & 18.3 & 0.0 & 9.7 & 114 & 79 & 0 \\
\hline $10 / 23 / 01$ & 13:38 & 18.5 & 18.6 & 18.7 & 18.3 & 0.0 & 9.7 & 100 & 79 & 0 \\
\hline $10 / 23 / 01$ & $13: 39$ & 18.2 & 18.6 & 18.7 & 18.2 & 0.0 & 9.6 & 137 & 79 & \\
\hline $10 / 23 / 01$ & $13: 40$ & 18.5 & 18.6 & 18.7 & 18.4 & 0.0 & 9.7 & 99 & 79 & \\
\hline $10 / 23 / 01$ & $13: 41$ & 18.1 & 18.6 & 18.7 & 18.1 & 0.0 & 9.7 & 101 & 79 & \\
\hline $10 / 23 / 01$ & $13: 42$ & 18.5 & 18.6 & 18.6 & 18.4 & 0.0 & 9.5 & 107 & 79 & 0. \\
\hline $10 / 23 / 01$ & $13: 43$ & 18.0 & 18.6 & 18.6 & 18.0 & 0.0 & 9.7 & 107 & 79 & 0 \\
\hline $10 / 23 / 01$ & $13: 44$ & 18.5 & 18.6 & 18.6 & 18.4 & 0.0 & 9.7 & 81 & 79 & 0 \\
\hline $10 / 23 / 01$ & $13: 45$ & 18.0 & 18.6 & 18.7 & 18.0 & 0.0 & 9.6 & 126 & 79 & 0 \\
\hline $10 / 23 / 01$ & $13: 46$ & 18.6 & 18.6 & 18.6 & 18.4 & 0.0 & 9.6 & 110 & 79 & 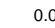 \\
\hline $10 / 23 / 01$ & $13: 47$ & 18.0 & 18.6 & 18.6 & 17.9 & 0.0 & 9.6 & 126 & 79 & \\
\hline $10 / 23 / 01$ & $13: 48$ & 18.6 & 18.6 & 18.6 & 18.5 & 0.0 & 9.7 & 128 & 79 & \\
\hline $10 / 23 / 01$ & $13: 49$ & 18.1 & 18.6 & 18.6 & 18.0 & 0.0 & 9.6 & 126 & 79 & 0 \\
\hline $10 / 23 / 01$ & 13:50 & 18.6 & 18.6 & 18.6 & 18.5 & 0.0 & 9.6 & 97 & 79 & 0 \\
\hline $10 / 23 / 01$ & 13:51 & 18.1 & 18.6 & 18.6 & 18.0 & 0.0 & 9.7 & 132 & 79 & 0.0 \\
\hline $10 / 23 / 01$ & 13:52 & 18.6 & 18.6 & 18.6 & 18.5 & 0.0 & 9.6 & 111 & 79 & 0 \\
\hline $10 / 23 / 01$ & 13:53 & 18.1 & 18.6 & 18.6 & 18.0 & 0.0 & 9.6 & 123 & 79 & 0. \\
\hline $10 / 23 / 01$ & 13:54 & 18.6 & 18.6 & 18.6 & 18.5 & 0.0 & 9.6 & 85 & 79 & \\
\hline $10 / 23 / 01$ & 13:55 & 18.2 & 18.6 & 18.6 & 18.0 & 0.0 & 9.7 & 118 & 79 & \\
\hline $10 / 23 / 01$ & 13:56 & 18.6 & 18.6 & 18.6 & 18.5 & 0.0 & 9.6 & 122 & 79 & 0 \\
\hline $10 / 23 / 01$ & $13: 57$ & 18.2 & $\mid 18.6$ & 18.6 & 18.0 & 0.0 & 9.6 & 104 & 79 & 0 \\
\hline $10 / 23 / 01$ & 13:58 & 18.6 & 18.6 & 18.6 & 18.5 & 0.0 & 9.7 & 117 & 79 & 0 \\
\hline $10 / 23 / 01$ & 13:59 & 18.2 & 18.6 & 18.6 & 18.1 & 0.0 & 9.8 & 136 & 79 & 0 \\
\hline $10 / 23 / 01$ & 14:00 & 18.6 & 18.6 & 18.6 & 18.5 & 0.0 & 9.6 & 122 & 79 & 0. \\
\hline $10 / 23 / 01$ & $14: 01$ & 18.2 & 18.6 & 18.6 & 18.1 & 0.0 & 9.7 & 113 & 79 & 0 \\
\hline $10 / 23 / 01$ & $14: 02$ & 18.6 & $\mid 18.6$ & 18.6 & 18.5 & .0 & .6 & 101 & 79 & 0 \\
\hline $10 / 23 / 01$ & 14:03 & 18.2 & 18.6 & 18.6 & 18.0 & 0.0 & 9.6 & 86 & 79 & 0 \\
\hline $10 / 23 / 01$ & $14: 04$ & 18.6 & 18.6 & 18.6 & 18.5 & 0.0 & 9.6 & 132 & 79 & 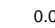 \\
\hline $10 / 23 / 01$ & $14: 05$ & 18.1 & 18.6 & 18.6 & 18.0 & 0.0 & 9.6 & 124 & 79 & 0 \\
\hline $10 / 23 / 01$ & $14: 06$ & 18.5 & 18.6 & 18.6 & 18.4 & 0.0 & 9.7 & 93 & 79 & 0 \\
\hline $10 / 23 / 01$ & $14: 07$ & 18.1 & 18.6 & 18.6 & 18.0 & 0.0 & 9.6 & 90 & 79 & 0 \\
\hline $10 / 23 / 01$ & $14: 08$ & 18.5 & 18.6 & 18.6 & 18.4 & 0.0 & 9.6 & 133 & 79 & 0. \\
\hline $10 / 23 / 01$ & $14: 09$ & 18.0 & 18.6 & 18.6 & 17.9 & 0.0 & 9.6 & 114 & 79 & 0. \\
\hline $10 / 23 / 01$ & $14: 10$ & 18.5 & 18.6 & 18.6 & 18.4 & 0.0 & 9.5 & 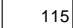 & 79 & 0. \\
\hline 10/23/01 & $14: 11$ & 18.0 & 18.6 & 18.6 & 17.9 & .0 & .7 & 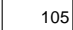 & 7 & \\
\hline $10 / 23 / 01$ & $14: 12$ & 18.5 & 18.6 & 18.6 & 18.4 & 0.0 & .7 & 125 & 79 & 0. \\
\hline $10 / 23 / 01$ & $14: 13$ & 18.0 & $\mid 18.6$ & 18.6 & 17.9 & 0.0 & 9.8 & 85 & 79 & 0 \\
\hline $10 / 23 / 01$ & $14: 14$ & 18.5 & 18.5 & 18.6 & 18.3 & 0.0 & 9.6 & 122 & 79 & 0 \\
\hline $10 / 23 / 01$ & $14: 15$ & 18.0 & 18.6 & 18.6 & 18.0 & 0.0 & 9.5 & 127 & 79 & 0. \\
\hline $10 / 23 / 01$ & $14: 16$ & 18.4 & 18.5 & 18.6 & 18.3 & 0.0 & 9.7 & 113 & 79 & 0 \\
\hline $10 / 23 / 01$ & $14: 17$ & 18.2 & 18.6 & 18.6 & 18.2 & 0.0 & 9.8 & 72 & 79 & 0.0 \\
\hline $10 / 23 / 01$ & $14: 18$ & 18.4 & \begin{tabular}{|l|}
18.5 \\
\end{tabular} & 18.6 & 18.2 & 0.0 & .5 & 107 & 79 & 0.0 \\
\hline $10 / 23 / 01$ & $14: 19$ & 18.4 & 18.5 & 18.6 & 18.4 & 0.0 & .6 & 112 & 19 & 0 \\
\hline $10 / 23 / 01$ & $14: 20$ & 18.3 & 18.5 & 18.5 & 18.2 & 0.0 & 6 & 78 & 79 & 0 \\
\hline $10 / 23 / 01$ & $14: 21$ & 18.5 & 18.5 & 18.5 & 18.4 & 0.0 & 9.6 & 74 & 79 & 0 \\
\hline $10 / 23 / 01$ & 14:22 & 18.2 & 18.5 & 18.5 & 18.1 & 0.0 & 9.6 & 119 & 79 & 0.0 \\
\hline $10 / 23 / 01$ & $14: 23$ & 18.5 & 18.5 & 18.5 & 18.4 & 0.0 & 9.6 & 91 & 79 & 0.0 \\
\hline
\end{tabular}

\begin{tabular}{|c|c|c|c|c|c|c|c|c|c|c|}
\hline \multicolumn{11}{|c|}{ PREC2_102301_0623 } \\
\hline DATE & TIME & $\begin{array}{c}\mathrm{HX} \\
\text { Outlet } \\
\left({ }^{\circ} \mathrm{C}\right) \\
\mathrm{TCO}\end{array}$ & $\begin{array}{c}\text { REC } \\
\text { PP } \\
\text { OUT } \\
\left({ }^{\circ} \mathrm{C}\right) \\
\text { TC1 }\end{array}$ & $\begin{array}{c}\text { TK } \\
\text { BOT } \\
\left({ }^{\circ} \mathrm{C}\right) \\
\text { TC2 }\end{array}$ & $\begin{array}{c}\mathrm{HX} \\
\text { OUT } \\
\left({ }^{\circ} \mathrm{C}\right) \\
\text { TC3 }\end{array}$ & $\begin{array}{c}\text { Mn } \\
\text { Flow } \\
(\mathrm{gpm})\end{array}$ & $\begin{array}{c}\text { Galigher } \\
\text { Flow } \\
\text { (gpm) }\end{array}$ & $\begin{array}{c}\text { Current } \\
\text { to } \mathrm{HX}\end{array}$ & $\begin{array}{c}\text { Voltage } \\
\text { to } \mathrm{HX}\end{array}$ & $\begin{array}{c}\mathrm{Sr} \\
\text { Flow } \\
(\mathrm{gpm})\end{array}$ \\
\hline $10 / 23 / 01$ & 14:24 & 18.1 & 18.5 & 18.5 & 17.9 & 0.0 & 9.6 & 69 & 79 & 0.0 \\
\hline $10 / 23 / 01$ & 14:25 & 18.5 & 18.5 & 18.5 & 18.4 & 0.0 & 9.5 & 100 & 79 & 0.0 \\
\hline $10 / 23 / 01$ & 14:26 & 17.9 & 18.5 & 18.5 & 17.8 & 0.0 & 9.6 & 103 & 79 & 0.0 \\
\hline $10 / 23 / 01$ & 14:27 & 18.4 & 18.5 & 18.5 & 18.3 & 0.0 & 9.7 & 119 & 79 & 0.0 \\
\hline $10 / 23 / 01$ & 14:28 & 17.9 & 18.5 & 18.5 & 17.9 & 0.0 & 9.6 & 120 & 79 & 0.0 \\
\hline $10 / 23 / 01$ & $14: 29$ & 18.4 & 18.5 & 18.5 & 18.3 & 0.0 & 9.6 & 102 & 79 & 0.0 \\
\hline 10/23/01 & $14: 30$ & 18.2 & 18.5 & 18.5 & 18.2 & 0.0 & 9.6 & 116 & 79 & 0.0 \\
\hline $10 / 23 / 01$ & $14: 31$ & 18.3 & 18.5 & 18.5 & 18.2 & 0.0 & 9.6 & 68 & 79 & 0.0 \\
\hline $10 / 23 / 01$ & 14:32 & 18.5 & 18.5 & 18.5 & 18.4 & 0.0 & 9.7 & 59 & 79 & 0.0 \\
\hline $10 / 23 / 01$ & 14:33 & 18.2 & 18.5 & 18.5 & 18.0 & 0.0 & 9.7 & 84 & 79 & 0.0 \\
\hline $10 / 23 / 01$ & 14:34 & 18.5 & 18.5 & 18.5 & 18.4 & 0.0 & 9.7 & 107 & 79 & 0.0 \\
\hline $10 / 23 / 01$ & 14:35 & 18.0 & 18.5 & 18.5 & 17.9 & 0.0 & 9.6 & 103 & 79 & 0.0 \\
\hline 10/23/01 & 14:36 & 18.4 & 18.5 & 18.5 & 18.3 & 0.0 & 9.4 & 55 & 79 & 0.0 \\
\hline $10 / 23 / 01$ & $14: 37$ & 17.9 & 18.5 & 18.5 & 17.8 & 0.0 & 9.6 & 115 & 79 & 0.0 \\
\hline $10 / 23 / 01$ & 14:38 & 18.4 & 18.5 & 18.5 & 18.3 & 0.0 & 9.6 & 66 & 79 & 0.0 \\
\hline 10/23/01 & 14:39 & 18.0 & 18.5 & 18.5 & 18.0 & 0.0 & 9.6 & 62 & 79 & 0.0 \\
\hline $10 / 23 / 01$ & 14:40 & 18.4 & 18.5 & 18.5 & 18.2 & 0.0 & 9.6 & 86 & 79 & 0.0 \\
\hline $10 / 23 / 01$ & 14:41 & 18.3 & 18.5 & 18.5 & 18.3 & 0.0 & 9.5 & 70 & 79 & 0.0 \\
\hline 10/23/01 & 14:42 & 18.3 & 18.5 & 18.5 & 18.1 & 0.0 & 9.7 & 99 & 79 & 0.0 \\
\hline 10/23/01 & 14:43 & 18.5 & 18.5 & 18.5 & 18.4 & 0.0 & 9.5 & 80 & 79 & 0.0 \\
\hline 10/23/01 & 14:44 & 18.2 & 18.5 & 18.5 & 18.0 & 0.0 & 9.6 & 69 & 79 & 0.0 \\
\hline $10 / 23 / 01$ & 14:45 & 18.5 & 18.5 & 18.5 & 18.4 & 0.0 & 9.7 & 58 & 79 & 0.0 \\
\hline $10 / 23 / 01$ & 14:46 & 18.1 & 18.5 & 18.5 & 18.0 & 0.0 & 9.5 & 54 & 79 & 0.0 \\
\hline $10 / 23 / 01$ & $14: 47$ & 18.5 & 18.5 & 18.5 & 18.4 & 0.0 & 9.7 & 85 & 79 & 0.0 \\
\hline $10 / 23 / 01$ & 14:48 & 18.2 & 18.5 & 18.5 & 18.0 & 0.0 & 9.7 & 75 & 79 & 0.0 \\
\hline 10/23/01 & 14:49 & 18.5 & 18.5 & 18.5 & 18.4 & 0.0 & 9.7 & 103 & 79 & 0.0 \\
\hline $10 / 23 / 01$ & 14:50 & 18.2 & 18.5 & 18.5 & 18.0 & 0.0 & 9.5 & 100 & 79 & 0.0 \\
\hline $10 / 23 / 01$ & 14:51 & 18.5 & 18.5 & 18.5 & 18.4 & 0.0 & 9.6 & 103 & 79 & 0.0 \\
\hline $10 / 23 / 01$ & 14:52 & 18.1 & 18.5 & 18.5 & 18.0 & 0.0 & 9.8 & 52 & 79 & 0.0 \\
\hline $10 / 23 / 01$ & 14:53 & 18.5 & 18.5 & 18.5 & 18.4 & 0.0 & 9.5 & 47 & 79 & 0.0 \\
\hline $10 / 23 / 01$ & 14:54 & 18.2 & 18.5 & 18.5 & 18.0 & 0.0 & 9.6 & 104 & 79 & 0.0 \\
\hline $10 / 23 / 01$ & 14:55 & 18.5 & 18.5 & 18.5 & 18.4 & 0.0 & 9.6 & 59 & 79 & 0.0 \\
\hline $10 / 23 / 01$ & 14:56 & 18.2 & 18.5 & 18.5 & 18.0 & 0.0 & 9.7 & 40 & 79 & 0.0 \\
\hline $10 / 23 / 01$ & 14:57 & 18.5 & 18.5 & 18.5 & 18.4 & 0.0 & 9.7 & 67 & 79 & 0.0 \\
\hline $10 / 23 / 01$ & 14:58 & 18.2 & 18.5 & 18.5 & 18.0 & 0.0 & 9.7 & 44 & 79 & 0.0 \\
\hline $10 / 23 / 01$ & 14:59 & 18.5 & 18.5 & 18.5 & 18.4 & 0.0 & 9.6 & 65 & 79 & 0.0 \\
\hline $10 / 23 / 01$ & $15: 00$ & 18.2 & 18.4 & 18.5 & 18.0 & 0.0 & 9.7 & 51 & 79 & 0.0 \\
\hline 10/23/01 & 15:01 & 18.4 & 18.4 & 18.5 & 18.4 & 0.0 & 9.6 & 95 & 79 & 0.0 \\
\hline $10 / 23 / 01$ & $15: 02$ & 18.2 & 18.5 & 18.5 & 18.0 & 0.0 & 9.7 & 41 & 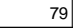 & 0.0 \\
\hline $10 / 23 / 01$ & $15: 03$ & 18.4 & 18.4 & 18.5 & 18.3 & 0.0 & 7 & 78 & 79 & .0 \\
\hline $10 / 23 / 01$ & 15:04 & 18.3 & 18.4 & 18.5 & 18.1 & 0.0 & 9.8 & 59 & 79 & 0.0 \\
\hline $10 / 23 / 01$ & 15:05 & 18.3 & 18.4 & 18.5 & 18.3 & 0.0 & 9.5 & 80 & 79 & 0.0 \\
\hline $10 / 23 / 01$ & 15:06 & 18.3 & 18.4 & 18.4 & 18.1 & 0.0 & 9.6 & 95 & 79 & 0.0 \\
\hline $10 / 23 / 01$ & 15:07 & 18.2 & 18.4 & 18.5 & 18.2 & 0.0 & 9.8 & 54 & 79 & 0.0 \\
\hline $10 / 23 / 01$ & $15: 08$ & 18.3 & 18.5 & 18.5 & 18.2 & 0.0 & 9.7 & 45 & 79 & 0.0 \\
\hline 10/23/01 & $15: 09$ & 18.1 & 18.5 & 18.5 & 18.1 & .0 & 9.7 & 98 & 79 & . \\
\hline $10 / 23 / 01$ & $15: 10$ & 18.3 & 18.4 & 18.4 & 18.2 & 0.0 & 97 & 78 & 79 & 0.0 \\
\hline $10 / 23 / 01$ & $15: 11$ & 18.0 & 18.4 & 18.5 & 18 & .0 & 9.6 & 49 & 79 & .0 \\
\hline $10 / 23 / 01$ & $15: 12$ & 18.3 & 18.4 & 18.4 & 18.2 & 0.0 & 9.6 & 35 & 79 & 0.0 \\
\hline 10/23/01 & $15: 13$ & 18.1 & 18.4 & 18.4 & 18.1 & 0.0 & 9.8 & 61 & 79 & 0.0 \\
\hline $10 / 23 / 01$ & $15: 14$ & 18.3 & 18.4 & 18.4 & 18.2 & 0.0 & 9.8 & 34 & 79 & 0.0 \\
\hline 10/23/01 & $15: 15$ & 18.1 & 18.4 & 18.5 & 18.1 & 0.0 & 9.6 & 34 & 79 & 0.0 \\
\hline $10 / 23 / 01$ & $15: 16$ & 18.3 & 18.4 & 18.4 & 18.2 & 0.0 & 9.6 & 33 & 79 & 0.0 \\
\hline $10 / 23 / 01$ & $15: 17$ & 18.1 & 18.4 & 18.4 & 18.1 & 0.0 & 9.6 & 32 & 71 & 0.0 \\
\hline 10/23/01 & $15: 18$ & 18.3 & 18.4 & 18.4 & 18.1 & 0.0 & 9.7 & 23 & 79 & 0.0 \\
\hline 10/23/01 & $15: 19$ & 18.1 & 18.4 & 18.4 & 18.1 & 0.0 & 9.6 & 30 & 79 & 0.0 \\
\hline $10 / 23 / 01$ & $15: 20$ & 18.3 & 18.4 & 18.4 & 18.1 & 0.0 & 9.7 & 22 & 79 & 0.0 \\
\hline $10 / 23 / 01$ & $15: 21$ & 18.0 & 18.4 & 18.4 & 18.0 & 0.0 & 9.7 & 65 & 79 & 0.0 \\
\hline 10/23/01 & $15: 22$ & 18.3 & 18.4 & 18.4 & 18.1 & 0.0 & 9.6 & 21 & 79 & 0.0 \\
\hline $10 / 23 / 01$ & $15: 23$ & 18.0 & 18.4 & 18.4 & 18.0 & 0.0 & 9.6 & 21 & 79 & 0.0 \\
\hline
\end{tabular}


WSRC-TR-2002-00459, Rev. 0 SRT-RPP-2002-00221

\begin{tabular}{|c|c|c|c|c|c|c|c|c|c|c|}
\hline \multicolumn{3}{|c|}{ PREC2_102301_0623 } & \multirow[b]{2}{*}{\begin{tabular}{|c|} 
REC \\
PP \\
OUT \\
$\left({ }^{\circ} \mathrm{C}\right)$ \\
TC1 \\
\end{tabular}} & \multirow[b]{2}{*}{$\begin{array}{c}\mathrm{TK} \\
\mathrm{BOT} \\
\left({ }^{\circ} \mathrm{C}\right) \\
\mathrm{TC} 2\end{array}$} & \multirow[b]{2}{*}{\begin{tabular}{|l|} 
HX \\
OUT \\
$\left({ }^{\circ} \mathrm{C}\right)$ \\
TC3
\end{tabular}} & \multirow[b]{2}{*}{$\begin{array}{c}\mathrm{Mn} \\
\text { Flow } \\
(\mathrm{gpm})\end{array}$} & \multirow[b]{2}{*}{$\begin{array}{c}\text { Galigher } \\
\text { Flow } \\
(\mathrm{gpm})\end{array}$} & \multirow[b]{2}{*}{$\begin{array}{c}\text { Current } \\
\text { to } \mathrm{HX}\end{array}$} & \multirow[b]{2}{*}{$\begin{array}{c}\text { Voltage } \\
\text { to } \mathrm{HX}\end{array}$} & \multirow[b]{2}{*}{$\begin{array}{c}\mathrm{Sr} \\
\text { Flow } \\
(\mathrm{gpm})\end{array}$} \\
\hline DATE & TIME & $\begin{array}{c}\mathrm{HX} \\
\text { Outlet } \\
\left({ }^{\circ} \mathrm{C}\right) \\
\mathrm{TCO}\end{array}$ & & & & & & & & \\
\hline $10 / 23 / 01$ & $15: 24$ & 18.3 & 18.4 & 18.4 & 18.2 & 0.0 & 9.6 & 22 & 79 & 0.0 \\
\hline $10 / 23 / 01$ & $15: 25$ & 18.0 & 18.4 & 18.4 & 17.9 & 0.0 & 9.7 & 22 & 79 & 0.0 \\
\hline $10 / 23 / 01$ & $15: 26$ & 18.3 & 18.4 & 18.4 & 18.2 & 0.0 & 9.7 & 22 & 79 & 0.0 \\
\hline $10 / 23 / 01$ & $15: 27$ & 17.9 & 18.4 & 18.4 & 17.9 & 0.0 & 9.6 & 21 & 79 & 0.0 \\
\hline $10 / 23 / 01$ & $15: 28$ & 18.3 & 18.4 & 18.4 & 18.2 & 0.0 & 9.7 & 21 & 79 & 0.0 \\
\hline 10/23/01 & 15:29 & 17.9 & 18.4 & 18.4 & 17.8 & 0.0 & 9.7 & 21 & 79 & 0.0 \\
\hline $10 / 23 / 01$ & $15: 30$ & 18.3 & 18.4 & 18.4 & 18.2 & 0.0 & 9.5 & 21 & 79 & 0.0 \\
\hline $10 / 23 / 01$ & $15: 31$ & 17.8 & 18.4 & 18.4 & 17.8 & 0.0 & 9.7 & 21 & 79 & 0.0 \\
\hline $10 / 23 / 01$ & 15:32 & 18.4 & 18.4 & 18.4 & 18.2 & 0.0 & 9.6 & 21 & 79 & 0.0 \\
\hline $10 / 23 / 01$ & $15: 33$ & 17.8 & 18.4 & 18.4 & 17.7 & 0.0 & 9.5 & 21 & 79 & 0.0 \\
\hline $10 / 23 / 01$ & $15: 34$ & 18.4 & 18.4 & 18.4 & 18.2 & 0.0 & 9.6 & 21 & 79 & 0.0 \\
\hline 10/23/01 & 15:35 & 17.9 & 18.4 & 18.4 & 17.7 & 0.0 & 9.6 & 21 & 79 & 0.0 \\
\hline $10 / 23 / 01$ & 15:36 & 18.4 & 18.4 & 18.4 & 18.2 & 0.0 & 9.6 & 21 & 79 & 0.0 \\
\hline $10 / 23 / 01$ & $15: 37$ & 17.9 & 18.4 & 18.4 & 17.7 & 0.0 & 9.7 & 21 & 79 & 0.0 \\
\hline $10 / 23 / 01$ & 15:38 & 18.4 & 18.4 & 18.4 & 18.2 & 0.0 & 9.5 & 21 & 79 & 0.0 \\
\hline $10 / 23 / 01$ & $15: 39$ & 17.9 & 18.4 & 18.4 & 17.8 & 0.0 & 9.6 & 21 & 79 & 0.0 \\
\hline $10 / 23 / 01$ & $15: 40$ & 18.4 & 18.4 & 18.4 & 18.3 & 0.0 & 9.6 & 21 & 79 & 0.0 \\
\hline $10 / 23 / 01$ & $15: 41$ & 18.0 & 18.4 & 18.4 & 17.8 & 0.0 & 9.6 & 21 & 79 & 0.0 \\
\hline 10/23/01 & 15:42 & 18.4 & 18.4 & 18.4 & 18.3 & 0.0 & 9.7 & 21 & 79 & 0.0 \\
\hline $10 / 23 / 01$ & $15: 43$ & 18.0 & 18.4 & 18.4 & 17.8 & 0.0 & 9.6 & 21 & 79 & 0.0 \\
\hline $10 / 23 / 01$ & $15: 44$ & 18.4 & 18.4 & 18.4 & 18.3 & 0.0 & 9.7 & 21 & 79 & 0.0 \\
\hline $10 / 23 / 01$ & $15: 45$ & 18.1 & 18.4 & 18.4 & 17.9 & 0.0 & 9.6 & 21 & 79 & 0.0 \\
\hline $10 / 23 / 01$ & $15: 46$ & 18.3 & 18.4 & 18.4 & 18.3 & 0.0 & 9.6 & 21 & 79 & 0.0 \\
\hline $10 / 23 / 01$ & $15: 47$ & 18.2 & 18.4 & 18.4 & 18.0 & 0.0 & 9.6 & 21 & 79 & 0.0 \\
\hline $10 / 23 / 01$ & $15: 48$ & 18.2 & 18.4 & 18.4 & 18.2 & 0.0 & 9.6 & 21 & 79 & 0.0 \\
\hline $10 / 23 / 01$ & $15: 49$ & 18.2 & 18.4 & 18.4 & 18.0 & 0.0 & 9.7 & 21 & 79 & 0.0 \\
\hline $10 / 23 / 01$ & 15:50 & 18.1 & 18.4 & 18.4 & 18.0 & 0.0 & 9.7 & 21 & 79 & 0.0 \\
\hline $10 / 23 / 01$ & $15: 51$ & 18.3 & 18.4 & 18.4 & 18.1 & 0.0 & 9.7 & 21 & 79 & 0.0 \\
\hline $10 / 23 / 01$ & 15:52 & 17.9 & 18.4 & 18.4 & 17.9 & 0.0 & 9.7 & 21 & 79 & 0.0 \\
\hline $10 / 23 / 01$ & 15:53 & 18.3 & 18.4 & 18.4 & 18.1 & 0.0 & 9.6 & 21 & 79 & 0.0 \\
\hline $10 / 23 / 01$ & 15:54 & 17.8 & 18.4 & 18.4 & 17.8 & 0.0 & 9.6 & 21 & 79 & 0.0 \\
\hline $10 / 23 / 01$ & $15: 55$ & 18.4 & 18.4 & 18.4 & 18.2 & 0.0 & 9.6 & 21 & 79 & 0.0 \\
\hline $10 / 23 / 01$ & $15: 56$ & 17.8 & 18.4 & 18.4 & 17.7 & 0.0 & 9.7 & 21 & 79 & 0.0 \\
\hline $10 / 23 / 01$ & 15:57 & 18.4 & 18.4 & 18.4 & 18.2 & 0.0 & 9.7 & 21 & 79 & 0.0 \\
\hline $10 / 23 / 01$ & 15:58 & 17.9 & 18.4 & 18.4 & 17.7 & 0.0 & 9.6 & 21 & 79 & 0.0 \\
\hline $10 / 23 / 01$ & $15: 59$ & 18.4 & 18.4 & 18.4 & 18.2 & 0.0 & 9.6 & 21 & 79 & 0.0 \\
\hline $10 / 23 / 01$ & $16: 00$ & 18.0 & 18.4 & 18.4 & 17.8 & 0.0 & 9.6 & 21 & 79 & 0.0 \\
\hline $10 / 23 / 01$ & 16:01 & 18.4 & 18.4 & 18.4 & 18.3 & 0.0 & 9.6 & 21 & 79 & 0.0 \\
\hline $10 / 23 / 01$ & 16:02 & 18.1 & 18.4 & 18.4 & 17.9 & 0.0 & 9.6 & 21 & 79 & 0.0 \\
\hline $10 / 23 / 01$ & 16:03 & 18.4 & 18.4 & 18.4 & 18.3 & 0.0 & 9.7 & 21 & 79 & 0.0 \\
\hline $10 / 23 / 01$ & $16: 04$ & 18.2 & 18.4 & 18.4 & 18.0 & 0.0 & 9.6 & 21 & 79 & 0.0 \\
\hline $10 / 23 / 01$ & 16:05 & 18.2 & 18.4 & 18.4 & 18.2 & 0.0 & 9.6 & 21 & 79 & 0.0 \\
\hline $10 / 23 / 01$ & 16:06 & 18.2 & 18.4 & 18.4 & 18.1 & 0.0 & 9.7 & 21 & 79 & 0.0 \\
\hline $10 / 23 / 01$ & 16:07 & 18.0 & 18.4 & 18.4 & 18.0 & 0.0 & 9.6 & 21 & 79 & 0.0 \\
\hline $10 / 23 / 01$ & 16:08 & 18.3 & 18.4 & 18.4 & 18.1 & 0.0 & 9.6 & 21 & 79 & 0.0 \\
\hline $10 / 23 / 01$ & 16:09 & 17.8 & 18.4 & 18.4 & 17.8 & 0.0 & 9.6 & 21 & 79 & 0.0 \\
\hline $10 / 23 / 01$ & $16: 10$ & 18.3 & 18.3 & 18.3 & 18.2 & 0.0 & 9.7 & 21 & 79 & 0.0 \\
\hline $10 / 23 / 01$ & $16: 11$ & 17.8 & 18.4 & 18.4 & 17.7 & 0.0 & 9.7 & 21 & 79 & 0.0 \\
\hline $10 / 23 / 01$ & $16: 12$ & 18.4 & 18.3 & 18.3 & 18.2 & 0.0 & 9.7 & 21 & 79 & 0.0 \\
\hline $10 / 23 / 01$ & $16: 13$ & 17.9 & 18.3 & 18.3 & 17.7 & 0.0 & 9.7 & 21 & 79 & 0.0 \\
\hline $10 / 23 / 01$ & 16:14 & 18.4 & 18.3 & 18.3 & 18.2 & 0.0 & 9.7 & 21 & 79 & 0.0 \\
\hline $10 / 23 / 01$ & $16: 15$ & 17.9 & 18.4 & 18.4 & 17.8 & 0.0 & 9.6 & 21 & 79 & 0.0 \\
\hline $10 / 23 / 01$ & $16: 16$ & 18.4 & 18.3 & 18.3 & 18.2 & 0.0 & 9.6 & 21 & 79 & 0.0 \\
\hline $10 / 23 / 01$ & 16:17 & 18.0 & 18.3 & 18.4 & 17.8 & 0.0 & 9.7 & 21 & 79 & 0.0 \\
\hline $10 / 23 / 01$ & $16: 18$ & 18.4 & 18.3 & 18.4 & 18.3 & 0.0 & 9.7 & 21 & 79 & 0.0 \\
\hline $10 / 23 / 01$ & $16: 19$ & 18.1 & 18.3 & 18.3 & 17.9 & 0.0 & 9.7 & 21 & 79 & 0.0 \\
\hline $10 / 23 / 01$ & $16: 20$ & 18.3 & 18.4 & $\mid 18.4$ & 18.2 & 0.0 & 9.6 & 21 & 79 & 0.0 \\
\hline $10 / 23 / 01$ & $16: 21$ & 18.2 & 18.3 & 18.4 & 18.0 & 0.0 & 9.7 & 21 & 79 & 0.0 \\
\hline $10 / 23 / 01$ & $16: 22$ & 18.1 & 18.3 & 18.4 & 18.1 & 0.0 & 9.6 & 21 & 79 & 0.0 \\
\hline $10 / 23 / 01$ & $16: 23$ & 18.3 & 18.3 & 18.3 & 18.1 & 0.0 & 9.7 & 21 & 79 & 0.0 \\
\hline
\end{tabular}

\begin{tabular}{|c|c|c|c|c|c|c|c|c|c|c|}
\hline \multicolumn{11}{|c|}{ PREC2_102301_0623 } \\
\hline DATE & TIME & $\begin{array}{c}\mathrm{HX} \\
\text { Outlet } \\
\left({ }^{\circ} \mathrm{C}\right) \\
\text { TC0 }\end{array}$ & \begin{tabular}{|c|} 
REC \\
PP \\
OUT \\
$\left({ }^{\circ} \mathrm{C}\right)$ \\
TC1 \\
\end{tabular} & $\begin{array}{c}\text { TK } \\
\text { BOT } \\
\left({ }^{\circ} \mathrm{C}\right) \\
\text { TC2 } \\
\end{array}$ & $\begin{array}{l}\mathrm{HX} \\
\text { OUT } \\
\left({ }^{\circ} \mathrm{C}\right) \\
\text { TC3 }\end{array}$ & $\begin{array}{c}\text { Mn } \\
\text { Flow } \\
\text { (gpm) }\end{array}$ & \begin{tabular}{|c} 
Galigher \\
Flow \\
(gpm)
\end{tabular} & $\begin{array}{c}\text { Current } \\
\text { to } \mathrm{HX}\end{array}$ & $\begin{array}{c}\text { Voltage } \\
\text { to } \mathrm{HX}\end{array}$ & $\begin{array}{c}\mathrm{Sr} \\
\text { Flow } \\
\text { (gpm) }\end{array}$ \\
\hline 10/23/01 & $16: 24$ & 17.9 & \begin{tabular}{|l|}
18.3 \\
\end{tabular} & 18.4 & 17.8 & 0.0 & 9.7 & 21 & 79 & 0.0 \\
\hline $10 / 23 / 01$ & $16: 25$ & 18.3 & $\mid 18.3$ & 18.3 & 18.1 & 0.0 & 9.7 & 21 & 79 & 0.0 \\
\hline 10/23/01 & $16: 26$ & 17.8 & \begin{tabular}{|l|}
18.3 \\
\end{tabular} & 18.4 & 17.7 & 0.0 & 9.7 & 21 & 79 & 0.0 \\
\hline 10/23/01 & $16: 27$ & 18.4 & \begin{tabular}{|l|}
18.3 \\
\end{tabular} & 18.3 & 18.2 & 0.0 & 9.7 & 21 & 79 & 0.0 \\
\hline 10/23/01 & $16: 28$ & 17.8 & \begin{tabular}{|l|} 
\\
\end{tabular} 8.4 & 18.4 & 17.7 & 0.0 & 9.6 & 21 & 79 & 0.0 \\
\hline 10/23/01 & $16: 29$ & 18.4 & 18.3 & 18.4 & 18.2 & 0.0 & 9.6 & 21 & 79 & 0.0 \\
\hline 10/23/01 & $16: 30$ & 17.9 & 18.3 & 18.4 & 17.8 & 0.0 & 9.7 & 21 & 79 & 0.0 \\
\hline $10 / 23 / 01$ & $16: 31$ & 18.4 & $\mid 18.3$ & 18.4 & 18.3 & 0.0 & 9.7 & 21 & 79 & 0.0 \\
\hline 10/23/01 & $16: 32$ & 18.0 & 18.3 & 18.3 & 17.8 & 0.0 & 9.7 & 21 & 79 & 0.0 \\
\hline 10/23/01 & $16: 33$ & 18.4 & \begin{tabular}{|l|} 
\\
\end{tabular} 8.3 & 18.4 & 18.3 & 0.0 & 9.7 & 21 & 79 & 0.0 \\
\hline 10/23/01 & $16: 34$ & 18.1 & \begin{tabular}{|l|}
18.3 \\
\end{tabular} & 18.3 & 18.0 & 0.0 & 9.6 & 21 & 79 & 0.0 \\
\hline 10/23/01 & $16: 35$ & 18.2 & \begin{tabular}{|l|} 
\\
\end{tabular} & 18.4 & 18.1 & 0.0 & 9.6 & 21 & 79 & 0.0 \\
\hline 10/23/01 & $16: 36$ & 18.2 & 18.3 & 18.3 & 18.0 & 0.0 & 9.6 & 21 & 79 & 0.0 \\
\hline 10/23/01 & $16: 37$ & 17.9 & \begin{tabular}{|l|} 
\\
\end{tabular} & 18.3 & 17.9 & 0.0 & 9.7 & 21 & 79 & 0.0 \\
\hline $10 / 23 / 01$ & $16: 38$ & 18.3 & 18.3 & 18.3 & 18.1 & 0.0 & 9.6 & 21 & 79 & 0.0 \\
\hline $10 / 23 / 01$ & $16: 39$ & 17.8 & 18.3 & 18.4 & 17.7 & 0.0 & 9.7 & 21 & 79 & 0.0 \\
\hline $10 / 23 / 01$ & $16: 40$ & 18.3 & \begin{tabular}{|l|}
18.3 \\
\end{tabular} & 18.3 & 18.2 & 0.0 & 9.6 & 21 & 79 & 0.0 \\
\hline $10 / 23 / 01$ & $16: 41$ & 17.8 & 18.3 & 18.3 & 17.7 & 0.0 & 9.7 & 21 & 79 & 0.0 \\
\hline $10 / 23 / 01$ & $16: 42$ & 18.4 & \begin{tabular}{|l|}
18.3 \\
\end{tabular} & 18.3 & 18.2 & 0.0 & 9.6 & 21 & 79 & 0.0 \\
\hline $10 / 23 / 01$ & $16: 43$ & 17.9 & 18.3 & 18.3 & 17.7 & 0.0 & 9.6 & 21 & 79 & 0.0 \\
\hline $10 / 23 / 01$ & $16: 44$ & 18.4 & 18.3 & 18.3 & 18.3 & 0.0 & 9.7 & 21 & 79 & 0.0 \\
\hline $10 / 23 / 01$ & $16: 45$ & 18.0 & 18.3 & 18.3 & 17.8 & 0.0 & 9.7 & 21 & 79 & 0.0 \\
\hline $10 / 23 / 01$ & $16: 46$ & 18.4 & 18.3 & 18.3 & 18.3 & 0.0 & 9.7 & 21 & 79 & 0.0 \\
\hline $10 / 23 / 01$ & $16: 47$ & 18.1 & \begin{tabular}{|l|}
18.3 \\
\end{tabular} & 18.3 & 17.9 & 0.0 & 9.7 & 21 & 79 & 0.0 \\
\hline $10 / 23 / 01$ & $16: 48$ & 18.4 & 18.3 & 18.3 & 18.3 & 0.0 & 9.7 & 21 & 79 & 0.0 \\
\hline $10 / 23 / 01$ & $16: 49$ & 17.9 & \begin{tabular}{|l|}
18.4 \\
\end{tabular} & 18.4 & 17.8 & 0.0 & 9.6 & 21 & 79 & 0.0 \\
\hline $10 / 23 / 01$ & $16: 50$ & 18.0 & \begin{tabular}{|l|}
18.3 \\
\end{tabular} & 18.3 & 17.8 & 0.0 & 9.7 & 21 & 79 & 0.0 \\
\hline $10 / 23 / 01$ & $16: 51$ & 18.0 & 18.3 & 18.3 & 17.9 & 0.0 & 9.6 & 21 & 79 & 0.0 \\
\hline $10 / 23 / 01$ & $16: 52$ & 18.1 & 18.3 & 18.3 & 17.9 & 0.0 & 9.6 & 21 & 79 & 0.0 \\
\hline $10 / 23 / 01$ & $16: 53$ & 18.1 & 18.3 & 18.3 & 17.9 & 0.0 & 9.7 & 21 & 79 & 0.0 \\
\hline $10 / 23 / 01$ & $16: 54$ & 18.2 & 18.3 & 18.3 & 18.0 & 0.0 & 9.5 & 21 & 79 & 0.0 \\
\hline $10 / 23 / 01$ & $16: 55$ & 18.0 & 18.3 & 18.3 & 17.9 & 0.0 & 9.5 & 21 & 79 & 0.0 \\
\hline $10 / 23 / 01$ & $16: 56$ & 18.2 & 18.3 & 18.3 & 18.1 & 0.0 & 9.6 & 21 & 79 & 0.0 \\
\hline $10 / 23 / 01$ & $16: 57$ & 18.0 & \begin{tabular}{|l|}
18.3 \\
\end{tabular} & 18.3 & 17.9 & 0.0 & 9.7 & 21 & 79 & 0.0 \\
\hline $10 / 23 / 01$ & $16: 58$ & 18.2 & | 18.3 & 18.3 & 18.1 & 0.0 & 9.7 & 21 & 79 & 0.0 \\
\hline $10 / 23 / 01$ & $16: 59$ & 18.0 & \begin{tabular}{|l|}
18.3 \\
\end{tabular} & 18.3 & 17.9 & 0.0 & 9.6 & 21 & 79 & 0.0 \\
\hline $10 / 23 / 01$ & $17: 00$ & 18.1 & \begin{tabular}{|l|}
18.3 \\
\end{tabular} & 18.3 & 18.0 & 0.0 & 9.7 & 21 & 79 & 0.0 \\
\hline $10 / 23 / 01$ & 17:01 & 18.1 & 18.3 & 18.3 & 17.9 & 0.0 & 9.7 & 21 & 79 & 0.0 \\
\hline $10 / 23 / 01$ & 17:02 & 18.1 & 18.3 & 18.3 & 18.0 & 0.0 & 9.6 & 21 & 79 & 0.0 \\
\hline $10 / 23 / 01$ & $17: 03$ & 18.1 & 18.3 & 18.3 & 18.0 & 0.0 & 9.7 & 21 & 79 & 0.0 \\
\hline $10 / 23 / 01$ & $17: 04$ & 18.0 & \begin{tabular}{|l|}
18.3 \\
\end{tabular} & 18.3 & 17.9 & 0.0 & 9.6 & 21 & 79 & 0.0 \\
\hline $10 / 23 / 01$ & $17: 05$ & 18.2 & \begin{tabular}{|l|}
18.3 \\
\end{tabular} & 18.3 & 18.1 & 0.0 & 9.7 & 21 & 79 & 0.0 \\
\hline $10 / 23 / 01$ & $17: 06$ & 18.2 & \begin{tabular}{|l|}
18.3 \\
\end{tabular} & 18.3 & 18.1 & 0.0 & 9.7 & 21 & 79 & 0.0 \\
\hline $10 / 23 / 01$ & $17: 07$ & 18.3 & 18.3 & 18.3 & 18.1 & 0.0 & 9.6 & 21 & 79 & 0.0 \\
\hline $10 / 23 / 01$ & $17: 08$ & 18.2 & \begin{tabular}{|l|}
18.3 \\
\end{tabular} & 18.3 & 18.1 & 0.0 & 9.7 & 21 & 79 & 0.0 \\
\hline $10 / 23 / 01$ & $17: 09$ & 18.3 & \begin{tabular}{|l|}
18.3 \\
\end{tabular} & 18.3 & 18.1 & 0.0 & 9.7 & 21 & 79 & 0.0 \\
\hline $10 / 23 / 01$ & $17: 10$ & 18.2 & \begin{tabular}{|l|}
18.3 \\
\end{tabular} & 18.3 & 18.1 & 0.0 & 9.7 & 21 & 79 & 0.0 \\
\hline $10 / 23 / 01$ & $17: 11$ & 18.3 & 18.3 & 18.3 & 18.2 & 0.0 & 9.6 & 21 & 79 & 0.0 \\
\hline $10 / 23 / 01$ & $17: 12$ & 18.2 & \begin{tabular}{|l|}
18.4 \\
\end{tabular} & 18.4 & 18.1 & 0.0 & 9.6 & 21 & 79 & 0.0 \\
\hline $10 / 23 / 01$ & $17: 13$ & 18.3 & \begin{tabular}{|l|}
18.4 \\
\end{tabular} & 18.4 & 18.2 & 0.0 & 9.6 & 21 & 79 & 0.0 \\
\hline $10 / 23 / 01$ & $17: 14$ & 18.3 & \begin{tabular}{|l|}
18.4 \\
\end{tabular} & 18.4 & 18.1 & 0.0 & 9.6 & 21 & 79 & 0.0 \\
\hline $10 / 23 / 01$ & $17: 15$ & 18.3 & \begin{tabular}{|l|} 
\\
\end{tabular} & 18.4 & 18.2 & 0.0 & 9.7 & 21 & 79 & 0.0 \\
\hline $10 / 23 / 01$ & $17: 16$ & 18.3 & \begin{tabular}{|l|}
18.4 \\
\end{tabular} & 18.4 & 18.2 & 0.0 & 9.6 & 21 & 79 & 0.0 \\
\hline $10 / 23 / 01$ & $17: 17$ & 18.3 & \begin{tabular}{|l|}
18.4 \\
\end{tabular} & 18.4 & 18.2 & 0.0 & 9.7 & 21 & 79 & 0.0 \\
\hline $10 / 23 / 01$ & $17: 18$ & 18.3 & \begin{tabular}{|l|} 
\\
\end{tabular} & 18.4 & 18.2 & 0.0 & 9.5 & 21 & 79 & 0.0 \\
\hline $10 / 23 / 01$ & $17: 19$ & 18.3 & \begin{tabular}{|l|}
18.4 \\
\end{tabular} & 18.4 & 18.2 & 0.0 & 9.6 & 21 & 79 & 0.0 \\
\hline $10 / 23 / 01$ & $17: 20$ & 18.3 & 18.4 & 18.4 & 18.2 & 0.0 & 9.7 & 21 & 79 & 0.0 \\
\hline $10 / 23 / 01$ & $17: 21$ & 18.3 & 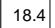 & 18.4 & 18.2 & 0.0 & 9.7 & 21 & 79 & 0.0 \\
\hline $10 / 23 / 01$ & $17: 22$ & 18.4 & \begin{tabular}{|l|} 
\\
\end{tabular} & 18.4 & 18.2 & 0.0 & 9.6 & 21 & 79 & 0.0 \\
\hline $10 / 23 / 01$ & $17: 23$ & 18.3 & \begin{tabular}{|l|}
$\mid 18.4$ \\
\end{tabular} & 18.4 & 18.2 & 0.0 & 9.7 & 21 & 79 & 0.0 \\
\hline
\end{tabular}


WSRC-TR-2002-00459, Rev. 0 SRT-RPP-2002-00221

\begin{tabular}{|c|c|c|c|c|c|c|c|c|c|c|}
\hline \multicolumn{11}{|c|}{ PREC2_102301_0623 } \\
\hline DATE & TIME & \begin{tabular}{|c|} 
HX \\
Outlet \\
$\left({ }^{\circ} \mathrm{C}\right)$ \\
TCO
\end{tabular} & $\begin{array}{c}\text { REC } \\
\text { PP } \\
\text { OUT } \\
\left({ }^{\circ} \mathrm{C}\right) \\
\text { TC1 }\end{array}$ & $\begin{array}{c}\text { TK } \\
\text { BOT } \\
\left({ }^{\circ} \mathrm{C}\right) \\
\text { TC2 }\end{array}$ & $\begin{array}{c}\mathrm{HX} \\
\text { OUT } \\
\left({ }^{\circ} \mathrm{C}\right) \\
\text { TC3 }\end{array}$ & $\begin{array}{c}\mathrm{Mn} \\
\text { Flow } \\
(\mathrm{gpm})\end{array}$ & $\begin{array}{c}\text { Galigher } \\
\text { Flow } \\
\text { (gpm) }\end{array}$ & $\begin{array}{c}\text { Current } \\
\text { to } \mathrm{HX}\end{array}$ & $\begin{array}{c}\text { Voltage } \\
\text { to } \mathrm{HX}\end{array}$ & $\begin{array}{c}\mathrm{Sr} \\
\text { Flow } \\
\text { (gpm) }\end{array}$ \\
\hline $10 / 23 / 01$ & $17: 24$ & 18.4 & \begin{tabular}{|l|} 
\\
\end{tabular} & 18.5 & 18.3 & 0.0 & 9.6 & 21 & 79 & 0 \\
\hline $10 / 23 / 01$ & $17: 25$ & 18.3 & \begin{tabular}{|l|} 
\\
\end{tabular} & 18.5 & 18.2 & 0.0 & 9.6 & 21 & 79 & 0 \\
\hline $10 / 23 / 01$ & $17: 26$ & 18.4 & 18.5 & 18.5 & 18.3 & 0.0 & 9.7 & 21 & 79 & 0 \\
\hline $10 / 23 / 01$ & $17: 27$ & 18.4 & 18.5 & 18.5 & 18.2 & 0.0 & 9.6 & 21 & 79 & 0 \\
\hline $10 / 23 / 01$ & $17: 28$ & 18.4 & 18.5 & 18.5 & 18.3 & 0.0 & 9.6 & 21 & 79 & 0 \\
\hline $10 / 23 / 01$ & $17: 29$ & 18.4 & 18.5 & 18.5 & 18.2 & 0.0 & 9.6 & 21 & 79 & 0 \\
\hline $10 / 23 / 01$ & $17: 30$ & 18.4 & 18.5 & 18.5 & 18.3 & 0.0 & 9.4 & 21 & 79 & 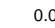 \\
\hline $10 / 23 / 01$ & $17: 31$ & 18.4 & 18.5 & 18.5 & 18.3 & 0.0 & 9.7 & 21 & 79 & 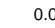 \\
\hline $10 / 23 / 01$ & $17: 32$ & 18.4 & 18.5 & 18.5 & 18.3 & 0.0 & 9.6 & 21 & 79 & 0. \\
\hline $10 / 23 / 01$ & $17: 33$ & 18.4 & 18.5 & 18.5 & 18.3 & 0.0 & 9.6 & 21 & 79 & 0. \\
\hline $10 / 23 / 01$ & $17: 34$ & 18.4 & 18.5 & 18.5 & 18.3 & 0.0 & 9.6 & 21 & 79 & 0. \\
\hline $10 / 23 / 01$ & $17: 35$ & 18.4 & 18.5 & 18.5 & 18.3 & 0.0 & 9.5 & 21 & 79 & 0 \\
\hline $10 / 23 / 01$ & $17: 36$ & 18.4 & 18.5 & 18.5 & 18.3 & 0.0 & 9.6 & 21 & 79 & 0 \\
\hline $10 / 23 / 01$ & $17: 37$ & 18.5 & 18.5 & 18.5 & 18.3 & 0.0 & 9.6 & 21 & 79 & 0.0 \\
\hline $10 / 23 / 01$ & $17: 38$ & 18.4 & 18.5 & 18.5 & 18.3 & 0.0 & 9.6 & 21 & 79 & 0.0 \\
\hline $10 / 23 / 01$ & $17: 39$ & 18.5 & 18.5 & 18.6 & 18.3 & 0.0 & 9.6 & 21 & 79 & 0. \\
\hline $10 / 23 / 01$ & $17: 40$ & 18.5 & 18.6 & 18.6 & 18.3 & 0.0 & 9.5 & 21 & 79 & \\
\hline $10 / 23 / 01$ & $17: 41$ & 18.5 & 18.6 & 18.6 & 18.4 & 0.0 & 9.7 & 21 & 79 & 0 \\
\hline $10 / 23 / 01$ & $17: 42$ & 18.5 & 18.6 & 18.6 & 18.3 & 0.0 & 9.8 & 21 & 79 & 0. \\
\hline $10 / 23 / 01$ & $17: 43$ & 18.5 & 18.6 & 18.6 & 18.4 & 0.0 & 9.7 & 21 & 79 & 0 \\
\hline $10 / 23 / 01$ & $17: 44$ & 18.5 & 18.6 & 18.6 & 18.3 & 0.0 & 9.6 & 21 & 79 & 0.0 \\
\hline $10 / 23 / 01$ & $17: 45$ & 18.5 & 18.6 & 18.6 & 18.4 & 0.0 & 9.7 & 21 & 79 & 0 \\
\hline $10 / 23 / 01$ & $17: 46$ & 18.5 & 18.6 & 18.6 & 18.4 & 0.0 & 9.6 & 21 & 79 & 0 \\
\hline $10 / 23 / 01$ & $17: 47$ & 18.6 & 18.6 & 18.6 & 18.4 & 0.0 & 9.6 & 21 & 79 & \\
\hline $10 / 23 / 01$ & $17: 48$ & 18.5 & \begin{tabular}{|l|}
18.6 \\
\end{tabular} & 18.6 & 18.4 & 0.0 & 9.6 & 21 & 79 & 0 \\
\hline $10 / 23 / 01$ & $17: 49$ & 18.6 & 18.6 & 18.6 & 18.4 & 0.0 & 9.7 & 21 & 79 & 0. \\
\hline $10 / 23 / 01$ & $17: 50$ & 18.5 & 18.6 & 18.6 & 18.4 & 0.0 & 9.8 & 21 & 79 & 0 \\
\hline $10 / 23 / 01$ & $17: 51$ & 18.6 & 18.6 & 18.6 & 18.4 & 0.0 & 9.7 & 21 & 79 & 0.0 \\
\hline $10 / 23 / 01$ & $17: 52$ & 18.6 & 18.6 & 18.6 & 18.4 & 0.0 & 9.5 & 21 & 79 & 0.0 \\
\hline $10 / 23 / 01$ & $17: 53$ & 18.6 & 18.6 & \begin{tabular}{|l|}
$\mid 18.7$ \\
\end{tabular} & 18.4 & 0.0 & 9.7 & 21 & 79 & 0 \\
\hline $10 / 23 / 01$ & $17: 54$ & 18.6 & 18.6 & $|18.7|$ & 18.4 & 0.0 & 9.6 & 21 & 79 & 0 \\
\hline $10 / 23 / 01$ & $17: 55$ & 18.6 & \begin{tabular}{|l|}
18.7 \\
\end{tabular} & 18.7 & 18.4 & 0.0 & 9.5 & 21 & 79 & O \\
\hline $10 / 23 / 01$ & 17:56 & 18.6 & 18.7 & \begin{tabular}{|l|}
18.7 \\
\end{tabular} & 18.5 & 0.0 & 9.5 & 21 & 79 & 0 \\
\hline $10 / 23 / 01$ & $17: 57$ & 18.6 & \begin{tabular}{|l|}
18.7 \\
\end{tabular} & 18.7 & 18.4 & 0.0 & 9.6 & 21 & 79 & 0.0 \\
\hline $10 / 23 / 01$ & 17:58 & 18.6 & 18.7 & \begin{tabular}{|l|}
18.7 \\
\end{tabular} & 18.5 & 0.0 & 9.6 & 21 & 79 & 0 \\
\hline $10 / 23 / 01$ & $17: 59$ & 18.6 & \begin{tabular}{|l|}
18.7 \\
\end{tabular} & 18.7 & 18.4 & 0.0 & 9.8 & 21 & 79 & 0.0 \\
\hline $10 / 23 / 01$ & 18:00 & 18.7 & $\mid 18.7$ & \begin{tabular}{|l|}
18.7 \\
\end{tabular} & 18.5 & 0.0 & 9.6 & 21 & 79 & 0. \\
\hline $10 / 23 / 01$ & 18:01 & 18.6 & \begin{tabular}{|l|}
18.7 \\
\end{tabular} & 18.7 & 18.5 & 0.0 & 9.7 & 21 & 79 & 0 \\
\hline $10 / 23 / 01$ & 18:02 & 18.7 & 18.7 & 18.7 & 18.5 & 0.0 & 9.7 & 21 & 79 & 0. \\
\hline $10 / 23 / 01$ & 18:03 & 18.6 & 18.7 & \begin{tabular}{|l|}
18.7 \\
\end{tabular} & 18.5 & 0.0 & 9.7 & 21 & 79 & 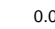 \\
\hline $10 / 23 / 01$ & 18:04 & 18.7 & 18.7 & 18.7 & 18.5 & 0.0 & 9.7 & 21 & 79 & 0 \\
\hline $10 / 23 / 01$ & $18: 05$ & 18.6 & \begin{tabular}{|l|}
18.7 \\
\end{tabular} & 18.7 & 18.5 & 0.0 & 9.6 & 21 & 79 & 0 \\
\hline $10 / 23 / 01$ & $18: 06$ & 18.7 & \begin{tabular}{|l|}
$\mid 18.7$ \\
\end{tabular} & $|18.7|$ & 18.5 & 0.0 & 9.6 & 21 & 79 & 0 \\
\hline $10 / 23 / 01$ & $18: 07$ & 18.7 & \begin{tabular}{|l|}
18.7 \\
\end{tabular} & 18.8 & 18.5 & 0.0 & 9.7 & 21 & 79 & 0 \\
\hline $10 / 23 / 01$ & $18: 08$ & 18.7 & $\mid 18.7$ & 18.8 & 18.5 & 0.0 & 9.6 & 21 & 79 & 0. \\
\hline $10 / 23 / 01$ & $18: 09$ & 18.7 & \begin{tabular}{|l|}
$\mid 18.7$ \\
\end{tabular} & $|18.7|$ & 18.5 & 0.0 & 9.6 & 21 & 79 & 0.0 \\
\hline $10 / 23 / 01$ & $18: 10$ & 18.7 & 18.8 & 18.8 & 18.5 & 0.0 & 9.6 & 21 & 79 & 0.0 \\
\hline $10 / 23 / 01$ & $18: 11$ & 18.7 & $\mid 18.8$ & 18.8 & 18.6 & 0.0 & 9.6 & 21 & 79 & 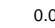 \\
\hline $10 / 23 / 01$ & $18: 12$ & 18.7 & 18.8 & 18.8 & 18.5 & 0.0 & .6 & 21 & 79 & 0. \\
\hline $10 / 23 / 01$ & $18: 13$ & 18.7 & \begin{tabular}{|l|}
18.8 \\
\end{tabular} & 18.8 & 18.6 & 0.0 & 9.6 & 21 & 79 & 0. \\
\hline $10 / 23 / 01$ & $18: 14$ & 18.7 & $\mid 18.8$ & 18.8 & 18.5 & 0.0 & 9.8 & 21 & 79 & 0 \\
\hline $10 / 23 / 01$ & $18: 15$ & 18.7 & 18.8 & 18.8 & 18.6 & 0.0 & 9.7 & 21 & 79 & 0 \\
\hline $10 / 23 / 01$ & $18: 16$ & 18.7 & \begin{tabular}{|l|}
18.8 \\
\end{tabular} & 18.8 & 18.6 & 0.0 & 9.5 & 21 & 79 & 0 \\
\hline $10 / 23 / 01$ & $18: 17$ & 18.8 & 18.8 & 18.8 & 18.6 & 0.0 & 9.7 & 21 & 79 & 0.0 \\
\hline $10 / 23 / 01$ & $18: 18$ & 18.7 & \begin{tabular}{|l|}
18.8 \\
\end{tabular} & 18.8 & 18.6 & 0.0 & .6 & 21 & 79 & 0.0 \\
\hline $10 / 23 / 01$ & $18: 19$ & 18.8 & \begin{tabular}{|l|}
18.8 \\
\end{tabular} & 18.8 & 18.6 & 0.0 & 9.6 & 21 & 79 & 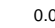 \\
\hline $10 / 23 / 01$ & $18: 20$ & 18.7 & 18.8 & 18.8 & 18.6 & 0.0 & .7 & 21 & 79 & 0 \\
\hline $10 / 23 / 01$ & $18: 21$ & 18.7 & $\mid 18.8$ & 18.8 & 18.6 & 0.0 & 9.6 & 21 & 79 & 0 \\
\hline $10 / 23 / 01$ & $18: 22$ & 18.8 & 18.8 & 18.8 & 18.6 & 0.0 & 9.7 & 21 & 79 & 0.0 \\
\hline $10 / 23 / 01$ & $18: 23$ & 18.8 & 18.8 & 18.8 & 18.6 & 0.0 & 9.6 & 21 & 79 & 0.0 \\
\hline
\end{tabular}

\begin{tabular}{|c|c|c|c|c|c|c|c|c|c|c|}
\hline \multicolumn{11}{|c|}{ PREC2_102301_0623 } \\
\hline DATE & TIME & $\begin{array}{c}\mathrm{HX} \\
\text { Outlet } \\
\left({ }^{\circ} \mathrm{C}\right) \\
\mathrm{TCO}\end{array}$ & $\begin{array}{c}\text { REC } \\
\text { PP } \\
\text { OUT } \\
\left({ }^{\circ} \mathrm{C}\right) \\
\text { TC1 }\end{array}$ & $\begin{array}{c}\text { TK } \\
\text { BOT } \\
\left({ }^{\circ} \mathrm{C}\right) \\
\text { TC2 }\end{array}$ & $\begin{array}{l}\text { HX } \\
\text { OUT } \\
\left({ }^{\circ} \mathrm{C}\right) \\
\text { TC3 }\end{array}$ & $\begin{array}{c}\text { Mn } \\
\text { Flow } \\
\text { (gpm) }\end{array}$ & $\begin{array}{c}\text { Galigher } \\
\text { Flow } \\
(\mathrm{gpm})\end{array}$ & $\begin{array}{c}\text { Current } \\
\text { to } \mathrm{HX}\end{array}$ & $\begin{array}{c}\text { Voltage } \\
\text { to } \mathrm{HX}\end{array}$ & $\begin{array}{c}\mathrm{Sr} \\
\text { Flow } \\
(\mathrm{gpm})\end{array}$ \\
\hline $10 / 23 / 01$ & $18: 24$ & 18.8 & 18.9 & 18.9 & 18.6 & 0.0 & 9.5 & 21 & 79 & 0.0 \\
\hline $10 / 23 / 01$ & $18: 25$ & 18.7 & 18.9 & 18.9 & 18.6 & 0.0 & 9.6 & 21 & 79 & 0.0 \\
\hline $10 / 23 / 01$ & $18: 26$ & 18.8 & 18.9 & 18.9 & 18.6 & 0.0 & 9.6 & 21 & 79 & 0.0 \\
\hline $10 / 23 / 01$ & $18: 27$ & 18.7 & 18.9 & 18.9 & 18.6 & 0.0 & 9.6 & 21 & 79 & 0.0 \\
\hline $10 / 23 / 01$ & $18: 28$ & 18.8 & 18.9 & 18.9 & 18.7 & 0.0 & 9.6 & 21 & 79 & 0.0 \\
\hline $10 / 23 / 01$ & $18: 29$ & 18.8 & 18.9 & 18.9 & 18.6 & 0.0 & 9.7 & 21 & 79 & 0.0 \\
\hline $10 / 23 / 01$ & $18: 30$ & 18.8 & 18.9 & 18.9 & 18.7 & 0.0 & 9.7 & 21 & 79 & 0.0 \\
\hline $10 / 23 / 01$ & 18:31 & 18.8 & 18.9 & 18.9 & 18.6 & 0.0 & 9.7 & 21 & 79 & 0.0 \\
\hline $10 / 23 / 01$ & 18:32 & 18.8 & 18.9 & 18.9 & 18.7 & 0.0 & 9.6 & 21 & 79 & 0.0 \\
\hline $10 / 23 / 01$ & $18: 33$ & 18.8 & 18.9 & 18.9 & 18.6 & 0.0 & 9.7 & 21 & 79 & 0.0 \\
\hline $10 / 23 / 01$ & $18: 34$ & 18.8 & 18.9 & 18.9 & 18.7 & 0.0 & 9.6 & 21 & 79 & 0.0 \\
\hline $10 / 23 / 01$ & $18: 35$ & 18.8 & 18.9 & 18.9 & 18.7 & 0.0 & 9.6 & 21 & 79 & 0.0 \\
\hline $10 / 23 / 01$ & $18: 36$ & 18.8 & 18.9 & 18.9 & 18.7 & 0.0 & 9.7 & 21 & 79 & 0.0 \\
\hline $10 / 23 / 01$ & 18:37 & 18.8 & 18.9 & 18.9 & 18.7 & 0.0 & 9.6 & 21 & 79 & 0.0 \\
\hline $10 / 23 / 01$ & $18: 38$ & 18.8 & 18.9 & 18.9 & 18.7 & 0.0 & 9.7 & 21 & 79 & 0.0 \\
\hline $10 / 23 / 01$ & $18: 39$ & 18.8 & 18.9 & 18.9 & 18.7 & 0.0 & 9.7 & 21 & 79 & 0.0 \\
\hline $10 / 23 / 01$ & $18: 40$ & 18.8 & 18.9 & 18.9 & 18.7 & 0.0 & 9.7 & 21 & 79 & 0.0 \\
\hline $10 / 23 / 01$ & $18: 41$ & 18.9 & 18.9 & 18.9 & 18.7 & 0.0 & 9.8 & 21 & 79 & 0.0 \\
\hline $10 / 23 / 01$ & $18: 42$ & 18.8 & 18.9 & 18.9 & 18.7 & 0.0 & 9.6 & 21 & 79 & 0.0 \\
\hline $10 / 23 / 01$ & $18: 43$ & 18.9 & 18.9 & 19.0 & 18.8 & 0.0 & 9.6 & 21 & 79 & 0.0 \\
\hline $10 / 23 / 01$ & $18: 44$ & 18.8 & 19.0 & 19.0 & 18.7 & 0.0 & 9.6 & 21 & 79 & 0.0 \\
\hline $10 / 23 / 01$ & $18: 45$ & 18.9 & 19.0 & 19.0 & 18.8 & 0.0 & 9.6 & 21 & 79 & 0.0 \\
\hline $10 / 23 / 01$ & $18: 46$ & 18.8 & 19.0 & 19.0 & 18.7 & 0.0 & 9.6 & 21 & 79 & 0.0 \\
\hline $10 / 23 / 01$ & $18: 47$ & 18.9 & 19.0 & 19.0 & 18.8 & 0.0 & 9.7 & 21 & $7 \mathrm{~s}$ & .0 \\
\hline $10 / 23 / 01$ & 18:48 & 18.9 & 19.0 & 19.0 & 18.7 & 0.0 & 9.6 & 21 & 79 & 0.0 \\
\hline $10 / 23 / 01$ & $18: 49$ & 18.9 & 19.0 & 19.0 & 18.8 & 0.0 & 9.5 & 21 & 79 & 0.0 \\
\hline $10 / 23 / 01$ & 18:50 & 18.9 & 19.0 & 19.0 & 18.7 & 0.0 & 9.6 & 21 & 79 & 0.0 \\
\hline $10 / 23 / 01$ & 18:51 & 18.9 & 19.0 & 19.0 & 18.8 & 0.0 & 9.7 & 21 & 79 & 0.0 \\
\hline $10 / 23 / 01$ & 18:52 & 18.9 & 19.0 & 19.0 & 18.7 & 0.0 & 9.7 & 21 & 79 & 0.0 \\
\hline $10 / 23 / 01$ & 18:53 & 18.9 & 19.0 & 19.0 & 18.8 & 0.0 & 9.7 & 21 & 79 & 0.0 \\
\hline $10 / 23 / 01$ & 18:54 & 18.9 & 19.0 & 19.0 & 18.8 & 0.0 & 9.7 & 21 & 79 & 0.0 \\
\hline $10 / 23 / 01$ & 18:55 & 18.9 & 19.0 & 19.0 & 18.8 & 0.0 & 9.6 & 21 & 7 & 0.0 \\
\hline $10 / 23 / 01$ & 18:56 & 18.9 & 19.0 & 19.0 & 18.8 & 0.0 & 9.6 & 21 & 79 & 0.0 \\
\hline $10 / 23 / 01$ & $18: 57$ & 18.9 & 19.0 & 19.0 & 18.7 & 0.0 & 9.7 & 21 & 79 & 0.0 \\
\hline $10 / 23 / 01$ & 18:58 & 18.9 & 19.0 & 19.0 & 18.8 & 0.0 & 9.6 & 21 & 79 & 0.0 \\
\hline $10 / 23 / 01$ & 18:59 & 18.9 & 19.0 & 19.0 & 18.7 & 0.0 & 9.6 & 21 & 79 & 0.0 \\
\hline $10 / 23 / 01$ & 19:00 & 18.9 & 19.0 & 19.0 & 18.8 & 0.0 & 9.6 & 21 & 79 & 0.0 \\
\hline $10 / 23 / 01$ & 19:01 & 18.9 & 19.0 & 19.0 & 18.8 & 0.0 & 9.5 & 21 & 79 & 0.0 \\
\hline $10 / 23 / 01$ & 19:02 & 18.9 & 19.0 & 19.0 & 18.8 & 0. & 9.6 & 21 & 15 & 0.0 \\
\hline $10 / 23 / 01$ & 19:03 & 18.9 & 19.0 & 19.0 & 18.8 & 0.0 & 9.6 & 21 & 79 & 0.0 \\
\hline $10 / 23 / 01$ & 19:04 & 18.9 & 19.0 & 19.0 & 18.8 & 0.0 & 9.7 & 21 & 79 & 0.0 \\
\hline $10 / 23 / 01$ & 19:05 & 18.9 & 19.1 & 19.0 & 18.8 & 0.0 & 9.7 & 21 & 79 & 0.0 \\
\hline $10 / 23 / 01$ & 19:06 & 18.9 & 19.0 & 19.1 & 18.8 & 0.0 & 9.7 & 21 & 79 & 0.0 \\
\hline $10 / 23 / 01$ & 19:07 & 19.0 & 19.1 & 19.1 & 18.8 & 0.0 & 9.5 & 21 & 79 & 0.0 \\
\hline $10 / 23 / 01$ & 19:08 & 18.9 & 19.0 & 19.0 & 18.8 & 0.0 & 9.7 & 21 & 79 & 0.0 \\
\hline $10 / 23 / 01$ & 19:09 & 19.0 & 19.1 & 19.1 & 18.8 & 0.0 & 9.7 & 21 & 79 & 0.0 \\
\hline $10 / 23 / 01$ & $19: 10$ & 18.9 & 19.1 & 19.1 & 18.8 & 0.0 & 9.6 & 21 & 79 & 0.0 \\
\hline $10 / 23 / 01$ & $19: 11$ & 19.0 & 19.1 & 19.1 & 18.8 & 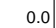 & 9.6 & 21 & 79 & 0.0 \\
\hline $10 / 23 / 01$ & $19: 12$ & 19.0 & 19.1 & 19.1 & 18.8 & 0.0 & 9.6 & 21 & 79 & 0.0 \\
\hline $10 / 23 / 01$ & $19: 13$ & 19.0 & 19.1 & 19.1 & 18.9 & 0.0 & 9.7 & 21 & 79 & 0.0 \\
\hline $10 / 23 / 01$ & $19: 14$ & 18.9 & 19.1 & 19.1 & 18.8 & 0.0 & 9.6 & 21 & 79 & 0.0 \\
\hline $10 / 23 / 01$ & $19: 15$ & 19.0 & 19.1 & 19.1 & 18.8 & 0.0 & 9.5 & 21 & 79 & 0.0 \\
\hline $10 / 23 / 01$ & $19: 16$ & 19.0 & 19.1 & 19.1 & 18.8 & 0.0 & 9.6 & 21 & 79 & 0.0 \\
\hline $10 / 23 / 01$ & 19:17 & 19.0 & 19.1 & 19.1 & 18.9 & 0.0 & 9.6 & 21 & 79 & 0.0 \\
\hline $10 / 23 / 01$ & $19: 18$ & 19.0 & 19.1 & 19.1 & 18.9 & 0.0 & 9.5 & 21 & 19 & 0.0 \\
\hline $10 / 23 / 01$ & $19: 19$ & 19.0 & 19.1 & 19.1 & 18.9 & 0.0 & 9.6 & 21 & 19 & 0.0 \\
\hline $10 / 23 / 01$ & $19: 20$ & 19.0 & 19.1 & 19.1 & 18.9 & 0.0 & 9.6 & 21 & 79 & 0.0 \\
\hline $10 / 23 / 01$ & $19: 21$ & 19.0 & 19.1 & 19.1 & 18.9 & 0.0 & 9.6 & 21 & 79 & 0.0 \\
\hline $10 / 23 / 01$ & 19:22 & 19.0 & 19.1 & 19.1 & 18.9 & 0.0 & 9.5 & 21 & 79 & 0.0 \\
\hline $10 / 23 / 01$ & 19:23 & 19.0 & 19.1 & 19.1 & 18.9 & 0.0 & 9.7 & 21 & 79 & 0.0 \\
\hline
\end{tabular}


WSRC-TR-2002-00459, Rev. 0 SRT-RPP-2002-00221

\begin{tabular}{|c|c|c|c|c|c|c|c|c|c|c|}
\hline \multicolumn{11}{|c|}{ PREC2_102301_0623 } \\
\hline DATE & TIME & \begin{tabular}{|c|} 
HX \\
Outlet \\
$\left({ }^{\circ} \mathrm{C}\right)$ \\
TCO
\end{tabular} & $\begin{array}{c}\text { REC } \\
\text { PP } \\
\text { OUT } \\
\left({ }^{\circ} \mathrm{C}\right) \\
\text { TC1 }\end{array}$ & $\begin{array}{c}\text { TK } \\
\text { BOT } \\
\left({ }^{\circ} \mathrm{C}\right) \\
\text { TC2 }\end{array}$ & $\begin{array}{c}\mathrm{HX} \\
\text { OUT } \\
\left({ }^{\circ} \mathrm{C}\right) \\
\text { TC3 }\end{array}$ & $\begin{array}{c}\mathrm{Mn} \\
\text { Flow } \\
(\mathrm{gpm})\end{array}$ & $\begin{array}{l}\text { Galigher } \\
\text { Flow } \\
\text { (gpm) }\end{array}$ & $\begin{array}{c}\text { Current } \\
\text { to } \mathrm{HX}\end{array}$ & $\begin{array}{c}\text { Voltage } \\
\text { to } \mathrm{HX}\end{array}$ & $\begin{array}{c}\mathrm{Sr} \\
\text { Flow } \\
\text { (gpm) }\end{array}$ \\
\hline $10 / 23 / 01$ & $19: 24$ & 19.0 & $\mid 19.1$ & 19.1 & 18.9 & 0.0 & 9.7 & 21 & 79 & 0 \\
\hline $10 / 23 / 01$ & $19: 25$ & 19.0 & $\mid 19.1$ & 19.1 & 18.9 & 0.0 & 9.7 & 21 & 79 & 0 \\
\hline $10 / 23 / 01$ & $19: 26$ & 19.1 & \begin{tabular}{|l|}
19.2 \\
\end{tabular} & 19.1 & 18.9 & 0.0 & 9.7 & 21 & 79 & 0 \\
\hline $10 / 23 / 01$ & $19: 27$ & 19.0 & \begin{tabular}{|l|}
19.2 \\
\end{tabular} & 19.2 & 18.9 & 0.0 & 9.5 & 21 & 79 & 0 \\
\hline $10 / 23 / 01$ & $19: 28$ & 19.0 & $\mid 19.1$ & 19.2 & 18.9 & 0.0 & 9.6 & 21 & 79 & 0 \\
\hline $10 / 23 / 01$ & $19: 29$ & 19.0 & \begin{tabular}{|l|}
19.2 \\
\end{tabular} & 19.2 & 18.9 & 0.0 & 9.7 & 21 & 79 & 0 \\
\hline $10 / 23 / 01$ & 19:30 & 19.0 & $\mid 19.2$ & 19.2 & 18.9 & 0.0 & 9.7 & 21 & 79 & 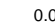 \\
\hline $10 / 23 / 01$ & 19:31 & 19.0 & \begin{tabular}{|l|}
19.2 \\
\end{tabular} & 19.2 & 18.9 & 0.0 & 9.5 & 21 & 79 & 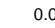 \\
\hline $10 / 23 / 01$ & 19:32 & 19.0 & \begin{tabular}{|l|}
19.2 \\
\end{tabular} & 19.2 & 18.9 & 0.0 & 9.6 & 21 & 79 & 0 \\
\hline $10 / 23 / 01$ & 19:33 & 19.1 & $\mid 19.2$ & 19.2 & 18.9 & 0.0 & 9.7 & 21 & 79 & 0. \\
\hline $10 / 23 / 01$ & $19: 34$ & 19.0 & \begin{tabular}{|l|}
19.2 \\
\end{tabular} & 19.2 & 18.9 & 0.0 & 9.7 & 21 & 79 & 0. \\
\hline $10 / 23 / 01$ & $19: 35$ & 19.1 & \begin{tabular}{|l|}
19.2 \\
\end{tabular} & 19.2 & 18.9 & 0.0 & 9.6 & 21 & 79 & 0 \\
\hline $10 / 23 / 01$ & $19: 36$ & 19.0 & $\mid 19.2$ & 19.2 & 18.9 & 0.0 & 9.6 & 20 & 79 & 0 \\
\hline $10 / 23 / 01$ & $19: 37$ & 19.1 & \begin{tabular}{|l|}
19.2 \\
\end{tabular} & 19.2 & 18.9 & 0.0 & 9.7 & 21 & 79 & 0 \\
\hline $10 / 23 / 01$ & 19:38 & 19.0 & 19.2 & \begin{tabular}{|l|}
19.2 \\
\end{tabular} & 18.9 & 0.0 & 9.6 & 21 & 79 & 0.0 \\
\hline $10 / 23 / 01$ & $19: 39$ & 19.1 & \begin{tabular}{|l|}
19.2 \\
\end{tabular} & 19.2 & 19.0 & 0.0 & 9.6 & 21 & 79 & 0. \\
\hline $10 / 23 / 01$ & $19: 40$ & 19.0 & \begin{tabular}{|l|}
19.2 \\
\end{tabular} & 19.2 & 18.9 & 0.0 & 9.7 & 21 & 79 & \\
\hline $10 / 23 / 01$ & $19: 41$ & 19.1 & \begin{tabular}{|l|}
19.2 \\
\end{tabular} & 19.2 & 18.9 & 0.0 & 9.6 & 21 & 79 & 0 \\
\hline $10 / 23 / 01$ & $19: 42$ & 19.0 & \begin{tabular}{|l|}
19.2 \\
\end{tabular} & 19.2 & 18.9 & 0.0 & 9.7 & 21 & 79 & 0. \\
\hline $10 / 23 / 01$ & $19: 43$ & 19.1 & \begin{tabular}{|l|}
19.2 \\
\end{tabular} & 19.2 & 19.0 & 0.0 & 9.7 & 21 & 79 & 0 \\
\hline $10 / 23 / 01$ & $19: 44$ & 19.1 & \begin{tabular}{|l|}
19.2 \\
\end{tabular} & 19.2 & 18.9 & 0.0 & 9.6 & 21 & 79 & 0.0 \\
\hline $10 / 23 / 01$ & 19:45 & 19.1 & \begin{tabular}{|l|}
19.2 \\
\end{tabular} & 19.2 & 19.0 & 0.0 & 9.7 & 21 & 79 & 0 \\
\hline $10 / 23 / 01$ & $19: 46$ & 19.1 & \begin{tabular}{|l|}
19.2 \\
\end{tabular} & 19.2 & 18.9 & 0.0 & 9.6 & 21 & 79 & 0 \\
\hline $10 / 23 / 01$ & $19: 47$ & 19.1 & \begin{tabular}{|l|}
19.2 \\
\end{tabular} & 19.2 & 18.9 & 0.0 & 9.6 & 21 & 79 & \\
\hline $10 / 23 / 01$ & $19: 48$ & 19.1 & \begin{tabular}{|l|}
19.2 \\
\end{tabular} & 19.2 & 18.9 & 0.0 & 9.7 & 21 & 79 & 0 \\
\hline $10 / 23 / 01$ & $19: 49$ & 19.1 & \begin{tabular}{|l|}
19.2 \\
\end{tabular} & 19.2 & 18.9 & 0.0 & 9.8 & 21 & 79 & 0. \\
\hline $10 / 23 / 01$ & 19:50 & 19.1 & \begin{tabular}{|l|}
19.2 \\
\end{tabular} & 19.2 & 19.0 & 0.0 & 9.5 & 21 & 79 & 0 \\
\hline $10 / 23 / 01$ & 19:51 & 19.1 & 19.2 & \begin{tabular}{|l|}
19.2 \\
\end{tabular} & 18.9 & 0.0 & 9.6 & 21 & 79 & 0.0 \\
\hline $10 / 23 / 01$ & 19:52 & 19.1 & \begin{tabular}{|l|}
19.2 \\
\end{tabular} & 19.2 & 19.0 & 0.0 & 9.7 & 21 & 79 & 0.0 \\
\hline $10 / 23 / 01$ & 19:53 & 19.1 & \begin{tabular}{|l|}
19.2 \\
\end{tabular} & 19.2 & 18.9 & 0.0 & 9.5 & 21 & 79 & 0 \\
\hline $10 / 23 / 01$ & 19:54 & 19.1 & 19.2 & 19.2 & 19.0 & 0.0 & 9.6 & 21 & 79 & 0 \\
\hline $10 / 23 / 01$ & $19: 55$ & 19.1 & \begin{tabular}{|l|}
19.2 \\
\end{tabular} & 19.2 & 19.0 & 0.0 & .6 & 21 & 79 & \\
\hline $10 / 23 / 01$ & 19:56 & 19.1 & 19.2 & 19.2 & 19.0 & 0.0 & 9.7 & 21 & 79 & 0 \\
\hline $10 / 23 / 01$ & $19: 57$ & 19.1 & \begin{tabular}{|l|}
19.2 \\
\end{tabular} & 19.2 & 19.0 & 0.0 & 9.6 & 21 & 79 & 0.0 \\
\hline $10 / 23 / 01$ & 19:58 & 19.1 & 19.3 & 19.2 & 19.0 & 0.0 & 9.7 & 21 & 79 & 0 \\
\hline $10 / 23 / 01$ & $19: 59$ & 19.1 & \begin{tabular}{|l|}
19.3 \\
\end{tabular} & 19.2 & 19.0 & 0.0 & 9.6 & 21 & 79 & 0.0 \\
\hline $10 / 23 / 01$ & 20:00 & 19.1 & 19.3 & 19.2 & 19.0 & 0.0 & 9.6 & 21 & 79 & 0. \\
\hline $10 / 23 / 01$ & 20:01 & 19.1 & \begin{tabular}{|l|}
19.3 \\
\end{tabular} & 19.3 & 19.0 & 0.0 & 9.7 & 21 & 79 & 0 \\
\hline $10 / 23 / 01$ & $20: 02$ & 19.1 & \begin{tabular}{|l|}
19.3 \\
\end{tabular} & 19.3 & 19.0 & 0.0 & 9.6 & 21 & 79 & 0 \\
\hline $10 / 23 / 01$ & 20:03 & 19.2 & 19.3 & 19.3 & 19.0 & 0.0 & 9.7 & 21 & 79 & 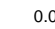 \\
\hline $10 / 23 / 01$ & 20:04 & 19.1 & \begin{tabular}{|l|}
19.3 \\
\end{tabular} & 19.3 & 19.0 & 0.0 & 9.6 & 21 & 79 & 0 \\
\hline $10 / 23 / 01$ & 20:05 & 19.2 & \begin{tabular}{|l|}
19.3 \\
\end{tabular} & 19.3 & 19.0 & 0.0 & 9.5 & 21 & 79 & 0 \\
\hline $10 / 23 / 01$ & 20:06 & 19.1 & $\mid 19.3$ & 19.3 & 19.0 & 0.0 & 9.5 & 21 & 79 & 0 \\
\hline $10 / 23 / 01$ & $20: 07$ & 19.2 & \begin{tabular}{|l|}
19.3 \\
\end{tabular} & 19.3 & 19.1 & 0.0 & 9.6 & 21 & 79 & 0 \\
\hline $10 / 23 / 01$ & 20:08 & 19.1 & 19.3 & 19.3 & 19.0 & 0.0 & 9.7 & 21 & 79 & 0. \\
\hline $10 / 23 / 01$ & 20:09 & 19.2 & $\mid 19.3$ & 19.3 & 19.1 & 0.0 & 9.6 & 21 & 79 & 0.0 \\
\hline $10 / 23 / 01$ & $20: 10$ & 19.1 & \begin{tabular}{|l|}
19.3 \\
\end{tabular} & 19.3 & 19.0 & 0.0 & 9.6 & 21 & 79 & 0. \\
\hline 10/23/01 & $20: 11$ & 19.2 & 19.3 & 19.3 & 19.1 & .0 & 6 & 21 & 19 & 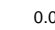 \\
\hline $10 / 23 / 01$ & $20: 12$ & 19.1 & \begin{tabular}{|l|}
19.3 \\
\end{tabular} & 19.3 & 19.0 & 0.0 & .7 & 21 & 79 & 0. \\
\hline $10 / 23 / 01$ & $20: 13$ & 19.2 & $\mid 19.3$ & 19.3 & 19.1 & 0.0 & 9.6 & 21 & 79 & 0. \\
\hline $10 / 23 / 01$ & $20: 14$ & 19.2 & 19.3 & 19.3 & 19.0 & 0.0 & 9.6 & 21 & 79 & 0 \\
\hline $10 / 23 / 01$ & $20: 15$ & 19.2 & \begin{tabular}{|l|}
19.3 \\
\end{tabular} & 19.3 & 19.0 & 0.0 & 9.7 & 21 & 79 & 0 \\
\hline $10 / 23 / 01$ & $20: 16$ & 19.2 & $\mid 19.3$ & 19.3 & 19.0 & 0.0 & 9.7 & 21 & 79 & 0 \\
\hline $10 / 23 / 01$ & $20: 17$ & 19.2 & \begin{tabular}{|l|}
19.3 \\
\end{tabular} & 19.3 & 19.0 & 0.0 & 9.6 & 21 & 79 & 0.0 \\
\hline $10 / 23 / 01$ & $20: 18$ & 19.2 & \begin{tabular}{|l|}
19.3 \\
\end{tabular} & 19.3 & 19.0 & 0.0 & 9.6 & 21 & 79 & 0.0 \\
\hline $10 / 23 / 01$ & $20: 19$ & 19.2 & $\mid 19.3$ & 19.3 & 19.0 & 0.0 & 9.5 & 21 & 79 & 0. \\
\hline $10 / 23 / 01$ & $20: 20$ & 19.2 & $\mid 19.3$ & 19.3 & 19.1 & 0.0 & .7 & 21 & 79 & 0 \\
\hline $10 / 23 / 01$ & $20: 21$ & 19.2 & \begin{tabular}{|l|}
19.3 \\
\end{tabular} & 19.3 & 19.0 & 0.0 & 9.7 & 21 & 79 & 0 \\
\hline $10 / 23 / 01$ & $20: 22$ & 19.2 & \begin{tabular}{|l|}
19.3 \\
\end{tabular} & 19.3 & 19.1 & 0.0 & 9.6 & 21 & 79 & 0.0 \\
\hline $10 / 23 / 01$ & $20: 23$ & 19.2 & \begin{tabular}{|l|}
19.3 \\
\end{tabular} & 19.3 & 19.0 & 0.0 & 9.6 & 21 & 79 & 0.0 \\
\hline
\end{tabular}

\begin{tabular}{|c|c|c|c|c|c|c|c|c|c|c|}
\hline \multicolumn{11}{|c|}{ PREC2_102301_0623 } \\
\hline DATE & TIME & $\begin{array}{c}\mathrm{HX} \\
\text { Outlet } \\
\left({ }^{\circ} \mathrm{C}\right) \\
\mathrm{TCO}\end{array}$ & $\begin{array}{c}\text { REC } \\
\text { PP } \\
\text { OUT } \\
\left({ }^{\circ} \mathrm{C}\right) \\
\text { TC1 }\end{array}$ & $\begin{array}{c}\text { TK } \\
\text { BOT } \\
\left({ }^{\circ} \mathrm{C}\right) \\
\text { TC2 }\end{array}$ & $\begin{array}{c}\text { HX } \\
\text { OUT } \\
\left({ }^{\circ} \mathrm{C}\right) \\
\text { TC3 }\end{array}$ & $\begin{array}{c}\text { Mn } \\
\text { Flow } \\
\text { (gpm) }\end{array}$ & $\begin{array}{c}\text { Galigher } \\
\text { Flow } \\
(\mathrm{gpm})\end{array}$ & $\begin{array}{c}\text { Current } \\
\text { to } \mathrm{HX}\end{array}$ & $\begin{array}{c}\text { Voltage } \\
\text { to } \mathrm{HX}\end{array}$ & $\begin{array}{c}\mathrm{Sr} \\
\text { Flow } \\
\text { (gpm) }\end{array}$ \\
\hline $10 / 23 / 01$ & $20: 24$ & 19.2 & 19.3 & 19.3 & 19.1 & 0.0 & 9.8 & 21 & 79 & 0.0 \\
\hline $10 / 23 / 01$ & $20: 25$ & 19.2 & 19.3 & 19.3 & 19.0 & 0.0 & 9.6 & 21 & 79 & 0.0 \\
\hline $10 / 23 / 01$ & $20: 26$ & 19.2 & 19.3 & 19.3 & 19.1 & 0.0 & 9.6 & 21 & 79 & 0.0 \\
\hline $10 / 23 / 01$ & $20: 27$ & 19.2 & 19.3 & 19.3 & 19.0 & 0.0 & 9.6 & 21 & 79 & 0.0 \\
\hline $10 / 23 / 01$ & $20: 28$ & 19.2 & 19.3 & 19.3 & 19.1 & 0.0 & 9.7 & 21 & 79 & 0.0 \\
\hline $10 / 23 / 01$ & $20: 29$ & 19.2 & 19.3 & 19.3 & 19.1 & 0.0 & 9.6 & 20 & 79 & 0.0 \\
\hline $10 / 23 / 01$ & $20: 30$ & 19.2 & 19.3 & 19.3 & 19.1 & 0.0 & 9.5 & 21 & 79 & 0.0 \\
\hline $10 / 23 / 01$ & $20: 31$ & 19.2 & 19.3 & 19.3 & 19.1 & 0.0 & 9.6 & 21 & 79 & 0.0 \\
\hline $10 / 23 / 01$ & $20: 32$ & 19.2 & 19.4 & 19.3 & 19.1 & 0.0 & 9.7 & 21 & 79 & 0.0 \\
\hline $10 / 23 / 01$ & $20: 33$ & 19.2 & 19.4 & 19.3 & 19.1 & 0.0 & 9.7 & 21 & 79 & 0.0 \\
\hline $10 / 23 / 01$ & $20: 34$ & 19.2 & 19.4 & 19.3 & 19.1 & 0.0 & 9.7 & 21 & 79 & 0.0 \\
\hline $10 / 23 / 01$ & $20: 35$ & 19.3 & 19.4 & 19.3 & 19.1 & 0.0 & 9.6 & 21 & 79 & 0.0 \\
\hline $10 / 23 / 01$ & $20: 36$ & 19.2 & 19.4 & 19.4 & 19.1 & 0.0 & 9.5 & 21 & 79 & 0.0 \\
\hline $10 / 23 / 01$ & 20:37 & 19.3 & 19.4 & 19.4 & 19.1 & 0.0 & 9.6 & 21 & 79 & 0.0 \\
\hline $10 / 23 / 01$ & $20: 38$ & 19.2 & 19.4 & 19.4 & 19.1 & 0.0 & 9.6 & 21 & 79 & 0.0 \\
\hline $10 / 23 / 01$ & $20: 39$ & 19.3 & 19.4 & 19.4 & 19.1 & 0.0 & 9.7 & 21 & 79 & 0.0 \\
\hline $10 / 23 / 01$ & $20: 40$ & 19.2 & 19.4 & 19.4 & 19.1 & 0.0 & 9.8 & 21 & 79 & 0.0 \\
\hline $10 / 23 / 01$ & $20: 41$ & 19.2 & 19.4 & 19.4 & 19.1 & 0.0 & 9.7 & 21 & 79 & 0.0 \\
\hline $10 / 23 / 01$ & $20: 42$ & 19.2 & 19.4 & 19.4 & 19.1 & 0.0 & 9.6 & 21 & 79 & 0.0 \\
\hline $10 / 23 / 01$ & $20: 43$ & 19.2 & 19.4 & 19.4 & 19.1 & 0.0 & 9.6 & 21 & 79 & 0.0 \\
\hline $10 / 23 / 01$ & $20: 44$ & 19.3 & 19.4 & 19.4 & 19.1 & 0.0 & 9.6 & 21 & 79 & 0.0 \\
\hline $10 / 23 / 01$ & $20: 45$ & 19.2 & 19.4 & 19.4 & 19.1 & 0.0 & 9.7 & 21 & 79 & 0.0 \\
\hline $10 / 23 / 01$ & $20: 46$ & 19.3 & 19.4 & 19.4 & 19.2 & 0.0 & 9.7 & 21 & 79 & 0.0 \\
\hline $10 / 23 / 01$ & $20: 47$ & 19.2 & 19.4 & 19.4 & 19.1 & 0.0 & 9.6 & 21 & 7 & .0 \\
\hline $10 / 23 / 01$ & $20: 48$ & 19.3 & 19.4 & 19.4 & 19.2 & 0.0 & 9.6 & 21 & 75 & 0.0 \\
\hline $10 / 23 / 01$ & $20: 49$ & 19.3 & 19.4 & 19.4 & 19.1 & 0.0 & 9.6 & 21 & 79 & 0.0 \\
\hline $10 / 23 / 01$ & $20: 50$ & 19.3 & 19.4 & 19.4 & 19.2 & 0.0 & 9.6 & 67 & 79 & 0.0 \\
\hline $10 / 23 / 01$ & $20: 51$ & 19.3 & 19.4 & 19.4 & 19.1 & 0.0 & 9.7 & 21 & 79 & 0.0 \\
\hline $10 / 23 / 01$ & 20:52 & 19.3 & 19.4 & 19.4 & 19.2 & 0.0 & 9.6 & 25 & 79 & 0.0 \\
\hline $10 / 23 / 01$ & 20:53 & 19.3 & 19.4 & 19.4 & 19.1 & 0.0 & 9.7 & 70 & 79 & 0.0 \\
\hline $10 / 23 / 01$ & 20:54 & 19.3 & 19.4 & 19.4 & 19.1 & 0.0 & 9.6 & 63 & 79 & 0.0 \\
\hline $10 / 23 / 01$ & 20:55 & 19.3 & 19.4 & 19.4 & 19.1 & 0.0 & 9.5 & 26 & 15 & 0.0 \\
\hline $10 / 23 / 01$ & $20: 56$ & 19.3 & 19.4 & 19.4 & 19.1 & 0.0 & 9.6 & 58 & 79 & 0.0 \\
\hline $10 / 23 / 01$ & 20:57 & 19.3 & 19.4 & 19.4 & 19.1 & 0.0 & 9.7 & 37 & 79 & 0.0 \\
\hline $10 / 23 / 01$ & 20:58 & 19.3 & 19.4 & 19.4 & 19.1 & 0.0 & 9.6 & 95 & 79 & 0.0 \\
\hline $10 / 23 / 01$ & 20:59 & 19.3 & 19.4 & 19.4 & 19.2 & 0.0 & 9.8 & 26 & 79 & 0.0 \\
\hline $10 / 23 / 01$ & 21:00 & 19.3 & 19.4 & 19.4 & 19.1 & 0.0 & 9.6 & 34 & 79 & 0.0 \\
\hline $10 / 23 / 01$ & 21:01 & 19.3 & 19.4 & 19.4 & 19.2 & 0.0 & 9.6 & 57 & 79 & 0.0 \\
\hline $10 / 23 / 01$ & 21:02 & 19.3 & 19.4 & 19.4 & 19.1 & 0. & 9.7 & 22 & 79 & 0.0 \\
\hline $10 / 23 / 01$ & $21: 03$ & 19.3 & 19.4 & 19.4 & 19.2 & 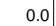 & 9.6 & 55 & 79 & 0.0 \\
\hline $10 / 23 / 01$ & $21: 04$ & 19.3 & 19.4 & 19.4 & 19.1 & 0.0 & 9.7 & 35 & 79 & 0.0 \\
\hline $10 / 23 / 01$ & 21:05 & 19.3 & 19.4 & 19.4 & 19.2 & 0.0 & 9.7 & 96 & 79 & 0.0 \\
\hline $10 / 23 / 01$ & $21: 06$ & 19.3 & 19.4 & 19.4 & 19.1 & 0.0 & 9.5 & 24 & 79 & 0.0 \\
\hline $10 / 23 / 01$ & 21:07 & 19.3 & 19.4 & 19.4 & 19.2 & 0.0 & 9.5 & 36 & 79 & 0.0 \\
\hline $10 / 23 / 01$ & 21:08 & 19.3 & 19.4 & 19.4 & 19.2 & 0.0 & 9.6 & 65 & 79 & 0.0 \\
\hline $10 / 23 / 01$ & 21:09 & 19.3 & 19.5 & 19.5 & 19.2 & 0.0 & 9.6 & 76 & 79 & 0.0 \\
\hline $10 / 23 / 01$ & $21: 10$ & 19.3 & 19.4 & 19.4 & 19.2 & 0 & 0.0 & 88 & 79 & 0.0 \\
\hline $10 / 23 / 01$ & 21:11 & 19.3 & 19.5 & 19.5 & 19. & 0.0 & 9. & 77 & 79 & 0.0 \\
\hline $10 / 23 / 01$ & $21: 12$ & 19.4 & 19.5 & 19.5 & 19.2 & 0. & 9.7 & 58 & 79 & 0.0 \\
\hline $10 / 23 / 01$ & $21: 13$ & 19.3 & 19.5 & 19.5 & 19.2 & 0.0 & 9.6 & 47 & 79 & 0.0 \\
\hline $10 / 23 / 01$ & 21:14 & 19.4 & 19.5 & 19.5 & 19.2 & 0.0 & 9.7 & 56 & 79 & 0.0 \\
\hline $10 / 23 / 01$ & $21: 15$ & 19.3 & 19.5 & 19.5 & 19.2 & 0.0 & 9.6 & 91 & 79 & 0.0 \\
\hline $10 / 23 / 01$ & $21: 16$ & 19.4 & 19.5 & 19.5 & 19.2 & 0.0 & 9.6 & 33 & 79 & 0.0 \\
\hline $10 / 23 / 01$ & $21: 17$ & 19.3 & 19.5 & 19.5 & 19.2 & 0.0 & 9.7 & 80 & 79 & 0.0 \\
\hline $10 / 23 / 01$ & $21: 18$ & 19.4 & 19.5 & 19.5 & 19.2 & 0.0 & 9.7 & 35 & 10 & 0.0 \\
\hline $10 / 23 / 01$ & $21: 19$ & 19.3 & 19.5 & 19.5 & 19.2 & 0. & 9.8 & 39 & 79 & 0.0 \\
\hline $10 / 23 / 01$ & $21: 20$ & 19.4 & 19.5 & 19.5 & 19.3 & 0.0 & 9.7 & 79 & 79 & 0.0 \\
\hline $10 / 23 / 01$ & 21:21 & 19.3 & 19.5 & 19.5 & 19.2 & 0.0 & 9.7 & 29 & 79 & 0.0 \\
\hline $10 / 23 / 01$ & $21: 22$ & 19.4 & 19.5 & 19.5 & 19.2 & 0.0 & 9.8 & 50 & 79 & 0.0 \\
\hline $10 / 23 / 01$ & $21: 23$ & 19.4 & 19.5 & 19.5 & 19.2 & 0.0 & 9.5 & 28 & 79 & 0.0 \\
\hline
\end{tabular}


WSRC-TR-2002-00459, Rev. 0 SRT-RPP-2002-00221

\begin{tabular}{|c|c|c|c|c|c|c|c|c|c|c|}
\hline \multicolumn{3}{|c|}{\begin{tabular}{|l|} 
PREC2_102301_0623 \\
\end{tabular}} & \multirow[b]{2}{*}{\begin{tabular}{|c} 
REC \\
PP \\
OUT \\
$\left({ }^{\circ} \mathrm{C}\right)$ \\
TC1
\end{tabular}} & \multirow[b]{2}{*}{\begin{tabular}{|c} 
TK \\
BOT \\
$\left({ }^{\circ} \mathrm{C}\right)$ \\
TC2 \\
\end{tabular}} & \multirow[b]{2}{*}{\begin{tabular}{|l|}
$\mathrm{HX}$ \\
OUT \\
$\left({ }^{\circ} \mathrm{C}\right)$ \\
$\mathrm{TC} 3$ \\
\end{tabular}} & \multirow[b]{2}{*}{$\begin{array}{c}\text { Mn } \\
\text { Flow } \\
\text { (gpm) }\end{array}$} & \multirow[b]{2}{*}{\begin{tabular}{|c|} 
Galigher \\
Flow \\
$(\mathrm{gpm})$
\end{tabular}} & \multirow[b]{2}{*}{$\begin{array}{c}\text { Current } \\
\text { to } \mathrm{HX}\end{array}$} & \multirow[b]{2}{*}{$\begin{array}{l}\text { Voltage } \\
\text { to } \mathrm{HX}\end{array}$} & \multirow[b]{2}{*}{$\begin{array}{c}\mathrm{Sr} \\
\text { Flow } \\
\text { (gpm) }\end{array}$} \\
\hline DATE & TIME & $\begin{array}{c}\mathrm{HX} \\
\text { Outlet } \\
\left({ }^{\circ} \mathrm{C}\right) \\
\mathrm{TCO}\end{array}$ & & & & & & & & \\
\hline $10 / 23 / 01$ & $21: 24$ & 19.4 & 19.5 & 19.5 & \begin{tabular}{|l|}
19.2 \\
\end{tabular} & 0.0 & 9.5 & 32 & 79 & 0.0 \\
\hline $10 / 23 / 01$ & $21: 25$ & 19.4 & 19.5 & 19.5 & 19.3 & 0.0 & 9.7 & 77 & 79 & 0.0 \\
\hline $10 / 23 / 01$ & $21: 26$ & 19.4 & 19.5 & 19.5 & \begin{tabular}{|l|}
19.2 \\
\end{tabular} & 0.0 & 9.7 & 56 & 79 & 0.0 \\
\hline $10 / 23 / 01$ & $21: 27$ & 19.4 & 19.5 & 19.5 & 19.3 & 0.0 & 9.7 & 80 & 79 & 0.0 \\
\hline $10 / 23 / 01$ & $21: 28$ & 19.4 & 19.5 & 19.5 & \begin{tabular}{|l|}
19.2 \\
\end{tabular} & 0.0 & 9.8 & 70 & 79 & 0.0 \\
\hline 10/23/01 & $21: 29$ & 19.4 & 19.5 & 19.5 & 19.3 & 0.0 & 9.5 & 100 & 79 & 0.0 \\
\hline 10/23/01 & $21: 30$ & 19.3 & 19.5 & 19.5 & 19.2 & 0.0 & 9.7 & 93 & 79 & 0.0 \\
\hline 10/23/01 & $21: 31$ & 19.4 & 19.5 & 19.5 & 19.3 & 0.0 & 9.6 & 59 & 79 & 0.0 \\
\hline $10 / 23 / 01$ & $21: 32$ & 19.4 & 19.5 & 19.5 & \begin{tabular}{|l|}
19.2 \\
\end{tabular} & 0.0 & 9.5 & 51 & 79 & 0.0 \\
\hline 10/23/01 & $21: 33$ & 19.4 & 19.5 & 19.5 & 19.3 & 0.0 & 9.7 & 76 & 79 & 0.0 \\
\hline $10 / 23 / 01$ & $21: 34$ & 19.4 & 19.5 & 19.5 & 19.2 & 0.0 & 9.7 & 32 & 79 & 0.0 \\
\hline 10/23/01 & $21: 35$ & 19.4 & 19.5 & 19.5 & 19.3 & 0.0 & 9.5 & 44 & 79 & 0.0 \\
\hline 10/23/01 & $21: 36$ & 19.4 & 19.5 & 19.5 & 19.2 & 0.0 & 9.6 & 95 & 79 & 0.0 \\
\hline 10/23/01 & $21: 37$ & 19.4 & 19.5 & 19.5 & 19.3 & 0.0 & 9.6 & 56 & 79 & 0.0 \\
\hline 10/23/01 & $21: 38$ & 19.4 & 19.5 & 19.5 & 19.2 & 0.0 & 9.8 & 99 & 79 & 0.0 \\
\hline 10/23/01 & $21: 39$ & 19.4 & 19.5 & 19.5 & 19.3 & 0.0 & 9.8 & 46 & 79 & 0.0 \\
\hline 10/23/01 & $21: 40$ & 19.4 & 19.5 & 19.5 & 19.3 & 0.0 & 9.6 & 47 & 79 & 0.0 \\
\hline 10/23/01 & $21: 41$ & 19.4 & 19.5 & \begin{tabular}{|l|}
19.5 \\
\end{tabular} & 19.3 & 0.0 & 9.7 & 65 & 79 & 0.0 \\
\hline 10/23/01 & $21: 42$ & 19.4 & 19.5 & 19.5 & 19.3 & 0.0 & 9.6 & 48 & 79 & 0.0 \\
\hline 10/23/01 & $21: 43$ & 19.4 & 19.5 & 19.5 & 19.2 & 0.0 & 9.7 & 64 & 79 & 0.0 \\
\hline $10 / 23 / 01$ & $21: 44$ & 19.4 & 19.5 & \begin{tabular}{|l|}
19.5 \\
\end{tabular} & 19.3 & 0.0 & 9.7 & 41 & 79 & 0.0 \\
\hline $10 / 23 / 01$ & $21: 45$ & 19.4 & 19.5 & \begin{tabular}{|l|}
19.5 \\
\end{tabular} & 19.3 & 0.0 & 9.7 & 44 & 79 & 0.0 \\
\hline $10 / 23 / 01$ & $21: 46$ & 19.4 & 19.5 & 19.5 & 19.3 & 0.0 & 9.6 & 50 & 79 & 0.0 \\
\hline 10/23/01 & $21: 47$ & 19.4 & 19.5 & 19.5 & 19.3 & 0.0 & 9.6 & 41 & 79 & 0.0 \\
\hline $10 / 23 / 01$ & $21: 48$ & 19.4 & \begin{tabular}{|l|}
19.6 \\
\end{tabular} & 19.5 & 19.3 & 0.0 & 9.5 & 52 & 79 & 0.0 \\
\hline 10/23/01 & $21: 49$ & 19.4 & 19.6 & \begin{tabular}{|l|}
19.6 \\
\end{tabular} & 19.3 & 0.0 & 9.6 & 53 & 79 & 0.0 \\
\hline 10/23/01 & $21: 50$ & 19.4 & 19.6 & \begin{tabular}{|l|}
19.6 \\
\end{tabular} & 19.3 & 0.0 & 9.8 & 89 & 79 & 0.0 \\
\hline $10 / 23 / 01$ & $21: 51$ & 19.4 & 19.6 & \begin{tabular}{|l|}
19.6 \\
\end{tabular} & 19.3 & 0.0 & 9.6 & 60 & 79 & 0.0 \\
\hline $10 / 23 / 01$ & $21: 52$ & 19.4 & 19.6 & 19.6 & 19.3 & 0.0 & 9.7 & 49 & 79 & 0.0 \\
\hline $10 / 23 / 01$ & $21: 53$ & 19.4 & 19.6 & 19.6 & 19.3 & 0.0 & 9.7 & 44 & 79 & 0.0 \\
\hline $10 / 23 / 01$ & $21: 54$ & 19.4 & 19.6 & \begin{tabular}{|l|}
19.6 \\
\end{tabular} & 19.3 & 0.0 & 9.7 & 98 & 79 & 0.0 \\
\hline $10 / 23 / 01$ & $21: 55$ & 19.5 & 19.6 & \begin{tabular}{|l|}
19.6 \\
\end{tabular} & 19.3 & 0.0 & 9.6 & 117 & 79 & 0.0 \\
\hline $10 / 23 / 01$ & $21: 56$ & 19.4 & 19.6 & \begin{tabular}{|l|}
19.6 \\
\end{tabular} & 19.3 & 0.0 & 9.6 & 44 & 79 & 0.0 \\
\hline $10 / 23 / 01$ & $21: 57$ & 19.5 & 19.6 & 19.6 & 19.3 & 0.0 & 9.6 & 48 & 79 & 0.0 \\
\hline $10 / 23 / 01$ & $21: 58$ & 19.4 & 19.6 & \begin{tabular}{|l|}
19.6 \\
\end{tabular} & 19.3 & 0.0 & 9.7 & 55 & 79 & 0.0 \\
\hline $10 / 23 / 01$ & $21: 59$ & 19.5 & 19.6 & \begin{tabular}{|l|}
19.6 \\
\end{tabular} & \begin{tabular}{|l}
19.4 \\
\end{tabular} & 0.0 & 9.7 & 62 & 79 & 0.0 \\
\hline $10 / 23 / 01$ & $22: 00$ & 19.4 & 19.6 & \begin{tabular}{|l|}
19.6 \\
\end{tabular} & 19.3 & 0.0 & 9.5 & 43 & 79 & 0.0 \\
\hline $10 / 23 / 01$ & $22: 01$ & 19.5 & 19.6 & 19.6 & \begin{tabular}{|l|}
19.4 \\
\end{tabular} & 0.0 & 9.7 & 76 & 79 & 0.0 \\
\hline $10 / 23 / 01$ & 22:02 & 19.4 & 19.6 & \begin{tabular}{|l|}
19.6 \\
\end{tabular} & 19.3 & 0.0 & 9.7 & 91 & 79 & 0.0 \\
\hline $10 / 23 / 01$ & 22:03 & 19.5 & 19.6 & \begin{tabular}{|l|}
19.6 \\
\end{tabular} & \begin{tabular}{|l|}
19.4 \\
\end{tabular} & 0.0 & 9.7 & 52 & 79 & 0.0 \\
\hline $10 / 23 / 01$ & $22: 04$ & 19.4 & 19.6 & \begin{tabular}{|l|}
19.6 \\
\end{tabular} & 19.3 & 0.0 & 9.6 & 112 & 79 & 0.0 \\
\hline $10 / 23 / 01$ & $22: 05$ & 19.5 & 19.6 & \begin{tabular}{|l|}
19.6 \\
\end{tabular} & 19.4 & 0.0 & 9.6 & 53 & 79 & 0.0 \\
\hline $10 / 23 / 01$ & $22: 06$ & 19.5 & 19.6 & \begin{tabular}{|l|}
19.6 \\
\end{tabular} & 19.3 & 0.0 & 9.6 & 69 & 79 & 0.0 \\
\hline $10 / 23 / 01$ & 22:07 & 19.5 & 19.6 & \begin{tabular}{|l|}
19.6 \\
\end{tabular} & \begin{tabular}{|l}
19.4 \\
\end{tabular} & 0.0 & 9.7 & 117 & 79 & 0.0 \\
\hline $10 / 23 / 01$ & $22: 08$ & 19.5 & 19.6 & \begin{tabular}{|l|}
19.6 \\
\end{tabular} & \begin{tabular}{|l|}
19.4 \\
\end{tabular} & 0.0 & 9.7 & 102 & 79 & 0.0 \\
\hline $10 / 23 / 01$ & $22: 09$ & 19.4 & 19.6 & \begin{tabular}{|l|}
19.6 \\
\end{tabular} & 19.3 & 0.0 & 9.5 & 70 & 79 & 0.0 \\
\hline $10 / 23 / 01$ & $22: 10$ & 19.5 & 19.6 & \begin{tabular}{|l|}
19.6 \\
\end{tabular} & \begin{tabular}{|l|}
19.4 \\
\end{tabular} & 0.0 & 9.6 & 52 & 79 & 0.0 \\
\hline $10 / 23 / 01$ & 22:11 & 19.4 & 19.6 & \begin{tabular}{|l|}
19.6 \\
\end{tabular} & 19.3 & 0.0 & 9.7 & 52 & 79 & 0.0 \\
\hline $10 / 23 / 01$ & 22:12 & 19.5 & 19.6 & 19.6 & \begin{tabular}{|l|}
19.4 \\
\end{tabular} & 0.0 & 9.6 & 74 & 79 & 0.0 \\
\hline $10 / 23 / 01$ & $22: 13$ & 19.4 & 19.6 & \begin{tabular}{|l|}
19.6 \\
\end{tabular} & 19.3 & 0.0 & 9.7 & 74 & 79 & 0.0 \\
\hline $10 / 23 / 01$ & $22: 14$ & 19.5 & 19.6 & \begin{tabular}{|l|}
19.6 \\
\end{tabular} & 19.4 & 0.0 & 9.7 & 56 & 79 & 0.0 \\
\hline $10 / 23 / 01$ & $22: 15$ & 19.5 & 19.6 & \begin{tabular}{|l|}
19.6 \\
\end{tabular} & 19.3 & 0.0 & 9.8 & 58 & 79 & 0.0 \\
\hline $10 / 23 / 01$ & $22: 16$ & 19.5 & 19.6 & \begin{tabular}{|l|}
19.6 \\
\end{tabular} & \begin{tabular}{|l|}
19.4 \\
\end{tabular} & 0.0 & 9.7 & 118 & 79 & 0.0 \\
\hline $10 / 23 / 01$ & $22: 17$ & 19.5 & 19.6 & \begin{tabular}{|l|}
19.6 \\
\end{tabular} & 19.3 & 0.0 & 9.7 & 103 & 79 & 0.0 \\
\hline 10/23/01 & $22: 18$ & 19.5 & 19.6 & \begin{tabular}{|l|}
19.6 \\
\end{tabular} & \begin{tabular}{|l}
19.4 \\
\end{tabular} & 0.0 & 9.8 & 93 & 79 & 0.0 \\
\hline $10 / 23 / 01$ & $22: 19$ & 19.5 & 19.6 & \begin{tabular}{|l|}
19.6 \\
\end{tabular} & 19.4 & 0.0 & 9.7 & 68 & 79 & 0.0 \\
\hline $10 / 23 / 01$ & 22:20 & 19.5 & 19.6 & \begin{tabular}{|l|}
19.6 \\
\end{tabular} & \begin{tabular}{|l|}
19.3 \\
\end{tabular} & 0.0 & 9.7 & 112 & 79 & 0.0 \\
\hline $10 / 23 / 01$ & $22: 21$ & 19.5 & 19.6 & \begin{tabular}{|l|}
19.6 \\
\end{tabular} & 19.4 & 0.0 & 9.6 & 60 & 79 & 0.0 \\
\hline $10 / 23 / 01$ & $22: 22$ & 19.5 & 19.6 & \begin{tabular}{|l|}
19.6 \\
\end{tabular} & 19.3 & 0.0 & 9.6 & 98 & 79 & 0.0 \\
\hline 10/23/01 & $22: 23$ & 19.5 & \begin{tabular}{|l|}
19.6 \\
\end{tabular} & \begin{tabular}{|l|}
19.6 \\
\end{tabular} & \begin{tabular}{|l|}
19.4 \\
\end{tabular} & 0.0 & 9.6 & 74 & 79 & 0.0 \\
\hline
\end{tabular}

\begin{tabular}{|c|c|c|c|c|c|c|c|c|c|c|}
\hline \multicolumn{11}{|c|}{ PREC2_102301_0623 } \\
\hline DATE & TIME & $\begin{array}{c}\mathrm{HX} \\
\text { Outlet } \\
\left({ }^{\circ} \mathrm{C}\right) \\
\mathrm{TCO}\end{array}$ & \begin{tabular}{|c|} 
REC \\
PP \\
OUT \\
$\left({ }^{\circ} \mathrm{C}\right)$ \\
TC1
\end{tabular} & $\begin{array}{c}\text { TK } \\
\text { BOT } \\
\left({ }^{\circ} \mathrm{C}\right) \\
\text { TC2 }\end{array}$ & $\begin{array}{l}\text { HX } \\
\text { OUT } \\
\left({ }^{\circ} \mathrm{C}\right) \\
\text { TC3 }\end{array}$ & \begin{tabular}{|c|} 
Mn \\
Flow \\
(gpm) \\
\end{tabular} & $\begin{array}{c}\text { Galigher } \\
\text { Flow } \\
(\mathrm{gpm})\end{array}$ & $\begin{array}{c}\text { Current } \\
\text { to } \mathrm{HX}\end{array}$ & $\begin{array}{c}\text { Voltage } \\
\text { to } \mathrm{HX}\end{array}$ & $\begin{array}{c}\mathrm{Sr} \\
\text { Flow } \\
(\mathrm{gpm})\end{array}$ \\
\hline $10 / 23 / 01$ & 22:24 & 19.4 & $\mid 19.6$ & 19.6 & 19.3 & 0.0 & 9.5 & 90 & 79 & 0. \\
\hline $10 / 23 / 01$ & 22:25 & 19.5 & $\mid 19.6$ & 19.6 & 19.4 & 0.0 & 9.7 & 61 & 79 & 0. \\
\hline $10 / 23 / 01$ & 22:26 & 19.5 & $\mid 19.6$ & 19.6 & 19.3 & 0.0 & 9.6 & 119 & 79 & 0. \\
\hline $10 / 23 / 01$ & 22:27 & 19.5 & 19.6 & 19.6 & 19.4 & 0.0 & 9.6 & 74 & 79 & 0 \\
\hline $10 / 23 / 01$ & 22:28 & 19.5 & $\mid 19.6$ & 19.6 & 19.3 & 0.0 & 9.8 & 104 & 79 & 0 \\
\hline $10 / 23 / 01$ & 22:29 & 19.5 & $\mid 19.6$ & 19.7 & 19.4 & 0.0 & 9.4 & 92 & 79 & 0. \\
\hline $10 / 23 / 01$ & 22:30 & 19.5 & 19.7 & 19.7 & 19.4 & 0.0 & 9.7 & 93 & 79 & 0 \\
\hline $10 / 23 / 01$ & 22:31 & 19.5 & $\mid 19.7$ & 19.7 & 19.4 & 0.0 & 9.6 & 84 & 79 & 0 \\
\hline $10 / 23 / 01$ & 22:32 & 19.5 & 19.7 & 19.7 & 19.4 & 0.0 & 9.5 & 64 & 79 & 0. \\
\hline $10 / 23 / 01$ & 22:33 & 19.5 & $|19.7|$ & 19.7 & 19.4 & 0.0 & 9.7 & 69 & 79 & 0. \\
\hline $10 / 23 / 01$ & 22:34 & 19.5 & $|19.7|$ & 19.7 & 19.4 & 0.0 & 9.7 & 109 & 79 & 0.0 \\
\hline $10 / 23 / 01$ & 22:35 & 19.5 & $\mid 19.7$ & 19.7 & 19.4 & 0.0 & 9.6 & 75 & 79 & 0 \\
\hline $10 / 23 / 01$ & 22:36 & 19.5 & $\mid 19.7$ & 19.7 & 19.4 & 0.0 & 9.7 & 65 & 79 & 0. \\
\hline $10 / 23 / 01$ & 22:37 & 19.5 & $|19.7|$ & 19.7 & 19.4 & 0.0 & 9.7 & 110 & 79 & 0. \\
\hline $10 / 23 / 01$ & 22:38 & 19.6 & $|19.7|$ & 19.7 & 19.4 & 0.0 & 9.5 & 66 & 79 & 0. \\
\hline $10 / 23 / 01$ & $22: 39$ & 19.5 & \begin{tabular}{|l|}
$\mid 19.7$ \\
\end{tabular} & 19.7 & 19.4 & 0.0 & 9.7 & 123 & 79 & 0. \\
\hline $10 / 23 / 01$ & $22: 40$ & 19.5 & $|19.7|$ & 19.7 & 19.4 & 0.0 & 9.8 & 90 & 79 & 0 \\
\hline $10 / 23 / 01$ & $22: 41$ & 19.5 & \begin{tabular}{|l|}
$\mid 19.7$ \\
\end{tabular} & 19.7 & 19.4 & 0.0 & 9.7 & 113 & 79 & \\
\hline $10 / 23 / 01$ & 22:42 & 19.5 & $|19.7|$ & 19.7 & 19.4 & 0.0 & 9.6 & 74 & 79 & 0. \\
\hline $10 / 23 / 01$ & $22: 43$ & 19.5 & $|19.7|$ & 19.7 & 19.4 & 0.0 & 9.7 & 89 & 79 & 0 \\
\hline $10 / 23 / 01$ & 22:44 & 19.5 & \begin{tabular}{|l|}
19.7 \\
\end{tabular} & 19.7 & 19.4 & 0.0 & 9.7 & 68 & 79 & 0.0 \\
\hline $10 / 23 / 01$ & 22:45 & 19.5 & \begin{tabular}{|l|}
19.7 \\
\end{tabular} & 19.7 & 19.4 & 0.0 & 9.6 & 94 & 79 & 0.0 \\
\hline $10 / 23 / 01$ & 22:46 & 19.5 & 19.7 & 19.7 & 19.4 & 0.0 & 9.6 & 77 & 79 & 0. \\
\hline $10 / 23 / 01$ & 22:47 & 19.6 & 19.7 & 19.7 & 19.4 & 0.0 & 9.7 & 74 & 79 & \\
\hline $10 / 23 / 01$ & $22: 48$ & 19.5 & \begin{tabular}{|l|}
$\mid 19.7$ \\
\end{tabular} & 19.7 & 19.4 & 0.0 & 9.7 & 107 & 79 & 0. \\
\hline $10 / 23 / 01$ & $22: 49$ & 19.6 & 19.7 & 19.7 & 19.5 & 0.0 & 9.7 & 112 & 79 & 0. \\
\hline $10 / 23 / 01$ & 22:50 & 19.5 & $|19.7|$ & 19.7 & 19.4 & 0.0 & 9.8 & 114 & 79 & 0.0 \\
\hline $10 / 23 / 01$ & 22:51 & 19.6 & $|19.7|$ & 19.7 & 19.5 & 0.0 & 9.6 & 95 & 79 & 0. \\
\hline $10 / 23 / 01$ & 22:52 & 19.5 & $|19.7|$ & 19.7 & 19.4 & 0.0 & 9.6 & 107 & 79 & 0. \\
\hline $10 / 23 / 01$ & 22:53 & 19.6 & \begin{tabular}{|l|}
19.7 \\
\end{tabular} & 19.7 & 19.5 & 0.0 & 9.6 & 126 & 79 & \\
\hline $10 / 23 / 01$ & 22:54 & 19.5 & \begin{tabular}{|l|}
19.7 \\
\end{tabular} & 19.7 & 19.4 & 0.0 & 9.7 & 112 & 79 & 0 \\
\hline $10 / 23 / 01$ & 22:55 & 19.6 & $\mid 19.7$ & 19.7 & 19.5 & 0.0 & 9.6 & 95 & 79 & 0.0 \\
\hline $10 / 23 / 01$ & 22:56 & 19.6 & $|19.7|$ & 19.7 & 19.4 & 0.0 & 9.8 & 114 & 79 & 0. \\
\hline $10 / 23 / 01$ & 22:57 & 19.6 & $|19.7|$ & 19.7 & 19.5 & 0.0 & 9.7 & 74 & 79 & 0 \\
\hline $10 / 23 / 01$ & 22:58 & 19.6 & $\mid$\begin{tabular}{|l|} 
\\
\end{tabular} & 19.7 & 19.4 & 0.0 & 9.6 & 75 & 79 & \\
\hline $10 / 23 / 01$ & 22:59 & 19.5 & $\mid 19.7$ & 19.7 & 19.5 & 0.0 & 9.7 & 75 & 79 & 0 \\
\hline $10 / 23 / 01$ & $23: 00$ & 19.6 & $\mid$\begin{tabular}{|l|}
$\mid 19.7$ \\
\end{tabular} & 19.7 & 19.5 & 0.0 & 9.7 & 127 & 79 & 0. \\
\hline $10 / 23 / 01$ & 23:01 & 19.5 & \begin{tabular}{|l|}
19.7 \\
\end{tabular} & 19.7 & 19.4 & 0.0 & 9.7 & 75 & 79 & 0. \\
\hline $10 / 23 / 01$ & 23:02 & 19.6 & \begin{tabular}{|l|}
19.7 \\
\end{tabular} & 19.7 & 19.5 & 0.0 & 9.8 & 86 & 79 & 0. \\
\hline $10 / 23 / 01$ & 23:03 & 19.5 & \begin{tabular}{|l|}
19.7 \\
\end{tabular} & 19.7 & 19.4 & 0.0 & 9.7 & 110 & 79 & 0. \\
\hline $10 / 23 / 01$ & 23:04 & 19.6 & 19.7 & 19.7 & 19.5 & 0.0 & 9.6 & 93 & 79 & 0.4 \\
\hline $10 / 23 / 01$ & 23:05 & 19.5 & 19.7 & 19.7 & 19.4 & 0.0 & 9.6 & 121 & 79 & 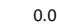 \\
\hline $10 / 23 / 01$ & 23:06 & 19.6 & \begin{tabular}{|l|}
19.7 \\
\end{tabular} & 19.7 & 19.5 & 0.0 & 9.7 & 78 & 79 & 0. \\
\hline $10 / 23 / 01$ & 23:07 & 19.6 & $|19.7|$ & 19.7 & 19.4 & 0.0 & 9.6 & 86 & 79 & 0. \\
\hline $10 / 23 / 01$ & 23:08 & 19.6 & $|19.7|$ & 19.7 & 19.5 & 0.0 & 9.7 & 123 & 79 & 0 \\
\hline $10 / 23 / 01$ & 23:09 & 19.6 & $\mid 19.7$ & 19.8 & 19.5 & 0.0 & 9.5 & 115 & 79 & 0. \\
\hline $10 / 23 / 01$ & $23: 10$ & 19.6 & \begin{tabular}{|l|}
$\mid 19.7$ \\
\end{tabular} & 19.7 & 19.5 & 0.0 & 9.8 & 50 & 79 & 0. \\
\hline $10 / 23 / 01$ & 23:11 & 19.6 & \begin{tabular}{|l|}
19.7 \\
\end{tabular} & 19.7 & 19.5 & 0.0 & 9.6 & 100 & 79 & 0. \\
\hline $10 / 23 / 01$ & $23: 12$ & 19.6 & $\mid 19.8$ & 19.7 & 19.5 & 0.0 & 9.6 & 104 & 79 & 0. \\
\hline $10 / 23 / 01$ & $23: 13$ & 19.6 & 19.8 & 19.7 & 19.5 & 0.0 & 9.6 & 99 & 79 & 0. \\
\hline $10 / 23 / 01$ & 23:14 & 19.6 & $\mid 19.8$ & 19.8 & 19.5 & 0.0 & 10.3 & 120 & 79 & 0 \\
\hline $10 / 23 / 01$ & $23: 15$ & 19.6 & 19.8 & 19.8 & 19.5 & 0.0 & 9.7 & 125 & 79 & 0. \\
\hline $10 / 23 / 01$ & $23: 16$ & 19.6 & 19.8 & 19.8 & 19.4 & 0.0 & 9.6 & 101 & 79 & 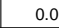 \\
\hline $10 / 23 / 01$ & 23:17 & 19.6 & $\mid 19.8$ & 19.8 & 19.5 & 0.0 & 9.7 & 81 & 79 & $0 . c$ \\
\hline $10 / 23 / 01$ & $23: 18$ & 19.6 & $\mid 19.8$ & 19.8 & 19.5 & 0.0 & 9.6 & 105 & 79 & 0. \\
\hline $10 / 23 / 01$ & $23: 19$ & 19.6 & 19.8 & 19.8 & 19.5 & 0.0 & 9.7 & 120 & 79 & 0. \\
\hline $10 / 23 / 01$ & $23: 20$ & 19.6 & 19.8 & 19.8 & 19.5 & 0.0 & 9.7 & 108 & 79 & 0. \\
\hline $10 / 23 / 01$ & 23:21 & 19.6 & 19.8 & 19.8 & 19.5 & 0.0 & 9.7 & 88 & 79 & 0.0 \\
\hline $10 / 23 / 01$ & 23:22 & 19.6 & $\mid 19.8$ & 19.8 & 19.5 & 0.0 & 9.7 & 82 & 79 & 0.0 \\
\hline 23/01 & $23: 23$ & 19.6 & 19.8 & 19.8 & 19.5 & 0.0 & 9.6 & 121 & 79 & 0.0 \\
\hline
\end{tabular}


WSRC-TR-2002-00459, Rev. 0 SRT-RPP-2002-00221

\begin{tabular}{|c|c|c|c|c|c|c|c|c|c|c|}
\hline \multicolumn{3}{|c|}{ |PREC2_102301_0623 } & \multirow[b]{2}{*}{$\mid \begin{array}{c}\text { REC } \\
\text { PP } \\
\text { OUT } \\
\left({ }^{\circ} \mathrm{C}\right) \\
\text { TC1 }\end{array}$} & \multirow[b]{2}{*}{\begin{tabular}{|c|} 
TK \\
BOT \\
$\left({ }^{\circ} \mathrm{C}\right)$ \\
TC2 \\
\end{tabular}} & \multirow[b]{2}{*}{\begin{tabular}{|c|} 
HX \\
OUT \\
$\left({ }^{\circ} \mathrm{C}\right)$ \\
TC3 \\
\end{tabular}} & \multirow[b]{2}{*}{$\begin{array}{c}\mathrm{Mn} \\
\text { Flow } \\
(\mathrm{gpm})\end{array}$} & \multirow[b]{2}{*}{\begin{tabular}{|c|} 
Galigher \\
Flow \\
(gpm)
\end{tabular}} & \multirow[b]{2}{*}{$\begin{array}{c}\text { Current } \\
\text { to } \mathrm{HX}\end{array}$} & \multirow[b]{2}{*}{$\begin{array}{c}\text { Voltage } \\
\text { to } \mathrm{HX}\end{array}$} & \multirow[b]{2}{*}{$\begin{array}{c}\mathrm{Sr} \\
\text { Flow } \\
\text { (gpm) }\end{array}$} \\
\hline DATE & TIME & $\begin{array}{c}\mathrm{HX} \\
\text { Outlet } \\
\left({ }^{\circ} \mathrm{C}\right) \\
\text { TCO }\end{array}$ & & & & & & & & \\
\hline $10 / 23 / 01$ & $23: 24$ & 19.6 & 19.8 & 19.8 & 19.5 & 0.0 & 9.7 & 112 & 79 & 0.0 \\
\hline 10/23/01 & $23: 25$ & 19.6 & 19.8 & 19.8 & 19.5 & 0.0 & 9.7 & 123 & 79 & 0.0 \\
\hline $10 / 23 / 01$ & $23: 26$ & 19.6 & $\mid 19.8$ & 19.8 & \begin{tabular}{|r|r} 
\\
\end{tabular} & 0.0 & 9.7 & 123 & 79 & 0.0 \\
\hline $10 / 23 / 01$ & $23: 27$ & 19.6 & \begin{tabular}{|l|}
19.8 \\
\end{tabular} & 19.8 & 19.5 & 0.0 & 9.8 & 91 & 79 & 0.0 \\
\hline 10/23/01 & $23: 28$ & 19.6 & $\mid 19.8$ & 19.8 & 19.5 & 0.0 & 9.6 & 130 & 79 & 0.0 \\
\hline 10/23/01 & $23: 29$ & 19.6 & $\mid 19.8$ & 19.8 & 19.5 & 0.0 & 9.6 & 95 & 79 & 0.0 \\
\hline 10/23/01 & $23: 30$ & 19.7 & $\mid 19.8$ & 19.8 & 19.5 & 0.0 & 9.6 & 133 & 79 & 0.0 \\
\hline $10 / 23 / 01$ & 23:31 & 19.6 & $\mid 19.8$ & 19.8 & 19.5 & 0.0 & 9.6 & 115 & 79 & 0.0 \\
\hline $10 / 23 / 01$ & $23: 32$ & 19.6 & $\mid 19.8$ & 19.8 & $\mid 19.6$ & 0.0 & 9.6 & 128 & 79 & 0.0 \\
\hline $10 / 23 / 01$ & 23:33 & 19.6 & \begin{tabular}{|l|}
19.8 \\
\end{tabular} & 19.8 & 19.5 & 0.0 & 9.6 & 103 & 79 & 0.0 \\
\hline 10/23/01 & $23: 34$ & 19.7 & $\mid 19.8$ & 19.8 & $\mid 19.6$ & 0.0 & 9.7 & 124 & 79 & 0.0 \\
\hline $10 / 23 / 01$ & $23: 35$ & 19.6 & \begin{tabular}{|l|}
19.8 \\
\end{tabular} & 19.8 & 19.5 & 0.0 & 9.7 & 90 & 79 & 0.0 \\
\hline $10 / 23 / 01$ & 23:36 & 19.7 & \begin{tabular}{|l|}
19.8 \\
\end{tabular} & \begin{tabular}{|l|} 
\\
\end{tabular} & 19.6 & 0.0 & 9.7 & 127 & 79 & 0.0 \\
\hline $10 / 23 / 01$ & 23:37 & 19.6 & $\mid 19.8$ & \begin{tabular}{|l|} 
\\
\end{tabular} & 19.5 & 0.0 & 9.6 & 96 & 79 & 0.0 \\
\hline $10 / 23 / 01$ & 23:38 & 19.7 & 19.8 & 19.8 & 19.6 & 0.0 & 9.6 & 117 & 79 & 0.0 \\
\hline $10 / 23 / 01$ & 23:39 & 19.7 & $\mid 19.8$ & 19.8 & 19.5 & 0.0 & 9.7 & 130 & 79 & 0.0 \\
\hline $10 / 23 / 01$ & $23: 40$ & 19.6 & $\mid 19.8$ & \begin{tabular}{|l|}
19.8 \\
\end{tabular} & \begin{tabular}{|l|}
19.5 \\
\end{tabular} & 0.0 & 9.6 & 117 & 79 & 0.0 \\
\hline 10/23/01 & 23:41 & 19.7 & \begin{tabular}{|l|}
19.8 \\
\end{tabular} & \begin{tabular}{|l|}
19.8 \\
\end{tabular} & \begin{tabular}{|l|}
19.6 \\
\end{tabular} & 0.0 & 9.6 & 127 & 79 & 0.0 \\
\hline $10 / 23 / 01$ & 23:42 & 19.6 & \begin{tabular}{|l|}
19.8 \\
\end{tabular} & \begin{tabular}{|l|}
19.8 \\
\end{tabular} & \begin{tabular}{|l|}
19.6 \\
\end{tabular} & 0.0 & 9.6 & 124 & 79 & 0.0 \\
\hline $10 / 23 / 01$ & $23: 43$ & 19.7 & $\mid 19.8$ & \begin{tabular}{|l|} 
\\
\end{tabular} & 19.6 & 0.0 & 9.6 & 129 & 79 & 0.0 \\
\hline $10 / 23 / 01$ & $23: 44$ & 19.6 & \begin{tabular}{|l|}
19.8 \\
\end{tabular} & \begin{tabular}{|l|}
19.8 \\
\end{tabular} & 19.6 & 0.0 & 9.6 & 122 & 79 & 0.0 \\
\hline $10 / 23 / 01$ & 23:45 & 19.7 & \begin{tabular}{|l|}
19.8 \\
\end{tabular} & \begin{tabular}{|l|}
19.8 \\
\end{tabular} & 19.6 & 0.0 & 9.6 & 98 & 79 & 0.0 \\
\hline $10 / 23 / 01$ & 23:46 & 19.6 & $\mid 19.8$ & \begin{tabular}{|l|} 
\\
\end{tabular} & 19.6 & 0.0 & 9.7 & 127 & 79 & 0.0 \\
\hline $10 / 23 / 01$ & 23:47 & 19.7 & 19.8 & \begin{tabular}{|l|}
19.8 \\
\end{tabular} & \begin{tabular}{|l|}
19.6 \\
\end{tabular} & 0.0 & 9.7 & 98 & 79 & 0.0 \\
\hline $10 / 23 / 01$ & 23:48 & 19.7 & \begin{tabular}{|l|}
19.8 \\
\end{tabular} & \begin{tabular}{|l|}
19.9 \\
\end{tabular} & 19.6 & 0.0 & 9.6 & 89 & 79 & 0.0 \\
\hline $10 / 23 / 01$ & $23: 49$ & 19.7 & 19.9 & \begin{tabular}{|l|} 
\\
\end{tabular} & 19.6 & 0.0 & 9.7 & 127 & 79 & 0.0 \\
\hline $10 / 23 / 01$ & 23:50 & 19.7 & $\mid 19.9$ & \begin{tabular}{|l|} 
\\
\end{tabular} & 19.6 & 0.0 & 9.7 & 97 & 79 & 0.0 \\
\hline $10 / 23 / 01$ & 23:51 & 19.7 & 19.9 & \begin{tabular}{|l|} 
\\
\end{tabular} & 19.6 & 0.0 & 9.6 & 129 & 79 & 0.0 \\
\hline $10 / 23 / 01$ & 23:52 & 19.7 & \begin{tabular}{|l|}
19.9 \\
\end{tabular} & \begin{tabular}{|l|}
19.9 \\
\end{tabular} & 19.6 & 0.0 & 9.7 & 136 & 79 & 0.0 \\
\hline $10 / 23 / 01$ & 23:53 & 19.7 & 19.9 & \begin{tabular}{|l|}
19.9 \\
\end{tabular} & 19.6 & 0.0 & 9.7 & 122 & 79 & 0.0 \\
\hline $10 / 23 / 01$ & 23:54 & 19.7 & $\mid 19.9$ & \begin{tabular}{|l|}
19.9 \\
\end{tabular} & 19.6 & 0.0 & 9.7 & 127 & 79 & 0.0 \\
\hline $10 / 23 / 01$ & 23:55 & 19.7 & $\mid 19.9$ & \begin{tabular}{|l|} 
\\
\end{tabular} & 19.6 & 0.0 & 9.7 & 123 & 79 & 0.0 \\
\hline $10 / 23 / 01$ & 23:56 & 19.7 & 19.8 & 19.9 & 19.6 & 0.0 & 9.6 & 118 & 79 & 0.0 \\
\hline $10 / 23 / 01$ & 23:57 & 19.7 & \begin{tabular}{|l|}
19.9 \\
\end{tabular} & \begin{tabular}{|l|}
19.9 \\
\end{tabular} & \begin{tabular}{|l|} 
\\
\end{tabular} & 0.0 & 9.6 & 103 & 79 & 0.0 \\
\hline $10 / 23 / 01$ & 23:58 & 19.7 & $\mid 19.8$ & 19.9 & 19.6 & 0.0 & 9.6 & 139 & 79 & 0.0 \\
\hline $10 / 23 / 01$ & 23:59 & 19.7 & 19.9 & \begin{tabular}{|l|} 
\\
\end{tabular} & 19.5 & 0.0 & 9.6 & 103 & 79 & 0.0 \\
\hline $10 / 24 / 01$ & $0: 00$ & 19.7 & 19.9 & \begin{tabular}{|l|} 
\\
\end{tabular} & 19.6 & 0.0 & 9.7 & 97 & 79 & 0.0 \\
\hline $10 / 24 / 01$ & 0:01 & 19.7 & $\mid 19.9$ & \begin{tabular}{|l|} 
\\
\end{tabular} & $\mid 19.6$ & 0.0 & 9.7 & 94 & 79 & 0.0 \\
\hline $10 / 24 / 01$ & $0: 02$ & 19.7 & \begin{tabular}{|l|}
19.9 \\
\end{tabular} & \begin{tabular}{|l|}
19.9 \\
\end{tabular} & 19.6 & 0.0 & 9.7 & 88 & 79 & 0.0 \\
\hline $10 / 24 / 01$ & 0:03 & 19.7 & $\mid 19.9$ & \begin{tabular}{|l|} 
\\
\end{tabular} & 19.6 & 0.0 & 9.6 & 124 & 79 & 0.0 \\
\hline $10 / 24 / 01$ & $0: 04$ & 19.7 & 19.9 & \begin{tabular}{|l|} 
\\
\end{tabular} & 19.6 & 0.0 & 9.6 & 136 & 79 & 0.0 \\
\hline $10 / 24 / 01$ & $0: 05$ & 19.7 & 19.9 & \begin{tabular}{|l|}
19.9 \\
\end{tabular} & \begin{tabular}{|l|}
19.6 \\
\end{tabular} & 0.0 & 9.6 & 134 & 79 & 0.0 \\
\hline $10 / 24 / 01$ & $0: 06$ & 19.7 & $\mid$\begin{tabular}{|l|} 
\\
\end{tabular} & \begin{tabular}{|l|} 
\\
\end{tabular} & 19.6 & 0.0 & 9.6 & 114 & 79 & 0.0 \\
\hline $10 / 24 / 01$ & $0: 07$ & 19.7 & 19.9 & \begin{tabular}{|l|} 
\\
\end{tabular} & 19.6 & 0.0 & .0 & 119 & 79 & 0.0 \\
\hline $10 / 24 / 01$ & $0: 08$ & 19.7 & 19.9 & \begin{tabular}{|l|} 
\\
\end{tabular} & 19.6 & 0.0 & 9.6 & 105 & 79 & 0.0 \\
\hline $10 / 24 / 01$ & 0:09 & 19.7 & $\mid 19.9$ & \begin{tabular}{|l|} 
\\
\end{tabular} & 19.6 & 0.0 & 9.7 & 124 & 79 & 0.0 \\
\hline $10 / 24 / 01$ & $0: 10$ & 19.7 & \begin{tabular}{|l|}
19.9 \\
\end{tabular} & \begin{tabular}{|l|}
19.9 \\
\end{tabular} & \begin{tabular}{|l|}
19.6 \\
\end{tabular} & 0.0 & 9.6 & 113 & 79 & 0.0 \\
\hline $10 / 24 / 01$ & $0: 11$ & 19.7 & $\mid 19.9$ & 19.9 & $\mid 19.6$ & 0.0 & 9.6 & 134 & 79 & 0.0 \\
\hline $10 / 24 / 01$ & $0: 12$ & 19.7 & $\mid 19.9$ & \begin{tabular}{|l|}
19.9 \\
\end{tabular} & 19.6 & 0.0 & 9.6 & 96 & 79 & 0.0 \\
\hline $10 / 24 / 01$ & $0: 13$ & 19.7 & 19.9 & 19.9 & 19.6 & 0.0 & 9.7 & 95 & 79 & 0.0 \\
\hline $10 / 24 / 01$ & $0: 14$ & 19.7 & $\mid 19.9$ & \begin{tabular}{|l|}
19.9 \\
\end{tabular} & $\mid 19.6$ & 0.0 & 9.6 & 113 & 79 & 0.0 \\
\hline $10 / 24 / 01$ & $0: 15$ & 19.7 & $\mid 19.9$ & \begin{tabular}{|l|} 
\\
\end{tabular} & 19.6 & 0.0 & 9.6 & 128 & 79 & 0.0 \\
\hline $10 / 24 / 01$ & $0: 16$ & 19.7 & 19.9 & \begin{tabular}{|l|} 
\\
\end{tabular} & 19.6 & 0.0 & 9.7 & 105 & 79 & 0.0 \\
\hline $10 / 24 / 01$ & $0: 17$ & 19.7 & 19.9 & \begin{tabular}{|l|} 
\\
\end{tabular} & 19.7 & 0.0 & 9.7 & 100 & 79 & 0.0 \\
\hline $10 / 24 / 01$ & $0: 18$ & 19.7 & 19.9 & \begin{tabular}{|l|} 
\\
\end{tabular} & 19.6 & 0.0 & 9.7 & 104 & 79 & 0.0 \\
\hline $10 / 24 / 01$ & $0: 19$ & 19.7 & 19.9 & \begin{tabular}{|l|} 
\\
\end{tabular} & 19.6 & 0.0 & 9.6 & 132 & 79 & 0.0 \\
\hline $10 / 24 / 01$ & $0: 20$ & 19.7 & $\mid 19.9$ & \begin{tabular}{|l|}
19.9 \\
\end{tabular} & 19.6 & 0.0 & 9.7 & 93 & 79 & 0.0 \\
\hline $10 / 24 / 01$ & $0: 21$ & 19.7 & $\mid 19.9$ & \begin{tabular}{|l|} 
\\
\end{tabular} & $\mid 19.6$ & 0.0 & 9.5 & 100 & 79 & 0.0 \\
\hline $10 / 24 / 01$ & $0: 22$ & 19.8 & $\mid 19.9$ & \begin{tabular}{|l|} 
\\
\end{tabular} & 19.7 & 0.0 & 9.6 & 123 & 79 & 0.0 \\
\hline $10 / 24 / 01$ & $0: 23$ & 19.7 & 19.9 & \begin{tabular}{|l|} 
\\
\end{tabular} & $\mid 19.6$ & 0.0 & 9.7 & 125 & 79 & 0.0 \\
\hline
\end{tabular}

\begin{tabular}{|c|c|c|c|c|c|c|c|c|c|c|}
\hline \multicolumn{11}{|c|}{ PREC2_102301_0623 } \\
\hline DATE & TIME & \begin{tabular}{|c|}
$\mathrm{HX}$ \\
Outlet \\
$\left({ }^{\circ} \mathrm{C}\right)$ \\
TCO
\end{tabular} & \begin{tabular}{|c|} 
REC \\
PP \\
OUT \\
$\left({ }^{\circ} \mathrm{C}\right)$ \\
TC1 \\
\end{tabular} & \begin{tabular}{|c|} 
TK \\
BOT \\
$\left({ }^{\circ} \mathrm{C}\right)$ \\
TC2 \\
\end{tabular} & $\begin{array}{l}\text { HX } \\
\text { OUT } \\
\left({ }^{\circ} \mathrm{C}\right) \\
\text { TC3 }\end{array}$ & \begin{tabular}{|c|} 
Mn \\
Flow \\
(gpm)
\end{tabular} & \begin{tabular}{|c|} 
Galigher \\
Flow \\
(gpm)
\end{tabular} & $\begin{array}{l}\text { Current } \\
\text { to } \mathrm{HX}\end{array}$ & $\begin{array}{l}\text { Voltage } \\
\text { to } \mathrm{HX}\end{array}$ & $\begin{array}{c}\text { Sr } \\
\text { Flow } \\
\text { (gpm) }\end{array}$ \\
\hline $10 / 24 / 01$ & $0: 24$ & 19.8 & 19.9 & 19.9 & 19.7 & 0.0 & 9.7 & 108 & 79 & 0.0 \\
\hline $10 / 24 / 01$ & $0: 25$ & 19.7 & 19.9 & 19.9 & 19.6 & 0.0 & 9.6 & 103 & 79 & 0.0 \\
\hline $10 / 24 / 01$ & $0: 26$ & 19.8 & 19.9 & 19.9 & 19.7 & 0.0 & 9.6 & 88 & 79 & 0.0 \\
\hline $10 / 24 / 01$ & $0: 27$ & 19.7 & 19.9 & 19.9 & 19.6 & 0.0 & 9.7 & 120 & 79 & 0.0 \\
\hline $10 / 24 / 01$ & $0: 28$ & 19.8 & 19.9 & 19.9 & 19.7 & 0.0 & 9.8 & 88 & 79 & 0.0 \\
\hline $10 / 24 / 01$ & $0: 29$ & 19.7 & 19.9 & 20.0 & 19.6 & 0.0 & 9.6 & 92 & 79 & 0.0 \\
\hline $10 / 24 / 01$ & $0: 30$ & 19.8 & 19.9 & 19.9 & 19.7 & 0.0 & 9.6 & 119 & 79 & 0.0 \\
\hline $10 / 24 / 01$ & $0: 31$ & 19.8 & 19.9 & 20.0 & 19.7 & 0.0 & 9.6 & 109 & 79 & 0.0 \\
\hline $10 / 24 / 01$ & $0: 32$ & 19.8 & 19.9 & 20.0 & 19.7 & 0.0 & 9.7 & 119 & 79 & 0.0 \\
\hline $10 / 24 / 01$ & $0: 33$ & 19.8 & 19.9 & 20.0 & 19.7 & 0.0 & 9.6 & 118 & 79 & 0.0 \\
\hline $10 / 24 / 01$ & $0: 34$ & 19.7 & 19.9 & 20.0 & 19.7 & 0.0 & 9.6 & 118 & 79 & 0.0 \\
\hline $10 / 24 / 01$ & $0: 35$ & 19.8 & 20.0 & 20.0 & 19.7 & 0.0 & 9.7 & 102 & 79 & 0.0 \\
\hline $10 / 24 / 01$ & $0: 36$ & 19.7 & 19.9 & 20.0 & 19.7 & 0.0 & 9.6 & 117 & 79 & 0.0 \\
\hline $10 / 24 / 01$ & $0: 37$ & 19.8 & 20.0 & 20.0 & 19.7 & 0.0 & 9.7 & 90 & 79 & 0.0 \\
\hline $10 / 24 / 01$ & $0: 38$ & 19.8 & 20.0 & 20.0 & 19.7 & 0.0 & 9.7 & 119 & 79 & 0.0 \\
\hline $10 / 24 / 01$ & $0: 39$ & 19.8 & 20.0 & 20.0 & 19.7 & 0.0 & 9.6 & 115 & 79 & 0.0 \\
\hline $10 / 24 / 01$ & $0: 40$ & 19.8 & 20.0 & 20.0 & 19.7 & 0.0 & 9.7 & 91 & 79 & 0.0 \\
\hline $10 / 24 / 01$ & $0: 41$ & 19.8 & 19.9 & 20.0 & 19.7 & 0.0 & 9.7 & 112 & 79 & 0.0 \\
\hline $10 / 24 / 01$ & $0: 42$ & 19.8 & 19.9 & 20.0 & 19.7 & 0.0 & 9.6 & 105 & 79 & 0.0 \\
\hline $10 / 24 / 01$ & $0: 43$ & 19.8 & 20.0 & 20.0 & 19.7 & 0.0 & 9.5 & 111 & 79 & 0.0 \\
\hline $10 / 24 / 01$ & $0: 44$ & 19.8 & 20.0 & 20.0 & 19.7 & 0.0 & 9.7 & 98 & 79 & 0.0 \\
\hline $10 / 24 / 01$ & $0: 45$ & 19.8 & 20.0 & 20.0 & 19.7 & 0.0 & 9.8 & 76 & 79 & 0.0 \\
\hline $10 / 24 / 01$ & $0: 46$ & 19.8 & 20.0 & 20.0 & 19.7 & 0.0 & 9.7 & 107 & 79 & 0.0 \\
\hline $10 / 24 / 01$ & $0: 47$ & 19.8 & 20.0 & 20.0 & 19.7 & 0.0 & 9.7 & 104 & 79 & 0.0 \\
\hline $10 / 24 / 01$ & $0: 48$ & 19.8 & 20.0 & 20.0 & 19.7 & 0.0 & 9.6 & 108 & 79 & 0.0 \\
\hline $10 / 24 / 01$ & $0: 49$ & 19.8 & 20.0 & 20.0 & 19.7 & 0.0 & 9.6 & 104 & 79 & 0.0 \\
\hline $10 / 24 / 01$ & $0: 50$ & 19.8 & 20.0 & 20.0 & 19.7 & 0.0 & 9.6 & 82 & 79 & 0.0 \\
\hline $10 / 24 / 01$ & $0: 51$ & 19.8 & 20.0 & 20.0 & 19.7 & 0.0 & 9.6 & 90 & 79 & 0.0 \\
\hline $10 / 24 / 01$ & 0:52 & 19.8 & 20.0 & 20.0 & 19.7 & 0.0 & 9.7 & 106 & 79 & 0.0 \\
\hline $10 / 24 / 01$ & $0: 53$ & 19.8 & 20.0 & 20.0 & 19.7 & 0.0 & 9.7 & 102 & 79 & 0.0 \\
\hline $10 / 24 / 01$ & $0: 54$ & 19.8 & 20.0 & 20.0 & 19.7 & 0.0 & 9.6 & 94 & 79 & 0.0 \\
\hline $10 / 24 / 01$ & $0: 55$ & 19.8 & 20.0 & 20.0 & 19.7 & 0.0 & 9.6 & 95 & 79 & 0.0 \\
\hline $10 / 24 / 01$ & $0: 56$ & 19.8 & 20.0 & 20.0 & 19.7 & 0.0 & 9.5 & 81 & 79 & 0.0 \\
\hline $10 / 24 / 01$ & $0: 57$ & 19.8 & 20.0 & 20.0 & 19.7 & 0.0 & 9.7 & 100 & 79 & 0.0 \\
\hline $10 / 24 / 01$ & 0:58 & 19.8 & 20.0 & 20.0 & 19.7 & 0.0 & 9.7 & 96 & 79 & 0.0 \\
\hline $10 / 24 / 01$ & $0: 59$ & 19.8 & 20.0 & 20.0 & 19.7 & 0.0 & 9.5 & 93 & 79 & 0.0 \\
\hline $10 / 24 / 01$ & 1:00 & 19.8 & 20.0 & \begin{tabular}{|l|} 
\\
\end{tabular} & 19.7 & 0.0 & 9.6 & 82 & 79 & 0.0 \\
\hline $10 / 24 / 01$ & 1:01 & 19.9 & 20.0 & 20.0 & 19.8 & 0.0 & 9.6 & 68 & 79 & 0.0 \\
\hline $10 / 24 / 01$ & 1:02 & 19.6 & 20.0 & 20.0 & 19.6 & 0.0 & 9.7 & 79 & 79 & 0.0 \\
\hline $10 / 24 / 01$ & 1:03 & 19.7 & 20.0 & 20.0 & 19.6 & 0.0 & 9.6 & 86 & 79 & 0.0 \\
\hline $10 / 24 / 01$ & 1:04 & 19.6 & 20.0 & 20.0 & 19.5 & 0.0 & 9.7 & 89 & 79 & 0.0 \\
\hline $10 / 24 / 01$ & 1:05 & 19.7 & 20.0 & 20.0 & 19.6 & 0.0 & 9.6 & 71 & 79 & 0.0 \\
\hline $10 / 24 / 01$ & 1:06 & 19.6 & 20.0 & 20.0 & 19.5 & 0.0 & 9.6 & 77 & 79 & 0.0 \\
\hline $10 / 24 / 01$ & 1:07 & 19.6 & 20.0 & 20.0 & 19.5 & 0.0 & 9.6 & 81 & 79 & 0.0 \\
\hline $10 / 24 / 01$ & 1:08 & 19.7 & 20.0 & 20.0 & 19.6 & 0.0 & 9.7 & 77 & 79 & 0.0 \\
\hline $10 / 24 / 01$ & 1:09 & 19.5 & 19.9 & 20.0 & 19.5 & 0.0 & 9.6 & 88 & 79 & 0.0 \\
\hline $10 / 24 / 01$ & $1: 10$ & 19.7 & 19.9 & \begin{tabular}{|l|}
19.9 \\
\end{tabular} & 19.6 & 0.0 & 9.6 & 77 & 79 & 0.0 \\
\hline $10 / 24 / 01$ & 1:11 & 19.5 & 19.9 & 19.9 & 19.4 & 0.0 & 9.6 & 71 & 79 & 0.0 \\
\hline $10 / 24 / 01$ & $1: 12$ & 19.7 & 19.9 & \begin{tabular}{|l|}
19.9 \\
\end{tabular} & 19.6 & 0.0 & 9.6 & 81 & 79 & 0.0 \\
\hline $10 / 24 / 01$ & 1:13 & 19.5 & 19.9 & 19.9 & 19.4 & 0.0 & 9.6 & 65 & 79 & 0.0 \\
\hline $10 / 24 / 01$ & $1: 14$ & 19.6 & 19.9 & \begin{tabular}{|l|}
19.9 \\
\end{tabular} & 19.6 & 0.0 & 9.6 & 65 & 79 & 0.0 \\
\hline $10 / 24 / 01$ & 1:15 & 19.5 & 19.9 & \begin{tabular}{|l|} 
\\
\end{tabular} & 19.4 & 0.0 & 9.6 & 65 & 79 & 0.0 \\
\hline $10 / 24 / 01$ & 1:16 & 19.5 & 19.9 & \begin{tabular}{|l|}
19.9 \\
\end{tabular} & 19.5 & 0.0 & 9.7 & 65 & 79 & 0.0 \\
\hline $10 / 24 / 01$ & 1:17 & 19.5 & 19.9 & 19.9 & 19.4 & 0.0 & 9.7 & 65 & 79 & 0.0 \\
\hline $10 / 24 / 01$ & $1: 18$ & 19.5 & 19.9 & \begin{tabular}{|l|} 
\\
\end{tabular} & 19.4 & 0.0 & 9.6 & 65 & 79 & 0.0 \\
\hline $10 / 24 / 01$ & 1:19 & 19.6 & 19.8 & \begin{tabular}{|l|} 
\\
\end{tabular} & 19.5 & 0.0 & 9.7 & 65 & 79 & 0.0 \\
\hline $10 / 24 / 01$ & $1: 20$ & 19.4 & 19.8 & \begin{tabular}{|l|}
19.9 \\
\end{tabular} & 19.4 & 0.0 & 9.6 & 65 & 79 & 0.0 \\
\hline $10 / 24 / 01$ & $1: 21$ & 19.6 & 19.8 & \begin{tabular}{|l|}
19.9 \\
\end{tabular} & 19.5 & 0.0 & 9.6 & 65 & 79 & 0.0 \\
\hline $10 / 24 / 01$ & $1: 22$ & 19.4 & 19.8 & \begin{tabular}{|l|}
19.8 \\
\end{tabular} & 19.3 & 0.0 & 9.7 & 65 & 79 & 0.0 \\
\hline $10 / 24 / 01$ & 1:23 & 19.5 & 19.8 & \begin{tabular}{|l|}
19.8 \\
\end{tabular} & 19.5 & 0.0 & 9.6 & 65 & 79 & 0.0 \\
\hline
\end{tabular}


WSRC-TR-2002-00459, Rev. 0 SRT-RPP-2002-00221

\begin{tabular}{|c|c|c|c|c|c|c|c|c|c|c|}
\hline \multicolumn{3}{|c|}{ |PREC2_102301_0623 } & \multirow[b]{2}{*}{$\mid \begin{array}{c}\text { REC } \\
\text { PP } \\
\text { OUT } \\
\left({ }^{\circ} \mathrm{C}\right) \\
\text { TC1 }\end{array}$} & \multirow[b]{2}{*}{\begin{tabular}{|c|} 
TK \\
BOT \\
$\left({ }^{\circ} \mathrm{C}\right)$ \\
TC2 \\
\end{tabular}} & \multirow[b]{2}{*}{\begin{tabular}{|c|} 
HX \\
OUT \\
$\left({ }^{\circ} \mathrm{C}\right)$ \\
TC3 \\
\end{tabular}} & \multirow[b]{2}{*}{$\begin{array}{c}\text { Mn } \\
\text { Flow } \\
(\mathrm{gpm})\end{array}$} & \multirow[b]{2}{*}{\begin{tabular}{|c|} 
Galigher \\
Flow \\
(gpm)
\end{tabular}} & \multirow[b]{2}{*}{$\begin{array}{c}\text { Current } \\
\text { to } \mathrm{HX}\end{array}$} & \multirow[b]{2}{*}{$\begin{array}{c}\text { Voltage } \\
\text { to } \mathrm{HX}\end{array}$} & \multirow[b]{2}{*}{$\begin{array}{c}\mathrm{Sr} \\
\text { Flow } \\
\text { (gpm) }\end{array}$} \\
\hline DATE & TIME & $\begin{array}{c}\mathrm{HX} \\
\text { Outlet } \\
\left({ }^{\circ} \mathrm{C}\right) \\
\text { TCO }\end{array}$ & & & & & & & & \\
\hline $10 / 24 / 01$ & $1: 24$ & 19.4 & 19.8 & 19.8 & 19.3 & 0.0 & 9.7 & 65 & 79 & 0.0 \\
\hline 10/24/01 & $1: 25$ & 19.5 & 19.8 & 19.8 & \begin{tabular}{|l|l}
19.4 \\
\end{tabular} & 0.0 & 9.6 & 65 & 79 & 0.0 \\
\hline $10 / 24 / 01$ & $1: 26$ & 19.4 & $\mid 19.8$ & 19.8 & 19.4 & 0.0 & 9.7 & 65 & 79 & 0.0 \\
\hline $10 / 24 / 01$ & $1: 27$ & 19.4 & \begin{tabular}{|c|}
19.8 \\
\end{tabular} & 19.8 & $\mid 19.4$ & 0.0 & 9.6 & 65 & 79 & 0.0 \\
\hline $10 / 24 / 01$ & $1: 28$ & 19.5 & $\mid 19.8$ & 19.8 & $\mid 19.4$ & 0.0 & 9.7 & 65 & 79 & 0.0 \\
\hline $10 / 24 / 01$ & $1: 29$ & 19.4 & $\mid 19.8$ & 19.8 & 19.3 & 0.0 & 9.6 & 65 & 79 & 0.0 \\
\hline $10 / 24 / 01$ & $1: 30$ & 19.5 & \begin{tabular}{|c|}
$\mid 19.7$ \\
\end{tabular} & 19.8 & $\mid 19.4$ & 0.0 & 9.6 & 65 & 79 & 0.0 \\
\hline $10 / 24 / 01$ & $1: 31$ & 19.3 & \begin{tabular}{|c|}
$\mid 19.7$ \\
\end{tabular} & 19.8 & 19.3 & 0.0 & 9.7 & 65 & 79 & 0.0 \\
\hline $10 / 24 / 01$ & $1: 32$ & 19.5 & \begin{tabular}{|c|}
$\mid 19.7$ \\
\end{tabular} & $\mid 19.7$ & $\mid 19.4$ & 0.0 & 9.6 & 65 & 79 & 0.0 \\
\hline $10 / 24 / 01$ & $1: 33$ & 19.3 & \begin{tabular}{|c|}
$\mid 19.7$ \\
\end{tabular} & 19.7 & 19.2 & 0.0 & 9.6 & 65 & 79 & 0.0 \\
\hline $10 / 24 / 01$ & $1: 34$ & 19.4 & \begin{tabular}{|c|}
$\mid 19.7$ \\
\end{tabular} & 19.7 & \begin{tabular}{|l|l}
19.4 \\
\end{tabular} & 0.0 & 9.7 & 65 & 79 & 0.0 \\
\hline $10 / 24 / 01$ & $1: 35$ & 19.3 & \begin{tabular}{|l|}
19.7 \\
\end{tabular} & $\mid 19.7$ & 19.3 & 0.0 & 9.7 & 65 & 79 & 0.0 \\
\hline $10 / 24 / 01$ & 1:36 & 19.4 & \begin{tabular}{|l|}
19.7 \\
\end{tabular} & \begin{tabular}{|l|}
19.7 \\
\end{tabular} & 19.3 & 0.0 & 9.5 & 65 & 79 & 0.0 \\
\hline $10 / 24 / 01$ & $1: 37$ & 19.4 & \begin{tabular}{|l|}
19.7 \\
\end{tabular} & \begin{tabular}{|l|}
19.7 \\
\end{tabular} & 19.3 & 0.0 & 9.8 & 65 & 79 & 0.0 \\
\hline $10 / 24 / 01$ & $1: 38$ & 19.3 & \begin{tabular}{|l|}
19.7 \\
\end{tabular} & \begin{tabular}{|l|}
19.7 \\
\end{tabular} & \begin{tabular}{|l|}
19.2 \\
\end{tabular} & 0.0 & 9.7 & 65 & 79 & 0.0 \\
\hline $10 / 24 / 01$ & 1:39 & 19.4 & \begin{tabular}{|l|}
$\mid 19.7$ \\
\end{tabular} & $\mid 19.7$ & 19.3 & 0.0 & 9.6 & 65 & 79 & 0.0 \\
\hline $10 / 24 / 01$ & $1: 40$ & 19.3 & $|19.7|$ & \begin{tabular}{|l|}
19.7 \\
\end{tabular} & \begin{tabular}{|l|}
19.2 \\
\end{tabular} & 0.0 & 9.6 & 65 & 79 & 0.0 \\
\hline $10 / 24 / 01$ & $1: 41$ & 19.4 & \begin{tabular}{|l|}
19.6 \\
\end{tabular} & 19.7 & 19.3 & 0.0 & 9.7 & 65 & 79 & 0.0 \\
\hline $10 / 24 / 01$ & $1: 42$ & 19.3 & \begin{tabular}{|l|} 
\\
\end{tabular} & \begin{tabular}{|l|}
19.7 \\
\end{tabular} & \begin{tabular}{|l|}
19.2 \\
\end{tabular} & 0.0 & 9.6 & 65 & 79 & 0.0 \\
\hline $10 / 24 / 01$ & $1: 43$ & 19.4 & \begin{tabular}{|l|}
19.6 \\
\end{tabular} & 19.6 & 19.3 & 0.0 & 9.7 & 65 & 79 & 0.0 \\
\hline $10 / 24 / 01$ & $1: 44$ & 19.3 & \begin{tabular}{|l|}
19.6 \\
\end{tabular} & \begin{tabular}{|l|} 
\\
\end{tabular} & \begin{tabular}{|l|}
19.2 \\
\end{tabular} & 0.0 & 9.7 & 65 & 79 & 0.0 \\
\hline $10 / 24 / 01$ & $1: 45$ & 19.3 & \begin{tabular}{|l|}
19.6 \\
\end{tabular} & \begin{tabular}{|l|}
19.6 \\
\end{tabular} & 19.3 & 0.0 & 9.6 & 65 & 79 & 0.0 \\
\hline $10 / 24 / 01$ & $1: 46$ & 19.3 & \begin{tabular}{|l|}
19.6 \\
\end{tabular} & \begin{tabular}{|l|} 
\\
\end{tabular} & \begin{tabular}{|l|}
19.2 \\
\end{tabular} & 0.0 & 9.6 & 65 & 79 & 0.0 \\
\hline $10 / 24 / 01$ & $1: 47$ & 19.3 & $\mid 19.6$ & \begin{tabular}{|l|}
19.6 \\
\end{tabular} & \begin{tabular}{|l|}
19.2 \\
\end{tabular} & 0.0 & 9.7 & 65 & 79 & 0.0 \\
\hline $10 / 24 / 01$ & $1: 48$ & 19.3 & \begin{tabular}{|l|}
19.6 \\
\end{tabular} & \begin{tabular}{|l|}
19.6 \\
\end{tabular} & \begin{tabular}{|l|}
19.2 \\
\end{tabular} & 0.0 & 9.6 & 65 & 79 & 0.0 \\
\hline $10 / 24 / 01$ & $1: 49$ & 19.2 & \begin{tabular}{|l|}
19.6 \\
\end{tabular} & \begin{tabular}{|l|} 
\\
\end{tabular} & \begin{tabular}{|l|}
19.1 \\
\end{tabular} & 0.0 & 9.7 & 65 & 79 & 0.0 \\
\hline $10 / 24 / 01$ & $1: 50$ & 19.3 & $\mid 19.5$ & \begin{tabular}{|l|}
19.6 \\
\end{tabular} & \begin{tabular}{|l|}
19.2 \\
\end{tabular} & 0.0 & 9.6 & 65 & 79 & 0.0 \\
\hline $10 / 24 / 01$ & 1:51 & 19.2 & \begin{tabular}{|l|}
19.6 \\
\end{tabular} & \begin{tabular}{|l|} 
\\
\end{tabular} & 19.1 & 0.0 & 9.6 & 65 & 79 & 0.0 \\
\hline $10 / 24 / 01$ & 1:52 & 19.3 & \begin{tabular}{|l|}
19.5 \\
\end{tabular} & \begin{tabular}{|l|}
19.6 \\
\end{tabular} & \begin{tabular}{|l|}
19.2 \\
\end{tabular} & 0.0 & 9.7 & 65 & 79 & 0.0 \\
\hline $10 / 24 / 01$ & $1: 53$ & 19.2 & \begin{tabular}{|l|} 
\\
\end{tabular} & \begin{tabular}{|l|}
19.6 \\
\end{tabular} & \begin{tabular}{|l|}
19.1 \\
\end{tabular} & 0.0 & 9.7 & 65 & 79 & 0.0 \\
\hline $10 / 24 / 01$ & $1: 54$ & 19.3 & \begin{tabular}{|l|}
19.5 \\
\end{tabular} & \begin{tabular}{|l|}
19.6 \\
\end{tabular} & \begin{tabular}{|l|}
19.2 \\
\end{tabular} & 0.0 & 9.6 & 65 & 79 & 0.0 \\
\hline $10 / 24 / 01$ & $1: 55$ & 19.2 & \begin{tabular}{|l|}
19.5 \\
\end{tabular} & \begin{tabular}{|l|}
19.6 \\
\end{tabular} & \begin{tabular}{|l|}
19.1 \\
\end{tabular} & 0.0 & 9.7 & 65 & 79 & 0.0 \\
\hline $10 / 24 / 01$ & $1: 56$ & 19.2 & 19.5 & 19.5 & \begin{tabular}{|l|}
19.2 \\
\end{tabular} & 0.0 & 9.5 & 65 & 79 & 0.0 \\
\hline $10 / 24 / 01$ & $1: 57$ & 19.2 & \begin{tabular}{|l|}
19.5 \\
\end{tabular} & \begin{tabular}{|l|}
19.5 \\
\end{tabular} & \begin{tabular}{|l|}
19.1 \\
\end{tabular} & 0.0 & 9.6 & 65 & 79 & 0.0 \\
\hline $10 / 24 / 01$ & $1: 58$ & 19.2 & 19.5 & 19.5 & 19.1 & 0.0 & 9.7 & 65 & 79 & 0.0 \\
\hline $10 / 24 / 01$ & $1: 59$ & 19.2 & 19.5 & 19.5 & \begin{tabular}{|l|}
19.1 \\
\end{tabular} & 0.0 & 9.6 & 65 & 79 & 0.0 \\
\hline $10 / 24 / 01$ & $2: 00$ & 19.1 & \begin{tabular}{|l|}
19.5 \\
\end{tabular} & \begin{tabular}{|l|}
19.5 \\
\end{tabular} & \begin{tabular}{|l|}
19.0 \\
\end{tabular} & 0.0 & 9.7 & 65 & 79 & 0.0 \\
\hline $10 / 24 / 01$ & 2:01 & 19.3 & 19.5 & 19.5 & \begin{tabular}{|l|}
19.2 \\
\end{tabular} & 0.0 & 9.7 & 65 & 79 & 0.0 \\
\hline $10 / 24 / 01$ & 2:02 & 19.1 & \begin{tabular}{|l|}
19.5 \\
\end{tabular} & \begin{tabular}{|l|}
19.5 \\
\end{tabular} & 19.0 & 0.0 & 9.7 & 65 & 79 & 0.0 \\
\hline $10 / 24 / 01$ & 2:03 & 19.2 & \begin{tabular}{|l|}
19.5 \\
\end{tabular} & 19.5 & \begin{tabular}{|l|}
19.2 \\
\end{tabular} & 0.0 & 9.6 & 65 & 79 & 0.0 \\
\hline $10 / 24 / 01$ & 2:04 & 19.1 & \begin{tabular}{|l|}
19.5 \\
\end{tabular} & 19.5 & 19.0 & 0.0 & 9.7 & 65 & 79 & 0.0 \\
\hline $10 / 24 / 01$ & 2:05 & 19.2 & $\mid 19.4$ & \begin{tabular}{|l|}
19.5 \\
\end{tabular} & \begin{tabular}{|l|}
19.1 \\
\end{tabular} & 0.0 & 9.8 & 65 & 79 & 0.0 \\
\hline $10 / 24 / 01$ & 2:06 & 19.1 & \begin{tabular}{|l|}
$\mid 19.4$ \\
\end{tabular} & 19.5 & \begin{tabular}{|l|}
19.0 \\
\end{tabular} & 0.0 & 9.7 & 65 & 79 & 0.0 \\
\hline $10 / 24 / 01$ & 2:07 & 19.1 & \begin{tabular}{|l|}
19.4 \\
\end{tabular} & 19.5 & 19.0 & 0.0 & 9.7 & 65 & 79 & 0.0 \\
\hline $10 / 24 / 01$ & 2:08 & 19.2 & \begin{tabular}{|l|} 
\\
\end{tabular} & \begin{tabular}{|l|} 
\\
\end{tabular} & 19.0 & 0.0 & 9.6 & 65 & 79 & 0.0 \\
\hline $10 / 24 / 01$ & $2: 09$ & 19.1 & \begin{tabular}{|l|}
19.4 \\
\end{tabular} & \begin{tabular}{|l|}
19.4 \\
\end{tabular} & \begin{tabular}{|l|}
19.0 \\
\end{tabular} & 0.0 & 9.7 & 65 & 79 & 0.0 \\
\hline $10 / 24 / 01$ & $2: 10$ & 19.2 & \begin{tabular}{|l|}
19.4 \\
\end{tabular} & \begin{tabular}{|l|}
19.4 \\
\end{tabular} & \begin{tabular}{|l|}
19.1 \\
\end{tabular} & 0.0 & 9.7 & 65 & 79 & 0.0 \\
\hline $10 / 24 / 01$ & 2:11 & 19.0 & $\mid 19.4$ & \begin{tabular}{|l|} 
\\
\end{tabular} & 19.0 & 0.0 & 9.5 & 65 & 79 & 0.0 \\
\hline $10 / 24 / 01$ & $2: 12$ & 19.2 & \begin{tabular}{|l|}
19.4 \\
\end{tabular} & \begin{tabular}{|l|}
19.4 \\
\end{tabular} & \begin{tabular}{|l|}
19.1 \\
\end{tabular} & 0.0 & 9.6 & 65 & 79 & 0.0 \\
\hline $10 / 24 / 01$ & 2:13 & 19.0 & \begin{tabular}{|l|} 
\\
\end{tabular} & 19.4 & 19.0 & 0.0 & 9.7 & 65 & 79 & 0.0 \\
\hline $10 / 24 / 01$ & 2:14 & 19.1 & \begin{tabular}{|l|}
19.4 \\
\end{tabular} & \begin{tabular}{|l|}
19.4 \\
\end{tabular} & \begin{tabular}{|l|}
19.1 \\
\end{tabular} & 0.0 & 9.6 & 65 & 79 & 0.0 \\
\hline $10 / 24 / 01$ & 2:15 & 19.1 & \begin{tabular}{|l|}
19.4 \\
\end{tabular} & \begin{tabular}{|l|} 
\\
\end{tabular} & 19.0 & 0.0 & 9.6 & 65 & 79 & 0.0 \\
\hline $10 / 24 / 01$ & $2: 16$ & 19.1 & \begin{tabular}{|l|}
19.4 \\
\end{tabular} & \begin{tabular}{|l|} 
\\
\end{tabular} & 19.0 & 0.0 & 9.6 & 65 & 79 & 0.0 \\
\hline $10 / 24 / 01$ & $2: 17$ & 19.1 & \begin{tabular}{|l|}
19.4 \\
\end{tabular} & \begin{tabular}{|l|} 
\\
\end{tabular} & 19.0 & 0.0 & 9.6 & 65 & 79 & 0.0 \\
\hline $10 / 24 / 01$ & $2: 18$ & 19.0 & \begin{tabular}{|l|}
19.4 \\
\end{tabular} & \begin{tabular}{|l|} 
\\
\end{tabular} & 19.0 & 0.0 & 9.6 & 65 & 79 & 0.0 \\
\hline $10 / 24 / 01$ & $2: 19$ & 19.1 & \begin{tabular}{|l|}
19.3 \\
\end{tabular} & \begin{tabular}{|l|}
19.4 \\
\end{tabular} & \begin{tabular}{|l|}
19.0 \\
\end{tabular} & 0.0 & 9.7 & 65 & 79 & 0.0 \\
\hline $10 / 24 / 01$ & $2: 20$ & 19.0 & \begin{tabular}{|l|} 
\\
\end{tabular} & \begin{tabular}{|l|}
19.4 \\
\end{tabular} & 18.9 & 0.0 & 9.6 & 65 & 79 & 0.0 \\
\hline $10 / 24 / 01$ & $2: 21$ & 19.1 & \begin{tabular}{|l|} 
\\
\end{tabular} & \begin{tabular}{|l|} 
\\
\end{tabular} & 19.0 & 0.0 & 9.6 & 65 & 79 & 0.0 \\
\hline $10 / 24 / 01$ & $2: 22$ & 19.0 & \begin{tabular}{|l|} 
\\
\end{tabular} & \begin{tabular}{|l|} 
\\
\end{tabular} & 18.9 & 0.0 & 9.7 & 65 & 79 & 0.0 \\
\hline $10 / 24 / 01$ & 2:23 & 19.1 & \begin{tabular}{|l|}
19.3 \\
\end{tabular} & \begin{tabular}{|l|}
19.4 \\
\end{tabular} & 19.0 & 0.0 & 9.5 & 65 & 79 & 0.0 \\
\hline
\end{tabular}

\begin{tabular}{|c|c|c|c|c|c|c|c|c|c|c|}
\hline \multicolumn{11}{|c|}{ PREC2_102301_0623 } \\
\hline DATE & TIME & \begin{tabular}{|c}
$\mathrm{HX}$ \\
Outlet \\
$\left({ }^{\circ} \mathrm{C}\right)$ \\
$\mathrm{TC} 0$
\end{tabular} & \begin{tabular}{|c} 
REC \\
PP \\
OUT \\
$\left({ }^{\circ} \mathrm{C}\right)$ \\
TC1
\end{tabular} & $\begin{array}{c}\text { TK } \\
\text { BOT } \\
\left({ }^{\circ} \mathrm{C}\right) \\
\text { TC2 }\end{array}$ & $\begin{array}{l}\text { HX } \\
\text { OUT } \\
\left({ }^{\circ} \mathrm{C}\right) \\
\text { TC3 }\end{array}$ & \begin{tabular}{|c|} 
Mn \\
Flow \\
(gpm)
\end{tabular} & $\begin{array}{c}\text { Galigher } \\
\text { Flow } \\
\text { (gpm) }\end{array}$ & $\begin{array}{c}\text { Current } \\
\text { to } \mathrm{HX}\end{array}$ & $\begin{array}{l}\text { Voltage } \\
\text { to } \mathrm{HX}\end{array}$ & $\begin{array}{c}\mathrm{Sr} \\
\text { Flow } \\
\text { (gpm) }\end{array}$ \\
\hline $10 / 24 / 01$ & 2:24 & 19.0 & 19.4 & 19.4 & 18.9 & 0.0 & 9.7 & 65 & 79 & 0.0 \\
\hline $10 / 24 / 01$ & 2:25 & 19.1 & 19.4 & 19.4 & 19.0 & 0.0 & 9.7 & 65 & 79 & 0.0 \\
\hline $10 / 24 / 01$ & $2: 26$ & 19.0 & 19.3 & 19.4 & 19.0 & 0.0 & 9.6 & 65 & 79 & 0.0 \\
\hline $10 / 24 / 01$ & 2:27 & 19.0 & 19.3 & 19.4 & 19.0 & 0.0 & 9.7 & 65 & 79 & 0.0 \\
\hline $10 / 24 / 01$ & $2: 28$ & 19.1 & 19.3 & 19.3 & 19.0 & 0.0 & 9.6 & 65 & 79 & 0.0 \\
\hline $10 / 24 / 01$ & $2: 29$ & 19.0 & 19.3 & 19.3 & 18.9 & 0.0 & 9.6 & 65 & 79 & 0.0 \\
\hline $10 / 24 / 01$ & $2: 30$ & 19.1 & 19.3 & 19.3 & 19.0 & 0.0 & 9.6 & 65 & 79 & 0.0 \\
\hline 10/24/01 & $2: 31$ & 18.9 & 19.3 & 19.3 & 18.9 & 0.0 & 9.6 & 65 & 79 & 0.0 \\
\hline $10 / 24 / 01$ & 2:32 & 19.1 & 19.3 & 19.3 & 19.0 & 0.0 & 9.7 & 69 & 79 & 0.0 \\
\hline $10 / 24 / 01$ & $2: 33$ & 18.9 & 19.3 & 19.3 & 18.9 & 0.0 & 9.6 & 70 & 79 & 0.0 \\
\hline $10 / 24 / 01$ & $2: 34$ & 19.0 & 19.3 & 19.3 & 19.0 & 0.0 & 9.7 & 71 & 79 & 0.0 \\
\hline $10 / 24 / 01$ & $2: 35$ & 18.9 & 19.3 & 19.3 & 18.8 & 0.0 & 9.7 & 79 & 79 & 0.0 \\
\hline $10 / 24 / 01$ & 2:36 & 19.0 & 19.3 & 19.3 & 18.9 & 0.0 & 9.7 & 71 & 79 & 0.0 \\
\hline $10 / 24 / 01$ & 2:37 & 19.0 & 19.3 & 19.3 & 18.9 & 0.0 & 9.6 & 72 & 79 & 0.0 \\
\hline $10 / 24 / 01$ & 2:38 & 18.9 & 19.3 & 19.3 & 18.9 & 0.0 & 9.7 & 77 & 79 & 0.0 \\
\hline $10 / 24 / 01$ & 2:39 & 19.0 & 19.3 & 19.3 & 18.9 & 0.0 & 9.6 & 73 & 79 & 0.0 \\
\hline $10 / 24 / 01$ & $2: 40$ & 18.9 & \begin{tabular}{|l|}
19.2 \\
\end{tabular} & 19.3 & 18.8 & 0.0 & 9.7 & 87 & 79 & 0.0 \\
\hline $10 / 24 / 01$ & 2:41 & 19.0 & 19.2 & 19.3 & 18.9 & 0.0 & 9.6 & 85 & 79 & 0.0 \\
\hline $10 / 24 / 01$ & 2:42 & 18.9 & 19.2 & 19.3 & 18.8 & 0.0 & 9.7 & 87 & 79 & 0.0 \\
\hline $10 / 24 / 01$ & 2:43 & 19.0 & 19.2 & 19.2 & 18.9 & 0.0 & 9.7 & 63 & 79 & 0.0 \\
\hline $10 / 24 / 01$ & 2:44 & 18.9 & 19.2 & 19.2 & 18.8 & 0.0 & 9.6 & 71 & 79 & 0.0 \\
\hline $10 / 24 / 01$ & $2: 45$ & 19.0 & 19.2 & 19.2 & 18.9 & 0.0 & 9.7 & 81 & 79 & 0.0 \\
\hline $10 / 24 / 01$ & $2: 46$ & 18.9 & 19.2 & 19.2 & 18.8 & 0.0 & 9.7 & 75 & 79 & 0.0 \\
\hline $10 / 24 / 01$ & $2: 47$ & 18.9 & 19.2 & 19.2 & 18.8 & 0.0 & 9.6 & 65 & 79 & 0.0 \\
\hline $10 / 24 / 01$ & 2:48 & 18.9 & \begin{tabular}{|l|}
19.2 \\
\end{tabular} & 19.2 & 18.8 & 0.0 & 9.6 & 93 & 79 & 0.0 \\
\hline $10 / 24 / 01$ & $2: 49$ & 18.8 & \begin{tabular}{|l|}
19.2 \\
\end{tabular} & 19.2 & 18.8 & 0.0 & 9.7 & 68 & 79 & 0.0 \\
\hline $10 / 24 / 01$ & 2:50 & 18.9 & 19.2 & 19.2 & 18.8 & 0.0 & 9.6 & 92 & 79 & 0.0 \\
\hline $10 / 24 / 01$ & 2:51 & 18.8 & 19.2 & 19.2 & 18.7 & 0.0 & 9.6 & 86 & 79 & 0.0 \\
\hline $10 / 24 / 01$ & 2:52 & 19.0 & \begin{tabular}{|l|}
19.2 \\
\end{tabular} & 19.2 & 18.9 & 0.0 & 9.6 & 83 & 79 & 0.0 \\
\hline $10 / 24 / 01$ & 2:53 & 18.8 & \begin{tabular}{|l|}
19.1 \\
\end{tabular} & 19.2 & 18.7 & 0.0 & 9.6 & 93 & 79 & 0.0 \\
\hline $10 / 24 / 01$ & 2:54 & 18.9 & \begin{tabular}{|l|} 
\\
\end{tabular} & 19.2 & 18.9 & 0.0 & 9.6 & 98 & 79 & 0.0 \\
\hline $10 / 24 / 01$ & 2:55 & 18.8 & 19.2 & 19.2 & 18.7 & 0.0 & 9.6 & 96 & 79 & 0.0 \\
\hline $10 / 24 / 01$ & 2:56 & 18.9 & 19.1 & 19.1 & 18.8 & 0.0 & 9.7 & 98 & 79 & 0.0 \\
\hline $10 / 24 / 01$ & 2:57 & 18.9 & \begin{tabular}{|l|}
19.1 \\
\end{tabular} & 19.1 & 18.7 & 0.0 & 9.6 & 87 & 79 & 0.0 \\
\hline $10 / 24 / 01$ & 2:58 & 18.8 & 19.1 & 19.1 & 18.8 & 0.0 & 9.7 & 97 & 79 & 0.0 \\
\hline $10 / 24 / 01$ & 2:59 & 18.9 & \begin{tabular}{|l|} 
\\
\end{tabular} & 19.1 & 18.8 & 0.0 & 9.6 & 70 & 79 & 0.0 \\
\hline $10 / 24 / 01$ & 3:00 & 18.8 & \begin{tabular}{|l|}
19.1 \\
\end{tabular} & 19.1 & 18.7 & 0.0 & 9.7 & 93 & 79 & 0.0 \\
\hline $\mid 10 / 24 / 01$ & 3:01 & 18.9 & \begin{tabular}{|l|} 
\\
\end{tabular} & 19.1 & 18.8 & 0.0 & 9.6 & 104 & 79 & 0.0 \\
\hline $10 / 24 / 01$ & 3:02 & 18.8 & \begin{tabular}{|l|} 
\\
\end{tabular} & 19.1 & 18.7 & 0.0 & 9.7 & 106 & 79 & 0.0 \\
\hline $10 / 24 / 01$ & 3:03 & 18.9 & 19.1 & 19.1 & 18.8 & 0.0 & 9.6 & 78 & 79 & 0.0 \\
\hline $10 / 24 / 01$ & 3:04 & 18.8 & $\mid 19.1$ & 19.1 & 18.7 & 0.0 & 9.6 & 104 & 79 & 0.0 \\
\hline $10 / 24 / 01$ & 3:05 & 18.9 & \begin{tabular}{|l|}
19.1 \\
\end{tabular} & 19.1 & 18.8 & 0.0 & 9.6 & 105 & 79 & 0.0 \\
\hline $\mid 10 / 24 / 01$ & 3:06 & 18.8 & \begin{tabular}{|l|} 
\\
\end{tabular} & 19.1 & 18.7 & 0.0 & 9.7 & 96 & 79 & 0.0 \\
\hline $10 / 24 / 01$ & 3:07 & 18.8 & 19.1 & 19.1 & 18.7 & 0.0 & 9.6 & 107 & 79 & 0.0 \\
\hline $10 / 24 / 01$ & 3:08 & 18.8 & 19.1 & 19.1 & 18.7 & 0.0 & 9.6 & 104 & 79 & 0.0 \\
\hline $10 / 24 / 01$ & 3:09 & 18.7 & 19.1 & 19.1 & 18.7 & 0.0 & 9.6 & 108 & 79 & 0.0 \\
\hline $10 / 24 / 01$ & $3: 10$ & 18.8 & \begin{tabular}{|l|}
19.1 \\
\end{tabular} & 19.1 & 18.7 & 0.0 & 9.8 & 94 & 79 & 0.0 \\
\hline $10 / 24 / 01$ & 3:11 & 18.7 & 19.1 & 19.1 & 18.6 & 0.0 & 9.7 & 95 & 79 & 0.0 \\
\hline $10 / 24 / 01$ & 3:12 & 18.9 & 19.0 & 19.1 & 18.8 & 0.0 & 9.6 & 109 & 79 & 0.0 \\
\hline $10 / 24 / 01$ & 3:13 & 18.7 & 19.1 & 19.1 & 18.6 & 0.0 & 9.6 & 110 & 79 & 0.0 \\
\hline $10 / 24 / 01$ & $3: 14$ & 18.8 & 19.0 & 19.1 & 18.8 & 0.0 & 9.6 & 105 & 79 & 0.0 \\
\hline $10 / 24 / 01$ & 3:15 & 18.7 & 19.0 & 19.1 & 18.6 & 0.0 & 9.7 & 88 & 79 & 0.0 \\
\hline $10 / 24 / 01$ & $3: 16$ & 18.8 & 19.0 & 19.1 & 18.7 & 0.0 & 9.7 & 113 & 79 & 0.0 \\
\hline $10 / 24 / 01$ & 3:17 & 18.7 & 19.0 & 19.0 & 18.6 & 0.0 & 9.6 & 116 & 79 & 0.0 \\
\hline $\mid 10 / 24 / 01$ & $3: 18$ & 18.7 & 19.0 & 19.0 & 18.7 & 0.0 & 9.6 & 101 & 79 & 0.0 \\
\hline $\mid 10 / 24 / 01$ & 3:19 & 18.8 & 19.0 & 19.0 & 18.7 & 0.0 & 9.6 & 117 & 79 & 0.0 \\
\hline $\mid 10 / 24 / 01$ & $3: 20$ & 18.7 & 19.0 & 19.0 & 18.6 & 0.0 & 9.7 & 120 & 79 & 0.0 \\
\hline $10 / 24 / 01$ & $3: 21$ & 18.8 & 19.0 & 19.0 & 18.7 & 0.0 & 9.6 & 118 & 79 & 0.0 \\
\hline $10 / 24 / 01$ & 3:22 & 18.7 & 19.0 & 19.0 & 18.6 & 0.0 & 9.6 & 92 & 79 & 0.0 \\
\hline $10 / 24 / 01$ & 3:23 & 18.8 & 19.0 & 19.0 & 18.7 & 0.0 & 9.6 & 114 & 79 & 0.0 \\
\hline
\end{tabular}


WSRC-TR-2002-00459, Rev. 0 SRT-RPP-2002-00221

\begin{tabular}{|c|c|c|c|c|c|c|c|c|c|c|}
\hline \multicolumn{3}{|c|}{ |PREC2_102301_0623 } & \multirow[b]{2}{*}{$\mid \begin{array}{c}\text { REC } \\
\text { PP } \\
\text { OUT } \\
\left({ }^{\circ} \mathrm{C}\right) \\
\text { TC1 }\end{array}$} & \multirow[b]{2}{*}{\begin{tabular}{|c|} 
TK \\
BOT \\
$\left({ }^{\circ} \mathrm{C}\right)$ \\
TC2 \\
\end{tabular}} & \multirow[b]{2}{*}{\begin{tabular}{|c|}
$\mathrm{HX}$ \\
OUT \\
$\left({ }^{\circ} \mathrm{C}\right)$ \\
$\mathrm{TC} 3$ \\
\end{tabular}} & \multirow[b]{2}{*}{$\begin{array}{c}\mathrm{Mn} \\
\text { Flow } \\
(\mathrm{gpm})\end{array}$} & \multirow[b]{2}{*}{\begin{tabular}{|c|} 
Galigher \\
Flow \\
(gpm)
\end{tabular}} & \multirow[b]{2}{*}{$\begin{array}{c}\text { Current } \\
\text { to } \mathrm{HX}\end{array}$} & \multirow[b]{2}{*}{$\begin{array}{c}\text { Voltage } \\
\text { to } \mathrm{HX}\end{array}$} & \multirow[b]{2}{*}{$\begin{array}{c}\mathrm{Sr} \\
\text { Flow } \\
\text { (gpm) }\end{array}$} \\
\hline DATE & TIME & $\begin{array}{c}\mathrm{HX} \\
\text { Outlet } \\
\left({ }^{\circ} \mathrm{C}\right) \\
\text { TCO }\end{array}$ & & & & & & & & \\
\hline $10 / 24 / 01$ & 3:24 & 18.7 & 19.0 & 19.0 & 18.6 & 0.0 & 9.7 & 118 & 79 & 0.0 \\
\hline 10/24/01 & $3: 25$ & 18.8 & 19.0 & 19.0 & \begin{tabular}{|l}
18.7 \\
\end{tabular} & 0.0 & 9.6 & 116 & 79 & 0.0 \\
\hline $10 / 24 / 01$ & $3: 26$ & 18.7 & 19.0 & 19.0 & 18.6 & 0.0 & 9.6 & 120 & 79 & 0.0 \\
\hline $10 / 24 / 01$ & 3:27 & 18.7 & 19.0 & 19.0 & $\mid 18.7$ & 0.0 & 9.6 & 121 & 79 & 0.0 \\
\hline $10 / 24 / 01$ & $3: 28$ & 18.7 & 19.0 & 19.0 & 18.6 & 0.0 & 9.6 & 100 & 79 & 0.0 \\
\hline $10 / 24 / 01$ & $3: 29$ & 18.7 & 19.0 & 19.0 & 18.6 & 0.0 & 9.7 & 89 & 79 & 0.0 \\
\hline $10 / 24 / 01$ & 3:30 & 18.8 & 19.0 & 19.0 & 18.6 & 0.0 & 9.6 & 123 & 79 & 0.0 \\
\hline $10 / 24 / 01$ & 3:31 & 18.6 & 19.0 & 19.0 & 18.6 & 0.0 & 9.7 & 103 & 79 & 0.0 \\
\hline $10 / 24 / 01$ & $3: 32$ & 18.8 & 19.0 & 19.0 & 18.7 & 0.0 & 9.6 & 110 & 79 & 0.0 \\
\hline $10 / 24 / 01$ & 3:33 & 18.6 & 19.0 & 19.0 & 18.5 & 0.0 & 9.6 & 131 & 79 & 0.0 \\
\hline $10 / 24 / 01$ & 3:34 & 18.8 & 18.9 & 18.9 & \begin{tabular}{|l|}
18.7 \\
\end{tabular} & 0.0 & 9.6 & 122 & 79 & 0.0 \\
\hline $10 / 24 / 01$ & 3:35 & 18.6 & 18.9 & 18.9 & 18.5 & 0.0 & 9.7 & 107 & 79 & 0.0 \\
\hline $10 / 24 / 01$ & 3:36 & 18.7 & 18.9 & 18.9 & 18.6 & 0.0 & 9.6 & 101 & 79 & 0.0 \\
\hline $10 / 24 / 01$ & 3:37 & 18.7 & 18.9 & 18.9 & $\mid 18.6$ & 0.0 & 9.6 & 132 & 79 & 0.0 \\
\hline $10 / 24 / 01$ & 3:38 & 18.6 & 18.9 & 18.9 & $\mid 18.6$ & 0.0 & 9.6 & 120 & 79 & 0.0 \\
\hline $10 / 24 / 01$ & 3:39 & 18.7 & 18.9 & 18.9 & \begin{tabular}{|l|}
18.6 \\
\end{tabular} & 0.0 & 9.7 & 121 & 79 & 0.0 \\
\hline $10 / 24 / 01$ & 3:40 & 18.6 & \begin{tabular}{|l|} 
\\
\end{tabular} & \begin{tabular}{|l|} 
\\
\end{tabular} & \begin{tabular}{|l|}
18.5 \\
\end{tabular} & 0.0 & 9.6 & 134 & 79 & 0.0 \\
\hline $10 / 24 / 01$ & 3:41 & 18.7 & 18.9 & 18.9 & 18.6 & 0.0 & 9.6 & 109 & 79 & 0.0 \\
\hline $10 / 24 / 01$ & 3:42 & 18.6 & \begin{tabular}{|l|} 
\\
\end{tabular} & \begin{tabular}{|l|}
18.9 \\
\end{tabular} & 18.5 & 0.0 & 9.6 & 87 & 79 & 0.0 \\
\hline $10 / 24 / 01$ & 3:43 & 18.7 & \begin{tabular}{|l|} 
\\
\end{tabular} & 18.9 & 18.6 & 0.0 & 9.7 & 124 & 79 & 0.0 \\
\hline $10 / 24 / 01$ & 3:44 & 18.6 & 18.9 & 18.9 & 18.5 & 0.0 & 9.7 & 94 & 79 & 0.0 \\
\hline $10 / 24 / 01$ & 3:45 & 18.7 & 18.9 & 18.9 & 18.6 & 0.0 & 9.5 & 131 & 79 & 0.0 \\
\hline $10 / 24 / 01$ & 3:46 & 18.6 & 18.9 & 18.9 & 18.5 & 0.0 & 9.7 & 116 & 79 & 0.0 \\
\hline $10 / 24 / 01$ & 3:47 & 18.6 & 18.9 & \begin{tabular}{|l|} 
\\
\end{tabular} & 18.6 & 0.0 & 9.7 & 94 & 79 & 0.0 \\
\hline $10 / 24 / 01$ & 3:48 & 18.7 & 18.9 & \begin{tabular}{|l|} 
\\
\end{tabular} & 18.5 & 0.0 & 9.6 & 126 & 79 & 0.0 \\
\hline $10 / 24 / 01$ & 3:49 & 18.6 & 18.9 & 18.9 & 18.5 & 0.0 & 9.7 & 118 & 79 & 0.0 \\
\hline $10 / 24 / 01$ & 3:50 & 18.7 & 18.9 & 18.9 & 18.6 & 0.0 & 9.6 & 136 & 79 & 0.0 \\
\hline $10 / 24 / 01$ & 3:51 & 18.6 & \begin{tabular}{|l|} 
\\
\end{tabular} & 18.9 & 18.5 & 0.0 & 9.6 & 125 & 79 & 0.0 \\
\hline $10 / 24 / 01$ & 3:52 & 18.7 & \begin{tabular}{|l|} 
\\
\end{tabular} & \begin{tabular}{|l|}
18.9 \\
\end{tabular} & 18.6 & 0.0 & 9.6 & 122 & 79 & 0.0 \\
\hline $10 / 24 / 01$ & 3:53 & 18.6 & 18.9 & \begin{tabular}{|l|} 
\\
\end{tabular} & 18.4 & 0.0 & 9.7 & 134 & 79 & 0.0 \\
\hline $10 / 24 / 01$ & 3:54 & 18.7 & 18.9 & \begin{tabular}{|l|} 
\\
\end{tabular} & 18.6 & 0.0 & 9.6 & 121 & 79 & 0.0 \\
\hline $10 / 24 / 01$ & 3:55 & 18.5 & 18.9 & 18.9 & \begin{tabular}{|l|}
18.4 \\
\end{tabular} & 0.0 & 9.6 & 136 & 79 & 0.0 \\
\hline $10 / 24 / 01$ & 3:56 & 18.6 & 18.9 & 18.9 & 18.5 & 0.0 & 9.6 & 109 & 79 & 0.0 \\
\hline $10 / 24 / 01$ & 3:57 & 18.6 & 18.9 & \begin{tabular}{|l|}
18.9 \\
\end{tabular} & 18.5 & 0.0 & 9.7 & 136 & 79 & 0.0 \\
\hline $10 / 24 / 01$ & 3:58 & 18.6 & \begin{tabular}{|l|} 
\\
\end{tabular} & 18.9 & 18.5 & 0.0 & 9.7 & 126 & 79 & 0.0 \\
\hline $10 / 24 / 01$ & 3:59 & 18.6 & \begin{tabular}{|l|} 
\\
\end{tabular} & \begin{tabular}{|l|} 
\\
\end{tabular} & 18.5 & 0.0 & 9.7 & 97 & 79 & 0.0 \\
\hline $10 / 24 / 01$ & 4:00 & 18.6 & \begin{tabular}{|l|}
18.9 \\
\end{tabular} & \begin{tabular}{|l|} 
\\
\end{tabular} & \begin{tabular}{|l|}
18.5 \\
\end{tabular} & 0.0 & 9.5 & 100 & 79 & 0.0 \\
\hline $10 / 24 / 01$ & 4:01 & 18.7 & \begin{tabular}{|l|} 
\\
\end{tabular} & \begin{tabular}{|l|} 
\\
\end{tabular} & \begin{tabular}{|l|}
18.5 \\
\end{tabular} & 0.0 & 9.7 & 100 & 79 & 0.0 \\
\hline $10 / 24 / 01$ & 4:02 & 18.5 & \begin{tabular}{|l|} 
\\
\end{tabular} & \begin{tabular}{|l|} 
\\
\end{tabular} & \begin{tabular}{|l|}
18.4 \\
\end{tabular} & 0.0 & 9.6 & 126 & 79 & 0.0 \\
\hline $10 / 24 / 01$ & 4:03 & 18.7 & \begin{tabular}{|l|} 
\\
\end{tabular} & 18.8 & 18.6 & 0.0 & 9.6 & 110 & 79 & 0.0 \\
\hline $10 / 24 / 01$ & 4:04 & 18.5 & \begin{tabular}{|l|} 
\\
\end{tabular} & 18.8 & \begin{tabular}{|l|}
18.4 \\
\end{tabular} & 0.0 & 9.7 & 132 & 79 & 0.0 \\
\hline $10 / 24 / 01$ & 4:05 & 18.6 & \begin{tabular}{|l|}
18.8 \\
\end{tabular} & \begin{tabular}{|l|}
18.8 \\
\end{tabular} & \begin{tabular}{|l|}
18.5 \\
\end{tabular} & 0.0 & 9.6 & 140 & 79 & 0.0 \\
\hline $10 / 24 / 01$ & 4:06 & 18.5 & \begin{tabular}{|l|} 
\\
\end{tabular} & \begin{tabular}{|l|} 
\\
\end{tabular} & \begin{tabular}{|l|}
18.4 \\
\end{tabular} & 0.0 & 9.7 & 110 & 79 & 0.0 \\
\hline $10 / 24 / 01$ & 4:07 & 18.6 & 18.8 & \begin{tabular}{|l|} 
\\
\end{tabular} & 18.5 & 0.0 & .0 & 106 & 79 & 0.0 \\
\hline $10 / 24 / 01$ & 4:08 & 18.6 & 18.8 & 18.8 & \begin{tabular}{|l|}
18.4 \\
\end{tabular} & 0.0 & 9.6 & 110 & 79 & 0.0 \\
\hline $10 / 24 / 01$ & 4:09 & 18.5 & \begin{tabular}{|l|}
18.8 \\
\end{tabular} & \begin{tabular}{|l|}
18.8 \\
\end{tabular} & \begin{tabular}{|l|}
18.4 \\
\end{tabular} & 0.0 & 9.6 & 129 & 79 & 0.0 \\
\hline $10 / 24 / 01$ & 4:10 & 18.6 & \begin{tabular}{|l|}
18.8 \\
\end{tabular} & \begin{tabular}{|l|} 
\\
\end{tabular} & \begin{tabular}{|l|}
18.5 \\
\end{tabular} & 0.0 & 9.7 & 135 & 79 & 0.0 \\
\hline $10 / 24 / 01$ & 4:11 & 18.5 & \begin{tabular}{|l|} 
\\
\end{tabular} & 18.8 & \begin{tabular}{|l|}
18.4 \\
\end{tabular} & 0.0 & 9.6 & 104 & 79 & 0.0 \\
\hline $10 / 24 / 01$ & 4:12 & 18.6 & \begin{tabular}{|l|} 
\\
\end{tabular} & \begin{tabular}{|l|} 
\\
\end{tabular} & 18.5 & 0.0 & 9.7 & 128 & 79 & 0.0 \\
\hline $10 / 24 / 01$ & 4:13 & 18.5 & 18.8 & 18.8 & 18.4 & 0.0 & 9.7 & 114 & 79 & 0.0 \\
\hline $10 / 24 / 01$ & 4:14 & 18.6 & \begin{tabular}{|l|}
18.8 \\
\end{tabular} & \begin{tabular}{|l|} 
\\
\end{tabular} & \begin{tabular}{|l|}
18.5 \\
\end{tabular} & 0.0 & 9.6 & 120 & 79 & 0.0 \\
\hline $10 / 24 / 01$ & 4:15 & 18.5 & \begin{tabular}{|l|} 
\\
\end{tabular} & 18.8 & 18.4 & 0.0 & 9.7 & 129 & 79 & 0.0 \\
\hline $10 / 24 / 01$ & 4:16 & 18.6 & 18.8 & 18.8 & 18.5 & 0.0 & 9.6 & 111 & 79 & 0.0 \\
\hline $10 / 24 / 01$ & 4:17 & 18.5 & \begin{tabular}{|l|} 
\\
\end{tabular} & 18.8 & $\mid 18.4$ & 0.0 & 9.6 & 138 & 79 & 0.0 \\
\hline $10 / 24 / 01$ & 4:18 & 18.5 & \begin{tabular}{|l|} 
\\
\end{tabular} & 18.8 & \begin{tabular}{|l|}
18.4 \\
\end{tabular} & 0.0 & 9.7 & 120 & 79 & 0.0 \\
\hline $10 / 24 / 01$ & 4:19 & 18.5 & \begin{tabular}{|l|}
18.8 \\
\end{tabular} & \begin{tabular}{|l|}
18.8 \\
\end{tabular} & \begin{tabular}{|l|}
18.4 \\
\end{tabular} & 0.0 & 9.6 & 131 & 79 & 0.0 \\
\hline $10 / 24 / 01$ & 4:20 & 18.5 & \begin{tabular}{|l|} 
\\
\end{tabular} & \begin{tabular}{|l|} 
\\
\end{tabular} & 18.4 & 0.0 & 9.6 & 120 & 79 & 0.0 \\
\hline $10 / 24 / 01$ & $4: 21$ & 18.6 & \begin{tabular}{|l|} 
\\
\end{tabular} & \begin{tabular}{|l|} 
\\
\end{tabular} & 18.5 & 0.0 & 9.6 & 91 & 79 & 0.0 \\
\hline $10 / 24 / 01$ & $4: 22$ & 18.5 & \begin{tabular}{|l|} 
\\
\end{tabular} & \begin{tabular}{|l|} 
\\
\end{tabular} & \begin{tabular}{|l|}
18.4 \\
\end{tabular} & 0.0 & 9.6 & 129 & 79 & 0.0 \\
\hline $10 / 24 / 01$ & $4: 23$ & 18.6 & \begin{tabular}{|l|} 
\\
\end{tabular} & \begin{tabular}{|l|} 
\\
\end{tabular} & 18.5 & 0.0 & 9.7 & 128 & 79 & 0.0 \\
\hline
\end{tabular}

\begin{tabular}{|c|c|c|c|c|c|c|c|c|c|c|}
\hline \multicolumn{11}{|c|}{ PREC2_102301_0623 } \\
\hline DATE & TIME & \begin{tabular}{|c}
$\mathrm{HX}$ \\
Outlet \\
$\left({ }^{\circ} \mathrm{C}\right)$ \\
$\mathrm{TC} 0$
\end{tabular} & \begin{tabular}{|c|} 
REC \\
PP \\
OUT \\
$\left({ }^{\circ} \mathrm{C}\right)$ \\
TC1 \\
\end{tabular} & \begin{tabular}{|c|} 
TK \\
BOT \\
$\left({ }^{\circ} \mathrm{C}\right)$ \\
TC2 \\
\end{tabular} & $\begin{array}{l}\text { HX } \\
\text { OUT } \\
\left({ }^{\circ} \mathrm{C}\right) \\
\text { TC3 }\end{array}$ & \begin{tabular}{|c|} 
Mn \\
Flow \\
(gpm)
\end{tabular} & $\begin{array}{c}\text { Galigher } \\
\text { Flow } \\
\text { (gpm) }\end{array}$ & $\begin{array}{l}\text { Current } \\
\text { to } \mathrm{HX}\end{array}$ & $\begin{array}{l}\text { Voltage } \\
\text { to } \mathrm{HX}\end{array}$ & $\begin{array}{c}\text { Sr } \\
\text { Flow } \\
\text { (gpm) }\end{array}$ \\
\hline $10 / 24 / 01$ & 4:24 & 18.5 & 18.8 & 18.8 & 18.3 & 0.0 & 9.7 & 96 & 79 & 0.0 \\
\hline $10 / 24 / 01$ & 4:25 & 18.6 & 18.8 & 18.8 & 18.5 & 0.0 & 9.6 & 92 & 79 & 0.0 \\
\hline $10 / 24 / 01$ & 4:26 & 18.5 & 18.8 & 18.7 & 18.3 & 0.0 & 9.7 & 116 & 79 & 0.0 \\
\hline $10 / 24 / 01$ & 4:27 & 18.5 & 18.8 & 18.7 & 18.4 & 0.0 & 9.6 & 126 & 79 & 0.0 \\
\hline $10 / 24 / 01$ & $4: 28$ & 18.5 & 18.8 & 18.8 & 18.4 & 0.0 & 8.8 & 135 & 79 & 0.0 \\
\hline $10 / 24 / 01$ & 4:29 & 18.6 & 18.9 & 18.8 & 18.5 & 0.0 & 9.7 & 97 & 79 & 0.0 \\
\hline $10 / 24 / 01$ & $4: 30$ & 18.6 & 18.8 & 18.8 & 18.4 & 0.0 & 9.6 & 119 & 79 & 0.0 \\
\hline $10 / 24 / 01$ & $4: 31$ & 18.5 & 18.8 & 18.8 & 18.4 & 0.0 & 9.6 & 131 & 79 & 0.0 \\
\hline $10 / 24 / 01$ & 4:32 & 18.6 & 18.8 & 18.8 & 18.5 & 0.0 & 9.6 & 132 & 79 & 0.0 \\
\hline $10 / 24 / 01$ & 4:33 & 18.4 & 18.8 & 18.7 & 18.3 & 0.0 & 9.6 & 90 & 79 & 0.0 \\
\hline $10 / 24 / 01$ & 4:34 & 18.6 & 18.8 & 18.7 & 18.5 & 0.0 & 9.7 & 131 & 79 & 0.0 \\
\hline $10 / 24 / 01$ & 4:35 & 18.5 & 18.7 & 18.7 & 18.3 & 0.0 & 9.6 & 121 & 79 & 0.0 \\
\hline $10 / 24 / 01$ & 4:36 & 18.5 & 18.7 & $\mid 18.7$ & 18.4 & 0.0 & 9.6 & 133 & 79 & 0.0 \\
\hline $10 / 24 / 01$ & 4:37 & 18.5 & 18.7 & $\mid 18.7$ & 18.3 & 0.0 & 9.7 & 128 & 79 & 0.0 \\
\hline $10 / 24 / 01$ & $4: 38$ & 18.5 & 18.7 & 18.7 & 18.4 & 0.0 & 9.7 & 93 & 79 & 0.0 \\
\hline $10 / 24 / 01$ & 4:39 & 18.5 & 18.7 & 18.7 & 18.4 & 0.0 & 9.7 & 133 & 79 & 0.0 \\
\hline $10 / 24 / 01$ & $4: 40$ & 18.4 & 18.7 & 18.7 & 18.4 & 0.0 & 9.6 & 127 & 79 & 0.0 \\
\hline $10 / 24 / 01$ & 4:41 & 18.5 & 18.7 & 18.7 & 18.4 & 0.0 & 9.6 & 107 & 79 & 0.0 \\
\hline $10 / 24 / 01$ & 4:42 & 18.4 & 18.7 & $\mid 18.7$ & 18.3 & 0.0 & 9.5 & 124 & 79 & 0.0 \\
\hline $10 / 24 / 01$ & 4:43 & 18.5 & 18.7 & 18.7 & 18.4 & 0.0 & 9.7 & 135 & 79 & 0.0 \\
\hline $10 / 24 / 01$ & 4:44 & 18.4 & 18.7 & \begin{tabular}{|l|} 
\\
\end{tabular} & 18.3 & 0.0 & 9.6 & 82 & 79 & 0.0 \\
\hline $10 / 24 / 01$ & 4:45 & 18.6 & 18.7 & 18.7 & 18.4 & 0.0 & 9.7 & 134 & 79 & 0.0 \\
\hline $10 / 24 / 01$ & $4: 46$ & 18.4 & 18.7 & 18.7 & 18.3 & 0.0 & 9.7 & 73 & 79 & 0.0 \\
\hline $10 / 24 / 01$ & $4: 47$ & 18.5 & 18.7 & $\mid 18.7$ & 18.4 & 0.0 & 9.7 & 118 & 79 & 0.0 \\
\hline $10 / 24 / 01$ & 4:48 & 18.4 & 18.7 & \begin{tabular}{|l|} 
\\
\end{tabular} & 18.3 & 0.0 & 9.6 & 89 & 79 & 0.0 \\
\hline $10 / 24 / 01$ & $4: 49$ & 18.5 & 18.7 & 18.7 & 18.4 & 0.0 & 9.6 & 108 & 79 & 0.0 \\
\hline $10 / 24 / 01$ & 4:50 & 18.5 & 18.7 & $\mid 18.7$ & 18.3 & 0.0 & 9.7 & 98 & 79 & 0.0 \\
\hline $10 / 24 / 01$ & 4:51 & 18.4 & 18.7 & \begin{tabular}{|l|} 
\\
\end{tabular} & 18.3 & 0.0 & 9.7 & 101 & 79 & 0.0 \\
\hline $10 / 24 / 01$ & 4:52 & 18.5 & 18.7 & \begin{tabular}{|l|} 
\\
\end{tabular} & 18.4 & 0.0 & 9.6 & 119 & 79 & 0.0 \\
\hline $10 / 24 / 01$ & 4:53 & 18.4 & 18.7 & 18.7 & 18.3 & 0.0 & 9.7 & 90 & 79 & 0.0 \\
\hline $10 / 24 / 01$ & 4:54 & 18.5 & 18.7 & \begin{tabular}{|l|} 
\\
\end{tabular} & 18.4 & 0.0 & 9.6 & 103 & 79 & 0.0 \\
\hline $10 / 24 / 01$ & 4:55 & 18.4 & 18.7 & $\mid 18.7$ & 18.3 & 0.0 & 9.7 & 78 & 79 & 0.0 \\
\hline $10 / 24 / 01$ & 4:56 & 18.5 & 18.7 & 18.7 & 18.4 & 0.0 & 9.6 & 97 & 79 & 0.0 \\
\hline $10 / 24 / 01$ & 4:57 & 18.4 & 18.7 & 18.7 & 18.3 & 0.0 & 9.6 & 123 & 79 & 0.0 \\
\hline $10 / 24 / 01$ & 4:58 & 18.5 & 18.7 & 18.7 & 18.4 & 0.0 & 9.7 & 127 & 79 & 0.0 \\
\hline $10 / 24 / 01$ & 4:59 & 18.4 & 18.7 & $\mid 18.7$ & 18.3 & 0.0 & 9.6 & 76 & 79 & 0.0 \\
\hline $10 / 24 / 01$ & 5:00 & 18.4 & 18.7 & \begin{tabular}{|l|}
18.7 \\
\end{tabular} & 18.3 & 0.0 & 9.6 & 82 & 79 & 0.0 \\
\hline $10 / 24 / 01$ & 5:01 & 18.5 & 18.7 & $\mid 18.7$ & 18.3 & 0.0 & 9.6 & 72 & 79 & 0.0 \\
\hline $10 / 24 / 01$ & 5:02 & 18.4 & 18.7 & \begin{tabular}{|l|} 
\\
\end{tabular} & 18.3 & 0.0 & 9.6 & 110 & 79 & 0.0 \\
\hline $10 / 24 / 01$ & 5:03 & 18.5 & 18.7 & $\mid 18.7$ & 18.4 & 0.0 & 9.7 & 115 & 79 & 0.0 \\
\hline $10 / 24 / 01$ & 5:04 & 18.4 & 18.7 & $\mid 18.7$ & 18.2 & 0.0 & 9.7 & 114 & 79 & 0.0 \\
\hline $10 / 24 / 01$ & 5:05 & 18.5 & \begin{tabular}{|l|}
18.7 \\
\end{tabular} & \begin{tabular}{|l|}
18.7 \\
\end{tabular} & 18.4 & 0.0 & 9.7 & 79 & 79 & 0.0 \\
\hline $10 / 24 / 01$ & 5:06 & 18.4 & 18.7 & 18.6 & 18.2 & 0.0 & 9.6 & 121 & 79 & 0.0 \\
\hline $10 / 24 / 01$ & 5:07 & 18.5 & 18.7 & 18.6 & 18.4 & 0.0 & 9.6 & 104 & 79 & 0.0 \\
\hline $10 / 24 / 01$ & 5:08 & 18.4 & 18.7 & 18.6 & 18.2 & 0.0 & 9.6 & 73 & 79 & 0.0 \\
\hline $10 / 24 / 01$ & 5:09 & 18.4 & 18.7 & $\mid 18.6$ & 18.3 & 0.0 & 9.6 & 102 & 79 & 0.0 \\
\hline $10 / 24 / 01$ & $5: 10$ & 18.4 & \begin{tabular}{|l|}
18.7 \\
\end{tabular} & \begin{tabular}{|l|} 
\\
\end{tabular} & 18.3 & 0.0 & 9.6 & 85 & 79 & 0.0 \\
\hline $10 / 24 / 01$ & $5: 11$ & 18.4 & 18.7 & 18.6 & 18.3 & 0.0 & 9.7 & 101 & 79 & 0.0 \\
\hline $10 / 24 / 01$ & $5: 12$ & 18.5 & 18.7 & \begin{tabular}{|l|} 
\\
\end{tabular} & 18.3 & 0.0 & 9.6 & 96 & 79 & 0.0 \\
\hline $10 / 24 / 01$ & $5: 13$ & 18.4 & 18.7 & 18.6 & 18.2 & 0.0 & 9.7 & 86 & 79 & 0.0 \\
\hline $10 / 24 / 01$ & $5: 14$ & 18.5 & 18.6 & \begin{tabular}{|l|} 
\\
\end{tabular} & 18.4 & 0.0 & 9.6 & 59 & 79 & 0.0 \\
\hline $10 / 24 / 01$ & $5: 15$ & 18.3 & 18.6 & 18.6 & 18.2 & 0.0 & 9.7 & 115 & 79 & 0.0 \\
\hline $10 / 24 / 01$ & $5: 16$ & 18.5 & 18.6 & 18.6 & 18.4 & 0.0 & 9.6 & 65 & 79 & 0.0 \\
\hline $10 / 24 / 01$ & $5: 17$ & 18.3 & 18.6 & 18.6 & 18.2 & 0.0 & 9.6 & 114 & 79 & 0.0 \\
\hline $10 / 24 / 01$ & $5: 18$ & 18.4 & 18.6 & 18.6 & 18.3 & 0.0 & 9.6 & 48 & 79 & 0.0 \\
\hline $10 / 24 / 01$ & $5: 19$ & 18.4 & 18.6 & 18.6 & 18.2 & 0.0 & 9.6 & 119 & 79 & 0.0 \\
\hline $10 / 24 / 01$ & $5: 20$ & 18.4 & 18.6 & 18.6 & 18.3 & 0.0 & 9.6 & 79 & 79 & 0.0 \\
\hline $10 / 24 / 01$ & $5: 21$ & 18.4 & 18.6 & \begin{tabular}{|l|} 
\\
\end{tabular} & 18.3 & 0.0 & 9.5 & 63 & 79 & 0.0 \\
\hline $10 / 24 / 01$ & $5: 22$ & 18.4 & 18.6 & 18.6 & 18.3 & 0.0 & 9.7 & 116 & 79 & 0.0 \\
\hline $10 / 24 / 01$ & 5:23 & 18.5 & 18.6 & \begin{tabular}{|l|} 
\\
\end{tabular} & 18.3 & 0.0 & 9.6 & 75 & 79 & 0.0 \\
\hline
\end{tabular}


WSRC-TR-2002-00459, Rev. 0 SRT-RPP-2002-00221

\begin{tabular}{|c|c|c|c|c|c|c|c|c|c|c|}
\hline \multicolumn{11}{|c|}{ PREC2_102301_0623 } \\
\hline DATE & TIME & $\begin{array}{c}\mathrm{HX} \\
\text { Outlet } \\
\left({ }^{\circ} \mathrm{C}\right) \\
\mathrm{TCO} \\
\end{array}$ & \begin{tabular}{|l} 
REC \\
PP \\
OUT \\
$\left({ }^{\circ} \mathrm{C}\right)$ \\
TC1 \\
\end{tabular} & $\begin{array}{c}\text { TK } \\
\text { BOT } \\
\left({ }^{\circ} \mathrm{C}\right) \\
\text { TC2 } \\
\end{array}$ & \begin{tabular}{|l} 
HX \\
OUT \\
$\left({ }^{\circ} \mathrm{C}\right)$ \\
TC3 \\
\end{tabular} & $\begin{array}{c}\text { Mn } \\
\text { Flow } \\
\text { (gpm) } \\
\end{array}$ & $\begin{array}{c}\text { Galigher } \\
\text { Flow } \\
\text { (gpm) }\end{array}$ & $\begin{array}{c}\text { Current } \\
\text { to } \mathrm{HX}\end{array}$ & $\begin{array}{l}\text { Voltage } \\
\text { to } \mathrm{HX}\end{array}$ & $\begin{array}{c}\mathrm{Sr} \\
\text { Flow } \\
\text { (gpm) }\end{array}$ \\
\hline $10 / 24 / 01$ & $5: 24$ & 18.3 & 18.6 & 18.6 & 18.2 & 0.0 & 9.6 & 77 & 79 & 0.0 \\
\hline $10 / 24 / 01$ & $5: 25$ & 18.5 & 18.6 & 18.6 & 18.3 & 0.0 & 9.6 & 76 & 79 & 0.0 \\
\hline $10 / 24 / 01$ & $5: 26$ & 18.3 & 18.6 & 18.6 & 18.2 & 0.0 & 9.7 & 100 & 79 & 0.0 \\
\hline $10 / 24 / 01$ & $5: 27$ & 18.5 & 18.6 & 18.6 & 18.3 & 0.0 & 9.6 & 117 & 79 & 0.0 \\
\hline $10 / 24 / 01$ & $5: 28$ & 18.4 & 18.6 & 18.6 & \begin{tabular}{|l|}
18.2 \\
\end{tabular} & 0.0 & 9.6 & 97 & 79 & 0.0 \\
\hline $10 / 24 / 01$ & $5: 29$ & 18.4 & 18.6 & 18.6 & \begin{tabular}{|l|}
18.3 \\
\end{tabular} & 0.0 & 9.7 & 70 & 79 & 0.0 \\
\hline $10 / 24 / 01$ & $5: 30$ & 18.4 & 18.6 & 18.6 & 18.2 & 0.0 & 9.6 & 88 & 79 & 0.0 \\
\hline $10 / 24 / 01$ & $5: 31$ & 18.4 & 18.6 & 18.6 & 18.2 & 0.0 & 9.6 & 51 & 79 & 0.0 \\
\hline $10 / 24 / 01$ & $5: 32$ & 18.4 & 18.6 & 18.6 & 18.3 & 0.0 & 9.7 & 76 & 79 & 0.0 \\
\hline $10 / 24 / 01$ & $5: 33$ & 18.3 & 18.6 & 18.6 & 18.2 & 0.0 & 9.6 & 70 & 79 & 0.0 \\
\hline $10 / 24 / 01$ & $5: 34$ & 18.5 & 18.6 & 18.6 & 18.3 & 0.0 & 9.6 & 45 & 79 & 0.0 \\
\hline $10 / 24 / 01$ & $5: 35$ & 18.3 & 18.6 & 18.6 & 18.2 & 0.0 & 9.6 & 48 & 79 & 0.0 \\
\hline $10 / 24 / 01$ & $5: 36$ & 18.5 & 18.6 & 18.6 & 18.4 & 0.0 & 9.7 & 106 & 79 & 0.0 \\
\hline $10 / 24 / 01$ & $5: 37$ & 18.5 & 18.6 & 18.6 & 18.3 & 0.0 & 9.7 & 110 & 79 & 0.0 \\
\hline $10 / 24 / 01$ & $5: 38$ & 18.5 & 18.6 & 18.6 & 18.4 & 0.0 & 9.7 & 85 & 79 & 0.0 \\
\hline $10 / 24 / 01$ & 5:39 & 18.5 & 18.6 & 18.6 & 18.3 & 0.0 & 9.6 & 43 & 79 & 0.0 \\
\hline $10 / 24 / 01$ & $5: 40$ & 18.5 & 18.6 & 18.6 & 18.4 & 0.0 & 9.6 & 61 & 79 & 0.0 \\
\hline $10 / 24 / 01$ & $5: 41$ & 18.5 & 18.6 & 18.6 & 18.4 & 0.0 & 9.6 & 46 & 79 & 0. \\
\hline $10 / 24 / 01$ & $5: 42$ & 18.5 & 18.6 & 18.6 & 18.4 & 0.0 & 9.7 & 101 & 79 & 0.0 \\
\hline $10 / 24 / 01$ & $5: 43$ & 18.5 & 18.7 & 18.6 & 18.4 & 0.0 & 9.7 & 102 & 79 & 0.0 \\
\hline $10 / 24 / 01$ & $5: 44$ & 18.5 & \begin{tabular}{|l|}
18.7 \\
\end{tabular} & \begin{tabular}{|l|}
18.7 \\
\end{tabular} & 18.4 & 0.0 & 9.6 & 76 & 79 & 0.0 \\
\hline $10 / 24 / 01$ & $5: 45$ & 18.6 & 18.7 & 18.7 & 18.4 & 0.0 & 9.7 & 37 & 79 & 0.0 \\
\hline $10 / 24 / 01$ & $5: 46$ & 18.5 & 18.7 & 18.7 & 18.4 & 0.0 & 9.5 & 66 & 79 & 0.0 \\
\hline $10 / 24 / 01$ & $5: 47$ & 18.6 & 18.7 & 18.7 & 18.4 & 0.0 & 9.6 & 74 & 79 & 0.0 \\
\hline $10 / 24 / 01$ & $5: 48$ & 18.5 & 18.7 & 18.7 & 18.4 & 0.0 & 9.6 & 71 & 79 & 0.0 \\
\hline $10 / 24 / 01$ & $5: 49$ & 18.6 & 18.7 & 18.7 & 18.4 & 0.0 & 9.8 & 74 & 79 & 0.0 \\
\hline $10 / 24 / 01$ & 5:50 & 18.5 & 18.7 & 18.7 & 18.4 & 0.0 & 9.7 & 81 & 79 & 0.0 \\
\hline $10 / 24 / 01$ & 5:51 & 18.6 & 18.7 & 18.7 & 18.4 & 0.0 & 9.6 & 35 & 7 & 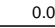 \\
\hline $10 / 24 / 01$ & 5:52 & 18.6 & 18.7 & 18.7 & 18.4 & 0.0 & 9.6 & 53 & 79 & 0.0 \\
\hline $10 / 24 / 01$ & 5:53 & 18.6 & 18.7 & \begin{tabular}{|l|}
18.7 \\
\end{tabular} & 18.5 & 0.0 & 9.6 & 78 & 79 & 0.0 \\
\hline $10 / 24 / 01$ & 5:54 & 18.6 & \begin{tabular}{|l|}
18.7 \\
\end{tabular} & 18.7 & 18.4 & 0.0 & 9.6 & 32 & 79 & 0.0 \\
\hline $10 / 24 / 01$ & $5: 55$ & 18.6 & 18.7 & 18.7 & 18.4 & 0.0 & 9.6 & 55 & 79 & 0.0 \\
\hline $10 / 24 / 01$ & $5: 56$ & 18.6 & 18.7 & 18.7 & 18.5 & 0.0 & 9.5 & 85 & 79 & 0.0 \\
\hline $10 / 24 / 01$ & $5: 57$ & 18.6 & 18.7 & 18.7 & 18.4 & 0.0 & 9.7 & 60 & 79 & 0.0 \\
\hline $10 / 24 / 01$ & 5:58 & 18.6 & \begin{tabular}{|l|}
18.8 \\
\end{tabular} & \begin{tabular}{|l|}
18.7 \\
\end{tabular} & 18.5 & 0.0 & 9.6 & 75 & 79 & 0.0 \\
\hline $10 / 24 / 01$ & 5:59 & 18.6 & \begin{tabular}{|l|}
18.8 \\
\end{tabular} & \begin{tabular}{|l|}
18.7 \\
\end{tabular} & 18.5 & 0.0 & 9.7 & 55 & 79 & 0.0 \\
\hline $10 / 24 / 01$ & 6:00 & 18.6 & 18.8 & 18.7 & 18.5 & 0.0 & 9.5 & 62 & 79 & 0.0 \\
\hline $10 / 24 / 01$ & 6:01 & 18.6 & 18.8 & 18.7 & 18.5 & 0.0 & 9.7 & 60 & 79 & 0.0 \\
\hline $10 / 24 / 01$ & 6:02 & 18.6 & 18.8 & 18.8 & 18.5 & 0.0 & 9.7 & 29 & 79 & 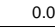 \\
\hline $10 / 24 / 01$ & 6:03 & 18.6 & 18.8 & 18.8 & 18.5 & 0.0 & 9.7 & 99 & 79 & 0.0 \\
\hline $10 / 24 / 01$ & 6:04 & 18.6 & 18.8 & 18.8 & 18.5 & 0.0 & 9.6 & 34 & 79 & 0.0 \\
\hline $10 / 24 / 01$ & 6:05 & 18.6 & \begin{tabular}{|l|}
18.8 \\
\end{tabular} & 18.8 & 18.5 & 0.0 & 9.6 & 100 & 79 & 0.0 \\
\hline $10 / 24 / 01$ & 6:06 & 18.6 & \begin{tabular}{|l|}
18.8 \\
\end{tabular} & \begin{tabular}{|l|} 
\\
\end{tabular} & 18.5 & 0.0 & 9.6 & 77 & 79 & 0.0 \\
\hline $10 / 24 / 01$ & 6:07 & 18.7 & 18.8 & 18.8 & 18.5 & 0.0 & 9.6 & 30 & 79 & 0.0 \\
\hline 10/24/01 & 6:08 & 18.7 & 18.8 & 18.8 & 18.5 & 0.0 & 9.8 & 26 & 79 & 0.0 \\
\hline $10 / 24 / 01$ & 6:09 & 18.7 & \begin{tabular}{|l|}
18.8 \\
\end{tabular} & 18.8 & 18.6 & 0.0 & 9.6 & 66 & 79 & 0.0 \\
\hline $10 / 24 / 01$ & $6: 10$ & 18.7 & \begin{tabular}{|l|}
18.8 \\
\end{tabular} & 18.8 & 18.5 & 0.0 & 9.6 & 98 & 79 & 0.0 \\
\hline $10 / 24 / 01$ & 6:11 & 18.7 & 18.8 & 18.8 & 18.6 & 0.0 & 9.6 & 30 & 79 & 0.0 \\
\hline $10 / 24 / 01$ & $6: 12$ & 18.7 & \begin{tabular}{|l|}
18.8 \\
\end{tabular} & 18.8 & 18.5 & 0.0 & 9.6 & 32 & 79 & 0.0 \\
\hline 10/24/01 & $6: 13$ & 18.7 & 18.8 & 18.8 & 18.6 & 0.0 & 9.7 & 63 & 79 & 0.0 \\
\hline $10 / 24 / 01$ & $6: 14$ & 18.7 & 18.8 & 18.8 & 18.5 & 0.0 & 9.6 & 46 & 7 & 0 \\
\hline $10 / 24 / 01$ & $6: 15$ & 18.7 & 18.9 & 18.8 & 18.6 & 0.0 & .6 & 51 & 79 & 0.0 \\
\hline $10 / 24 / 01$ & $6: 16$ & 18.7 & 18.9 & 18.8 & 18.6 & 0.0 & 9.6 & 32 & 79 & 0.0 \\
\hline $10 / 24 / 01$ & $6: 17$ & 18.7 & 18.9 & 18.8 & 18.6 & 0.0 & 9.7 & 68 & 79 & 0.0 \\
\hline $10 / 24 / 01$ & $6: 18$ & 18.7 & 18.9 & 18.9 & 18.6 & 0.0 & 9.6 & 24 & 79 & 0.0 \\
\hline $10 / 24 / 01$ & $6: 19$ & 18.7 & \begin{tabular}{|l|}
18.9 \\
\end{tabular} & 18.9 & 18.6 & 0.0 & 9.7 & 23 & 79 & 0.0 \\
\hline $10 / 24 / 01$ & 6:20 & 18.7 & 18.9 & 18.9 & 18.6 & 0.0 & 9.6 & 26 & 79 & 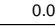 \\
\hline $10 / 24 / 01$ & $6: 21$ & 18.7 & 18.9 & 18.9 & 18.6 & 0.0 & .6 & 38 & 79 & 0.0 \\
\hline $10 / 24 / 01$ & $6: 22$ & 18.8 & 18.9 & 18.9 & 18.6 & 0.0 & 9.7 & 97 & 79 & 0.0 \\
\hline $10 / 24 / 01$ & $6: 23$ & 18.7 & 18.9 & 18.9 & 18.6 & 0.0 & 9.6 & 25 & 79 & 0.0 \\
\hline
\end{tabular}


WSRC-TR-2002-00459, Rev. 0 SRT-RPP-2002-00221

\begin{tabular}{|c|c|c|c|c|c|c|c|c|c|c|}
\hline \multicolumn{3}{|c|}{ PREC2_102401_0627 } & \multirow[b]{2}{*}{\begin{tabular}{|c|} 
REC \\
PP \\
OUT \\
$\left({ }^{\circ} \mathrm{C}\right)$ \\
TC1
\end{tabular}} & \multirow[b]{2}{*}{\begin{tabular}{|c|} 
TK \\
BOT \\
$\left({ }^{\circ} \mathrm{C}\right)$ \\
TC2 \\
\end{tabular}} & \multirow[b]{2}{*}{\begin{tabular}{|c|}
$\mathrm{HX}$ \\
OUT \\
$\left({ }^{\circ} \mathrm{C}\right)$ \\
TC3
\end{tabular}} & \multirow[b]{2}{*}{$\begin{array}{c}\mathrm{Mn} \\
\text { Flow } \\
(\mathrm{gpm})\end{array}$} & \multirow[b]{2}{*}{\begin{tabular}{|c|} 
Galigher \\
Flow \\
(gpm)
\end{tabular}} & \multirow[b]{2}{*}{$\begin{array}{c}\text { Current } \\
\text { to } \mathrm{HX}\end{array}$} & \multirow[b]{2}{*}{$\begin{array}{c}\text { Voltage } \\
\text { to } \mathrm{HX}\end{array}$} & \multirow[b]{2}{*}{$\begin{array}{c}\mathrm{Sr} \\
\text { Flow } \\
(\mathrm{gpm})\end{array}$} \\
\hline DATE & TIME & $\begin{array}{c}\mathrm{HX} \\
\text { Outlet } \\
\left({ }^{\circ} \mathrm{C}\right) \\
\text { TCO }\end{array}$ & & & & & & & & \\
\hline $10 / 24 / 01$ & $6: 27$ & 18.8 & \begin{tabular}{|l|}
18.9 \\
\end{tabular} & 18.9 & 18.7 & 0.0 & 9.6 & 99 & 79 & 0.0 \\
\hline $10 / 24 / 01$ & $6: 28$ & 18.8 & 18.9 & 18.9 & 18.7 & 0.0 & 9.6 & 99 & 79 & 0.0 \\
\hline $10 / 24 / 01$ & $6: 29$ & 18.8 & 18.9 & 18.9 & 18.6 & 0.0 & 9.8 & 23 & 79 & 0.0 \\
\hline $10 / 24 / 01$ & $6: 30$ & 18.8 & 18.9 & 18.9 & 18.6 & 0.0 & 9.7 & 29 & 79 & 0.0 \\
\hline $10 / 24 / 01$ & 6:31 & 18.8 & 18.9 & 18.9 & 18.7 & 0.0 & 9.6 & 35 & 79 & 0.0 \\
\hline $10 / 24 / 01$ & 6:32 & 18.8 & 19.0 & 18.9 & 18.6 & 0.0 & 9.6 & 21 & 79 & 0.0 \\
\hline $10 / 24 / 01$ & 6:33 & 18.8 & 19.0 & 18.9 & 18.7 & 0.0 & 9.7 & 97 & 79 & 0.0 \\
\hline $10 / 24 / 01$ & 6:34 & 18.8 & 19.0 & 18.9 & 18.7 & 0.0 & 9.7 & 23 & 79 & 0.0 \\
\hline $10 / 24 / 01$ & 6:35 & 18.9 & 19.0 & 18.9 & 18.7 & 0.0 & 9.6 & 26 & 79 & 0.0 \\
\hline $10 / 24 / 01$ & $6: 36$ & 18.8 & 19.0 & 19.0 & 18.7 & 0.0 & 9.6 & 40 & 79 & 0.0 \\
\hline $10 / 24 / 01$ & 6:37 & 18.9 & 19.0 & 19.0 & 18.7 & 0.0 & 9.6 & 29 & 79 & 0.0 \\
\hline $10 / 24 / 01$ & $6: 38$ & 18.8 & 19.0 & 19.0 & 18.7 & 0.0 & 9.7 & 26 & 79 & 0.0 \\
\hline $10 / 24 / 01$ & $6: 39$ & 18.9 & 19.0 & 19.0 & 18.7 & 0.0 & 9.7 & 50 & 79 & 0.0 \\
\hline $10 / 24 / 01$ & $6: 40$ & 18.8 & 19.0 & 19.0 & 18.7 & 0.0 & 9.6 & 25 & 79 & 0.0 \\
\hline $10 / 24 / 01$ & $6: 41$ & 18.8 & 19.0 & 19.0 & 18.7 & 0.0 & 9.6 & 21 & 79 & 0.0 \\
\hline $10 / 24 / 01$ & $6: 42$ & 18.9 & 19.0 & \begin{tabular}{|l|}
19.0 \\
\end{tabular} & 18.7 & 0.0 & 9.6 & 37 & 79 & 0.0 \\
\hline $10 / 24 / 01$ & $6: 43$ & 18.8 & 19.0 & 19.0 & 18.7 & 0.0 & 9.6 & 46 & 79 & 0.0 \\
\hline $10 / 24 / 01$ & $6: 44$ & 18.9 & 19.0 & 19.0 & 18.7 & 0.0 & 9.7 & 28 & 79 & 0.0 \\
\hline $10 / 24 / 01$ & $6: 45$ & 18.9 & 19.0 & 19.0 & 18.7 & 0.0 & 9.6 & 20 & 79 & 0.0 \\
\hline $10 / 24 / 01$ & $6: 46$ & 18.9 & 19.0 & \begin{tabular}{|l|} 
\\
\end{tabular} & 18.8 & 0.0 & 9.7 & 20 & 79 & 0.0 \\
\hline $10 / 24 / 01$ & $6: 47$ & 18.9 & 19.0 & 19.0 & 18.7 & 0.0 & 9.6 & 21 & 79 & 0.0 \\
\hline $10 / 24 / 01$ & $6: 48$ & 18.9 & \begin{tabular}{|l|} 
\\
\end{tabular} & 19.0 & 18.8 & 0.0 & 9.6 & 21 & 79 & 0.0 \\
\hline $10 / 24 / 01$ & 6:49 & 18.9 & 19.1 & 19.0 & 18.7 & 0.0 & 9.5 & 21 & 79 & 0.0 \\
\hline $10 / 24 / 01$ & 6:50 & 18.9 & 19.1 & 19.0 & 18.8 & 0.0 & 9.7 & 37 & 79 & 0.0 \\
\hline $10 / 24 / 01$ & 6:51 & 18.9 & 19.1 & 19.0 & 18.7 & 0.0 & 9.6 & 21 & 79 & 0.0 \\
\hline $10 / 24 / 01$ & 6:52 & 18.9 & 19.1 & 19.0 & 18.8 & 0.0 & 9.6 & 21 & 79 & 0.0 \\
\hline $10 / 24 / 01$ & $6: 53$ & 18.9 & 19.1 & \begin{tabular}{|l|}
19.0 \\
\end{tabular} & 18.8 & 0.0 & 9.7 & 21 & 79 & 0.0 \\
\hline $10 / 24 / 01$ & 6:54 & 18.9 & \begin{tabular}{|l|} 
\\
\end{tabular} & 19.1 & 18.8 & 0.0 & 9.6 & 21 & 79 & 0.0 \\
\hline $10 / 24 / 01$ & 6:55 & 18.9 & 19.1 & 19.0 & 18.8 & 0.0 & 9.7 & 21 & 79 & 0.0 \\
\hline $10 / 24 / 01$ & 6:56 & 18.9 & 19.1 & 19.0 & 18.8 & 0.0 & 9.7 & 21 & 79 & 0.0 \\
\hline $10 / 24 / 01$ & 6:57 & 19.0 & 19.1 & 19.1 & 18.8 & 0.0 & 9.6 & 21 & 79 & 0.0 \\
\hline $10 / 24 / 01$ & 6:58 & 18.9 & \begin{tabular}{|l|} 
\\
\end{tabular} & \begin{tabular}{|l|}
19.1 \\
\end{tabular} & 18.8 & 0.0 & 9.6 & 21 & 79 & 0.0 \\
\hline $10 / 24 / 01$ & 6:59 & 19.0 & 19.1 & 19.1 & 18.8 & 0.0 & 9.6 & 21 & 79 & 0.0 \\
\hline $10 / 24 / 01$ & 7:00 & 18.9 & 19.1 & 19.1 & 18.8 & 0.0 & 9.6 & 21 & 79 & 0.0 \\
\hline $10 / 24 / 01$ & 7:01 & 19.0 & \begin{tabular}{|l|} 
\\
\end{tabular} & 19.1 & 18.8 & 0.0 & 9.6 & 21 & 79 & 0.0 \\
\hline $10 / 24 / 01$ & 7:02 & 18.9 & 19.1 & 19.1 & 18.8 & 0.0 & 9.7 & 21 & 79 & 0.0 \\
\hline $10 / 24 / 01$ & 7:03 & 19.0 & 19.1 & 19.1 & 18.8 & 0.0 & 9.6 & 21 & 79 & 0.0 \\
\hline $10 / 24 / 01$ & 7:04 & 18.9 & 19.1 & \begin{tabular}{|l|} 
\\
\end{tabular} & 18.8 & 0.0 & 9.6 & 21 & 79 & 0.0 \\
\hline $10 / 24 / 01$ & 7:05 & 19.0 & \begin{tabular}{|l|} 
\\
\end{tabular} & \begin{tabular}{|l|}
19.1 \\
\end{tabular} & 18.8 & 0.0 & 9.7 & 21 & 79 & 0.0 \\
\hline $10 / 24 / 01$ & 7:06 & 19.0 & 19.1 & \begin{tabular}{|l|} 
\\
\end{tabular} & 18.8 & 0.0 & 9.7 & 21 & 79 & 0.0 \\
\hline $10 / 24 / 01$ & 7:07 & 19.0 & 19.1 & 19.1 & 18.8 & 0.0 & 9.7 & 21 & 79 & 0.0 \\
\hline $10 / 24 / 01$ & 7:08 & 19.0 & \begin{tabular}{|l|} 
\\
\end{tabular} & 19.1 & 18.9 & 0.0 & 9.7 & 21 & 79 & 0.0 \\
\hline $10 / 24 / 01$ & 7:09 & 19.0 & 19.2 & 19.1 & 18.8 & 0.0 & 9.5 & 21 & 79 & 0.0 \\
\hline $10 / 24 / 01$ & 7:10 & 19.0 & 19.2 & 19.1 & 18.9 & 0.0 & 9.7 & 21 & 79 & 0.0 \\
\hline $10 / 24 / 01$ & 7:11 & 19.0 & \begin{tabular}{|l|}
19.2 \\
\end{tabular} & \begin{tabular}{|l|}
19.2 \\
\end{tabular} & 18.8 & 0.0 & 9.6 & 21 & 79 & 0.0 \\
\hline $10 / 24 / 01$ & 7:12 & 19.1 & \begin{tabular}{|l|}
19.2 \\
\end{tabular} & \begin{tabular}{|l|}
19.2 \\
\end{tabular} & 18.9 & 0.0 & 9.7 & 20 & 79 & 0.0 \\
\hline $10 / 24 / 01$ & 7:13 & 19.0 & \begin{tabular}{|l|}
19.2 \\
\end{tabular} & 19.2 & 18.8 & 0.0 & 9.6 & 20 & 79 & 0.0 \\
\hline $10 / 24 / 01$ & 7:14 & 19.0 & 19.2 & 19.1 & 18.9 & 0.0 & 9.6 & 20 & 79 & 0.0 \\
\hline $10 / 24 / 01$ & 7:15 & 19.0 & 19.2 & 19.2 & 18.8 & 0.0 & 9.7 & 21 & 79 & 0.0 \\
\hline $10 / 24 / 01$ & 7:16 & 19.0 & \begin{tabular}{|l|}
19.2 \\
\end{tabular} & 19.2 & 18.9 & 0.0 & 9.6 & 21 & 79 & 0.0 \\
\hline $10 / 24 / 01$ & 7:17 & 19.0 & 19.2 & 19.2 & 18.9 & 0.0 & 9.7 & 20 & 79 & 0.0 \\
\hline $10 / 24 / 01$ & 7:18 & 19.0 & \begin{tabular}{|l|}
19.2 \\
\end{tabular} & \begin{tabular}{|l|}
19.2 \\
\end{tabular} & 18.9 & 0.0 & 9.7 & 20 & 79 & 0.0 \\
\hline $10 / 24 / 01$ & 7:19 & 19.0 & \begin{tabular}{|l|}
19.2 \\
\end{tabular} & \begin{tabular}{|l|}
19.2 \\
\end{tabular} & 18.9 & 0.0 & 9.7 & 21 & 79 & 0.0 \\
\hline $10 / 24 / 01$ & 7:20 & 19.0 & \begin{tabular}{|l|}
19.2 \\
\end{tabular} & 19.2 & 18.9 & 0.0 & 9.6 & 20 & 79 & 0.0 \\
\hline $10 / 24 / 01$ & 7:21 & 19.1 & \begin{tabular}{|l|}
19.2 \\
\end{tabular} & \begin{tabular}{|l|} 
\\
\end{tabular} & 18.9 & 0.0 & 9.6 & 20 & 79 & 0.0 \\
\hline $10 / 24 / 01$ & 7:22 & 19.0 & 19.2 & \begin{tabular}{|l|}
19.2 \\
\end{tabular} & 18.9 & 0.0 & 9.6 & 20 & 79 & 0.0 \\
\hline $10 / 24 / 01$ & 7:23 & 19.1 & \begin{tabular}{|l|}
19.2 \\
\end{tabular} & \begin{tabular}{|l|} 
\\
\end{tabular} & 18.9 & 0.0 & 9.6 & 21 & 79 & 0.0 \\
\hline 10/24/01 & 7:24 & 19.0 & 19.2 & 19.2 & 18.9 & 0.0 & 9.5 & 20 & 79 & 0.0 \\
\hline $10 / 24 / 01$ & 7:25 & 19.1 & \begin{tabular}{|l|} 
\\
\end{tabular} & 19.2 & 19.0 & 0.0 & 9.6 & 35 & 79 & 0.0 \\
\hline $10 / 24 / 01$ & 7:26 & 19.1 & 19.2 & 19.2 & 18.9 & 0.0 & 9.6 & 20 & 79 & 0.0 \\
\hline
\end{tabular}

\begin{tabular}{|c|c|c|c|c|c|c|c|c|c|c|}
\hline \multicolumn{11}{|c|}{ PREC2_102401_0627 } \\
\hline DATE & TIME & $\begin{array}{c}\mathrm{HX} \\
\text { Outlet } \\
\left({ }^{\circ} \mathrm{C}\right) \\
\text { TCO }\end{array}$ & $\begin{array}{c}\text { REC } \\
\text { PP } \\
\text { OUT } \\
\left({ }^{\circ} \mathrm{C}\right) \\
\text { TC } 1\end{array}$ & $\begin{array}{c}\text { TK } \\
\text { BOT } \\
\left({ }^{\circ} \mathrm{C}\right) \\
\text { TC2 }\end{array}$ & $\begin{array}{l}\mathrm{HX} \\
\text { OUT } \\
\left({ }^{\circ} \mathrm{C}\right) \\
\text { TC3 }\end{array}$ & $\begin{array}{c}\text { Mn } \\
\text { Flow } \\
\text { (gpm) }\end{array}$ & $\begin{array}{c}\text { Galigher } \\
\text { Flow } \\
(\mathrm{gpm})\end{array}$ & $\begin{array}{l}\text { Current } \\
\text { to } \mathrm{HX}\end{array}$ & $\begin{array}{c}\text { Voltage } \\
\text { to } \mathrm{HX}\end{array}$ & $\begin{array}{c}\mathrm{Sr} \\
\text { Flow } \\
\text { (gpm) }\end{array}$ \\
\hline $10 / 24 / 01$ & 7:27 & 19.1 & 19.2 & 19.2 & 19.0 & 0.0 & 9.6 & 20 & 79 & 0.0 \\
\hline $10 / 24 / 01$ & 7:28 & 19.1 & 19.2 & 19.2 & 18.9 & 0.0 & 9.7 & 50 & 79 & 0.0 \\
\hline $10 / 24 / 01$ & 7:29 & 19.1 & 19.3 & 19.2 & 19.0 & 0.0 & 9.6 & 96 & 79 & 0.0 \\
\hline $10 / 24 / 01$ & $7: 30$ & 19.1 & 19.3 & 19.2 & 18.9 & 0.0 & 9.6 & 38 & 79 & 0.0 \\
\hline $10 / 24 / 01$ & $7: 31$ & 19.1 & 19.3 & 19.2 & 19.0 & 0.0 & 9.7 & 20 & 79 & 0.0 \\
\hline $10 / 24 / 01$ & 7:32 & 19.1 & 19.3 & 19.2 & 19.0 & 0.0 & 9.6 & 23 & 79 & 0.0 \\
\hline $10 / 24 / 01$ & 7:33 & 19.1 & 19.3 & 19.3 & 19.0 & 0.0 & 9.6 & 70 & 79 & 0.0 \\
\hline $10 / 24 / 01$ & $7: 34$ & 19.1 & 19.3 & 19.3 & 19.0 & 0.0 & 9.6 & 22 & 79 & 0.0 \\
\hline $10 / 24 / 01$ & $7: 35$ & 19.1 & 19.3 & 19.2 & 19.0 & 0.0 & 9.7 & 20 & 79 & 0.0 \\
\hline $10 / 24 / 01$ & 7:36 & 19.2 & 19.3 & 19.3 & 19.0 & 0.0 & 9.7 & 71 & 79 & 0.0 \\
\hline $10 / 24 / 01$ & $7: 37$ & 19.1 & 19.3 & 19.3 & 19.0 & 0.0 & 9.6 & 22 & 79 & 0.0 \\
\hline $10 / 24 / 01$ & 7:38 & 19.2 & 19.3 & 19.3 & 19.0 & 0.0 & 9.6 & 23 & 79 & 0.0 \\
\hline $10 / 24 / 01$ & $7: 39$ & 19.1 & 19.3 & 19.3 & 19.0 & 0.0 & 9.7 & 21 & 79 & 0.0 \\
\hline $10 / 24 / 01$ & $7: 40$ & 19.2 & 19.3 & 19.3 & 19.0 & 0.0 & 9.7 & 34 & 79 & 0.0 \\
\hline $10 / 24 / 01$ & $7: 41$ & 19.1 & 19.3 & 19.3 & 19.0 & 0.0 & 9.6 & 32 & 79 & 0.0 \\
\hline $10 / 24 / 01$ & 7:42 & 19.2 & 19.3 & 19.3 & 19.0 & 0.0 & 9.7 & 69 & 79 & 0.0 \\
\hline $10 / 24 / 01$ & $7: 43$ & 19.1 & 19.3 & 19.3 & 19.0 & 0.0 & 9.6 & 71 & 79 & 0.0 \\
\hline $10 / 24 / 01$ & 7:44 & 19.1 & 19.3 & 19.3 & 19.0 & 0.0 & 9.6 & 37 & 79 & 0.0 \\
\hline $10 / 24 / 01$ & 7:45 & 19.2 & 19.3 & 19.3 & 19.0 & 0.0 & 9.6 & 43 & 79 & 0.0 \\
\hline $10 / 24 / 01$ & 7:46 & 19.1 & 19.3 & 19.3 & 19.0 & 0.0 & 9.6 & 82 & 79 & 0.0 \\
\hline $10 / 24 / 01$ & $7: 47$ & 19.2 & 19.3 & 19.3 & 19.0 & 0.0 & 9.6 & 41 & 79 & 0.0 \\
\hline $10 / 24 / 01$ & 7:48 & 19.1 & 19.3 & 19.3 & 19.0 & 0.0 & 9.6 & 95 & 79 & 0.0 \\
\hline $10 / 24 / 01$ & 7:49 & 19.2 & 19.3 & 19.3 & 19.1 & 0.0 & 9.7 & 98 & 79 & 0.0 \\
\hline $10 / 24 / 01$ & 7:50 & 19.1 & 19.4 & 19.3 & 19.0 & 0.0 & 9.7 & 47 & 79 & 0.0 \\
\hline $10 / 24 / 01$ & $7: 51$ & 19.2 & 19.3 & 19.3 & 19.1 & 0.0 & 9.7 & 27 & 79 & 0.0 \\
\hline $10 / 24 / 01$ & 7:52 & 19.2 & 19.4 & 19.3 & 19.0 & 0.0 & 9.5 & 48 & 79 & 0.0 \\
\hline $10 / 24 / 01$ & 7:53 & 19.2 & 19.4 & 19.3 & 19.1 & 0.0 & 9.6 & 31 & 79 & 0.0 \\
\hline $10 / 24 / 01$ & 7:54 & 19.2 & 19.4 & 19.4 & 19.0 & 0.0 & 9.7 & 79 & 79 & 0.0 \\
\hline $10 / 24 / 01$ & 7:55 & 19.2 & 19.4 & 19.4 & 19.1 & 0.0 & 9.6 & 28 & 79 & 0.0 \\
\hline $10 / 24 / 01$ & 7:56 & 19.2 & 19.4 & 19.4 & 19.1 & 0.0 & 9.6 & 27 & 79 & 0.0 \\
\hline $10 / 24 / 01$ & 7:57 & 19.2 & 19.4 & 19.4 & 19.1 & 0.0 & 9.6 & 24 & 79 & 0.0 \\
\hline $10 / 24 / 01$ & 7:58 & 19.2 & 19.4 & 19.4 & 19.1 & 0.0 & 9.7 & 51 & 79 & 0.0 \\
\hline $10 / 24 / 01$ & $7: 59$ & 19.2 & 19.4 & 19.4 & 19.1 & 0.0 & 9.7 & 24 & 79 & 0.0 \\
\hline $10 / 24 / 01$ & 8:00 & 19.3 & 19.4 & 19.4 & 19.1 & 0.0 & 9.7 & 37 & 79 & 0.0 \\
\hline $10 / 24 / 01$ & 8:01 & 19.2 & 19.4 & 19.4 & 19.1 & 0.0 & 9.6 & 93 & 79 & 0.0 \\
\hline $10 / 24 / 01$ & 8:02 & 19.3 & 19.4 & 19.4 & 19.1 & 0.0 & 9.6 & 30 & 79 & 0.0 \\
\hline $10 / 24 / 01$ & 8:03 & 19.2 & 19.4 & 19.4 & 19.1 & 0.0 & 9.7 & 90 & 79 & 0.0 \\
\hline $10 / 24 / 01$ & 8:04 & 19.3 & 19.4 & 19.4 & 19.1 & 0.0 & 9.6 & 36 & 79 & 0.0 \\
\hline $10 / 24 / 01$ & 8:05 & 19.2 & 19.4 & 19.4 & 19.1 & 0.0 & 9.5 & 30 & 79 & 0.0 \\
\hline $10 / 24 / 01$ & 8:06 & 19.2 & 19.4 & 19.4 & 19.1 & 0.0 & 9.6 & 71 & 79 & 0.0 \\
\hline $10 / 24 / 01$ & 8:07 & 19.2 & 19.4 & 19.4 & 19.1 & 0.0 & 9.7 & 28 & 79 & 0.0 \\
\hline $10 / 24 / 01$ & 8:08 & 19.2 & 19.4 & 19.4 & 19.1 & 0.0 & 9.6 & 84 & 79 & 0.0 \\
\hline $10 / 24 / 01$ & 8:09 & 19.3 & 19.4 & 19.4 & 19.1 & 0.0 & 9.7 & 62 & 79 & 0.0 \\
\hline $10 / 24 / 01$ & $8: 10$ & 19.2 & 19.4 & 19.4 & 19.1 & 0.0 & 9.6 & 39 & 79 & 0.0 \\
\hline $10 / 24 / 01$ & $8: 11$ & 19.3 & 19.4 & 19.4 & 19.1 & 0.0 & 9.6 & 57 & 79 & 0.0 \\
\hline $10 / 24 / 01$ & $8: 12$ & 19.3 & 19.5 & 19.4 & 19.1 & 0.0 & 9.6 & 26 & 79 & 0.0 \\
\hline $10 / 24 / 01$ & 8:13 & 19.3 & 19.5 & 19.4 & 19.2 & 0.0 & 9.7 & 51 & 79 & 0.0 \\
\hline $10 / 24 / 01$ & 8:14 & 19.3 & 19.5 & 19.4 & 19.2 & 0.0 & 9.7 & 70 & 79 & 0.0 \\
\hline $10 / 24 / 01$ & $8: 15$ & 19.3 & 19.5 & 19.4 & 19.2 & 0.0 & 9.7 & 44 & 79 & 0.0 \\
\hline $10 / 24 / 01$ & 8:16 & 19.3 & 19.5 & 19.5 & 19.2 & 0.0 & 9.6 & 32 & 79 & 0.0 \\
\hline $10 / 24 / 01$ & 8:17 & 19.4 & 19.5 & 19.5 & 19.2 & 0.0 & 9.7 & 29 & 79 & 0.0 \\
\hline $10 / 24 / 01$ & 8:18 & 19.3 & 19.5 & 19.5 & 19.2 & 0.0 & 9.6 & 49 & 79 & 0.0 \\
\hline $10 / 24 / 01$ & $8: 19$ & 19.4 & 19.5 & 19.5 & 19.2 & 0.0 & 9.7 & 47 & 79 & 0.0 \\
\hline $10 / 24 / 01$ & 8:20 & 19.3 & 19.5 & 19.5 & 19.2 & 0.0 & 9.6 & 31 & 79 & 0.0 \\
\hline $10 / 24 / 01$ & 8:21 & 19.3 & 19.5 & 19.5 & 19.2 & 0.0 & 9.7 & 91 & 79 & 0.0 \\
\hline $10 / 24 / 01$ & 8:22 & 19.3 & 19.5 & 19.5 & 19.2 & 0.0 & 9.6 & 84 & 79 & 0.0 \\
\hline $10 / 24 / 01$ & 8:23 & 19.3 & 19.5 & 19.5 & 19.2 & 0.0 & 9.7 & 34 & 79 & 0.0 \\
\hline $10 / 24 / 01$ & 8:24 & 19.4 & 19.5 & 19.5 & 19.2 & 0.0 & 9.6 & 81 & 79 & 0.0 \\
\hline $10 / 24 / 01$ & 8:25 & 19.3 & 19.5 & 19.5 & 19.2 & 0.0 & 9.7 & 98 & 79 & 0.0 \\
\hline $10 / 24 / 01$ & 8:26 & 19.4 & 19.5 & 19.5 & 19.2 & 0.0 & 9.7 & 54 & 79 & 0.0 \\
\hline
\end{tabular}


WSRC-TR-2002-00459, Rev. 0 SRT-RPP-2002-00221

\begin{tabular}{|c|c|c|c|c|c|c|c|c|c|c|}
\hline \multicolumn{11}{|c|}{ PREC2_102401_0627 } \\
\hline DATE & TIME & $\begin{array}{c}\mathrm{HX} \\
\text { Outlet } \\
\left({ }^{\circ} \mathrm{C}\right) \\
\mathrm{TCO}\end{array}$ & \begin{tabular}{|c|} 
REC \\
PP \\
OUT \\
$\left({ }^{\circ} \mathrm{C}\right)$ \\
TC1
\end{tabular} & $\begin{array}{c}\text { TK } \\
\text { BOT } \\
\left({ }^{\circ} \mathrm{C}\right) \\
\text { TC2 }\end{array}$ & $\begin{array}{c}\mathrm{HX} \\
\text { OUT } \\
\left({ }^{\circ} \mathrm{C}\right) \\
\text { TC3 }\end{array}$ & $\begin{array}{c}\text { Mn } \\
\text { Flow } \\
\text { (gpm) }\end{array}$ & $\begin{array}{c}\text { Galigher } \\
\text { Flow } \\
\text { (gpm) }\end{array}$ & $\begin{array}{c}\text { Current } \\
\text { to } \mathrm{HX}\end{array}$ & $\begin{array}{c}\text { Voltage } \\
\text { to } \mathrm{HX}\end{array}$ & $\begin{array}{c}\mathrm{Sr} \\
\text { Flow } \\
\text { (gpm) }\end{array}$ \\
\hline $10 / 24 / 01$ & $8: 27$ & 19.3 & 19.5 & 19.5 & 19.2 & 0.0 & 9.7 & 65 & 79 & 0.0 \\
\hline $10 / 24 / 01$ & $8: 28$ & 19.4 & 19.5 & 19.5 & 19.2 & 0.0 & 9.7 & 84 & 79 & 0.0 \\
\hline $10 / 24 / 01$ & $8: 29$ & 19.3 & 19.5 & 19.5 & 19.2 & 0.0 & 9.6 & 37 & 79 & 0.0 \\
\hline $10 / 24 / 01$ & $8: 30$ & 19.4 & 19.5 & 19.5 & 19.2 & 0.0 & 9.6 & 57 & 79 & 0.0 \\
\hline $10 / 24 / 01$ & $8: 31$ & 19.3 & 19.5 & 19.5 & 19.2 & 0.0 & 9.7 & 60 & 79 & 0.0 \\
\hline $10 / 24 / 01$ & $8: 32$ & 19.4 & 19.5 & 19.5 & 19.2 & 0.0 & 9.6 & 100 & 79 & 0.0 \\
\hline $10 / 24 / 01$ & $8: 33$ & 19.4 & 19.5 & 19.5 & 19.2 & 0.0 & 9.7 & 94 & 79 & 0.0 \\
\hline $10 / 24 / 01$ & $8: 34$ & 19.4 & 19.5 & 19.5 & 19.2 & 0.0 & 9.6 & 115 & 79 & 0.0 \\
\hline $10 / 24 / 01$ & $8: 35$ & 19.4 & 19.5 & 19.5 & 19.2 & 0.0 & 9.5 & 38 & 79 & 0.0 \\
\hline $10 / 24 / 01$ & $8: 36$ & 19.3 & 19.5 & 19.5 & 19.2 & 0.0 & 9.6 & 77 & 79 & 0.0 \\
\hline $10 / 24 / 01$ & $8: 37$ & 19.4 & 19.5 & 19.5 & 19.2 & 0.0 & 9.6 & 50 & 79 & 0.0 \\
\hline $10 / 24 / 01$ & $8: 38$ & 19.3 & 19.5 & 19.5 & 19.2 & 0.0 & 9.6 & 55 & 79 & 0.0 \\
\hline $10 / 24 / 01$ & $8: 39$ & 19.4 & 19.5 & 19.5 & 19.3 & 0.0 & 9.7 & 54 & 79 & 0.0 \\
\hline $10 / 24 / 01$ & $8: 40$ & 19.3 & 19.5 & 19.5 & 19.2 & 0.0 & 9.7 & 104 & 79 & 0.0 \\
\hline $10 / 24 / 01$ & $8: 41$ & 19.4 & 19.5 & 19.5 & 19.3 & 0.0 & 9.7 & 102 & 79 & 0.0 \\
\hline $10 / 24 / 01$ & $8: 42$ & 19.3 & 19.5 & 19.5 & 19.2 & 0.0 & 9.6 & 81 & 79 & 0.0 \\
\hline $10 / 24 / 01$ & $8: 43$ & 19.4 & 19.5 & 19.5 & 19.3 & 0.0 & 9.7 & 99 & 79 & 0.0 \\
\hline $10 / 24 / 01$ & $8: 44$ & 19.4 & 19.5 & 19.5 & 19.2 & 0.0 & 9.7 & 72 & 79 & 0.0 \\
\hline $10 / 24 / 01$ & $8: 45$ & 19.4 & 19.5 & 19.5 & 19.2 & 0.0 & 9.7 & 65 & 79 & 0.0 \\
\hline $10 / 24 / 01$ & $8: 46$ & 19.4 & 19.6 & 19.5 & 19.2 & 0.0 & 9.7 & 69 & 79 & 0.0 \\
\hline $10 / 24 / 01$ & $8: 47$ & 19.4 & 19.5 & 19.5 & 19.2 & 0.0 & 9.7 & 87 & 79 & 0.0 \\
\hline $10 / 24 / 01$ & $8: 48$ & 19.4 & 19.5 & 19.5 & 19.2 & 0.0 & 9.5 & 102 & 79 & 0.0 \\
\hline $10 / 24 / 01$ & $8: 49$ & 19.3 & 19.5 & 19.5 & 19.2 & 0.0 & 9.6 & 63 & 79 & 0.0 \\
\hline $10 / 24 / 01$ & 8:50 & 19.4 & 19.5 & 19.5 & 19.3 & 0.0 & 9.6 & 61 & 79 & 0.0 \\
\hline $10 / 24 / 01$ & $8: 51$ & 19.4 & 19.6 & 19.6 & 19.2 & 0.0 & 9.6 & 57 & 79 & 0.0 \\
\hline $10 / 24 / 01$ & 8:52 & 19.4 & 19.6 & 19.6 & 19.3 & 0.0 & 9.6 & 65 & 79 & 0.0 \\
\hline $10 / 24 / 01$ & 8:53 & 19.4 & 19.6 & 19.6 & 19.2 & 0.0 & 9.7 & 92 & 79 & 0.0 \\
\hline $10 / 24 / 01$ & $8: 54$ & 19.4 & 19.6 & 19.6 & 19.3 & 0.0 & 9.5 & 65 & 79 & 0.0 \\
\hline $10 / 24 / 01$ & $8: 55$ & 19.4 & 19.6 & 19.6 & 19.2 & 0.0 & 9.7 & 53 & 79 & 0.0 \\
\hline $10 / 24 / 01$ & 8:56 & 19.4 & 19.6 & 19.6 & 19.3 & 0.0 & 9.6 & 68 & 79 & 0.0 \\
\hline $10 / 24 / 01$ & 8:57 & 19.4 & 19.6 & 19.6 & 19.2 & 0.0 & 9.7 & 94 & 79 & 0.0 \\
\hline $10 / 24 / 01$ & 8:58 & 19.4 & 19.6 & 19.6 & 19.3 & 0.0 & 9.6 & 79 & 79 & 0.0 \\
\hline $10 / 24 / 01$ & $8: 59$ & 19.4 & \begin{tabular}{|l|}
19.6 \\
\end{tabular} & 19.6 & 19.3 & 0.0 & 9.7 & 99 & 79 & 0.0 \\
\hline $10 / 24 / 01$ & 9:00 & 19.4 & 19.6 & 19.6 & 19.3 & 0.0 & 9.7 & 99 & 79 & 0.0 \\
\hline $10 / 24 / 01$ & 9:01 & 19.4 & 19.6 & 19.6 & 19.3 & 0.0 & 9.7 & 42 & 79 & 0.0 \\
\hline $10 / 24 / 01$ & 9:02 & 19.4 & 19.6 & 19.6 & 19.2 & 0.0 & 9.6 & 59 & 79 & 0.0 \\
\hline $10 / 24 / 01$ & 9:03 & 19.4 & 19.6 & 19.6 & 19.3 & 0.0 & 9.6 & 85 & 79 & 0.0 \\
\hline $10 / 24 / 01$ & 9:04 & 19.4 & 19.6 & 19.6 & 19.2 & 0.0 & 9.6 & 57 & 79 & 0.0 \\
\hline $10 / 24 / 01$ & 9:05 & 19.4 & 19.6 & 19.6 & 19.3 & 0.0 & 9.6 & 99 & 79 & 0.0 \\
\hline $10 / 24 / 01$ & 9:06 & 19.4 & 19.6 & 19.6 & 19.2 & 0.0 & 9.6 & 95 & 79 & 0.0 \\
\hline $10 / 24 / 01$ & 9:07 & 19.4 & 19.6 & 19.6 & 19.3 & 0.0 & 9.5 & 77 & 79 & 0.0 \\
\hline $10 / 24 / 01$ & 9:08 & 19.4 & 19.6 & 19.6 & 19.2 & 0.0 & 9.6 & 94 & 79 & 0.0 \\
\hline $10 / 24 / 01$ & 9:09 & 19.4 & 19.6 & 19.6 & 19.3 & 0.0 & 9.6 & 82 & 79 & 0.0 \\
\hline $10 / 24 / 01$ & $9: 10$ & 19.4 & 19.6 & 19.6 & 19.2 & 0.0 & 9.7 & 86 & 79 & 0.0 \\
\hline $10 / 24 / 01$ & $9: 11$ & 19.4 & 19.6 & 19.6 & 19.3 & 0.0 & 9.6 & 104 & 79 & 0.0 \\
\hline $10 / 24 / 01$ & $9: 12$ & 19.4 & 19.6 & 19.6 & 19.3 & 0.0 & 9.6 & 102 & 79 & 0.0 \\
\hline $10 / 24 / 01$ & 9:13 & 19.4 & 19.6 & 19.6 & 19.2 & 0.0 & 9.7 & 111 & 79 & 0.0 \\
\hline $10 / 24 / 01$ & $9: 14$ & 19.4 & \begin{tabular}{|l|}
19.6 \\
\end{tabular} & 19.6 & 19.3 & 0.0 & 9.6 & 104 & 79 & 0.0 \\
\hline $10 / 24 / 01$ & 9:15 & 19.4 & 19.6 & 19.6 & 19.2 & 0.0 & 9.6 & 64 & 79 & 0.0 \\
\hline $10 / 24 / 01$ & $9: 16$ & 19.5 & \begin{tabular}{|l|}
19.6 \\
\end{tabular} & 19.6 & 19.3 & 0.0 & 9.8 & 81 & 79 & 0.0 \\
\hline $10 / 24 / 01$ & 9:17 & 19.4 & 19.6 & 19.6 & 19.2 & 0.0 & 9.6 & 110 & 79 & 0.0 \\
\hline $10 / 24 / 01$ & $9: 18$ & 19.5 & 19.6 & 19.6 & 19.3 & 0.0 & 9.6 & 72 & 79 & 0.0 \\
\hline $10 / 24 / 01$ & $9: 19$ & 19.4 & \begin{tabular}{|l|}
19.6 \\
\end{tabular} & 19.6 & 19.2 & 0.0 & 9.7 & 102 & 79 & 0.0 \\
\hline $10 / 24 / 01$ & $9: 20$ & 19.4 & 19.6 & 19.6 & 19.3 & 0.0 & 9.6 & 115 & 79 & 0.0 \\
\hline 10/24/01 & $9: 21$ & 19.4 & 19.6 & 19.6 & 19.3 & 0.0 & 9.7 & 92 & 79 & 0.0 \\
\hline $10 / 24 / 01$ & $9: 22$ & 19.4 & 19.6 & 19.6 & 19.3 & 0.0 & 9.6 & 119 & 79 & 0.0 \\
\hline $10 / 24 / 01$ & $9: 23$ & 19.4 & 19.6 & 19.6 & 19.3 & 0.0 & 9.5 & 96 & 79 & 0.0 \\
\hline $10 / 24 / 01$ & $9: 24$ & 19.4 & 19.6 & 19.6 & 19.3 & 0.0 & 9.5 & 111 & 79 & 0.0 \\
\hline $10 / 24 / 01$ & 9:25 & 19.5 & 19.6 & 19.6 & 19.3 & 0.0 & 9.6 & 73 & 79 & 0.0 \\
\hline $0 / 24 / 01$ & 9:26 & 19.4 & .6 & 19.6 & 19.3 & 0.0 & 9.6 & 84 & 79 & 0.0 \\
\hline
\end{tabular}

\begin{tabular}{|c|c|c|c|c|c|c|c|c|c|c|}
\hline \multicolumn{11}{|c|}{ PREC2_102401_0627 } \\
\hline DATE & TIME & \begin{tabular}{|c|}
$\mathrm{HX}$ \\
Outlet \\
$\left({ }^{\circ} \mathrm{C}\right)$ \\
$\mathrm{TCO}$ \\
\end{tabular} & \begin{tabular}{|c|} 
REC \\
PP \\
OUT \\
$\left({ }^{\circ} \mathrm{C}\right)$ \\
TC1
\end{tabular} & $\begin{array}{c}\text { TK } \\
\text { BOT } \\
\left({ }^{\circ} \mathrm{C}\right) \\
\text { TC2 }\end{array}$ & $\begin{array}{c}\mathrm{HX} \\
\text { OUT } \\
\left({ }^{\circ} \mathrm{C}\right) \\
\text { TC3 }\end{array}$ & \begin{tabular}{|c|} 
Mn \\
Flow \\
(gpm)
\end{tabular} & \begin{tabular}{|c|} 
Galigher \\
Flow \\
(gpm)
\end{tabular} & $\begin{array}{c}\text { Current } \\
\text { to } \mathrm{HX}\end{array}$ & $\begin{array}{c}\text { Voltage } \\
\text { to } \mathrm{HX} \\
\end{array}$ & $\begin{array}{c}\mathrm{Sr} \\
\text { Flow } \\
(\mathrm{gpm})\end{array}$ \\
\hline $10 / 24 / 01$ & 9:27 & 19.5 & 19.6 & 19.6 & 19.3 & 0.0 & 9.6 & 117 & 79 & 0.0 \\
\hline $10 / 24 / 01$ & $9: 28$ & 19.4 & 19.6 & 19.6 & 19.2 & 0.0 & 9.6 & 109 & 79 & 0.0 \\
\hline $10 / 24 / 01$ & 9:29 & 19.5 & 19.6 & 19.6 & 19.3 & 0.0 & 9.7 & 71 & 79 & 0.0 \\
\hline $10 / 24 / 01$ & 9:30 & 19.4 & 19.6 & 19.6 & 19.2 & 0.0 & 9.7 & 117 & 79 & 0.0 \\
\hline $10 / 24 / 01$ & 9:31 & 19.4 & 19.6 & 19.6 & 19.3 & 0.0 & 9.6 & 89 & 79 & 0.0 \\
\hline $10 / 24 / 01$ & 9:32 & 19.4 & 19.6 & 19.6 & 19.2 & 0.0 & 9.8 & 100 & 79 & 0.0 \\
\hline $10 / 24 / 01$ & 9:33 & 19.4 & 19.6 & 19.6 & 19.3 & 0.0 & 9.6 & 85 & 79 & 0.0 \\
\hline $10 / 24 / 01$ & 9:34 & 19.4 & 19.6 & 19.6 & 19.3 & 0.0 & 9.7 & 72 & 79 & 0.0 \\
\hline $10 / 24 / 01$ & 9:35 & 19.4 & 19.6 & 19.6 & 19.3 & 0.0 & 9.7 & 118 & 79 & 0.0 \\
\hline $10 / 24 / 01$ & 9:36 & 19.4 & 19.6 & 19.6 & 19.3 & 0.0 & 9.6 & 114 & 79 & 0.0 \\
\hline $10 / 24 / 01$ & 9:37 & 19.4 & 19.6 & 19.6 & 19.2 & 0.0 & 9.6 & 82 & 79 & 0.0 \\
\hline $10 / 24 / 01$ & 9:38 & 19.4 & 19.6 & 19.6 & 19.3 & 0.0 & 9.8 & 65 & 79 & 0.0 \\
\hline $10 / 24 / 01$ & 9:39 & 19.4 & 19.6 & 19.6 & 19.2 & 0.0 & 9.7 & 122 & 79 & 0.0 \\
\hline $10 / 24 / 01$ & 9:40 & 19.4 & 19.6 & 19.6 & 19.3 & 0.0 & 9.6 & 91 & 79 & 0.0 \\
\hline $10 / 24 / 01$ & $9: 41$ & 19.4 & 19.6 & 19.6 & 19.2 & 0.0 & 9.7 & 120 & 79 & 0.0 \\
\hline $10 / 24 / 01$ & 9:42 & 19.4 & 19.6 & 19.6 & 19.3 & 0.0 & 9.7 & 76 & 79 & 0.0 \\
\hline $10 / 24 / 01$ & $9: 43$ & 19.4 & 19.6 & 19.6 & 19.2 & 0.0 & 9.7 & 73 & 79 & 0.0 \\
\hline $10 / 24 / 01$ & $9: 44$ & 19.4 & 19.6 & 19.6 & 19.3 & 0.0 & 9.5 & 116 & 79 & 0.0 \\
\hline $10 / 24 / 01$ & 9:45 & 19.4 & 19.6 & 19.6 & 19.2 & 0.0 & 9.6 & 74 & 79 & 0.0 \\
\hline $10 / 24 / 01$ & $9: 46$ & 19.4 & 19.6 & 19.6 & 19.2 & 0.0 & 9.6 & 97 & 79 & 0.0 \\
\hline $10 / 24 / 01$ & 9:47 & 19.4 & 19.6 & 19.6 & 19.3 & 0.0 & 9.6 & 115 & 79 & 0.0 \\
\hline $10 / 24 / 01$ & 9:48 & 19.4 & 19.6 & 19.6 & 19.2 & 0.0 & 9.5 & 102 & 79 & 0.0 \\
\hline $10 / 24 / 01$ & 9:49 & 19.4 & 19.6 & 19.6 & 19.3 & 0.0 & 9.6 & 105 & 79 & 0.0 \\
\hline $10 / 24 / 01$ & 9:50 & 19.4 & 19.6 & 19.6 & 19.2 & 0.0 & 9.6 & 81 & 79 & 0.0 \\
\hline $10 / 24 / 01$ & 9:51 & 19.4 & 19.6 & 19.6 & 19.3 & 0.0 & 9.6 & 112 & 79 & 0.0 \\
\hline $10 / 24 / 01$ & 9:52 & 19.4 & 19.6 & 19.6 & 19.2 & 0.0 & 9.7 & 99 & 79 & 0.0 \\
\hline $10 / 24 / 01$ & 9:53 & 19.4 & 19.6 & 19.6 & 19.3 & 0.0 & 9.7 & 131 & 79 & 0.0 \\
\hline $10 / 24 / 01$ & 9:54 & 19.4 & 19.6 & 19.5 & 19.2 & 0.0 & 9.7 & 67 & 79 & 0.0 \\
\hline $10 / 24 / 01$ & 9:55 & 19.4 & 19.6 & 19.5 & 19.2 & 0.0 & 9.7 & 96 & 79 & 0.0 \\
\hline $10 / 24 / 01$ & 9:56 & 19.4 & 19.6 & 19.5 & 19.2 & 0.0 & 9.7 & 97 & 79 & 0.0 \\
\hline $10 / 24 / 01$ & 9:57 & 19.4 & 19.6 & 19.6 & 19.2 & 0.0 & 9.6 & 86 & 79 & 0.0 \\
\hline $10 / 24 / 01$ & 9:58 & 19.4 & 19.6 & 19.5 & 19.2 & 0.0 & 9.7 & 131 & 79 & 0.0 \\
\hline $10 / 24 / 01$ & 9:59 & 19.3 & 19.6 & 19.5 & 19.2 & 0.0 & 9.7 & 85 & 79 & 0.0 \\
\hline $10 / 24 / 01$ & $10: 00$ & 19.4 & 19.5 & 19.5 & 19.3 & 0.0 & 9.6 & 116 & 79 & 0.0 \\
\hline $10 / 24 / 01$ & 10:01 & 19.3 & 19.5 & 19.5 & 19.2 & 0.0 & 9.6 & 129 & 79 & 0.0 \\
\hline $10 / 24 / 01$ & 10:02 & 19.4 & 19.5 & 19.5 & 19.3 & 0.0 & 9.6 & 73 & 79 & 0.0 \\
\hline $10 / 24 / 01$ & 10:03 & 19.3 & 19.5 & 19.5 & 19.2 & 0.0 & 9.6 & 71 & 79 & 0.0 \\
\hline $10 / 24 / 01$ & 10:04 & 19.4 & 19.5 & 19.5 & 19.3 & 0.0 & 9.7 & 124 & 79 & 0.0 \\
\hline $10 / 24 / 01$ & 10:05 & 19.3 & 19.5 & 19.5 & 19.2 & 0.0 & 9.7 & 101 & 79 & 0.0 \\
\hline $10 / 24 / 01$ & 10:06 & 19.3 & 19.5 & 19.5 & 19.2 & 0.0 & 9.7 & 71 & 79 & 0.0 \\
\hline $10 / 24 / 01$ & 10:07 & 19.3 & 19.5 & 19.5 & 19.2 & 0.0 & 9.6 & 113 & 79 & 0.0 \\
\hline $10 / 24 / 01$ & 10:08 & 19.3 & 19.5 & 19.5 & 19.2 & 0.0 & 9.7 & 85 & 79 & 0.0 \\
\hline $10 / 24 / 01$ & 10:09 & 19.4 & 19.5 & 19.5 & 19.2 & 0.0 & 9.6 & 73 & 79 & 0.0 \\
\hline $10 / 24 / 01$ & $10: 10$ & 19.3 & 19.5 & 19.5 & 19.2 & 0.0 & 9.5 & 108 & 79 & 0.0 \\
\hline $10 / 24 / 01$ & 10:11 & 19.4 & 19.5 & 19.5 & 19.2 & 0.0 & 9.7 & 91 & 79 & 0.0 \\
\hline $10 / 24 / 01$ & $10: 12$ & 19.3 & 19.5 & 19.5 & 19.2 & 0.0 & 9.5 & 85 & 79 & 0.0 \\
\hline $10 / 24 / 01$ & $10: 13$ & 19.4 & 19.6 & 19.5 & 19.3 & 0.0 & 9.8 & 91 & 79 & 0.0 \\
\hline 10/24/01 & $10: 14$ & 19.3 & \begin{tabular}{|l|} 
\\
\end{tabular} & 19.5 & 19.2 & 0.0 & 9.6 & 93 & 79 & 0.0 \\
\hline $10 / 24 / 01$ & $10: 15$ & 19.4 & 19.6 & 19.5 & 19.3 & 0.0 & 9.6 & 102 & 79 & 0.0 \\
\hline $10 / 24 / 01$ & $10: 16$ & 19.3 & 19.6 & 19.5 & 19.2 & 0.0 & 9.7 & 92 & 79 & 0.0 \\
\hline $10 / 24 / 01$ & $10: 17$ & 19.4 & 19.6 & 19.5 & 19.3 & 0.0 & 9.7 & 72 & 79 & 0.0 \\
\hline $10 / 24 / 01$ & $10: 18$ & 19.4 & 19.5 & 19.5 & 19.3 & 0.0 & 9.6 & 126 & 79 & 0.0 \\
\hline $10 / 24 / 01$ & $10: 19$ & 19.4 & 19.6 & 19.6 & 19.3 & 0.0 & 9.6 & 125 & 79 & 0.0 \\
\hline $10 / 24 / 01$ & $10: 20$ & 19.4 & 19.6 & 19.5 & 19.3 & 0.0 & 9.8 & 113 & 79 & 0.0 \\
\hline $10 / 24 / 01$ & $10: 21$ & 19.3 & 19.6 & 19.6 & 19.3 & 0.0 & 9.6 & 93 & 79 & 0.0 \\
\hline $10 / 24 / 01$ & $10: 22$ & 19.4 & 19.6 & 19.5 & 19.3 & 0.0 & 9.6 & 75 & 79 & 0.0 \\
\hline $10 / 24 / 01$ & $10: 23$ & 19.3 & 19.6 & 19.5 & 19.2 & 0.0 & 9.6 & 95 & 79 & 0.0 \\
\hline $10 / 24 / 01$ & $10: 24$ & 19.4 & 19.5 & 19.5 & 19.3 & 0.0 & 9.6 & 129 & 79 & 0.0 \\
\hline $10 / 24 / 01$ & $10: 25$ & 19.3 & 19.6 & 19.5 & 19.2 & 0.0 & 9.7 & 78 & 79 & 0.0 \\
\hline $10 / 24 / 01$ & $10: 26$ & 19.4 & 19.5 & 19.5 & 19.3 & 0.0 & 9.6 & 74 & 79 & 0.0 \\
\hline
\end{tabular}


WSRC-TR-2002-00459, Rev. 0 SRT-RPP-2002-00221

\begin{tabular}{|c|c|c|c|c|c|c|c|c|c|c|}
\hline \multicolumn{11}{|c|}{ PREC2_102401_0627 } \\
\hline DATE & TIME & \begin{tabular}{|c}
$\mathrm{HX}$ \\
Outlet \\
$\left({ }^{\circ} \mathrm{C}\right)$ \\
$\mathrm{TCO}$
\end{tabular} & \begin{tabular}{|c|} 
REC \\
PP \\
OUT \\
$\left({ }^{\circ} \mathrm{C}\right)$ \\
TC1 1
\end{tabular} & \begin{tabular}{|c|} 
TK \\
BOT \\
$\left({ }^{\circ} \mathrm{C}\right)$ \\
TC2
\end{tabular} & $\begin{array}{l}\text { HX } \\
\text { OUT } \\
\left({ }^{\circ} \mathrm{C}\right) \\
\text { TC3 }\end{array}$ & $\begin{array}{c}\text { Mn } \\
\text { Flow } \\
\text { (gpm) }\end{array}$ & $\begin{array}{l}\text { Galigher } \\
\text { Flow } \\
\text { (gpm) }\end{array}$ & $\begin{array}{c}\text { Current } \\
\text { to } \mathrm{HX}\end{array}$ & $\begin{array}{l}\text { Voltage } \\
\text { to } \mathrm{HX}\end{array}$ & $\begin{array}{c}\mathrm{Sr} \\
\text { Flow } \\
\text { (gpm) }\end{array}$ \\
\hline $10 / 24 / 01$ & 10:27 & 19.3 & 19.5 & 19.5 & 19.2 & 0.0 & 9.6 & 79 & 79 & 0.0 \\
\hline $10 / 24 / 01$ & $10: 28$ & 19.4 & 19.5 & 19.5 & 19.3 & 0.0 & 9.6 & 98 & 79 & 0.0 \\
\hline $10 / 24 / 01$ & 10:29 & 19.3 & 19.5 & 19.5 & 19.2 & 0.0 & 9.7 & 135 & 79 & 0.0 \\
\hline $10 / 24 / 01$ & $10: 30$ & 19.3 & 19.5 & \begin{tabular}{|l|}
19.5 \\
\end{tabular} & \begin{tabular}{|l|}
19.2 \\
\end{tabular} & 0.0 & 9.6 & 82 & 79 & 0.0 \\
\hline $10 / 24 / 01$ & 10:31 & 19.3 & 19.5 & \begin{tabular}{|l|}
19.5 \\
\end{tabular} & \begin{tabular}{|l|}
19.2 \\
\end{tabular} & 0.0 & 9.6 & 129 & 79 & 0.0 \\
\hline $10 / 24 / 01$ & 10:32 & 19.3 & 19.5 & 19.5 & 19.2 & 0.0 & 9.6 & 111 & 79 & 0.0 \\
\hline $10 / 24 / 01$ & $10: 33$ & 19.3 & 19.5 & 19.5 & 19.2 & 0.0 & 9.7 & 76 & 79 & 0.0 \\
\hline $10 / 24 / 01$ & $10: 34$ & 19.3 & 19.5 & 19.5 & 19.2 & 0.0 & 9.6 & 97 & 79 & 0.0 \\
\hline $10 / 24 / 01$ & $10: 35$ & 19.3 & 19.5 & 19.5 & \begin{tabular}{|l|}
19.2 \\
\end{tabular} & 0.0 & 9.6 & 94 & 79 & 0.0 \\
\hline $10 / 24 / 01$ & $10: 36$ & 19.3 & 19.5 & 19.5 & \begin{tabular}{|l|}
19.1 \\
\end{tabular} & 0.0 & 9.8 & 95 & 79 & 0.0 \\
\hline $10 / 24 / 01$ & $10: 37$ & 19.3 & 19.5 & 19.5 & 19.2 & 0.0 & 9.7 & 87 & 79 & 0.0 \\
\hline $10 / 24 / 01$ & $10: 38$ & 19.3 & 19.5 & 19.5 & \begin{tabular}{|l|}
19.2 \\
\end{tabular} & 0.0 & 9.6 & 79 & 79 & 0.0 \\
\hline $10 / 24 / 01$ & $10: 39$ & 19.3 & 19.5 & 19.5 & \begin{tabular}{|l|}
19.2 \\
\end{tabular} & 0.0 & 9.7 & 80 & 79 & 0.0 \\
\hline $10 / 24 / 01$ & $10: 40$ & 19.3 & 19.5 & 19.5 & 19.1 & 0.0 & 9.6 & 97 & 79 & 0.0 \\
\hline $10 / 24 / 01$ & $10: 41$ & 19.3 & 19.4 & 19.5 & \begin{tabular}{|l|}
19.2 \\
\end{tabular} & 0.0 & 9.6 & 130 & 79 & 0.0 \\
\hline $10 / 24 / 01$ & 10:42 & 19.3 & 19.4 & 19.5 & 19.2 & 0.0 & 9.7 & 94 & 79 & 0.0 \\
\hline $10 / 24 / 01$ & 10:43 & 19.2 & 19.4 & 19.5 & $\mid 19.1$ & 0.0 & 9.7 & 81 & 79 & 0.0 \\
\hline $10 / 24 / 01$ & $10: 44$ & 19.3 & 19.4 & 19.5 & 19.2 & 0.0 & 9.6 & 127 & 79 & 0.0 \\
\hline $10 / 24 / 01$ & $10: 45$ & 19.2 & 19.4 & 19.4 & \begin{tabular}{|l|}
19.1 \\
\end{tabular} & 0.0 & 9.6 & 84 & 79 & 0.0 \\
\hline $10 / 24 / 01$ & 10:46 & 19.3 & 19.4 & 19.4 & 19.2 & 0.0 & 9.6 & 111 & 79 & 0.0 \\
\hline $10 / 24 / 01$ & 10:47 & 19.2 & 19.4 & 19.4 & $\mid 19.1$ & 0.0 & 9.6 & 109 & 79 & 0.0 \\
\hline $10 / 24 / 01$ & $10: 48$ & 19.3 & 19.4 & 19.4 & 19.2 & 0.0 & 9.6 & 95 & 79 & 0.0 \\
\hline $10 / 24 / 01$ & $10: 49$ & 19.2 & 19.4 & 19.4 & 19.1 & 0.0 & 9.6 & 122 & 79 & 0.0 \\
\hline $10 / 24 / 01$ & $10: 50$ & 19.3 & 19.4 & \begin{tabular}{|l|}
19.4 \\
\end{tabular} & 19.2 & 0.0 & 9.7 & 103 & 79 & 0.0 \\
\hline $10 / 24 / 01$ & 10:51 & 19.2 & 19.4 & \begin{tabular}{|l|}
19.4 \\
\end{tabular} & $\mid 19.1$ & 0.0 & 9.7 & 101 & 79 & 0.0 \\
\hline $10 / 24 / 01$ & 10:52 & 19.3 & 19.4 & 19.4 & $\mid 19.1$ & 0.0 & 9.7 & 115 & 79 & 0.0 \\
\hline $10 / 24 / 01$ & 10:53 & 19.3 & 19.4 & 19.4 & $\mid 19.1$ & 0.0 & 9.6 & 85 & 79 & 0.0 \\
\hline $10 / 24 / 01$ & 10:54 & 19.2 & 19.4 & 19.4 & 19.1 & 0.0 & 9.6 & 133 & 79 & 0.0 \\
\hline $10 / 24 / 01$ & 10:55 & 19.3 & 19.4 & 19.4 & 19.2 & 0.0 & 9.6 & 93 & 79 & 0.0 \\
\hline $10 / 24 / 01$ & $10: 56$ & 19.2 & 19.4 & 19.4 & $\mid 19.1$ & 0.0 & 9.7 & 97 & 79 & 0.0 \\
\hline $10 / 24 / 01$ & $10: 57$ & 19.3 & 19.4 & 19.4 & \begin{tabular}{|l|}
19.2 \\
\end{tabular} & 0.0 & 9.7 & 137 & 79 & 0.0 \\
\hline $10 / 24 / 01$ & $10: 58$ & 19.2 & \begin{tabular}{|l|} 
\\
\end{tabular} & \begin{tabular}{|l|}
19.4 \\
\end{tabular} & 19.1 & 0.0 & 9.7 & 102 & 79 & 0.0 \\
\hline $10 / 24 / 01$ & 10:59 & 19.3 & 19.4 & 19.4 & 19.2 & 0.0 & 9.6 & 102 & 79 & 0.0 \\
\hline $10 / 24 / 01$ & $11: 00$ & 19.2 & 19.4 & \begin{tabular}{|l|}
19.4 \\
\end{tabular} & \begin{tabular}{|l|}
19.1 \\
\end{tabular} & 0.0 & 9.6 & 130 & 79 & 0.0 \\
\hline $10 / 24 / 01$ & 11:01 & 19.3 & 19.4 & 19.4 & 19.2 & 0.0 & 9.6 & 90 & 79 & 0.0 \\
\hline $10 / 24 / 01$ & $11: 02$ & 19.2 & 19.4 & 19.4 & 19.1 & 0.0 & 9.6 & 108 & 79 & 0.0 \\
\hline $10 / 24 / 01$ & $11: 03$ & 19.2 & 19.4 & 19.4 & 19.1 & 0.0 & 9.7 & 115 & 79 & 0.0 \\
\hline $10 / 24 / 01$ & 11:05 & 19.2 & 19.4 & \begin{tabular}{|l|}
19.4 \\
\end{tabular} & 19.1 & 0.0 & 9.6 & 130 & 79 & 0.0 \\
\hline $10 / 24 / 01$ & 11:06 & 19.2 & \begin{tabular}{|l|} 
\\
$\mid 19.4$
\end{tabular} & \begin{tabular}{|l|}
19.4 \\
\end{tabular} & $\mid 19.1$ & 0.0 & 9.8 & 84 & 79 & 0.0 \\
\hline $10 / 24 / 01$ & 11:07 & 19.2 & 19.4 & \begin{tabular}{|l|}
19.4 \\
\end{tabular} & $\mid 19.1$ & 0.0 & 9.6 & 115 & 79 & 0.0 \\
\hline $10 / 24 / 01$ & $11: 08$ & 19.2 & 19.4 & 19.4 & \begin{tabular}{|l|}
19.1 \\
\end{tabular} & 0.0 & 9.6 & 102 & 79 & 0.0 \\
\hline $10 / 24 / 01$ & $11: 09$ & 19.2 & 19.4 & 19.4 & 19.1 & 0.0 & 9.7 & 106 & 79 & 0.0 \\
\hline $10 / 24 / 01$ & $11: 10$ & 19.2 & 19.4 & 19.4 & $\mid 19.1$ & 0.0 & 9.6 & 123 & 79 & 0.0 \\
\hline $10 / 24 / 01$ & 11:11 & 19.2 & 19.4 & \begin{tabular}{|l|}
19.4 \\
\end{tabular} & $\mid 19.1$ & 0.0 & 9.6 & 84 & 79 & 0.0 \\
\hline $10 / 24 / 01$ & $11: 12$ & 19.2 & \begin{tabular}{|l|} 
\\
\end{tabular} & \begin{tabular}{|l|}
19.4 \\
\end{tabular} & $\mid 19.1$ & 0.0 & 9.5 & 126 & 79 & 0.0 \\
\hline $10 / 24 / 01$ & $11: 13$ & 19.2 & \begin{tabular}{|l|} 
\\
$\mid 19.4$
\end{tabular} & \begin{tabular}{|l|}
19.4 \\
\end{tabular} & $\mid 19.1$ & 0.0 & 9.7 & 78 & 79 & 0.0 \\
\hline $10 / 24 / 01$ & $11: 14$ & 19.2 & 19.4 & \begin{tabular}{|l|}
19.4 \\
\end{tabular} & $\mid 19.1$ & 0.0 & 9.7 & 124 & 79 & 0.0 \\
\hline $10 / 24 / 01$ & $11: 15$ & 19.2 & 19.4 & \begin{tabular}{|l|}
19.4 \\
\end{tabular} & 19.1 & 0.0 & 9.7 & 80 & 79 & 0.0 \\
\hline $10 / 24 / 01$ & $11: 16$ & 19.2 & 19.4 & 19.4 & $\mid 19.1$ & 0.0 & 9.6 & 135 & 79 & 0.0 \\
\hline $10 / 24 / 01$ & $11: 17$ & 19.2 & 19.4 & \begin{tabular}{|l|}
19.4 \\
\end{tabular} & $\mid 19.1$ & 0.0 & 9.6 & 76 & 79 & 0.0 \\
\hline $10 / 24 / 01$ & $11: 18$ & 19.2 & \begin{tabular}{|l|} 
\\
\end{tabular} & \begin{tabular}{|l|}
19.4 \\
\end{tabular} & $\mid 19.1$ & 0.0 & 9.7 & 118 & 79 & 0.0 \\
\hline $10 / 24 / 01$ & $11: 19$ & 19.2 & \begin{tabular}{|l|} 
\\
\end{tabular} & \begin{tabular}{|l|}
19.4 \\
\end{tabular} & \begin{tabular}{|l|}
19.1 \\
\end{tabular} & 0.0 & 9.6 & 97 & 79 & 0.0 \\
\hline $10 / 24 / 01$ & $11: 20$ & 19.2 & \begin{tabular}{|l|} 
\\
\end{tabular} & \begin{tabular}{|l|}
19.4 \\
\end{tabular} & $\mid 19.1$ & 0.0 & 9.7 & 108 & 79 & 0.0 \\
\hline $10 / 24 / 01$ & $11: 21$ & 19.2 & 19.4 & 19.4 & 19.1 & 0.0 & 9.6 & 112 & 79 & 0.0 \\
\hline $10 / 24 / 01$ & $11: 22$ & 19.2 & 19.4 & \begin{tabular}{|l|}
19.4 \\
\end{tabular} & \begin{tabular}{|l|}
19.1 \\
\end{tabular} & 0.0 & 9.6 & 84 & 79 & 0.0 \\
\hline $10 / 24 / 01$ & $11: 23$ & 19.2 & 19.4 & \begin{tabular}{|l|}
19.4 \\
\end{tabular} & 19.0 & 0.0 & 9.7 & 124 & 79 & 0.0 \\
\hline $10 / 24 / 01$ & $11: 24$ & 19.2 & 19.3 & \begin{tabular}{|l|}
19.4 \\
\end{tabular} & $\mid 19.1$ & 0.0 & 9.6 & 103 & 79 & 0.0 \\
\hline $10 / 24 / 01$ & $11: 25$ & 19.2 & 19.3 & \begin{tabular}{|l|}
19.4 \\
\end{tabular} & $\mid 19.1$ & 0.0 & 9.5 & 81 & 79 & 0.0 \\
\hline $10 / 24 / 01$ & $11: 26$ & 19.2 & 19.3 & \begin{tabular}{|l|}
19.4 \\
\end{tabular} & $\mid 19.1$ & 0.0 & 9.7 & 113 & 79 & 0.0 \\
\hline $10 / 24 / 01$ & $11: 27$ & 19.2 & 19.3 & \begin{tabular}{|l|}
19.4 \\
\end{tabular} & \begin{tabular}{|l|}
19.1 \\
\end{tabular} & 0.0 & 9.5 & 131 & 79 & 0.0 \\
\hline
\end{tabular}

\begin{tabular}{|c|c|c|c|c|c|c|c|c|c|c|}
\hline \multicolumn{11}{|c|}{ PREC2_102401_0627 } \\
\hline DATE & TIME & \begin{tabular}{|c|}
$\mathrm{HX}$ \\
Outlet \\
$\left({ }^{\circ} \mathrm{C}\right)$ \\
TCO \\
\end{tabular} & \begin{tabular}{|c|} 
REC \\
PP \\
OUT \\
$\left({ }^{\circ} \mathrm{C}\right)$ \\
TC1
\end{tabular} & $\begin{array}{c}\text { TK } \\
\text { BOT } \\
\left({ }^{\circ} \mathrm{C}\right) \\
\text { TC2 }\end{array}$ & $\begin{array}{c}\mathrm{HX} \\
\text { OUT } \\
\left({ }^{\circ} \mathrm{C}\right) \\
\text { TC3 }\end{array}$ & $\begin{array}{c}\text { Mn } \\
\text { Flow } \\
(\mathrm{gpm})\end{array}$ & $\begin{array}{c}\text { Galigher } \\
\text { Flow } \\
\text { (gpm) }\end{array}$ & $\begin{array}{c}\text { Current } \\
\text { to } \mathrm{HX}\end{array}$ & $\begin{array}{c}\text { Voltage } \\
\text { to } \mathrm{HX}\end{array}$ & $\begin{array}{c}\text { Sr } \\
\text { Flow } \\
(\mathrm{gpm})\end{array}$ \\
\hline $10 / 24 / 01$ & $11: 28$ & 19.2 & 19.4 & 19.4 & 19.1 & 0.0 & 9.6 & 109 & 79 & 0.0 \\
\hline $10 / 24 / 01$ & $11: 29$ & 19.2 & 19.3 & 19.4 & 19.1 & 0.0 & 9.7 & 80 & 79 & 0.0 \\
\hline $10 / 24 / 01$ & $11: 30$ & 19.1 & 19.3 & 19.4 & 19.0 & 0.0 & 9.6 & 92 & 79 & 0.0 \\
\hline 10/24/01 & 11:31 & 19.2 & 19.3 & 19.3 & 19.1 & 0.0 & 9.6 & 125 & 79 & 0.0 \\
\hline $10 / 24 / 01$ & $11: 32$ & 19.1 & 19.3 & 19.4 & 19.0 & 0.0 & 9.6 & 131 & 79 & 0.0 \\
\hline $10 / 24 / 01$ & $11: 33$ & 19.2 & 19.3 & 19.3 & 19.1 & 0.0 & 9.6 & 137 & 79 & 0.0 \\
\hline 10/24/01 & $11: 34$ & 19.1 & 19.3 & 19.3 & 19.0 & 0.0 & 9.7 & 116 & 79 & 0.0 \\
\hline $10 / 24 / 01$ & $11: 35$ & 19.2 & 19.3 & 19.3 & 19.1 & 0.0 & 9.7 & 123 & 79 & 0.0 \\
\hline $10 / 24 / 01$ & $11: 36$ & 19.1 & 19.3 & 19.3 & 19.0 & 0.0 & 9.6 & 128 & 79 & 0.0 \\
\hline $10 / 24 / 01$ & $11: 37$ & 19.2 & 19.3 & 19.3 & 19.1 & 0.0 & 9.6 & 94 & 79 & 0.0 \\
\hline $10 / 24 / 01$ & $11: 38$ & 19.2 & 19.3 & 19.3 & 19.0 & 0.0 & 9.6 & 80 & 79 & 0.0 \\
\hline 10/24/01 & 11:39 & 19.1 & 19.3 & 19.3 & 19.0 & 0.0 & 9.6 & 97 & 79 & 0.0 \\
\hline $10 / 24 / 01$ & $11: 40$ & 19.2 & 19.3 & \begin{tabular}{|c|}
19.3 \\
\end{tabular} & 19.1 & 0.0 & 9.7 & 127 & 79 & 0.0 \\
\hline $10 / 24 / 01$ & 11:41 & 19.1 & 19.3 & 19.3 & 19.0 & 0.0 & 9.6 & 121 & 79 & 0.0 \\
\hline 10/24/01 & $11: 42$ & 19.2 & 19.3 & 19.3 & 19.1 & 0.0 & 9.7 & 111 & 79 & 0.0 \\
\hline $10 / 24 / 01$ & $11: 43$ & 19.1 & 19.3 & 19.3 & 19.0 & 0.0 & 9.7 & 84 & 79 & 0.0 \\
\hline $10 / 24 / 01$ & 11:44 & 19.2 & 19.3 & 19.3 & 19.1 & 0.0 & 9.5 & 87 & 79 & 0.0 \\
\hline $10 / 24 / 01$ & $11: 45$ & 19.1 & 19.3 & 19.3 & 19.0 & 0.0 & 9.8 & 81 & 79 & 0.0 \\
\hline $10 / 24 / 01$ & 11:46 & 19.2 & 19.3 & 19.3 & 19.1 & 0.0 & 9.6 & 124 & 79 & 0.0 \\
\hline 10/24/01 & 11:47 & 19.1 & 19.3 & 19.3 & 19.0 & 0.0 & 9.7 & 129 & 79 & 0.0 \\
\hline $10 / 24 / 01$ & 11:48 & 19.1 & 19.3 & 19.3 & 19.0 & 0.0 & 9.6 & 129 & 79 & 0.0 \\
\hline $10 / 24 / 01$ & 11:49 & 19.2 & 19.3 & 19.3 & 19.0 & 0.0 & 9.6 & 126 & 79 & 0.0 \\
\hline $10 / 24 / 01$ & 11:50 & 19.1 & 19.3 & 19.3 & 19.0 & 0.0 & 9.6 & 92 & 79 & 0.0 \\
\hline $10 / 24 / 01$ & 11:51 & 19.2 & 19.3 & 19.3 & 19.0 & 0.0 & 9.6 & 110 & 79 & 0.0 \\
\hline $10 / 24 / 01$ & 11:52 & 19.1 & 19.3 & 19.3 & 19.0 & 0.0 & 9.7 & 136 & 79 & 0.0 \\
\hline $10 / 24 / 01$ & 11:53 & 19.2 & 19.3 & \begin{tabular}{|l|}
19.3 \\
\end{tabular} & 19.1 & 0.0 & 9.6 & 89 & 79 & 0.0 \\
\hline $10 / 24 / 01$ & 11:54 & 19.1 & 19.3 & 19.3 & 19.0 & 0.0 & 9.6 & 103 & 79 & 0.0 \\
\hline $10 / 24 / 01$ & 11:55 & 19.2 & 19.3 & 19.3 & 19.1 & 0.0 & 9.6 & 128 & 79 & 0.0 \\
\hline $10 / 24 / 01$ & 11:56 & 19.1 & 19.3 & 19.3 & 19.0 & 0.0 & 9.7 & 88 & 79 & 0.0 \\
\hline $10 / 24 / 01$ & 11:57 & 19.2 & 19.3 & 19.3 & 19.1 & 0.0 & 9.7 & 108 & 79 & 0.0 \\
\hline $10 / 24 / 01$ & 11:58 & 19.1 & 19.3 & 19.3 & 19.0 & 0.0 & 9.6 & 120 & 79 & 0.0 \\
\hline $10 / 24 / 01$ & 11:59 & 19.1 & 19.3 & \begin{tabular}{|l|}
19.3 \\
\end{tabular} & 19.0 & 0.0 & 9.7 & 84 & 79 & 0.0 \\
\hline $10 / 24 / 01$ & 12:00 & 19.2 & 19.3 & 19.3 & 19.0 & 0.0 & 9.7 & 133 & 79 & 0.0 \\
\hline $10 / 24 / 01$ & 12:01 & 19.1 & 19.3 & 19.3 & 19.0 & 0.0 & 9.7 & 82 & 79 & 0.0 \\
\hline $10 / 24 / 01$ & 12:02 & 19.2 & 19.3 & 19.3 & 19.0 & 0.0 & 9.7 & 112 & 79 & 0.0 \\
\hline $10 / 24 / 01$ & 12:03 & 19.1 & 19.3 & 19.3 & 19.0 & 0.0 & 9.6 & 119 & 79 & 0.0 \\
\hline $10 / 24 / 01$ & 12:04 & 19.2 & 19.3 & \begin{tabular}{|l|}
19.3 \\
\end{tabular} & 19.0 & 0.0 & 9.6 & 96 & 79 & 0.0 \\
\hline $10 / 24 / 01$ & 12:05 & 19.1 & 19.3 & 19.3 & 19.0 & 0.0 & 9.6 & 111 & 79 & 0.0 \\
\hline $10 / 24 / 01$ & 12:06 & 19.2 & 19.3 & \begin{tabular}{|l|}
19.3 \\
\end{tabular} & 19.0 & 0.0 & 9.7 & 84 & 79 & 0.0 \\
\hline $10 / 24 / 01$ & 12:07 & 19.1 & 19.3 & 19.3 & 19.0 & 0.0 & 9.6 & 99 & 79 & 0.0 \\
\hline $10 / 24 / 01$ & 12:08 & 19.1 & 19.3 & 19.3 & 19.0 & 0.0 & 9.7 & 120 & 79 & 0.0 \\
\hline $10 / 24 / 01$ & 12:09 & 19.1 & 19.3 & 19.3 & 19.0 & 0.0 & 9.6 & 81 & 79 & 0.0 \\
\hline $10 / 24 / 01$ & $12: 10$ & 19.1 & 19.3 & 19.3 & 19.0 & 0.0 & 9.8 & 129 & 79 & 0.0 \\
\hline $10 / 24 / 01$ & 12:11 & 19.1 & 19.3 & \begin{tabular}{|l|}
19.3 \\
\end{tabular} & 19.0 & 0.0 & 9.7 & 86 & 79 & 0.0 \\
\hline $10 / 24 / 01$ & $12: 12$ & 19.1 & 19.3 & \begin{tabular}{|l|}
19.3 \\
\end{tabular} & 19.0 & 0.0 & 9.6 & 91 & 79 & 0.0 \\
\hline $10 / 24 / 01$ & 12:13 & 19.1 & 19.3 & 19.3 & 19.0 & 0.0 & 9.6 & 137 & 79 & 0.0 \\
\hline $10 / 24 / 01$ & 12:14 & 19.1 & 19.3 & 19.3 & 19.0 & 0.0 & 9.7 & 95 & 79 & 0.0 \\
\hline $10 / 24 / 01$ & 12:15 & 19.2 & 19.3 & 19.3 & 19.0 & 0.0 & 9.7 & 88 & 79 & 0.0 \\
\hline $10 / 24 / 01$ & $12: 16$ & 19.1 & 19.3 & 19.3 & 19.0 & 0.0 & 9.6 & 128 & 79 & 0.0 \\
\hline $10 / 24 / 01$ & 12:17 & 19.1 & 19.3 & \begin{tabular}{|l|}
19.3 \\
\end{tabular} & 19.0 & 0.0 & 9.7 & 103 & 79 & 0.0 \\
\hline $10 / 24 / 01$ & 12:18 & 19.1 & 19.3 & \begin{tabular}{|l|}
19.3 \\
\end{tabular} & 19.0 & 0.0 & 9.7 & 86 & 79 & 0.0 \\
\hline $10 / 24 / 01$ & 12:19 & 19.1 & 19.3 & 19.3 & 19.0 & 0.0 & 9.6 & 109 & 79 & 0.0 \\
\hline $10 / 24 / 01$ & 12:20 & 19.1 & 19.3 & 19.3 & 19.0 & 0.0 & 9.6 & 126 & 79 & 0.0 \\
\hline $10 / 24 / 01$ & 12:21 & 19.1 & 19.3 & 19.3 & 19.0 & 0.0 & 9.8 & 114 & 79 & 0.0 \\
\hline $10 / 24 / 01$ & 12:22 & 19.1 & 19.3 & \begin{tabular}{|l|}
19.3 \\
\end{tabular} & 19.0 & 0.0 & 9.7 & 92 & 79 & 0.0 \\
\hline $10 / 24 / 01$ & 12:23 & 19.1 & 19.3 & \begin{tabular}{|l|}
19.3 \\
\end{tabular} & 19.0 & 0.0 & 9.6 & 87 & 79 & 0.0 \\
\hline $10 / 24 / 01$ & 12:24 & 19.1 & 19.3 & 19.3 & 19.0 & 0.0 & 9.7 & 95 & 79 & 0.0 \\
\hline $10 / 24 / 01$ & 12:25 & 19.1 & 19.3 & \begin{tabular}{|l|}
19.3 \\
\end{tabular} & 19.0 & 0.0 & 9.7 & 112 & 79 & 0.0 \\
\hline $10 / 24 / 01$ & 12:26 & 19.1 & 19.3 & 19.3 & 19.0 & 0.0 & 9.6 & 122 & 79 & 0.0 \\
\hline $10 / 24 / 01$ & 12:27 & 19.1 & 19.3 & 19.3 & 19.0 & 0.0 & 9.6 & 126 & 79 & 0.0 \\
\hline
\end{tabular}


WSRC-TR-2002-00459, Rev. 0 SRT-RPP-2002-00221

\begin{tabular}{|c|c|c|c|c|c|c|c|c|c|c|}
\hline \multicolumn{11}{|c|}{ PREC2_102401_0627 } \\
\hline DATE & TIME & $\begin{array}{c}\mathrm{HX} \\
\text { Outlet } \\
\left({ }^{\circ} \mathrm{C}\right) \\
\mathrm{TCO}\end{array}$ & \begin{tabular}{|c|} 
REC \\
PP \\
OUT \\
$\left({ }^{\circ} \mathrm{C}\right)$ \\
TC1
\end{tabular} & \begin{tabular}{|c|}
$\mathrm{TK}$ \\
$\mathrm{BOT}$ \\
$\left({ }^{\circ} \mathrm{C}\right)$ \\
$\mathrm{TC} 2$ \\
\end{tabular} & \begin{tabular}{|c} 
HX \\
OUT \\
$\left({ }^{\circ} \mathrm{C}\right)$ \\
TC3 \\
\end{tabular} & \begin{tabular}{|c} 
Mn \\
Flow \\
(gpm)
\end{tabular} & \begin{tabular}{|c|} 
Galigher \\
Flow \\
(gpm)
\end{tabular} & $\begin{array}{c}\text { Current } \\
\text { to } \mathrm{HX}\end{array}$ & $\begin{array}{l}\text { Voltage } \\
\text { to } \mathrm{HX}\end{array}$ & $\begin{array}{c}\text { Sr } \\
\text { Flow } \\
\text { (gpm) }\end{array}$ \\
\hline $10 / 24 / 01$ & 12:28 & 19.1 & 19.3 & \begin{tabular}{|l|} 
\\
\end{tabular} & 19.0 & 0.0 & 9.5 & 120 & 79 & 0. \\
\hline $10 / 24 / 01$ & $12: 29$ & 19.1 & 19.3 & 19.3 & 19.0 & 0.0 & 9.6 & 102 & 79 & 0. \\
\hline $10 / 24 / 01$ & $12: 30$ & 19.1 & 19.3 & \begin{tabular}{|l|}
19.3 \\
\end{tabular} & 19.0 & 0.0 & 9.6 & 81 & 79 & 0. \\
\hline $10 / 24 / 01$ & 12:31 & 19.1 & \begin{tabular}{|l|} 
\\
\end{tabular} & \begin{tabular}{|l|}
19.3 \\
\end{tabular} & 19.0 & 0.0 & 9.7 & 79 & 79 & 0. \\
\hline $10 / 24 / 01$ & $12: 32$ & 19.1 & 19.3 & \begin{tabular}{|l|}
$\mid 19.3$ \\
\end{tabular} & 19.0 & 0.0 & 9.7 & 81 & 79 & 0. \\
\hline $10 / 24 / 01$ & $12: 33$ & 19.1 & 19.3 & \begin{tabular}{|l|} 
\\
\end{tabular} & 19.0 & 0.0 & 9.6 & 94 & 79 & 0. \\
\hline $10 / 24 / 01$ & $12: 34$ & 19.1 & 19.3 & \begin{tabular}{|l|}
19.3 \\
\end{tabular} & 19.0 & 0.0 & 9.7 & 92 & 79 & 0. \\
\hline $10 / 24 / 01$ & 12:35 & 19.1 & 19.3 & \begin{tabular}{|l|}
19.3 \\
\end{tabular} & 19.0 & 0.0 & 9.5 & 102 & 79 & 0. \\
\hline $10 / 24 / 01$ & $12: 36$ & 19.0 & \begin{tabular}{|l|}
19.3 \\
\end{tabular} & \begin{tabular}{|l|}
19.3 \\
\end{tabular} & 19.0 & 0.0 & 9.6 & 112 & 79 & 0. \\
\hline $10 / 24 / 01$ & $12: 37$ & 19.1 & 19.3 & \begin{tabular}{|l|} 
\\
\end{tabular} & 19.0 & 0.0 & 9.6 & 121 & 79 & 0. \\
\hline $10 / 24 / 01$ & $12: 38$ & 19.1 & 19.2 & \begin{tabular}{|l|} 
\\
\end{tabular} & 18.9 & 0.0 & 9.6 & 137 & 79 & 0. \\
\hline $10 / 24 / 01$ & $12: 39$ & 19.1 & 19.2 & \begin{tabular}{|l|} 
\\
\end{tabular} & 19.0 & 0.0 & 9.6 & 135 & 79 & 0. \\
\hline $10 / 24 / 01$ & $12: 40$ & 19.1 & 19.2 & \begin{tabular}{|l|}
19.3 \\
\end{tabular} & 19.0 & 0.0 & 9.6 & 120 & 79 & 0. \\
\hline $10 / 24 / 01$ & $12: 41$ & 19.1 & 19.2 & \begin{tabular}{|l|}
19.3 \\
\end{tabular} & 19.0 & 0.0 & 9.5 & 104 & 79 & 0. \\
\hline $10 / 24 / 01$ & 12:42 & 19.1 & 19.2 & \begin{tabular}{|l|} 
\\
\end{tabular} & 19.0 & 0.0 & 9.5 & 106 & 79 & 0. \\
\hline $10 / 24 / 01$ & $12: 43$ & 19.1 & 19.2 & \begin{tabular}{|l|} 
\\
\end{tabular} & 19.0 & 0.0 & 9.5 & 91 & 79 & 0. \\
\hline $10 / 24 / 01$ & $12: 44$ & 19.1 & 19.2 & \begin{tabular}{|l|} 
\\
\end{tabular} & 19.0 & 0.0 & 9.6 & 83 & 79 & 0. \\
\hline $10 / 24 / 01$ & $12: 45$ & 19.0 & 19.2 & \begin{tabular}{|l|}
19.3 \\
\end{tabular} & 19.0 & 0.0 & 9.7 & 77 & 79 & 0. \\
\hline $10 / 24 / 01$ & $12: 46$ & 19.1 & 19.2 & \begin{tabular}{|l|}
19.2 \\
\end{tabular} & 19.0 & 0.0 & 9.7 & 90 & 79 & 0. \\
\hline $10 / 24 / 01$ & $12: 47$ & 19.0 & 19.2 & \begin{tabular}{|l|}
19.2 \\
\end{tabular} & 18.9 & 0.0 & 9.6 & 121 & 79 & 0. \\
\hline $10 / 24 / 01$ & $12: 48$ & 19.1 & \begin{tabular}{|l|}
19.2 \\
\end{tabular} & \begin{tabular}{|l|}
19.2 \\
\end{tabular} & 19.0 & 0.0 & 9.6 & 125 & 79 & 0. \\
\hline $10 / 24 / 01$ & $12: 49$ & 19.1 & 19.2 & \begin{tabular}{|l|}
19.2 \\
\end{tabular} & 18.9 & 0.0 & 9.7 & 132 & 79 & 0. \\
\hline $10 / 24 / 01$ & $12: 50$ & 19.1 & 19.2 & \begin{tabular}{|l|}
19.3 \\
\end{tabular} & 19.0 & 0.0 & 9.6 & 114 & 79 & 0. \\
\hline $10 / 24 / 01$ & 12:51 & 19.1 & 19.2 & \begin{tabular}{|l|}
19.3 \\
\end{tabular} & 19.0 & 0.0 & 9.6 & 75 & 79 & 0. \\
\hline $10 / 24 / 01$ & 12:52 & 19.1 & 19.2 & \begin{tabular}{|l|}
19.3 \\
\end{tabular} & 19.0 & 0.0 & 9.7 & 83 & 79 & 0. \\
\hline $10 / 24 / 01$ & $12: 53$ & 19.1 & 19.2 & \begin{tabular}{|l|} 
\\
\end{tabular} & 19.0 & 0.0 & 9.7 & 113 & 79 & 0. \\
\hline $10 / 24 / 01$ & 12:54 & 19.1 & 19.2 & \begin{tabular}{|l|}
19.2 \\
\end{tabular} & 19.0 & 0.0 & 9.6 & 127 & 79 & 0. \\
\hline $10 / 24 / 01$ & 12:55 & 19.1 & 19.2 & \begin{tabular}{|l|}
19.2 \\
\end{tabular} & 19.0 & 0.0 & 9.7 & 85 & 79 & 0. \\
\hline $10 / 24 / 01$ & 12:56 & 19.0 & 19.2 & \begin{tabular}{|l|}
19.3 \\
\end{tabular} & 18.9 & 0.0 & 9.6 & 98 & 79 & 0. \\
\hline $10 / 24 / 01$ & 12:57 & 19.1 & 19.2 & \begin{tabular}{|l|}
19.2 \\
\end{tabular} & 19.0 & 0.0 & 9.6 & 117 & 79 & 0. \\
\hline $10 / 24 / 01$ & 12:58 & 19.0 & 19.2 & \begin{tabular}{|l|} 
\\
\end{tabular} & 18.9 & 0.0 & 9.7 & 75 & 79 & 0. \\
\hline $10 / 24 / 01$ & $12: 59$ & 19.1 & 19.2 & \begin{tabular}{|l|} 
\\
\end{tabular} & 19.0 & 0.0 & 9.7 & 132 & 79 & 0. \\
\hline $10 / 24 / 01$ & 13:00 & 19.1 & \begin{tabular}{|l|}
19.2 \\
\end{tabular} & \begin{tabular}{|l|}
19.2 \\
\end{tabular} & \begin{tabular}{|l|}
18.9 \\
\end{tabular} & 0.0 & 9.7 & 79 & 79 & 0. \\
\hline $10 / 24 / 01$ & 13:01 & 19.1 & \begin{tabular}{|l|}
19.2 \\
\end{tabular} & \begin{tabular}{|l|}
19.2 \\
\end{tabular} & 19.0 & 0.0 & 9.6 & 129 & 79 & 0. \\
\hline $10 / 24 / 01$ & 13:02 & 19.1 & 19.2 & \begin{tabular}{|l|}
19.2 \\
\end{tabular} & 18.9 & 0.0 & 9.7 & 128 & 79 & 0. \\
\hline $10 / 24 / 01$ & 13:03 & 19.0 & 19.2 & \begin{tabular}{|l|} 
\\
\end{tabular} & 18.9 & 0.0 & 9.7 & 79 & 79 & 0. \\
\hline $10 / 24 / 01$ & 13:04 & 19.1 & \begin{tabular}{|l|}
19.2 \\
\end{tabular} & \begin{tabular}{|l|}
19.2 \\
\end{tabular} & 19.0 & 0.0 & 9.6 & 118 & 79 & 0. \\
\hline $10 / 24 / 01$ & 13:05 & 19.0 & 19.2 & \begin{tabular}{|l|}
19.2 \\
\end{tabular} & 18.9 & 0.0 & 9.6 & 132 & 79 & 0. \\
\hline $10 / 24 / 01$ & 13:06 & 19.1 & \begin{tabular}{|l|}
19.2 \\
\end{tabular} & \begin{tabular}{|l|}
19.2 \\
\end{tabular} & 19.0 & 0.0 & 9.7 & 113 & 79 & 0. \\
\hline $10 / 24 / 01$ & 13:07 & 19.0 & 19.2 & \begin{tabular}{|l|} 
\\
\end{tabular} & 18.9 & 0.0 & 9.7 & 78 & 79 & 0. \\
\hline $10 / 24 / 01$ & 13:08 & 19.1 & 19.2 & \begin{tabular}{|l|}
19.2 \\
\end{tabular} & 19.0 & 0.0 & 9.7 & 71 & 79 & 0. \\
\hline $10 / 24 / 01$ & 13:09 & 19.0 & 19.2 & \begin{tabular}{|l|}
19.2 \\
\end{tabular} & \begin{tabular}{|l|}
18.9 \\
\end{tabular} & 0.0 & 9.7 & 112 & 79 & 0. \\
\hline $10 / 24 / 01$ & $13: 10$ & 19.1 & 19.2 & \begin{tabular}{|l|}
19.2 \\
\end{tabular} & 19.0 & 0.0 & 9.7 & 89 & 79 & 0. \\
\hline $10 / 24 / 01$ & 13:11 & 19.1 & 19.2 & \begin{tabular}{|l|}
19.2 \\
\end{tabular} & 19.0 & 0.0 & 9.7 & 92 & 79 & 0. \\
\hline $10 / 24 / 01$ & 13:12 & 19.1 & 19.3 & \begin{tabular}{|l|}
19.2 \\
\end{tabular} & 19.0 & 0.0 & 9.6 & 87 & 79 & 0. \\
\hline $10 / 24 / 01$ & $13: 13$ & 19.1 & 19.3 & \begin{tabular}{|l|}
19.2 \\
\end{tabular} & 19.0 & 0.0 & 9.6 & 106 & 79 & 0. \\
\hline $10 / 24 / 01$ & $13: 14$ & 19.1 & 19.2 & \begin{tabular}{|l|} 
\\
\end{tabular} & 19.0 & 0.0 & 9.6 & 115 & 79 & 0. \\
\hline $10 / 24 / 01$ & $13: 15$ & 19.1 & 19.2 & \begin{tabular}{|l|}
19.2 \\
\end{tabular} & 19.0 & 0.0 & 9.6 & 121 & 79 & 0. \\
\hline $10 / 24 / 01$ & 13:16 & 19.0 & 19.2 & \begin{tabular}{|l|}
19.2 \\
\end{tabular} & \begin{tabular}{|l|}
18.9 \\
\end{tabular} & 0.0 & 9.7 & 89 & 79 & 0. \\
\hline $10 / 24 / 01$ & $13: 17$ & 19.1 & 19.2 & \begin{tabular}{|l|}
19.2 \\
\end{tabular} & 19.0 & 0.0 & 9.6 & 89 & 79 & 0. \\
\hline $10 / 24 / 01$ & $13: 18$ & 19.0 & 19.2 & \begin{tabular}{|l|} 
\\
\end{tabular} & 18.9 & 0.0 & 9.7 & 113 & 79 & 0. \\
\hline $10 / 24 / 01$ & $13: 19$ & 19.1 & \begin{tabular}{|l|}
19.2 \\
\end{tabular} & \begin{tabular}{|l|}
19.2 \\
\end{tabular} & \begin{tabular}{|l|}
19.0 \\
\end{tabular} & 0.0 & 9.6 & 83 & 79 & 0. \\
\hline $10 / 24 / 01$ & $13: 20$ & 19.0 & 19.2 & \begin{tabular}{|l|}
19.2 \\
\end{tabular} & 18.9 & 0.0 & 9.7 & 102 & 79 & 0. \\
\hline $10 / 24 / 01$ & $13: 21$ & 19.1 & 19.2 & \begin{tabular}{|l|}
19.2 \\
\end{tabular} & 19.0 & 0.0 & 9.7 & 122 & 79 & 0. \\
\hline $10 / 24 / 01$ & 13:22 & 19.0 & 19.2 & \begin{tabular}{|l|} 
\\
\end{tabular} & 18.9 & 0.0 & 9.6 & 132 & 79 & 0. \\
\hline $10 / 24 / 01$ & 13:23 & 19.0 & 19.2 & \begin{tabular}{|l|} 
\\
\end{tabular} & 18.9 & 0.0 & 9.7 & 114 & 79 & 0. \\
\hline $10 / 24 / 01$ & 13:24 & 19.0 & \begin{tabular}{|l|}
19.2 \\
\end{tabular} & \begin{tabular}{|l|}
19.2 \\
\end{tabular} & 18.9 & 0.0 & 9.6 & 112 & 79 & 0. \\
\hline $10 / 24 / 01$ & $13: 25$ & 19.0 & 19.2 & \begin{tabular}{|l|}
19.2 \\
\end{tabular} & 18.9 & 0.0 & 9.7 & 93 & 79 & 0. \\
\hline $10 / 24 / 01$ & 13:26 & 19.1 & 19.2 & \begin{tabular}{|l|}
19.2 \\
\end{tabular} & 18.9 & 0.0 & 9.7 & 131 & 79 & 0. \\
\hline $10 / 24 / 01$ & 13:27 & 19.0 & 19.2 & 19.2 & 18.9 & 0.0 & 9.7 & 112 & 79 & 0. \\
\hline
\end{tabular}

\begin{tabular}{|c|c|c|c|c|c|c|c|c|c|c|}
\hline \multicolumn{3}{|c|}{ PREC2_102401_0627 } & & & & & & & & \\
\hline DATE & TIME & $\begin{array}{c}\mathrm{HX} \\
\text { Outlet } \\
\left({ }^{\circ} \mathrm{C}\right) \\
\mathrm{TCO}\end{array}$ & $\begin{array}{c}\text { REC } \\
\text { PP } \\
\text { OUT } \\
\left({ }^{\circ} \mathrm{C}\right) \\
\text { TC1 }\end{array}$ & $\begin{array}{c}\text { TK } \\
\text { BOT } \\
\left({ }^{\circ} \mathrm{C}\right) \\
\text { TC2 }\end{array}$ & $\begin{array}{l}\text { HX } \\
\text { OUT } \\
\left({ }^{\circ} \mathrm{C}\right) \\
\text { TC3 }\end{array}$ & $\begin{array}{c}\mathrm{Mn} \\
\text { Flow } \\
\text { (gpm) }\end{array}$ & $\begin{array}{c}\text { Galigher } \\
\text { Flow } \\
\text { (gpm) }\end{array}$ & $\begin{array}{c}\text { Current } \\
\text { to } \mathrm{HX}\end{array}$ & $\begin{array}{c}\text { Voltage } \\
\text { to } \mathrm{HX}\end{array}$ & $\begin{array}{c}\mathrm{Sr} \\
\text { Flow } \\
(\mathrm{gpm})\end{array}$ \\
\hline $10 / 24 / 01$ & $13: 28$ & 19.1 & 19.2 & 19.2 & 19.0 & 0.0 & 9.6 & 78 & 79 & 0.0 \\
\hline $10 / 24 / 01$ & $13: 29$ & 19.0 & 19.2 & 19.2 & 19.0 & 0.0 & 9.6 & 106 & 79 & 0.0 \\
\hline $10 / 24 / 01$ & 13:30 & 19.1 & 19.2 & 19.2 & 19.0 & 0.0 & 9.7 & 84 & 79 & 0.0 \\
\hline $10 / 24 / 01$ & 13:31 & 19.0 & 19.2 & 19.2 & 19.0 & 0.0 & 9.7 & 94 & 79 & 0.0 \\
\hline $10 / 24 / 01$ & $13: 32$ & 19.0 & 19.2 & 19.2 & 19.0 & 0.0 & 9.6 & 129 & 79 & 0.0 \\
\hline $10 / 24 / 01$ & $13: 33$ & 19.0 & 19.2 & 19.2 & 18.9 & 0.0 & 9.6 & 99 & 79 & 0.0 \\
\hline $10 / 24 / 01$ & $13: 34$ & 19.0 & 19.2 & 19.2 & 18.9 & 0.0 & 9.6 & 77 & 79 & 0.0 \\
\hline $10 / 24 / 01$ & 13:35 & 19.0 & 19.2 & 19.2 & 18.9 & 0.0 & 9.6 & 78 & 79 & 0.0 \\
\hline $10 / 24 / 01$ & 13:36 & 19.0 & 19.2 & 19.2 & 18.9 & 0.0 & 9.6 & 99 & 79 & 0.0 \\
\hline $10 / 24 / 01$ & 13:37 & 19.0 & 19.2 & 19.2 & 18.9 & 0.0 & 9.6 & 110 & 79 & 0.0 \\
\hline $10 / 24 / 01$ & 13:38 & 19.0 & 19.2 & 19.2 & 18.9 & 0.0 & 9.7 & 89 & 79 & 0.0 \\
\hline $10 / 24 / 01$ & $13: 39$ & 19.0 & 19.2 & 19.2 & 18.9 & 0.0 & 9.7 & 119 & 79 & 0.0 \\
\hline $10 / 24 / 01$ & $13: 40$ & 19.0 & 19.2 & 19.2 & 18.9 & 0.0 & 9.6 & 100 & 79 & 0.0 \\
\hline $10 / 24 / 01$ & 13:41 & 19.0 & 19.2 & 19.2 & 18.9 & 0.0 & 9.5 & 129 & 79 & 0.0 \\
\hline $10 / 24 / 01$ & 13:42 & 19.0 & 19.2 & 19.2 & 18.9 & 0.0 & 9.8 & 128 & 79 & 0.0 \\
\hline $10 / 24 / 01$ & $13: 43$ & 19.0 & 19.2 & 19.2 & 18.9 & 0.0 & 9.6 & 97 & 79 & 0.0 \\
\hline $10 / 24 / 01$ & $13: 44$ & 19.0 & 19.1 & 19.2 & 18.9 & 0.0 & 9.5 & 129 & 79 & 0.0 \\
\hline $10 / 24 / 01$ & 13:45 & 19.0 & 19.2 & 19.2 & 18.9 & 0.0 & 9.7 & 123 & 79 & 0.0 \\
\hline $10 / 24 / 01$ & 13:46 & 19.0 & 19.2 & 19.2 & 18.9 & 0.0 & 9.6 & 77 & 79 & 0.0 \\
\hline $10 / 24 / 01$ & 13:47 & 19.0 & 19.1 & 19.2 & 18.9 & 0.0 & 9.7 & 76 & 79 & 0.0 \\
\hline $10 / 24 / 01$ & $13: 48$ & 19.0 & 19.1 & 19.2 & 18.9 & 0.0 & 9.7 & 91 & 79 & 0.0 \\
\hline $10 / 24 / 01$ & $13: 49$ & 19.0 & 19.1 & 19.2 & 18.9 & 0.0 & 9.7 & 90 & 79 & 0.0 \\
\hline $10 / 24 / 01$ & 13:50 & 19.0 & 19.1 & 19.2 & 18.9 & 0.0 & 9.6 & 86 & 79 & 0.0 \\
\hline $10 / 24 / 01$ & 13:51 & 19.0 & 19.1 & 19.2 & 18.9 & 0.0 & 9.8 & 108 & 79 & 0.0 \\
\hline $10 / 24 / 01$ & 13:52 & 19.0 & 19.1 & 19.2 & 18.9 & 0.0 & 9.6 & 91 & 79 & 0.0 \\
\hline $10 / 24 / 01$ & 13:53 & 19.0 & 19.1 & 19.2 & 18.9 & 0.0 & 9.5 & 102 & 79 & 0.0 \\
\hline $10 / 24 / 01$ & 13:54 & 19.0 & 19.1 & 19.2 & 18.9 & 0.0 & 9.7 & 75 & 79 & 0.0 \\
\hline $10 / 24 / 01$ & 13:55 & 19.0 & 19.1 & 19.2 & 18.9 & 0.0 & 9.6 & 82 & 79 & 0.0 \\
\hline $10 / 24 / 01$ & 13:56 & 19.0 & 19.1 & 19.2 & 18.9 & 0.0 & 9.7 & 121 & 79 & 0.0 \\
\hline $10 / 24 / 01$ & 13:57 & 19.0 & 19.1 & 19.1 & 18.9 & 0.0 & 9.7 & 75 & 79 & 0.0 \\
\hline $10 / 24 / 01$ & 13:58 & 18.9 & 19.1 & 19.2 & 18.8 & 0.0 & 9.7 & 87 & 79 & 0.0 \\
\hline $10 / 24 / 01$ & 13:59 & 19.0 & 19.1 & 19.2 & 18.9 & 0.0 & 9.7 & 95 & 79 & 0.0 \\
\hline $10 / 24 / 01$ & $14: 00$ & 18.9 & 19.1 & 19.1 & 18.8 & 0.0 & 9.6 & 91 & 79 & 0.0 \\
\hline $10 / 24 / 01$ & 14:01 & 19.0 & 19.1 & 19.1 & 18.9 & 0.0 & 9.6 & 91 & 79 & 0.0 \\
\hline $10 / 24 / 01$ & 14:02 & 18.9 & 19.1 & 19.1 & 18.8 & 0.0 & 9.5 & 95 & 79 & 0.0 \\
\hline $10 / 24 / 01$ & $14: 03$ & 19.0 & 19.1 & $\mid 19.1$ & 18.9 & 0.0 & 9.5 & 83 & 79 & 0.0 \\
\hline $10 / 24 / 01$ & $14: 04$ & 19.0 & 19.1 & $\mid 19.1$ & 18.9 & 0.0 & 9.6 & 94 & 79 & 0.0 \\
\hline $10 / 24 / 01$ & 14:05 & 18.9 & 19.1 & 19.1 & 18.8 & 0.0 & 9.6 & 70 & 79 & 0.0 \\
\hline $10 / 24 / 01$ & $14: 06$ & 19.0 & 19.1 & 19.1 & 18.9 & 0.0 & 9.6 & 73 & 79 & 0.0 \\
\hline $10 / 24 / 01$ & $14: 07$ & 18.9 & 19.1 & $\mid 19.1$ & 18.8 & 0.0 & 9.7 & 98 & 79 & 0.0 \\
\hline $10 / 24 / 01$ & $14: 08$ & 19.0 & 19.1 & 19.1 & 18.9 & 0.0 & 9.6 & 113 & 79 & 0.0 \\
\hline $10 / 24 / 01$ & $14: 09$ & 18.9 & 19.1 & 19.1 & 18.8 & 0.0 & 9.7 & 96 & 79 & 0.0 \\
\hline $10 / 24 / 01$ & $14: 10$ & 19.0 & 19.1 & 19.1 & 18.9 & 0.0 & 9.8 & 103 & 79 & 0.0 \\
\hline $10 / 24 / 01$ & $14: 11$ & 18.9 & 19.1 & $\mid 19.1$ & 18.8 & 0.0 & 9.6 & 127 & 79 & 0.0 \\
\hline $10 / 24 / 01$ & $14: 12$ & 19.0 & 19.1 & 19.1 & 18.9 & 0.0 & 9.6 & 83 & 79 & 0.0 \\
\hline $10 / 24 / 01$ & $14: 13$ & 18.9 & 19.1 & 19.1 & 18.8 & 0.0 & 9.7 & 91 & 79 & 0.0 \\
\hline $10 / 24 / 01$ & $14: 14$ & 18.9 & 19.1 & $\mid 19.1$ & 18.9 & 0.0 & 9.6 & 109 & 79 & 0.0 \\
\hline $10 / 24 / 01$ & $14: 15$ & 19.0 & 19.1 & 19.1 & 18.8 & 0.0 & 9.7 & 65 & 79 & 0.0 \\
\hline $10 / 24 / 01$ & $14: 16$ & 18.9 & 19.1 & 19.1 & 18.8 & 0.0 & 9.6 & 84 & 79 & 0.0 \\
\hline $10 / 24 / 01$ & $14: 17$ & 19.0 & 19.1 & 19.1 & 18.9 & 0.0 & 9.8 & 68 & 79 & 0.0 \\
\hline $10 / 24 / 01$ & $14: 18$ & 18.9 & 19.1 & $\mid 19.1$ & 18.8 & 0.0 & 9.6 & 81 & 79 & 0.0 \\
\hline $10 / 24 / 01$ & $14: 19$ & 19.0 & 19.1 & 19.1 & 18.9 & 0.0 & 9.6 & 74 & 79 & 0.0 \\
\hline $10 / 24 / 01$ & $14: 20$ & 18.9 & 19.1 & 19.1 & 18.8 & 0.0 & 9.6 & 108 & 79 & 0.0 \\
\hline $10 / 24 / 01$ & $14: 21$ & 19.0 & 19.1 & 19.1 & 18.9 & 0.0 & 9.6 & 78 & 79 & 0.0 \\
\hline $10 / 24 / 01$ & $14: 22$ & 18.9 & 19.1 & $\mid 19.1$ & 18.8 & 0.0 & 9.6 & 63 & 79 & 0.0 \\
\hline $10 / 24 / 01$ & $14: 23$ & 18.9 & 19.1 & 19.1 & 18.8 & 0.0 & 9.6 & 99 & 79 & 0.0 \\
\hline $10 / 24 / 01$ & $14: 24$ & 18.9 & 19.1 & 19.1 & 18.8 & 0.0 & 9.7 & 98 & 79 & 0.0 \\
\hline $10 / 24 / 01$ & $14: 25$ & 18.9 & 19.1 & 19.1 & 18.8 & 0.0 & 9.7 & 80 & 79 & 0.0 \\
\hline $10 / 24 / 01$ & $14: 26$ & 19.0 & 19.1 & 19.1 & 18.8 & 0.0 & 9.7 & 107 & 79 & 0.0 \\
\hline $10 / 24 / 01$ & $14: 27$ & 18.9 & 19.1 & 19.1 & 18.8 & 0.0 & 9.6 & 91 & 79 & 0.0 \\
\hline
\end{tabular}


WSRC-TR-2002-00459, Rev. 0 SRT-RPP-2002-00221

\begin{tabular}{|c|c|c|c|c|c|c|c|c|c|c|}
\hline \multicolumn{11}{|c|}{ PREC2_102401_0627 } \\
\hline DATE & TIME & \begin{tabular}{|c}
$\mathrm{HX}$ \\
Outlet \\
$\left({ }^{\circ} \mathrm{C}\right)$ \\
$\mathrm{TCO}$
\end{tabular} & \begin{tabular}{|c|} 
REC \\
PP \\
OUT \\
$\left({ }^{\circ} \mathrm{C}\right)$ \\
TC1 \\
\end{tabular} & \begin{tabular}{|c} 
TK \\
BOT \\
$\left({ }^{\circ} \mathrm{C}\right)$ \\
TC2 \\
\end{tabular} & \begin{tabular}{|c|} 
HX \\
OUT \\
$\left({ }^{\circ} \mathrm{C}\right)$ \\
TC3
\end{tabular} & \begin{tabular}{|c|} 
Mn \\
Flow \\
(gpm)
\end{tabular} & $\begin{array}{c}\text { Galigher } \\
\text { Flow } \\
\text { (gpm) }\end{array}$ & $\begin{array}{c}\text { Current } \\
\text { to } \mathrm{HX}\end{array}$ & $\begin{array}{l}\text { Voltage } \\
\text { to } \mathrm{HX}\end{array}$ & $\begin{array}{c}\mathrm{Sr} \\
\text { Flow } \\
\text { (gpm) }\end{array}$ \\
\hline $10 / 24 / 01$ & $14: 28$ & 19.0 & 19.1 & 19.1 & 18.8 & 0.0 & 9.6 & 85 & 79 & $0 . c$ \\
\hline $10 / 24 / 01$ & $14: 29$ & 18.9 & 19.1 & 19.1 & 18.8 & 0.0 & 9.6 & 130 & 79 & $0 . c$ \\
\hline $10 / 24 / 01$ & $14: 30$ & 19.0 & 19.1 & 19.1 & 18.8 & 0.0 & 9.7 & 67 & 79 & $0 . c$ \\
\hline $10 / 24 / 01$ & $14: 31$ & 18.9 & 19.1 & 19.1 & 18.8 & 0.0 & 9.7 & 72 & 79 & $0 . c$ \\
\hline $10 / 24 / 01$ & $14: 32$ & 18.9 & 19.1 & 19.1 & $\mid 18.8$ & 0.0 & 9.6 & 86 & 79 & 0. \\
\hline $10 / 24 / 01$ & $14: 33$ & 18.9 & 19.1 & 19.1 & 18.8 & 0.0 & 9.7 & 117 & 79 & $0 . c$ \\
\hline $10 / 24 / 01$ & $14: 34$ & 18.9 & 19.1 & 19.1 & 18.8 & 0.0 & 9.6 & 76 & 79 & $0 . c$ \\
\hline $10 / 24 / 01$ & $14: 35$ & 18.9 & 19.1 & 19.1 & $\mid 18.8$ & 0.0 & 9.9 & 116 & 79 & 0. \\
\hline $10 / 24 / 01$ & $14: 36$ & 18.9 & 19.1 & 19.1 & \begin{tabular}{|l|} 
\\
\end{tabular} & 0.0 & 9.7 & 98 & 79 & $0 . c$ \\
\hline $10 / 24 / 01$ & $14: 37$ & 19.0 & 19.1 & 19.1 & 18.9 & 0.0 & 9.7 & 95 & 79 & $0 . c$ \\
\hline $10 / 24 / 01$ & $14: 38$ & 18.9 & 19.1 & 19.1 & 18.8 & 0.0 & 9.6 & 119 & 79 & $0 . c$ \\
\hline $10 / 24 / 01$ & $14: 39$ & 19.0 & 19.1 & 19.1 & 18.8 & 0.0 & 9.7 & 86 & 79 & $0 . c$ \\
\hline $10 / 24 / 01$ & $14: 40$ & 18.9 & 19.1 & 19.1 & 18.8 & 0.0 & 9.6 & 67 & 79 & 0. \\
\hline $10 / 24 / 01$ & $14: 41$ & 18.9 & 19.1 & 19.1 & \begin{tabular}{|l|} 
\\
\end{tabular} & 0.0 & 9.7 & 64 & 79 & $0 . c$ \\
\hline $10 / 24 / 01$ & $14: 42$ & 18.9 & 19.1 & 19.1 & 18.8 & 0.0 & 9.6 & 121 & 79 & $0 . c$ \\
\hline $10 / 24 / 01$ & $14: 43$ & 18.9 & 19.1 & 19.1 & 18.8 & 0.0 & 9.6 & 123 & 79 & $0 . c$ \\
\hline $10 / 24 / 01$ & $14: 44$ & 18.9 & 19.1 & 19.1 & 18.8 & 0.0 & 9.7 & 82 & 79 & $0 . c$ \\
\hline $10 / 24 / 01$ & $14: 45$ & 18.9 & 19.0 & 19.1 & 18.8 & 0.0 & 9.7 & 57 & 79 & 0. \\
\hline $10 / 24 / 01$ & $14: 46$ & 18.9 & 19.0 & 19.1 & \begin{tabular}{|l|} 
\\
\end{tabular} & 0.0 & 9.7 & 66 & 79 & $0 . c$ \\
\hline $10 / 24 / 01$ & $14: 47$ & 18.9 & 19.0 & 19.1 & \begin{tabular}{|l|}
18.8 \\
\end{tabular} & 0.0 & 9.6 & 68 & 79 & $0 . c$ \\
\hline $10 / 24 / 01$ & $14: 48$ & 18.9 & 19.0 & 19.0 & 18.8 & 0.0 & 9.6 & 99 & 79 & $0 . c$ \\
\hline $10 / 24 / 01$ & $14: 49$ & 18.9 & 19.0 & 19.0 & $\mid 18.7$ & 0.0 & 9.6 & 70 & 79 & $0 . c$ \\
\hline $10 / 24 / 01$ & $14: 50$ & 18.9 & 19.0 & 19.0 & \begin{tabular}{|l|} 
\\
\end{tabular} & 0.0 & 9.7 & 58 & 79 & $0 . c$ \\
\hline $10 / 24 / 01$ & $14: 51$ & 18.8 & 19.0 & 19.0 & $\mid 18.7$ & 0.0 & 9.7 & 65 & 79 & $0 . c$ \\
\hline $10 / 24 / 01$ & 14:52 & 18.9 & 19.0 & 19.0 & 18.8 & 0.0 & 9.6 & 103 & 79 & $0 . c$ \\
\hline $10 / 24 / 01$ & $14: 53$ & 18.8 & 19.0 & 19.0 & 18.7 & 0.0 & 9.7 & 58 & 79 & $0 . c$ \\
\hline $10 / 24 / 01$ & $14: 54$ & 18.9 & 19.0 & 19.0 & \begin{tabular}{|l|}
18.7 \\
\end{tabular} & 0.0 & 9.6 & 70 & 79 & $0 . c$ \\
\hline $10 / 24 / 01$ & $14: 55$ & 18.9 & \begin{tabular}{|l|}
19.0 \\
\end{tabular} & 19.0 & 18.7 & 0.0 & 9.7 & 77 & 79 & $0 . c$ \\
\hline $10 / 24 / 01$ & $14: 56$ & 18.8 & 19.0 & 19.0 & $\mid 18.7$ & 0.0 & 9.6 & 62 & 79 & $0 . c$ \\
\hline $10 / 24 / 01$ & 14:57 & 18.9 & 19.0 & 19.0 & 18.7 & 0.0 & 9.6 & 103 & 79 & $0 . c$ \\
\hline $10 / 24 / 01$ & $14: 58$ & 18.8 & 19.0 & 19.0 & $\mid 18.7$ & 0.0 & 9.6 & 84 & 79 & $0 . c$ \\
\hline $10 / 24 / 01$ & $14: 59$ & 18.8 & 19.0 & 19.0 & $\mid 18.7$ & 0.0 & 9.7 & 78 & 79 & $0 . c$ \\
\hline $10 / 24 / 01$ & $15: 00$ & 18.8 & 19.0 & 19.0 & 18.7 & 0.0 & 9.6 & 59 & 79 & $0 . c$ \\
\hline $10 / 24 / 01$ & 15:01 & 18.8 & 19.0 & 19.0 & \begin{tabular}{|l|}
18.7 \\
\end{tabular} & 0.0 & 9.7 & 70 & 79 & $0 . c$ \\
\hline $10 / 24 / 01$ & 15:02 & 18.8 & 18.9 & 19.0 & 18.7 & 0.0 & 9.6 & 50 & 79 & $0 . c$ \\
\hline $10 / 24 / 01$ & $15: 03$ & 18.8 & 18.9 & 19.0 & 18.7 & 0.0 & 9.6 & 95 & 79 & $0 . c$ \\
\hline $10 / 24 / 01$ & $15: 04$ & 18.8 & 18.9 & 19.0 & $\mid 18.7$ & 0.0 & 9.6 & 94 & 79 & $0 . c$ \\
\hline $10 / 24 / 01$ & $15: 05$ & 18.8 & 18.9 & 18.9 & $\mid 18.7$ & 0.0 & 9.6 & 88 & 79 & $0 . c$ \\
\hline $10 / 24 / 01$ & $15: 06$ & 18.8 & 18.9 & 18.9 & $\mid 18.7$ & 0.0 & 9.6 & 59 & 79 & $0 . c$ \\
\hline $10 / 24 / 01$ & $15: 07$ & 18.8 & 18.9 & 18.9 & $\mid 18.7$ & 0.0 & 9.7 & 98 & 79 & $0 . c$ \\
\hline $10 / 24 / 01$ & $15: 08$ & 18.8 & 18.9 & 18.9 & 18.7 & 0.0 & 9.6 & 73 & 79 & $0 . c$ \\
\hline $10 / 24 / 01$ & 15:09 & 18.8 & 18.9 & 18.9 & \begin{tabular}{|l|}
18.6 \\
\end{tabular} & 0.0 & 9.6 & 52 & 79 & $0 . c$ \\
\hline $10 / 24 / 01$ & $15: 10$ & 18.8 & 18.9 & 18.9 & 18.7 & 0.0 & 9.7 & 61 & 79 & $0 . c$ \\
\hline $10 / 24 / 01$ & $15: 11$ & 18.8 & 18.9 & 18.9 & \begin{tabular}{|l|} 
\\
\end{tabular} & 0.0 & 9.6 & 60 & 79 & $0 . c$ \\
\hline $10 / 24 / 01$ & $15: 12$ & 18.8 & 18.9 & \begin{tabular}{|l|l}
18.9 \\
\end{tabular} & 18.7 & 0.0 & 9.6 & 63 & 79 & $0 . c$ \\
\hline $10 / 24 / 01$ & $15: 13$ & 18.8 & 18.9 & 18.9 & 18.6 & 0.0 & 9.6 & 100 & 79 & $0 . c$ \\
\hline $10 / 24 / 01$ & $15: 14$ & 18.8 & 18.9 & 18.9 & 18.7 & 0.0 & 9.7 & 63 & 79 & $0 . c$ \\
\hline $10 / 24 / 01$ & $15: 15$ & 18.8 & 18.9 & 18.9 & 18.6 & 0.0 & 9.7 & 61 & 79 & $0 . c$ \\
\hline $10 / 24 / 01$ & $15: 16$ & 18.8 & 18.9 & 18.9 & \begin{tabular}{|l|}
18.6 \\
\end{tabular} & 0.0 & 9.6 & 49 & 79 & $0 . c$ \\
\hline $10 / 24 / 01$ & $15: 17$ & 18.8 & 18.9 & 18.9 & 18.7 & 0.0 & 9.7 & 64 & 79 & $0 . c$ \\
\hline $10 / 24 / 01$ & $15: 18$ & 18.7 & 18.9 & 18.9 & 18.6 & 0.0 & 9.7 & 112 & 79 & $0 . c$ \\
\hline $10 / 24 / 01$ & $15: 19$ & 18.8 & 18.9 & 18.9 & $\mid 18.7$ & 0.0 & 9.7 & 73 & 79 & $0 . c$ \\
\hline $10 / 24 / 01$ & $15: 20$ & 18.7 & 18.9 & 18.9 & 18.6 & 0.0 & 9.7 & 84 & 79 & $0 . c$ \\
\hline $10 / 24 / 01$ & $15: 21$ & 18.8 & 18.9 & 18.9 & \begin{tabular}{|l|}
18.7 \\
\end{tabular} & 0.0 & 9.7 & 79 & 79 & $0 . c$ \\
\hline $10 / 24 / 01$ & $15: 22$ & 18.7 & 18.9 & 18.9 & 18.6 & 0.0 & 9.7 & 107 & 79 & $0 . c$ \\
\hline $10 / 24 / 01$ & $15: 23$ & 18.7 & 18.9 & 18.9 & 18.6 & 0.0 & 9.6 & 53 & 79 & $0 . c$ \\
\hline $10 / 24 / 01$ & $15: 24$ & 18.7 & \begin{tabular}{|l|}
18.9 \\
\end{tabular} & \begin{tabular}{|l|}
18.9 \\
\end{tabular} & 18.6 & 0.0 & 9.6 & 50 & 79 & $0 . c$ \\
\hline $10 / 24 / 01$ & $15: 25$ & 18.7 & 18.9 & 18.9 & 18.6 & 0.0 & 9.6 & 73 & 79 & $0 . c$ \\
\hline $10 / 24 / 01$ & $15: 26$ & 18.7 & 18.8 & 18.9 & \begin{tabular}{|l|} 
\\
\end{tabular} & 0.0 & 9.6 & 43 & 79 & $0 . c$ \\
\hline $10 / 24 / 01$ & $15: 27$ & 18.7 & 18.8 & 18.9 & 18.6 & 0.0 & 9.6 & 102 & 79 & 0. \\
\hline
\end{tabular}

\begin{tabular}{|c|c|c|c|c|c|c|c|c|c|c|}
\hline \multicolumn{11}{|c|}{ PREC2_102401_0627 } \\
\hline DATE & TIME & $\begin{array}{c}\mathrm{HX} \\
\text { Outlet } \\
\left({ }^{\circ} \mathrm{C}\right) \\
\mathrm{TCO}\end{array}$ & \begin{tabular}{|c|} 
REC \\
PP \\
OUT \\
$\left({ }^{\circ} \mathrm{C}\right)$ \\
TC1 \\
\end{tabular} & $\begin{array}{c}\text { TK } \\
\text { BOT } \\
\left({ }^{\circ} \mathrm{C}\right) \\
\text { TC2 }\end{array}$ & \begin{tabular}{|c|}
$\mathrm{HX}$ \\
OUT \\
$\left({ }^{\circ} \mathrm{C}\right)$ \\
TC3
\end{tabular} & \begin{tabular}{|c|} 
Mn \\
Flow \\
(gpm) \\
\end{tabular} & \begin{tabular}{|c|} 
Galigher \\
Flow \\
(gpm)
\end{tabular} & $\begin{array}{c}\text { Current } \\
\text { to } \mathrm{HX}\end{array}$ & $\begin{array}{c}\text { Voltage } \\
\text { to } \mathrm{HX}\end{array}$ & $\begin{array}{c}\mathrm{Sr} \\
\text { Flow } \\
\text { (gpm) }\end{array}$ \\
\hline $10 / 24 / 01$ & $15: 28$ & 18.7 & 18.8 & 18.8 & 18.6 & 0.0 & 9.7 & 51 & 79 & 0.0 \\
\hline $10 / 24 / 01$ & $15: 29$ & 18.7 & 18.8 & 18.9 & 18.6 & 0.0 & 9.7 & 45 & 79 & 0.0 \\
\hline $10 / 24 / 01$ & $15: 30$ & 18.7 & 18.8 & 18.8 & 18.6 & 0.0 & 9.6 & 78 & 79 & 0.0 \\
\hline $10 / 24 / 01$ & $15: 31$ & 18.7 & 18.8 & 18.8 & 18.6 & 0.0 & 9.6 & 96 & 79 & 0.0 \\
\hline $10 / 24 / 01$ & 15:32 & 18.7 & \begin{tabular}{|l|}
18.8 \\
\end{tabular} & 18.8 & 18.6 & 0.0 & 9.6 & 58 & 79 & 0.0 \\
\hline $10 / 24 / 01$ & $15: 33$ & 18.7 & $\mid 18.8$ & 18.8 & 18.6 & 0.0 & 9.6 & 64 & 79 & 0.0 \\
\hline $10 / 24 / 01$ & $15: 34$ & 18.7 & 18.8 & 18.8 & 18.6 & 0.0 & 9.6 & 64 & 79 & 0.0 \\
\hline $10 / 24 / 01$ & $15: 35$ & 18.7 & 18.8 & 18.8 & 18.6 & 0.0 & 9.6 & 95 & 79 & 0.0 \\
\hline $10 / 24 / 01$ & $15: 36$ & 18.7 & 18.8 & 18.8 & 18.5 & 0.0 & 9.7 & 41 & 79 & 0.0 \\
\hline $10 / 24 / 01$ & $15: 37$ & 18.7 & 18.8 & 18.8 & 18.6 & 0.0 & 9.6 & 101 & 79 & 0.0 \\
\hline $10 / 24 / 01$ & $15: 38$ & 18.7 & 18.8 & 18.8 & 18.5 & 0.0 & 9.7 & 52 & 79 & 0.0 \\
\hline $10 / 24 / 01$ & $15: 39$ & 18.7 & 18.8 & 18.8 & 18.6 & 0.0 & 9.6 & 66 & 79 & 0.0 \\
\hline $10 / 24 / 01$ & $15: 40$ & 18.7 & $\mid 18.8$ & 18.8 & 18.5 & 0.0 & 9.6 & 38 & 79 & 0.0 \\
\hline $10 / 24 / 01$ & $15: 41$ & 18.7 & 18.8 & 18.8 & 18.6 & 0.0 & 9.6 & 37 & 79 & 0.0 \\
\hline $10 / 24 / 01$ & $15: 42$ & 18.7 & \begin{tabular}{|l|}
18.8 \\
\end{tabular} & 18.8 & 18.5 & 0.0 & 9.7 & 54 & 79 & 0.0 \\
\hline $10 / 24 / 01$ & $15: 43$ & 18.7 & 18.8 & 18.8 & 18.5 & 0.0 & 9.6 & 51 & 79 & 0.0 \\
\hline $10 / 24 / 01$ & $15: 44$ & 18.7 & 18.8 & 18.8 & 18.5 & 0.0 & 9.6 & 38 & 79 & 0.0 \\
\hline $10 / 24 / 01$ & $15: 45$ & 18.6 & 18.8 & 18.8 & 18.5 & 0.0 & 9.6 & 66 & 79 & 0.0 \\
\hline $10 / 24 / 01$ & $15: 46$ & 18.7 & \begin{tabular}{|l|}
18.8 \\
\end{tabular} & 18.8 & 18.6 & 0.0 & 9.7 & 35 & 79 & 0.0 \\
\hline $10 / 24 / 01$ & $15: 47$ & 18.6 & 18.8 & 18.8 & 18.5 & 0.0 & 9.6 & 41 & 79 & 0.0 \\
\hline $10 / 24 / 01$ & $15: 48$ & 18.7 & 18.8 & 18.8 & 18.6 & 0.0 & 9.6 & 34 & 79 & 0.0 \\
\hline $10 / 24 / 01$ & $15: 49$ & 18.6 & 18.8 & 18.8 & 18.5 & 0.0 & 9.6 & 35 & 79 & 0.0 \\
\hline $10 / 24 / 01$ & 15:50 & 18.7 & 18.8 & 18.8 & 18.6 & 0.0 & 9.6 & 39 & 79 & 0.0 \\
\hline $10 / 24 / 01$ & 15:51 & 18.6 & \begin{tabular}{|l|}
18.8 \\
\end{tabular} & 18.8 & 18.5 & 0.0 & 9.6 & 35 & 79 & 0.0 \\
\hline $10 / 24 / 01$ & 15:52 & 18.6 & $\mid 18.8$ & 18.8 & 18.5 & 0.0 & 9.6 & 87 & 79 & 0.0 \\
\hline $10 / 24 / 01$ & 15:53 & 18.6 & 18.8 & 18.8 & 18.5 & 0.0 & 9.6 & 87 & 79 & 0.0 \\
\hline $10 / 24 / 01$ & 15:54 & 18.6 & 18.8 & 18.8 & 18.5 & 0.0 & 9.6 & 59 & 79 & 0.0 \\
\hline $10 / 24 / 01$ & 15:55 & 18.7 & 18.8 & 18.8 & 18.5 & 0.0 & 9.6 & 46 & 79 & 0.0 \\
\hline $10 / 24 / 01$ & $15: 56$ & 18.6 & \begin{tabular}{|l|}
18.7 \\
\end{tabular} & 18.8 & 18.5 & 0.0 & 9.7 & 99 & 79 & 0.0 \\
\hline $10 / 24 / 01$ & $15: 57$ & 18.7 & \begin{tabular}{|l|}
$\mid 18.7$ \\
\end{tabular} & 18.8 & 18.5 & 0.0 & 9.6 & 31 & 79 & 0.0 \\
\hline $10 / 24 / 01$ & 15:58 & 18.6 & \begin{tabular}{|l|}
18.7 \\
\end{tabular} & 18.8 & 18.5 & 0.0 & 9.7 & 33 & 79 & 0.0 \\
\hline $10 / 24 / 01$ & $15: 59$ & 18.6 & \begin{tabular}{|l|}
$\mid 18.7$ \\
\end{tabular} & 18.8 & 18.5 & 0.0 & 9.7 & 80 & 79 & 0.0 \\
\hline $10 / 24 / 01$ & 16:00 & 18.6 & \begin{tabular}{|l|}
$\mid 18.7$ \\
\end{tabular} & 18.8 & 18.5 & 0.0 & 9.6 & 82 & 79 & 0.0 \\
\hline $10 / 24 / 01$ & 16:01 & 18.6 & \begin{tabular}{|l|}
18.7 \\
\end{tabular} & 18.8 & 18.5 & 0.0 & 9.6 & 26 & 79 & 0.0 \\
\hline $10 / 24 / 01$ & $16: 02$ & 18.6 & \begin{tabular}{|l|}
$\mid 18.7$ \\
\end{tabular} & \begin{tabular}{|l|}
18.7 \\
\end{tabular} & 18.5 & 0.0 & 9.6 & 28 & 79 & 0.0 \\
\hline $10 / 24 / 01$ & 16:03 & 18.6 & \begin{tabular}{|l|}
18.7 \\
\end{tabular} & 18.8 & 18.5 & 0.0 & 9.7 & 34 & 79 & 0.0 \\
\hline $10 / 24 / 01$ & $16: 04$ & 18.6 & \begin{tabular}{|l|}
18.7 \\
\end{tabular} & \begin{tabular}{|l|}
18.7 \\
\end{tabular} & 18.5 & 0.0 & 9.7 & 50 & 79 & 0.0 \\
\hline $10 / 24 / 01$ & $16: 05$ & 18.6 & \begin{tabular}{|l|}
$\mid 18.7$ \\
\end{tabular} & \begin{tabular}{|l|}
$\mid 18.7$ \\
\end{tabular} & 18.5 & 0.0 & 9.6 & 24 & 79 & 0.0 \\
\hline $10 / 24 / 01$ & $16: 06$ & 18.6 & \begin{tabular}{|l|}
18.7 \\
\end{tabular} & \begin{tabular}{|l|}
18.7 \\
\end{tabular} & 18.5 & 0.0 & 9.7 & 37 & 79 & 0.0 \\
\hline $10 / 24 / 01$ & $16: 07$ & 18.6 & \begin{tabular}{|l|}
18.7 \\
\end{tabular} & 18.8 & 18.5 & 0.0 & 9.6 & 26 & 79 & 0.0 \\
\hline $10 / 24 / 01$ & $16: 08$ & 18.7 & \begin{tabular}{|l|}
18.7 \\
\end{tabular} & \begin{tabular}{|l|}
18.7 \\
\end{tabular} & 18.5 & 0.0 & 9.6 & 33 & 79 & 0.0 \\
\hline $10 / 24 / 01$ & $16: 09$ & 18.6 & \begin{tabular}{|l|}
18.7 \\
\end{tabular} & \begin{tabular}{|l|}
18.7 \\
\end{tabular} & 18.5 & 0.0 & 9.6 & 30 & 79 & 0.0 \\
\hline $10 / 24 / 01$ & $16: 10$ & 18.6 & \begin{tabular}{|l|}
$\mid 18.7$ \\
\end{tabular} & 18.8 & 18.5 & 0.0 & 9.7 & 25 & 79 & 0.0 \\
\hline $10 / 24 / 01$ & $16: 11$ & 18.6 & \begin{tabular}{|l|}
18.7 \\
\end{tabular} & \begin{tabular}{|l|}
18.7 \\
\end{tabular} & 18.5 & 0.0 & 9.6 & 24 & 79 & 0.0 \\
\hline $10 / 24 / 01$ & $16: 12$ & 18.6 & \begin{tabular}{|l|}
18.7 \\
\end{tabular} & \begin{tabular}{|l|}
18.7 \\
\end{tabular} & 18.5 & 0.0 & 9.6 & 39 & 79 & 0.0 \\
\hline $10 / 24 / 01$ & $16: 13$ & 18.6 & \begin{tabular}{|l|}
$\mid 18.7$ \\
\end{tabular} & \begin{tabular}{|l|}
$\mid 18.7$ \\
\end{tabular} & 18.5 & 0.0 & 9.6 & 23 & 79 & 0.0 \\
\hline $10 / 24 / 01$ & $16: 14$ & 18.6 & \begin{tabular}{|l|}
$\mid 18.7$ \\
\end{tabular} & \begin{tabular}{|l|}
$\mid 18.7$ \\
\end{tabular} & 18.5 & 0.0 & 9.6 & 23 & 79 & 0.0 \\
\hline $10 / 24 / 01$ & $16: 15$ & 18.6 & \begin{tabular}{|l|}
$\mid 18.7$ \\
\end{tabular} & \begin{tabular}{|l|}
18.7 \\
\end{tabular} & 18.5 & 0.0 & 9.6 & 33 & 79 & 0.0 \\
\hline $10 / 24 / 01$ & $16: 16$ & 18.6 & \begin{tabular}{|l|}
$\mid 18.7$ \\
\end{tabular} & \begin{tabular}{|l|}
18.7 \\
\end{tabular} & 18.4 & 0.0 & 9.6 & 54 & 79 & 0.0 \\
\hline $10 / 24 / 01$ & $16: 17$ & 18.6 & \begin{tabular}{|l|}
18.7 \\
\end{tabular} & \begin{tabular}{|l|}
18.7 \\
\end{tabular} & 18.5 & 0.0 & 9.6 & 37 & 79 & 0.0 \\
\hline $10 / 24 / 01$ & $16: 18$ & 18.6 & \begin{tabular}{|l|}
$\mid 18.7$ \\
\end{tabular} & \begin{tabular}{|l|}
$\mid 18.7$ \\
\end{tabular} & 18.4 & 0.0 & 9.7 & 87 & 79 & 0.0 \\
\hline $10 / 24 / 01$ & $16: 19$ & 18.6 & \begin{tabular}{|l|}
18.7 \\
\end{tabular} & \begin{tabular}{|l|}
18.7 \\
\end{tabular} & 18.5 & 0.0 & 9.7 & 86 & 79 & 0.0 \\
\hline $10 / 24 / 01$ & $16: 20$ & 18.6 & \begin{tabular}{|l|}
18.7 \\
\end{tabular} & \begin{tabular}{|l|}
18.7 \\
\end{tabular} & 18.4 & 0.0 & 9.6 & 21 & 79 & 0.0 \\
\hline $10 / 24 / 01$ & $16: 21$ & 18.6 & \begin{tabular}{|l|}
18.7 \\
\end{tabular} & \begin{tabular}{|l|}
18.7 \\
\end{tabular} & 18.5 & 0.0 & 9.7 & 21 & 79 & 0.0 \\
\hline $10 / 24 / 01$ & $16: 22$ & 18.6 & \begin{tabular}{|l|}
18.7 \\
\end{tabular} & \begin{tabular}{|l|}
18.7 \\
\end{tabular} & 18.4 & 0.0 & 9.6 & 21 & 79 & 0.0 \\
\hline $10 / 24 / 01$ & $16: 23$ & 18.6 & \begin{tabular}{|l|}
18.7 \\
\end{tabular} & \begin{tabular}{|l|}
18.7 \\
\end{tabular} & 18.5 & 0.0 & 9.7 & 21 & 79 & 0.0 \\
\hline $10 / 24 / 01$ & $16: 24$ & 18.6 & \begin{tabular}{|l|}
$\mid 18.7$ \\
\end{tabular} & \begin{tabular}{|l|}
$\mid 18.7$ \\
\end{tabular} & 18.4 & 0.0 & 9.7 & 21 & 79 & 0.0 \\
\hline $10 / 24 / 01$ & $16: 25$ & 18.5 & 18.7 & \begin{tabular}{|l|}
18.7 \\
\end{tabular} & 18.4 & 0.0 & 9.7 & 21 & 79 & 0.0 \\
\hline $10 / 24 / 01$ & $16: 26$ & 18.6 & \begin{tabular}{|l|}
$\mid 18.7$ \\
\end{tabular} & \begin{tabular}{|l|}
$\mid 18.7$ \\
\end{tabular} & 18.5 & 0.0 & 9.7 & 21 & 79 & 0.0 \\
\hline $10 / 24 / 01$ & $16: 27$ & 18.5 & \begin{tabular}{|l|}
18.7 \\
\end{tabular} & \begin{tabular}{|l|}
18.7 \\
\end{tabular} & \begin{tabular}{|l|}
18.4 \\
\end{tabular} & 0.0 & 9.7 & 21 & 79 & 0.0 \\
\hline
\end{tabular}


WSRC-TR-2002-00459, Rev. 0 SRT-RPP-2002-00221

\begin{tabular}{|c|c|c|c|c|c|c|c|c|c|c|}
\hline \multicolumn{3}{|c|}{ PREC2_102401_0627 } & \multirow[b]{2}{*}{\begin{tabular}{|c|} 
REC \\
PP \\
OUT \\
$\left({ }^{\circ} \mathrm{C}\right)$ \\
TC1
\end{tabular}} & \multirow[b]{2}{*}{\begin{tabular}{|c} 
TK \\
BOT \\
$\left({ }^{\circ} \mathrm{C}\right)$ \\
TC2 \\
\end{tabular}} & \multirow[b]{2}{*}{\begin{tabular}{|c|} 
HX \\
OUT \\
$\left({ }^{\circ} \mathrm{C}\right)$ \\
TC3 \\
\end{tabular}} & \multirow[b]{2}{*}{$\begin{array}{c}\text { Mn } \\
\text { Flow } \\
\text { (gpm) }\end{array}$} & \multirow[b]{2}{*}{$\begin{array}{c}\text { Galigher } \\
\text { Flow } \\
\text { (gpm) }\end{array}$} & \multirow[b]{2}{*}{$\begin{array}{c}\text { Current } \\
\text { to } \mathrm{HX}\end{array}$} & \multirow[b]{2}{*}{$\begin{array}{l}\text { Voltage } \\
\text { to } \mathrm{HX}\end{array}$} & \multirow[b]{2}{*}{$\begin{array}{c}\mathrm{Sr} \\
\text { Flow } \\
(\mathrm{gpm})\end{array}$} \\
\hline DATE & TIME & \begin{tabular}{|c}
$\mathrm{HX}$ \\
Outlet \\
$\left({ }^{\circ} \mathrm{C}\right)$ \\
$\mathrm{TCO}$
\end{tabular} & & & & & & & & \\
\hline $10 / 24 / 01$ & $16: 28$ & 18.6 & 18.7 & 18.7 & 18.5 & 0.0 & 9.6 & 21 & 79 & 0.0 \\
\hline $10 / 24 / 01$ & $16: 29$ & 18.5 & 18.7 & \begin{tabular}{|l|}
18.7 \\
\end{tabular} & \begin{tabular}{|l|}
18.4 \\
\end{tabular} & 0.0 & 9.6 & 21 & 79 & 0.0 \\
\hline $10 / 24 / 01$ & $16: 30$ & 18.6 & 18.7 & 18.7 & 18.4 & 0.0 & 9.7 & 21 & 79 & 0.0 \\
\hline $10 / 24 / 01$ & $16: 31$ & 18.5 & 18.7 & 18.7 & 18.4 & 0.0 & 9.6 & 21 & 79 & 0.0 \\
\hline $10 / 24 / 01$ & $16: 32$ & 18.5 & 18.7 & \begin{tabular}{|l|}
18.7 \\
\end{tabular} & \begin{tabular}{|l|}
18.4 \\
\end{tabular} & 0.0 & 9.6 & 21 & 79 & 0.0 \\
\hline $10 / 24 / 01$ & $16: 33$ & 18.6 & 18.6 & \begin{tabular}{|l|}
18.7 \\
\end{tabular} & \begin{tabular}{|l|}
18.4 \\
\end{tabular} & 0.0 & 9.6 & 21 & 79 & 0.0 \\
\hline $10 / 24 / 01$ & $16: 34$ & 18.5 & 18.7 & \begin{tabular}{|l|}
18.7 \\
\end{tabular} & \begin{tabular}{|l|}
18.4 \\
\end{tabular} & 0.0 & 9.6 & 21 & 79 & 0.0 \\
\hline $10 / 24 / 01$ & $16: 35$ & 18.6 & 18.7 & \begin{tabular}{|l|}
18.7 \\
\end{tabular} & \begin{tabular}{|l|}
18.4 \\
\end{tabular} & 0.0 & 9.6 & 21 & 79 & 0.0 \\
\hline $10 / 24 / 01$ & $16: 36$ & 18.5 & 18.7 & 18.7 & \begin{tabular}{|l|}
18.4 \\
\end{tabular} & 0.0 & 9.6 & 21 & 79 & 0.0 \\
\hline $10 / 24 / 01$ & $16: 37$ & 18.6 & 18.7 & 18.7 & 18.4 & 0.0 & 9.7 & 21 & 79 & 0.0 \\
\hline $10 / 24 / 01$ & $16: 38$ & 18.5 & 18.7 & 18.7 & \begin{tabular}{|l|}
18.4 \\
\end{tabular} & 0.0 & 9.6 & 21 & 79 & 0.0 \\
\hline $10 / 24 / 01$ & $16: 39$ & 18.6 & 18.6 & 18.7 & 18.4 & 0.0 & 9.7 & 21 & 79 & 0.0 \\
\hline $10 / 24 / 01$ & $16: 40$ & 18.5 & 18.7 & \begin{tabular}{|l|}
18.7 \\
\end{tabular} & \begin{tabular}{|l|}
18.4 \\
\end{tabular} & 0.0 & 9.6 & 21 & 79 & 0.0 \\
\hline $10 / 24 / 01$ & $16: 41$ & 18.5 & 18.7 & \begin{tabular}{|l|}
18.7 \\
\end{tabular} & \begin{tabular}{|l|}
18.4 \\
\end{tabular} & 0.0 & 9.6 & 21 & 79 & 0.0 \\
\hline $10 / 24 / 01$ & $16: 42$ & 18.5 & 18.6 & 18.7 & \begin{tabular}{|l|}
18.4 \\
\end{tabular} & 0.0 & 9.6 & 21 & 79 & 0.0 \\
\hline $10 / 24 / 01$ & $16: 43$ & 18.5 & 18.6 & 18.7 & 18.4 & 0.0 & 9.7 & 21 & 79 & 0.0 \\
\hline $10 / 24 / 01$ & $16: 44$ & 18.5 & 18.6 & 18.7 & \begin{tabular}{|l|}
18.4 \\
\end{tabular} & 0.0 & 9.7 & 21 & 79 & 0.0 \\
\hline $10 / 24 / 01$ & $16: 45$ & 18.5 & 18.6 & 18.7 & 18.4 & 0.0 & 9.7 & 21 & 79 & 0.0 \\
\hline $10 / 24 / 01$ & $16: 46$ & 18.6 & 18.6 & \begin{tabular}{|l|}
18.7 \\
\end{tabular} & \begin{tabular}{|l|}
18.4 \\
\end{tabular} & 0.0 & 9.7 & 21 & 79 & 0.0 \\
\hline $10 / 24 / 01$ & $16: 47$ & 18.5 & 18.6 & 18.6 & \begin{tabular}{|l|}
18.4 \\
\end{tabular} & 0.0 & 9.6 & 21 & 79 & 0.0 \\
\hline $10 / 24 / 01$ & $16: 48$ & 18.6 & 18.6 & 18.6 & \begin{tabular}{|l|}
18.4 \\
\end{tabular} & 0.0 & 9.7 & 21 & 79 & 0.0 \\
\hline $10 / 24 / 01$ & $16: 49$ & 18.5 & 18.6 & 18.6 & \begin{tabular}{|l|}
18.4 \\
\end{tabular} & 0.0 & 9.6 & 21 & 79 & 0.0 \\
\hline $10 / 24 / 01$ & $16: 50$ & 18.5 & 18.6 & 18.6 & \begin{tabular}{|l|}
18.4 \\
\end{tabular} & 0.0 & 9.7 & 21 & 79 & 0.0 \\
\hline $10 / 24 / 01$ & 16:51 & 18.5 & 18.7 & 18.6 & \begin{tabular}{|l|}
18.4 \\
\end{tabular} & 0.0 & 9.7 & 21 & 79 & 0.0 \\
\hline $10 / 24 / 01$ & $16: 52$ & 18.5 & $\mid 18.7$ & 18.6 & \begin{tabular}{|l|}
18.4 \\
\end{tabular} & 0.0 & 9.6 & 21 & 79 & 0.0 \\
\hline $10 / 24 / 01$ & $16: 53$ & 18.6 & 18.7 & 18.6 & \begin{tabular}{|l|}
18.4 \\
\end{tabular} & 0.0 & 9.7 & 21 & 79 & 0.0 \\
\hline $10 / 24 / 01$ & $16: 54$ & 18.5 & 18.6 & 18.7 & \begin{tabular}{|l|}
18.4 \\
\end{tabular} & 0.0 & 9.6 & 21 & 79 & 0.0 \\
\hline $10 / 24 / 01$ & $16: 55$ & 18.5 & 18.6 & 18.6 & \begin{tabular}{|l|}
18.4 \\
\end{tabular} & 0.0 & 9.6 & 21 & 79 & 0.0 \\
\hline $10 / 24 / 01$ & $16: 56$ & 18.5 & 18.6 & 18.6 & 18.4 & 0.0 & 9.7 & 20 & 79 & 0.0 \\
\hline $10 / 24 / 01$ & $16: 57$ & 18.6 & 18.6 & 18.6 & \begin{tabular}{|l|}
18.4 \\
\end{tabular} & 0.0 & 9.6 & 21 & 79 & 0.0 \\
\hline $10 / 24 / 01$ & $16: 58$ & 18.5 & 18.6 & 18.6 & 18.4 & 0.0 & 9.6 & 21 & 79 & 0.0 \\
\hline $10 / 24 / 01$ & $16: 59$ & 18.5 & 18.6 & 18.6 & \begin{tabular}{|l|}
18.4 \\
\end{tabular} & 0.0 & 9.6 & 21 & 79 & 0.0 \\
\hline $10 / 24 / 01$ & $17: 00$ & 18.5 & 18.6 & 18.6 & 18.4 & 0.0 & 9.6 & 21 & 79 & 0.0 \\
\hline $10 / 24 / 01$ & $17: 01$ & 18.5 & 18.6 & 18.6 & 18.4 & 0.0 & 9.7 & 21 & 79 & 0.0 \\
\hline $10 / 24 / 01$ & $17: 02$ & 18.5 & 18.6 & 18.6 & 18.4 & 0.0 & 9.7 & 21 & 79 & 0.0 \\
\hline $10 / 24 / 01$ & $17: 03$ & 18.5 & 18.6 & 18.6 & \begin{tabular}{|l|}
18.4 \\
\end{tabular} & 0.0 & 9.6 & 21 & 79 & 0.0 \\
\hline $10 / 24 / 01$ & $17: 04$ & 18.5 & $\mid 18.6$ & 18.6 & \begin{tabular}{|l|}
18.4 \\
\end{tabular} & 0.0 & 9.6 & 21 & 79 & 0.0 \\
\hline $10 / 24 / 01$ & $17: 05$ & 18.5 & $\mid 18.6$ & 18.6 & \begin{tabular}{|l|}
18.4 \\
\end{tabular} & 0.0 & 9.6 & 21 & 79 & 0.0 \\
\hline $10 / 24 / 01$ & $17: 06$ & 18.5 & $\mid 18.6$ & 18.6 & \begin{tabular}{|l|}
18.4 \\
\end{tabular} & 0.0 & 9.6 & 21 & 79 & 0.0 \\
\hline $10 / 24 / 01$ & $17: 07$ & 18.5 & 18.6 & 18.6 & 18.4 & 0.0 & 9.6 & 21 & 79 & 0.0 \\
\hline $10 / 24 / 01$ & $17: 08$ & 18.5 & 18.6 & 18.6 & \begin{tabular}{|l|}
18.4 \\
\end{tabular} & 0.0 & 9.6 & 21 & 79 & 0.0 \\
\hline $10 / 24 / 01$ & $17: 09$ & 18.5 & 18.6 & 18.6 & \begin{tabular}{|l|}
18.4 \\
\end{tabular} & 0.0 & 9.6 & 21 & 79 & 0.0 \\
\hline $10 / 24 / 01$ & $17: 10$ & 18.5 & 18.6 & 18.6 & 18.4 & 0.0 & 9.6 & 21 & 79 & 0.0 \\
\hline $10 / 24 / 01$ & $17: 11$ & 18.5 & 18.6 & 18.6 & \begin{tabular}{|l|}
18.4 \\
\end{tabular} & 0.0 & 9.7 & 21 & 79 & 0.0 \\
\hline $10 / 24 / 01$ & $17: 12$ & 18.5 & 18.6 & 18.6 & \begin{tabular}{|l|}
18.4 \\
\end{tabular} & 0.0 & 9.6 & 21 & 79 & 0.0 \\
\hline $10 / 24 / 01$ & $17: 13$ & 18.5 & $\mid 18.6$ & 18.6 & 18.4 & 0.0 & 9.6 & 21 & 79 & 0.0 \\
\hline $10 / 24 / 01$ & $17: 14$ & 18.5 & 18.6 & 18.6 & \begin{tabular}{|l|}
18.4 \\
\end{tabular} & 0.0 & 9.6 & 21 & 79 & 0.0 \\
\hline $10 / 24 / 01$ & $17: 15$ & 18.5 & 18.6 & 18.6 & \begin{tabular}{|l|}
18.4 \\
\end{tabular} & 0.0 & 9.7 & 21 & 79 & 0.0 \\
\hline $10 / 24 / 01$ & $17: 16$ & 18.5 & 18.6 & 18.6 & 18.4 & 0.0 & 9.6 & 21 & 79 & 0.0 \\
\hline $10 / 24 / 01$ & $17: 17$ & 18.5 & 18.6 & 18.6 & \begin{tabular}{|l|}
18.4 \\
\end{tabular} & 0.0 & 9.7 & 21 & 79 & 0.0 \\
\hline $10 / 24 / 01$ & $17: 18$ & 18.5 & 18.6 & 18.6 & \begin{tabular}{|l|}
18.4 \\
\end{tabular} & 0.0 & 9.6 & 21 & 79 & 0.0 \\
\hline $10 / 24 / 01$ & $17: 19$ & 18.5 & 18.6 & 18.6 & 18.4 & 0.0 & 9.7 & 21 & 79 & 0.0 \\
\hline $10 / 24 / 01$ & $17: 20$ & 18.5 & 18.6 & 18.6 & \begin{tabular}{|l|}
18.4 \\
\end{tabular} & 0.0 & 9.7 & 21 & 79 & 0.0 \\
\hline $10 / 24 / 01$ & $17: 21$ & 18.5 & 18.6 & 18.6 & \begin{tabular}{|l|}
18.4 \\
\end{tabular} & 0.0 & 9.6 & 21 & 79 & 0.0 \\
\hline $10 / 24 / 01$ & $17: 22$ & 18.5 & 18.6 & 18.6 & 18.4 & 0.0 & 9.6 & 21 & 79 & 0.0 \\
\hline $10 / 24 / 01$ & $17: 23$ & 18.5 & 18.6 & 18.6 & \begin{tabular}{|l|}
18.4 \\
\end{tabular} & 0.0 & 9.6 & 21 & 79 & 0.0 \\
\hline $10 / 24 / 01$ & $17: 24$ & 18.5 & 18.6 & 18.6 & \begin{tabular}{|l|}
18.4 \\
\end{tabular} & 0.0 & 9.6 & 21 & 79 & 0.0 \\
\hline $10 / 24 / 01$ & $17: 25$ & 18.5 & 18.6 & 18.6 & 18.3 & 0.0 & 9.7 & 21 & 79 & 0.0 \\
\hline $10 / 24 / 01$ & $17: 26$ & 18.5 & 18.6 & 18.6 & \begin{tabular}{|l|}
18.4 \\
\end{tabular} & 0.0 & 9.7 & 21 & 79 & 0.0 \\
\hline $10 / 24 / 01$ & $17: 27$ & 18.5 & 18.6 & 18.6 & 18.3 & 0.0 & 9.6 & 21 & 79 & 0.0 \\
\hline
\end{tabular}

\begin{tabular}{|c|c|c|c|c|c|c|c|c|c|c|}
\hline \multicolumn{3}{|c|}{ PREC2_102401_0627 } & & & & & & & & \\
\hline DATE & TIME & $\begin{array}{c}\mathrm{HX} \\
\text { Outlet } \\
\left({ }^{\circ} \mathrm{C}\right) \\
\mathrm{TCO}\end{array}$ & $\begin{array}{c}\text { REC } \\
\text { PP } \\
\text { OUT } \\
\left({ }^{\circ} \mathrm{C}\right) \\
\text { TC1 }\end{array}$ & $\begin{array}{c}\text { TK } \\
\text { BOT } \\
\left({ }^{\circ} \mathrm{C}\right) \\
\text { TC2 }\end{array}$ & $\begin{array}{c}\text { HX } \\
\text { OUT } \\
\left({ }^{\circ} \mathrm{C}\right) \\
\text { TC3 }\end{array}$ & $\begin{array}{c}\text { Mn } \\
\text { Flow } \\
(\mathrm{gpm})\end{array}$ & $\begin{array}{c}\text { Galigher } \\
\text { Flow } \\
\text { (gpm) }\end{array}$ & $\begin{array}{c}\text { Current } \\
\text { to } \mathrm{HX}\end{array}$ & $\begin{array}{l}\text { Voltage } \\
\text { to } \mathrm{HX}\end{array}$ & $\begin{array}{c}\mathrm{Sr} \\
\text { Flow } \\
\text { (gpm) }\end{array}$ \\
\hline 10/24/01 & $17: 28$ & 18.5 & 18.6 & 18.6 & 18.4 & 0.0 & 9.6 & 21 & 79 & 0.0 \\
\hline $10 / 24 / 01$ & $17: 29$ & 18.5 & 18.6 & 18.6 & 18.4 & 0.0 & 9.7 & 21 & 79 & 0.0 \\
\hline $10 / 24 / 01$ & $17: 30$ & 18.5 & 18.6 & 18.6 & 18.4 & 0.0 & 9.7 & 21 & 79 & 0.0 \\
\hline 10/24/01 & $17: 31$ & 18.5 & 18.6 & 18.6 & 18.4 & 0.0 & 9.6 & 21 & 79 & 0.0 \\
\hline $10 / 24 / 01$ & $17: 32$ & 18.5 & 18.6 & 18.6 & 18.3 & 0.0 & 9.7 & 21 & 79 & 0.0 \\
\hline $10 / 24 / 01$ & $17: 33$ & 18.5 & 18.6 & 18.6 & 18.4 & 0.0 & 9.7 & 21 & 79 & 0.0 \\
\hline $10 / 24 / 01$ & $17: 34$ & 18.5 & 18.6 & 18.6 & 18.3 & 0.0 & 9.7 & 20 & 79 & 0.0 \\
\hline $10 / 24 / 01$ & $17: 35$ & 18.5 & 18.6 & 18.6 & 18.4 & 0.0 & 9.6 & 20 & 79 & 0.0 \\
\hline $10 / 24 / 01$ & $17: 36$ & 18.5 & 18.6 & 18.6 & 18.3 & 0.0 & 9.7 & 21 & 79 & 0.0 \\
\hline $10 / 24 / 01$ & $17: 37$ & 18.5 & 18.6 & 18.6 & 18.4 & 0.0 & 9.6 & 20 & 79 & 0.0 \\
\hline 10/24/01 & $17: 38$ & 18.5 & 18.6 & 18.6 & 18.3 & 0.0 & 9.7 & 21 & 79 & 0.0 \\
\hline $10 / 24 / 01$ & $17: 39$ & 18.5 & 18.6 & 18.6 & 18.4 & 0.0 & 9.7 & 21 & 79 & 0.0 \\
\hline $10 / 24 / 01$ & $17: 40$ & 18.5 & 18.6 & 18.6 & 18.4 & 0.0 & 9.6 & 21 & 79 & 0.0 \\
\hline $10 / 24 / 01$ & $17: 41$ & 18.5 & 18.6 & 18.6 & 18.4 & 0.0 & 9.7 & 21 & 79 & 0.0 \\
\hline $10 / 24 / 01$ & $17: 42$ & 18.6 & 18.6 & 18.6 & 18.4 & 0.0 & 9.6 & 21 & 79 & 0.0 \\
\hline $10 / 24 / 01$ & $17: 43$ & 18.5 & 18.6 & 18.6 & 18.4 & 0.0 & 9.6 & 21 & 79 & 0.0 \\
\hline $10 / 24 / 01$ & $17: 44$ & 18.6 & 18.6 & 18.6 & 18.4 & 0.0 & 9.7 & 20 & 79 & 0.0 \\
\hline $10 / 24 / 01$ & $17: 45$ & 18.5 & 18.6 & 18.6 & 18.4 & 0.0 & 9.7 & 21 & 79 & 0.0 \\
\hline $10 / 24 / 01$ & $17: 46$ & 18.5 & 18.6 & 18.6 & 18.4 & 0.0 & 9.7 & 21 & 79 & 0.0 \\
\hline $10 / 24 / 01$ & $17: 47$ & 18.5 & 18.6 & 18.6 & 18.4 & 0.0 & 9.6 & 21 & 79 & 0.0 \\
\hline $10 / 24 / 01$ & $17: 48$ & 18.5 & 18.6 & 18.6 & 18.4 & 0.0 & 9.6 & 21 & 79 & 0.0 \\
\hline $10 / 24 / 01$ & $17: 49$ & 18.5 & 18.6 & 18.6 & 18.4 & 0.0 & 9.6 & 21 & 79 & 0.0 \\
\hline $10 / 24 / 01$ & $17: 50$ & 18.5 & 18.6 & 18.6 & 18.4 & 0.0 & 9.6 & 21 & 79 & 0.0 \\
\hline $10 / 24 / 01$ & $17: 51$ & 18.5 & 18.6 & 18.6 & 18.4 & 0.0 & 9.6 & 21 & 79 & 0.0 \\
\hline $10 / 24 / 01$ & $17: 52$ & 18.5 & 18.6 & 18.6 & 18.4 & 0.0 & 9.6 & 20 & 79 & 0.0 \\
\hline $10 / 24 / 01$ & $17: 53$ & 18.5 & 18.6 & 18.6 & 18.4 & 0.0 & 9.6 & 21 & 79 & 0.0 \\
\hline $10 / 24 / 01$ & $17: 54$ & 18.5 & 18.6 & 18.6 & 18.4 & 0.0 & 9.7 & 21 & 79 & 0.0 \\
\hline $10 / 24 / 01$ & $17: 55$ & 18.5 & 18.6 & 18.6 & 18.4 & 0.0 & 9.7 & 20 & 79 & 0.0 \\
\hline $10 / 24 / 01$ & $17: 56$ & 18.5 & 18.6 & 18.6 & 18.3 & 0.0 & 9.7 & 20 & 79 & 0.0 \\
\hline $10 / 24 / 01$ & $17: 57$ & 18.5 & 18.6 & 18.6 & 18.4 & 0.0 & 9.7 & 21 & 79 & 0.0 \\
\hline $10 / 24 / 01$ & $17: 58$ & 18.5 & 18.6 & 18.6 & 18.4 & 0.0 & 9.7 & 21 & 79 & 0.0 \\
\hline $10 / 24 / 01$ & $17: 59$ & 18.5 & 18.6 & 18.6 & 18.4 & 0.0 & 9.7 & 20 & 79 & 0.0 \\
\hline $10 / 24 / 01$ & $18: 00$ & 18.5 & 18.6 & 18.6 & 18.4 & 0.0 & 9.7 & 20 & 79 & 0.0 \\
\hline $10 / 24 / 01$ & 18:01 & 18.5 & 18.6 & 18.6 & 18.3 & 0.0 & 9.7 & 21 & 79 & 0.0 \\
\hline $10 / 24 / 01$ & $18: 02$ & 18.5 & 18.6 & 18.6 & 18.4 & 0.0 & 9.7 & 21 & 79 & 0.0 \\
\hline $10 / 24 / 01$ & $18: 03$ & 18.5 & 18.6 & 18.6 & 18.3 & 0.0 & 9.7 & 21 & 79 & 0.0 \\
\hline $10 / 24 / 01$ & $18: 04$ & 18.5 & 18.6 & 18.6 & 18.4 & 0.0 & 9.6 & 21 & 79 & 0.0 \\
\hline $10 / 24 / 01$ & $18: 05$ & 18.5 & 18.6 & 18.6 & 18.3 & 0.0 & 9.6 & 21 & 79 & 0.0 \\
\hline $10 / 24 / 01$ & $18: 06$ & 18.5 & 18.6 & 18.6 & 18.4 & 0.0 & 9.6 & 21 & 79 & 0.0 \\
\hline $10 / 24 / 01$ & $18: 07$ & 18.5 & 18.6 & 18.6 & 18.4 & 0.0 & 9.6 & 21 & 79 & 0.0 \\
\hline $10 / 24 / 01$ & $18: 08$ & 18.5 & 18.6 & 18.6 & 18.4 & 0.0 & 9.6 & 21 & 79 & 0.0 \\
\hline $10 / 24 / 01$ & $18: 09$ & 18.5 & 18.6 & 18.6 & 18.4 & 0.0 & 9.6 & 21 & 79 & 0.0 \\
\hline $10 / 24 / 01$ & $18: 10$ & 18.5 & 18.6 & 18.6 & 18.3 & 0.0 & 9.7 & 21 & 79 & 0.0 \\
\hline $10 / 24 / 01$ & $18: 11$ & 18.5 & 18.6 & 18.6 & 18.4 & 0.0 & 9.6 & 21 & 79 & 0.0 \\
\hline $10 / 24 / 01$ & $18: 12$ & 18.5 & 18.6 & 18.6 & 18.3 & 0.0 & 9.6 & 20 & 79 & 0.0 \\
\hline $10 / 24 / 01$ & $18: 13$ & 18.5 & 18.6 & 18.6 & 18.4 & 0.0 & 9.7 & 21 & 79 & 0.0 \\
\hline $10 / 24 / 01$ & $18: 14$ & 18.5 & 18.6 & 18.6 & 18.3 & 0.0 & 9.7 & 21 & 79 & 0.0 \\
\hline $10 / 24 / 01$ & $18: 15$ & 18.5 & 18.6 & 18.6 & 18.4 & 0.0 & 9.7 & 21 & 79 & 0.0 \\
\hline $10 / 24 / 01$ & $18: 16$ & 18.5 & 18.6 & 18.6 & 18.3 & 0.0 & 9.7 & 21 & 79 & 0.0 \\
\hline $10 / 24 / 01$ & $18: 17$ & 18.5 & 18.6 & 18.6 & 18.3 & 0.0 & 9.7 & 21 & 79 & 0.0 \\
\hline $10 / 24 / 01$ & $18: 18$ & 18.5 & 18.6 & 18.6 & 18.3 & 0.0 & 9.6 & 21 & 79 & 0.0 \\
\hline $10 / 24 / 01$ & $18: 19$ & 18.5 & 18.6 & 18.6 & 18.3 & 0.0 & 9.6 & 21 & 79 & 0.0 \\
\hline $10 / 24 / 01$ & $18: 20$ & 18.5 & 18.6 & 18.6 & 18.4 & 0.0 & 9.6 & 21 & 79 & 0.0 \\
\hline $10 / 24 / 01$ & $18: 21$ & 18.4 & 18.6 & 18.6 & 18.3 & 0.0 & 9.6 & 21 & 79 & 0.0 \\
\hline $10 / 24 / 01$ & $18: 22$ & 18.5 & 18.6 & 18.6 & 18.4 & 0.0 & 9.7 & 21 & 79 & 0.0 \\
\hline $10 / 24 / 01$ & $18: 23$ & 18.4 & 18.6 & 18.6 & 18.3 & 0.0 & 9.7 & 21 & 79 & 0.0 \\
\hline $10 / 24 / 01$ & $18: 24$ & 18.5 & 18.6 & 18.6 & 18.4 & 0.0 & 9.7 & 21 & 79 & 0.0 \\
\hline $10 / 24 / 01$ & $18: 25$ & 18.5 & 18.6 & 18.6 & 18.3 & 0.0 & 9.7 & 20 & 79 & 0.0 \\
\hline $10 / 24 / 01$ & $18: 26$ & 18.5 & 18.6 & 18.6 & 18.3 & 0.0 & 9.7 & 21 & 79 & 0.0 \\
\hline $10 / 24 / 01$ & $18: 27$ & 18.5 & 18.6 & 18.6 & 18.3 & 0.0 & 9.6 & 21 & 79 & 0.0 \\
\hline
\end{tabular}


WSRC-TR-2002-00459, Rev. 0 SRT-RPP-2002-00221

\begin{tabular}{|c|c|c|c|c|c|c|c|c|c|c|}
\hline \multicolumn{11}{|c|}{ PREC2_102401_0627 } \\
\hline DATE & TIME & $\begin{array}{c}\mathrm{HX} \\
\text { Outlet } \\
\left({ }^{\circ} \mathrm{C}\right) \\
\mathrm{TCO}\end{array}$ & $\begin{array}{c}\text { REC } \\
\text { PP } \\
\text { OUT } \\
\left({ }^{\circ} \mathrm{C}\right) \\
\text { TC1 }\end{array}$ & $\begin{array}{c}\text { TK } \\
\text { BOT } \\
\left({ }^{\circ} \mathrm{C}\right) \\
\text { TC2 }\end{array}$ & \begin{tabular}{|l|} 
HX \\
OUT \\
$\left({ }^{\circ} \mathrm{C}\right)$ \\
TC3
\end{tabular} & $\begin{array}{c}\mathrm{Mn} \\
\text { Flow } \\
(\mathrm{gpm})\end{array}$ & $\begin{array}{c}\text { Galigher } \\
\text { Flow } \\
\text { (gpm) }\end{array}$ & $\begin{array}{c}\text { Current } \\
\text { to } \mathrm{HX}\end{array}$ & $\begin{array}{c}\text { Voltage } \\
\text { to } \mathrm{HX}\end{array}$ & $\begin{array}{c}\mathrm{Sr} \\
\text { Flow } \\
(\mathrm{gpm})\end{array}$ \\
\hline $10 / 24 / 01$ & $18: 28$ & 18.4 & 18.6 & 18.6 & \begin{tabular}{|l|}
18.3 \\
\end{tabular} & 0.0 & 9.7 & 21 & 79 & 0.0 \\
\hline $10 / 24 / 01$ & $18: 29$ & 18.5 & 18.6 & 18.6 & 18.3 & 0.0 & 9.7 & 21 & 79 & 0.0 \\
\hline $10 / 24 / 01$ & 18:30 & 18.4 & 18.6 & 18.6 & 18.3 & 0.0 & 9.6 & 21 & 79 & 0.0 \\
\hline $10 / 24 / 01$ & $18: 31$ & 18.5 & 18.6 & 18.6 & 18.4 & 0.0 & 9.6 & 20 & 79 & 0.0 \\
\hline $10 / 24 / 01$ & 18:32 & 18.4 & 18.6 & 18.6 & \begin{tabular}{|l|}
18.3 \\
\end{tabular} & 0.0 & 9.6 & 21 & 79 & 0.0 \\
\hline $10 / 24 / 01$ & 18:33 & 18.5 & 18.6 & 18.6 & 18.3 & 0.0 & 9.6 & 21 & 79 & 0.0 \\
\hline $10 / 24 / 01$ & $18: 34$ & 18.4 & 18.6 & 18.6 & 18.3 & 0.0 & 9.7 & 20 & 79 & 0.0 \\
\hline $10 / 24 / 01$ & 18:35 & 18.5 & 18.6 & 18.6 & 18.3 & 0.0 & 9.7 & 21 & 79 & 0.0 \\
\hline $10 / 24 / 01$ & $18: 36$ & 18.5 & 18.5 & 18.6 & 18.3 & 0.0 & 9.6 & 20 & 79 & 0.0 \\
\hline $10 / 24 / 01$ & $18: 37$ & 18.4 & 18.6 & 18.6 & \begin{tabular}{|l|}
18.3 \\
\end{tabular} & 0.0 & 9.7 & 21 & 79 & 0.0 \\
\hline $10 / 24 / 01$ & 18:38 & 18.5 & 18.5 & 18.6 & 18.3 & 0.0 & 9.6 & 21 & 79 & 0.0 \\
\hline $10 / 24 / 01$ & 18:39 & 18.4 & 18.6 & 18.6 & \begin{tabular}{|l|}
18.3 \\
\end{tabular} & 0.0 & 9.6 & 20 & 79 & 0.0 \\
\hline $10 / 24 / 01$ & $18: 40$ & 18.5 & 18.6 & 18.6 & 18.3 & 0.0 & 9.6 & 21 & 79 & 0.0 \\
\hline $10 / 24 / 01$ & $18: 41$ & 18.4 & 18.6 & 18.6 & 18.3 & 0.0 & 9.7 & 21 & 79 & 0.0 \\
\hline $10 / 24 / 01$ & $18: 42$ & 18.5 & 18.6 & 18.6 & 18.3 & 0.0 & 9.7 & 21 & 79 & 0.0 \\
\hline $10 / 24 / 01$ & $18: 43$ & 18.4 & 18.5 & 18.5 & \begin{tabular}{|l|}
18.3 \\
\end{tabular} & 0.0 & 9.7 & 21 & 79 & 0.0 \\
\hline $10 / 24 / 01$ & $18: 44$ & 18.5 & 18.5 & 18.6 & 18.3 & 0.0 & 9.7 & 21 & 79 & 0.0 \\
\hline $10 / 24 / 01$ & $18: 45$ & 18.5 & 18.5 & 18.6 & 18.3 & 0.0 & 9.7 & 21 & 79 & 0.0 \\
\hline $10 / 24 / 01$ & $18: 46$ & 18.4 & 18.5 & 18.6 & 18.3 & 0.0 & 9.6 & 21 & 79 & 0.0 \\
\hline $10 / 24 / 01$ & $18: 47$ & 18.5 & 18.5 & 18.5 & 18.3 & 0.0 & 9.7 & 21 & 79 & 0.0 \\
\hline $10 / 24 / 01$ & $18: 48$ & 18.4 & 18.5 & 18.6 & 18.3 & 0.0 & 9.6 & 20 & 79 & 0.0 \\
\hline $10 / 24 / 01$ & $18: 49$ & 18.5 & 18.5 & 18.5 & 18.3 & 0.0 & 9.6 & 21 & 79 & 0.0 \\
\hline $10 / 24 / 01$ & 18:50 & 18.4 & 18.5 & 18.5 & 18.3 & 0.0 & 9.6 & 21 & 79 & 0.0 \\
\hline $10 / 24 / 01$ & 18:51 & 18.5 & 18.5 & 18.5 & 18.3 & 0.0 & 9.6 & 21 & 79 & 0.0 \\
\hline $10 / 24 / 01$ & 18:52 & 18.4 & 18.5 & 18.5 & 18.3 & 0.0 & 9.6 & 20 & 79 & 0.0 \\
\hline $10 / 24 / 01$ & 18:53 & 18.4 & 18.5 & 18.5 & 18.3 & 0.0 & 9.6 & 21 & 79 & 0.0 \\
\hline $10 / 24 / 01$ & 18:54 & 18.4 & 18.5 & 18.5 & 18.3 & 0.0 & 9.6 & 21 & 79 & 0.0 \\
\hline $10 / 24 / 01$ & 18:55 & 18.4 & 18.5 & 18.5 & 18.3 & 0.0 & 9.7 & 21 & 79 & 0.0 \\
\hline $10 / 24 / 01$ & 18:56 & 18.4 & 18.5 & 18.5 & 18.3 & 0.0 & 9.6 & 21 & 79 & 0.0 \\
\hline $10 / 24 / 01$ & 18:57 & 18.4 & 18.5 & 18.5 & 18.3 & 0.0 & 9.6 & 21 & 79 & 0.0 \\
\hline $10 / 24 / 01$ & 18:58 & 18.5 & 18.5 & 18.5 & 18.3 & 0.0 & 9.6 & 21 & 79 & 0.0 \\
\hline $10 / 24 / 01$ & 18:59 & 18.4 & 18.5 & 18.5 & \begin{tabular}{|l|}
18.3 \\
\end{tabular} & 0.0 & 9.7 & 20 & 79 & 0.0 \\
\hline $10 / 24 / 01$ & 19:00 & 18.4 & 18.5 & 18.5 & 18.3 & 0.0 & 9.6 & 21 & 79 & 0.0 \\
\hline $10 / 24 / 01$ & 19:01 & 18.4 & 18.5 & 18.5 & 18.3 & 0.0 & 9.7 & 21 & 79 & 0.0 \\
\hline $10 / 24 / 01$ & 19:02 & 18.4 & 18.5 & 18.5 & 18.3 & 0.0 & 9.6 & 20 & 79 & 0.0 \\
\hline $10 / 24 / 01$ & 19:03 & 18.4 & 18.5 & 18.5 & 18.3 & 0.0 & 9.6 & 20 & 79 & 0.0 \\
\hline $10 / 24 / 01$ & 19:04 & 18.4 & 18.5 & 18.5 & 18.3 & 0.0 & 9.7 & 20 & 79 & 0.0 \\
\hline $10 / 24 / 01$ & 19:05 & 18.4 & 18.5 & 18.5 & 18.3 & 0.0 & 9.6 & 21 & 79 & 0.0 \\
\hline $10 / 24 / 01$ & 19:06 & 18.4 & 18.5 & 18.5 & \begin{tabular}{|l|}
18.2 \\
\end{tabular} & 0.0 & 9.6 & 21 & 79 & 0.0 \\
\hline $10 / 24 / 01$ & 19:07 & 18.4 & 18.5 & 18.5 & 18.3 & 0.0 & 9.6 & 20 & 79 & 0.0 \\
\hline $10 / 24 / 01$ & 19:08 & 18.4 & 18.5 & 18.5 & 18.3 & 0.0 & 9.6 & 21 & 79 & 0.0 \\
\hline $10 / 24 / 01$ & 19:09 & 18.5 & 18.5 & 18.5 & \begin{tabular}{|l|}
18.3 \\
\end{tabular} & 0.0 & 9.7 & 20 & 79 & 0.0 \\
\hline $10 / 24 / 01$ & $19: 10$ & 18.4 & 18.5 & 18.5 & 18.3 & 0.0 & 9.6 & 21 & 79 & 0.0 \\
\hline $10 / 24 / 01$ & 19:11 & 18.4 & 18.5 & 18.5 & \begin{tabular}{|l|}
18.3 \\
\end{tabular} & 0.0 & 9.7 & 21 & 79 & 0.0 \\
\hline $10 / 24 / 01$ & $19: 12$ & 18.4 & 18.5 & 18.5 & 18.3 & 0.0 & 9.7 & 20 & 79 & 0.0 \\
\hline $10 / 24 / 01$ & $19: 13$ & 18.4 & 18.5 & 18.5 & 18.3 & 0.0 & 9.6 & 21 & 79 & 0.0 \\
\hline $10 / 24 / 01$ & 19:14 & 18.4 & 18.5 & 18.5 & 18.3 & 0.0 & 9.6 & 20 & 79 & 0.0 \\
\hline $10 / 24 / 01$ & 19:15 & 18.4 & 18.5 & 18.5 & \begin{tabular}{|l|}
18.3 \\
\end{tabular} & 0.0 & 9.7 & 20 & 79 & 0.0 \\
\hline $10 / 24 / 01$ & $19: 16$ & 18.4 & 18.5 & 18.5 & \begin{tabular}{|l|}
18.3 \\
\end{tabular} & 0.0 & 9.7 & 20 & 79 & 0.0 \\
\hline $10 / 24 / 01$ & 19:17 & 18.4 & 18.5 & 18.5 & \begin{tabular}{|l|}
18.2 \\
\end{tabular} & 0.0 & 9.6 & 20 & 79 & 0.0 \\
\hline $10 / 24 / 01$ & 19:18 & 18.4 & 18.5 & 18.5 & 18.3 & 0.0 & 9.7 & 20 & 79 & 0.0 \\
\hline $10 / 24 / 01$ & $19: 19$ & 18.4 & 18.5 & 18.5 & \begin{tabular}{|l|}
18.2 \\
\end{tabular} & 0.0 & 9.7 & 20 & 79 & 0.0 \\
\hline $10 / 24 / 01$ & $19: 20$ & 18.4 & 18.5 & 18.5 & 18.3 & 0.0 & 9.7 & 20 & 79 & 0.0 \\
\hline $10 / 24 / 01$ & 19:21 & 18.4 & 18.5 & 18.5 & \begin{tabular}{|l|}
18.2 \\
\end{tabular} & 0.0 & 9.6 & 21 & 79 & 0.0 \\
\hline $10 / 24 / 01$ & 19:22 & 18.4 & 18.5 & 18.5 & \begin{tabular}{|l|}
18.2 \\
\end{tabular} & 0.0 & 9.7 & 20 & 79 & 0.0 \\
\hline $10 / 24 / 01$ & 19:23 & 18.4 & 18.5 & 18.5 & \begin{tabular}{|l|}
18.2 \\
\end{tabular} & 0.0 & 9.7 & 20 & 79 & 0.0 \\
\hline $10 / 24 / 01$ & 19:24 & 18.4 & 18.5 & 18.5 & \begin{tabular}{|l|}
18.2 \\
\end{tabular} & 0.0 & 9.6 & 20 & 79 & 0.0 \\
\hline $10 / 24 / 01$ & 19:25 & 18.4 & 18.5 & 18.5 & \begin{tabular}{|l|}
18.2 \\
\end{tabular} & 0.0 & 9.6 & 20 & 79 & 0.0 \\
\hline $10 / 24 / 01$ & 19:26 & 18.3 & 18.5 & 18.5 & 18.2 & 0.0 & 9.6 & 20 & 79 & 0.0 \\
\hline $10 / 24 / 01$ & 19:27 & 18.4 & 18.5 & 18.5 & \begin{tabular}{|l|l}
18.3 \\
\end{tabular} & 0.0 & 9.6 & 20 & 79 & 0.0 \\
\hline
\end{tabular}

\begin{tabular}{|c|c|c|c|c|c|c|c|c|c|c|}
\hline \multicolumn{11}{|c|}{ PREC2_102401_0627 } \\
\hline DATE & TIME & $\begin{array}{c}\mathrm{HX} \\
\text { Outlet } \\
\left({ }^{\circ} \mathrm{C}\right) \\
\text { TCO }\end{array}$ & $\begin{array}{c}\text { REC } \\
\text { PP } \\
\text { OUT } \\
\left({ }^{\circ} \mathrm{C}\right) \\
\text { TC1 }\end{array}$ & $\begin{array}{c}\text { TK } \\
\text { BOT } \\
\left({ }^{\circ} \mathrm{C}\right) \\
\text { TC2 }\end{array}$ & $\begin{array}{c}\mathrm{HX} \\
\text { OUT } \\
\left({ }^{\circ} \mathrm{C}\right) \\
\text { TC3 }\end{array}$ & $\begin{array}{c}\text { Mn } \\
\text { Flow } \\
\text { (gpm) }\end{array}$ & $\begin{array}{c}\text { Galigher } \\
\text { Flow } \\
(\mathrm{gpm})\end{array}$ & $\begin{array}{c}\text { Current } \\
\text { to } \mathrm{HX}\end{array}$ & $\begin{array}{c}\text { Voltage } \\
\text { to } \mathrm{HX}\end{array}$ & $\begin{array}{c}\mathrm{Sr} \\
\text { Flow } \\
(\mathrm{gpm})\end{array}$ \\
\hline $10 / 24 / 01$ & 19:28 & 18.4 & 18.5 & 18.5 & 18.2 & 0.0 & 9.7 & 20 & 79 & 0.0 \\
\hline $10 / 24 / 01$ & $19: 29$ & 18.4 & 18.5 & 18.5 & 18.3 & 0.0 & 9.6 & 21 & 79 & 0.0 \\
\hline $10 / 24 / 01$ & 19:30 & 18.3 & 18.5 & 18.5 & 18.2 & 0.0 & 9.6 & 20 & 79 & 0.0 \\
\hline $10 / 24 / 01$ & 19:31 & 18.4 & 18.5 & 18.5 & 18.2 & 0.0 & 9.7 & 21 & 79 & 0.0 \\
\hline $10 / 24 / 01$ & 19:32 & 18.4 & 18.5 & 18.5 & 18.2 & 0.0 & 9.6 & 20 & 79 & 0.0 \\
\hline $10 / 24 / 01$ & 19:33 & 18.3 & 18.5 & 18.5 & 18.2 & 0.0 & 9.6 & 20 & 79 & 0.0 \\
\hline $10 / 24 / 01$ & 19:34 & 18.4 & 18.5 & 18.5 & 18.2 & 0.0 & 9.6 & 20 & 79 & 0.0 \\
\hline $10 / 24 / 01$ & 19:35 & 18.3 & 18.4 & 18.5 & 18.2 & 0.0 & 9.6 & 20 & 79 & 0.0 \\
\hline $10 / 24 / 01$ & $19: 36$ & 18.4 & 18.4 & 18.5 & 18.3 & 0.0 & 9.6 & 21 & 79 & 0.0 \\
\hline $10 / 24 / 01$ & 19:37 & 18.3 & 18.5 & 18.5 & 18.2 & 0.0 & 9.6 & 20 & 79 & 0.0 \\
\hline $10 / 24 / 01$ & 19:38 & 18.4 & 18.4 & 18.5 & 18.2 & 0.0 & 9.6 & 21 & 79 & 0.0 \\
\hline $10 / 24 / 01$ & $19: 39$ & 18.3 & 18.4 & 18.5 & 18.2 & 0.0 & 9.6 & 20 & 79 & 0.0 \\
\hline $10 / 24 / 01$ & $19: 40$ & 18.4 & 18.4 & 18.5 & 18.2 & 0.0 & 9.7 & 21 & 79 & 0.0 \\
\hline $10 / 24 / 01$ & 19:41 & 18.3 & 18.4 & 18.4 & 18.2 & 0.0 & 9.6 & 20 & 79 & 0.0 \\
\hline $10 / 24 / 01$ & 19:42 & 18.3 & 18.4 & 18.5 & 18.2 & 0.0 & 9.6 & 20 & 79 & 0.0 \\
\hline $10 / 24 / 01$ & $19: 43$ & 18.4 & 18.4 & 18.4 & 18.2 & 0.0 & 9.7 & 20 & 79 & 0.0 \\
\hline $10 / 24 / 01$ & 19:44 & 18.3 & 18.4 & 18.4 & 18.2 & 0.0 & 9.6 & 21 & 79 & 0.0 \\
\hline $10 / 24 / 01$ & 19:45 & 18.4 & 18.4 & 18.4 & 18.2 & 0.0 & 9.7 & 21 & 79 & 0.0 \\
\hline $10 / 24 / 01$ & 19:46 & 18.3 & 18.4 & 18.4 & 18.2 & 0.0 & 9.6 & 20 & 79 & 0.0 \\
\hline $10 / 24 / 01$ & 19:47 & 18.4 & 18.4 & 18.4 & 18.2 & 0.0 & 9.6 & 21 & 79 & 0.0 \\
\hline $10 / 24 / 01$ & 19:48 & 18.3 & 18.4 & 18.4 & 18.2 & 0.0 & 9.6 & 20 & 79 & 0.0 \\
\hline $10 / 24 / 01$ & 19:49 & 18.3 & 18.4 & 18.4 & 18.2 & 0.0 & 9.7 & 20 & 79 & 0.0 \\
\hline $10 / 24 / 01$ & 19:50 & 18.3 & 18.4 & 18.4 & 18.2 & 0.0 & 9.7 & 20 & 79 & 0.0 \\
\hline $10 / 24 / 01$ & 19:51 & 18.3 & 18.4 & 18.4 & 18.2 & 0.0 & 9.7 & 20 & 79 & 0.0 \\
\hline $10 / 24 / 01$ & 19:52 & 18.3 & 18.4 & 18.4 & 18.2 & 0.0 & 9.6 & 21 & 79 & 0.0 \\
\hline $10 / 24 / 01$ & 19:53 & 18.3 & 18.4 & 18.4 & 18.2 & 0.0 & 9.6 & 20 & 79 & 0.0 \\
\hline $10 / 24 / 01$ & 19:54 & 18.3 & 18.4 & 18.4 & 18.2 & 0.0 & 9.7 & 20 & 79 & 0.0 \\
\hline $10 / 24 / 01$ & 19:55 & 18.3 & 18.4 & 18.4 & 18.2 & 0.0 & 9.7 & 21 & 79 & 0.0 \\
\hline $10 / 24 / 01$ & 19:56 & 18.3 & 18.4 & 18.4 & 18.2 & 0.0 & 9.7 & 20 & 79 & 0.0 \\
\hline $10 / 24 / 01$ & 19:57 & 18.3 & 18.4 & 18.4 & 18.2 & 0.0 & 9.6 & 21 & 79 & 0.0 \\
\hline $10 / 24 / 01$ & 19:58 & 18.3 & 18.4 & 18.4 & 18.2 & 0.0 & 9.6 & 20 & 79 & 0.0 \\
\hline $10 / 24 / 01$ & 19:59 & 18.3 & 18.4 & 18.4 & 18.2 & 0.0 & 9.6 & 20 & 79 & 0.0 \\
\hline $10 / 24 / 01$ & $20: 00$ & 18.3 & 18.4 & 18.4 & 18.2 & 0.0 & 9.6 & 20 & 79 & 0.0 \\
\hline $10 / 24 / 01$ & 20:01 & 18.3 & 18.4 & 18.4 & 18.2 & 0.0 & 9.6 & 20 & 79 & 0.0 \\
\hline $10 / 24 / 01$ & $20: 02$ & 18.3 & 18.4 & 18.4 & 18.2 & 0.0 & 9.7 & 21 & 79 & 0.0 \\
\hline $10 / 24 / 01$ & $20: 03$ & 18.4 & 18.4 & 18.4 & 18.2 & 0.0 & 9.7 & 20 & 79 & 0.0 \\
\hline $10 / 24 / 01$ & 20:04 & 18.3 & 18.4 & 18.4 & 18.2 & 0.0 & 9.7 & 20 & 79 & 0.0 \\
\hline $10 / 24 / 01$ & $20: 05$ & 18.4 & 18.4 & 18.4 & 18.2 & 0.0 & 9.7 & 20 & 79 & 0.0 \\
\hline $10 / 24 / 01$ & $20: 06$ & 18.3 & 18.4 & 18.4 & 18.2 & 0.0 & 9.6 & 21 & 79 & 0.0 \\
\hline $10 / 24 / 01$ & $20: 07$ & 18.3 & 18.4 & 18.4 & 18.2 & 0.0 & 9.6 & 20 & 79 & 0.0 \\
\hline $10 / 24 / 01$ & $20: 08$ & 18.3 & 18.4 & 18.4 & 18.2 & 0.0 & 9.6 & 21 & 79 & 0.0 \\
\hline $10 / 24 / 01$ & $20: 09$ & 18.3 & 18.4 & 18.4 & 18.2 & 0.0 & 9.6 & 20 & 79 & 0.0 \\
\hline $10 / 24 / 01$ & $20: 10$ & 18.3 & 18.4 & 18.4 & 18.2 & 0.0 & 9.6 & 20 & 79 & 0.0 \\
\hline $10 / 24 / 01$ & $20: 11$ & 18.3 & 18.4 & 18.4 & 18.2 & 0.0 & 9.7 & 20 & 79 & 0.0 \\
\hline $10 / 24 / 01$ & $20: 12$ & 18.3 & 18.4 & 18.4 & 18.2 & 0.0 & 9.6 & 20 & 79 & 0.0 \\
\hline $10 / 24 / 01$ & $20: 13$ & 18.3 & 18.4 & 18.4 & 18.1 & 0.0 & 9.7 & 21 & 79 & 0.0 \\
\hline $10 / 24 / 01$ & $20: 14$ & 18.3 & 18.4 & 18.4 & 18.2 & 0.0 & 9.6 & 21 & 79 & 0.0 \\
\hline $10 / 24 / 01$ & $20: 15$ & 18.3 & 18.4 & 18.4 & 18.1 & 0.0 & 9.6 & 20 & 79 & 0.0 \\
\hline $10 / 24 / 01$ & $20: 16$ & 18.3 & 18.4 & 18.4 & 18.2 & 0.0 & 9.6 & 20 & 79 & 0.0 \\
\hline $10 / 24 / 01$ & $20: 17$ & 18.3 & 18.4 & 18.4 & 18.1 & 0.0 & 9.6 & 21 & 79 & 0.0 \\
\hline $10 / 24 / 01$ & $20: 18$ & 18.3 & 18.4 & 18.4 & 18.2 & 0.0 & 9.6 & 20 & 79 & 0.0 \\
\hline $10 / 24 / 01$ & $20: 19$ & 18.3 & 18.4 & 18.4 & 18.1 & 0.0 & 9.7 & 20 & 79 & 0.0 \\
\hline $10 / 24 / 01$ & $20: 20$ & 18.3 & 18.4 & 18.4 & 18.1 & 0.0 & 9.6 & 21 & 79 & 0.0 \\
\hline $10 / 24 / 01$ & $20: 21$ & 18.3 & 18.4 & 18.4 & 18.1 & 0.0 & 9.6 & 20 & 79 & 0.0 \\
\hline $10 / 24 / 01$ & $20: 22$ & 18.3 & 18.4 & 18.4 & 18.1 & 0.0 & 9.6 & 20 & 79 & 0.0 \\
\hline $10 / 24 / 01$ & $20: 23$ & 18.3 & 18.4 & 18.4 & 18.2 & 0.0 & 9.7 & 21 & 79 & 0.0 \\
\hline $10 / 24 / 01$ & $20: 24$ & 18.2 & 18.4 & 18.4 & 18.1 & 0.0 & 9.6 & 20 & 79 & 0.0 \\
\hline $10 / 24 / 01$ & $20: 25$ & 18.3 & 18.4 & 18.4 & 18.2 & 0.0 & 9.7 & 20 & 79 & 0.0 \\
\hline $10 / 24 / 01$ & $20: 26$ & 18.2 & 18.4 & 18.4 & 18.1 & 0.0 & 9.6 & 21 & 79 & 0.0 \\
\hline $10 / 24 / 01$ & $20: 27$ & 18.3 & 18.3 & 18.4 & 18.1 & 0.0 & 9.7 & 20 & 79 & 0.0 \\
\hline
\end{tabular}


WSRC-TR-2002-00459, Rev. 0 SRT-RPP-2002-00221

\begin{tabular}{|c|c|c|c|c|c|c|c|c|c|c|}
\hline \multicolumn{11}{|c|}{ PREC2_102401_0627 } \\
\hline DATE & TIME & \begin{tabular}{|c}
$\mathrm{HX}$ \\
Outlet \\
$\left({ }^{\circ} \mathrm{C}\right)$ \\
TCO
\end{tabular} & $\begin{array}{c}\text { REC } \\
\text { PP } \\
\text { OUT } \\
\left({ }^{\circ} \mathrm{C}\right) \\
\text { TC1 }\end{array}$ & \begin{tabular}{|c|} 
TK \\
BOT \\
$\left({ }^{\circ} \mathrm{C}\right)$ \\
TC2 \\
\end{tabular} & \begin{tabular}{|c|} 
HX \\
OUT \\
$\left({ }^{\circ} \mathrm{C}\right)$ \\
TC3 \\
\end{tabular} & $\begin{array}{c}\text { Mn } \\
\text { Flow } \\
\text { (gpm) }\end{array}$ & $\begin{array}{c}\text { Galigher } \\
\text { Flow } \\
\text { (gpm) }\end{array}$ & $\begin{array}{c}\text { Current } \\
\text { to } \mathrm{HX}\end{array}$ & $\begin{array}{l}\text { Voltage } \\
\text { to } \mathrm{HX}\end{array}$ & $\begin{array}{c}\mathrm{Sr} \\
\text { Flow } \\
\text { (gpm) }\end{array}$ \\
\hline $10 / 24 / 01$ & $20: 28$ & 18.3 & 18.3 & 18.4 & 18.1 & 0.0 & 9.6 & 21 & 79 & 0.0 \\
\hline $10 / 24 / 01$ & $20: 29$ & 18.2 & 18.4 & \begin{tabular}{|l|} 
\\
\end{tabular} & 18.1 & 0.0 & 9.6 & 21 & 79 & 0.0 \\
\hline $10 / 24 / 01$ & $20: 30$ & 18.3 & 18.3 & 18.3 & 18.1 & 0.0 & 9.6 & 20 & 79 & 0.0 \\
\hline $10 / 24 / 01$ & $20: 31$ & 18.2 & 18.3 & \begin{tabular}{|l|}
18.4 \\
\end{tabular} & 18.1 & 0.0 & 9.7 & 21 & 79 & 0.0 \\
\hline $10 / 24 / 01$ & $20: 32$ & 18.3 & 18.3 & \begin{tabular}{|l|}
18.4 \\
\end{tabular} & 18.1 & 0.0 & 9.7 & 20 & 79 & 0.0 \\
\hline $10 / 24 / 01$ & $20: 33$ & 18.2 & 18.4 & 18.4 & 18.1 & 0.0 & 9.6 & 20 & 79 & 0.0 \\
\hline $10 / 24 / 01$ & $20: 34$ & 18.3 & 18.3 & \begin{tabular}{|l|} 
\\
\end{tabular} & 18.1 & 0.0 & 9.7 & 21 & 79 & 0.0 \\
\hline $10 / 24 / 01$ & $20: 35$ & 18.2 & 18.4 & \begin{tabular}{|l|}
18.4 \\
\end{tabular} & 18.1 & 0.0 & 9.7 & 21 & 79 & 0.0 \\
\hline $10 / 24 / 01$ & $20: 36$ & 18.3 & 18.4 & \begin{tabular}{|l|}
18.4 \\
\end{tabular} & 18.1 & 0.0 & 9.6 & 20 & 79 & 0.0 \\
\hline $10 / 24 / 01$ & $20: 37$ & 18.3 & 18.4 & 18.4 & 18.1 & 0.0 & 9.6 & 21 & 79 & 0.0 \\
\hline $10 / 24 / 01$ & 20:38 & 18.2 & 18.4 & 18.4 & 18.1 & 0.0 & 9.7 & 20 & 79 & 0.0 \\
\hline $10 / 24 / 01$ & $20: 39$ & 18.3 & 18.3 & 18.4 & 18.1 & 0.0 & 9.6 & 20 & 79 & 0.0 \\
\hline $10 / 24 / 01$ & $20: 40$ & 18.2 & 18.4 & $\mid 18.4$ & 18.1 & 0.0 & 9.6 & 20 & 79 & 0.0 \\
\hline $10 / 24 / 01$ & $20: 41$ & 18.3 & 18.4 & 18.4 & 18.1 & 0.0 & 9.6 & 20 & 79 & 0.0 \\
\hline $10 / 24 / 01$ & $20: 42$ & 18.2 & 18.4 & \begin{tabular}{|l|}
18.4 \\
\end{tabular} & 18.1 & 0.0 & 9.7 & 20 & 79 & 0.0 \\
\hline $10 / 24 / 01$ & $20: 43$ & 18.3 & 18.4 & 18.4 & 18.2 & 0.0 & 9.6 & 21 & 79 & 0.0 \\
\hline $10 / 24 / 01$ & $20: 44$ & 18.3 & 18.4 & 18.4 & 18.1 & 0.0 & 9.6 & 20 & 79 & 0.0 \\
\hline $10 / 24 / 01$ & $20: 45$ & 18.3 & 18.4 & 18.4 & 18.2 & 0.0 & 9.6 & 21 & 79 & 0.0 \\
\hline $10 / 24 / 01$ & $20: 46$ & 18.3 & 18.4 & \begin{tabular}{|l|}
18.4 \\
\end{tabular} & 18.1 & 0.0 & 9.7 & 20 & 79 & 0.0 \\
\hline $10 / 24 / 01$ & $20: 47$ & 18.3 & 18.4 & \begin{tabular}{|l|}
18.4 \\
\end{tabular} & 18.1 & 0.0 & 9.6 & 20 & 79 & 0.0 \\
\hline $10 / 24 / 01$ & $20: 48$ & 18.3 & 18.4 & \begin{tabular}{|l|}
18.4 \\
\end{tabular} & 18.1 & 0.0 & 9.7 & 21 & 79 & 0.0 \\
\hline $10 / 24 / 01$ & $20: 49$ & 18.2 & 18.4 & 18.4 & 18.1 & 0.0 & 9.6 & 21 & 79 & 0.0 \\
\hline $10 / 24 / 01$ & $20: 50$ & 18.3 & 18.4 & 18.4 & 18.2 & 0.0 & 9.7 & 20 & 79 & 0.0 \\
\hline $10 / 24 / 01$ & 20:51 & 18.2 & 18.3 & \begin{tabular}{|l|}
18.4 \\
\end{tabular} & 18.1 & 0.0 & 9.7 & 20 & 79 & 0.0 \\
\hline $10 / 24 / 01$ & 20:52 & 18.3 & 18.3 & \begin{tabular}{|l|}
18.3 \\
\end{tabular} & 18.1 & 0.0 & 9.7 & 20 & 79 & 0.0 \\
\hline $10 / 24 / 01$ & 20:53 & 18.2 & 18.3 & \begin{tabular}{|l|}
18.3 \\
\end{tabular} & 18.1 & 0.0 & 9.6 & 20 & 79 & 0.0 \\
\hline $10 / 24 / 01$ & $20: 54$ & 18.2 & 18.3 & 18.3 & 18.1 & 0.0 & 9.6 & 21 & 79 & 0.0 \\
\hline $10 / 24 / 01$ & 20:55 & 18.2 & 18.3 & 18.3 & 18.1 & 0.0 & 9.6 & 20 & 79 & 0.0 \\
\hline $10 / 24 / 01$ & $20: 56$ & 18.2 & 18.3 & \begin{tabular}{|l|}
18.3 \\
\end{tabular} & 18.1 & 0.0 & 9.6 & 21 & 79 & 0.0 \\
\hline $10 / 24 / 01$ & 20:57 & 18.2 & 18.3 & 18.3 & 18.1 & 0.0 & 9.7 & 21 & 79 & 0.0 \\
\hline $10 / 24 / 01$ & $20: 58$ & 18.2 & 18.3 & \begin{tabular}{|l|} 
\\
\end{tabular} & 18.1 & 0.0 & 9.6 & 20 & 79 & 0.0 \\
\hline $10 / 24 / 01$ & $20: 59$ & 18.3 & 18.3 & \begin{tabular}{|l|}
18.3 \\
\end{tabular} & 18.1 & 0.0 & 9.6 & 21 & 79 & 0.0 \\
\hline $10 / 24 / 01$ & $21: 00$ & 18.2 & 18.3 & \begin{tabular}{|l|} 
\\
\end{tabular} & 18.0 & 0.0 & 9.6 & 21 & 79 & 0.0 \\
\hline $10 / 24 / 01$ & 21:01 & 18.3 & 18.3 & 18.3 & 18.1 & 0.0 & 9.7 & 20 & 79 & 0.0 \\
\hline $10 / 24 / 01$ & 21:02 & 18.2 & 18.4 & 18.3 & 18.1 & 0.0 & 9.6 & 20 & 79 & 0.0 \\
\hline $10 / 24 / 01$ & $21: 03$ & 18.3 & 18.3 & \begin{tabular}{|l|}
18.3 \\
\end{tabular} & 18.1 & 0.0 & 9.6 & 21 & 79 & 0.0 \\
\hline $10 / 24 / 01$ & $21: 04$ & 18.2 & 18.3 & \begin{tabular}{|l|} 
\\
\end{tabular} & 18.1 & 0.0 & 9.6 & 20 & 79 & 0.0 \\
\hline $10 / 24 / 01$ & 21:05 & 18.2 & 18.3 & \begin{tabular}{|l|}
18.3 \\
\end{tabular} & 18.1 & 0.0 & 9.6 & 20 & 79 & 0.0 \\
\hline $10 / 24 / 01$ & $21: 06$ & 18.2 & 18.3 & \begin{tabular}{|l|} 
\\
\end{tabular} & 18.1 & 0.0 & 9.7 & 21 & 79 & 0.0 \\
\hline $10 / 24 / 01$ & $21: 07$ & 18.2 & 18.3 & 18.3 & 18.1 & 0.0 & 9.7 & 21 & 79 & 0.0 \\
\hline $10 / 24 / 01$ & $21: 08$ & 18.2 & 18.3 & 18.3 & 18.1 & 0.0 & 9.6 & 21 & 79 & 0.0 \\
\hline $10 / 24 / 01$ & $21: 09$ & 18.2 & 18.3 & \begin{tabular}{|l|}
18.3 \\
\end{tabular} & 18.0 & 0.0 & 9.6 & 21 & 79 & 0.0 \\
\hline $10 / 24 / 01$ & $21: 10$ & 18.2 & 18.3 & \begin{tabular}{|l|}
18.3 \\
\end{tabular} & 18.1 & 0.0 & 9.7 & 21 & 79 & 0.0 \\
\hline $10 / 24 / 01$ & $21: 11$ & 18.2 & 18.3 & \begin{tabular}{|l|}
18.3 \\
\end{tabular} & 18.0 & 0.0 & 9.7 & 21 & 79 & 0.0 \\
\hline $10 / 24 / 01$ & $21: 12$ & 18.2 & 18.3 & \begin{tabular}{|l|}
18.3 \\
\end{tabular} & 18.1 & 0.0 & 9.7 & 20 & 79 & 0.0 \\
\hline $10 / 24 / 01$ & $21: 13$ & 18.2 & 18.3 & \begin{tabular}{|l|} 
\\
\end{tabular} & 18.0 & 0.0 & 9.7 & 21 & 79 & 0.0 \\
\hline $10 / 24 / 01$ & $21: 14$ & 18.2 & 18.3 & \begin{tabular}{|l|} 
\\
\end{tabular} & 18.1 & 0.0 & 9.6 & 21 & 79 & 0.0 \\
\hline $10 / 24 / 01$ & $21: 15$ & 18.2 & 18.3 & 18.3 & 18.0 & 0.0 & 9.6 & 21 & 79 & 0.0 \\
\hline $10 / 24 / 01$ & $21: 16$ & 18.2 & 18.3 & \begin{tabular}{|l|} 
\\
\end{tabular} & 18.0 & 0.0 & 9.7 & 21 & 79 & 0.0 \\
\hline $10 / 24 / 01$ & $21: 17$ & 18.2 & 18.3 & \begin{tabular}{|l|} 
\\
\end{tabular} & 18.1 & 0.0 & 9.7 & 21 & 79 & 0.0 \\
\hline $10 / 24 / 01$ & $21: 18$ & 18.2 & 18.3 & \begin{tabular}{|l|} 
\\
\end{tabular} & 18.0 & 0.0 & 9.6 & 21 & 79 & 0.0 \\
\hline $10 / 24 / 01$ & $21: 19$ & 18.2 & 18.3 & 18.3 & 18.1 & 0.0 & 9.7 & 21 & 79 & 0.0 \\
\hline $10 / 24 / 01$ & $21: 20$ & 18.2 & 18.3 & \begin{tabular}{|l|}
18.3 \\
\end{tabular} & 18.0 & 0.0 & 9.6 & 21 & 79 & 0.0 \\
\hline $10 / 24 / 01$ & $21: 21$ & 18.2 & 18.3 & \begin{tabular}{|l|}
18.3 \\
\end{tabular} & 18.1 & 0.0 & 9.7 & 21 & 79 & 0.0 \\
\hline $10 / 24 / 01$ & $21: 22$ & 18.2 & 18.3 & 18.3 & 18.0 & 0.0 & 9.7 & 21 & 79 & 0.0 \\
\hline $10 / 24 / 01$ & $21: 23$ & 18.2 & 18.3 & \begin{tabular}{|l|} 
\\
\end{tabular} & 18.1 & 0.0 & 9.6 & 21 & 79 & 0.0 \\
\hline $10 / 24 / 01$ & $21: 24$ & 18.2 & 18.3 & \begin{tabular}{|l|} 
\\
\end{tabular} & 18.0 & 0.0 & 9.6 & 21 & 79 & 0.0 \\
\hline $10 / 24 / 01$ & $21: 25$ & 18.2 & 18.3 & 18.3 & 18.0 & 0.0 & 9.6 & 20 & 79 & 0.0 \\
\hline $10 / 24 / 01$ & $21: 26$ & 18.2 & 18.3 & \begin{tabular}{|l|} 
\\
\end{tabular} & 18.1 & 0.0 & 9.6 & 20 & 79 & 0.0 \\
\hline $10 / 24 / 01$ & $21: 27$ & 18.2 & 18.3 & 18.3 & 18.0 & 0.0 & 9.6 & 21 & 79 & 0.0 \\
\hline
\end{tabular}

\begin{tabular}{|c|c|c|c|c|c|c|c|c|c|c|}
\hline \multicolumn{11}{|c|}{ PREC2_102401_0627 } \\
\hline DATE & TIME & $\begin{array}{c}\mathrm{HX} \\
\text { Outlet } \\
\left({ }^{\circ} \mathrm{C}\right) \\
\mathrm{TCO}\end{array}$ & \begin{tabular}{|c|} 
REC \\
PP \\
OUT \\
$\left({ }^{\circ} \mathrm{C}\right)$ \\
TC1
\end{tabular} & \begin{tabular}{|c|} 
TK \\
BOT \\
$\left({ }^{\circ} \mathrm{C}\right)$ \\
TC2 \\
\end{tabular} & $\begin{array}{l}\text { HX } \\
\text { OUT } \\
\left(^{\circ} \mathrm{C}\right) \\
\text { TC3 }\end{array}$ & $\begin{array}{c}\mathrm{Mn} \\
\text { Flow } \\
(\mathrm{gpm})\end{array}$ & \begin{tabular}{|c|} 
Galigher \\
Flow \\
(gpm)
\end{tabular} & $\begin{array}{c}\text { Current } \\
\text { to } \mathrm{HX}\end{array}$ & $\begin{array}{c}\text { Voltage } \\
\text { to } \mathrm{HX}\end{array}$ & $\begin{array}{c}\mathrm{Sr} \\
\text { Flow } \\
\text { (gpm) }\end{array}$ \\
\hline $10 / 24 / 01$ & $21: 28$ & 18.2 & 18.3 & 18.3 & 18.1 & 0.0 & 9.6 & 21 & 79 & 0.0 \\
\hline $10 / 24 / 01$ & $21: 29$ & 18.2 & 18.3 & 18.3 & 18.0 & 0.0 & 9.6 & 20 & 79 & 0.0 \\
\hline $10 / 24 / 01$ & $21: 30$ & 18.2 & 18.3 & 18.3 & 18.1 & 0.0 & 9.6 & 21 & 79 & 0.0 \\
\hline $10 / 24 / 01$ & $21: 31$ & 18.2 & 18.3 & 18.3 & 18.0 & 0.0 & 9.6 & 20 & 79 & 0.0 \\
\hline $10 / 24 / 01$ & $21: 32$ & 18.2 & \begin{tabular}{|l|} 
\\
\end{tabular} & 18.3 & 18.1 & 0.0 & 9.6 & 20 & 79 & 0.0 \\
\hline $10 / 24 / 01$ & $21: 33$ & 18.2 & $\mid$\begin{tabular}{|l|}
$\mid 18.3$ \\
\end{tabular} & $\mid 18.3$ & 18.1 & 0.0 & 9.6 & 20 & 79 & 0.0 \\
\hline $10 / 24 / 01$ & $21: 34$ & 18.2 & 18.3 & 18.3 & 18.1 & 0.0 & 9.7 & 20 & 79 & 0.0 \\
\hline $10 / 24 / 01$ & $21: 35$ & 18.2 & 18.3 & 18.3 & 18.1 & 0.0 & 9.7 & 21 & 79 & 0.0 \\
\hline $10 / 24 / 01$ & $21: 36$ & 18.2 & 18.3 & 18.3 & 18.0 & 0.0 & 9.6 & 20 & 79 & 0.0 \\
\hline $10 / 24 / 01$ & $21: 37$ & 18.2 & 18.3 & 18.3 & 18.1 & 0.0 & 9.6 & 21 & 79 & 0.0 \\
\hline $10 / 24 / 01$ & $21: 38$ & 18.1 & 18.3 & 18.3 & 18.0 & 0.0 & 9.6 & 20 & 79 & 0.0 \\
\hline $10 / 24 / 01$ & $21: 39$ & 18.2 & 18.3 & 18.3 & 18.1 & 0.0 & 9.6 & 21 & 79 & 0.0 \\
\hline $10 / 24 / 01$ & $21: 40$ & 18.1 & 18.3 & 18.3 & 18.0 & 0.0 & 9.7 & 20 & 79 & 0.0 \\
\hline $10 / 24 / 01$ & $21: 41$ & 18.2 & \begin{tabular}{|l|} 
\\
\end{tabular} & $\mid$\begin{tabular}{|l|} 
\\
\end{tabular} & 18.0 & 0.0 & 9.7 & 20 & 79 & 0.0 \\
\hline $10 / 24 / 01$ & $21: 42$ & 18.2 & 18.3 & 18.3 & 18.0 & 0.0 & 9.6 & 20 & 79 & 0.0 \\
\hline $10 / 24 / 01$ & $21: 43$ & 18.2 & \begin{tabular}{|l|} 
\\
\end{tabular} & 18.3 & 18.0 & 0.0 & 9.6 & 20 & 79 & 0.0 \\
\hline $10 / 24 / 01$ & $21: 44$ & 18.2 & 18.2 & 18.3 & 18.0 & 0.0 & 9.6 & 20 & 79 & 0.0 \\
\hline $10 / 24 / 01$ & $21: 45$ & 18.1 & 18.2 & 18.3 & 18.0 & 0.0 & 9.6 & 21 & 79 & 0.0 \\
\hline $10 / 24 / 01$ & $21: 46$ & 18.2 & 18.2 & 18.3 & 18.0 & 0.0 & 9.6 & 21 & 79 & 0.0 \\
\hline $10 / 24 / 01$ & $21: 47$ & 18.1 & 18.2 & 18.3 & 18.0 & 0.0 & 9.6 & 21 & 79 & 0.0 \\
\hline $10 / 24 / 01$ & $21: 48$ & 18.2 & \begin{tabular}{|l|} 
\\
\end{tabular} & 18.3 & 18.0 & 0.0 & 9.6 & 20 & 79 & 0.0 \\
\hline $10 / 24 / 01$ & $21: 49$ & 18.1 & \begin{tabular}{|l|} 
\\
\end{tabular} & 18.3 & 18.0 & 0.0 & 9.6 & 21 & 79 & 0.0 \\
\hline $10 / 24 / 01$ & $21: 50$ & 18.2 & \begin{tabular}{|l|}
18.2 \\
\end{tabular} & 18.2 & 18.0 & 0.0 & 9.6 & 20 & 79 & 0.0 \\
\hline $10 / 24 / 01$ & $21: 51$ & 18.1 & 18.2 & 18.2 & 18.0 & 0.0 & 9.6 & 21 & 79 & 0.0 \\
\hline $10 / 24 / 01$ & $21: 52$ & 18.1 & 18.2 & 18.2 & 18.0 & 0.0 & 9.7 & 21 & 79 & 0.0 \\
\hline $10 / 24 / 01$ & $21: 53$ & 18.1 & 18.2 & 18.2 & 18.0 & 0.0 & 9.7 & 21 & 79 & 0.0 \\
\hline $10 / 24 / 01$ & $21: 54$ & 18.1 & \begin{tabular}{|l|}
18.2 \\
\end{tabular} & 18.2 & 18.0 & 0.0 & 9.7 & 21 & 79 & 0.0 \\
\hline $10 / 24 / 01$ & $21: 55$ & 18.2 & \begin{tabular}{|l|}
18.2 \\
\end{tabular} & 18.2 & 18.0 & 0.0 & 9.6 & 21 & 79 & 0.0 \\
\hline $10 / 24 / 01$ & $21: 56$ & 18.1 & \begin{tabular}{|l|} 
\\
\end{tabular} & 18.2 & 18.0 & 0.0 & 9.6 & 21 & 79 & 0.0 \\
\hline $10 / 24 / 01$ & $21: 57$ & 18.2 & 18.2 & 18.2 & 18.0 & 0.0 & 9.7 & 21 & 79 & 0.0 \\
\hline $10 / 24 / 01$ & $21: 58$ & 18.1 & 18.2 & 18.2 & 18.0 & 0.0 & 9.6 & 20 & 79 & 0.0 \\
\hline $10 / 24 / 01$ & $21: 59$ & 18.2 & 18.2 & 18.2 & 18.0 & 0.0 & 9.6 & 21 & 79 & 0.0 \\
\hline $10 / 24 / 01$ & 22:00 & 18.1 & \begin{tabular}{|l|}
18.2 \\
\end{tabular} & 18.2 & 18.0 & 0.0 & 9.6 & 21 & 79 & 0.0 \\
\hline $10 / 24 / 01$ & 22:01 & 18.1 & \begin{tabular}{|l|}
18.2 \\
\end{tabular} & 18.2 & 18.0 & 0.0 & 9.6 & 21 & 79 & 0.0 \\
\hline $10 / 24 / 01$ & 22:02 & 18.2 & \begin{tabular}{|l|} 
\\
\end{tabular} & 18.2 & 18.0 & 0.0 & 9.6 & 21 & 79 & 0.0 \\
\hline $10 / 24 / 01$ & 22:03 & 18.1 & \begin{tabular}{|l|}
18.2 \\
\end{tabular} & 18.2 & 18.0 & 0.0 & 9.6 & 20 & 79 & 0.0 \\
\hline $10 / 24 / 01$ & 22:04 & 18.2 & \begin{tabular}{|l|} 
\\
\end{tabular} & 18.2 & 18.0 & 0.0 & 9.6 & 21 & 79 & 0.0 \\
\hline $10 / 24 / 01$ & 22:05 & 18.1 & \begin{tabular}{|l|}
18.2 \\
\end{tabular} & 18.2 & 18.0 & 0.0 & 9.6 & 21 & 79 & 0.0 \\
\hline $10 / 24 / 01$ & 22:06 & 18.2 & 18.2 & 18.3 & 18.0 & 0.0 & 9.6 & 21 & 79 & 0.0 \\
\hline $10 / 24 / 01$ & 22:07 & 18.1 & 18.3 & 18.3 & 18.0 & 0.0 & 9.6 & 21 & 79 & 0.0 \\
\hline $10 / 24 / 01$ & 22:08 & 18.2 & 18.3 & 18.3 & 18.0 & 0.0 & 9.6 & 21 & 79 & 0.0 \\
\hline $10 / 24 / 01$ & 22:09 & 18.1 & 18.3 & 18.3 & 18.0 & 0.0 & 9.7 & 21 & 79 & 0.0 \\
\hline $10 / 24 / 01$ & 22:10 & 18.1 & 18.3 & 18.3 & 18.0 & 0.0 & 9.6 & 21 & 79 & 0.0 \\
\hline $10 / 24 / 01$ & 22:11 & 18.2 & \begin{tabular}{|l|} 
\\
\end{tabular} & 18.2 & 18.0 & 0.0 & 9.6 & 21 & 79 & 0.0 \\
\hline $10 / 24 / 01$ & 22:12 & 18.1 & \begin{tabular}{|l|} 
\\
\end{tabular} & 18.3 & 18.0 & 0.0 & 9.6 & 20 & 79 & 0.0 \\
\hline $10 / 24 / 01$ & $22: 13$ & 18.2 & 18.2 & 18.3 & 18.0 & 0.0 & 9.6 & 21 & 79 & 0.0 \\
\hline $10 / 24 / 01$ & 22:14 & 18.1 & \begin{tabular}{|l|}
18.2 \\
\end{tabular} & 18.2 & 18.0 & 0.0 & 9.7 & 21 & 79 & 0.0 \\
\hline $10 / 24 / 01$ & 22:15 & 18.2 & \begin{tabular}{|l|}
18.2 \\
\end{tabular} & 18.2 & 18.0 & 0.0 & 9.6 & 21 & 79 & 0.0 \\
\hline $10 / 24 / 01$ & 22:16 & 18.1 & 18.2 & 18.2 & 18.0 & 0.0 & 9.7 & 21 & 79 & 0.0 \\
\hline $10 / 24 / 01$ & 22:17 & 18.1 & \begin{tabular}{|l|}
18.2 \\
\end{tabular} & 18.2 & 18.0 & 0.0 & 9.7 & 21 & 79 & 0.0 \\
\hline $10 / 24 / 01$ & $22: 18$ & 18.1 & \begin{tabular}{|l|}
18.2 \\
\end{tabular} & \begin{tabular}{|l|}
18.2 \\
\end{tabular} & 18.0 & 0.0 & 9.6 & 21 & 79 & 0.0 \\
\hline $10 / 24 / 01$ & 22:19 & 18.1 & \begin{tabular}{|l|} 
\\
\end{tabular} & 18.2 & 18.0 & 0.0 & 9.6 & 20 & 79 & 0.0 \\
\hline $10 / 24 / 01$ & 22:20 & 18.2 & \begin{tabular}{|l|} 
\\
\end{tabular} & 18.2 & 18.0 & 0.0 & 9.7 & 21 & 79 & 0.0 \\
\hline $10 / 24 / 01$ & $22: 21$ & 18.1 & \begin{tabular}{|l|} 
\\
\end{tabular} & 18.2 & 18.0 & 0.0 & 9.6 & 20 & 79 & 0.0 \\
\hline $10 / 24 / 01$ & 22:22 & 18.2 & 18.2 & 18.2 & 18.0 & 0.0 & 9.6 & 21 & 79 & 0.0 \\
\hline $10 / 24 / 01$ & $22: 23$ & 18.1 & \begin{tabular}{|l|}
18.2 \\
\end{tabular} & $\mid$\begin{tabular}{|l|}
$\mid 18.2$ \\
\end{tabular} & 18.0 & 0.0 & 9.6 & 21 & 79 & 0.0 \\
\hline $10 / 24 / 01$ & 22:24 & 18.2 & 18.2 & 18.2 & 18.0 & 0.0 & 9.6 & 20 & 79 & 0.0 \\
\hline $10 / 24 / 01$ & 22:25 & 18.1 & \begin{tabular}{|l|}
18.2 \\
\end{tabular} & 18.2 & 17.9 & 0.0 & 9.7 & 20 & 79 & 0.0 \\
\hline $10 / 24 / 01$ & 22:26 & 18.1 & \begin{tabular}{|l|} 
\\
\end{tabular} & 18.2 & 18.0 & 0.0 & 9.7 & 20 & 79 & 0.0 \\
\hline $10 / 24 / 01$ & 22:27 & 18.1 & 18.2 & 18.2 & 17.9 & 0.0 & 9.7 & 21 & 79 & 0.0 \\
\hline
\end{tabular}


WSRC-TR-2002-00459, Rev. 0 SRT-RPP-2002-00221

\begin{tabular}{|c|c|c|c|c|c|c|c|c|c|c|}
\hline \multicolumn{3}{|c|}{ PREC2_102401_0627 } & \multirow[b]{2}{*}{$\begin{array}{l}\text { REC } \\
\text { PP } \\
\text { OUT } \\
\left({ }^{\circ} \mathrm{C}\right) \\
\text { TC1 }\end{array}$} & \multirow[b]{2}{*}{$\begin{array}{c}\text { TK } \\
\text { BOT } \\
\left({ }^{\circ} \mathrm{C}\right) \\
\text { TC2 }\end{array}$} & \multirow[b]{2}{*}{$\begin{array}{c}\mathrm{HX} \\
\text { OUT } \\
\left({ }^{\circ} \mathrm{C}\right) \\
\text { TC3 }\end{array}$} & \multirow[b]{2}{*}{$\begin{array}{c}\text { Mn } \\
\text { Flow } \\
\text { (gpm) }\end{array}$} & \multirow[b]{2}{*}{$\begin{array}{c}\text { Galigher } \\
\text { Flow } \\
(\mathrm{gpm})\end{array}$} & \multirow[b]{2}{*}{$\begin{array}{c}\text { Current } \\
\text { to } \mathrm{HX}\end{array}$} & \multirow[b]{2}{*}{$\begin{array}{c}\text { Voltage } \\
\text { to } \mathrm{HX}\end{array}$} & \multirow[b]{2}{*}{$\begin{array}{c}\mathrm{Sr} \\
\text { Flow } \\
(\mathrm{gpm})\end{array}$} \\
\hline DATE & TIME & $\begin{array}{c}\mathrm{HX} \\
\text { Outlet } \\
\left({ }^{\circ} \mathrm{C}\right) \\
\mathrm{TCO}\end{array}$ & & & & & & & & \\
\hline $10 / 24 / 01$ & $22: 28$ & 18.1 & 18.2 & 18.2 & 18.0 & 0.0 & 9.6 & 21 & 79 & 0.0 \\
\hline $10 / 24 / 01$ & $22: 29$ & 18.1 & 18.2 & 18.2 & 18.0 & 0.0 & 9.6 & 21 & 79 & 0.0 \\
\hline $10 / 24 / 01$ & 22:30 & 18.1 & 18.2 & 18.2 & 17.9 & 0.0 & 9.7 & 21 & 79 & 0.0 \\
\hline $10 / 24 / 01$ & 22:31 & 18.1 & 18.2 & 18.2 & 18.0 & 0.0 & 9.6 & 21 & 79 & 0.0 \\
\hline $10 / 24 / 01$ & 22:32 & 18.1 & 18.2 & 18.2 & 17.9 & 0.0 & 9.6 & 21 & 79 & 0.0 \\
\hline $10 / 24 / 01$ & $22: 33$ & 18.1 & 18.2 & 18.2 & 18.0 & 0.0 & 9.6 & 21 & 79 & 0.0 \\
\hline $10 / 24 / 01$ & 22:34 & 18.1 & 18.2 & 18.2 & 18.0 & 0.0 & 9.7 & 21 & 79 & 0.0 \\
\hline $10 / 24 / 01$ & 22:35 & 18.1 & 18.2 & 18.2 & 18.0 & 0.0 & 9.6 & 21 & 79 & 0.0 \\
\hline $10 / 24 / 01$ & 22:36 & 18.1 & 18.2 & 18.2 & 18.0 & 0.0 & 9.7 & 21 & 79 & 0.0 \\
\hline $10 / 24 / 01$ & 22:37 & 18.1 & 18.2 & 18.2 & 18.0 & 0.0 & 9.6 & 21 & 79 & 0.0 \\
\hline $10 / 24 / 01$ & 22:38 & 18.1 & 18.2 & 18.2 & 18.0 & 0.0 & 9.6 & 20 & 79 & 0.0 \\
\hline $10 / 24 / 01$ & 22:39 & 18.1 & 18.2 & 18.2 & 18.0 & 0.0 & 9.6 & 21 & 79 & 0.0 \\
\hline $10 / 24 / 01$ & 22:40 & 18.2 & 18.2 & 18.2 & 18.0 & 0.0 & 9.6 & 21 & 79 & 0.0 \\
\hline $10 / 24 / 01$ & 22:41 & 18.1 & 18.2 & 18.2 & 18.0 & 0.0 & 9.7 & 20 & 79 & 0.0 \\
\hline $10 / 24 / 01$ & 22:42 & 18.1 & 18.2 & 18.2 & 18.0 & 0.0 & 9.7 & 21 & 79 & 0.0 \\
\hline $10 / 24 / 01$ & $22: 43$ & 18.1 & 18.2 & 18.2 & 18.0 & 0.0 & 9.6 & 20 & 79 & 0.0 \\
\hline $10 / 24 / 01$ & 22:44 & 18.1 & 18.2 & 18.2 & 18.0 & 0.0 & 9.6 & 21 & 79 & 0.0 \\
\hline $10 / 24 / 01$ & 22:45 & 18.1 & 18.2 & 18.2 & 18.0 & 0.0 & 9.7 & 21 & 79 & 0.0 \\
\hline $10 / 24 / 01$ & 22:46 & 18.1 & 18.2 & 18.2 & 18.0 & 0.0 & 9.6 & 21 & 79 & 0.0 \\
\hline $10 / 24 / 01$ & 22:47 & 18.1 & 18.2 & 18.2 & 18.0 & 0.0 & 9.6 & 21 & 79 & 0.0 \\
\hline $10 / 24 / 01$ & 22:48 & 18.1 & 18.2 & 18.2 & 18.0 & 0.0 & 9.6 & 21 & 79 & 0.0 \\
\hline $10 / 24 / 01$ & $22: 49$ & 18.2 & 18.2 & 18.2 & 18.0 & 0.0 & 9.7 & 21 & 79 & 0.0 \\
\hline $10 / 24 / 01$ & 22:50 & 18.1 & 18.2 & 18.2 & 18.0 & 0.0 & 9.6 & 21 & 79 & 0.0 \\
\hline $10 / 24 / 01$ & 22:51 & 18.2 & 18.3 & 18.2 & 18.1 & 0.0 & 9.7 & 21 & 79 & 0.0 \\
\hline $10 / 24 / 01$ & 22:52 & 18.2 & 18.3 & 18.3 & 18.0 & 0.0 & 9.6 & 21 & 79 & 0.0 \\
\hline $10 / 24 / 01$ & 22:53 & 18.2 & 18.2 & 18.3 & 18.0 & 0.0 & 9.7 & 21 & 79 & 0.0 \\
\hline $10 / 24 / 01$ & 22:54 & 18.1 & 18.2 & 18.2 & 18.0 & 0.0 & 9.7 & 21 & 79 & 0.0 \\
\hline $10 / 24 / 01$ & 22:55 & 18.1 & 18.2 & 18.2 & 18.0 & 0.0 & 9.6 & 21 & 79 & 0.0 \\
\hline $10 / 24 / 01$ & 22:56 & 18.1 & 18.2 & 18.2 & 18.0 & 0.0 & 9.6 & 21 & 79 & 0.0 \\
\hline $10 / 24 / 01$ & 22:57 & 18.1 & 18.2 & 18.2 & 18.0 & 0.0 & 9.6 & 21 & 79 & 0.0 \\
\hline $10 / 24 / 01$ & 22:58 & 18.2 & 18.2 & 18.2 & 18.0 & 0.0 & 9.6 & 21 & 79 & 0.0 \\
\hline $10 / 24 / 01$ & 22:59 & 18.1 & 18.2 & 18.2 & 18.0 & 0.0 & 9.7 & 21 & 79 & 0.0 \\
\hline $10 / 24 / 01$ & $23: 00$ & 18.1 & 18.2 & 18.2 & 18.0 & 0.0 & 9.6 & 21 & 79 & 0.0 \\
\hline $10 / 24 / 01$ & 23:01 & 18.1 & 18.2 & 18.2 & 18.0 & 0.0 & 9.7 & 21 & 79 & 0.0 \\
\hline $10 / 24 / 01$ & 23:02 & 18.1 & 18.2 & 18.2 & 18.0 & 0.0 & 9.7 & 21 & 79 & 0.0 \\
\hline $10 / 24 / 01$ & 23:03 & 18.1 & 18.2 & 18.2 & 18.0 & 0.0 & 9.7 & 21 & 79 & 0.0 \\
\hline $10 / 24 / 01$ & 23:04 & 18.1 & 18.2 & 18.2 & 18.0 & 0.0 & 9.7 & 21 & 79 & 0.0 \\
\hline $10 / 24 / 01$ & 23:05 & 18.1 & 18.2 & 18.2 & 18.0 & 0.0 & 9.7 & 21 & 79 & 0.0 \\
\hline $10 / 24 / 01$ & 23:06 & 18.1 & 18.2 & 18.2 & 17.9 & 0.0 & 9.6 & 21 & 79 & 0.0 \\
\hline $10 / 24 / 01$ & 23:07 & 18.1 & 18.2 & 18.2 & 18.0 & 0.0 & 9.7 & 21 & 79 & 0.0 \\
\hline $10 / 24 / 01$ & 23:08 & 18.1 & 18.2 & 18.2 & 17.9 & 0.0 & 9.6 & 21 & 79 & 0.0 \\
\hline $10 / 24 / 01$ & 23:09 & 18.1 & 18.2 & 18.2 & 18.0 & 0.0 & 9.7 & 21 & 79 & 0.0 \\
\hline $10 / 24 / 01$ & 23:10 & 18.1 & 18.2 & 18.2 & 17.9 & 0.0 & 9.6 & 21 & 79 & 0.0 \\
\hline $10 / 24 / 01$ & 23:11 & 18.1 & 18.2 & 18.2 & 18.0 & 0.0 & 9.6 & 21 & 79 & 0.0 \\
\hline $10 / 24 / 01$ & 23:12 & 18.1 & 18.2 & 18.2 & 17.9 & 0.0 & 9.6 & 21 & 79 & 0.0 \\
\hline $10 / 24 / 01$ & 23:13 & 18.1 & 18.2 & 18.2 & 18.0 & 0.0 & 9.6 & 21 & 79 & 0.0 \\
\hline $10 / 24 / 01$ & 23:14 & 18.1 & 18.2 & 18.2 & 18.0 & 0.0 & 9.6 & 21 & 79 & 0.0 \\
\hline $10 / 24 / 01$ & 23:15 & 18.1 & 18.2 & 18.2 & 18.0 & 0.0 & 9.6 & 20 & 79 & 0.0 \\
\hline $10 / 24 / 01$ & 23:16 & 18.1 & 18.2 & 18.2 & 18.0 & 0.0 & 9.7 & 21 & 79 & 0.0 \\
\hline $10 / 24 / 01$ & 23:17 & 18.1 & 18.2 & 18.2 & 18.0 & 0.0 & 9.7 & 21 & 79 & 0.0 \\
\hline $10 / 24 / 01$ & 23:18 & 18.1 & 18.2 & 18.2 & 18.0 & 0.0 & 9.6 & 21 & 79 & 0.0 \\
\hline $10 / 24 / 01$ & 23:19 & 18.1 & 18.2 & 18.2 & 18.0 & 0.0 & 9.7 & 21 & 79 & 0.0 \\
\hline $10 / 24 / 01$ & $23: 20$ & 18.1 & 18.2 & 18.2 & 18.0 & 0.0 & 9.6 & 20 & 79 & 0.0 \\
\hline $10 / 24 / 01$ & $23: 21$ & 18.1 & 18.2 & 18.2 & 18.0 & 0.0 & 9.6 & 21 & 79 & 0.0 \\
\hline $10 / 24 / 01$ & $23: 22$ & 18.1 & 18.2 & 18.2 & 18.0 & 0.0 & 9.7 & 21 & 79 & 0.0 \\
\hline $10 / 24 / 01$ & $23: 23$ & 18.1 & 18.2 & 18.2 & 18.0 & 0.0 & 9.7 & 20 & 79 & 0.0 \\
\hline $10 / 24 / 01$ & $23: 24$ & 18.1 & 18.2 & 18.2 & 18.0 & 0.0 & 9.7 & 20 & 79 & 0.0 \\
\hline $10 / 24 / 01$ & $23: 25$ & 18.2 & 18.2 & 18.2 & 18.0 & 0.0 & 9.6 & 21 & 79 & 0.0 \\
\hline $10 / 24 / 01$ & $23: 26$ & 18.1 & 18.2 & 18.2 & 18.0 & 0.0 & 9.7 & 20 & 79 & 0.0 \\
\hline $10 / 24 / 01$ & $23: 27$ & 18.2 & 18.2 & 18.2 & 18.0 & 0.0 & 9.7 & 21 & 79 & 0.0 \\
\hline
\end{tabular}

\begin{tabular}{|c|c|c|c|c|c|c|c|c|c|c|}
\hline \multicolumn{3}{|c|}{ PREC2_102401_0627 } & & & & & & & & \\
\hline DATE & TIME & $\begin{array}{c}\mathrm{HX} \\
\text { Outlet } \\
\left({ }^{\circ} \mathrm{C}\right) \\
\text { TCO }\end{array}$ & $\begin{array}{l}\text { REC } \\
\text { PP } \\
\text { OUT } \\
\left({ }^{\circ} \mathrm{C}\right) \\
\text { TC1 }\end{array}$ & $\begin{array}{c}\text { TK } \\
\text { BOT } \\
\left({ }^{\circ} \mathrm{C}\right) \\
\text { TC2 }\end{array}$ & $\begin{array}{l}\text { HX } \\
\text { OUT } \\
\left({ }^{\circ} \mathrm{C}\right) \\
\text { TC3 }\end{array}$ & $\begin{array}{c}\text { Mn } \\
\text { Flow } \\
\text { (gpm) }\end{array}$ & $\begin{array}{c}\text { Galigher } \\
\text { Flow } \\
\text { (gpm) }\end{array}$ & $\begin{array}{l}\text { Current } \\
\text { to } \mathrm{HX}\end{array}$ & $\begin{array}{c}\text { Voltage } \\
\text { to } \mathrm{HX}\end{array}$ & $\begin{array}{c}\mathrm{Sr} \\
\text { Flow } \\
\text { (gpm) }\end{array}$ \\
\hline $10 / 24 / 01$ & $23: 28$ & 18.1 & 18.2 & 18.2 & 18.0 & 0.0 & 9.7 & 21 & 79 & 0.0 \\
\hline $10 / 24 / 01$ & $23: 29$ & 18.2 & 18.2 & 18.2 & 18.0 & 0.0 & 9.6 & 21 & 79 & 0.0 \\
\hline $10 / 24 / 01$ & $23: 30$ & 18.1 & 18.2 & 18.2 & 18.0 & 0.0 & 9.6 & 21 & 79 & 0.0 \\
\hline $10 / 24 / 01$ & $23: 31$ & 18.1 & 18.2 & 18.2 & 18.0 & 0.0 & 9.7 & 20 & 79 & 0.0 \\
\hline $10 / 24 / 01$ & $23: 32$ & 18.1 & 18.2 & 18.2 & $\mid 18.0$ & 0.0 & 9.6 & 20 & 79 & 0.0 \\
\hline $10 / 24 / 01$ & $23: 33$ & 18.1 & 18.2 & 18.2 & 18.0 & 0.0 & 9.7 & 21 & 79 & 0.0 \\
\hline $10 / 24 / 01$ & $23: 34$ & 18.2 & 18.2 & 18.2 & 18.0 & 0.0 & 9.6 & 20 & 79 & 0.0 \\
\hline $10 / 24 / 01$ & $23: 35$ & 18.1 & 18.2 & 18.2 & 18.0 & 0.0 & 9.6 & 21 & 79 & 0.0 \\
\hline $10 / 24 / 01$ & $23: 36$ & 18.2 & 18.2 & 18.2 & 18.0 & 0.0 & 9.7 & 20 & 79 & 0.0 \\
\hline $10 / 24 / 01$ & $23: 37$ & 18.1 & 18.2 & 18.2 & 18.0 & 0.0 & 9.7 & 21 & 79 & 0.0 \\
\hline $10 / 24 / 01$ & $23: 38$ & 18.1 & 18.2 & 18.2 & 18.0 & 0.0 & 9.6 & 21 & 79 & 0.0 \\
\hline $10 / 24 / 01$ & $23: 39$ & 18.1 & 18.2 & 18.2 & 18.0 & 0.0 & 9.7 & 21 & 79 & 0.0 \\
\hline $10 / 24 / 01$ & $23: 40$ & 18.1 & 18.2 & 18.2 & 18.0 & 0.0 & 9.6 & 21 & 79 & 0.0 \\
\hline $10 / 24 / 01$ & $23: 41$ & 18.1 & 18.2 & 18.2 & 18.0 & 0.0 & 9.7 & 20 & 79 & 0.0 \\
\hline $10 / 24 / 01$ & $23: 42$ & 18.1 & 18.2 & 18.2 & 17.9 & 0.0 & 9.6 & 21 & 79 & 0.0 \\
\hline $10 / 24 / 01$ & $23: 43$ & 18.1 & 18.2 & 18.2 & 18.0 & 0.0 & 9.6 & 21 & 79 & 0.0 \\
\hline $10 / 24 / 01$ & 23:44 & 18.1 & 18.2 & 18.2 & 17.9 & 0.0 & 9.6 & 21 & 79 & 0.0 \\
\hline $10 / 24 / 01$ & $23: 45$ & 18.1 & 18.2 & 18.2 & 18.0 & 0.0 & 9.6 & 21 & 79 & 0.0 \\
\hline $10 / 24 / 01$ & $23: 46$ & 18.1 & 18.2 & 18.2 & \begin{tabular}{|l|}
17.9 \\
\end{tabular} & 0.0 & 9.6 & 21 & 79 & 0.0 \\
\hline $10 / 24 / 01$ & $23: 47$ & 18.1 & 18.2 & 18.2 & 18.0 & 0.0 & 9.6 & 21 & 79 & 0.0 \\
\hline $10 / 24 / 01$ & $23: 48$ & 18.1 & 18.2 & 18.2 & \begin{tabular}{|l|}
17.9 \\
\end{tabular} & 0.0 & 9.7 & 21 & 79 & 0.0 \\
\hline $10 / 24 / 01$ & $23: 49$ & 18.1 & 18.2 & 18.2 & \begin{tabular}{|l|}
17.9 \\
\end{tabular} & 0.0 & 9.7 & 21 & 79 & 0.0 \\
\hline $10 / 24 / 01$ & 23:50 & 18.1 & 18.2 & 18.2 & 18.0 & 0.0 & 9.6 & 20 & 79 & 0.0 \\
\hline $10 / 24 / 01$ & 23:51 & 18.1 & 18.2 & 18.2 & 17.9 & 0.0 & 9.6 & 21 & 79 & 0.0 \\
\hline $10 / 24 / 01$ & 23:52 & 18.1 & 18.2 & \begin{tabular}{|l|}
18.2 \\
\end{tabular} & \begin{tabular}{|l|}
18.0 \\
\end{tabular} & 0.0 & 9.7 & 21 & 79 & 0.0 \\
\hline $10 / 24 / 01$ & 23:53 & 18.1 & 18.2 & 18.2 & 17.9 & 0.0 & 9.6 & 21 & 79 & 0.0 \\
\hline $10 / 24 / 01$ & 23:54 & 18.1 & \begin{tabular}{|l|}
18.2 \\
\end{tabular} & 18.2 & 18.0 & 0.0 & 9.6 & 21 & 79 & 0.0 \\
\hline $10 / 24 / 01$ & 23:55 & 18.1 & 18.2 & 18.2 & 18.0 & 0.0 & 9.7 & 21 & 79 & 0.0 \\
\hline $10 / 24 / 01$ & 23:56 & 18.1 & 18.2 & 18.2 & 18.0 & 0.0 & 9.7 & 21 & 79 & 0.0 \\
\hline $10 / 24 / 01$ & 23:57 & 18.1 & 18.2 & 18.2 & 18.0 & 0.0 & 9.7 & 21 & 79 & 0.0 \\
\hline $10 / 24 / 01$ & 23:58 & 18.1 & 18.2 & 18.2 & 18.0 & 0.0 & 9.7 & 21 & 79 & 0.0 \\
\hline $10 / 24 / 01$ & 23:59 & 18.1 & \begin{tabular}{|l|}
18.2 \\
\end{tabular} & 18.2 & 18.0 & 0.0 & 9.6 & 20 & 79 & 0.0 \\
\hline $10 / 25 / 01$ & $0: 00$ & 18.1 & 18.2 & 18.2 & 18.0 & 0.0 & 9.6 & 21 & 79 & 0.0 \\
\hline $10 / 25 / 01$ & $0: 01$ & 18.1 & 18.2 & 18.2 & 18.0 & 0.0 & 9.7 & 20 & 79 & 0.0 \\
\hline $10 / 25 / 01$ & 0:02 & 18.1 & 18.2 & 18.2 & 18.0 & 0.0 & 9.6 & 21 & 79 & 0.0 \\
\hline $10 / 25 / 01$ & 0:03 & 18.1 & 18.2 & 18.2 & 18.0 & 0.0 & 9.7 & 20 & 79 & 0.0 \\
\hline $10 / 25 / 01$ & 0:04 & 18.1 & 18.2 & 18.2 & \begin{tabular}{|l|}
18.0 \\
\end{tabular} & 0.0 & 9.7 & 21 & 79 & 0.0 \\
\hline $10 / 25 / 01$ & 0:05 & 18.1 & 18.2 & \begin{tabular}{|l|}
18.2 \\
\end{tabular} & \begin{tabular}{|l|}
18.0 \\
\end{tabular} & 0.0 & 9.7 & 20 & 79 & 0.0 \\
\hline $10 / 25 / 01$ & 0:06 & 18.1 & 18.2 & 18.2 & 18.0 & 0.0 & 9 & 20 & 79 & 0.0 \\
\hline $10 / 25 / 01$ & 0:07 & 18.1 & 18.2 & 18.2 & 18.0 & 0.0 & 9.7 & 20 & 79 & 0.0 \\
\hline $10 / 25 / 01$ & 0:08 & 18.1 & 18.2 & 18.2 & 18.0 & 0.0 & 9.6 & 20 & 79 & 0.0 \\
\hline $10 / 25 / 01$ & 0:09 & 18.1 & 18.2 & 18.2 & 18.0 & 0.0 & 9.6 & 20 & 79 & 0.0 \\
\hline $10 / 25 / 01$ & $0: 10$ & 18.1 & 18.2 & 18.2 & 18.0 & 0.0 & 9.6 & 20 & 79 & 0.0 \\
\hline $10 / 25 / 01$ & $0: 11$ & 18.1 & 18.2 & 18.2 & 18.0 & 0.0 & 0.0 & 20 & 79 & 0.0 \\
\hline $10 / 25 / 01$ & $0: 12$ & 18.1 & 18.2 & 18.2 & 18.0 & 0.0 & 9.6 & 21 & 79 & 0.0 \\
\hline $10 / 25 / 01$ & $0: 13$ & 18.1 & 18.2 & 18.2 & 18.0 & 0.0 & 9.6 & 20 & 79 & 0.0 \\
\hline 10/25/01 & $0: 14$ & 18.1 & 18.2 & 18.2 & 18.0 & 0.0 & 9.7 & 20 & 79 & 0.0 \\
\hline $10 / 25 / 01$ & $0: 15$ & 18.1 & 18.2 & 18.2 & 18.0 & 0.0 & 9.6 & 21 & 79 & 0.0 \\
\hline $10 / 25 / 01$ & $0: 16$ & 18.1 & 18.2 & 18.2 & 18.0 & 0.0 & 9.6 & 21 & 79 & 0.0 \\
\hline $10 / 25 / 01$ & $0: 17$ & 18.1 & 18.2 & 18.2 & 18.0 & 0.0 & 9.6 & 21 & 79 & 0.0 \\
\hline 10/25/01 & $0: 18$ & 18.1 & 18.2 & 18.2 & 18.0 & 0.0 & 9.6 & 20 & 79 & 0.0 \\
\hline $10 / 25 / 01$ & $0: 19$ & 18.1 & 18.2 & 18.2 & 18.0 & 0.0 & 9.6 & 20 & 79 & 0.0 \\
\hline $10 / 25 / 01$ & $0: 20$ & 18.1 & 18.2 & 18.2 & 18.0 & 0.0 & 9.6 & 21 & 79 & 0.0 \\
\hline $10 / 25 / 01$ & $0: 21$ & 18.1 & 18.2 & 18.2 & 18.0 & 0.0 & 9.7 & 21 & 79 & 0.0 \\
\hline $10 / 25 / 01$ & $0: 22$ & 18.1 & 18.2 & 18.2 & 18.0 & 0.0 & 9.6 & 20 & 79 & 0.0 \\
\hline $10 / 25 / 01$ & $0: 23$ & 18.1 & 18.2 & 18.2 & 18.0 & 0.0 & 9.6 & 20 & 79 & 0.0 \\
\hline $10 / 25 / 01$ & 0:24 & 18.1 & 18.2 & 18.2 & 18.0 & 0.0 & 9.6 & 21 & 79 & 0.0 \\
\hline 10/25/01 & $0: 25$ & 18.1 & 18.2 & 18.3 & 18.0 & 0.0 & 9.6 & 21 & 79 & 0.0 \\
\hline $10 / 25 / 01$ & $0: 26$ & 18.1 & \begin{tabular}{|l|}
18.2 \\
\end{tabular} & 18.2 & 18.0 & 0.0 & 9.6 & 21 & 79 & 0.0 \\
\hline$/ / 25 / 01$ & $0: 27$ & 18.1 & 18.2 & 18.3 & 18.0 & 0.0 & 9.6 & 21 & 79 & 0. \\
\hline
\end{tabular}


WSRC-TR-2002-00459, Rev. 0 SRT-RPP-2002-00221

\begin{tabular}{|c|c|c|c|c|c|c|c|c|c|c|}
\hline \multicolumn{3}{|c|}{ PREC2_102401_0627 } & \multirow[b]{2}{*}{$\begin{array}{c}\text { REC } \\
\text { PP } \\
\text { OUT } \\
\left({ }^{\circ} \mathrm{C}\right) \\
\text { TC1 }\end{array}$} & \multirow[b]{2}{*}{$\begin{array}{c}\text { TK } \\
\text { BOT } \\
\left({ }^{\circ} \mathrm{C}\right) \\
\text { TC2 }\end{array}$} & \multirow[b]{2}{*}{\begin{tabular}{|c|} 
HX \\
OUT \\
$\left({ }^{\circ} \mathrm{C}\right)$ \\
TC3
\end{tabular}} & \multirow[b]{2}{*}{$\begin{array}{c}\text { Mn } \\
\text { Flow } \\
(\mathrm{gpm})\end{array}$} & \multirow[b]{2}{*}{$\begin{array}{c}\text { Galigher } \\
\text { Flow } \\
(\mathrm{gpm})\end{array}$} & \multirow[b]{2}{*}{$\begin{array}{c}\text { Current } \\
\text { to } \mathrm{HX}\end{array}$} & \multirow[b]{2}{*}{$\begin{array}{c}\text { Voltage } \\
\text { to } \mathrm{HX}\end{array}$} & \multirow[b]{2}{*}{$\begin{array}{c}\text { Sr } \\
\text { Flow } \\
\text { (gpm) }\end{array}$} \\
\hline DATE & TIME & $\begin{array}{c}\mathrm{HX} \\
\text { Outlet } \\
\left({ }^{\circ} \mathrm{C}\right) \\
\mathrm{TCO}\end{array}$ & & & & & & & & \\
\hline $10 / 25 / 01$ & $0: 28$ & 18.2 & 18.2 & 18.2 & 18.0 & 0.0 & 9.6 & 21 & 79 & 0.0 \\
\hline $10 / 25 / 01$ & $0: 29$ & 18.1 & 18.2 & 18.2 & 18.0 & 0.0 & 9.7 & 20 & 79 & 0.0 \\
\hline $10 / 25 / 01$ & $0: 30$ & 18.1 & 18.2 & 18.2 & 18.0 & 0.0 & 9.7 & 20 & 79 & 0.0 \\
\hline $10 / 25 / 01$ & $0: 31$ & 18.1 & 18.2 & 18.3 & 18.0 & 0.0 & 9.6 & 20 & 79 & 0.0 \\
\hline $10 / 25 / 01$ & $0: 32$ & 18.1 & 18.2 & 18.3 & 18.0 & 0.0 & 9.6 & 21 & 79 & 0.0 \\
\hline $10 / 25 / 01$ & $0: 33$ & 18.1 & 18.2 & 18.3 & 18.0 & 0.0 & 9.6 & 21 & 79 & 0.0 \\
\hline $10 / 25 / 01$ & 0:34 & 18.1 & 18.2 & 18.3 & 18.0 & 0.0 & 9.7 & 20 & 79 & 0.0 \\
\hline $10 / 25 / 01$ & $0: 35$ & 18.2 & 18.2 & 18.3 & 18.0 & 0.0 & 9.7 & 20 & 79 & 0.0 \\
\hline $10 / 25 / 01$ & $0: 36$ & 18.1 & 18.3 & 18.3 & 18.0 & 0.0 & 9.7 & 20 & 79 & 0.0 \\
\hline $10 / 25 / 01$ & $0: 37$ & 18.2 & 18.3 & 18.3 & 18.0 & 0.0 & 9.7 & 21 & 79 & 0.0 \\
\hline $10 / 25 / 01$ & 0:38 & 18.1 & 18.3 & 18.3 & 18.0 & 0.0 & 9.6 & 20 & 79 & 0.0 \\
\hline $10 / 25 / 01$ & $0: 39$ & 18.2 & 18.3 & 18.3 & 18.0 & 0.0 & 9.7 & 20 & 79 & 0.0 \\
\hline $10 / 25 / 01$ & $0: 40$ & 18.1 & 18.3 & 18.3 & 18.0 & 0.0 & 9.6 & 21 & 79 & 0.0 \\
\hline $10 / 25 / 01$ & $0: 41$ & 18.1 & 18.3 & 18.3 & 18.0 & 0.0 & 9.7 & 20 & 79 & 0.0 \\
\hline $10 / 25 / 01$ & $0: 42$ & 18.2 & 18.3 & 18.3 & 18.0 & 0.0 & 9.7 & 21 & 79 & 0.0 \\
\hline $10 / 25 / 01$ & $0: 43$ & 18.1 & 18.3 & 18.3 & 18.0 & 0.0 & 9.6 & 20 & 79 & 0.0 \\
\hline $10 / 25 / 01$ & 0:44 & 18.2 & 18.3 & 18.3 & 18.1 & 0.0 & 9.7 & 21 & 79 & 0.0 \\
\hline $10 / 25 / 01$ & $0: 45$ & 18.1 & 18.3 & 18.3 & 18.0 & 0.0 & 9.6 & 20 & 79 & 0.0 \\
\hline $10 / 25 / 01$ & $0: 46$ & 18.2 & 18.3 & 18.3 & 18.1 & 0.0 & 9.7 & 21 & 79 & 0.0 \\
\hline $10 / 25 / 01$ & $0: 47$ & 18.1 & 18.3 & 18.3 & 18.0 & 0.0 & 9.6 & 20 & 79 & 0.0 \\
\hline $10 / 25 / 01$ & $0: 48$ & 18.2 & 18.3 & 18.3 & 18.1 & 0.0 & 9.7 & 20 & 79 & 0.0 \\
\hline $10 / 25 / 01$ & $0: 49$ & 18.2 & 18.3 & 18.3 & 18.0 & 0.0 & 9.6 & 21 & 79 & 0.0 \\
\hline $10 / 25 / 01$ & $0: 50$ & 18.2 & 18.3 & 18.3 & 18.1 & 0.0 & 9.6 & 21 & 79 & 0.0 \\
\hline $10 / 25 / 01$ & $0: 51$ & 18.2 & 18.3 & 18.3 & 18.1 & 0.0 & 9.6 & 21 & 79 & 0.0 \\
\hline $10 / 25 / 01$ & 0:52 & 18.1 & 18.3 & 18.3 & 18.0 & 0.0 & 9.7 & 21 & 79 & 0.0 \\
\hline $10 / 25 / 01$ & 0:53 & 18.2 & 18.3 & 18.3 & 18.1 & 0.0 & 9.6 & 21 & 79 & 0.0 \\
\hline $10 / 25 / 01$ & $0: 54$ & 18.2 & 18.3 & 18.3 & 18.0 & 0.0 & 9.6 & 20 & 79 & 0.0 \\
\hline $10 / 25 / 01$ & $0: 55$ & 18.2 & 18.3 & 18.3 & 18.1 & 0.0 & 9.6 & 20 & 79 & 0.0 \\
\hline $10 / 25 / 01$ & $0: 56$ & 18.2 & 18.3 & 18.3 & 18.0 & 0.0 & 9.6 & 21 & 79 & 0.0 \\
\hline $10 / 25 / 01$ & $0: 57$ & 18.2 & 18.3 & 18.3 & 18.1 & 0.0 & 9.7 & 21 & 79 & 0.0 \\
\hline $10 / 25 / 01$ & 0:58 & 18.2 & 18.3 & 18.3 & 18.1 & 0.0 & 9.7 & 21 & 79 & 0.0 \\
\hline $10 / 25 / 01$ & $0: 59$ & 18.2 & 18.3 & 18.3 & 18.1 & 0.0 & 9.7 & 20 & 79 & 0.0 \\
\hline $10 / 25 / 01$ & 1:00 & 18.2 & 18.3 & 18.3 & 18.1 & 0.0 & 9.6 & 20 & 79 & 0.0 \\
\hline $10 / 25 / 01$ & 1:01 & 18.2 & 18.3 & 18.3 & 18.1 & 0.0 & 9.6 & 20 & 79 & 0.0 \\
\hline $10 / 25 / 01$ & 1:02 & 18.2 & 18.3 & 18.3 & 18.1 & 0.0 & 9.7 & 20 & 79 & 0.0 \\
\hline $10 / 25 / 01$ & 1:03 & 18.2 & 18.3 & 18.3 & 18.0 & 0.0 & 9.7 & 20 & 79 & 0.0 \\
\hline $10 / 25 / 01$ & $1: 04$ & 18.2 & 18.3 & 18.3 & 18.1 & 0.0 & 9.7 & 20 & 79 & 0.0 \\
\hline $10 / 25 / 01$ & 1:05 & 18.2 & 18.3 & 18.3 & 18.1 & 0.0 & 9.6 & 21 & 79 & 0.0 \\
\hline $10 / 25 / 01$ & 1:06 & 18.3 & 18.4 & 18.3 & 18.1 & 0.0 & 9.6 & 20 & 79 & 0.0 \\
\hline 10/25/01 & 1:07 & 18.3 & 18.4 & 18.4 & 18.1 & 0.0 & 9.6 & 20 & 79 & 0.0 \\
\hline $10 / 25 / 01$ & 1:08 & 18.3 & 18.4 & 18.4 & 18.1 & 0.0 & 9.6 & 20 & 79 & 0.0 \\
\hline $10 / 25 / 01$ & 1:09 & 18.2 & 18.3 & 18.3 & 18.0 & 0.0 & 9.6 & 20 & 79 & 0.0 \\
\hline $10 / 25 / 01$ & $1: 10$ & 18.2 & 18.3 & 18.3 & 18.0 & 0.0 & 9.7 & 21 & 79 & 0.0 \\
\hline $10 / 25 / 01$ & 1:11 & 18.1 & 18.3 & 18.3 & 17.9 & 0.0 & 9.6 & 21 & 79 & 0.0 \\
\hline $10 / 25 / 01$ & 1:12 & 18.1 & 18.2 & 18.3 & 18.0 & 0.0 & 9.7 & 20 & 79 & 0.0 \\
\hline $10 / 25 / 01$ & 1:13 & 18.0 & 18.2 & 18.2 & 17.9 & 0.0 & 9.6 & 21 & 79 & 0.0 \\
\hline $10 / 25 / 01$ & 1:14 & 18.0 & 18.2 & 18.2 & 17.9 & 0.0 & 9.6 & 21 & 79 & 0.0 \\
\hline $10 / 25 / 01$ & $1: 15$ & 18.0 & 18.2 & 18.2 & 17.9 & 0.0 & 9.6 & 20 & 79 & 0.0 \\
\hline $10 / 25 / 01$ & $1: 16$ & 18.0 & 18.1 & 18.1 & 17.8 & 0.0 & 9.7 & 20 & 79 & 0.0 \\
\hline $10 / 25 / 01$ & 1:17 & 18.0 & 18.1 & 18.1 & 17.8 & 0.0 & 9.7 & 20 & 79 & 0.0 \\
\hline $10 / 25 / 01$ & 1:18 & 17.9 & 18.1 & 18.1 & 17.8 & 0.0 & 9.6 & 20 & 79 & 0.0 \\
\hline $10 / 25 / 01$ & 1:19 & 17.9 & 18.0 & 18.1 & 17.8 & 0.0 & 9.6 & 20 & 79 & 0.0 \\
\hline $10 / 25 / 01$ & $1: 20$ & 17.8 & 18.0 & 18.0 & 17.7 & 0.0 & 9.7 & 20 & 79 & 0.0 \\
\hline $10 / 25 / 01$ & $1: 21$ & 17.9 & 18.0 & 18.0 & 17.7 & 0.0 & 9.7 & 20 & 79 & 0.0 \\
\hline 10/25/01 & 1:22 & 17.8 & 18.0 & 18.0 & 17.7 & 0.0 & 9.6 & 20 & 79 & 0.0 \\
\hline $10 / 25 / 01$ & 1:23 & 17.8 & 18.0 & 18.0 & 17.7 & 0.0 & 9.7 & 20 & 79 & 0.0 \\
\hline $10 / 25 / 01$ & 1:24 & 17.8 & 18.0 & 18.0 & 17.6 & 0.0 & 9.7 & 20 & 79 & 0.0 \\
\hline $10 / 25 / 01$ & 1:25 & 17.8 & 17.9 & 17.9 & 17.7 & 0.0 & 9.7 & 20 & 79 & 0.0 \\
\hline $10 / 25 / 01$ & $1: 26$ & 17.8 & 17.9 & 17.9 & 17.6 & 0.0 & 9.7 & 20 & 79 & 0.0 \\
\hline 10/25/01 & 1:27 & 17.7 & 17.9 & 17.9 & 17.6 & 0.0 & 9.6 & 20 & 79 & 0.0 \\
\hline
\end{tabular}

\begin{tabular}{|c|c|c|c|c|c|c|c|c|c|c|}
\hline \multicolumn{11}{|c|}{ PREC2_102401_0627 } \\
\hline DATE & TIME & \begin{tabular}{|c}
$\mathrm{HX}$ \\
Outlet \\
$\left({ }^{\circ} \mathrm{C}\right)$ \\
$\mathrm{TCO}$ \\
\end{tabular} & \begin{tabular}{|c|} 
REC \\
PP \\
OUT \\
$\left({ }^{\circ} \mathrm{C}\right)$ \\
TC1 \\
\end{tabular} & \begin{tabular}{|c|} 
TK \\
BOT \\
$\left({ }^{\circ} \mathrm{C}\right)$ \\
TC2 \\
\end{tabular} & $\begin{array}{c}\text { HX } \\
\text { OUT } \\
\left({ }^{\circ} \mathrm{C}\right) \\
\text { TC3 }\end{array}$ & $\begin{array}{c}\text { Mn } \\
\text { Flow } \\
(\mathrm{gpm})\end{array}$ & \begin{tabular}{|c|} 
Galigher \\
Flow \\
(gpm)
\end{tabular} & $\begin{array}{c}\text { Current } \\
\text { to } \mathrm{HX}\end{array}$ & $\begin{array}{c}\text { Voltage } \\
\text { to } \mathrm{HX}\end{array}$ & $\begin{array}{c}\mathrm{Sr} \\
\text { Flow } \\
\text { (gpm) }\end{array}$ \\
\hline $10 / 25 / 01$ & $1: 28$ & 17.7 & 17.9 & 17.9 & 17.6 & 0.0 & 9.6 & 20 & 79 & 0.0 \\
\hline $10 / 25 / 01$ & $1: 29$ & 17.7 & 17.9 & 17.9 & 17.6 & 0.0 & 9.6 & 21 & 79 & 0.0 \\
\hline $10 / 25 / 01$ & $1: 30$ & 17.7 & 17.8 & 17.9 & 17.6 & 0.0 & 9.6 & 21 & 79 & 0.0 \\
\hline $10 / 25 / 01$ & $1: 31$ & 17.6 & 17.8 & \begin{tabular}{|r|r}
17.8 \\
\end{tabular} & 17.5 & 0.0 & 9.6 & 20 & 79 & 0.0 \\
\hline 10/25/01 & $1: 32$ & 17.7 & 17.8 & \begin{tabular}{|r|r}
17.8 \\
\end{tabular} & 17.5 & 0.0 & 9.7 & 21 & 79 & 0.0 \\
\hline 10/25/01 & $1: 33$ & 17.6 & 17.8 & 17.8 & 17.5 & 0.0 & 9.6 & 20 & 79 & 0.0 \\
\hline $10 / 25 / 01$ & $1: 34$ & 17.7 & 17.8 & 17.8 & 17.5 & 0.0 & 9.6 & 20 & 79 & 0.0 \\
\hline 10/25/01 & $1: 35$ & 17.6 & 17.8 & 17.8 & 17.5 & 0.0 & 9.7 & 20 & 79 & 0.0 \\
\hline $10 / 25 / 01$ & $1: 36$ & 17.6 & 17.8 & 17.8 & 17.5 & 0.0 & 9.7 & 21 & 79 & 0.0 \\
\hline 10/25/01 & $1: 37$ & 17.6 & 17.7 & 17.7 & 17.4 & 0.0 & 9.7 & 20 & 79 & 0.0 \\
\hline 10/25/01 & $1: 38$ & 17.6 & 17.7 & 17.7 & 17.4 & 0.0 & 9.7 & 20 & 79 & 0.0 \\
\hline $10 / 25 / 01$ & 1:39 & 17.6 & 17.7 & \begin{tabular}{|r|r} 
\\
\end{tabular} & 17.4 & 0.0 & 9.6 & 20 & 79 & 0.0 \\
\hline 10/25/01 & $1: 40$ & 17.5 & 17.7 & 17.7 & 17.4 & 0.0 & 9.6 & 20 & 79 & 0.0 \\
\hline 10/25/01 & $1: 41$ & 17.6 & 17.7 & 17.7 & 17.4 & 0.0 & 9.7 & 21 & 79 & 0.0 \\
\hline $10 / 25 / 01$ & 1:42 & 17.5 & 17.7 & 17.7 & 17.4 & 0.0 & 9.6 & 21 & 79 & 0.0 \\
\hline $10 / 25 / 01$ & $1: 43$ & 17.5 & 17.7 & 17.7 & 17.4 & 0.0 & 9.6 & 20 & 79 & 0.0 \\
\hline $10 / 25 / 01$ & $1: 44$ & 17.5 & 17.7 & 17.7 & 17.4 & 0.0 & 9.7 & 20 & 79 & 0.0 \\
\hline $10 / 25 / 01$ & $1: 45$ & 17.5 & 17.7 & 17.7 & 17.4 & 0.0 & 9.6 & 21 & 79 & 0.0 \\
\hline $10 / 25 / 01$ & $1: 46$ & 17.5 & 17.7 & 17.7 & 17.3 & 0.0 & 9.6 & 20 & 79 & 0.0 \\
\hline $10 / 25 / 01$ & $1: 47$ & 17.5 & 17.6 & 17.6 & 17.4 & 0.0 & 9.7 & 21 & 79 & 0.0 \\
\hline $10 / 25 / 01$ & $1: 48$ & 17.5 & 17.6 & \begin{tabular}{|l|}
17.7 \\
\end{tabular} & 17.4 & 0.0 & 9.6 & 21 & 79 & 0.0 \\
\hline $10 / 25 / 01$ & $1: 49$ & 17.5 & 17.6 & 17.6 & 17.4 & 0.0 & 9.7 & 20 & 79 & 0.0 \\
\hline $10 / 25 / 01$ & $1: 50$ & 17.5 & 17.6 & 17.6 & 17.3 & 0.0 & 9.7 & 21 & 79 & 0.0 \\
\hline $10 / 25 / 01$ & $1: 51$ & 17.5 & 17.6 & 17.6 & 17.3 & 0.0 & 9.6 & 20 & 79 & 0.0 \\
\hline $10 / 25 / 01$ & 1:52 & 17.5 & 17.6 & 17.6 & 17.3 & 0.0 & 9.7 & 21 & 79 & 0.0 \\
\hline $10 / 25 / 01$ & 1:53 & 17.4 & 17.6 & 17.6 & 17.3 & 0.0 & 9.6 & 20 & 79 & 0.0 \\
\hline $10 / 25 / 01$ & $1: 54$ & 17.5 & 17.6 & \begin{tabular}{|l|}
17.6 \\
\end{tabular} & 17.3 & 0.0 & 9.7 & 21 & 79 & 0.0 \\
\hline $10 / 25 / 01$ & $1: 55$ & 17.4 & 17.6 & \begin{tabular}{|l|}
17.6 \\
\end{tabular} & 17.3 & 0.0 & 9.6 & 21 & 79 & 0.0 \\
\hline $10 / 25 / 01$ & 1:56 & 17.5 & 17.6 & \begin{tabular}{|l|}
17.6 \\
\end{tabular} & 17.3 & 0.0 & 9.7 & 20 & 79 & 0.0 \\
\hline $10 / 25 / 01$ & 1:57 & 17.4 & 17.6 & 17.6 & 17.3 & 0.0 & 9.6 & 21 & 79 & 0.0 \\
\hline $10 / 25 / 01$ & 1:58 & 17.4 & 17.6 & 17.6 & 17.3 & 0.0 & 9.6 & 21 & 79 & 0.0 \\
\hline $10 / 25 / 01$ & $1: 59$ & 17.4 & 17.6 & 17.6 & 17.2 & 0.0 & 9.7 & 20 & 79 & 0.0 \\
\hline $10 / 25 / 01$ & 2:00 & 17.4 & 17.5 & 17.6 & 17.3 & 0.0 & 9.6 & 21 & 79 & 0.0 \\
\hline $10 / 25 / 01$ & 2:01 & 17.4 & 17.5 & 17.6 & 17.3 & 0.0 & 9.6 & 21 & 79 & 0.0 \\
\hline $10 / 25 / 01$ & 2:02 & 17.4 & 17.5 & \begin{tabular}{|l|}
17.6 \\
\end{tabular} & 17.3 & 0.0 & 9.7 & 21 & 79 & 0.0 \\
\hline $10 / 25 / 01$ & 2:03 & 17.4 & 17.5 & 17.5 & 17.3 & 0.0 & 9.7 & 21 & 79 & 0.0 \\
\hline $10 / 25 / 01$ & 2:04 & 17.4 & 17.5 & \begin{tabular}{|l|}
17.5 \\
\end{tabular} & 17.2 & 0.0 & 9.7 & 20 & 79 & 0.0 \\
\hline $10 / 25 / 01$ & 2:05 & 17.4 & 17.5 & 17.5 & 17.3 & 0.0 & 9.7 & 20 & 79 & 0.0 \\
\hline $10 / 25 / 01$ & 2:06 & 17.4 & 17.5 & 17.5 & 17.2 & 0.0 & 9.6 & 20 & 79 & 0.0 \\
\hline $10 / 25 / 01$ & 2:07 & 17.4 & 17.5 & 17.5 & 17.2 & 0.0 & 9.6 & 20 & 79 & 0.0 \\
\hline $10 / 25 / 01$ & 2:08 & 17.3 & 17.5 & 17.5 & 17.2 & 0.0 & 9.7 & 20 & 79 & 0.0 \\
\hline $10 / 25 / 01$ & 2:09 & 17.4 & 17.5 & 17.5 & 17.2 & 0.0 & 9.7 & 20 & 79 & 0.0 \\
\hline $10 / 25 / 01$ & 2:10 & 17.3 & 17.5 & 17.5 & 17.2 & 0.0 & 9.8 & 21 & 79 & 0.0 \\
\hline $10 / 25 / 01$ & 2:11 & 17.3 & 17.5 & 17.5 & 17.2 & 0.0 & 9.7 & 20 & 79 & 0.0 \\
\hline $10 / 25 / 01$ & 2:12 & 17.3 & 17.5 & \begin{tabular}{|l|}
17.5 \\
\end{tabular} & 17.2 & 0.0 & 9.6 & 20 & 79 & 0.0 \\
\hline $10 / 25 / 01$ & 2:13 & 17.3 & 17.5 & 17.5 & 17.2 & 0.0 & 9.6 & 20 & 79 & 0.0 \\
\hline $10 / 25 / 01$ & 2:14 & 17.3 & 17.5 & 17.5 & 17.2 & 0.0 & 9.6 & 20 & 79 & 0.0 \\
\hline $10 / 25 / 01$ & 2:15 & 17.3 & 17.5 & 17.5 & 17.2 & 0.0 & 9.6 & 21 & 79 & 0.0 \\
\hline $10 / 25 / 01$ & 2:16 & 17.4 & 17.5 & 17.5 & 17.2 & 0.0 & 9.7 & 21 & 79 & 0.0 \\
\hline $10 / 25 / 01$ & 2:17 & 17.3 & 17.5 & \begin{tabular}{|l|}
17.5 \\
\end{tabular} & 17.2 & 0.0 & 9.6 & 20 & 79 & 0.0 \\
\hline $10 / 25 / 01$ & 2:18 & 17.4 & 17.5 & 17.5 & 17.2 & 0.0 & 9.6 & 20 & 79 & 0.0 \\
\hline 10/25/01 & 2:19 & 17.3 & 17.5 & 17.5 & 17.2 & 0.0 & 9.7 & 20 & 79 & 0.0 \\
\hline $10 / 25 / 01$ & 2:20 & 17.3 & 17.4 & \begin{tabular}{|l|}
17.5 \\
\end{tabular} & 17.2 & 0.0 & 9.7 & 20 & 79 & 0.0 \\
\hline $10 / 25 / 01$ & $2: 21$ & 17.3 & 17.5 & 17.5 & 17.1 & 0.0 & 9.6 & 21 & 79 & 0.0 \\
\hline $10 / 25 / 01$ & 2:22 & 17.3 & 17.5 & \begin{tabular}{|l|}
17.5 \\
\end{tabular} & 17.2 & 0.0 & 9.7 & 20 & 79 & 0.0 \\
\hline $10 / 25 / 01$ & $2: 23$ & 17.3 & 17.5 & \begin{tabular}{|l|}
17.5 \\
\end{tabular} & 17.2 & 0.0 & 9.7 & 20 & 79 & 0.0 \\
\hline $10 / 25 / 01$ & 2:24 & 17.3 & 17.5 & 17.5 & 17.2 & 0.0 & 9.7 & 20 & 79 & 0.0 \\
\hline $10 / 25 / 01$ & 2:25 & 17.3 & 17.4 & 17.5 & 17.2 & 0.0 & 9.7 & 20 & 79 & 0.0 \\
\hline $10 / 25 / 01$ & 2:26 & 17.3 & 17.5 & 17.5 & 17.2 & 0.0 & 9.6 & 20 & 79 & 0.0 \\
\hline $10 / 25 / 01$ & 2:27 & 17.3 & 17.5 & 17.5 & 17.2 & 0.0 & 9.7 & 20 & 79 & 0.0 \\
\hline
\end{tabular}


WSRC-TR-2002-00459, Rev. 0 SRT-RPP-2002-00221

\begin{tabular}{|c|c|c|c|c|c|c|c|c|c|c|}
\hline \multicolumn{3}{|c|}{ PREC2_102401_0627 } & & \multirow[b]{2}{*}{\begin{tabular}{|c|} 
TK \\
BOT \\
$\left({ }^{\circ} \mathrm{C}\right)$ \\
TC2 \\
\end{tabular}} & \multirow[b]{2}{*}{$\begin{array}{c}\mathrm{HX} \\
\text { OUT } \\
\left({ }^{\circ} \mathrm{C}\right) \\
\mathrm{TC} 3\end{array}$} & \multirow[b]{2}{*}{$\begin{array}{c}\mathrm{Mn} \\
\text { Flow } \\
(\mathrm{gpm})\end{array}$} & \multirow[b]{2}{*}{$\begin{array}{c}\text { Galigher } \\
\text { Flow } \\
\text { (gpm) }\end{array}$} & \multirow[b]{2}{*}{$\begin{array}{c}\text { Current } \\
\text { to } \mathrm{HX}\end{array}$} & \multirow[b]{2}{*}{$\begin{array}{c}\text { Voltage } \\
\text { to } \mathrm{HX}\end{array}$} & \multirow[b]{2}{*}{$\begin{array}{c}\mathrm{Sr} \\
\text { Flow } \\
(\mathrm{gpm})\end{array}$} \\
\hline DATE & TIME & $\begin{array}{c}\mathrm{HX} \\
\text { Outlet } \\
\left({ }^{\circ} \mathrm{C}\right) \\
\mathrm{TC} 0\end{array}$ & $\begin{array}{c}\text { REC } \\
\text { PP } \\
\text { OUT } \\
\left({ }^{\circ} \mathrm{C}\right) \\
\text { TC1 }\end{array}$ & & & & & & & \\
\hline $10 / 25 / 01$ & 2:28 & 17.3 & 17.5 & 17.5 & \begin{tabular}{|l|}
17.2 \\
\end{tabular} & 0.0 & 9.6 & 20 & 79 & 0.0 \\
\hline $10 / 25 / 01$ & $2: 29$ & 17.3 & 17.4 & 17.5 & 17.2 & 0.0 & 9.6 & 20 & 79 & 0.0 \\
\hline $10 / 25 / 01$ & 2:30 & 17.3 & 17.5 & 17.5 & 17.1 & 0.0 & 9.6 & 20 & 79 & 0.0 \\
\hline $10 / 25 / 01$ & 2:31 & 17.3 & 17.4 & 17.5 & \begin{tabular}{|l|}
17.2 \\
\end{tabular} & 0.0 & 9.6 & 20 & 79 & 0.0 \\
\hline $10 / 25 / 01$ & 2:32 & 17.3 & 17.5 & 17.5 & \begin{tabular}{|r|}
17.2 \\
\end{tabular} & 0.0 & 9.6 & 20 & 79 & 0.0 \\
\hline $10 / 25 / 01$ & $2: 33$ & 17.3 & 17.4 & 17.5 & \begin{tabular}{|l|}
17.2 \\
\end{tabular} & 0.0 & 9.7 & 20 & 79 & 0.0 \\
\hline $10 / 25 / 01$ & 2:34 & 17.3 & 17.4 & 17.5 & 17.2 & 0.0 & 9.6 & 20 & 79 & 0.0 \\
\hline $10 / 25 / 01$ & 2:35 & 17.3 & 17.4 & 17.5 & \begin{tabular}{|l|}
17.2 \\
\end{tabular} & 0.0 & 9.7 & 20 & 79 & 0.0 \\
\hline $10 / 25 / 01$ & $2: 36$ & 17.3 & 17.4 & 17.4 & \begin{tabular}{|l|}
17.2 \\
\end{tabular} & 0.0 & 9.6 & 20 & 79 & 0.0 \\
\hline $10 / 25 / 01$ & $2: 37$ & 17.3 & 17.4 & 17.5 & \begin{tabular}{|l|}
17.2 \\
\end{tabular} & 0.0 & 9.6 & 20 & 79 & 0.0 \\
\hline $10 / 25 / 01$ & 2:38 & 17.3 & 17.4 & 17.4 & \begin{tabular}{|l|}
17.2 \\
\end{tabular} & 0.0 & 9.6 & 20 & 79 & 0.0 \\
\hline $10 / 25 / 01$ & 2:39 & 17.3 & 17.4 & 17.5 & 17.1 & 0.0 & 9.7 & 20 & 79 & 0.0 \\
\hline $10 / 25 / 01$ & $2: 40$ & 17.3 & 17.4 & 17.4 & 17.2 & 0.0 & 9.6 & 20 & 79 & 0.0 \\
\hline $10 / 25 / 01$ & $2: 41$ & 17.3 & 17.4 & 17.4 & 17.1 & 0.0 & 9.6 & 21 & 79 & 0.0 \\
\hline $10 / 25 / 01$ & $2: 42$ & 17.3 & 17.4 & 17.5 & \begin{tabular}{|l|}
17.2 \\
\end{tabular} & 0.0 & 9.7 & 20 & 79 & 0.0 \\
\hline $10 / 25 / 01$ & $2: 43$ & 17.3 & 17.5 & 17.5 & 17.1 & 0.0 & 9.7 & 20 & 79 & 0.0 \\
\hline $10 / 25 / 01$ & $2: 44$ & 17.3 & 17.4 & 17.5 & \begin{tabular}{|l|}
17.2 \\
\end{tabular} & 0.0 & 9.6 & 20 & 79 & 0.0 \\
\hline $10 / 25 / 01$ & $2: 45$ & 17.3 & 17.5 & 17.5 & \begin{tabular}{|l|}
17.2 \\
\end{tabular} & 0.0 & 9.6 & 20 & 79 & 0.0 \\
\hline $10 / 25 / 01$ & $2: 46$ & 17.3 & 17.5 & 17.5 & \begin{tabular}{|l|}
17.2 \\
\end{tabular} & 0.0 & 9.6 & 20 & 79 & 0.0 \\
\hline $10 / 25 / 01$ & $2: 47$ & 17.3 & 17.4 & 17.5 & 17.2 & 0.0 & 9.7 & 20 & 79 & 0.0 \\
\hline $10 / 25 / 01$ & $2: 48$ & 17.3 & 17.5 & 17.5 & 17.2 & 0.0 & 9.7 & 20 & 79 & 0.0 \\
\hline $10 / 25 / 01$ & $2: 49$ & 17.3 & 17.4 & 17.5 & \begin{tabular}{|l|}
17.2 \\
\end{tabular} & 0.0 & 9.6 & 20 & 79 & 0.0 \\
\hline $10 / 25 / 01$ & $2: 50$ & 17.3 & 17.5 & 17.5 & 17.1 & 0.0 & 9.6 & 20 & 79 & 0.0 \\
\hline $10 / 25 / 01$ & 2:51 & 17.3 & 17.4 & 17.5 & 17.2 & 0.0 & 9.6 & 20 & 79 & 0.0 \\
\hline $10 / 25 / 01$ & 2:52 & 17.3 & 17.4 & 17.4 & 17.1 & 0.0 & 9.7 & 21 & 79 & 0.0 \\
\hline $10 / 25 / 01$ & 2:53 & 17.3 & 17.4 & 17.4 & \begin{tabular}{|l|}
17.2 \\
\end{tabular} & 0.0 & 9.6 & 20 & 79 & 0.0 \\
\hline $10 / 25 / 01$ & $2: 54$ & 17.3 & 17.4 & 17.4 & 17.1 & 0.0 & 9.7 & 20 & 79 & 0.0 \\
\hline $10 / 25 / 01$ & 2:55 & 17.3 & 17.4 & 17.4 & 17.1 & 0.0 & 9.6 & 20 & 79 & 0.0 \\
\hline $10 / 25 / 01$ & 2:56 & 17.3 & 17.4 & 17.4 & 17.1 & 0.0 & 9.7 & 20 & 79 & 0.0 \\
\hline $10 / 25 / 01$ & 2:57 & 17.3 & 17.4 & 17.4 & 17.1 & 0.0 & 9.7 & 20 & 79 & 0.0 \\
\hline $10 / 25 / 01$ & 2:58 & 17.3 & 17.4 & 17.4 & 17.1 & 0.0 & 9.6 & 20 & 79 & 0.0 \\
\hline $10 / 25 / 01$ & 2:59 & 17.3 & 17.4 & 17.4 & 17.1 & 0.0 & 9.6 & 20 & 79 & 0.0 \\
\hline $10 / 25 / 01$ & 3:00 & 17.3 & 17.4 & 17.4 & 17.1 & 0.0 & 9.6 & 21 & 79 & 0.0 \\
\hline $10 / 25 / 01$ & 3:01 & 17.3 & 17.4 & 17.4 & 17.1 & 0.0 & 9.6 & 20 & 79 & 0.0 \\
\hline $10 / 25 / 01$ & 3:02 & 17.3 & 17.4 & 17.4 & 17.1 & 0.0 & 9.7 & 20 & 79 & 0.0 \\
\hline $10 / 25 / 01$ & 3:03 & 17.3 & 17.4 & 17.4 & 17.1 & 0.0 & 9.6 & 20 & 79 & 0.0 \\
\hline $10 / 25 / 01$ & 3:04 & 17.3 & 17.4 & 17.4 & 17.1 & 0.0 & 9.6 & 20 & 79 & 0.0 \\
\hline $10 / 25 / 01$ & 3:05 & 17.2 & 17.4 & 17.4 & 17.1 & 0.0 & 9.6 & 20 & 79 & 0.0 \\
\hline $10 / 25 / 01$ & 3:06 & 17.3 & 17.4 & 17.4 & 17.1 & 0.0 & 9.6 & 20 & 79 & 0.0 \\
\hline $10 / 25 / 01$ & 3:07 & 17.2 & 17.4 & 17.4 & 17.1 & 0.0 & 9.6 & 20 & 79 & 0.0 \\
\hline $10 / 25 / 01$ & 3:08 & 17.3 & 17.4 & 17.4 & \begin{tabular}{|l|}
17.1 \\
\end{tabular} & 0.0 & 9.7 & 20 & 79 & 0.0 \\
\hline $10 / 25 / 01$ & 3:09 & 17.3 & 17.4 & 17.4 & 17.1 & 0.0 & 9.7 & 20 & 79 & 0.0 \\
\hline $10 / 25 / 01$ & $3: 10$ & 17.3 & 17.4 & 17.4 & 17.1 & 0.0 & 9.7 & 20 & 79 & 0.0 \\
\hline $10 / 25 / 01$ & $3: 11$ & 17.3 & 17.4 & 17.4 & 17.1 & 0.0 & 9.6 & 20 & 79 & 0.0 \\
\hline $10 / 25 / 01$ & $3: 12$ & 17.2 & 17.4 & 17.4 & 17.1 & 0.0 & 9.6 & 20 & 79 & 0.0 \\
\hline $10 / 25 / 01$ & $3: 13$ & 17.3 & 17.3 & 17.3 & 17.1 & 0.0 & 9.7 & 20 & 79 & 0.0 \\
\hline $10 / 25 / 01$ & $3: 14$ & 17.2 & 17.3 & 17.3 & \begin{tabular}{|l|}
17.0 \\
\end{tabular} & 0.0 & 9.6 & 20 & 79 & 0.0 \\
\hline $10 / 25 / 01$ & $3: 15$ & 17.3 & 17.4 & 17.4 & 17.1 & 0.0 & 9.6 & 20 & 79 & 0.0 \\
\hline $10 / 25 / 01$ & $3: 16$ & 17.3 & 17.4 & 17.4 & 17.1 & 0.0 & 10.8 & 20 & 79 & 0.0 \\
\hline $10 / 25 / 01$ & $3: 17$ & 17.3 & 17.4 & 17.4 & 17.1 & 0.0 & 9.6 & 20 & 79 & 0.0 \\
\hline $10 / 25 / 01$ & $3: 18$ & 17.3 & 17.4 & 17.4 & 17.1 & 0.0 & 9.6 & 20 & 79 & 0.0 \\
\hline $10 / 25 / 01$ & $3: 19$ & 17.4 & 17.4 & 17.4 & \begin{tabular}{|l|}
17.2 \\
\end{tabular} & 0.0 & 9.6 & 20 & 79 & 0.0 \\
\hline $10 / 25 / 01$ & $3: 20$ & 17.5 & 17.5 & 17.5 & \begin{tabular}{|l|}
17.3 \\
\end{tabular} & 0.0 & 9.6 & 20 & 79 & 0.0 \\
\hline $10 / 25 / 01$ & $3: 21$ & 17.6 & 17.6 & 17.6 & \begin{tabular}{|l|}
17.4 \\
\end{tabular} & 0.0 & 9.6 & 20 & 79 & 0.0 \\
\hline $10 / 25 / 01$ & 3:22 & 17.6 & 17.6 & 17.6 & $\mid 17.4$ & 0.0 & 9.7 & 20 & 79 & 0.0 \\
\hline $10 / 25 / 01$ & $3: 23$ & 17.7 & 17.7 & 17.7 & \begin{tabular}{|r|}
17.5 \\
\end{tabular} & 0.0 & 9.7 & 20 & 79 & 0.0 \\
\hline $10 / 25 / 01$ & 3:24 & 17.8 & 17.8 & 17.8 & \begin{tabular}{|r|}
17.6 \\
\end{tabular} & 0.0 & 9.6 & 20 & 79 & 0.0 \\
\hline $10 / 25 / 01$ & 3:25 & 17.8 & 17.9 & 17.9 & \begin{tabular}{|l|}
$\mid 17.7$ \\
\end{tabular} & 0.0 & 9.6 & 20 & 79 & 0.0 \\
\hline $10 / 25 / 01$ & 3:26 & 17.9 & 17.9 & 17.9 & \begin{tabular}{|l|}
$\mid 17.7$ \\
\end{tabular} & 0.0 & 9.6 & 20 & 79 & 0.0 \\
\hline $10 / 25 / 01$ & 3:27 & 18.0 & 18.0 & 18.0 & 17.8 & 0.0 & 9.7 & 20 & 79 & 0.0 \\
\hline
\end{tabular}

\begin{tabular}{|c|c|c|c|c|c|c|c|c|c|c|}
\hline \multicolumn{11}{|c|}{ PREC2_102401_0627 } \\
\hline DATE & TIME & $\begin{array}{c}\mathrm{HX} \\
\text { Outlet } \\
\left({ }^{\circ} \mathrm{C}\right) \\
\mathrm{TCO}\end{array}$ & $\begin{array}{l}\text { REC } \\
\text { PP } \\
\text { OUT } \\
\left({ }^{\circ} \mathrm{C}\right) \\
\text { TC1 }\end{array}$ & $\begin{array}{c}\text { TK } \\
\text { BOT } \\
\left({ }^{\circ} \mathrm{C}\right) \\
\text { TC2 }\end{array}$ & $\begin{array}{l}\text { HX } \\
\text { OUT } \\
\left({ }^{\circ} \mathrm{C}\right) \\
\text { TC3 }\end{array}$ & $\begin{array}{c}\text { Mn } \\
\text { Flow } \\
(\mathrm{gpm})\end{array}$ & $\begin{array}{c}\text { Galigher } \\
\text { Flow } \\
\text { (gpm) }\end{array}$ & $\begin{array}{c}\text { Current } \\
\text { to } \mathrm{HX}\end{array}$ & $\begin{array}{c}\text { Voltage } \\
\text { to } \mathrm{HX}\end{array}$ & $\begin{array}{c}\mathrm{Sr} \\
\text { Flow } \\
(\mathrm{gpm})\end{array}$ \\
\hline $10 / 25 / 01$ & 3:28 & 18.0 & 18.1 & 18.1 & 17.9 & 0.0 & 9.7 & 20 & 79 & 0.0 \\
\hline $10 / 25 / 01$ & $3: 29$ & 18.1 & 18.1 & 18.1 & 17.9 & 0.0 & 9.7 & 20 & 79 & 0.0 \\
\hline $10 / 25 / 01$ & $3: 30$ & 18.1 & 18.2 & 18.2 & 18.0 & 0.0 & 9.7 & 20 & 79 & 0.0 \\
\hline $10 / 25 / 01$ & 3:31 & 18.2 & 18.2 & 18.2 & 18.0 & 0.0 & 9.7 & 20 & 79 & 0.0 \\
\hline $10 / 25 / 01$ & 3:32 & 18.3 & 18.3 & 18.3 & 18.1 & 0.0 & 9.6 & 21 & 79 & 0.0 \\
\hline $10 / 25 / 01$ & 3:33 & 18.3 & 18.3 & 18.3 & 18.1 & 0.0 & 9.6 & 21 & 79 & 0.0 \\
\hline $10 / 25 / 01$ & $3: 34$ & 18.4 & 18.4 & 18.4 & 18.2 & 0.0 & 9.6 & 20 & 79 & 0.0 \\
\hline $10 / 25 / 01$ & 3:35 & 18.4 & 18.5 & 18.5 & 18.3 & 0.0 & 9.7 & 20 & 79 & 0.0 \\
\hline $10 / 25 / 01$ & $3: 36$ & 18.5 & 18.5 & 18.5 & 18.3 & 0.0 & 9.6 & 21 & 79 & 0.0 \\
\hline $10 / 25 / 01$ & $3: 37$ & 18.5 & 18.6 & 18.6 & 18.4 & 0.0 & 9.7 & 21 & 79 & 0.0 \\
\hline $10 / 25 / 01$ & 3:38 & 18.6 & 18.6 & 18.6 & 18.4 & 0.0 & 9.6 & 21 & 79 & 0.0 \\
\hline $10 / 25 / 01$ & $3: 39$ & 18.6 & 18.6 & 18.7 & 18.5 & 0.0 & 9.6 & 21 & 79 & 0.0 \\
\hline $10 / 25 / 01$ & $3: 40$ & 18.7 & 18.7 & 18.7 & 18.5 & 0.0 & 9.7 & 21 & 79 & 0.0 \\
\hline $10 / 25 / 01$ & $3: 41$ & 18.7 & 18.7 & 18.7 & 18.5 & 0.0 & 9.7 & 20 & 79 & 0.0 \\
\hline $10 / 25 / 01$ & $3: 42$ & 18.7 & 18.8 & 18.8 & 18.6 & 0.0 & 9.7 & 21 & 79 & 0.0 \\
\hline $10 / 25 / 01$ & 3:43 & 18.8 & 18.8 & 18.8 & 18.6 & 0.0 & 9.6 & 21 & 79 & 0.0 \\
\hline $10 / 25 / 01$ & $3: 44$ & 18.8 & 18.9 & 18.9 & 18.7 & 0.0 & 9.6 & 20 & 79 & 0.0 \\
\hline $10 / 25 / 01$ & $3: 45$ & 18.9 & 18.9 & 18.9 & 18.7 & 0.0 & 9.6 & 21 & 79 & 0.0 \\
\hline $10 / 25 / 01$ & $3: 46$ & 18.9 & 18.9 & 19.0 & 18.8 & 0.0 & 9.6 & 21 & 79 & 0.0 \\
\hline $10 / 25 / 01$ & $3: 47$ & 18.9 & 19.0 & 19.0 & 18.8 & 0.0 & 9.6 & 20 & 79 & 0.0 \\
\hline $10 / 25 / 01$ & $3: 48$ & 19.0 & 19.0 & 19.0 & 18.8 & 0.0 & 9.6 & 20 & 79 & 0.0 \\
\hline $10 / 25 / 01$ & $3: 49$ & 19.0 & 19.0 & 19.1 & 18.9 & 0.0 & 9.6 & 21 & 79 & 0.0 \\
\hline $10 / 25 / 01$ & 3:50 & 19.0 & 19.1 & 19.1 & 18.9 & 0.0 & 9.7 & 21 & 79 & 0.0 \\
\hline $10 / 25 / 01$ & 3:51 & 19.1 & 19.1 & 19.1 & 18.9 & 0.0 & 9.6 & 20 & 79 & 0.0 \\
\hline $10 / 25 / 01$ & 3:52 & 19.1 & 19.1 & 19.2 & 18.9 & 0.0 & 9.6 & 21 & 79 & 0.0 \\
\hline $10 / 25 / 01$ & 3:53 & 19.1 & 19.2 & 19.2 & 19.0 & 0.0 & 9.6 & 20 & 79 & 0.0 \\
\hline $10 / 25 / 01$ & 3:54 & 19.2 & 19.2 & 19.2 & 19.0 & 0.0 & 9.6 & 21 & 79 & 0.0 \\
\hline $10 / 25 / 01$ & 3:55 & 19.2 & 19.3 & 19.3 & 19.1 & 0.0 & 9.7 & 21 & 79 & 0.0 \\
\hline $10 / 25 / 01$ & 3:56 & 19.2 & 19.3 & 19.3 & 19.1 & 0.0 & 9.7 & 21 & 79 & 0.0 \\
\hline $10 / 25 / 01$ & 3:57 & 19.3 & 19.3 & 19.3 & 19.1 & 0.0 & 9.7 & 21 & 79 & 0.0 \\
\hline $10 / 25 / 01$ & 3:58 & 19.3 & 19.3 & 19.3 & 19.1 & 0.0 & 9.7 & 20 & 79 & 0.0 \\
\hline $10 / 25 / 01$ & 3:59 & 19.3 & 19.4 & 19.4 & 19.2 & 0.0 & 9.6 & 21 & 79 & 0.0 \\
\hline $10 / 25 / 01$ & 4:00 & 19.3 & 19.4 & 19.4 & 19.2 & 0.0 & 9.6 & 20 & 79 & 0.0 \\
\hline $10 / 25 / 01$ & $4: 01$ & 19.4 & 19.4 & 19.4 & 19.2 & 0.0 & 9.7 & 20 & 79 & 0.0 \\
\hline $10 / 25 / 01$ & 4:02 & 19.4 & 19.4 & 19.4 & 19.2 & 0.0 & 9.6 & 20 & 79 & 0.0 \\
\hline $10 / 25 / 01$ & 4:03 & 19.4 & 19.5 & 19.5 & 19.3 & 0.0 & 9.6 & 21 & 79 & 0.0 \\
\hline $10 / 25 / 01$ & 4:04 & 19.4 & 19.5 & 19.5 & 19.3 & 0.0 & 9.6 & 21 & 79 & 0.0 \\
\hline $10 / 25 / 01$ & 4:05 & 19.4 & 19.5 & 19.5 & 19.3 & 0.0 & 9.7 & 21 & 79 & 0.0 \\
\hline $10 / 25 / 01$ & 4:06 & 19.5 & 19.5 & 19.5 & 19.3 & 0.0 & 9.6 & 20 & 79 & 0.0 \\
\hline $10 / 25 / 01$ & $4: 07$ & 19.5 & 19.5 & 19.6 & 19.3 & 0.0 & 9.7 & 21 & 79 & 0.0 \\
\hline $10 / 25 / 01$ & 4:08 & 19.5 & 19.6 & 19.6 & 19.4 & 0.0 & 9.7 & 21 & 79 & 0.0 \\
\hline $10 / 25 / 01$ & 4:09 & 19.5 & 19.6 & 19.6 & 19.4 & 0.0 & 9.6 & 21 & 79 & 0.0 \\
\hline $10 / 25 / 01$ & $4: 10$ & 19.5 & 19.6 & 19.6 & 19.4 & 0.0 & 9.6 & 21 & 79 & 0.0 \\
\hline $10 / 25 / 01$ & $4: 11$ & 19.6 & 19.6 & 19.6 & 19.4 & 0.0 & 9.6 & 21 & 79 & 0.0 \\
\hline $10 / 25 / 01$ & 4:12 & 19.6 & 19.6 & 19.7 & 19.4 & 0.0 & 9.7 & 21 & 79 & 0.0 \\
\hline $10 / 25 / 01$ & 4:13 & 19.6 & 19.7 & 19.7 & 19.5 & 0.0 & 9.7 & 21 & 79 & 0.0 \\
\hline $10 / 25 / 01$ & 4:14 & 19.6 & 19.7 & 19.7 & 19.5 & 0.0 & 9.6 & 20 & 79 & 0.0 \\
\hline $10 / 25 / 01$ & 4:15 & 19.7 & 19.7 & 19.7 & 19.5 & 0.0 & 9.7 & 21 & 79 & 0.0 \\
\hline $10 / 25 / 01$ & 4:16 & 19.7 & 19.7 & 19.7 & 19.5 & 0.0 & 9.7 & 20 & 79 & 0.0 \\
\hline $10 / 25 / 01$ & $4: 17$ & 19.7 & 19.7 & 19.7 & 19.6 & 0.0 & 9.7 & 21 & 79 & 0.0 \\
\hline $10 / 25 / 01$ & $4: 18$ & 19.7 & 19.8 & 19.8 & 19.6 & 0.0 & 9.7 & 21 & 79 & 0.0 \\
\hline $10 / 25 / 01$ & $4: 19$ & 19.7 & 19.8 & 19.8 & 19.6 & 0.0 & 9.6 & 23 & 79 & 0.0 \\
\hline $10 / 25 / 01$ & $4: 20$ & 19.7 & 19.8 & 19.8 & 19.6 & 0.0 & 9.7 & 95 & 79 & 0.0 \\
\hline $10 / 25 / 01$ & $4: 21$ & 19.7 & 19.8 & 19.8 & 19.6 & 0.0 & 9.7 & 21 & 79 & 0.0 \\
\hline $10 / 25 / 01$ & 4:22 & 19.8 & 19.8 & 19.8 & 19.6 & 0.0 & 9.6 & 24 & 79 & 0.0 \\
\hline $10 / 25 / 01$ & $4: 23$ & 19.8 & 19.8 & 19.8 & 19.6 & 0.0 & 9.7 & 61 & 79 & 0.0 \\
\hline $10 / 25 / 01$ & $4: 24$ & 19.8 & 19.9 & 19.9 & 19.7 & 0.0 & 9.6 & 99 & 79 & 0.0 \\
\hline $10 / 25 / 01$ & $4: 25$ & 19.8 & 19.9 & 19.9 & 19.7 & 0.0 & 9.6 & 67 & 79 & 0.0 \\
\hline $10 / 25 / 01$ & $4: 26$ & 19.8 & 19.9 & 19.9 & $\mid 19.7$ & 0.0 & 9.7 & 32 & 79 & 0.0 \\
\hline $10 / 25 / 01$ & $4: 27$ & 19.8 & 19.9 & 19.9 & 19.7 & 0.0 & 9.7 & 43 & 79 & 0.0 \\
\hline
\end{tabular}


WSRC-TR-2002-00459, Rev. 0 SRT-RPP-2002-00221

\begin{tabular}{|c|c|c|c|c|c|c|c|c|c|c|}
\hline \multicolumn{11}{|c|}{ PREC2_102401_0627 } \\
\hline DATE & TIME & \begin{tabular}{|c|}
$\mathrm{HX}$ \\
Outlet \\
$\left({ }^{\circ} \mathrm{C}\right)$ \\
$\mathrm{TCO}$ \\
\end{tabular} & $\begin{array}{l}\text { REC } \\
\text { PP } \\
\text { OUT } \\
\left({ }^{\circ} \mathrm{C}\right) \\
\text { TC1 }\end{array}$ & \begin{tabular}{|c|}
$\mathrm{TK}$ \\
$\mathrm{BOT}$ \\
$\left({ }^{\circ} \mathrm{C}\right)$ \\
$\mathrm{TC} 2$ \\
\end{tabular} & \begin{tabular}{|c|} 
HX \\
OUT \\
$\left({ }^{\circ} \mathrm{C}\right)$ \\
TC3 \\
\end{tabular} & $\begin{array}{c}\text { Mn } \\
\text { Flow } \\
\text { (gpm) }\end{array}$ & $\begin{array}{c}\text { Galigher } \\
\text { Flow } \\
\text { (gpm) }\end{array}$ & $\begin{array}{l}\text { Current } \\
\text { to } \mathrm{HX}\end{array}$ & $\begin{array}{c}\text { Voltage } \\
\text { to } \mathrm{HX}\end{array}$ & $\begin{array}{c}\mathrm{Sr} \\
\text { Flow } \\
(\mathrm{gpm})\end{array}$ \\
\hline $10 / 25 / 01$ & 4:28 & 19.8 & 19.9 & 19.9 & 19.7 & 0.0 & 9.6 & 81 & 79 & 0.0 \\
\hline $10 / 25 / 01$ & 4:29 & 19.8 & 19.9 & 19.9 & 19.7 & 0.0 & 9.6 & 39 & 79 & 0.0 \\
\hline $10 / 25 / 01$ & 4:30 & 19.9 & 19.9 & 19.9 & 19.8 & 0.0 & 9.7 & 55 & 79 & 0.0 \\
\hline $10 / 25 / 01$ & 4:31 & 19.9 & 19.9 & 20.0 & 19.7 & 0.0 & 9.6 & 60 & 79 & 0.0 \\
\hline $10 / 25 / 01$ & 4:32 & 19.9 & 19.9 & 20.0 & 19.8 & 0.0 & 9.6 & 58 & 79 & 0.0 \\
\hline $10 / 25 / 01$ & 4:33 & 19.9 & 20.0 & 20.0 & \begin{tabular}{|l|}
19.8 \\
\end{tabular} & 0.0 & 9.6 & 35 & 79 & 0.0 \\
\hline $10 / 25 / 01$ & $4: 34$ & 19.9 & 20.0 & 20.0 & 19.8 & 0.0 & 9.6 & 40 & 79 & 0.0 \\
\hline $10 / 25 / 01$ & 4:35 & 19.9 & 20.0 & 20.0 & 19.8 & 0.0 & 9.6 & 74 & 79 & 0.0 \\
\hline $10 / 25 / 01$ & 4:36 & 19.9 & 20.0 & 20.0 & 19.8 & 0.0 & 9.6 & 83 & 79 & 0.0 \\
\hline $10 / 25 / 01$ & 4:37 & 19.9 & 20.0 & \begin{tabular}{|l|} 
\\
\end{tabular} & 19.8 & 0.0 & 9.6 & 90 & 79 & 0.0 \\
\hline $10 / 25 / 01$ & 4:38 & 19.9 & 20.0 & 20.0 & 19.8 & 0.0 & 9.6 & 89 & 79 & 0.0 \\
\hline $10 / 25 / 01$ & 4:39 & 20.0 & 20.0 & 20.0 & 19.8 & 0.0 & 9.6 & 77 & 79 & 0.0 \\
\hline $10 / 25 / 01$ & $4: 40$ & 20.0 & 20.0 & 20.0 & 19.8 & 0.0 & 9.7 & 35 & 79 & 0.0 \\
\hline $10 / 25 / 01$ & $4: 41$ & 20.0 & 20.0 & 20.1 & 19.9 & 0.0 & 9.7 & 74 & 79 & 0.0 \\
\hline $10 / 25 / 01$ & 4:42 & 20.0 & 20.0 & 20.1 & 19.9 & 0.0 & 9.6 & 54 & 79 & 0.0 \\
\hline $10 / 25 / 01$ & 4:43 & 20.0 & 20.1 & 20.1 & 19.9 & 0.0 & 9.6 & 92 & 79 & 0.0 \\
\hline $10 / 25 / 01$ & 4:44 & 20.0 & 20.1 & 20.1 & 19.9 & 0.0 & 9.6 & 58 & 79 & 0.0 \\
\hline $10 / 25 / 01$ & 4:45 & 20.0 & 20.1 & 20.1 & 19.9 & 0.0 & 9.7 & 69 & 79 & 0.0 \\
\hline $10 / 25 / 01$ & 4:46 & 20.0 & 20.1 & 20.1 & 19.9 & 0.0 & 9.6 & 90 & 79 & 0.0 \\
\hline $10 / 25 / 01$ & $4: 47$ & 20.0 & 20.1 & 20.1 & 19.9 & 0.0 & 9.6 & 90 & 79 & 0.0 \\
\hline $10 / 25 / 01$ & 4:48 & 20.1 & 20.1 & 20.1 & 20.0 & 0.0 & 9.6 & 85 & 79 & 0.0 \\
\hline $10 / 25 / 01$ & 4:49 & 20.1 & 20.1 & 20.2 & 20.0 & 0.0 & 9.6 & 52 & 79 & 0.0 \\
\hline $10 / 25 / 01$ & 4:50 & 20.1 & 20.2 & 20.2 & 20.0 & 0.0 & 9.6 & 46 & 79 & 0.0 \\
\hline $10 / 25 / 01$ & 4:51 & 20.1 & 20.2 & 20.2 & 20.0 & 0.0 & 9.6 & 100 & 79 & 0.0 \\
\hline $10 / 25 / 01$ & 4:52 & 20.1 & 20.2 & 20.2 & 20.0 & 0.0 & 9.6 & 84 & 79 & 0.0 \\
\hline $10 / 25 / 01$ & 4:53 & 20.1 & 20.2 & 20.2 & 20.0 & 0.0 & 9.6 & 54 & 79 & 0.0 \\
\hline $10 / 25 / 01$ & $4: 54$ & 20.1 & 20.2 & 20.2 & 20.1 & 0.0 & 9.6 & 80 & 79 & 0.0 \\
\hline $10 / 25 / 01$ & 4:55 & 20.1 & 20.2 & 20.3 & 20.1 & 0.0 & 9.6 & 83 & 79 & 0.0 \\
\hline $10 / 25 / 01$ & 4:56 & 20.2 & 20.2 & 20.3 & 20.1 & 0.0 & 9.6 & 88 & 79 & 0.0 \\
\hline $10 / 25 / 01$ & 4:57 & 20.2 & 20.2 & 20.3 & 20.1 & 0.0 & 9.6 & 57 & 79 & 0.0 \\
\hline $10 / 25 / 01$ & 4:58 & 20.2 & 20.3 & 20.3 & 20.1 & 0.0 & 8.2 & 91 & 79 & 0.0 \\
\hline $10 / 25 / 01$ & 4:59 & 20.2 & 20.3 & 20.3 & 20.1 & 0.0 & 9.6 & 62 & 79 & 0.0 \\
\hline $10 / 25 / 01$ & 5:00 & 20.3 & 20.4 & 20.4 & 20.2 & 0.0 & 9.3 & 102 & 79 & 0.0 \\
\hline $10 / 25 / 01$ & 5:01 & 20.4 & 20.6 & 20.4 & 20.2 & 0.0 & 9.7 & 79 & 79 & 0.0 \\
\hline $10 / 25 / 01$ & 5:02 & 20.5 & 20.6 & 20.6 & 20.4 & 0.0 & 9.3 & 82 & 79 & 0.0 \\
\hline $10 / 25 / 01$ & 5:03 & 20.5 & 20.6 & 20.6 & 20.4 & 0.0 & 9.0 & 112 & 79 & 0.0 \\
\hline $10 / 25 / 01$ & 5:04 & 20.4 & 20.5 & 20.5 & 20.3 & 0.0 & 8.0 & 89 & 79 & 0.0 \\
\hline $10 / 25 / 01$ & 5:05 & 20.3 & 20.4 & 20.5 & 20.2 & 0.0 & 8.0 & 80 & 79 & 0.0 \\
\hline $10 / 25 / 01$ & 5:06 & 20.3 & 20.4 & 20.4 & 20.2 & 0.0 & 8.0 & 109 & 79 & 0.0 \\
\hline $10 / 25 / 01$ & 5:07 & 20.3 & 20.4 & 20.4 & 20.2 & 0.0 & 8.0 & 115 & 79 & 0.0 \\
\hline $10 / 25 / 01$ & 5:08 & 20.2 & 20.3 & 20.4 & 20.1 & 0.0 & 8.0 & 94 & 79 & 0.0 \\
\hline $10 / 25 / 01$ & $5: 09$ & 20.2 & 20.3 & 20.3 & 20.1 & 0.0 & 7.9 & 114 & 79 & 0.0 \\
\hline $10 / 25 / 01$ & $5: 10$ & 20.1 & 20.2 & 20.3 & 20.0 & 0.0 & 8.0 & 80 & 79 & 0.0 \\
\hline $10 / 25 / 01$ & 5:11 & 20.1 & 20.2 & 20.2 & 20.0 & 0.0 & 8.0 & 128 & 79 & 0.0 \\
\hline $10 / 25 / 01$ & $5: 12$ & 20.1 & 20.1 & 20.2 & 20.0 & 0.0 & 8.0 & 106 & 79 & 0.0 \\
\hline $10 / 25 / 01$ & $5: 13$ & 20.0 & 20.1 & 20.2 & \begin{tabular}{|l|}
19.9 \\
\end{tabular} & 0.0 & 7.9 & 99 & 79 & 0.0 \\
\hline $10 / 25 / 01$ & 5:14 & 20.0 & 20.1 & 20.1 & 19.9 & 0.0 & 7.9 & 127 & 79 & 0.0 \\
\hline $10 / 25 / 01$ & 5:15 & 19.9 & 20.0 & 20.1 & \begin{tabular}{|l|}
19.8 \\
\end{tabular} & 0.0 & 7.9 & 127 & 79 & 0.0 \\
\hline $10 / 25 / 01$ & $5: 16$ & 19.9 & 20.0 & 20.1 & 19.8 & 0.0 & 8.0 & 105 & 79 & 0.0 \\
\hline $10 / 25 / 01$ & 5:17 & 19.9 & 20.0 & 20.0 & 19.8 & 0.0 & 8.0 & 92 & 79 & 0.0 \\
\hline $10 / 25 / 01$ & 5:18 & 19.9 & 19.9 & 20.0 & 19.8 & 0.0 & 8.0 & 79 & 79 & 0.0 \\
\hline $10 / 25 / 01$ & $5: 19$ & 19.8 & 19.9 & 20.0 & 19.7 & 0.0 & 8.0 & 79 & 79 & 0.0 \\
\hline $10 / 25 / 01$ & 5:20 & 19.8 & 19.9 & \begin{tabular}{|l|} 
\\
\end{tabular} & \begin{tabular}{|l|}
19.7 \\
\end{tabular} & 0.0 & 8.0 & 82 & 79 & 0.0 \\
\hline $10 / 25 / 01$ & $5: 21$ & 19.8 & 19.8 & 19.9 & $\mid 19.6$ & 0.0 & 8.0 & 94 & 79 & 0.0 \\
\hline $10 / 25 / 01$ & $5: 22$ & 19.7 & 19.8 & 19.9 & 19.6 & 0.0 & 8.0 & 116 & 79 & 0.0 \\
\hline $10 / 25 / 01$ & $5: 23$ & 19.7 & 19.8 & \begin{tabular}{|l|}
19.8 \\
\end{tabular} & 19.6 & 0.0 & 8.0 & 80 & 79 & 0.0 \\
\hline $10 / 25 / 01$ & 5:24 & 19.7 & 19.8 & 19.8 & 19.6 & 0.0 & 8.1 & 116 & 79 & 0.0 \\
\hline $10 / 25 / 01$ & 5:25 & 19.6 & 19.7 & 19.8 & 19.6 & 0.0 & 8.0 & 91 & 79 & 0.0 \\
\hline $10 / 25 / 01$ & $5: 26$ & 19.6 & 19.7 & 19.8 & 19.5 & 0.0 & 8.0 & 86 & 79 & 0.0 \\
\hline $10 / 25 / 01$ & 5:27 & 19.6 & \begin{tabular}{|l|}
19.7 \\
\end{tabular} & 19.8 & 19.5 & 0.0 & 8.0 & 120 & 79 & 0.0 \\
\hline
\end{tabular}

\begin{tabular}{|c|c|c|c|c|c|c|c|c|c|c|}
\hline \multicolumn{11}{|c|}{ PREC2_102401_0627 } \\
\hline DATE & TIME & $\begin{array}{c}\mathrm{HX} \\
\text { Outlet } \\
\left({ }^{\circ} \mathrm{C}\right) \\
\mathrm{TCO}\end{array}$ & \begin{tabular}{|c|} 
REC \\
PP \\
OUT \\
$\left({ }^{\circ} \mathrm{C}\right)$ \\
TC1 \\
\end{tabular} & $\begin{array}{c}\text { TK } \\
\text { BOT } \\
\left({ }^{\circ} \mathrm{C}\right) \\
\text { TC2 }\end{array}$ & \begin{tabular}{|l|} 
HX \\
OUT \\
$\left({ }^{\circ} \mathrm{C}\right)$ \\
TC3
\end{tabular} & $\begin{array}{c}\mathrm{Mn} \\
\text { Flow } \\
(\mathrm{gpm})\end{array}$ & $\begin{array}{c}\text { Galigher } \\
\text { Flow } \\
(\mathrm{gpm})\end{array}$ & $\begin{array}{c}\text { Current } \\
\text { to } \mathrm{HX}\end{array}$ & $\begin{array}{c}\text { Voltage } \\
\text { to } \mathrm{HX}\end{array}$ & $\begin{array}{c}\mathrm{Sr} \\
\text { Flow } \\
\text { (gpm) }\end{array}$ \\
\hline $10 / 25 / 01$ & $5: 28$ & 19.6 & 19.7 & 19.7 & 19.5 & 0.0 & 8.0 & 130 & 79 & 0.0 \\
\hline $10 / 25 / 01$ & $5: 29$ & 19.6 & 19.7 & 19.7 & \begin{tabular}{|l|}
19.5 \\
\end{tabular} & 0.0 & 8.0 & 110 & 79 & 0.0 \\
\hline $10 / 25 / 01$ & $5: 30$ & 19.5 & 19.6 & \begin{tabular}{|l|}
19.7 \\
\end{tabular} & \begin{tabular}{|l|}
19.5 \\
\end{tabular} & 0.0 & 8.0 & 86 & 79 & 0.0 \\
\hline $10 / 25 / 01$ & $5: 31$ & 19.5 & 19.6 & 19.7 & 19.4 & 0.0 & 8.0 & 77 & 79 & 0.0 \\
\hline $10 / 25 / 01$ & 5:32 & 19.5 & 19.6 & 19.7 & \begin{tabular}{|l|}
19.4 \\
\end{tabular} & 0.0 & 7.9 & 89 & 79 & 0.0 \\
\hline $10 / 25 / 01$ & $5: 33$ & 19.5 & 19.6 & 19.6 & \begin{tabular}{|l|}
19.4 \\
\end{tabular} & 0.0 & 8.0 & 110 & 79 & 0.0 \\
\hline $10 / 25 / 01$ & $5: 34$ & 19.5 & 19.6 & 19.6 & \begin{tabular}{|l|}
19.4 \\
\end{tabular} & 0.0 & 8.0 & 111 & 79 & 0.0 \\
\hline $10 / 25 / 01$ & $5: 35$ & 19.5 & 19.6 & 19.6 & 19.4 & 0.0 & 8.0 & 133 & 79 & 0.0 \\
\hline $10 / 25 / 01$ & $5: 36$ & 19.5 & 19.6 & 19.6 & \begin{tabular}{|l|}
19.4 \\
\end{tabular} & 0.0 & 8.0 & 135 & 79 & 0.0 \\
\hline $10 / 25 / 01$ & $5: 37$ & 19.5 & 19.6 & 19.6 & \begin{tabular}{|l|}
19.4 \\
\end{tabular} & 0.0 & 8.1 & 117 & 79 & 0.0 \\
\hline $10 / 25 / 01$ & $5: 38$ & 19.5 & 19.5 & 19.6 & \begin{tabular}{|l|}
19.4 \\
\end{tabular} & 0.0 & 8.1 & 111 & 79 & 0.0 \\
\hline $10 / 25 / 01$ & $5: 39$ & 19.4 & 19.5 & 19.6 & \begin{tabular}{|l|}
19.3 \\
\end{tabular} & 0.0 & 8.0 & 86 & 79 & 0.0 \\
\hline $10 / 25 / 01$ & $5: 40$ & 19.5 & 19.5 & 19.6 & \begin{tabular}{|l|}
19.4 \\
\end{tabular} & 0.0 & 8.0 & 94 & 79 & 0.0 \\
\hline $10 / 25 / 01$ & $5: 41$ & 19.4 & 19.5 & 19.6 & 19.3 & 0.0 & 8.0 & 114 & 79 & 0.0 \\
\hline $10 / 25 / 01$ & $5: 42$ & 19.4 & 19.5 & 19.6 & 19.3 & 0.0 & 8.0 & 128 & 79 & 0.0 \\
\hline $10 / 25 / 01$ & $5: 43$ & 19.4 & 19.5 & 19.5 & \begin{tabular}{|l|}
19.3 \\
\end{tabular} & 0.0 & 8.0 & 94 & 79 & 0.0 \\
\hline $10 / 25 / 01$ & $5: 44$ & 19.4 & 19.5 & 19.5 & \begin{tabular}{|l|}
19.3 \\
\end{tabular} & 0.0 & 8.0 & 83 & 79 & 0.0 \\
\hline $10 / 25 / 01$ & $5: 45$ & 19.4 & 19.5 & 19.5 & 19.3 & 0.0 & 8.0 & 120 & 79 & 0.0 \\
\hline $10 / 25 / 01$ & $5: 46$ & 19.4 & 19.4 & 19.5 & \begin{tabular}{|l|}
19.3 \\
\end{tabular} & 0.0 & 8.0 & 115 & 79 & 0.0 \\
\hline $10 / 25 / 01$ & $5: 47$ & 19.3 & 19.4 & 19.5 & \begin{tabular}{|l|}
19.2 \\
\end{tabular} & 0.0 & 8.0 & 82 & 79 & 0.0 \\
\hline $10 / 25 / 01$ & $5: 48$ & 19.3 & 19.4 & 19.5 & \begin{tabular}{|l|}
19.2 \\
\end{tabular} & 0.0 & 8.0 & 105 & 79 & 0.0 \\
\hline $10 / 25 / 01$ & $5: 49$ & 19.3 & 19.4 & 19.5 & \begin{tabular}{|l|}
19.2 \\
\end{tabular} & 0.0 & 8.0 & 124 & 79 & 0.0 \\
\hline $10 / 25 / 01$ & $5: 50$ & 19.3 & 19.4 & 19.5 & \begin{tabular}{|l|}
19.2 \\
\end{tabular} & 0.0 & 7.9 & 75 & 79 & 0.0 \\
\hline $10 / 25 / 01$ & $5: 51$ & 19.3 & 19.4 & 19.4 & \begin{tabular}{|l|}
19.2 \\
\end{tabular} & 0.0 & 8.0 & 108 & 79 & 0.0 \\
\hline $10 / 25 / 01$ & $5: 52$ & 19.3 & 19.4 & \begin{tabular}{|l|}
19.4 \\
\end{tabular} & \begin{tabular}{|l|}
19.2 \\
\end{tabular} & 0.0 & 7.9 & 125 & 79 & 0.0 \\
\hline $10 / 25 / 01$ & 5:53 & 19.3 & 19.3 & 19.4 & \begin{tabular}{|l|}
19.2 \\
\end{tabular} & 0.0 & 8.0 & 110 & 79 & 0.0 \\
\hline $10 / 25 / 01$ & $5: 54$ & 19.3 & 19.3 & 19.4 & \begin{tabular}{|l|}
19.2 \\
\end{tabular} & 0.0 & 8.0 & 93 & 79 & 0.0 \\
\hline $10 / 25 / 01$ & 5:55 & 19.2 & 19.3 & 19.4 & $\mid 19.1$ & 0.0 & 8.0 & 82 & 79 & 0.0 \\
\hline $10 / 25 / 01$ & $5: 56$ & 19.2 & 19.3 & 19.4 & \begin{tabular}{|l|} 
\\
\end{tabular} & 0.0 & 8.0 & 82 & 79 & 0.0 \\
\hline $10 / 25 / 01$ & $5: 57$ & 19.2 & 19.3 & 19.4 & \begin{tabular}{|l|}
19.1 \\
\end{tabular} & 0.0 & 8.0 & 90 & 79 & 0.0 \\
\hline $10 / 25 / 01$ & $5: 58$ & 19.2 & 19.3 & 19.4 & \begin{tabular}{|l|} 
\\
\end{tabular} & 0.0 & 8.0 & 101 & 79 & 0.0 \\
\hline $10 / 25 / 01$ & 5:59 & 19.2 & 19.3 & 19.3 & \begin{tabular}{|l|}
19.1 \\
\end{tabular} & 0.0 & 8.0 & 96 & 79 & 0.0 \\
\hline $10 / 25 / 01$ & $6: 00$ & 19.2 & 19.3 & 19.3 & 19.1 & 0.0 & 8.0 & 109 & 79 & 0.0 \\
\hline $10 / 25 / 01$ & 6:01 & 19.2 & 19.3 & 19.3 & \begin{tabular}{|l|}
19.1 \\
\end{tabular} & 0.0 & 8.0 & 112 & 79 & 0.0 \\
\hline $10 / 25 / 01$ & 6:02 & 19.2 & 19.3 & 19.3 & 19.1 & 0.0 & 8.1 & 99 & 79 & 0.0 \\
\hline $10 / 25 / 01$ & 6:03 & 19.2 & 19.3 & 19.3 & \begin{tabular}{|l|}
19.1 \\
\end{tabular} & 0.0 & 8.0 & 96 & 79 & 0.0 \\
\hline $10 / 25 / 01$ & 6:04 & 19.2 & 19.2 & 19.3 & \begin{tabular}{|l|} 
\\
\end{tabular} & 0.0 & 8.0 & 91 & 79 & 0.0 \\
\hline $10 / 25 / 01$ & $6: 05$ & 19.1 & 19.2 & 19.3 & 19.0 & 0.0 & 8.0 & 98 & 79 & 0.0 \\
\hline $10 / 25 / 01$ & $6: 06$ & 19.2 & 19.2 & 19.3 & \begin{tabular}{|l|}
19.1 \\
\end{tabular} & 0.0 & 8.0 & 84 & 79 & 0.0 \\
\hline $10 / 25 / 01$ & $6: 07$ & 19.1 & 19.2 & 19.3 & 19.0 & 0.0 & 8.0 & 81 & 79 & 0.0 \\
\hline $10 / 25 / 01$ & $6: 08$ & 19.1 & 19.2 & 19.3 & 19.0 & 0.0 & 8.0 & 80 & 79 & 0.0 \\
\hline $10 / 25 / 01$ & 6:09 & 19.1 & 19.2 & 19.3 & 19.0 & 0.0 & 8.0 & 83 & 79 & 0.0 \\
\hline $10 / 25 / 01$ & $6: 10$ & 19.1 & 19.2 & 19.3 & $\mid 19.0$ & 0.0 & 8.0 & 100 & 79 & 0.0 \\
\hline $10 / 25 / 01$ & $6: 11$ & 19.1 & 19.2 & 19.3 & 19.0 & 0.0 & 8.0 & 115 & 79 & 0.0 \\
\hline $10 / 25 / 01$ & $6: 12$ & 19.1 & 19.2 & 19.3 & 19.0 & 0.0 & 7.9 & 120 & 79 & 0.0 \\
\hline $10 / 25 / 01$ & $6: 13$ & 19.1 & 19.2 & 19.3 & $\mid 19.0$ & 0.0 & 8.0 & 126 & 79 & 0.0 \\
\hline $10 / 25 / 01$ & $6: 14$ & 19.1 & 19.2 & 19.3 & 19.0 & 0.0 & 8.0 & 85 & 79 & 0.0 \\
\hline $10 / 25 / 01$ & $6: 15$ & 19.1 & 19.2 & 19.3 & \begin{tabular}{|l|}
19.0 \\
\end{tabular} & 0.0 & 8.0 & 82 & 79 & 0.0 \\
\hline $10 / 25 / 01$ & $6: 16$ & 19.1 & 19.2 & 19.3 & $\mid 19.0$ & 0.0 & 8.0 & 97 & 79 & 0.0 \\
\hline $10 / 25 / 01$ & $6: 17$ & 19.1 & 19.2 & 19.3 & $\mid 19.0$ & 0.0 & 8.0 & 121 & 79 & 0.0 \\
\hline $10 / 25 / 01$ & $6: 18$ & 19.1 & 19.2 & 19.2 & $\mid 19.0$ & 0.0 & 8.1 & 114 & 79 & 0.0 \\
\hline $10 / 25 / 01$ & $6: 19$ & 19.1 & 19.2 & 19.2 & 19.0 & 0.0 & 8.0 & 88 & 79 & 0.0 \\
\hline $10 / 25 / 01$ & $6: 20$ & 19.1 & 19.2 & 19.2 & $\mid 19.0$ & 0.0 & 8.0 & 125 & 79 & 0.0 \\
\hline $10 / 25 / 01$ & $6: 21$ & 19.1 & 19.2 & 19.2 & $\mid 19.0$ & 0.0 & 8.1 & 85 & 79 & 0.0 \\
\hline $10 / 25 / 01$ & $6: 22$ & 19.1 & 19.2 & 19.2 & 19.0 & 0.0 & 8.1 & 90 & 79 & 0.0 \\
\hline $10 / 25 / 01$ & $6: 23$ & 19.1 & 19.2 & 19.2 & $\mid 19.0$ & 0.0 & 8.0 & 129 & 79 & 0.0 \\
\hline $10 / 25 / 01$ & $6: 24$ & 19.1 & 19.2 & 19.2 & $\mid 19.0$ & 0.0 & 8.0 & 109 & 79 & 0.0 \\
\hline $10 / 25 / 01$ & $6: 25$ & 19.1 & 19.2 & 19.2 & $\mid 19.0$ & 0.0 & 8.0 & 107 & 79 & 0.0 \\
\hline $10 / 25 / 01$ & $6: 26$ & 19.1 & 19.2 & 19.2 & 19.0 & 0.0 & 8.0 & 123 & 79 & 0.0 \\
\hline $10 / 25 / 01$ & $6: 27$ & 19.1 & 19.2 & 19.2 & $\mid 19.0$ & 0.0 & 8.0 & 115 & 79 & 0.0 \\
\hline
\end{tabular}


WSRC-TR-2002-00459, Rev. 0 SRT-RPP-2002-00221

\begin{tabular}{|c|c|c|c|c|c|c|c|c|c|c|}
\hline \multicolumn{11}{|c|}{ PREC2_102401_0627 } \\
\hline DATE & TIME & $\begin{array}{c}\mathrm{HX} \\
\text { Outlet } \\
\left({ }^{\circ} \mathrm{C}\right) \\
\mathrm{TC} 0\end{array}$ & $\begin{array}{l}\text { REC } \\
\text { PP } \\
\text { OUT } \\
\left({ }^{\circ} \mathrm{C}\right) \\
\text { TC1 }\end{array}$ & \begin{tabular}{|c} 
TK \\
BOT \\
$\left({ }^{\circ} \mathrm{C}\right)$ \\
TC2 \\
\end{tabular} & $\begin{array}{l}\mathrm{HX} \\
\text { OUT } \\
\left({ }^{\circ} \mathrm{C}\right) \\
\text { TC3 } \\
\end{array}$ & $\begin{array}{c}\text { Mn } \\
\text { Flow } \\
\text { (gpm) }\end{array}$ & $\begin{array}{c}\text { Galigher } \\
\text { Flow } \\
\text { (gpm) }\end{array}$ & $\begin{array}{l}\text { Current } \\
\text { to } \mathrm{HX}\end{array}$ & $\begin{array}{l}\text { Voltage } \\
\text { to } \mathrm{HX}\end{array}$ & $\begin{array}{c}\mathrm{Sr} \\
\text { Flow } \\
\text { (gpm) }\end{array}$ \\
\hline $10 / 25 / 01$ & $6: 28$ & 19.1 & 19.2 & 19.2 & 19.0 & 0.0 & 8.0 & 73 & 79 & 0.0 \\
\hline $10 / 25 / 01$ & $6: 29$ & 19.1 & 19.1 & 19.2 & 19.0 & 0.0 & 8.0 & 128 & 79 & 0.0 \\
\hline $10 / 25 / 01$ & $6: 30$ & 19.1 & 19.1 & 19.2 & 19.0 & 0.0 & 8.0 & 83 & 79 & 0.0 \\
\hline $10 / 25 / 01$ & $6: 31$ & 19.0 & 19.1 & 19.2 & 18.9 & 0.0 & 8.0 & 96 & 79 & 0.0 \\
\hline $10 / 25 / 01$ & 6:32 & 19.0 & 19.1 & 19.2 & \begin{tabular}{|l|}
18.9 \\
\end{tabular} & 0.0 & 8.0 & 94 & 79 & 0.0 \\
\hline $10 / 25 / 01$ & $6: 33$ & 19.0 & 19.1 & 19.2 & 18.9 & 0.0 & 8.0 & 83 & 79 & 0.0 \\
\hline $10 / 25 / 01$ & $6: 34$ & 19.0 & 19.1 & 19.2 & 18.9 & 0.0 & 8.0 & 110 & 79 & 0.0 \\
\hline $10 / 25 / 01$ & $6: 35$ & 19.0 & 19.1 & 19.2 & \begin{tabular}{|l|}
18.9 \\
\end{tabular} & 0.0 & 8.0 & 116 & 79 & 0. \\
\hline $10 / 25 / 01$ & $6: 36$ & 19.0 & 19.1 & 19.2 & 18.9 & 0.0 & 8.0 & 114 & 79 & 0.0 \\
\hline $10 / 25 / 01$ & $6: 37$ & 19.0 & 19.1 & 19.1 & 18.9 & 0.0 & 8.0 & 94 & 79 & 0 \\
\hline $10 / 25 / 01$ & $6: 38$ & 19.0 & 19.1 & 19.1 & 18.9 & 0.0 & 8.0 & 71 & 79 & 0.0 \\
\hline $10 / 25 / 01$ & $6: 39$ & 19.0 & 19.1 & 19.1 & 18.9 & 0.0 & 8.1 & 119 & 79 & 0 \\
\hline $10 / 25 / 01$ & $6: 40$ & 19.0 & 19.1 & 19.1 & 18.9 & 0.0 & 8.0 & 113 & 79 & 0 \\
\hline $10 / 25 / 01$ & $6: 41$ & 19.0 & 19.1 & 19.1 & 18.9 & 0.0 & 8.1 & 101 & 79 & 0 \\
\hline $10 / 25 / 01$ & $6: 42$ & 19.0 & 19.1 & 19.1 & 18.9 & 0.0 & . & 104 & 79 & 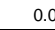 \\
\hline $10 / 25 / 01$ & $6: 43$ & 19.0 & 19.1 & 19.1 & 18.9 & 0.0 & 8.0 & 116 & 10 & 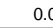 \\
\hline $10 / 25 / 01$ & $6: 44$ & 19.0 & 19.1 & 19.1 & 18.9 &. & & 93 & 10 & 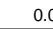 \\
\hline $10 / 25 / 01$ & $6: 45$ & 19.0 & 19.1 & 19.1 & 18.9 & 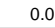 & & 101 & 0 & 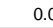 \\
\hline $10 / 25 / 01$ & $6: 46$ & 19.0 & 19.1 & 19.1 & 10.0 &. & & 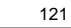 & 10 & 0.5 \\
\hline $10 / 25 / 01$ & $6: 47$ & 19.1 & 19.2 & 19.2 & 10.0 & 0.0 & . & r. & 10 & 0.6 \\
\hline $10 / 25 / 01$ & $6: 48$ & 19.1 & 19.3 & 19.3 & 19.0 & 0.0 & . & 200 & 10 & 0.6 \\
\hline $10 / 25 / 01$ & $6: 49$ & 19.3 & 19.5 & 19.4 & 19.2 & 0.0 & 1.4 & 100 & 79 & 0.6 \\
\hline $10 / 25 / 01$ & 6:50 & 19.3 & 19.6 & 19.4 & 19.2 & 0.0 & 1.0 & $10<$ & 19 & 0.0 \\
\hline $10 / 25 / 01$ & 6:51 & 19.8 & 20.1 & 19.7 & 19.6 & 0.0 & 0.4 & $1<0$ & 19 & 0.0 \\
\hline $10 / 25 / 01$ & 6:52 & 20.1 & 20.4 & 20.0 & 20.0 & 0.0 & 6.5 & 119 & 79 & 0.0 \\
\hline $10 / 25 / 01$ & 6:53 & 20.0 & 20.3 & 20.2 & 19.9 & 0.0 & 5.8 & 81 & 79 & 0.0 \\
\hline $10 / 25 / 01$ & 6:54 & 19.9 & 19.7 & 20.9 & 20.9 & 0.0 & -0.1 & 96 & 79 & 0.0 \\
\hline $10 / 25 / 01$ & 6:55 & 20.0 & 20.1 & 20.8 & 20.5 & 0.0 & -0.1 & 95 & 79 & 0.0 \\
\hline $10 / 25 / 01$ & 6:56 & 20.0 & 20.1 & 20.8 & 20.5 & 0.0 & -0.1 & 113 & 79 & 0.0 \\
\hline $10 / 25 / 01$ & $6: 57$ & 20.0 & 20.3 & 20.8 & 20.5 & 0.0 & -0.1 & 91 & 79 & 0.0 \\
\hline
\end{tabular}




\section{Appendix C}

\section{Experimental Data: Crossflow Test Rig Operations Data}

Appendix Contents

Nomenclature for Data Sheets

- Solenoid, $1=$ yes and $0=$ no for pressure to the backpulse piston

- $\quad$ FLTRT $\left({ }^{\circ} \mathrm{C}\right) \mathrm{T} 2$, Filtrate temperature in filter at exit of the housing

- CL LOOP $\left({ }^{\circ} \mathrm{C}\right) \mathrm{T} 3$, Temperature of the liquid in the cleaning loop

- SL LOOP $\left({ }^{\circ} \mathrm{C}\right) \mathrm{T} 1$, Temperature of the liquid in the slurry loop at the slurry reservoir

- UP AMB $\left({ }^{\circ} \mathrm{C}\right) \mathrm{T} 4$, Ambient temperature at the top of the crossflow test rig-3rd level

- BOT AMB $\left({ }^{\circ} \mathrm{C}\right) \mathrm{T} 5$, Ambient temperature at the bottom of the crossflow test rig-1st level

- BOT DP (psid) dP2, Differential pressure between the filter slurry entrance and the bottom filtrate exit

- FLTR (psig) P1, pressure at the filter slurry entrance

- FLTR DP (psid) dP1, Differential pressure between the filter slurry entrance and exit

- TOP DP (psid) dP3, Differential pressure between the filter slurry exit and the top filtrate exit

- FLTRATE (psig) P2, Pressure at the filtrate exit

- BP (psig) P3, Air pressure applied to the backpulse piston

- SL FLOW (gpm) Q1, Flow rate of the slurry

- FLTR FLOW (gpm) Q2, flow rate of the filtrate (low range meter used for slurry runs)

- HI FLTR FLOW (gpm) Q3, Flow rate of the filtrate (high range meter used for water runs)

- Temp corr flow $\left(\mathrm{gpm} / \mathrm{ft}^{2}\right)$, Filtrate flow per unit area of filter calculated by dividing the total filtrate flow by the area of the filter $\left(2.29 \mathrm{ft}^{2}\right)$ and correcting for temperature variation from $25^{\circ} \mathrm{C}$ by multiplying by $\mathrm{e}^{(2500)(1 /(273+\mathrm{T} 1)-1 / 298))}$

- Axial Vel (ft/sec), Axial tube velocity calculated by dividing the total slurry loop flow by the crosectional area of seven $3 / 8$ " ID tubes $\left(0.415 \mathrm{ft}^{2}\right)$

- Avg TMP (psid), calculated by averaging BOT DP and TOP DP 
Experimental data:

$\begin{array}{llr}\text { Data Set } & \text { Solution } & \text { Done on } \\ \text { Xflow2_102301_1011 } & \text { AN-107 Simulant } & 10 / 23 / 01 \\ \text { Xflow2_102401_0630 } & \text { AN-107 Simulant } & 10 / 24 / 01 \\ \text { Xflow2_102501_0700 } & \text { AN-107 Simulant } & 10 / 25 / 01 \\ \text { Xflow1_101501_0837 } & \text { DIF Water } & 10 / 15 / 01 \\ \text { Xflow2_110101_1045 } & \text { DIF Water } & 11 / 01 / 01\end{array}$

Note: Data plotted in the body of the report is highlighted for ease of reference. 
WSRC-TR-2002-00459, Rev. 0 SRT-RPP-2002-00221

Xflow2_102301_1011

\begin{tabular}{|c|c|c|c|c|c|c|c|c|c|c|c|c|c|c|c|c|c|c|}
\hline DAt & TIME & Sol & $\begin{array}{c}\text { FLTRT } \\
\left({ }^{\circ} \mathrm{C}\right) \\
\mathrm{T} 2\end{array}$ & $\begin{array}{c}\mathrm{CL} \\
\text { LOOP } \\
\left({ }^{\circ} \mathrm{C}\right) \\
\text { T3 }\end{array}$ & $\begin{array}{c}\text { SL } \\
\text { LOOP } \\
\left({ }^{\circ} \mathrm{C}\right) \\
\mathrm{T} 1\end{array}$ & $\begin{array}{c}\text { UP } \\
\text { AMB } \\
\left({ }^{\circ} \mathrm{C}\right) \\
\text { T4 }\end{array}$ & $\begin{array}{c}\text { BOT } \\
\text { AMB } \\
\left({ }^{\circ} \mathrm{C}\right) \\
\text { T5 }\end{array}$ & $\begin{array}{c}\text { BOT } \\
\text { DP } \\
\text { (psid) } \\
\text { dP2 }\end{array}$ & $\begin{array}{c}\text { FLTR } \\
\text { (psig) } \\
\text { P1 }\end{array}$ & $\begin{array}{c}\text { FLTR } \\
\text { DP } \\
\text { (psid) } \\
\text { dP1 }\end{array}$ & $\begin{array}{c}\text { TOP } \\
\text { DP } \\
\text { (psig) } \\
\text { dP3 }\end{array}$ & $\begin{array}{c}\text { FLTRATE } \\
\text { (psig) } \\
\text { P2 }\end{array}$ & $\begin{array}{c}\text { BP } \\
\text { (psig) } \\
\text { P3 }\end{array}$ & $\begin{array}{c}\text { SL } \\
\text { FLOW } \\
\text { (gpm) } \\
\text { Q1 }\end{array}$ & $\begin{array}{c}\text { FLTR } \\
\text { FLOW } \\
\text { (gpm) } \\
\text { Q2 }\end{array}$ & $\begin{array}{c}\text { Temp } \\
\text { corr flow } \\
\left(\mathrm{gpm} / \mathrm{ft}^{2}\right)\end{array}$ & & Avg \\
\hline$/ 23 / 01$ & $10: 55$ & 0 & 22.7 & 22.0 & 23.1 & 23.4 & 22.0 & 17.0 & 20.8 & 13.6 & 3.2 & & 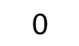 & 46.1 & 0.021 & 0.0097 & & \\
\hline & $10 \cdot 56$ & 0 & 2.7 & 2.1 & 23.4 & 23.4 & 22.1 & 41.8 & 7.0 & 4.0 & 17 & 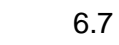 & & 4.7 & & & & \\
\hline$/ 23 / 01$ & $: 57$ & 0 & 22.8 & 22.1 & 23.7 & 23.4 & 22.2 & 50.4 & 8.5 & 1.8 & 38.0 & 9.3 & 0 & 42.6 & 0.018 & & & \\
\hline$/ 23 / 01$ & $: 58$ & 0 & 22.8 & 22.1 & 23.9 & 23.5 & 22.2 & 47.1 & 8.5 & 1.6 & 35.2 & 12.3 & & 42.4 & 0.018 & & & \\
\hline & $: 59$ & 0 & 22.1 & 22.1 & 24.2 & 23.5 & 22.2 & 54.2 & 58.3 & 1.6 & 42.5 & 4.9 & & 42.7 & 10 & & & \\
\hline & :00 & 0 & 22.3 & 22.1 & 24.4 & 23.5 & 22.2 & 54.0 & 8.2 & 1.7 & 42.3 & .9 & & 42.8 & & & & \\
\hline & & 0 & 22.4 & 22.1 & 24.6 & 23.6 & 22.3 & 54.2 & 8.9 & .5 & 42.7 & .9 & & 42.1 & 10 & & & \\
\hline 1 & :02 & 0 & 22.4 & 22.1 & 24.8 & 23.6 & 22.3 & 53.7 & 58.5 & 1.3 & 42.8 & 4.9 & & 41.9 & 0.010 & & & \\
\hline & :03 & 0 & 23.0 & 22.1 & 25.1 & 23.8 & 22.4 & 53.3 & 58.3 & 1.7 & 42.1 & 5.1 & & 42.6 & 0.010 & & & \\
\hline & 4 & 0 & 3.5 & 22.1 & 25.2 & 24.0 & 22.4 & 52.3 & 8.3 & 1.5 & 1. & 6 & & 2.3 & & & & \\
\hline & & 0 & 4.7 & 22.1 & 25.4 & 24.1 & 22.4 & 50.8 & 8.2 & .3 & 0.2 & 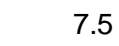 & & 2.0 & & & & \\
\hline 1 & :06 & 0 & 24.9 & 22.1 & 25.6 & 24.1 & 22.5 & 50.0 & 8.3 & 1.4 & 39.2 & 8.4 & & 42.4 & 0.010 & & & \\
\hline 1 & :07 & 0 & 25.2 & 22.1 & 25.7 & 24.2 & 22.5 & 49.8 & 8.7 & 1.2 & 38.9 & 8. & & 41.8 & & & & \\
\hline 1 & :08 & 0 & 25.3 & 22.1 & 25.9 & 24.2 & 22.6 & 49.7 & 58.3 & 3 & - & 9 & & 42.0 & 12 & & & \\
\hline 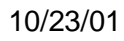 & & 0 & & 22.2 & 26.0 & 24.2 & 22.6 & 49.6 & & .1 & & & & .7 & & & & \\
\hline 4 & 0 & 0 & 25.7 & 22.2 & 26.2 & 24.3 & 22.6 & 49.6 & 8.3 & 1.2 & 9.0 & 8.9 & & 1.7 & 10 & & & \\
\hline 1 & 11 & 0 & 25.8 & 22.2 & 26.4 & 24.3 & 22.7 & 49.6 & 8.4 & 1.2 & 38.7 & 8 & & 1.9 & & & & \\
\hline 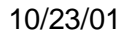 & 2 & 0 & 26.0 & 22.2 & 26.5 & 24.3 & 22.8 & 49.8 & 58.6 & 2 & 3 & $\varepsilon$ & & 2.0 & 10 & & & \\
\hline & & 0 & 6.2 & 22.2 & 26.6 & 24.4 & 22.8 & 49.5 & 2 & .2 & & & & .7 & & & & \\
\hline 1 & & 0 & 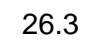 & 22.2 & 26.8 & 24.4 & 22.9 & 49.6 & .4 & .2 & 3 & & & .3 & & & & \\
\hline 1 & & 0 & 26 & 22.2 & 26.9 & 24.6 & 22.9 & 49.4 & 8.3 & 1.2 & 387 & 8.9 & & 1.3 & 0.107 & & & \\
\hline 1 & 6 & 0 & 26.6 & 22.3 & 27.0 & 24.6 & 22.9 & 50.1 & 59.0 & 1.2 & & $\varepsilon$ & & .7 & 6 & & & \\
\hline & & 0 & 6.7 & 22.3 & 27.1 & 24.7 & 23.0 & 49.3 & 8.0 & & & & & .4 & & & & \\
\hline 1 & & 0 & 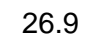 & 22.3 & 27.2 & 24.7 & 23.1 & 49.2 & .0 & & & & & 2.2 & & & & \\
\hline 1 & & 0 & 27.0 & 22.3 & 27.3 & 24.8 & 23.1 & 49.1 & .8 & & 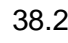 & 8 & & 2.2 & & & & \\
\hline 1 & & 0 & 271 & 22.3 & 27.4 & 24.8 & 23.1 & 49.7 & 8.5 & 1.0 & 8.8 & 9 & & 1.6 & & & & \\
\hline 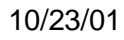 & 21 & 0 & 27.2 & 22.3 & 27.5 & 24.8 & 23.1 & 49.7 & 5 & & & & & & & & & \\
\hline / & & 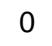 & 27.2 & 22.3 & 27.6 & 24.8 & 23.1 & 49.3 & .1 & & & & & & & & & \\
\hline & & 0 & 4 & 22.4 & 27.6 & 24.7 & 23.0 & 9.8 & 8.6 & 1.0 & 9.2 & .0 & & .5 & & & & \\
\hline & & 0 & 27 & 22.4 & 27.7 & 24.6 & 23.0 & 49.6 & 58.3 & 10.9 & 3 & 9 & & .8 & 3 & & & \\
\hline & & & & & & & & & & & & & & & & & & \\
\hline & & 0 & & 2.4 & 27 & $L_{2}$ & 22.9 & 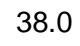 & & & & & & & & & & \\
\hline & & 0 & 7.6 & 22.4 & 27.7 & 24.6 & 22.9 & 23.9 & 6 & 6.7 & 1 & & & & & & & \\
\hline & & 0 & 27.6 & 22.4 & 27.6 & 24.5 & 22.9 & 21.7 & 30.3 & 6.2 & & & & & & & & \\
\hline$/ 23 / 01$ & :28 & 0 & 27.5 & 22.4 & 27.4 & 24.6 & 22.9 & 19.3 & 30.2 & 6.2 & & & & .8 & & & & \\
\hline 1 & 29 & 0 & 27.2 & 22.4 & 27.3 & 24.6 & 22.8 & 15 & 3 & 6.2 & 10 & & & 9.8 & & & & \\
\hline 1 & & 0 & 7.2 & 22.4 & 27.2 & 24.5 & 22.8 & 10.3 & 0 & 3.2 & 4 & & & .8 & & & & \\
\hline & & 0 & 26. & 22.4 & 27.1 & 24.5 & 22.8 & 17.5 & 30.2 & 6.3 & & 12.8 & & & & & & \\
\hline 01 & :32 & 0 & 27 & 22.5 & 27.0 & 24.4 & 22.8 & 24.1 & 30.6 & 6.3 & & & & .9 & & & & \\
\hline & & 0 & 0 & 22.5 & 26 & 24.4 & 22.7 & 23.7 & & 6.2 & & & & 9.8 & & & & \\
\hline & & 0 & & 22.5 & 26.8 & 24.4 & 22.7 & 24.0 & & .0 & & & & .9 & & & & \\
\hline & & 0 & 27.2 & 22.5 & 26.7 & 24.4 & 22.7 & 31.4 & 37.9 & .6 & & & & 3.2 & & & & \\
\hline /23/01 & $11: 36$ & 0 & 27.2 & 22.5 & 26.8 & 24.4 & 22.7 & 42.2 & 48.8 & 15.8 & 26.7 & & & 1.0 & & & & \\
\hline$/ 23 / 01$ & $1: 37$ & 0 & 27.1 & 22.5 & 26.9 & 24.4 & 22.7 & 38.9 & 5.3 & & 2 & & & 0.2 & & & & \\
\hline & & 0 & 27 & 22.5 & 27 & 24.3 & 22.6 & 38.8 & 2 & & 2 & & & 0.3 & & & & \\
\hline & & 0 & 27.1 & 22.5 & 27.0 & 24.3 & 22.6 & 38.8 & & 5.2 & 23. & & & 0.0 & & & & \\
\hline$/ 23 / 01$ & $11: 40$ & 0 & 27.1 & 22.5 & 27.1 & 24.3 & 22.5 & 38.7 & 45.0 & 15.2 & 24 & & & 0.2 & 05 & & & \\
\hline 01 & $11: 41$ & 0 & 27.1 & 22.5 & 27.2 & 24.3 & 22.5 & 32.2 & 38.3 & 15.0 & 18.0 & 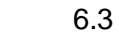 & & 9.0 & 0.086 & & 3 & \\
\hline & & 0 & 27.1 & 22.5 & 27.1 & 24.3 & 22.6 & 26.7 & 32.9 & 15.3 & 12 & & & 9.5 & & & & \\
\hline (2) & 12 & 0 & 27.1 & 22.5 & 27.1 & 24.3 & 20 & 22.5 & 0.3 & 15.4 & 7.5 & 7.9 & v & 52.0 & 0.049 & 02 & 21.6 & \\
\hline
\end{tabular}


WSRC-TR-2002-00459, Rev. 0 SRT-RPP-2002-00221

\author{
Xflow2_102301_1011
}

\begin{tabular}{|c|c|c|c|c|c|c|c|c|c|c|c|c|c|c|c|c|c|c|}
\hline DATE & TIME & Sol & $\begin{array}{c}\text { FLTRT } \\
\left({ }^{\circ} \mathrm{C}\right) \\
\text { T2 }\end{array}$ & $\begin{array}{c}\mathrm{CL} \\
\text { LOOP } \\
\left({ }^{\circ} \mathrm{C}\right) \\
\mathrm{T} 3\end{array}$ & $\begin{array}{c}\text { SL } \\
\text { LOOP } \\
\left({ }^{\circ} \mathrm{C}\right) \\
\mathrm{T} 1\end{array}$ & $\begin{array}{c}\text { UP } \\
\text { AMB } \\
\left({ }^{\circ} \mathrm{C}\right) \\
\text { T4 }\end{array}$ & $\begin{array}{c}\text { BOT } \\
\text { AMB } \\
\left({ }^{\circ} \mathrm{C}\right) \\
\text { T5 }\end{array}$ & $\begin{array}{l}\text { BOT } \\
\text { DP } \\
\text { (psid) } \\
\text { dP2 }\end{array}$ & $\begin{array}{l}\text { FLTR } \\
\text { (psig) } \\
\text { P1 }\end{array}$ & $\begin{array}{c}\text { FLTR } \\
\text { DP } \\
\text { (psid) } \\
\text { dP1 }\end{array}$ & $\begin{array}{c}\text { TOP } \\
\text { DP } \\
\text { (psig) } \\
\text { dP3 }\end{array}$ & $\begin{array}{l}\text { FLTRATE } \\
\text { (psig) } \\
\text { P2 }\end{array}$ & $\begin{array}{c}\text { BP } \\
\text { (psig) } \\
\text { P3 }\end{array}$ & $\begin{array}{c}\mathrm{SL} \\
\text { FLOW } \\
\text { (gpm) } \\
\text { Q1 }\end{array}$ & $\begin{array}{c}\text { FLTR } \\
\text { FLOW } \\
\text { (gpm) } \\
\text { Q2 }\end{array}$ & $\begin{array}{l}\text { Temp } \\
\text { corr flow } \\
\left(\mathrm{gpm} / \mathrm{ft}^{2}\right)\end{array}$ & $\begin{array}{l}\text { Axial Vel } \\
(\mathrm{ft} / \mathrm{sec})\end{array}$ & $\begin{array}{l}\text { Avg } \\
\text { TMP } \\
\text { (psid) }\end{array}$ \\
\hline$/ 23 / 01$ & $11: 44$ & 0 & 27.2 & 22.5 & 27.1 & 24.3 & 22.7 & 22.5 & 30.5 & 15.4 & 7.7 & 7.9 & 0 & 50.1 & 0.050 & 0.0206 & 20.8 & 15.1 \\
\hline$/ 23 / 01$ & $11: 45$ & 0 & 27.2 & 22.6 & 27.1 & 24.4 & 22.7 & 22.5 & 30.4 & 15.4 & 7.8 & 7.9 & 0 & 50.7 & 0.049 & 0.0202 & 21.0 & 15.2 \\
\hline o/23/01 & $11: 46$ & 0 & 27.1 & 22.6 & 27.0 & 24.4 & 22.7 & 22.7 & 30.7 & 15.3 & 8.1 & 7.9 & 0 & 50.5 & 0.050 & 0.0206 & 20.9 & 15.4 \\
\hline 0/23/01 & $11: 47$ & 0 & 27.1 & 22.6 & 27.0 & 24.5 & 22.8 & 17.0 & 24.9 & 12.7 & 4.7 & 7.9 & 0 & 45.6 & 0.039 & 0.0161 & 18.9 & 10.8 \\
\hline D/23/01 & $11: 48$ & 0 & 27.0 & 22.6 & 26.9 & 24.5 & 22.8 & 11.1 & 19.0 & 12.5 & -1.3 & 7.8 & 0 & 45.6 & 0.014 & 0.0058 & 18.9 & 4.9 \\
\hline /23/01 & $11: 49$ & 0 & 26.9 & 22.6 & 26.7 & 24.6 & 22.9 & 9.6 & 17.4 & 12.5 & -2.4 & 7.8 & 0 & 45.2 & 0.010 & 0.0042 & 18.7 & 3.6 \\
\hline 0/23/01 & $11: 50$ & 0 & 26.9 & 22.6 & 26.6 & 24.6 & 22.9 & 13.7 & 21.5 & 15.4 & -1.0 & 7.8 & 0 & 50.0 & 0.017 & 0.0 & .7 & 6.3 \\
\hline 0/23/01 & $11: 51$ & 0 & 26.8 & 22.6 & 26.6 & 24.6 & 23.0 & 14.0 & 21.9 & 15.5 & -0.8 & 7.8 & 0 & 50.1 & 0.017 & 0.0071 & 20.8 & 6.6 \\
\hline O/23/01 & $11: 52$ & 0 & 26.8 & 22.6 & 26.5 & 24.7 & 23.0 & 14.6 & 22.4 & 15.6 & -0.5 & 7.8 & 0 & 50.7 & 0.019 & 0.0080 & 21.1 & 7.0 \\
\hline /23/01 & $1: 53$ & 0 & 26.9 & 22.6 & 26.5 & 24.8 & 23.1 & 26.7 & 34.6 & 12.2 & 15.2 & 8.0 & 0 & 44.0 & 0.079 & 0. & 18.3 & 20.9 \\
\hline $0 / 2$ & :54 & 0 & 26.8 & 22.6 & 26.5 & 24.8 & 23.1 & 26.8 & 34.7 & 12.3 & 14.9 & 7.9 & c & 44.4 & 0.076 & & 4 & 0.9 \\
\hline $0 / 23 / 01$ & $11: 55$ & 0 & 26.8 & 22.6 & 26.5 & 24.8 & 23.1 & 26.8 & 34.6 & 12.4 & 15.1 & 7.9 & 0 & 44.4 & 0.076 & & .4 & 20.9 \\
\hline 0/23/01 & $11: 56$ & 0 & 26.7 & 22.7 & 26.4 & 24.9 & 23.1 & 26.8 & 34.8 & 12.4 & 14.9 & 7.9 & 0 & 44.3 & 0.075 & 0.0 & 18.4 & 20.9 \\
\hline 0/23/01 & $11: 57$ & 0 & 26.7 & 22.7 & 26.4 & 24.9 & 23.2 & 26.7 & 34.6 & 12.5 & 14.8 & 7.9 & 0 & 44.4 & 0.074 & 10 & 4 & 20.7 \\
\hline 1 & 58 & 0 & 26.7 & 22.7 & 26.4 & 25.0 & 23.2 & 27.0 & 34.9 & 12.4 & 14.9 & I & c & 44.2 & 0.074 & & $T$ & 20.9 \\
\hline $0 / 2$ & :59 & 0 & 26.7 & 22.7 & 26.4 & 25.0 & 23.3 & 26.8 & 34.8 & 12.4 & 14.9 & 7.9 & 0 & 44.6 & 0.074 & & 5 & 20.8 \\
\hline 0/23/01 & $12: 00$ & 0 & 26.7 & 22.7 & 26.4 & 25.0 & 23.3 & 26.7 & 34.6 & 12.4 & 15.0 & 7.9 & 0 & 44.2 & 0.074 & & 3 & 20.9 \\
\hline 0/23/01 & $12: 01$ & 0 & 26.6 & 22.7 & 26.4 & 25.1 & 23.3 & 26.9 & 34.8 & 12.5 & 14.8 & 7.9 & 0 & 44.1 & 0.073 & 0. & 3 & 20.8 \\
\hline 1 & :02 & 0 & 26.6 & 22.7 & 26.4 & 5.2 & 3.4 & 26.8 & 34.7 & 12.3 & 15.0 & 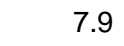 & 0 & 44.4 & 0.073 & & 4 & 20.9 \\
\hline $0 / 2$ & :03 & 0 & 26.6 & 22.7 & 26.4 & 25.2 & 3.4 & 26.9 & 34.8 & 12.5 & 14.9 & 7.9 & c & 44.3 & 0.073 & & 4 & 20.9 \\
\hline 0/23/01 & 2:04 & 0 & 26.6 & 22.8 & 25.9 & 25.2 & 23.4 & 26.7 & 34.6 & 12.5 & 15.1 & 7.9 & 0 & 44.3 & 0.072 & 06 & 4 & 20.9 \\
\hline 0/23/01 & 2:05 & 0 & 26.5 & 22.8 & 25.1 & 25.2 & 23.4 & 27.3 & 35.1 & 12.4 & 15.2 & 7.9 & 0 & 44.1 & 0.071 & & 3 & 21.2 \\
\hline //23/01 & :06 & 0 & 26.4 & 22.8 & 24.6 & 25.1 & 23.4 & 27.1 & 34.9 & 12.5 & 15.4 & 7 & 0 & 44.1 & 0.070 & & 3 & 21.2 \\
\hline 1 & 07 & 0 & 26.1 & 22.8 & 24.6 & 25.1 & 23.3 & 27.2 & 35.1 & 12.5 & 15.2 & 7.9 & c & 44.3 & 0.070 & & 4 & 21.2 \\
\hline $0 / 2$ & $: 08$ & 0 & 26.1 & 22.8 & 24.6 & 25.1 & 23.3 & 27.2 & 35.1 & 12.5 & 15.2 & 7.9 & 0 & 44.1 & 0.070 & & 3 & 21.2 \\
\hline 0/23/01 & :09 & 0 & 26.0 & 22.8 & 24.6 & 25.0 & 23.2 & 27.3 & 35.2 & 12.5 & 15.3 & 7.9 & 0 & 44.0 & 0.070 & & 3 & 21.3 \\
\hline O/23/01 & $: 10$ & 0 & 26.0 & 22.8 & 24.7 & 24.9 & 23.2 & 27.2 & 35.1 & 12.5 & 15.2 & 7.9 & 0 & 44.3 & 0.070 & 8 & 4 & 21.2 \\
\hline 1 & 11 & 0 & 26.0 & 22.8 & 24.7 & 24.9 & 23.2 & 27.3 & 35.2 & 12.5 & 15.3 & 7 & c & 44.3 & 0.070 & & 4 & 21.3 \\
\hline $0 / 2$ & $: 12$ & 0 & 25.8 & 22.8 & 24.7 & 25.0 & 23.2 & 27.1 & 34.9 & 12.4 & 15.6 & 7.9 & c & 43.9 & 0.070 & & 2 & 21.4 \\
\hline 0/23/01 & $2: 13$ & 0 & 25.7 & 22.9 & 24.8 & 25.0 & 23.1 & 27.2 & 35.1 & 12.4 & 15.3 & 7.9 & 0 & 44.1 & 0.070 & & 3 & 21.2 \\
\hline 0/23/01 & $: 14$ & 0 & 25.6 & 22.9 & 24.8 & 25.0 & 23.1 & 27.2 & 35.1 & 12.5 & 15.3 & 7.9 & 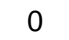 & 44.0 & 0.070 & 0. & 3 & 21.2 \\
\hline o/23/01 & 5 & 0 & 25.5 & 22.9 & 24.8 & 24.9 & 23.0 & 27.2 & 35.1 & 12.5 & 15.3 & 7 & 0 & 44.1 & 0.069 & & 18.3 & 21.3 \\
\hline $0 / 23$ & 16 & 0 & 25.4 & 22.9 & 24.8 & 24.9 & 23.0 & 27.2 & 35.1 & 12.5 & 15.0 & $t$ & c & 44.6 & 0.069 & & 5 & 21.1 \\
\hline $0 / 2$ & $: 17$ & 0 & 25.3 & 22.9 & 24.9 & 24.9 & 23.0 & 27.3 & 35.2 & 12.4 & 15.4 & 7.9 & 0 & 44.1 & 0.069 & & 18.3 & 21.3 \\
\hline 0/23/01 & :18 & 0 & 25.3 & 22.9 & 24.9 & 25.0 & 23.0 & 27.3 & 35.1 & 12.5 & 15.5 & 7.9 & 0 & 44.3 & 0.069 & & 4 & 21.4 \\
\hline /23/01 & $: 19$ & 0 & 25.3 & 22.9 & 24.9 & 24.9 & 23.0 & 27.2 & 35.1 & 12.5 & 15.1 & 7.9 & 0 & 44.6 & 0.069 & & 5 & 21.1 \\
\hline /23 & 20 & 0 & 25.2 & 22.9 & 24.9 & 24.9 & 23.0 & 27.2 & 35.1 & 12.4 & 15.1 & 7. & 0 & 44.2 & 0.069 & & 3 & 21.2 \\
\hline $0 / 2$ & $2: 21$ & 0 & 25.2 & 22.9 & 24.9 & 24.9 & 22.9 & 27.1 & 35.0 & 12.5 & 15.1 & 7.9 & 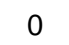 & 44.2 & 0.069 & & 18.3 & 21.1 \\
\hline 0/23/01 & 2:22 & 0 & 25.2 & 22.9 & 25.0 & 24.9 & 22.9 & 27.3 & 35.2 & 12.5 & 15.2 & 7.9 & 0 & 43.1 & 0.069 & 0.0 & 17.9 & 21.3 \\
\hline 0/23/01 & 2:23 & 0 & 25.2 & 22.9 & 24.9 & 24.9 & 22.8 & 27.2 & 35.2 & 12.5 & 15.2 & 7.9 & 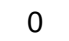 & 44.0 & 0.069 & 0.0 & 18.3 & 21.2 \\
\hline 1 & :24 & 0 & 25.2 & 22.9 & 25.0 & 24.9 & 22.8 & 27.0 & 34.8 & 12.4 & 15.2 & T & 0 & 44.3 & 0.069 & & 4 & 21.1 \\
\hline 0/23/01 & $2: 25$ & 0 & 25.2 & 22.9 & 25.0 & 24.9 & 22.8 & 27.2 & 35.1 & 12.5 & 15.1 & 7.9 & 0 & 44.3 & 0.069 & 02 & 4 & 21.2 \\
\hline 0/23/01 & |2:26 & 0 & 25.2 & 22.9 & 25.0 & 24.9 & 22.8 & 27.2 & 35.0 & 12.4 & 15.1 & 7.9 & 0 & 44.1 & 0.069 & 0.0301 & 18.3 & 21.1 \\
\hline 0/23/01 & 12:27 & 0 & 25.2 & 22.9 & 25.0 & 24.9 & 22.8 & 27.4 & 35.3 & 12.5 & 15.2 & 7.9 & 0 & 44.1 & 0.069 & 0.0 & 18.3 & 21.3 \\
\hline 0/23/01 & 28 & 0 & 25.2 & 22.9 & 25.0 & 24.9 & 22.8 & 27.1 & 34.8 & 12.5 & 15.3 & 7.9 & 0 & 44.3 & 0.068 & & 4 & 21.2 \\
\hline $10 / 23 / 01$ & 2:29 & 0 & 25.2 & 22.9 & 25.1 & 24.9 & 22.8 & 27.3 & 35.2 & 12.4 & 15.3 & 7.9 & 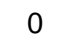 & 44.2 & 0.068 & 0.0 & 18.4 & 21.3 \\
\hline $10 / 23 / 01$ & 12:30 & 0 & 25.2 & 23.0 & 25.1 & 24.9 & 22.7 & 27.3 & 35.2 & 12.5 & 15.1 & 7.9 & 0 & 44.3 & 0.068 & 0.0296 & 18.4 & 21.2 \\
\hline 0/23/01 & 12:31 & 0 & 25.2 & 23.0 & 25.0 & 24.9 & 22.7 & 27.1 & 34.9 & 12.4 & 15.1 & 7.9 & 0 & 44.1 & 0.068 & 0.0297 & 18.3 & 21.1 \\
\hline $0 / 23 / 01$ & & 0 & 25.2 & 23.0 & 25.1 & 24.9 & 22.7 & 27.2 & 35.1 & 12.6 & 15.0 & 7.9 & 0 & 44.5 & 0.068 & & 18.5 & 21.1 \\
\hline $0 / 23 / 01$ & 12:33 & 0 & 25.3 & 23.0 & 25.1 & 24.9 & 2.7 & 27.3 & 35.1 & 12.5 & 15.2 & 7.9 & 0 & 44.3 & 0.068 & 0.0296 & 18.4 & 21.3 \\
\hline
\end{tabular}


WSRC-TR-2002-00459, Rev. 0 SRT-RPP-2002-00221

\author{
Xflow2_102301_1011
}

\begin{tabular}{ccccccccccc} 
CL & SL & UP & BOT & BOT & \multicolumn{3}{c}{ FLTR } & TOP & SL & FLTR \\
FLTRT LOOP LOOP & AMB & AMB & DP & FLTR & DP & DP & FLTRATE & BP & FLOW & FLOW
\end{tabular}

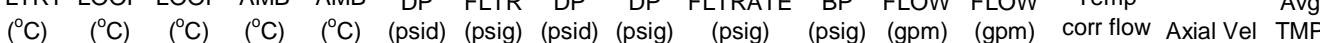

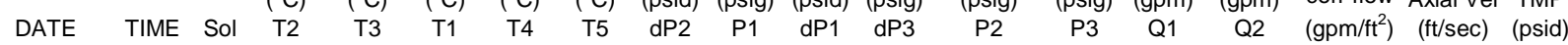

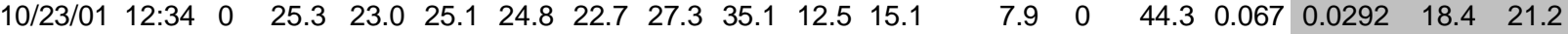

$\begin{array}{llllllllllll}10 / 23 / 01 & 12: 35 & 0 & 25.3 & 23.0 & 25.1 & 24.9 & 22.7 & 27.1 & 35.0 & 12.5 & 15.0\end{array}$

$\begin{array}{llllllllllll}10 / 23 / 01 & 12: 36 & 0 & 25.3 & 23.0 & 25.1 & 24.8 & 22.7 & 27.1 & 34.9 & 12.4 & 15.2\end{array}$

$\begin{array}{llllllllllll}10 / 23 / 01 & 12: 37 & 0 & 25.3 & 23.0 & 25.1 & 24.9 & 22.7 & 27.2 & 35.0 & 12.6 & 15.0\end{array}$

$\begin{array}{llllllllllll}10 / 23 / 01 & 12: 38 & 0 & 25.3 & 23.0 & 25.2 & 24.9 & 22.7 & 27.3 & 35.1 & 12.5 & 14.9\end{array}$

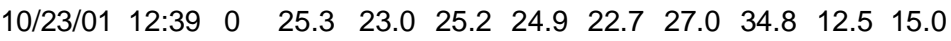

$\begin{array}{llllllllllll}10 / 23 / 01 & 12: 40 & 0 & 25.4 & 23.0 & 25.3 & 24.8 & 22.7 & 27.1 & 35.0 & 12.5 & 15.1\end{array}$

$\begin{array}{llllllllllll}10 / 23 / 01 & 12: 41 & 0 & 25.4 & 23.0 & 25.3 & 24.7 & 22.7 & 27.2 & 35.0 & 12.4 & 15.1\end{array}$

$\begin{array}{llllllllllll}10 / 23 / 01 & 12: 42 & 0 & 25.4 & 23.0 & 25.4 & 24.7 & 22.7 & 27.2 & 35.0 & 12.6 & 15.2\end{array}$

$\begin{array}{llllllllllll}10 / 23 / 01 & 12: 43 & 0 & 25.4 & 23.0 & 25.5 & 24.6 & 22.7 & 27.1 & 34.9 & 12.5 & 15.1\end{array}$

$\begin{array}{llllllllllll}10 / 23 / 01 & 12: 44 & 0 & 25.5 & 23.0 & 25.5 & 24.7 & 22.8 & 27.1 & 34.9 & 12.4 & 15.1\end{array}$

$\begin{array}{llllllllllll}10 / 23 / 01 & 12: 45 & 0 & 25.5 & 23.0 & 25.6 & 24.7 & 22.9 & 27.2 & 35.1 & 12.5 & 15.2\end{array}$

$10 / 23 / 01 \quad 12: 46 \quad 0$

$\begin{array}{lllllllll}25.5 & 23.0 & 25.6 & 24.8 & 22.9 & 27.2 & 35.0 & 12.3 & 15.2\end{array}$

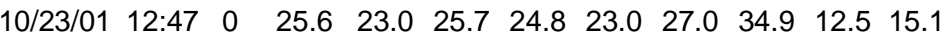

$10 / 23 / 01 \quad 12: 48 \quad 0$

$\begin{array}{lllllllll}25.7 & 23.0 & 25.7 & 24.9 & 23.1 & 27.0 & 34.8 & 12.4 & 15.2\end{array}$

$10 / 23 / 01 \quad 12: 49 \quad 0$

$\begin{array}{lllllllll}25.7 & 23.0 & 25.8 & 25.0 & 23.1 & 27.2 & 35.0 & 12.5 & 15.0\end{array}$

$\begin{array}{llllllllllll}10 / 23 / 01 & 12: 50 & 0 & 25.6 & 23.1 & 25.8 & 25.1 & 23.2 & 27.1 & 35.0 & 12.4 & 15.2\end{array}$

$\begin{array}{llllllllllll}10 / 23 / 01 & 12: 51 & 0 & 25.7 & 23.1 & 25.9 & 25.2 & 23.3 & 26.9 & 34.7 & 12.6 & 15.1\end{array}$

$10 / 23 / 01 \quad 12: 52 \quad 0$

$\begin{array}{lllllllll}25.8 & 23.1 & 25.9 & 25.3 & 23.3 & 27.1 & 34.8 & 12.5 & 15.1\end{array}$

$\begin{array}{llllllllllll}10 / 23 / 01 & 12: 53 & 0 & 25.8 & 23.1 & 26.0 & 25.3 & 23.4 & 27.2 & 35.1 & 12.4 & 15.1\end{array}$

$\begin{array}{llllllllllll}10 / 23 / 01 & 12: 54 & 0 & 25.9 & 23.1 & 26.0 & 25.4 & 23.4 & 27.3 & 35.1 & 12.4 & 15.1\end{array}$

$\begin{array}{llllllllllll}10 / 23 / 01 & 12: 55 & 0 & 25.9 & 23.1 & 26.1 & 25.4 & 23.4 & 27.2 & 35.0 & 12.4 & 15.2\end{array}$

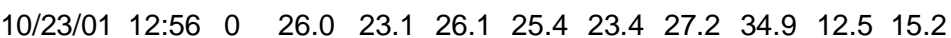

$\begin{array}{llllllllllll}10 / 23 / 01 & 12: 57 & 0 & 26.0 & 23.1 & 26.1 & 25.4 & 23.4 & 27.1 & 35.0 & 12.3 & 15.2\end{array}$

$\begin{array}{llllllllllll}10 / 23 / 01 & 12: 58 & 0 & 26.0 & 23.2 & 26.2 & 25.4 & 23.4 & 27.0 & 34.8 & 12.4 & 15.3\end{array}$

$\begin{array}{llllllllllll}10 / 23 / 01 & 12: 59 & 0 & 26.0 & 23.2 & 26.2 & 25.4 & 23.3 & 27.2 & 35.0 & 12.6 & 15.0\end{array}$

$\begin{array}{llllllllllll}10 / 23 / 01 & 13: 00 & 0 & 26.2 & 23.2 & 26.3 & 25.3 & 23.3 & 27.0 & 34.9 & 12.4 & 15.1\end{array}$

$\begin{array}{llllllllllll}10 / 23 / 01 & 13: 01 & 0 & 26.2 & 23.2 & 26.3 & 25.3 & 23.3 & 27.2 & 35.1 & 12.4 & 15.1\end{array}$

$\begin{array}{llllllllllll}10 / 23 / 01 & 13: 02 & 0 & 26.2 & 23.2 & 26.4 & 25.3 & 23.3 & 27.1 & 35.0 & 12.4 & 15.3\end{array}$

$\begin{array}{llllllllllll}10 / 23 / 01 & 13: 03 & 0 & 26.3 & 23.2 & 26.4 & 25.3 & 23.3 & 26.9 & 34.8 & 12.4 & 15.1\end{array}$

$\begin{array}{llllllllllll}10 / 23 / 01 & 13: 04 & 0 & 26.2 & 23.2 & 26.4 & 25.3 & 23.3 & 27.1 & 34.9 & 12.5 & 15.0\end{array}$

$\begin{array}{llllllllllll}10 / 23 / 01 & 13: 05 & 0 & 26.3 & 23.2 & 26.4 & 25.3 & 23.2 & 27.2 & 35.0 & 12.5 & 15.1\end{array}$

$\begin{array}{llllllllllll}10 / 23 / 01 & 13: 06 & 0 & 26.4 & 23.2 & 26.5 & 25.3 & 23.2 & 27.2 & 35.1 & 12.4 & 15.4\end{array}$

$\begin{array}{llllllllllll}10 / 23 / 01 & 13: 07 & 0 & 26.4 & 23.3 & 26.5 & 25.3 & 23.2 & 27.1 & 35.0 & 12.4 & 15.0\end{array}$

$\begin{array}{llllllllllll}10 / 23 / 01 & 13: 08 & 0 & 26.4 & 23.2 & 26.5 & 25.2 & 23.2 & 27.0 & 34.9 & 12.4 & 15.0\end{array}$

$\begin{array}{llllllllllll}10 / 23 / 01 & 13: 09 & 0 & 26.6 & 23.2 & 26.6 & 25.3 & 23.2 & 27.4 & 35.2 & 12.4 & 15.2\end{array}$

$\begin{array}{llllllllllll}10 / 23 / 01 & 13: 10 & 0 & 26.5 & 23.3 & 26.6 & 25.2 & 23.2 & 27.2 & 35.0 & 12.4 & 15.1\end{array}$

$10 / 23 / 01 \quad 13: 11 \quad 0$

$\begin{array}{lllllllll}26.5 & 23.3 & 26.7 & 25.2 & 23.2 & 27.0 & 34.9 & 12.5 & 15.1\end{array}$

$10 / 23 / 01 \quad 13: 12 \quad 0$ $10 / 23 / 01 \quad 13: 13 \quad 0$ $10 / 23 / 01 \quad 13: 14 \quad 0$ $10 / 23 / 01 \quad 13: 15 \quad 0$ $10 / 23 / 01 \quad 13: 16 \quad 0$ $10 / 23 / 01 \quad 13: 17 \quad 0$ $10 / 23 / 01 \quad 13: 18 \quad 0$ $10 / 23 / 01 \quad 13: 19 \quad 0$ $10 / 23 / 01 \quad 13: 20 \quad 0$ $10 / 23 / 01 \quad 13: 21 \quad 0$

10/23/01 13:22 0

$\begin{array}{lllllllll}26.6 & 23.3 & 26.7 & 25.2 & 23.1 & 27.2 & 35.1 & 12.4 & 15.2\end{array}$ $\begin{array}{lllllllll}26.7 & 23.3 & 26.7 & 25.2 & 23.1 & 27.0 & 34.8 & 12.4 & 15.3\end{array}$ $\begin{array}{lllllllll}26.6 & 23.3 & 26.7 & 25.2 & 23.1 & 27.1 & 34.9 & 12.5 & 15.1\end{array}$ $\begin{array}{lllllllll}26.7 & 23.3 & 26.8 & 25.1 & 23.1 & 27.1 & 34.9 & 12.4 & 15.0\end{array}$ $\begin{array}{lllllllll}26.8 & 23.3 & 26.8 & 25.2 & 23.1 & 27.2 & 35.0 & 12.4 & 15.2\end{array}$ $\begin{array}{lllllllll}26.8 & 23.3 & 26.8 & 25.2 & 23.1 & 27.1 & 34.9 & 12.5 & 15.2\end{array}$ $\begin{array}{lllllllll}26.8 & 23.3 & 26.8 & 25.2 & 23.0 & 27.2 & 35.0 & 12.5 & 15.1\end{array}$ $\begin{array}{lllllllll}26.8 & 23.3 & 26.9 & 25.2 & 23.0 & 27.0 & 34.7 & 12.3 & 15.0\end{array}$ $\begin{array}{lllllllll}26.7 & 23.3 & 26.9 & 25.1 & 23.0 & 26.9 & 34.8 & 12.4 & 15.1\end{array}$ $\begin{array}{lllllllll}26.8 & 23.3 & 26.9 & 25.1 & 23.0 & 27.1 & 34.9 & 12.4 & 15.2\end{array}$ $\begin{array}{lllllllll}26.9 & 23.3 & 26.9 & 25.1 & 22.9 & 27.2 & 35.1 & 12.4 & 15.2\end{array}$

$\begin{array}{lllllll}7.9 & 0 & 43.9 & 0.068 & 0.0296 & 18.2 & 21.1\end{array}$

$\begin{array}{llllllll}7.9 & 0 & 44.3 & 0.068 & 0.0296 & 18.4 & 21.1\end{array}$

$\begin{array}{llllllll}7.9 & 0 & 44.2 & 0.067 & 0.0291 & 18.4 & 21.1\end{array}$

$\begin{array}{lllllll}7.9 & 0 & 44.0 & 0.067 & 0.0291 & 18.3 & 21.1\end{array}$

$\begin{array}{llllllll}7.9 & 0 & 44.4 & 0.067 & 0.0291 & 18.4 & 21.0\end{array}$

$\begin{array}{llllllll}7.9 & 0 & 44.2 & 0.068 & 0.0295 & 18.4 & 21.1\end{array}$

$\begin{array}{lllllll}7.9 & 0 & 44.1 & 0.068 & 0.0294 & 18.3 & 21.1\end{array}$

$\begin{array}{llllllll}7.9 & 0 & 44.4 & 0.068 & 0.0294 & 18.4 & 21.2\end{array}$

$\begin{array}{llllllll}7.9 & 0 & 44.2 & 0.068 & 0.0293 & 18.4 & 21.1\end{array}$

$\begin{array}{lllllll}7.9 & 0 & 44.2 & 0.068 & 0.0293 & 18.3 & 21.1\end{array}$

$\begin{array}{llllllll}7.9 & 0 & 44.2 & 0.068 & 0.0292 & 18.3 & 21.2\end{array}$

$\begin{array}{llllllll}7.9 & 0 & 44.3 & 0.068 & 0.0292 & 18.4 & 21.2\end{array}$

$\begin{array}{llllllll}7.9 & 0 & 44.1 & 0.068 & 0.0291 & 18.3 & 21.0\end{array}$

$\begin{array}{llllllll}7.9 & 0 & 44.1 & 0.068 & 0.0291 & 18.3 & 21.1\end{array}$

$\begin{array}{llllllll}7.9 & 0 & 44.2 & 0.068 & 0.0291 & 18.3 & 21.1\end{array}$

$\begin{array}{llllllll}7.9 & 0 & 44.1 & 0.068 & 0.0290 & 18.3 & 21.1\end{array}$

$\begin{array}{llllllll}7.9 & 0 & 44.2 & 0.067 & 0.0285 & 18.4 & 21.0\end{array}$

$\begin{array}{llllllll}7.9 & 0 & 44.2 & 0.068 & 0.0289 & 18.3 & 21.1\end{array}$

$\begin{array}{llllllll}7.9 & 0 & 44.3 & 0.068 & 0.0289 & 18.4 & 21.1\end{array}$

$\begin{array}{llllllll}7.9 & 0 & 44.2 & 0.068 & 0.0289 & 18.3 & 21.2\end{array}$

$\begin{array}{llllllll}7.9 & 0 & 43.9 & 0.068 & 0.0288 & 18.2 & 21.2\end{array}$

$\begin{array}{llllllll}7.9 & 0 & 44.8 & 0.068 & 0.0288 & 18.6 & 21.2\end{array}$

$\begin{array}{llllllll}7.9 & 0 & 44.2 & 0.068 & 0.0288 & 18.4 & 21.2\end{array}$

$\begin{array}{llllllll}7.9 & 0 & 44.3 & 0.068 & 0.0287 & 18.4 & 21.2\end{array}$

$\begin{array}{llllllll}7.9 & 0 & 44.3 & 0.068 & 0.0287 & 18.4 & 21.1\end{array}$

$\begin{array}{llllllll}7.9 & 0 & 44.2 & 0.068 & 0.0287 & 18.3 & 21.1\end{array}$

$\begin{array}{llllllll}7.9 & 0 & 44.5 & 0.068 & 0.0286 & 18.5 & 21.1\end{array}$

$\begin{array}{llllllll}7.9 & 0 & 44.0 & 0.068 & 0.0286 & 18.3 & 21.2\end{array}$

$\begin{array}{llllllll}7.9 & 0 & 44.2 & 0.068 & 0.0286 & 18.3 & 21.0\end{array}$

$\begin{array}{llllllll}7.9 & 0 & 44.4 & 0.068 & 0.0285 & 18.4 & 21.0\end{array}$

$\begin{array}{llllllll}7.9 & 0 & 44.2 & 0.068 & 0.0285 & 18.3 & 21.1\end{array}$

$\begin{array}{llllllll}7.9 & 0 & 44.1 & 0.068 & 0.0285 & 18.3 & 21.3\end{array}$

$\begin{array}{llllllll}7.9 & 0 & 44.1 & 0.068 & 0.0285 & 18.3 & 21.1\end{array}$

$\begin{array}{lllllll}7.9 & 0 & 44.2 & 0.068 & 0.0285 & 18.3 & 21.0\end{array}$

$\begin{array}{llllllll}7.9 & 0 & 44.3 & 0.068 & 0.0284 & 18.4 & 21.3\end{array}$

$\begin{array}{llllllll}7.9 & 0 & 44.3 & 0.068 & 0.0284 & 18.4 & 21.2\end{array}$

$\begin{array}{lllllll}7.9 & 0 & 44.3 & 0.068 & 0.0283 & 18.4 & 21.1\end{array}$ Average $18.3 \quad 21.1$

$\begin{array}{lllllll}7.9 & 0 & 44.3 & 0.071 & 0.0296 & 18.4 & 21.2\end{array}$

$\begin{array}{lllllll}7.9 & 0 & 44.3 & 0.068 & 0.0283 & 18.4 & 21.2\end{array}$

$\begin{array}{lllllll}7.9 & 0 & 44.4 & 0.068 & 0.0283 & 18.4 & 21.1\end{array}$

$\begin{array}{lllllll}7.9 & 0 & 44.3 & 0.068 & 0.0283 & 18.4 & 21.0\end{array}$

$\begin{array}{lllllll}7.9 & 0 & 44.2 & 0.069 & 0.0287 & 18.3 & 21.2\end{array}$

$\begin{array}{lllllll}7.9 & 0 & 44.2 & 0.070 & 0.0291 & 18.3 & 21.1\end{array}$

$\begin{array}{lllllll}7.9 & 0 & 44.1 & 0.068 & 0.0282 & 18.3 & 21.1\end{array}$

$\begin{array}{lllllll}7.9 & 0 & 44.4 & 0.069 & 0.0286 & 18.4 & 21.0\end{array}$

$\begin{array}{lllllll}7.9 & 0 & 44.1 & 0.068 & 0.0282 & 18.3 & 21.0\end{array}$

$\begin{array}{lllllll}7.9 & 0 & 44.2 & 0.068 & 0.0281 & 18.3 & 21.1\end{array}$

$\begin{array}{lllllll}7.9 & 0 & 44.2 & 0.069 & 0.0285 & 18.4 & 21.2\end{array}$ 
WSRC-TR-2002-00459, Rev. 0 SRT-RPP-2002-00221

Xflow2_102301_1011

\begin{tabular}{|c|c|c|c|c|c|c|c|c|c|c|c|c|c|c|c|c|c|c|}
\hline DATE & TIME & Sol & $\begin{array}{l}\left({ }^{\circ} \mathrm{C}\right) \\
\mathrm{T} 2\end{array}$ & $\begin{array}{c}\mathrm{CL} \\
\text { LOOP } \\
\left({ }^{\circ} \mathrm{C}\right) \\
\text { T3 }\end{array}$ & $\begin{array}{c}\text { SL } \\
\text { LOOP } \\
\left({ }^{\circ} \mathrm{C}\right) \\
\mathrm{T} 1\end{array}$ & $\begin{array}{c}\text { UP } \\
\text { AMB } \\
\left({ }^{\circ} \mathrm{C}\right) \\
\mathrm{T} 4\end{array}$ & $\begin{array}{c}\text { BOT } \\
\text { AMB } \\
\left({ }^{\circ} \mathrm{C}\right) \\
\text { T5 }\end{array}$ & $\begin{array}{c}\text { BOT } \\
\text { DP } \\
\text { (psid) } \\
\text { dP2 }\end{array}$ & $\begin{array}{l}\text { FLTR } \\
\text { (psig) } \\
\text { P1 }\end{array}$ & $\begin{array}{l}\text { FLTR } \\
\text { DP } \\
\text { (psid) } \\
\text { dP1 }\end{array}$ & $\begin{array}{c}\text { TOP } \\
\text { DP } \\
\text { (psig) } \\
\text { dP3 }\end{array}$ & $\begin{array}{l}\text { FLTRATE } \\
\text { (psig) } \\
\text { P2 }\end{array}$ & $\begin{array}{c}\text { BP } \\
\text { (psig) } \\
\text { P3 }\end{array}$ & $\begin{array}{l}\text { SL } \\
\text { FLOW } \\
\text { (gpm) } \\
\text { Q1 }\end{array}$ & $\begin{array}{c}\text { FLTR } \\
\text { FLOW } \\
\text { (gpm) } \\
\text { Q2 }\end{array}$ & $\begin{array}{l}\text { Temp } \\
\text { corr flow } \\
\left(\mathrm{gpm} / \mathrm{ft}^{2}\right)\end{array}$ & $\begin{array}{l}\text { Axial Vel } \\
\text { (ft/sec) }\end{array}$ & $\begin{array}{l}\text { Avg } \\
\text { TMP } \\
\text { (psid) }\end{array}$ \\
\hline 10/23/01 & $13: 23$ & 0 & 26.9 & 23.3 & 27.0 & 25.0 & 22.9 & 27.0 & 34.9 & 12.5 & 15.0 & 7.9 & 0 & 44.4 & 0.068 & 0.0281 & 18.4 & 21.0 \\
\hline /23/01 & $13: 24$ & 0 & 26.8 & 23.3 & 27.0 & 25.1 & 23.0 & 27.2 & 34.9 & 12.4 & 14.9 & 7.9 & 0 & 44.2 & 0.069 & 0.0285 & 18.3 & 11 \\
\hline /23/01 & $13: 25$ & 0 & 26.9 & 23.3 & 27.0 & 25.0 & 23.0 & 27.1 & 34.8 & 12.3 & 15.1 & 7.9 & 0 & 44.3 & 0.069 & 0.0285 & 18.4 & 1.1. \\
\hline 0/23/01 & $13: 26$ & 0 & 27.0 & 23.3 & 27.0 & 25.0 & 23.0 & 21.8 & 34.8 & 12.4 & 9.9 & 13.1 & 0 & 44.3 & 0.010 & 0.0041 & 18.4 & 15.8 \\
\hline & $13: 27$ & 0 & 26.9 & 23.4 & 27.1 & 25.1 & 23.0 & 10.2 & 30.9 & 11.1 & -0.5 & & 0 & 41.6 & & & & 8 \\
\hline 0/23/01 & $13: 28$ & 0 & 27.0 & 23.4 & 27.0 & 25.1 & 23.0 & 8.9 & 30.0 & 11.8 & -2.1 & 21.0 & 0 & 42.7 & 0.010 & 0.0041 & 17.7 & 3.4 \\
\hline /23/01 & $13: 29$ & 0 & 27.0 & 23.3 & 27.0 & 25.1 & 22.9 & 16.9 & 30.0 & 11.5 & 5.7 & 13.1 & 0 & 42.4 & 0.010 & 0.0041 & 17.6 & 113 \\
\hline 0/23/01 & $13: 30$ & 0 & 27.1 & 23.3 & 27.0 & 25.1 & 22.9 & 21.3 & 30.2 & 11.7 & 9.9 & 8.9 & 0 & 42.5 & 0.121 & 0.0500 & 17.7 & 15.6 \\
\hline 0/23/01 & 13:31 & 0 & 27.1 & 23.4 & 27.0 & 25.1 & 22.9 & 21.9 & 30.1 & 11.4 & 11.1 & 8.3 & 0 & 42.1 & 0.082 & 0.0339 & 17.5 & 16.5 \\
\hline & $13: 32$ & 0 & 27.1 & 23.4 & 27.0 & 25.1 & 22.9 & 22.1 & 30.3 & 11.4 & 11.2 & .2 & 0 & 42.1 & 0.077 & & & 66 \\
\hline$/ 23 / 01$ & $13: 33$ & 0 & 27.1 & 23.4 & 27.0 & 25.1 & 23.0 & 21.9 & 30.1 & 11.2 & 11.1 & 8.2 & 0 & 42.0 & 0.074 & 0.0306 & 17.4 & 16.5 \\
\hline /23/01 & $13: 34$ & 0 & 27.1 & 23.4 & 26.9 & 25.1 & 23.0 & 22.2 & 30.4 & 11.5 & 11.2 & 8.2 & 0 & 42.0 & 0.072 & 0.0298 & 17.4 & 16.7 \\
\hline & 13:35 & 0 & 27.1 & 23.4 & 26.9 & 25.1 & 22.9 & 21.9 & 30.1 & 11.4 & 11.1 & 82 & 0 & 42.0 & 0.070 & & 7.4 & 165 \\
\hline 11 & $13: 36$ & 0 & 27.0 & 23.4 & 27.0 & 25.1 & 22.9 & 22.2 & 30.4 & 11.4 & 11.2 & 8.1 & 0 & 42.0 & 0.069 & 85 & 17.4 & 16.7 \\
\hline 1 & 13:37 & 0 & 27.1 & 23.4 & 26.9 & 25.1 & 22.9 & 22.2 & 30.3 & 11.5 & 11.2 & 8.1 & 0 & 42.1 & 0.068 & 81 & 17.5 & 16.7 \\
\hline & $13: 38$ & 0 & 27.1 & 23.4 & 26.9 & 25.1 & 22.9 & 25.5 & 33.8 & 7.9 & 18.0 & 3 & 0 & 33.9 & 0.088 & & 14.1 & 21.7 \\
\hline 1 & $13: 39$ & 0 & 27.1 & 23.4 & 26.9 & 25.1 & 22.9 & 35.4 & 43.9 & 8.0 & 28.0 & 8.6 & 0 & 34.3 & 0.111 & 60 & 14.2 & 31.7 \\
\hline & $13: 40$ & 0 & 27.1 & 23.4 & 27.0 & 25.1 & 22.9 & 37.1 & 45.5 & 6.4 & 31.3 & 8.5 & & 30.1 & 67 & & .5 & \\
\hline 1 & $13: 41$ & 0 & 27.1 & 23.4 & 26.9 & 25.1 & 22.9 & 37.1 & 45.4 & 6.4 & 31.0 & 8.4 & 0 & 29.9 & 0.101 & 18 & 12.4 & 34.1 \\
\hline 1 & $13: 42$ & 0 & 27.1 & 23.4 & 27.0 & 25.1 & 22.9 & 36.9 & 45.3 & 6.4 & 31. & 8.4 & & 30.2 & 0.098 & 0. & 12 & 34. \\
\hline & $13: 43$ & 0 & 27.1 & 23.4 & 27.0 & 25.1 & 22.9 & 36.9 & 45.2 & 6.3 & 31.1 & 8.3 & 0 & 30.0 & 0.095 & 92 & 12.5 & 34.0 \\
\hline & $13: 44$ & 0 & 27.2 & 23.4 & 27.0 & 25.1 & 22.9 & 23.6 & 31.7 & 4.7 & 19.3 & 8.1 & 0 & 25.0 & 0.064 & 64 & 10.4 & 21.5 \\
\hline 1 & $13: 45$ & 0 & 27.2 & 23.4 & 27.0 & 25.1 & 22.9 & 21.9 & 30.0 & 6.3 & 16.3 & 8.0 & & 30.1 & 0. & & .5 & 19. \\
\hline & $13: 46$ & 0 & 27.2 & 23.4 & 26.9 & 25.1 & 22.9 & 22.2 & 30.2 & 6.4 & 16. & 8.0 & & 30.0 & 0. & & & 19.3 \\
\hline 1 & $13: 47$ & 0 & 27.1 & 23.4 & 26.9 & 25.1 & 22.9 & 22.1 & 30.2 & 6.3 & 16.4 & 8.0 & 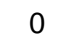 & 29.8 & 0.056 & 32 & 12.4 & 19.3 \\
\hline & $13: 48$ & 0 & 27.1 & 23.4 & 26.8 & 25.2 & 22.9 & 5.6 & 13.6 & 6.4 & -0.4 & 7.8 & 0 & 29.8 & 13 & 0 & 12.4 & 6 \\
\hline & $13: 49$ & 0 & 27.1 & 23.4 & 26.7 & 25.1 & 22.9 & 6.8 & 14.7 & 7.3 & 0.0 & 7.8 & & 32.1 & 0.013 & & 13.3 & 4 \\
\hline 1 & $13: 50$ & 0 & 27.0 & 23.4 & 26.6 & 25.2 & 22.9 & 7.2 & 15.1 & 6.5 & 1.2 & 7.8 & & 30.3 & 17 & 71 & 6 & 4.2 \\
\hline & & 0 & 27.0 & 23.4 & 26.5 & 25.1 & 22.9 & 7.2 & 15.1 & 6. & 1.2 & & & & & & & \\
\hline & $13: 52$ & 0 & 26.9 & 3.4 & 26.4 & 25.1 & 22.9 & 7.2 & 15.1 & 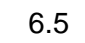 & 1.2 & .8 & & 30.3 & 0.017 & 71 & 12.6 & 4.2 \\
\hline & $13: 53$ & 0 & 26.9 & 23.4 & 26.3 & 25.1 & 22.9 & 7.1 & 15.0 & 6.5 & 1.3 & 8 & 0 & 30.2 & 0.017 & 72 & 12.5 & 4.2 \\
\hline & & 0 & 26.9 & 3.4 & 26.2 & 25.1 & 22.9 & 7.2 & 15.2 & 65 & 1.2 & & & & 0.017 & & & \\
\hline & $13: 55$ & 0 & 26.9 & 23.4 & 26.1 & 25.1 & 22.8 & 7.2 & 15.1 & 6.5 & 1.2 & 8 & 0 & 30.3 & 0.017 & 72 & 6 & 4.2 \\
\hline & 13: & 0 & 26.8 & 23.4 & 26.0 & 25.1 & 22.8 & 7.3 & 15.2 & 6.6 & 1.2 & & & 30.3 & & & & \\
\hline & $13: 5$ & 0 & 26.8 & 3.4 & 25.9 & 25.2 & 22.8 & 7.3 & 15.2 & 6.5 & 1. & & & 30.3 & 7 & & 12 & \\
\hline 11 & 13:58 & 0 & 26.8 & 23.5 & 25.9 & 25.2 & 22.8 & 7.2 & 15.1 & 6.5 & 1.2 & 8 & 0 & 30.3 & 0.017 & 72 & 12.6 & 4.2 \\
\hline & & 0 & 26.7 & .5 & 25.8 & 25.1 & & 7.3 & 15.2 & - & 1. & & & 1.2 & & & & \\
\hline & $14: 00$ & U & 26.6 & 3.4 & 25.7 & 5.2 & 22.7 & 7.3 & 15.2 & 6.5 & 1.1 & & 0 & 30.2 & 17 & 73 & 12.5 & \\
\hline & $14:$ & 0 & 26.5 & 23.4 & 25.6 & 25.1 & 22.7 & 7.2 & 15.1 & 6.5 & 1.2 & & & 30.2 & 0. & & 5 & \\
\hline & & 0 & 26.4 & & & & & 5 & & & & & & & & & & \\
\hline & $14: 03$ & 0 & 26.4 & 23.5 & 25.4 & 25.2 & 22.8 & 7.2 & 15.1 & 2.1 & 5.7 & 7.9 & 0 & 14.9 & 0.025 & 08 & 6.2 & 4 \\
\hline & & 0 & 26.3 & 3.5 & 25.4 & 25.2 & & 7.1 & 15.0 & 2.3 & 5.2 & & & 4 & & & & \\
\hline & 14 & 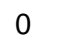 & 26.2 & & 25.3 & & 22.8 & 7.2 & 15.1 & & 5.4 & & 0 & 15.4 & & & & \\
\hline & $14: 06$ & 0 & 26.1 & 23.5 & 25.2 & 25.2 & 22.8 & 7.1 & 15.1 & 2.2 & 5.4 & & & 15.3 & 0.025 & & 4 & \\
\hline & & 0 & 26. & 23.5 & & & & & & & . & & & 15.3 & & & & \\
\hline & $14: 08$ & 0 & 26.0 & 23.5 & 25.0 & 25.2 & 22.8 & 7.5 & 15.3 & 2.3 & 5.3 & .9 & 0 & 15.3 & 0.025 & 0.0109 & .4 & 4 \\
\hline & 14: & 0 & 25.9 & 3.5 & & 25.2 & & 7.2 & & 2.2 & 5. & & & 15.3 & 0.025 & & & \\
\hline & 1 & 0 & 25.8 & 20.5 & & & & 1.5 & & 2.3 & b. & & 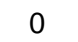 & .2 & 26 & & & \\
\hline & & 0 & 25. & & & & & 7.0 & & 2. & 5. & & 0 & 15.3 & & & 6.4 & \\
\hline & & & & & & & & & & & 24 & & & & & & 3 & \\
\hline
\end{tabular}


WSRC-TR-2002-00459, Rev. 0 SRT-RPP-2002-00221

Xflow2_102301_1011

\begin{tabular}{|c|c|c|c|c|c|c|c|c|c|c|c|c|c|c|c|c|c|c|}
\hline DATE & TIME & Sol & $\begin{array}{c}\text { FLTRT } \\
\left({ }^{\circ} \mathrm{C}\right) \\
\text { T2 }\end{array}$ & $\begin{array}{c}\mathrm{CL} \\
\text { LOOP } \\
\left({ }^{\circ} \mathrm{C}\right) \\
\text { T3 }\end{array}$ & $\begin{array}{c}\text { SL } \\
\text { LOOP } \\
\left({ }^{\circ} \mathrm{C}\right) \\
\mathrm{T} 1\end{array}$ & $\begin{array}{c}\text { UP } \\
\text { AMB } \\
\left({ }^{\circ} \mathrm{C}\right) \\
\text { T4 }\end{array}$ & $\begin{array}{c}\text { BOT } \\
\text { AMB } \\
\left({ }^{\circ} \mathrm{C}\right) \\
\text { T5 }\end{array}$ & $\begin{array}{c}\text { BOT } \\
\text { DP } \\
\text { (psid) } \\
\text { dP2 }\end{array}$ & $\begin{array}{l}\text { FLTR } \\
\text { (psig) } \\
\text { P1 }\end{array}$ & $\begin{array}{l}\text { FLTR } \\
\text { DP } \\
\text { (psid) } \\
\text { dP1 }\end{array}$ & $\begin{array}{l}\text { TOP } \\
\text { DP } \\
\text { (psig) } \\
\text { dP3 }\end{array}$ & $\begin{array}{l}\text { FLTRATE } \\
\text { (psig) } \\
\text { P2 }\end{array}$ & $\begin{array}{c}\mathrm{BP} \\
\text { (psig) } \\
\text { P3 }\end{array}$ & $\begin{array}{c}\mathrm{SL} \\
\text { FLOW } \\
\text { (gpm) } \\
\text { Q1 }\end{array}$ & $\begin{array}{c}\text { FLTR } \\
\text { FLOW } \\
\text { (gpm) } \\
\text { Q2 }\end{array}$ & $\begin{array}{c}\text { Temp } \\
\text { corr flow } \\
\left(\mathrm{gpm} / \mathrm{ft}^{2}\right)\end{array}$ & $\begin{array}{c}\text { Axial Vel } \\
(\mathrm{ft} / \mathrm{sec})\end{array}$ & $\begin{array}{l}\text { Avg } \\
\text { TMP } \\
\text { (psid) }\end{array}$ \\
\hline 0/23/01 & $14: 13$ & 0 & 25.6 & 23.4 & 24.6 & 25.2 & 22.7 & 7.1 & 15.0 & 2.2 & 5.6 & 7.9 & 0 & 15.3 & 0.025 & 0.0110 & 6.3 & 6.3 \\
\hline /23/01 & $14: 14$ & 0 & 25.5 & 23.5 & 24.6 & 25.2 & 22.7 & 7.6 & 15.6 & 2.2 & 56 & 7.9 & 0 & 15.1 & 0.026 & & 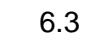 & 8.6 \\
\hline 0/23/01 & $14: 15$ & 0 & 25.5 & 23.5 & 24.5 & 25.2 & 22.7 & 7.5 & 15.5 & 2.2 & 5.5 & 7.9 & 0 & 15.0 & 0.026 & 0.0115 & 6.2 & 6.5 \\
\hline 0/23/01 & $14: 16$ & 0 & 25.4 & 23.5 & 24.5 & 25.1 & 22.7 & 7.5 & 15.4 & 2.2 & 5.6 & 79 & 0 & 15.1 & 0.026 & 0.0115 & 6.3 & 6.5 \\
\hline /23/01 & $14: 17$ & 0 & 25.3 & 23.5 & 24.5 & 25.1 & 22.7 & 7.2 & 15.1 & 2.2 & 5.5 & 7.9 & 0 & 15.1 & 0.026 & 0.0115 & 8 & 6.4 \\
\hline $10 / 23 / 01$ & $14: 18$ & 0 & 25.3 & 23.5 & 24.4 & 25.1 & 22.7 & 7.1 & 15.0 & 2.2 & 5.3 & 7.9 & 0 & 15.1 & 0.026 & 0.0116 & .3 & 6.2 \\
\hline & $14: 19$ & 0 & 25.2 & & 24.3 & 25.0 & 22.7 & 7.2 & 15.2 & 2.2 & 5.7 & 9 & 0 & 15.0 & & & & 4 \\
\hline /23/01 & $14: 20$ & 0 & 25.2 & 23.5 & 24.3 & 25.0 & 22.7 & 13.5 & 21.5 & 2.9 & 11.0 & 8.0 & 0 & 17.9 & 0.042 & 0.0187 & .4 & 12.2 \\
\hline 0/23/01 & $14: 21$ & 0 & 25.1 & 23.5 & 24.2 & 25.0 & 22.7 & 2.3 & 10.3 & 1.4 & 0.5 & 7.8 & 0 & 12.3 & 0.010 & 0.0045 & 5.1 & 1.4 \\
\hline & $14: 22$ & 0 & 25.0 & 23.5 & 24.1 & 25.0 & 22.7 & 1.9 & 9.8 & 1.4 & 0 & 78 & & 10.8 & 0.012 & & & \\
\hline 10/23/01 & $14: 23$ & 0 & 25.0 & 23.5 & 24.1 & 25.0 & 22.7 & 6.3 & 14.2 & 1.1 & 5.6 & 7.9 & 0 & 9.0 & 0.025 & 0.0112 & .7 & 5.9 \\
\hline 1 & $14: 24$ & 0 & 24.9 & 3.5 & 24.0 & 25.0 & 22.7 & 6.9 & 14.8 & 1.2 & 6.0 & 7.9 & 0 & 9.6 & 0.027 & & & .5 \\
\hline 1 & $14: 25$ & 0 & 24.9 & 23.5 & 24.0 & 25.0 & 22.7 & 6.9 & 14.8 & 1.3 & 6.1 & 7.9 & 0 & 10.1 & 0.027 & 0.0121 & 4.2 & .5 \\
\hline $0 / 23$ & $14: 26$ & 0 & 24.8 & 23.5 & 23.9 & 25.0 & 22.7 & 7.0 & 14.9 & 1.3 & 5.9 & 7.9 & 0 & 10.1 & 0.027 & 22 & 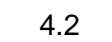 & 5.4 \\
\hline & $14: 27$ & 0 & 24.8 & 23.5 & 23.9 & 25.0 & 22.7 & 7.0 & 14.9 & 1.3 & 6. & 9 & & 10.1 & 0.027 & & & \\
\hline$/ 2$ & $14: 28$ & 0 & 24.7 & 23.5 & 23.8 & 24.9 & 22.7 & 14.7 & 22.6 & 1.4 & 13.6 & .0 & 0 & 11.3 & 0.046 & & 4.7 & 4.1 \\
\hline 1 & $14: 29$ & 0 & 24.7 & 23.5 & 23.8 & 24.9 & 22.6 & 19.0 & 27.1 & 1.3 & 18.2 & 1 & 0 & 10.1 & 0.059 & 66 & 4.2 & 18.6 \\
\hline & $14: 30$ & 0 & 24.8 & 23.5 & 23.8 & 24.9 & 22.6 & 21.4 & 29.5 & 1.3 & 20.7 & 8.1 & 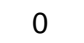 & 10.2 & 0.063 & & 2 & 21.1 \\
\hline$/ 2$ & $14: 31$ & 0 & 24.7 & 23.5 & 23.8 & 24.9 & 22.6 & 21.8 & 29.9 & 1.3 & 20.8 & .1 & 0 & 10.1 & 0.060 & 71 & 4.2 & 21.3 \\
\hline $1 / 2$ & $14: 32$ & 0 & 24.7 & 23.5 & 23.8 & 25.0 & 22.6 & 21.9 & 30.0 & 1.3 & 20. & 8 & & 10.0 & 0.058 & & & 21.2 \\
\hline ) & $14: 33$ & 0 & 24.6 & 3.5 & 23.8 & 24.9 & 22.6 & 21.9 & 29.9 & 1.3 & 20. & .0 & 0 & 10.0 & 0.056 & & & 21.4 \\
\hline 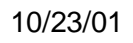 & $14: 34$ & 0 & 24.7 & 23.5 & 23.9 & 24.9 & 22.6 & 36.9 & 45.1 & 1.3 & 35. & 8.2 & & 9.9 & 0.076 & 43 & 1 & 6.4 \\
\hline & $14: 35$ & 0 & 24.6 & 3.5 & 23.9 & 24.9 & 22.6 & 37.3 & 45.5 & 1.3 & 36 & 8.1 & & 9.8 & 0.068 & & .1 & 6.8 \\
\hline 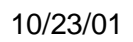 & $14: 36$ & 0 & 24.5 & 23.5 & 23.9 & 25.0 & 22.6 & 37.0 & 45.0 & 1.3 & 36. & 8.1 & 0 & 9.6 & 0.063 & 84 & 4.0 & 6.7 \\
\hline $8 / 2$ & $14: 37$ & 0 & 24.5 & 23.5 & 24.0 & 24.9 & 22.6 & 37.3 & 45.2 & 1.3 & 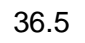 & 8.1 & & 9.4 & 0.059 & 55 & $g^{-}$ & 0 \\
\hline 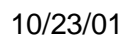 & & 0 & 24.4 & 23.5 & 24.1 & 25.0 & 22.6 & 55.9 & 63.8 & 1.6 & & & & 11.4 & & & & \\
\hline $0 / 23$ & $14: 39$ & 0 & 24.4 & 3.5 & 24.2 & 24.9 & 22.6 & 52.4 & 60.4 & 1.5 & 50 & 8.1 & & 10.4 & 60 & 68 & 43 & 1.5 \\
\hline & $14: 40$ & 0 & 24.5 & 3.5 & 24.3 & 25.0 & 22.6 & 52.4 & 60.4 & 1.5 & $\Gamma-1$ & 8.0 & 0 & 10.4 & 57 & 54 & 4.3 & 51.9 \\
\hline & $14: 41$ & 0 & 24.6 & 3.5 & 24.4 & 25.0 & 22.6 & 51.6 & 59.4 & 1.5 & 50 & & & 10.3 & 0. & & & .9 \\
\hline $0 / 2$ & $14: 42$ & 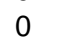 & 24.7 & 3.5 & 24.6 & 25.0 & 22.6 & 52.0 & 60.0 & 1.5 & - & 8.0 & & 10.4 & 0.053 & 34 & .3 & 51.6 \\
\hline & 14 & 0 & 24.8 & 3.5 & 24.6 & 25.0 & 22.6 & 51.9 & 59.7 & 1.5 & 5 & & & 10.4 & & & & 51.7 \\
\hline & $14: 44$ & 0 & 24.9 & 3.5 & 24.9 & 5.0 & 22.6 & 36.6 & 44.6 & 13.8 & & 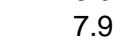 & & 43.4 & & & 180 & \\
\hline & $14: 45$ & 0 & 24.9 & 3.5 & 25.0 & 25.0 & 22.6 & 36.1 & 43.9 & 3.8 & 22.9 & 7.9 & 0 & 43.6 & 0.032 & 40 & 8.1 & 29.5 \\
\hline & & . & 250 & .5 & 25.1 & 25.0 & 22.6 & 26.9 & 34.8 & 12.7 & & & & & & & & \\
\hline & $14: 47$ & 0 & 25.0 & 3.5 & 25.2 & 25.1 & 22.7 & 22.8 & 30.7 & 10.7 & 12. & 7.9 & C & 37.6 & 0.023 & 00 & 15.6 & 17.7 \\
\hline & $14: 4$ & 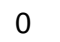 & 25.1 & 23.5 & 25.2 & 25.0 & 22.7 & 22.2 & 30.9 & 10.7 & 11. & 8.6 & & 37.7 & 0.016 & & 15 & 17.1 \\
\hline & & 0 & & & & & & 17.4 & & 98 & & & & & & & & \\
\hline & $14: 50$ & 0 & 25.2 & 3.5 & 25.3 & 25.2 & 22.8 & 19.1 & 30.5 & 9.8 & 9.8 & 1.4 & 0 & 38.2 & 0.097 & 21 & 15.9 & 14.4 \\
\hline & & 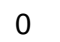 & 25.2 & .5 & 25.3 & 25.3 & 22.9 & 21.7 & 32.1 & 8.5 & 12. & & & 5.7 & & & & \\
\hline & 14 & U & 25.2 & .5 & 25.3 & 25.3 & 23.0 & 46.0 & 56.8 & 6.0 & 39 & 10 & 0 & 28.6 & 45 & 27 & 11.9 & 42.9 \\
\hline & 14 & 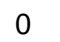 & 25.4 & 3.5 & 25.5 & 25.5 & 23.1 & 41.7 & 51.5 & 12.5 & 29 & 9. & & 44.2 & 20 & & & 35.6 \\
\hline & & 0 & - & .5 & & & & 50.6 & & 10 & & & & & & & & \\
\hline & $14: 55$ & 0 & 25.6 & .5 & 25.8 & 25.6 & 23.2 & 51.1 & 60.7 & 4.7 & 46.9 & 9. & 0 & 24.7 & 0.109 & 66 & 10.2 & 49.0 \\
\hline & 11 & 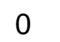 & 25.7 & 3.5 & 25.9 & 25.7 & 23.3 & 51.4 & 60.8 & 4.6 & . & & & 24.2 & 0.103 & & 10 & 48.9 \\
\hline & 14 & 0 & 2 & & & 25.8 & & 51.4 & & 4. & (1) & & 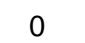 & & & & & \\
\hline /2 & $14: 58$ & 0 & 25.9 & 3.5 & 26.2 & 25.8 & 23.4 & 51.4 & 60.6 & 4.5 & 47. & & 0 & 24.0 & 0.095 & 01 & 10.0 & 49.6 \\
\hline & & 0 & 26. & 20 & & & & & & & & & & & & & & \\
\hline$/ 23 / 01$ & $15: 00$ & 0 & 26.2 & & 26.4 & & & 51.9 & 61.0 & 4.5 & 47.5 & & 0 & 24.0 & 0.089 & 373 & 10.0 & 49.7 \\
\hline & & 0 & 26.3 & & & & & & & & 47 & & & 0 & 088 & & 100 & 49.8 \\
\hline & & & & & & & & & & & & & & & & & & \\
\hline
\end{tabular}


WSRC-TR-2002-00459, Rev. 0 SRT-RPP-2002-00221

Xflow2_102301_1011

\begin{tabular}{|c|c|c|c|c|c|c|c|c|c|c|c|c|c|c|c|c|c|c|}
\hline DATE & TIME & Sol & $\begin{array}{l}\left({ }^{\circ} \mathrm{C}\right) \\
\mathrm{T} 2\end{array}$ & $\begin{array}{c}\mathrm{CL} \\
\text { LOOP } \\
\left({ }^{\circ} \mathrm{C}\right) \\
\text { T3 }\end{array}$ & $\begin{array}{c}\text { SL } \\
\text { LOOP } \\
\left({ }^{\circ} \mathrm{C}\right) \\
\mathrm{T} 1\end{array}$ & $\begin{array}{c}\text { UP } \\
\text { AMB } \\
\left({ }^{\circ} \mathrm{C}\right) \\
\text { T4 }\end{array}$ & $\begin{array}{c}\text { BOT } \\
\text { AMB } \\
\left({ }^{\circ} \mathrm{C}\right) \\
\text { T5 }\end{array}$ & $\begin{array}{c}\text { BOT } \\
\text { DP } \\
\text { (psid) } \\
\text { dP2 }\end{array}$ & $\begin{array}{c}\text { FLTR } \\
\text { (psig) } \\
\text { P1 }\end{array}$ & $\begin{array}{c}\text { FLTR } \\
\text { DP } \\
\text { (psid) } \\
\text { dP1 }\end{array}$ & $\begin{array}{c}\text { TOP } \\
\text { DP } \\
\text { (psig) } \\
\text { dP3 }\end{array}$ & $\begin{array}{c}\text { FLTRATE } \\
\text { (psig) } \\
\text { P2 }\end{array}$ & $\begin{array}{c}\text { BP } \\
\text { (psig) } \\
\text { P3 }\end{array}$ & $\begin{array}{l}\text { SL } \\
\text { FLOW } \\
\text { (gpm) } \\
\text { Q1 }\end{array}$ & $\begin{array}{c}\text { FLTR } \\
\text { FLOW } \\
\text { (gpm) } \\
\text { Q2 }\end{array}$ & $\begin{array}{c}\text { Temp } \\
\text { corr flow } \\
\left(\mathrm{gpm} / \mathrm{ft}^{2}\right)\end{array}$ & $\begin{array}{c}\text { Axial Vel } \\
(\mathrm{ft} / \mathrm{sec})\end{array}$ & $\begin{array}{l}\text { Avg } \\
\text { TMP } \\
\text { (psid) }\end{array}$ \\
\hline 10/23/01 & $15: 03$ & 0 & 26.5 & 23.6 & 26.7 & 25.8 & 23.3 & 52.2 & 60.9 & 4.5 & 48.1 & 8.9 & 0 & 24.0 & 0.086 & 0.0358 & 10.0 & 50.1 \\
\hline /23/01 & $15: 04$ & 0 & 26.7 & 23.6 & 26.9 & 25.7 & 23.3 & 51.7 & 60.3 & 4.4 & 48.4 & 8.7 & 0 & 24.0 & 0.085 & 0.0352 & 9.9 & 0.0 \\
\hline$/ 23 / 01$ & $15: 05$ & 0 & 26.8 & 23.6 & 27.0 & 25.7 & 23.3 & 52.8 & 61.1 & 4.5 & 48.1 & 8.5 & 0 & 23.9 & 0.087 & 0.0359 & 9.9 & 50.4 \\
\hline $0 / 23 / 01$ & $15: 06$ & 0 & 26.9 & 23.6 & 27.1 & 25.7 & 23.3 & 52.3 & 60.4 & 4.4 & 48.5 & 8.3 & 0 & 23.8 & 0.083 & 0.0342 & 9.9 & 50.4 \\
\hline /23/01 & $15: 07$ & 0 & 27.0 & 23.6 & 27.2 & 25.6 & 23.3 & 53.0 & 61.1 & 4.5 & & 83 & 0 & 23.9 & 0.082 & & 0 & \\
\hline$/ 23 / 01$ & $15: 08$ & 0 & 27.1 & 23.6 & 27.3 & 25.6 & 23.3 & 52.6 & 61.0 & 4.5 & 48.6 & 8.3 & 0 & 23.9 & 0.081 & 0.0332 & 9.9 & 50.6 \\
\hline /23/01 & $15: 09$ & 0 & 27.2 & 23.6 & 27.3 & 25.6 & 23.3 & 52.4 & 60.6 & 4.4 & 48.8 & 8.3 & 0 & 23.9 & 0.080 & 0.0328 & 9.9 & 50.6 \\
\hline$/ 23 / 01$ & $15: 10$ & 0 & 27.3 & 23.6 & 26.3 & 25.6 & 23.3 & 53.0 & 61.1 & 4.4 & 48.5 & 8.2 & 0 & 23.7 & 0.077 & 0.0324 & 9.8 & 50.8 \\
\hline $\mathrm{D} / 23 / 01$ & $15: 11$ & 0 & 27.1 & 23.6 & 25.6 & 25.6 & 23.3 & 52.9 & 61.0 & 4.4 & 49.3 & 8.2 & 0 & 23.7 & 0.075 & 0.0322 & 9.8 & 51.1 \\
\hline /23/01 & $15: 12$ & 0 & 27.0 & 23.6 & 25.3 & 25.7 & 23.2 & 53.5 & 61.6 & 4.4 & & 8.2 & & 23.6 & 0.074 & & & 1.4 \\
\hline /23/01 & $15: 13$ & 0 & 26.9 & 23.6 & 25.3 & 25.8 & 23.3 & 53.4 & 61.4 & 4.4 & 49.2 & 8.2 & 0 & 23.6 & 0.074 & 0.0320 & 9. & 51.3 \\
\hline$/ 23 / 01$ & $15: 14$ & 0 & 26.8 & 23.6 & 25.5 & 25.8 & 23.3 & 53.0 & 61.1 & 4.4 & 49. & 8.2 & 0 & 23.6 & 0.073 & $0 . c$ & 9.8 & 51.1 \\
\hline /23/01 & $15: 15$ & 0 & 26.7 & 23.6 & 25.6 & 25.8 & 23.3 & 53.5 & 61.7 & 4.4 & 48 & 82 & 0 & 23.6 & 0.073 & & & 515 \\
\hline$/ 23 / 01$ & $15: 16$ & 0 & 26.5 & 23.6 & 25.7 & 25.8 & 23.3 & 53.3 & 61.3 & 4.3 & 49.2 & 8.2 & 0 & 23.6 & 0.072 & 08 & 9. & 51.3 \\
\hline$/ 23 / 01$ & $15: 17$ & 0 & 26.4 & 23.6 & 25.8 & 25.8 & 23.3 & 53.1 & 61.2 & 4.4 & 49.0 & 8.2 & c & 23.6 & 0.072 & 0. & 9.8 & 51.1 \\
\hline /23/01 & $15: 18$ & 0 & 26.3 & 23.6 & 25.9 & 25.9 & 23.3 & 53.2 & 61.4 & 4.4 & 45 & 8.2 & 0 & 23.6 & 0.072 & & & 51.2 \\
\hline 1 & $15: 19$ & 0 & 26.3 & 23.6 & 26.0 & 26.0 & 23.3 & 53.6 & 61.6 & 4.4 & 49 & 8.2 & 0 & 23.6 & 0.072 & 0. & 9.8 & 51.6 \\
\hline 1 & $15: 20$ & 0 & 26.3 & 23.7 & 26.1 & 26.0 & 23.3 & 53.1 & 61.3 & 4.3 & 4 & - & & 23.6 & 0.071 & & & 51.2 \\
\hline 1 & $15: 21$ & 0 & 26.3 & 23.7 & 26.2 & 26.0 & 23.3 & 53.4 & 61.5 & 4.4 & $4 \varepsilon$ & 8.2 & 0 & 23.6 & 0.071 & 0. & 9.8 & 51.1 \\
\hline 11 & $15: 22$ & 0 & 26.4 & 23.7 & 26.3 & 26.0 & 23.3 & 53.2 & 61.4 & 4.3 & 48 & 8. & & 23.5 & 0.071 & & 0 & 51.0 \\
\hline & $15: 23$ & 0 & 26.4 & 3.7 & 26.4 & & 23.3 & & 61.1 & 4.3 & & 8.2 & ? & 23.5 & 0.071 & & & 51.1 \\
\hline 1 & $15: 24$ & 0 & 26.5 & 23.7 & 26.5 & 26.1 & 23.3 & 52.7 & 60.8 & 4.3 & 49 & 8.2 & 0 & 23.5 & 0.070 & 93 & 9.7 & 51.0 \\
\hline 11 & $15: 25$ & 0 & 26.5 & 23.7 & 26.5 & 6.1 & 23.3 & 53.7 & 61.9 & 4.3 & 45 & 0 & & 23.5 & 0.070 & & 9.7 & 51.5 \\
\hline & $15: 26$ & 0 & 26.7 & 23.7 & 26.6 & 26.1 & 23.3 & 53.3 & 61.2 & 4.3 & & 8. & 0 & 23.5 & 0.070 & & & 5 \\
\hline 1 & $15: 27$ & 0 & 26.8 & 23.7 & 26.7 & 6.0 & 23.3 & 53.7 & 61.8 & 4.4 & 49 & 0 & c & 23.5 & 0.070 & 0. & 9.8 & 51 \\
\hline & $15: 28$ & 0 & 26.9 & 23.7 & 26.8 & 26.0 & 23.3 & 52.7 & 60.7 & 4.3 & 4 & 8.2 & 0 & 23.5 & 0.070 & 90 & 9.8 & 5 \\
\hline & $15: 29$ & 0 & 27.0 & 3.7 & 26.9 & 25.9 & 23.3 & 53.4 & 61.7 & 4.3 & 4 & 8.2 & 0 & 23.5 & 0.069 & & & 51.4 \\
\hline 11 & $15: 30$ & 0 & 27.0 & 23.7 & 27.0 & 5.9 & 23.2 & 53.4 & 61.4 & 4.3 & 4 & 0 & c & 23.5 & 0.069 & 0 & 9.7 & 51.5 \\
\hline & & 0 & 27.0 & 23.7 & 27.0 & .9 & 23.3 & 53.0 & 60.9 & 4.3 & & 8. & & & & & & \\
\hline 1 & $15: 32$ & 0 & 27.1 & 23.7 & 27.1 & 5.9 & 23.3 & 53.5 & 61.3 & 4.3 & 49 & 8. & c & 23.5 & 0.069 & 84 & 9.8 & 51.3 \\
\hline & $15: 33$ & 0 & 27.1 & 23.7 & 27.2 & 25.9 & 23.3 & 53.3 & 61.4 & 4.3 & 45 & 8.2 & 0 & 23.5 & 0.069 & 83 & 9.8 & 51.1 \\
\hline & $15: 34$ & 0 & 27.3 & 3.7 & 27.3 & 25.9 & 23.3 & 53.3 & 61.2 & 4.3 & & 0 & & 23.5 & 0.069 & & & 515 \\
\hline & $15: 35$ & 0 & 27.3 & 23.7 & 27.3 & 5.8 & 23.3 & 53.4 & 61.2 & 4.3 & 4 & 8. & & 23.4 & 0.068 & 78 & 9.7 & 51.3 \\
\hline & 15: & 0 & 27.4 & 3.7 & 27.4 & 5.9 & 23.3 & 53.2 & 61.2 & 4.3 & & 8.2 & & 23.4 & 0. & & & 5 \\
\hline & $15:$ & 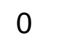 & 27.4 & .7 & 27.5 & 8 & 23.3 & 53. & 61.4 & 4.3 & & & & 23.4 & 0. & & & 514 \\
\hline & $15: 38$ & 0 & 27.5 & 23.7 & 27.6 & 25.9 & 23.3 & 53.2 & 61.3 & 4.3 & 45 & 8.2 & 0 & 23.5 & 0.068 & 0.0276 & 8 & 51.3 \\
\hline & 15: & 0 & 27.6 & & 27.6 & 25.8 & 23.2 & 53.5 & 615 & 4.3 & & & & & & & & \\
\hline & 15: & 0 & 27.7 & .7 & 27.7 & 5.8 & 23.2 & 54.1 & 62.2 & 4.3 & 4 & 8. & & 23.5 & 68 & 76 & & 5 \\
\hline & 15: & 0 & 27.7 & 23.7 & 27.7 & 25.7 & 23.3 & 53.5 & 61.6 & 4.3 & 4 & 8.2 & & 23.5 & 0.068 & & & 51.7 \\
\hline & 15: & 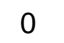 & 27.7 & & & & & & & 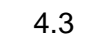 & & & & & & & & \\
\hline & $15: 43$ & 0 & 27.8 & 23.7 & 27.9 & 25.7 & 23.2 & 52.8 & 60.8 & 4.3 & 4 & 8.1 & 1 & 23.6 & 0.067 & 70 & 9.8 & 51.1 \\
\hline & $15: 4$ & 0 & 27.9 & .7 & 27.9 & 25.6 & 23.2 & 52.7 & 60.5 & 4.3 & & 3.1 & & 23 & & & & \\
\hline & 15: & 0 & 27.9 & & 28.0 & & 23.2 & & 612 & 43 & & 8 & & 2 & 67 & & & \\
\hline & $15: 4$ & 0 & 28.0 & .8 & 28.0 & 25.7 & 23.2 & 52.4 & 60.4 & 4.3 & $4 ؟$ & 8.1 & & 23.6 & 0.067 & & & 51.1 \\
\hline & 15: & 0 & 28. & & 28.1 & & & & & & & & & & & & & \\
\hline 1 & $15: 48$ & 0 & 28.1 & 23.8 & 28.2 & 25.8 & 23.2 & 53.5 & 61.5 & 4.4 & 45 & 8.1 & 1 & 23.6 & 0.067 & 0.0268 & 9.8 & 51.4 \\
\hline & $15:$ & 0 & 28.2 & .7 & 28.2 & 25.7 & 23.2 & 52.9 & 61 & 4. & & 8.1 & & 23 & 67 & & & \\
\hline & 15 & 0 & 28.3 & $<0.0$ & 8.3 & 20. & 2 & . & & 4. & & & 0 & & 7 & & & \\
\hline & & 0 & 28. & & & & & & & 1 & & 8. & 0 & 23 & 067 & & 9.8 & \\
\hline & & & 84 & & & & & & & 4.3 & & 0 & & & & & & \\
\hline
\end{tabular}


WSRC-TR-2002-00459, Rev. 0 SRT-RPP-2002-00221

Xflow2_102301_1011

\begin{tabular}{|c|c|c|c|c|c|c|c|c|c|c|c|c|c|c|c|c|c|c|}
\hline DATE & TIME & Sol & $\begin{array}{l}\left({ }^{\circ} \mathrm{C}\right) \\
\mathrm{T} 2\end{array}$ & $\begin{array}{c}\mathrm{CL} \\
\text { LOOP } \\
\left({ }^{\circ} \mathrm{C}\right) \\
\text { T3 }\end{array}$ & $\begin{array}{c}\text { SL } \\
\text { LOOP } \\
\left({ }^{\circ} \mathrm{C}\right) \\
\mathrm{T} 1\end{array}$ & $\begin{array}{c}\text { UP } \\
\text { AMB } \\
\left({ }^{\circ} \mathrm{C}\right) \\
\text { T4 }\end{array}$ & $\begin{array}{c}\text { BOT } \\
\text { AMB } \\
\left({ }^{\circ} \mathrm{C}\right) \\
\text { T5 }\end{array}$ & $\begin{array}{c}\text { BOT } \\
\text { DP } \\
\text { (psid) } \\
\text { dP2 }\end{array}$ & $\begin{array}{c}\text { FLTR } \\
\text { (psig) } \\
\text { P1 }\end{array}$ & $\begin{array}{l}\text { FLTR } \\
\text { DP } \\
\text { (psid) } \\
\text { dP1 }\end{array}$ & $\begin{array}{c}\text { TOP } \\
\text { DP } \\
\text { (psig) } \\
\text { dP3 }\end{array}$ & $\begin{array}{l}\text { FLTRATE } \\
\text { (psig) } \\
\text { P2 }\end{array}$ & $\begin{array}{c}\text { BP } \\
\text { (psig) } \\
\text { P3 }\end{array}$ & $\begin{array}{l}\text { SL } \\
\text { FLOW } \\
\text { (gpm) } \\
\text { Q1 }\end{array}$ & $\begin{array}{c}\text { FLTR } \\
\text { FLOW } \\
\text { (gpm) } \\
\text { Q2 }\end{array}$ & $\begin{array}{c}\text { Temp } \\
\text { corr flow } \\
\left(\mathrm{gpm} / \mathrm{ft}^{2}\right)\end{array}$ & $\begin{array}{c}\text { Axial Vel } \\
(\mathrm{ft} / \mathrm{sec})\end{array}$ & $\begin{array}{l}\text { Avg } \\
\text { TMP } \\
\text { (psid) }\end{array}$ \\
\hline 10/23/01 & $15: 53$ & 0 & 28.4 & 23.8 & 28.5 & 25.8 & 23.2 & 53.1 & 61.1 & 4.3 & 49.2 & 8.1 & 0 & 23.6 & 0.067 & 0.0266 & 9.8 & 51.1 \\
\hline 0/23/01 & $15: 54$ & 0 & 28.5 & 23.8 & 28.5 & 25.8 & 23.2 & 52.9 & 60.8 & 4.3 & 49.1 & 8.1 & 0 & 23.5 & 0.067 & 0.0266 & 9.8 & 51.0 \\
\hline /23/01 & $15: 55$ & 0 & 28.5 & 23.8 & 28.5 & 25.9 & 23.2 & 53.3 & 61.3 & 4.4 & 49.1 & 8.1 & 0 & 23.6 & 0.067 & 0.0265 & 9.8 & 51.2 \\
\hline 10/23/01 & $15: 56$ & 0 & 28.6 & 23.8 & 28.6 & 26.0 & 23.2 & 53.2 & 61.3 & 4.3 & 48.9 & 8.1 & 0 & 23.6 & 0.067 & 0.0265 & 9.8 & 51.1 \\
\hline$/ 23 / 01$ & $15: 57$ & 0 & 28.6 & 23.8 & 28.7 & & 23.3 & & 61.2 & 4.3 & & 8.1 & 0 & 23.6 & 0.067 & & & \\
\hline 0/23/01 & $15: 58$ & 0 & 28.7 & 23.8 & 28.7 & 26.1 & 23.2 & 53.2 & 61.3 & 4.3 & 49.6 & 8.1 & 0 & 23.6 & 0.067 & 0.0264 & 9.8 & 51.4 \\
\hline /23/01 & $15: 59$ & 0 & 28.7 & 23.8 & 28.7 & 26.2 & 23.3 & 53.2 & 61.1 & 4.3 & 48.5 & 8.1 & 0 & 23.5 & 0.067 & 0.0264 & 9.7 & 50.9 \\
\hline 0/23/01 & $16: 00$ & 0 & 28.8 & 23.8 & 28.8 & 26.2 & 23.4 & 53.3 & 61.5 & 4.3 & 49.3 & 8.1 & 0 & 23.5 & 0.067 & 0.0263 & 9.8 & 51.3 \\
\hline 10/23/01 & $16: 01$ & 0 & 28.8 & 23.8 & 28.8 & 26.1 & 23.3 & 52.7 & 60.7 & 4.3 & 48.9 & 8.1 & 0 & 23.5 & 0.067 & 0.0263 & 9.7 & 50.8 \\
\hline /23/01 & $16: 02$ & 0 & 28.9 & 23.8 & 28.9 & 6.0 & 23.3 & 53.5 & 61.6 & 4.3 & & 8.1 & 0 & 23.5 & 0.067 & 0.0263 & & \\
\hline$/ 23 / 01$ & $16: 03$ & 0 & 28.9 & 23.8 & 28.9 & 26.0 & 23.3 & 53.3 & 61.2 & 4.3 & 49.3 & 8.1 & 0 & 23.5 & 0.067 & 0.0262 & 9.7 & 51.3 \\
\hline /23/01 & $16: 04$ & 0 & 29.0 & 23.8 & 29.0 & 25.9 & 23.3 & 53.0 & 60.9 & 4.3 & 49.3 & 8.1 & 0 & 23.4 & 0.067 & 0.0262 & 9.7 & 51.1 \\
\hline$/ 23 / 01$ & $16: 05$ & 0 & 29.0 & 23.8 & 29.0 & 25.9 & 23.3 & 53.7 & 61.7 & 4.3 & 49.4 & 8.1 & 0 & 23.5 & 0.067 & & & 516 \\
\hline 0/23/01 & $16: 06$ & 0 & 29.1 & 23.8 & 29.0 & 25.9 & 23.3 & 52.8 & 60.8 & 4.3 & 49.2 & 8.1 & 0 & 23.5 & 0.067 & 0.0261 & 9.8 & 51.0 \\
\hline /23/01 & $16: 07$ & 0 & 29.1 & 23.8 & 29.0 & 25.8 & 23.3 & 52.8 & 60.9 & 4.2 & 49.5 & 8.1 & ( & 23.5 & 0.067 & 0.0261 & 9.7 & 51.1 \\
\hline /23/01 & $16: 08$ & 0 & 29.1 & 23.8 & 29.1 & 25.8 & 23.3 & 52.4 & 60.1 & 4.2 & 49 & 8.1 & 0 & 23.4 & 0.067 & 0.0261 & a & 50.9 \\
\hline /23/01 & $16: 09$ & 0 & 29.2 & 23.8 & 29.1 & 25.8 & 23.3 & 53.6 & 61.6 & 4.3 & 49.8 & 8.1 & 0 & 23.5 & 0.067 & 0.0261 & 9.8 & 51.7 \\
\hline 11 & $16: 10$ & 0 & 29.2 & 23.8 & 29.2 & 5.8 & 23.3 & 52.9 & 60.8 & 4.3 & 49 & 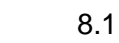 & ( & 23.4 & 0.067 & 261 & & 511 \\
\hline$/ 23 / 01$ & $16: 11$ & 0 & 29.3 & 3.8 & 29.2 & 25.8 & 23.2 & 52.9 & 60.8 & 4.3 & 48 & 8.1 & 0 & 23.4 & 0.067 & 0.0260 & 9.7 & 50.9 \\
\hline & $16: 12$ & 0 & 29.3 & 23.8 & 29.3 & 25.9 & 23.3 & 53.6 & 61.6 & 4.3 & 49. & 8.1 & 0 & 23.4 & .067 & 0. & 9.7 & 51.4 \\
\hline & & & & & & & & & & & & & & & & & & \\
\hline & $16: 13$ & 0 & 29.3 & 23.8 & 29.2 & 25.8 & 23.2 & 22.0 & 30.0 & 2.5 & 20. & 7.9 & 0 & 16.5 & 0.034 & 0.0132 & 6 & 21.0 \\
\hline /23/01 & $16: 14$ & 0 & 29.3 & 23.8 & 29.1 & 5.8 & 23.2 & 22.0 & 30.0 & 2.5 & 20 & 7. & ( & 16.6 & 33 & 29 & 6. & 21.0 \\
\hline & $16: 1$ & 0 & 29.3 & 23.8 & 29.0 & 5.7 & 23.2 & 18.2 & 30.2 & 2.5 & 16 & 12.0 & 0 & 16.6 & 0.119 & & & 1 \\
\hline 11 & $16: 16$ & 0 & 29.3 & 23.8 & 28.9 & 25.7 & 23.2 & 36.5 & 43.4 & 3.4 & 34 & 6. & 0 & 20.0 & 0.014 & 55 & 8.3 & 35 \\
\hline 01 & $16: 17$ & 0 & 29.2 & 23.8 & 29.0 & 25.7 & 23.2 & 52.4 & 61.0 & 4.3 & 4 & 8.6 & 0 & 23.3 & 18 & 61 & 9.7 & 5 \\
\hline 01 & $16: 18$ & 0 & 29.2 & 3.8 & 29.0 & 25.7 & 23.2 & 53.1 & 61.5 & 4.4 & 4 & 8.4 & 0 & 23.6 & 0.103 & 02 & & 51.1 \\
\hline$/ 23 / 01$ & $16: 19$ & 0 & 29.2 & 23.9 & 29.1 & 25.7 & 23.2 & 52.6 & 60.8 & 4.4 & $4 \varepsilon$ & 8. & ( & 23.5 & 0.097 & 78 & 9.8 & 50.7 \\
\hline & $16: 20$ & 0 & 29.1 & 23.8 & 29.1 & 25.7 & 23.2 & 53.4 & 61.6 & 4.5 & & 8. & & 23.6 & & & & \\
\hline 11 & $16: 21$ & 0 & 29.4 & 3.9 & 29.2 & 5.7 & 23.3 & 52.8 & 60.9 & 4.4 & 49 & 8. & 0 & 23.5 & 0.091 & 0. & 9.7 & 5 \\
\hline$/ 23 / 01$ & $16: 22$ & 0 & 29.3 & 23.9 & 29.2 & 25.8 & 23.2 & 52.9 & 60.9 & 4.4 & 4 & 8.2 & 0 & 23.6 & 38 & 342 & 9.8 & 50.8 \\
\hline & $16: 23$ & 0 & 29.3 & 3.9 & 29.3 & 5.9 & 23.2 & 53.2 & 61.2 & 4.4 & & & 0 & & & & & 51.0 \\
\hline 11 & $16: 24$ & 0 & 29.4 & 3.9 & 29.3 & 5.9 & 23.2 & 52.8 & 60.7 & 4.4 & 4 & 8.2 & ( & 23.6 & 0.085 & 29 & 9.8 & 50.8 \\
\hline & $16: 2$ & 0 & 29.4 & 3.9 & 29.4 & 26.0 & 23.2 & 53.1 & 61.0 & 4.4 & 4 & 8.2 & & 23.5 & 84 & & 9. & 5 \\
\hline & $16:$ & 0 & 29.5 & & 29.4 & 1 & 23.2 & 53.2 & 61.2 & 4.4 & & & & 23.4 & & & & 512 \\
\hline$/ 23 / 01$ & $16: 27$ & 0 & 29.6 & 3.9 & 29.5 & 26.1 & 23.2 & 53.2 & 61.4 & 4.4 & 4 & 8.2 & 0 & 23.4 & 0.082 & 316 & .7 & 51.1 \\
\hline & 16: & 0 & 29.6 & .9 & 29.5 & 26.2 & 23.2 & 53.2 & 61.4 & 4.4 & & 8.4 & & & & & & \\
\hline & $16: 29$ & 0 & 29.6 & .9 & 29.5 & .2 & 23.3 & .3 & 61.5 & 4.4 & 4 & 8.2 & ( & 23.4 & 81 & 12 & 9.7 & 5 \\
\hline & 16: & 0 & 29.6 & 23.9 & 29.6 & 26.2 & 23.3 & 52.9 & 60.9 & 4.4 & 4 & 8.2 & & 23.4 & 99 & & & 5 \\
\hline & 16: & 0 & 29.7 & & & & & & & 4 & & & & & & & & \\
\hline 11 & $16: 32$ & 0 & 29.7 & 23.9 & 29.6 & 26.1 & 23.2 & 53.5 & 61.7 & 4.4 & & 8. & 0 & 23.5 & 0.078 & 300 & 9.8 & 51.3 \\
\hline & 16: & 0 & 29.7 & .9 & 29.7 & 26.2 & 23.2 & 53.2 & 61.5 & 4.4 & & 5.3 & & & & & & . \\
\hline & 16 & 0 & 29.7 & & 29.6 & & 23.2 & & 618 & 44 & & & 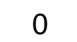 & 23.4 & 75 & & & \\
\hline & $16: 3$ & 0 & 29.8 & 3.9 & 29.7 & 6.2 & 23.2 & 52.8 & 61.2 & 4.4 & 4 & 8.6 & & 23.4 & 0.074 & 283 & 9.1 & 50.9 \\
\hline & & 0 & $29 . \varepsilon$ & 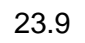 & & & ror & & & & & & & & & & & \\
\hline 11 & $16: 37$ & 0 & 29.9 & 3.9 & 29.8 & 26.2 & 23.3 & 51.9 & 60.5 & 4.3 & & 8.8 & 0 & 23.4 & 0.073 & 0.0279 & 9.7 & 50.1 \\
\hline & $16:$ & 0 & 29.9 & , & & & 23.2 & & 61.6 & 4. & & 9.1 & & & & & 9. & \\
\hline & & 0 & 29.9 & 0.5 & & 0.2 &.$<$ & 51.7 & 00.1 & 4. & & & 0 & & 5 & & & \\
\hline & 10 & 0 & 29.9 & & & & & & 611 & 43 & & 9. & 0 & 23 & 74 & & 9.7 & \\
\hline & & & 195 & & & & & & & 4.3 & & 0 & & & & & & \\
\hline
\end{tabular}


WSRC-TR-2002-00459, Rev. 0 SRT-RPP-2002-00221

Xflow2_102301_1011

\begin{tabular}{|c|c|c|c|c|c|c|c|c|c|c|c|c|c|c|c|c|c|c|}
\hline DATE & TIME & Sol & $\begin{array}{c}\text { FLTRT } \\
\left({ }^{\circ} \mathrm{C}\right) \\
\text { T2 }\end{array}$ & $\begin{array}{c}\mathrm{CL} \\
\text { LOOP } \\
\left({ }^{\circ} \mathrm{C}\right) \\
\text { T3 }\end{array}$ & $\begin{array}{c}\mathrm{SL} \\
\text { LOOP } \\
\left({ }^{\circ} \mathrm{C}\right) \\
\mathrm{T} 1\end{array}$ & $\begin{array}{c}\text { UP } \\
\text { AMB } \\
\left(^{\circ} \mathrm{C}\right) \\
\text { T4 }\end{array}$ & $\begin{array}{c}\text { BOT } \\
\text { AMB } \\
\left({ }^{\circ} \mathrm{C}\right) \\
\text { T5 }\end{array}$ & $\begin{array}{c}\text { BOT } \\
\text { DP } \\
\text { (psid) } \\
\text { dP2 }\end{array}$ & $\begin{array}{c}\text { FLTR } \\
\text { (psig) } \\
\text { P1 }\end{array}$ & $\begin{array}{c}\text { FLTR } \\
\text { DP } \\
\text { (psid) } \\
\text { dP1 }\end{array}$ & $\begin{array}{c}\text { TOP } \\
\text { DP } \\
\text { (psig) } \\
\text { dP3 }\end{array}$ & $\begin{array}{l}\text { FLTRATE } \\
\text { (psig) } \\
\text { P2 }\end{array}$ & $\begin{array}{c}\mathrm{BP} \\
\text { (psig) } \\
\text { P3 }\end{array}$ & $\begin{array}{c}\mathrm{SL} \\
\text { FLOW } \\
\text { (gpm) } \\
\text { Q1 }\end{array}$ & $\begin{array}{c}\text { FLTR } \\
\text { FLOW } \\
\text { (gpm) } \\
\text { Q2 }\end{array}$ & $\begin{array}{c}\text { Temp } \\
\text { corr flow } \\
\left(\mathrm{gpm} / \mathrm{ft}^{2}\right)\end{array}$ & $\begin{array}{c}\text { Axial Vel } \\
(\mathrm{ft} / \mathrm{sec})\end{array}$ & $\begin{array}{l}\text { Avg } \\
\text { TMP } \\
\text { (psid) }\end{array}$ \\
\hline 0/23/01 & $16: 42$ & 0 & 30.0 & 23.9 & 29.9 & 26.1 & 23.2 & 51.8 & 60.8 & 4.3 & 48.3 & 9.1 & 0 & 23.4 & 0.074 & 0.0282 & 9.7 & 50.1 \\
\hline |0/23/01 & $16: 43$ & 0 & 30.0 & 23.9 & 29.9 & 26.1 & 23.2 & 51.9 & 61.2 & 4.3 & 48.0 & 9.1 & 0 & 23.4 & 0.073 & 0.0278 & 97 & 0.0 \\
\hline $10 / 23 / 01$ & $16: 44$ & 0 & 30.0 & 23.9 & 30.0 & 26.2 & 23.2 & 52.2 & 61.3 & 4.3 & 48.0 & 9.1 & 0 & 23.4 & 0.073 & 0.0278 & 9.7 & 50.1 \\
\hline $10 / 23 / 01$ & $16: 45$ & 0 & 30.1 & 23.9 & 29.9 & 26.1 & 23.2 & 52.4 & 61.4 & 4.3 & 48.5 & 9.1 & 0 & 23.4 & 0.073 & 0.0278 & 9.7 & 50.5 \\
\hline $10 / 23 / 01$ & $16: 46$ & 0 & 30.1 & 23.9 & 30.0 & 26.1 & 23.2 & 52.4 & 61.5 & 4.3 & 48.5 & 9.1 & 0 & 23.4 & 0.073 & 0.0277 & 9.7 & 50.4 \\
\hline $10 / 23 / 01$ & $16: 47$ & 0 & 30.1 & 23.9 & 30.0 & 26.1 & 23.2 & 52.3 & 61.2 & 4.4 & 48.7 & 9.1 & 0 & 23.4 & 0.073 & 0.0277 & 9.7 & 50.5 \\
\hline $10 / 23 / 01$ & $16: 48$ & 0 & 30.1 & 23.9 & .0 & 26.0 & 23.2 & 52.5 & 61.4 & 4.4 & 48.4 & 9.1 & 0 & 23.4 & 0.072 & & & \\
\hline $10 / 23 / 01$ & $16: 49$ & 0 & 30.2 & 23.9 & 30.0 & 26.0 & 23.3 & 52.8 & 61.8 & 4.4 & 48.3 & 9.1 & 0 & 23.4 & 0.072 & 0.0274 & 9.7 & 50.5 \\
\hline $10 / 23 / 01$ & $16: 50$ & 0 & 30.2 & 23.9 & 29.9 & 26.1 & 23.3 & 52.3 & 61.5 & 4.4 & 48.4 & 9.1 & 0 & 23.4 & 0.072 & 0.0274 & 9.7 & 50.4 \\
\hline /23/01 & $16: 51$ & 0 & 30.2 & 23.9 & 29.7 & 26.1 & 23.2 & 52.6 & 61.6 & 4.4 & 48.1 & 9.1 & 0 & 23.3 & 0.071 & 0. & & \\
\hline $10 / 23 / 01$ & $16: 52$ & 0 & 30.1 & 23.9 & 29.7 & 26.1 & 23.2 & 51.7 & 60.4 & 4.3 & 48.6 & 9.1 & 0 & 23.4 & 0.071 & 0.0272 & .7 & 50.1 \\
\hline |0/23/01 & $16: 53$ & 0 & 30.1 & 23.9 & 29.4 & 26.1 & 23.3 & 52.3 & 61.3 & 4.3 & 48.0 & 9.1 & 0 & 23.3 & 0.070 & 70 & 9.7 & 50.2 \\
\hline $10 / 23 / 01$ & $16: 54$ & 0 & 30.0 & 23.9 & 29.4 & 26.1 & 23.3 & 52.3 & 61.3 & 4.3 & 48.3 & 9.1 & 0 & 23.2 & 0.070 & 0.0270 & & 50.3 \\
\hline |0/23/01 & $16: 55$ & 0 & 29.9 & 23.9 & 29.2 & 26.1 & 23.3 & 52.6 & 61.5 & 4.4 & 48.7 & 9.0 & 0 & 23.3 & 0.070 & 0.0272 & 9.7 & 50.7 \\
\hline$\pi / 2$ & $16: 56$ & 0 & 29.9 & 23.9 & 29.2 & 26.2 & 23.2 & 52.1 & 60.9 & 4.3 & 48.0 & 9.0 & 0 & 23.3 & 0.069 & & & \\
\hline $10 / 23 / 01$ & $16: 57$ & 0 & 29.8 & 23.9 & 29.0 & 26.1 & 23.2 & 52.2 & 61.2 & 4.3 & 48.0 & 9.0 & 0 & 23.3 & 0.069 & 0.0270 & 7 & 50.1 \\
\hline |0/23/01 & $16: 58$ & 0 & 29.7 & 23.9 & 28.9 & 26.2 & 23.2 & 52.8 & 61.6 & 4.4 & 48.6 & 9.0 & 0 & 23.3 & 0.068 & 0.0 & 9.7 & 50.7 \\
\hline$/ 2$ & $16: 59$ & 0 & 29.6 & 23.9 & 28.8 & 26.2 & 23.3 & 52.7 & 61.6 & 4.4 & 48.0 & 9.0 & 0 & 23.3 & 0.068 & 67 & .7 & \\
\hline $10 / 23 / 01$ & $17: 00$ & 0 & 29.6 & 23.9 & 28.6 & 26.2 & 23.3 & 52.8 & 61.9 & 4.4 & 48.8 & 9.0 & 0 & 23.4 & 0.068 & $0 . c$ & 9.7 & 50.8 \\
\hline $10 / 23$ & $17: 01$ & 0 & 29.5 & 23.9 & 28.6 & 26.2 & 23.3 & 52.0 & 60.6 & 4.3 & 48. & 9.0 & 0 & 23.3 & 0.068 & 0.0 & & 50.1 \\
\hline $10 / 23 / 01$ & $17: 02$ & 0 & 29.4 & 24.0 & & 26.2 & 23.3 & 52.1 & 60.8 & 4.4 & 48. & 9.0 & 0 & 23.3 & 0.067 & & & \\
\hline $0 / 2$ & $17: 03$ & 0 & 29.3 & 23.9 & 28.4 & 26.3 & 23.2 & 52.9 & 61.8 & 4.4 & 48.5 & 9.0 & 0 & 23.2 & 0.067 & 66 & 9.6 & 50.7 \\
\hline $10 / 23 / 01$ & $17: 04$ & 0 & 29.2 & 24.0 & 28.3 & 26.3 & 23.3 & 51.9 & 60.9 & 4.3 & 48. & 9.0 & 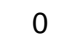 & 23.2 & 0.067 & 0. & $g$ & 50.2 \\
\hline $10 / 23 / 01$ & $17: 05$ & 0 & 29.2 & 23.9 & 28.1 & 26.4 & 23.3 & 52.8 & 61.6 & 4.4 & 48 & 9.0 & 0 & 23.2 & 0.066 & 0. & & 50.6 \\
\hline $10 / 23$ & $17: 06$ & 0 & 29.1 & 23.9 & 28.1 & 26.4 & 23.3 & 52.8 & 61.9 & 4.4 & 48.7 & 9.0 & 0 & 23.3 & 0.066 & 0. & 9.1 & 50.7 \\
\hline & $17: 07$ & 0 & 29.1 & 24.0 & 28.0 & 26.5 & 23.3 & 51.7 & 60.5 & 4.3 & 48.5 & .0 & 0 & 23.3 & 66 & & 9.7 & .1 \\
\hline 10/2 & $17: 08$ & 0 & 29.0 & 24.0 & 27.9 & 26.4 & 23.3 & 52.0 & 60.7 & 4.4 & 49.0 & 9.0 & 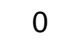 & 23.3 & 0.066 & 0. & 9.7 & 50.5 \\
\hline $10 / 23 / 01$ & $17: 09$ & 0 & 28.9 & 24.0 & 27.8 & 26.3 & 23.2 & 52.6 & 61.5 & 4.4 & 48. & 9.0 & 0 & 23.2 & 0.065 & 0. & 9.6 & 50.7 \\
\hline & $17: 10$ & 0 & 28.9 & 24.0 & 27.7 & 26.3 & 23.3 & 53. & 61.7 & 4.4 & 48 & 9.0 & 0 & 23.3 & & & & 50.9 \\
\hline 10/2 & $17: 11$ & 0 & 28.8 & 24.0 & 27.6 & 26.2 & 23.3 & 52.8 & 61.6 & 4.4 & 48 & 9.0 & 0 & 23.2 & 0.065 & 64 & 9. & 50.9 \\
\hline $10 / 23 / 01$ & $17: 12$ & 0 & 28.7 & 24.0 & 27.5 & 26.1 & 23.3 & 52.9 & 61.6 & 4.4 & 48.7 & 0 & 0 & 23.2 & 0.065 & 64 & .6 & .8 \\
\hline & $17: 13$ & 0 & 28.6 & 4.0 & 27.4 & 26.0 & 23.3 & 52.4 & 61.3 & 4.4 & $4 \varepsilon$ & 8.9 & 0 & 23.2 & 0. & & & 50.5 \\
\hline $10 / 23$ & $17: 14$ & 0 & 28.5 & 24.0 & 27.4 & 26.0 & 23.2 & 52.6 & 61.3 & 4.4 & 48. & 8.9 & 0 & 23.2 & 0.064 & 0. & 9.6 & 50.7 \\
\hline & $17: 15$ & 0 & 28.5 & 24.0 & 26.6 & 26.0 & 23.2 & 52.7 & 61.7 & 4.4 & 4 & & 0 & 23.1 & & & & \\
\hline $10 / 2$ & $17: 16$ & 0 & 28.2 & 24.0 & 25.7 & 26.0 & 23.2 & 53.7 & 62.6 & 4.4 & 45 & 8.9 & V & 23.1 & 0.062 & 65 & 9.6 & 51.5 \\
\hline $10 / 23 / 01$ & $17: 17$ & 0 & 28.0 & 24.0 & 25.3 & 26.0 & 23.2 & 53.3 & 62.1 & 4.4 & 48.7 & 8.9 & 0 & 23.1 & 0.061 & 64 & .6 & 51.0 \\
\hline & $17: 18$ & 0 & 27.6 & 24.0 & 25.3 & 26.1 & 23.2 & 52.3 & 61.0 & 4.3 & $4 \varepsilon$ & .9 & 0 & 23.0 & & & & \\
\hline $10 / 2$ & $17: 19$ & 1 & 27.6 & 24.0 & 25.4 & 26.1 & 23.2 & 6.2 & 56.7 & 7.4 & -0.7 & 50.2 & 53 & 32.5 & 0.017 & 0. & 13.5 & 2.7 \\
\hline & $17: 20$ & 0 & 27.6 & 24.0 & 25.3 & 26.1 & 23.3 & 46.7 & 56.5 & 7.5 & 39 & 9.8 & 0 & 32.6 & 0.159 & & 13.5 & 43.2 \\
\hline & $17: 21$ & 0 & 27. & 1.0 & 5.3 & 26.1 & 23.2 & 47.6 & 56.8 & 7.5 & 40 & & 0 & 32.5 & 0.133 & & 135 & 440 \\
\hline $10 / 23 / 01$ & $17: 22$ & 0 & 27.1 & 24.0 & 25.3 & 26.1 & 23.2 & 47.8 & 56.6 & 7.4 & 40.9 & 8.7 & 0 & 32.3 & 0.123 & 32 & 13.4 & 44.4 \\
\hline & $17: 23$ & 0 & 26.8 & 24.0 & 253 & 26.1 & 23.2 & 48.5 & & 7.4 & & & 0 & 32.1 & 0123 & & 13.3 & 45.0 \\
\hline & $17: 24$ & 0 & 26.6 & 1.0 & 25.2 & 26.2 & 23.3 & 48.6 & 56.8 & 7.3 & 4 & 0 & 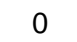 & 32.1 & 0.113 & 90 & 13.3 & 45.2 \\
\hline & $17: 25$ & 0 & 26.5 & 24.0 & 25.3 & 26.2 & 23.3 & 49.0 & 57.0 & 7.4 & 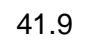 & 8.1 & 0 & 32.1 & 0.109 & 72 & 13.3 & 45.5 \\
\hline & $17: 26$ & 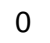 & 26.3 & .0 & & 26.2 & & & & & & & & & & & & \\
\hline 10/23/01 & $17: 27$ & 0 & 26.3 & 24.0 & 25.2 & 26.1 & 23.2 & 48.8 & 56.8 & 7.4 & 9 & 8.1 & 0 & 32.1 & 0.104 & 0.0451 & 13.3 & 45.3 \\
\hline & $17: 28$ & 0 & 26.2 & 24.0 & & 26.1 & & 48.6 & & 7. & & & 0 & 32.2 & & & 13 & \\
\hline & $17: 29$ & 0 & 26 & 5 & & & & & & 7. & & & 0 & & 100 & & 13.3 & \\
\hline & $17: 30$ & 0 & 26.0 & 24.0 & & 26.1 & 3.2 & 48. & & 7.4 & & 8. & 0 & 32.1 & 0.099 & & 13.3 & 45.0 \\
\hline$/ 01$ & $17: 31$ & 0 & 25.9 & 24.0 & & & & 48.6 & & 7.2 & 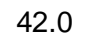 & 8.3 & 0 & 31.8 & $0.0 \varsigma$ & 0.0431 & 13.2 & 45.3 \\
\hline
\end{tabular}


WSRC-TR-2002-00459, Rev. 0 SRT-RPP-2002-00221

Xflow2_102301_1011

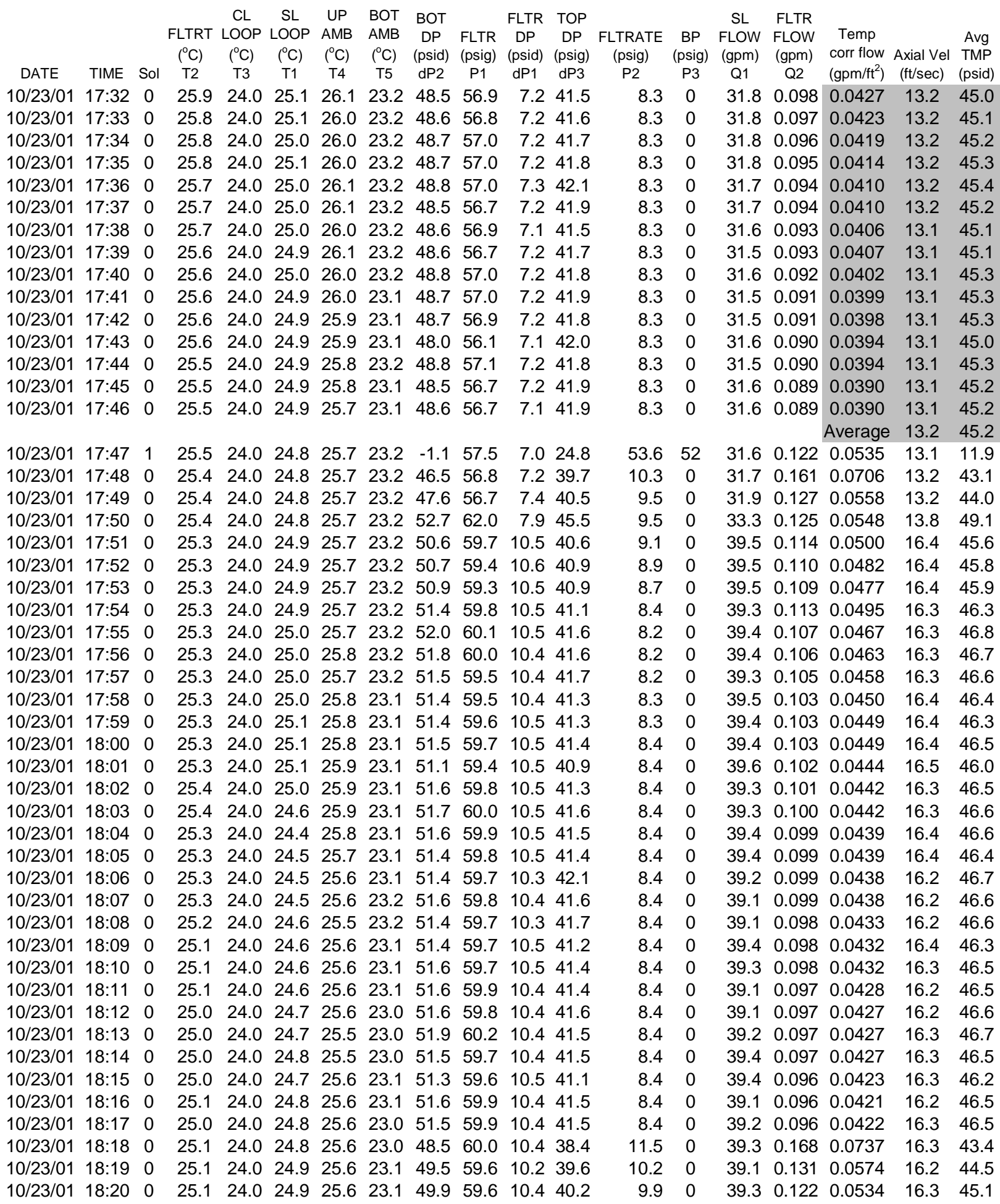


WSRC-TR-2002-00459, Rev. 0 SRT-RPP-2002-00221

Xflow2_102301_1011

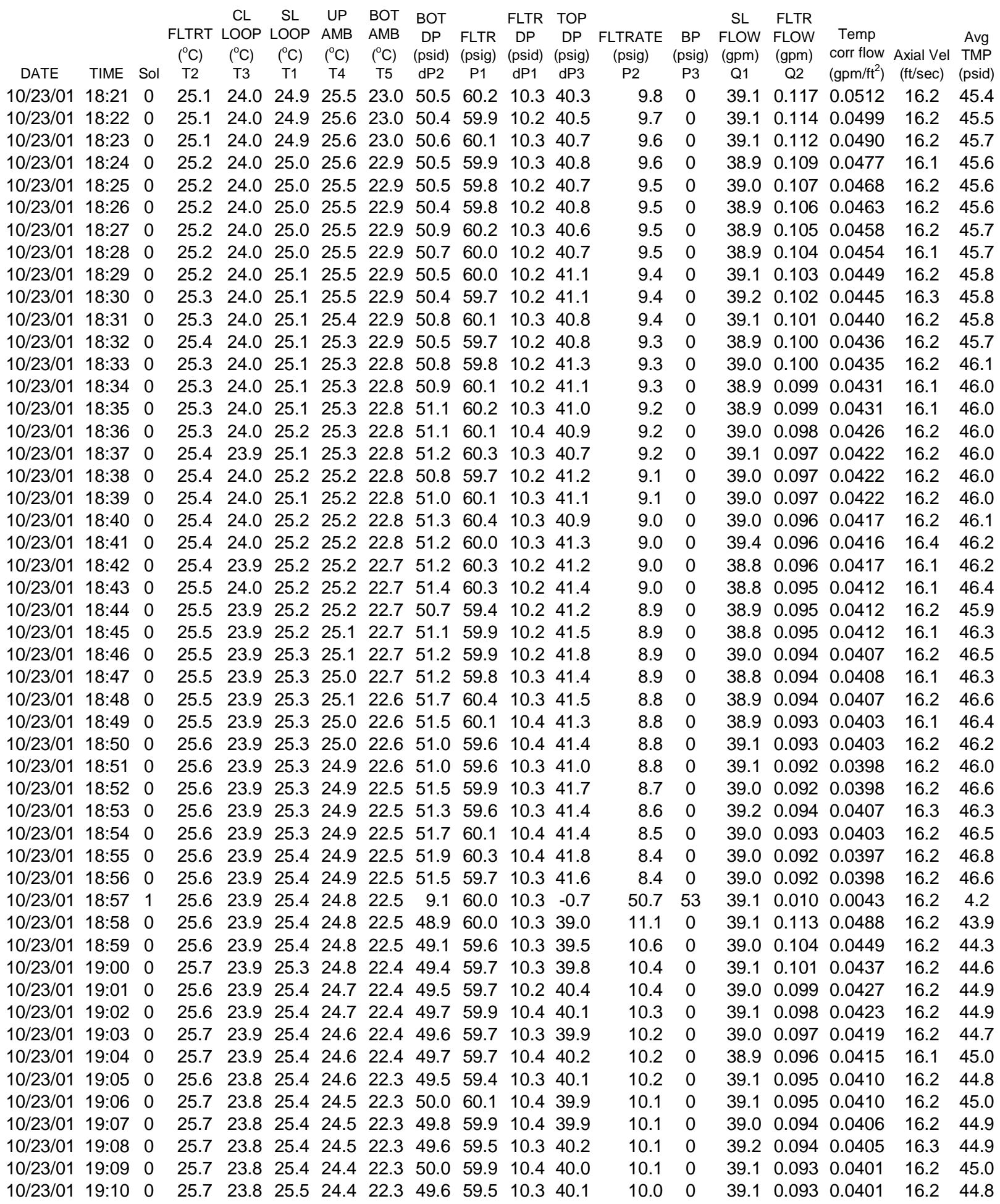


WSRC-TR-2002-00459, Rev. 0 SRT-RPP-2002-00221

Xflow2_102301_1011

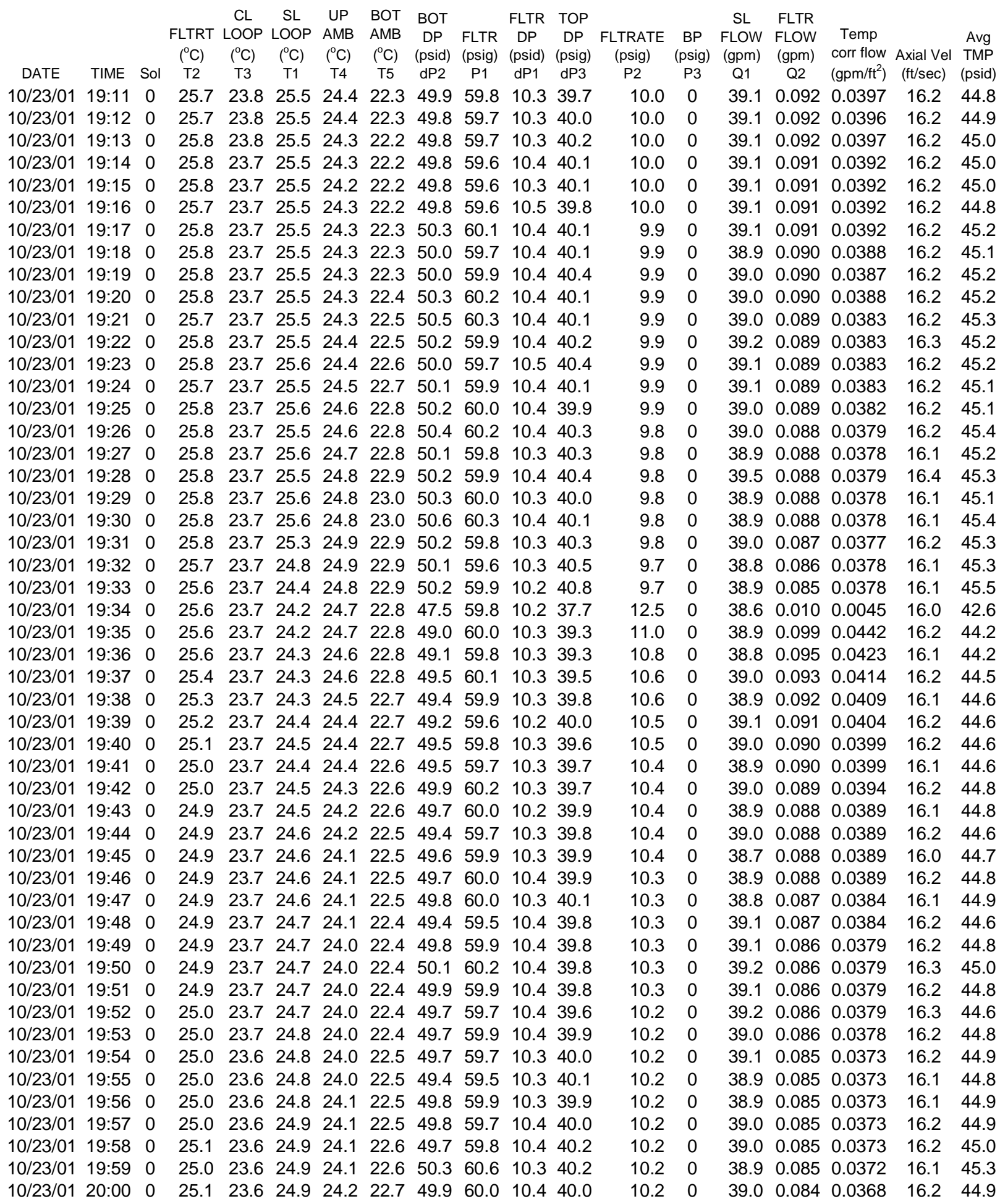


WSRC-TR-2002-00459, Rev. 0 SRT-RPP-2002-00221

Xflow2_102301_1011

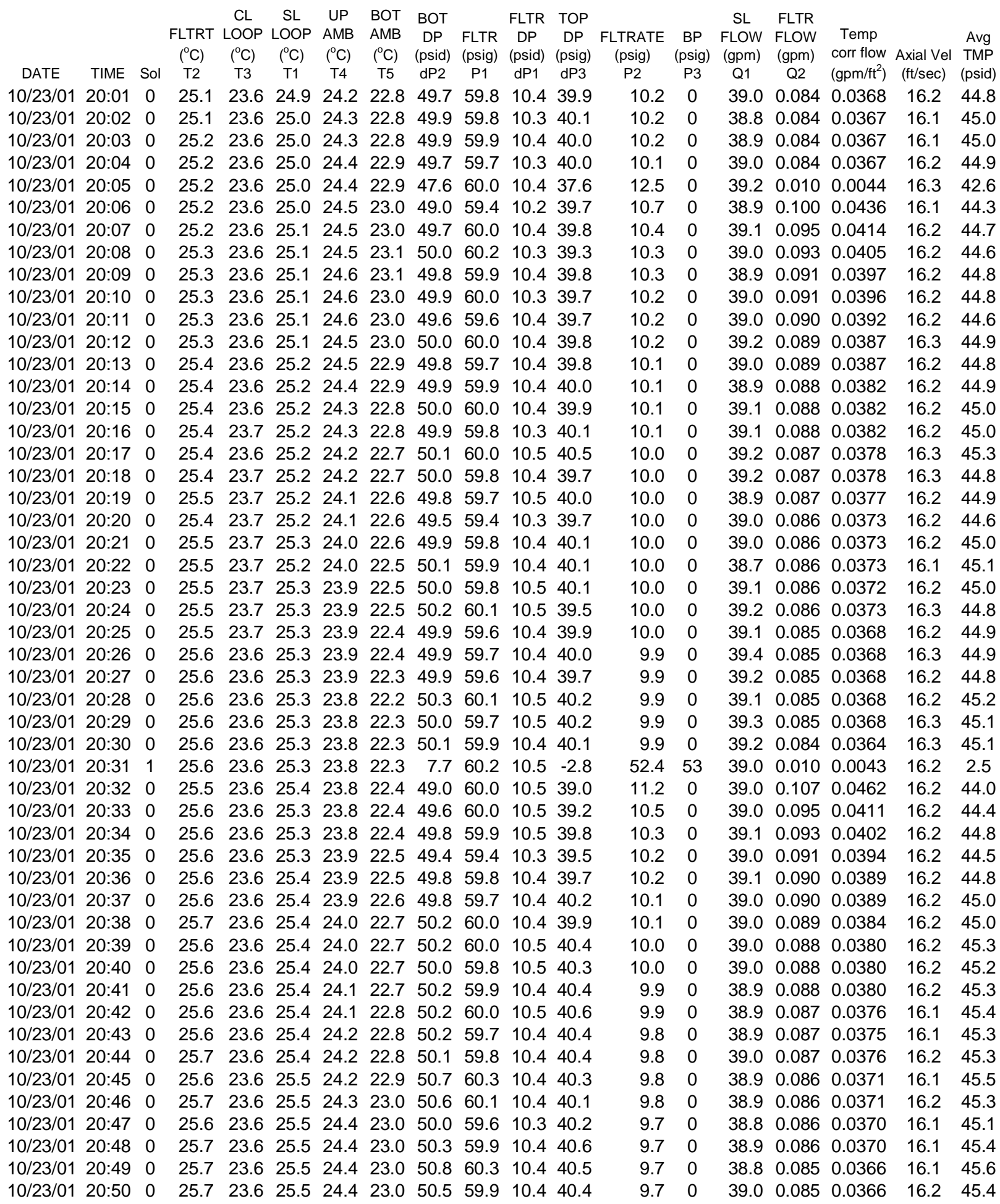


WSRC-TR-2002-00459, Rev. 0 SRT-RPP-2002-00221

Xflow2_102301_1011

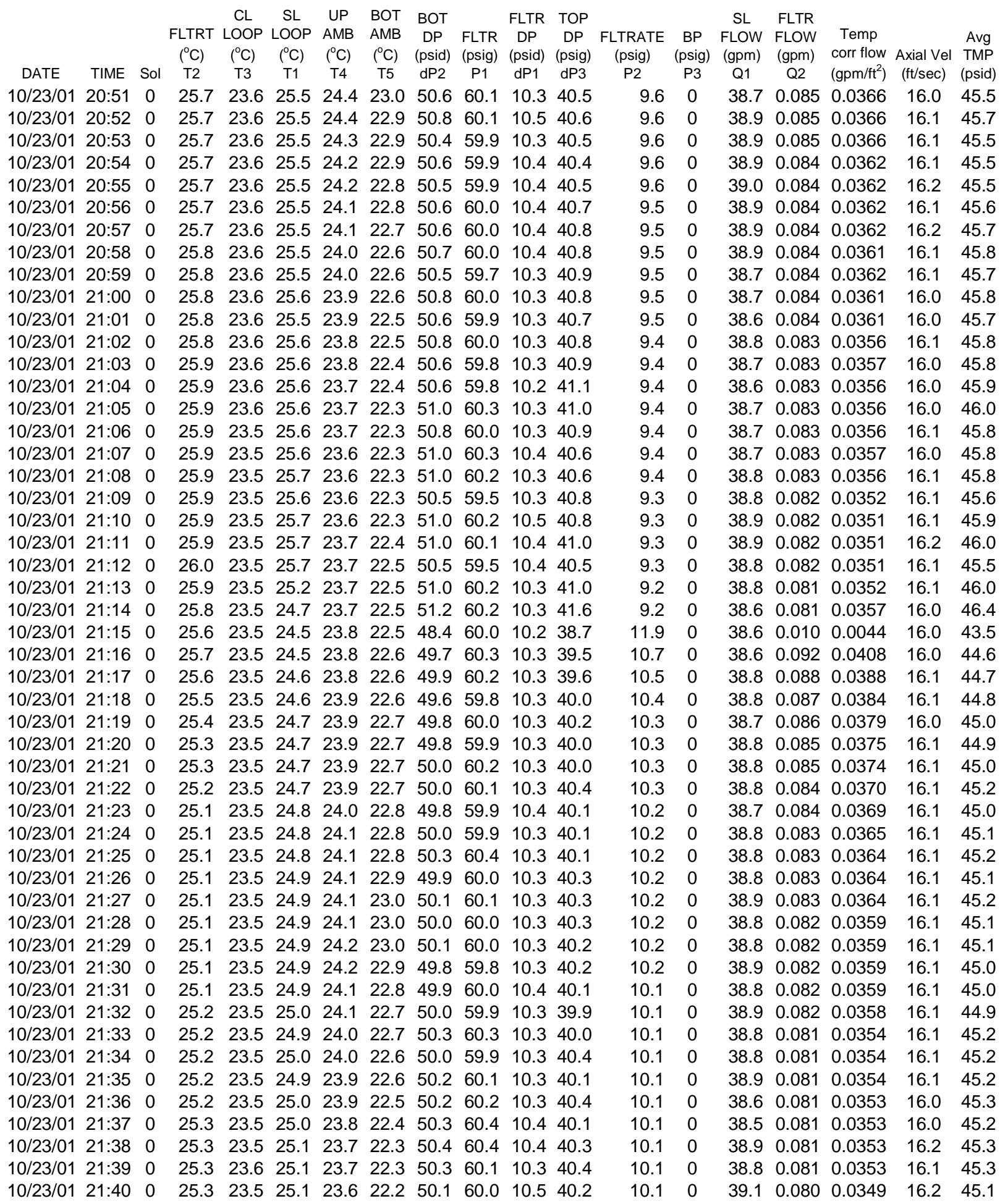


WSRC-TR-2002-00459, Rev. 0 SRT-RPP-2002-00221

Xflow2_102301_1011

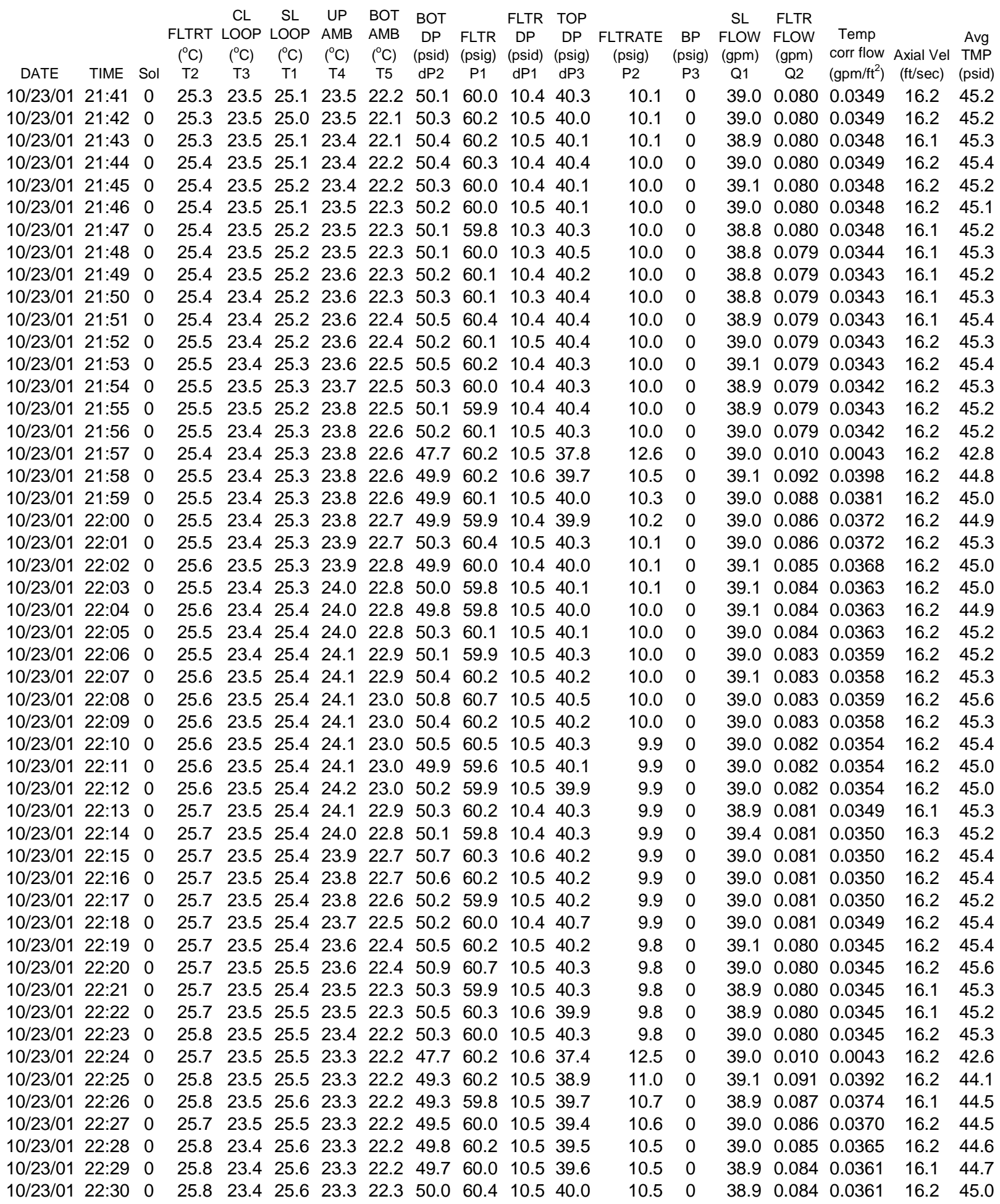


WSRC-TR-2002-00459, Rev. 0 SRT-RPP-2002-00221

Xflow2_102301_1011

\begin{tabular}{|c|c|c|c|c|c|c|c|c|c|c|c|c|c|c|c|c|c|c|}
\hline & & & & CL & SL & UP & OT & BOT & & $\mathrm{F}$ & & & & & & & & \\
\hline & & & & & & & & $\begin{array}{l}\text { PP } \\
\text { sid) }\end{array}$ & $\begin{array}{l}\text { FLTR } \\
\text { (psig) }\end{array}$ & $\begin{array}{c}\text { DP } \\
\text { (psid) }\end{array}$ & $\begin{array}{c}\text { DP } \\
\text { (psig) }\end{array}$ & $\begin{array}{l}\text { FLTRATE } \\
\text { (psig) }\end{array}$ & $\begin{array}{c}\text { BP } \\
\text { (psig) }\end{array}$ & $\begin{array}{l}\text { FLOW } \\
\text { (gpm) }\end{array}$ & $\begin{array}{l}\text { FLOW } \\
\text { (gom) }\end{array}$ & $\begin{array}{l}\text { Temp } \\
\text { corr flow }\end{array}$ & & $\begin{array}{l}\text { Avg } \\
\text { TMPP }\end{array}$ \\
\hline DATE & TIME & Sol & T2 & T3 & $\mathrm{T} 1$ & T4 & T5 & dP2 & P1 & dP1 & $\mathrm{dP3}$ & P2 & P3 & Q1 & Q2 & $\left(\mathrm{gpm} / \mathrm{ft}^{2}\right)$ & (ft/sec) & (psid) \\
\hline 0/23/01 & 22:31 & 0 & 25.8 & 23.4 & 25.6 & 23.3 & 22.3 & 50.0 & 60.2 & 10.4 & 40.2 & 10.4 & 0 & 38.8 & 0.083 & 0.0357 & 16.1 & 45.1 \\
\hline 0/23/01 & 22:32 & 0 & 25.9 & 23.4 & 25.6 & 23.4 & 22.3 & 50.2 & 60.4 & 10.5 & 40.0 & 10.4 & 0 & 38.9 & 0.083 & 0.0356 & 16.1 & 451 \\
\hline /23/01 & 22:33 & 0 & 25.8 & 23.4 & 25.6 & 23.4 & 22.3 & 49.8 & 60.0 & 10.5 & 39.7 & 10.4 & 0 & 38.9 & 0.083 & 0.0356 & 16.1 & 44.8 \\
\hline D/23/01 & 22:34 & 0 & 25.8 & 23.4 & 25.6 & 23.4 & 22.4 & 49.9 & 60.0 & 10.4 & 39.9 & 10.4 & 0 & 38.9 & 0.082 & 0.0352 & 16.1 & 44.9 \\
\hline & & 0 & 25.8 & 23.4 & & & & & & & & & 0 & 3.9 & & & & \\
\hline D/23/01 & 22:36 & 0 & 25.9 & 23.4 & 25.6 & 23.5 & 22.4 & 50.1 & 60.2 & 10.6 & 39.9 & 10.3 & 0 & 38.9 & 0.082 & 0.0352 & 16.1 & 45.0 \\
\hline$/ 23 / 01$ & 22:37 & 0 & 25.9 & 23.4 & 25.7 & 23.5 & 22.5 & 50.1 & 60.2 & 10.5 & 39.8 & & 0 & 38.9 & 0.082 & & 16.1 & 49 \\
\hline $0 / 23 / 01$ & 22:38 & 0 & 25.9 & 23.4 & 25.7 & 23.5 & 22.5 & 50.0 & 60.1 & 10.5 & 39.9 & 10.3 & 0 & 38.9 & 0.081 & & 16.1 & 45.0 \\
\hline 0/23/01 & 22:39 & 0 & 25.9 & 23.4 & 25.7 & 23.5 & 22.5 & 50.0 & 60.2 & 10.5 & 39.9 & 10.3 & 0 & 38.8 & 0.081 & 0.0347 & 16.1 & 45.0 \\
\hline & 22:40 & 0 & 25.9 & 23.4 & 25.7 & & 2.6 & .8 & 59.9 & 10.5 & 40.1 & & & 38.9 & & & 6.1 & \\
\hline /23/01 & 22:41 & 0 & 25.9 & 23.4 & 25.7 & 23.6 & 22.6 & 50.2 & 60.3 & 10.4 & 40.1 & 10.3 & 0 & 38.9 & 0.081 & 0. & 16.1 & 45.1 \\
\hline $0 / 23 / 01$ & 22:42 & 0 & 25.9 & 23.4 & 25.7 & 23.7 & 22.6 & 50.4 & 60.5 & 10.5 & 40.1 & 0.2 & 0 & 38.7 & 0.081 & 0. & 16.1 & 45.2 \\
\hline & 22:43 & 0 & 25.9 & 23.4 & 25.7 & 23.6 & 22.6 & 0.1 & 60.2 & 10.4 & 39.5 & & 0 & 38.8 & 0.080 & & 16.1 & \\
\hline & 22:44 & 0 & 25.9 & 23.4 & 25.7 & 23.6 & 22.7 & 50.2 & 60.1 & 10.5 & 40.2 & 10.2 & 0 & 38.9 & 0.080 & & 6.1 & 45.2 \\
\hline 1 & 22:45 & 0 & 26.0 & 23.4 & 25.7 & 23.7 & 22.7 & 50.1 & 60.1 & 10.5 & 40.1 & 0.2 & & 38.9 & 0.080 & & 16.2 & 45.1 \\
\hline & 22:46 & 0 & 26.0 & 23.4 & 25.8 & 23.8 & 22.7 & 49.8 & 59.8 & 10.5 & 40.0 & 10.2 & 0 & 38.9 & 0.080 & & 16.1 & \\
\hline 1 & 22:47 & 0 & 26.0 & 23.4 & 25.7 & 23.8 & 22.7 & 50.4 & 60.5 & 10.5 & 40. & 10.2 & 0 & 39.0 & 0.080 & & 6.2 & 45.2 \\
\hline & 22:48 & 0 & 26.0 & 23.4 & 25.8 & 23.8 & 22.8 & 50.4 & 60.4 & 10.4 & 40. & & & 38.8 & 0.080 & & .1 & 4 \\
\hline & 22:49 & 0 & 26.0 & 23.4 & 25.7 & 23.8 & 22.8 & 50.0 & 60.0 & 10.5 & 40 & 10.2 & 0 & 38.8 & 0.080 & & 16.1 & 45.1 \\
\hline 1 & 22:50 & 0 & 26.0 & 23.4 & 25.8 & 23.8 & 22.8 & 50.5 & 60.6 & 10.6 & 40 & 10.2 & & 38.9 & 0.080 & 0. & 16.2 & 45 \\
\hline & 22:51 & 0 & 26.0 & 23.4 & 25.8 & 23.8 & 22.8 & 50.2 & 60.2 & 10.4 & 40 & 0.2 & 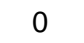 & 38.9 & 79 & & 6.1 & 45.2 \\
\hline & 22:52 & 0 & 26.0 & 23.4 & 25.8 & 23.8 & 22.9 & 50.2 & 60.2 & 10.5 & 40. & 0.2 & 0 & 38.9 & 0.079 & & 6.1 & 45.1 \\
\hline 1 & 22:53 & 0 & 26.0 & 23.4 & 25.8 & 23.8 & 22.9 & 0.4 & 60.4 & 10.5 & $3 s$ & & 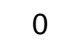 & 38.8 & 9 & & 16.1 & 4 \\
\hline & 22:54 & 0 & 26.1 & 23.4 & 25.8 & 23.8 & 22.9 & 50.3 & 60.3 & 10.6 & & & 0 & 38.8 & & & 6.1 & 4 \\
\hline 1 & 22:55 & 0 & 26.1 & 23.4 & 25.8 & 23.9 & 22.9 & 50.4 & 60.4 & 10. & 40 & 10.1 & 0 & 39.2 & 0.079 & 0. & 16.3 & 45.4 \\
\hline & 22:56 & 0 & 26.1 & 23.4 & 25.8 & 23.8 & 22.9 & 50.0 & 59.9 & 10.6 & Ty & 0.1 & & 38.9 & 9 & & .1 & 45 \\
\hline & 22:57 & 0 & 26.1 & 23.4 & 25.8 & 23.9 & 23.0 & 50.2 & 60.0 & 10.5 & $4 C$ & & 0 & 39.0 & 0. & & 16.2 & 45.1 \\
\hline 1 & 22:58 & 0 & 26.1 & 23.4 & 25.8 & 3.9 & 23.0 & 50.3 & 60.3 & 10.5 & 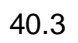 & 1 & ( & 39.0 & 0. & & 16.2 & 45.3 \\
\hline & 22:59 & 0 & 26.1 & 23.4 & 25.8 & & 23.0 & 50.1 & 60.1 & 10.5 & & & & & & & & \\
\hline & $23: 0$ & 0 & 26.1 & 23.4 & 25.8 & 23.9 & 23.0 & 50.2 & 60.3 & 10.4 & 4 & 10.1 & 0 & 38.9 & 0. & & .1 & 45.3 \\
\hline & 23:01 & 0 & 26.1 & 23.4 & 25.9 & 23.9 & 3.0 & 50.5 & 60.5 & 10.5 & 40.2 & 0.1 & 0 & 38.9 & 8 & 32 & 6.2 & 45.3 \\
\hline & 23:02 & 0 & 26.1 & 3.4 & 25.8 & 23.9 & 22.9 & 50.4 & 60.2 & 10.5 & & & 0 & & & & 1 & \\
\hline & 23:03 & 0 & 26.1 & 23.4 & 25.9 & 23.8 & 22.8 & 50.6 & 60.6 & 10.6 & 4 & 1 & 0 & 38.8 & 0.078 & & 6.1 & 45.5 \\
\hline & 23 & 0 & 26.1 & 23.4 & 25.8 & 23.8 & 22.8 & 50.1 & 60.0 & 10.5 & 1 & & & 38.8 & & & 16.1 & 4 \\
\hline & 23: & 0 & 26.1 & 23.4 & 25.9 & 23.7 & 22.7 & 50.2 & 60.2 & 10 & & & ( & 8 & 0. & & 16.1 & \\
\hline & 23:06 & 0 & 26.1 & 23.4 & 25.8 & 23.6 & 22.6 & 50.5 & 60.4 & 10.5 & 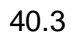 & .1 & 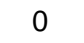 & 38.9 & 0.078 & 33 & 16.1 & 45.4 \\
\hline & 23: & 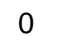 & 26.1 & .5 & 25.9 & 23.6 & 22.5 & 50.7 & 60.8 & 10 & & & & & & & & \\
\hline & 23:08 & U & 26.2 & .5 & 25.9 & 23.5 & 22.5 & 50.4 & 60.4 & 10 & & & 0 & 38.9 & 8 & & 16.2 & 45.3 \\
\hline & $23:($ & 0 & 26.1 & 23.5 & 25.9 & 23.4 & 22.4 & 50.4 & 60.3 & 10. & 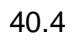 & & 0 & 38.8 & 0. & & 16.1 & 45.4 \\
\hline & 23 & 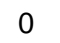 & 26.2 & 3.5 & 25.9 & 23.4 & 22.3 & 50.3 & 60.2 & 10 & & & & & & & & \\
\hline & 23:11 & 0 & 26.2 & 3.5 & 25.9 & 3.3 & 22.3 & 50.7 & 60.7 & ( & 7 & & 0 & 38.8 & 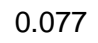 & & 161 & 45.7 \\
\hline & 23: & 0 & 26.2 & 3.5 & 25.9 & 23.3 & 22.2 & 50.2 & 60.1 & 10 & 1 & & & 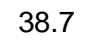 & & & & \\
\hline & $23: 13$ & 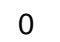 & 26.2 & & 25.7 & 23.3 & 22.2 & 50.5 & 60.4 & 1 & & & 0 & & & & 16.0 & 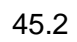 \\
\hline & $23: 14$ & 0 & 26.1 & 23.5 & 25.1 & 23.2 & 22.2 & 50.4 & 60.3 & 10.3 & 4 & 10.0 & 0 & 38.6 & 0.076 & & 16.0 & 45.3 \\
\hline & 23 & 0 & 26.0 & 23.4 & 24.6 & 23.2 & 22.2 & 50 & 60.1 & & & & & & & & & \\
\hline & $23: 16$ & 0 & 25.9 & 23.4 & & 23.1 & & & 60.4 & 10 & 3 & 12.6 & 0 & 38.5 & 0.010 & 44 & 16.0 & 43.0 \\
\hline & 23 & 0 & 25.9 & 23.4 & & 20 & & & 60.3 & & & & & v & & & 16.0 & 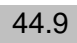 \\
\hline & 23 & 0 & 25.8 & 23.4 & & 23 & 22.3 & 50.0 & 60.3 & 10 & & & U & & 33 & & 16.0 & \\
\hline & & 0 & 25.7 & & & & & & & & & 10. & 0 & 38.6 & & & 16.0 & \\
\hline & & & 5.1 & & & & & & & & & 10. & & 8.6 & & & 6. & \\
\hline
\end{tabular}


WSRC-TR-2002-00459, Rev. 0 SRT-RPP-2002-00221

\author{
Xflow2_102301_1011
} \begin{tabular}{cccccccccccccc} 
CL & SL & UP & BOT & BOT & \multicolumn{1}{c}{ FLTR } & TOP & \multicolumn{3}{c}{ SL } & FLTR & \\
FLTRT LOOP LOOP & AMB & AMB & DP & FLTR & DP & DP & FLTRATE & BP & FLOW & FLOW & Temp & Avg
\end{tabular} $\begin{array}{llllllllllllllllll} & \left({ }^{\circ} \mathrm{C}\right) & \left({ }^{\circ} \mathrm{C}\right) & \left({ }^{\circ} \mathrm{C}\right) & \left({ }^{\circ} \mathrm{C}\right) & \left({ }^{\circ} \mathrm{C}\right) & \text { (psid) } & \text { (psig) } & \text { (psid) } & \text { (psig) } & \text { (psig) } & \text { (psig) } & \text { (gpm) } & \text { (gpm) } & \text { corr flow } & \text { Axial Vel TMP }\end{array}$

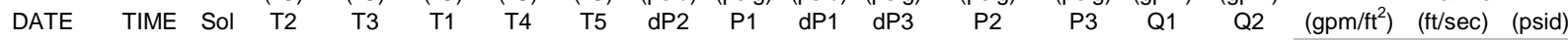

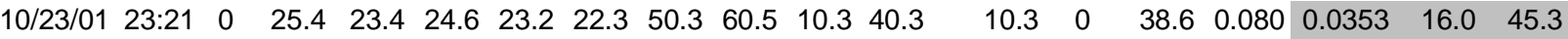
$\begin{array}{llllllllllll}10 / 23 / 01 & 23: 22 & 0 & 25.3 & 23.4 & 24.7 & 23.2 & 22.4 & 50.2 & 60.3 & 10.4 & 40.2\end{array}$

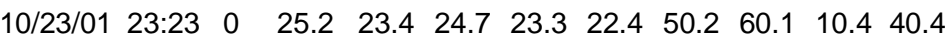

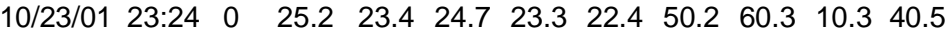

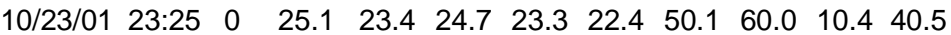

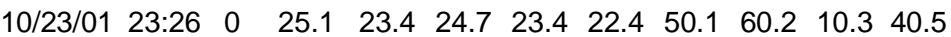

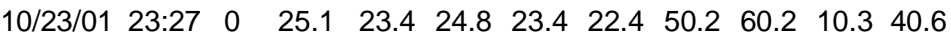

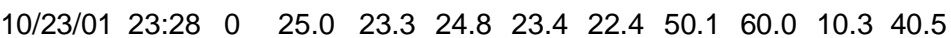

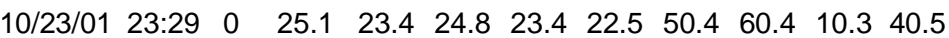

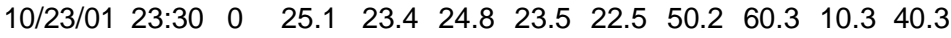

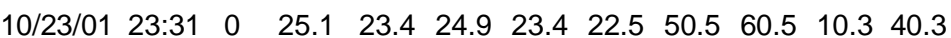

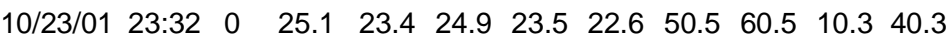

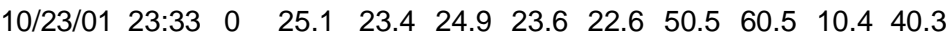

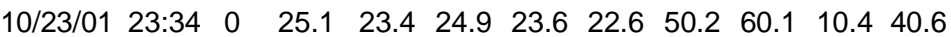

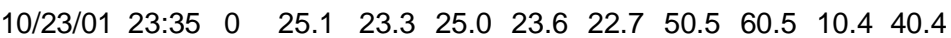

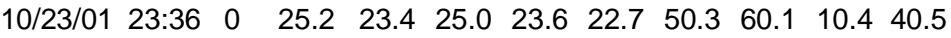

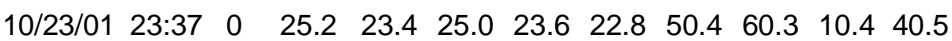

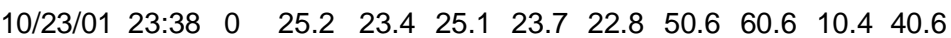

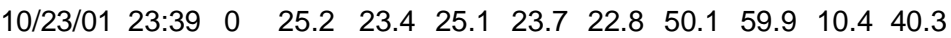

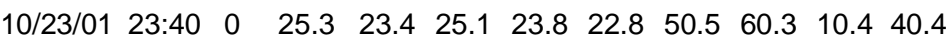

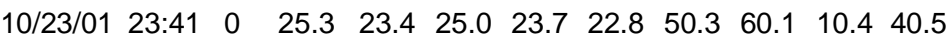

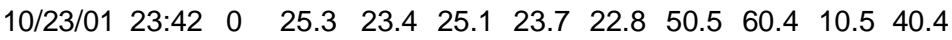

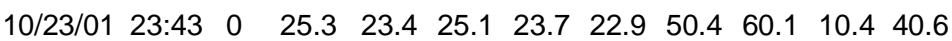

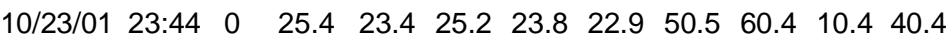

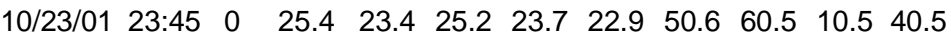

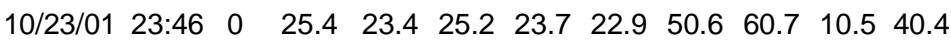

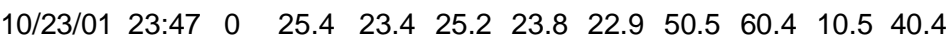

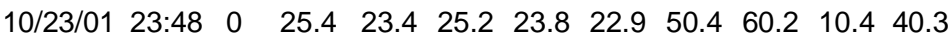

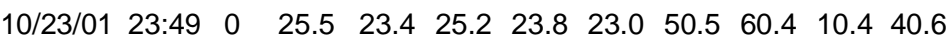

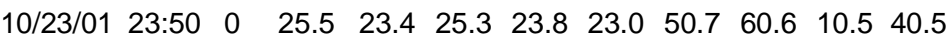

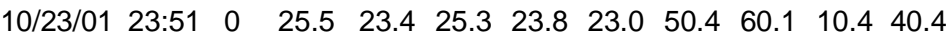

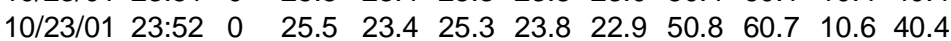

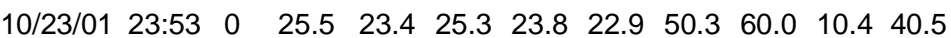

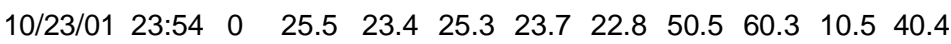
$\begin{array}{llllllllllll}10 / 23 / 01 & 23: 55 & 0 & 25.6 & 23.4 & 25.3 & 23.7 & 22.7 & 50.3 & 60.2 & 10.5 & 40.3\end{array}$

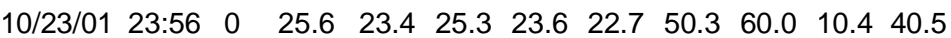

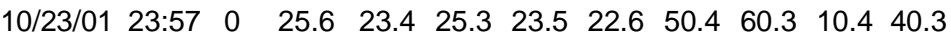

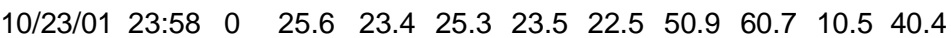

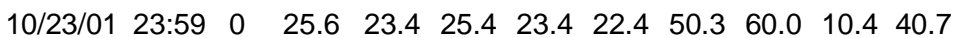

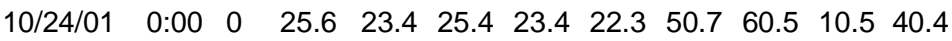

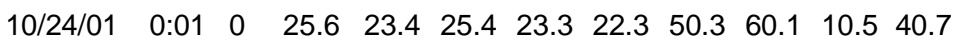

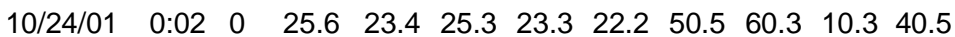

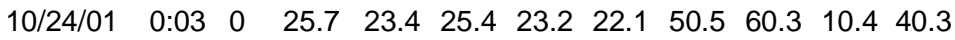

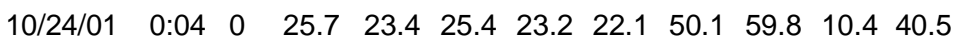

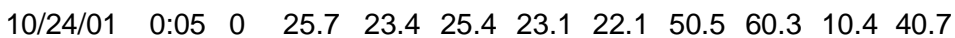

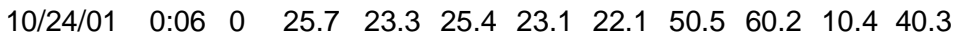

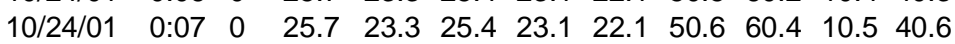
$\begin{array}{llllllllllll}10 / 24 / 01 & 0: 08 & 0 & 25.7 & 23.3 & 25.5 & 23.1 & 22.1 & 50.3 & 60.1 & 10.4 & 40.6\end{array}$

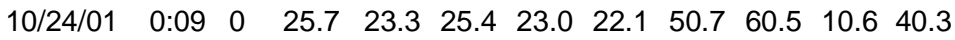

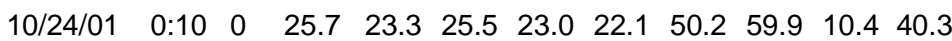

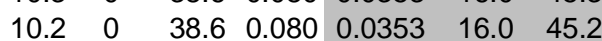

$\begin{array}{llllllll}10.2 & 0 & 38.6 & 0.080 & 0.0352 & 16.0 & 45.3\end{array}$

$\begin{array}{llllllll}10.2 & 0 & 38.6 & 0.079 & 0.0348 & 16.0 & 45.4\end{array}$

$\begin{array}{llllllll}10.2 & 0 & 38.6 & 0.079 & 0.0347 & 16.0 & 45.3\end{array}$

$\begin{array}{llllllll}10.2 & 0 & 38.7 & 0.079 & 0.0347 & 16.1 & 45.3\end{array}$

$\begin{array}{lllllllll}10.2 & 0 & 38.7 & 0.078 & 0.0343 & 16.0 & 45.4\end{array}$

$\begin{array}{llllllll}10.1 & 0 & 38.6 & 0.078 & 0.0343 & 16.0 & 45.3\end{array}$

$\begin{array}{llllllll}10.1 & 0 & 38.7 & 0.078 & 0.0342 & 16.0 & 45.4\end{array}$

$\begin{array}{lllllll}10.1 & 0 & 38.5 & 0.078 & 0.0342 & 16.0 & 45.3\end{array}$

$\begin{array}{llllllll}10.1 & 0 & 38.6 & 0.078 & 0.0342 & 16.0 & 45.4\end{array}$

$\begin{array}{llllllll}10.1 & 0 & 38.7 & 0.078 & 0.0342 & 16.1 & 45.4\end{array}$

$\begin{array}{lllllllll}10.1 & 0 & 38.7 & 0.078 & 0.0341 & 16.1 & 45.4\end{array}$

$\begin{array}{llllllll}10.1 & 0 & 38.7 & 0.077 & 0.0337 & 16.1 & 45.4\end{array}$

$\begin{array}{llllllll}10.1 & 0 & 38.8 & 0.077 & 0.0336 & 16.1 & 45.5\end{array}$

$\begin{array}{llllllll}10.1 & 0 & 38.8 & 0.077 & 0.0336 & 16.1 & 45.4\end{array}$

$\begin{array}{lllllll}10.1 & 0 & 38.7 & 0.077 & 0.0336 & 16.1 & 45.4\end{array}$

$\begin{array}{llllllll}10.1 & 0 & 38.9 & 0.077 & 0.0336 & 16.1 & 45.6\end{array}$

$\begin{array}{llllllll}10.1 & 0 & 38.7 & 0.077 & 0.0336 & 16.1 & 45.2\end{array}$

$\begin{array}{llllllll}10.0 & 0 & 39.0 & 0.077 & 0.0336 & 16.2 & 45.5\end{array}$

$\begin{array}{llllllll}10.0 & 0 & 38.8 & 0.077 & 0.0336 & 16.1 & 45.4\end{array}$

$\begin{array}{llllllll}10.0 & 0 & 38.9 & 0.077 & 0.0335 & 16.1 & 45.4\end{array}$

$\begin{array}{lllllll}10.0 & 0 & 38.9 & 0.076 & 0.0331 & 16.1 & 45.5\end{array}$

$\begin{array}{llllllll}10.0 & 0 & 38.8 & 0.076 & 0.0330 & 16.1 & 45.5\end{array}$

$\begin{array}{llllllll}10.0 & 0 & 38.9 & 0.076 & 0.0330 & 16.1 & 45.5\end{array}$

$\begin{array}{llllllll}10.0 & 0 & 38.7 & 0.076 & 0.0330 & 16.1 & 45.5\end{array}$

$\begin{array}{llllllll}10.0 & 0 & 38.8 & 0.076 & 0.0330 & 16.1 & 45.5\end{array}$

$\begin{array}{llllllll}10.0 & 0 & 38.9 & 0.076 & 0.0330 & 16.1 & 45.3\end{array}$

$\begin{array}{llllllll}10.0 & 0 & 38.8 & 0.076 & 0.0330 & 16.1 & 45.5\end{array}$

$\begin{array}{llllllll}10.0 & 0 & 38.8 & 0.076 & 0.0329 & 16.1 & 45.6\end{array}$

$\begin{array}{llllllll}10.0 & 0 & 38.8 & 0.076 & 0.0329 & 16.1 & 45.4\end{array}$

$\begin{array}{llllllll}10.0 & 0 & 38.9 & 0.076 & 0.0329 & 16.2 & 45.6\end{array}$

\begin{tabular}{lllll|l|l|}
10.0 & 0 & 39.0 & 0.076 & 0.0329 & 16.2 & 45.4
\end{tabular}

$\begin{array}{llllllll}10.0 & 0 & 38.9 & 0.076 & 0.0329 & 16.1 & 45.4\end{array}$

$\begin{array}{llllllll}10.0 & 0 & 38.9 & 0.076 & 0.0329 & 16.1 & 45.3\end{array}$

$\begin{array}{llllllll}10.0 & 0 & 38.8 & 0.075 & 0.0325 & 16.1 & 45.4\end{array}$

$\begin{array}{llllllll}10.0 & 0 & 38.8 & 0.075 & 0.0324 & 16.1 & 45.4\end{array}$

$\begin{array}{llllllll}10.0 & 0 & 38.7 & 0.075 & 0.0325 & 16.1 & 45.6\end{array}$

$\begin{array}{llllllll}10.0 & 0 & 38.9 & 0.075 & 0.0324 & 16.2 & 45.5\end{array}$

$\begin{array}{llllllll}9.9 & 0 & 38.8 & 0.075 & 0.0324 & 16.1 & 45.5\end{array}$

$\begin{array}{llllllll}9.9 & 0 & 38.8 & 0.075 & 0.0324 & 16.1 & 45.5\end{array}$

$\begin{array}{llllllll}9.9 & 0 & 38.7 & 0.075 & 0.0324 & 16.1 & 45.5\end{array}$

$\begin{array}{llllllll}9.9 & 0 & 38.9 & 0.075 & 0.0324 & 16.2 & 45.4\end{array}$

$\begin{array}{llllllll}9.9 & 0 & 38.9 & 0.075 & 0.0324 & 16.2 & 45.3\end{array}$

$\begin{array}{llllllll}9.9 & 0 & 38.8 & 0.075 & 0.0324 & 16.1 & 45.6\end{array}$

$\begin{array}{llllllll}9.9 & 0 & 38.9 & 0.075 & 0.0324 & 16.1 & 45.4\end{array}$

$\begin{array}{llllllll}9.9 & 0 & 38.9 & 0.075 & 0.0324 & 16.1 & 45.6\end{array}$

$\begin{array}{llllllll}9.9 & 0 & 38.9 & 0.075 & 0.0323 & 16.2 & 45.5\end{array}$

$\begin{array}{lllllllll}9.9 & 0 & 38.8 & 0.075 & 0.0323 & 16.1 & 45.5\end{array}$

$\begin{array}{lllllll}9.9 & 0 & 38.9 & 0.075 & 0.0323 & 16.2 & 45.3\end{array}$ 
WSRC-TR-2002-00459, Rev. 0 SRT-RPP-2002-00221

Xflow2_102301_1011

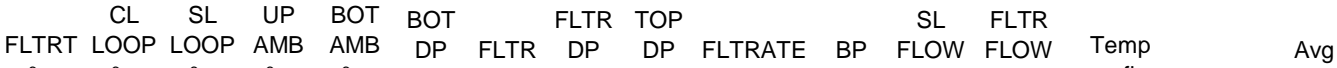
$\begin{array}{lllllllllllllll}\left({ }^{\circ} \mathrm{C}\right) & \left({ }^{\circ} \mathrm{C}\right) & \left({ }^{\circ} \mathrm{C}\right) & \left({ }^{\circ} \mathrm{C}\right) & \left({ }^{\circ} \mathrm{C}\right) & (\mathrm{psid}) & (\mathrm{psig}) & \text { (psid) } & \text { (psig) } & \text { (psig) } & \text { (psig) } & \text { (gpm) } & \text { (gpm) } & \text { corr flow } & \text { Axial Vel TMP }\end{array}$

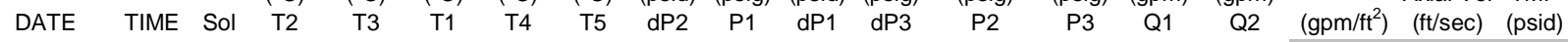

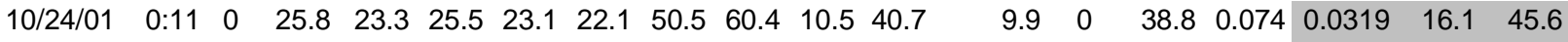

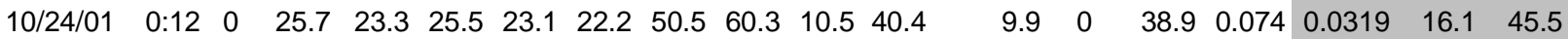
$\begin{array}{llllllllllll}10 / 24 / 01 & 0: 13 & 0 & 25.8 & 23.3 & 25.5 & 23.1 & 22.2 & 50.8 & 60.5 & 10.5 & 40.5\end{array}$ $\begin{array}{llllllllllll}10 / 24 / 01 & 0: 14 & 0 & 25.8 & 23.3 & 25.6 & 23.1 & 22.2 & 50.6 & 60.3 & 10.5 & 40.2\end{array}$ $\begin{array}{llllllllllll}10 / 24 / 01 & 0: 15 & 0 & 25.8 & 23.3 & 25.5 & 23.1 & 22.3 & 50.1 & 59.7 & 10.5 & 40.5\end{array}$ $\begin{array}{llllllllllll}10 / 24 / 01 & 0: 16 & 0 & 25.8 & 23.3 & 25.6 & 23.1 & 22.3 & 50.7 & 60.4 & 10.5 & 40.3\end{array}$ $\begin{array}{llllllllllll}10 / 24 / 01 & 0: 17 & 0 & 25.8 & 23.3 & 25.6 & 23.2 & 22.3 & 50.8 & 60.6 & 10.5 & 40.6\end{array}$ $\begin{array}{llllllllllll}10 / 24 / 01 & 0: 18 & 0 & 25.8 & 23.3 & 25.6 & 23.3 & 22.4 & 50.4 & 60.1 & 10.4 & 40.6\end{array}$ $\begin{array}{llllllllllll}10 / 24 / 01 & 0: 19 & 0 & 25.8 & 23.3 & 25.6 & 23.3 & 22.4 & 50.5 & 60.3 & 10.5 & 40.4\end{array}$ $\begin{array}{llllllllllll}10 / 24 / 01 & 0: 20 & 0 & 25.8 & 23.3 & 25.6 & 23.3 & 22.5 & 50.6 & 60.4 & 10.6 & 40.6\end{array}$ $\begin{array}{llllllllllll}10 / 24 / 01 & 0: 21 & 0 & 25.9 & 23.3 & 25.6 & 23.3 & 22.5 & 50.4 & 60.2 & 10.5 & 40.6\end{array}$ $\begin{array}{llllllllllll}10 / 24 / 01 & 0: 22 & 0 & 25.9 & 23.3 & 25.6 & 23.3 & 22.5 & 50.6 & 60.2 & 10.5 & 40.5\end{array}$ $\begin{array}{llllllllllll}10 / 24 / 01 & 0: 23 & 0 & 25.9 & 23.3 & 25.7 & 23.4 & 22.6 & 50.4 & 60.1 & 10.5 & 40.8\end{array}$

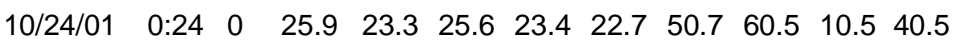

$\begin{array}{llllllllllll}10 / 24 / 01 & 0: 25 & 0 & 25.9 & 23.3 & 25.7 & 23.5 & 22.7 & 50.4 & 60.0 & 10.5 & 40.7\end{array}$ $\begin{array}{llllllllllll}10 / 24 / 01 & 0: 26 & 0 & 25.9 & 23.3 & 25.7 & 23.5 & 22.8 & 50.6 & 60.4 & 10.6 & 40.5\end{array}$ $\begin{array}{llllllllllll}10 / 24 / 01 & 0: 27 & 0 & 25.9 & 23.3 & 25.7 & 23.5 & 22.7 & 50.6 & 60.3 & 10.5 & 40.7\end{array}$ $\begin{array}{llllllllllll}10 / 24 / 01 & 0: 28 & 1 & 25.8 & 23.3 & 25.7 & 23.5 & 22.8 & 9.5 & 60.4 & 10.5 & -0.4\end{array}$ $\begin{array}{llllllllllll}10 / 24 / 01 & 0: 29 & 0 & 25.9 & 23.3 & 25.7 & 23.5 & 22.8 & 49.6 & 60.2 & 10.5 & 39.5\end{array}$ $\begin{array}{llllllllllll}10 / 24 / 01 & 0: 30 & 0 & 25.9 & 23.3 & 25.7 & 23.6 & 22.8 & 49.9 & 60.2 & 10.5 & 39.8\end{array}$ $\begin{array}{llllllllllll}10 / 24 / 01 & 0: 31 & 0 & 25.9 & 23.3 & 25.7 & 23.5 & 22.8 & 49.7 & 59.9 & 10.5 & 40.2\end{array}$ $\begin{array}{llllllllllll}10 / 24 / 01 & 0: 32 & 0 & 25.9 & 23.3 & 25.7 & 23.5 & 22.8 & 50.0 & 59.9 & 10.5 & 40.3\end{array}$ $\begin{array}{llllllllllll}10 / 24 / 01 & 0: 33 & 0 & 25.9 & 23.3 & 25.7 & 23.5 & 22.8 & 49.9 & 59.9 & 10.6 & 40.2\end{array}$ $\begin{array}{llllllllllll}10 / 24 / 01 & 0: 34 & 0 & 25.9 & 23.3 & 25.7 & 23.5 & 22.8 & 50.4 & 60.3 & 10.5 & 40.5\end{array}$ $\begin{array}{llllllllllll}10 / 24 / 01 & 0: 35 & 0 & 25.9 & 23.3 & 25.7 & 23.5 & 22.9 & 50.3 & 60.3 & 10.5 & 40.3\end{array}$

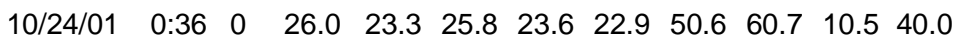
$\begin{array}{llllllllllll}10 / 24 / 01 & 0: 37 & 0 & 26.0 & 23.3 & 25.8 & 23.6 & 22.9 & 50.1 & 60.0 & 10.6 & 40.1\end{array}$ $\begin{array}{llllllllllll}10 / 24 / 01 & 0: 38 & 0 & 26.0 & 23.3 & 25.8 & 23.6 & 22.9 & 50.2 & 60.2 & 10.6 & 40.3\end{array}$ $\begin{array}{llllllllllll}10 / 24 / 01 & 0: 39 & 0 & 26.0 & 23.3 & 25.8 & 23.6 & 22.9 & 50.6 & 60.6 & 10.6 & 40.2\end{array}$ $\begin{array}{llllllllllll}10 / 24 / 01 & 0: 40 & 0 & 26.0 & 23.3 & 25.8 & 23.6 & 22.8 & 50.3 & 60.2 & 10.5 & 40.6\end{array}$

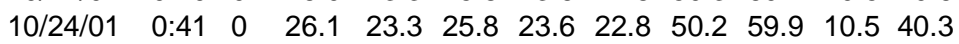
$\begin{array}{llllllllllll}10 / 24 / 01 & 0: 42 & 0 & 26.0 & 23.3 & 25.8 & 23.5 & 22.7 & 50.4 & 60.2 & 10.5 & 40.3\end{array}$ $\begin{array}{llllllllllll}10 / 24 / 01 & 0: 43 & 0 & 26.1 & 23.3 & 25.8 & 23.4 & 22.6 & 50.6 & 60.4 & 10.6 & 40.2\end{array}$ $\begin{array}{llllllllllll}10 / 24 / 01 & 0: 44 & 0 & 26.1 & 23.4 & 25.8 & 23.4 & 22.5 & 50.1 & 60.0 & 10.5 & 40.3\end{array}$ $\begin{array}{llllllllllll}10 / 24 / 01 & 0: 45 & 0 & 26.0 & 23.4 & 25.8 & 23.3 & 22.5 & 50.6 & 60.4 & 10.5 & 40.5\end{array}$ $\begin{array}{llllllllllll}10 / 24 / 01 & 0: 46 & 0 & 26.1 & 23.4 & 25.8 & 23.3 & 22.4 & 50.5 & 60.3 & 10.6 & 40.7\end{array}$

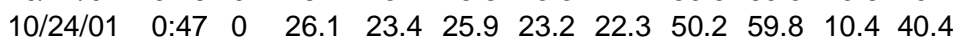
$\begin{array}{llllllllllll}10 / 24 / 01 & 0: 48 & 0 & 26.1 & 23.4 & 25.8 & 23.1 & 22.2 & 50.5 & 60.4 & 10.6 & 40.4\end{array}$ $\begin{array}{llllllllllll}10 / 24 / 01 & 0: 49 & 0 & 26.1 & 23.4 & 25.9 & 23.0 & 22.2 & 50.8 & 60.5 & 10.5 & 40.4\end{array}$ $\begin{array}{llllllllllll}10 / 24 / 01 & 0: 50 & 0 & 26.2 & 23.4 & 25.9 & 23.0 & 22.1 & 50.1 & 59.9 & 10.5 & 40.4\end{array}$ $\begin{array}{llllllllllll}10 / 24 / 01 & 0: 51 & 0 & 26.2 & 23.4 & 25.9 & 23.0 & 22.1 & 50.6 & 60.5 & 10.5 & 40.4\end{array}$ $\begin{array}{llllllllllll}10 / 24 / 01 & 0: 52 & 0 & 26.2 & 23.4 & 25.9 & 22.9 & 22.1 & 50.5 & 60.3 & 10.5 & 40.6\end{array}$ $\begin{array}{llllllllllll}10 / 24 / 01 & 0: 53 & 0 & 26.2 & 23.3 & 25.9 & 22.9 & 22.1 & 50.7 & 60.4 & 10.5 & 40.3\end{array}$ $\begin{array}{llllllllllll}10 / 24 / 01 & 0: 54 & 0 & 26.2 & 23.3 & 25.9 & 22.9 & 22.2 & 50.8 & 60.4 & 10.6 & 40.5\end{array}$ $\begin{array}{llllllllllll}10 / 24 / 01 & 0: 55 & 0 & 26.2 & 23.3 & 25.9 & 22.9 & 22.1 & 50.9 & 60.7 & 10.6 & 40.2\end{array}$

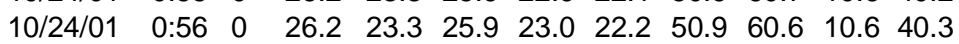
$\begin{array}{llllllllllll}10 / 24 / 01 & 0: 57 & 0 & 26.2 & 23.3 & 25.9 & 22.9 & 22.2 & 51.1 & 60.8 & 10.6 & 40.6\end{array}$ $\begin{array}{llllllllllll}10 / 24 / 01 & 0: 58 & 0 & 26.2 & 23.3 & 25.9 & 22.9 & 22.2 & 50.5 & 60.2 & 10.6 & 40.6\end{array}$

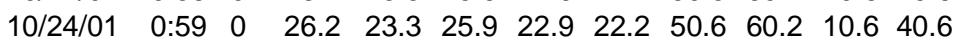

$\begin{array}{lllllll}9.9 & 0 & 39.0 & 0.074 & 0.0319 & 16.2 & 45.6\end{array}$ $\begin{array}{lllllll}9.9 & 0 & 38.8 & 0.074 & 0.0318 & 16.1 & 45.4\end{array}$ $\begin{array}{lllllll}9.9 & 0 & 38.8 & 0.074 & 0.0319 & 16.1 & 45.3\end{array}$ $\begin{array}{llllllll}9.9 & 0 & 38.9 & 0.074 & 0.0318 & 16.1 & 45.5\end{array}$ $\begin{array}{llllllll}9.9 & 0 & 38.9 & 0.074 & 0.0318 & 16.1 & 45.7\end{array}$ $\begin{array}{llllllll}9.9 & 0 & 38.9 & 0.074 & 0.0318 & 16.1 & 45.5\end{array}$ $\begin{array}{llllllll}9.9 & 0 & 38.8 & 0.074 & 0.0318 & 16.1 & 45.5\end{array}$ $\begin{array}{lllllll}9.9 & 0 & 38.9 & 0.074 & 0.0318 & 16.1 & 45.6\end{array}$ $\begin{array}{lllllll}9.9 & 0 & 38.9 & 0.074 & 0.0318 & 16.1 & 45.5\end{array}$ $\begin{array}{llllllll}9.9 & 0 & 38.9 & 0.074 & 0.0318 & 16.2 & 45.5\end{array}$ $\begin{array}{llllllll}9.9 & 0 & 38.7 & 0.074 & 0.0317 & 16.1 & 45.6\end{array}$ $\begin{array}{lllllll}9.9 & 0 & 38.7 & 0.074 & 0.0318 & 16.0 & 45.6\end{array}$ $\begin{array}{llllll} & \text { Average } & 16.1 & 45.4\end{array}$

$\begin{array}{lllllll}9.9 & 0 & 38.9 & 0.075 & 0.0322 & 16.1 & 45.5\end{array}$

$\begin{array}{lllllll}9.9 & 0 & 38.9 & 0.074 & 0.0317 & 16.1 & 45.7\end{array}$

$\begin{array}{lllllll}50.8 & 53 & 38.8 & 0.010 & 0.0043 & 16.1 & 4.5\end{array}$

$\begin{array}{lllllll}10.8 & 0 & 38.9 & 0.089 & 0.0381 & 16.1 & 44.5\end{array}$

$\begin{array}{lllllll}10.4 & 0 & 38.9 & 0.083 & 0.0355 & 16.1 & 44.8\end{array}$

$\begin{array}{lllllll}10.3 & 0 & 38.9 & 0.082 & 0.0351 & 16.1 & 45.0\end{array}$

$\begin{array}{lllllll}10.2 & 0 & 38.8 & 0.081 & 0.0347 & 16.1 & 45.1\end{array}$

$\begin{array}{lllllll}10.2 & 0 & 38.9 & 0.081 & 0.0347 & 16.1 & 45.0\end{array}$

$\begin{array}{lllllll}10.2 & 0 & 38.9 & 0.080 & 0.0342 & 16.1 & 45.4\end{array}$

$\begin{array}{lllllll}10.1 & 0 & 38.8 & 0.080 & 0.0342 & 16.1 & 45.3\end{array}$

$\begin{array}{lllllll}10.1 & 0 & 38.8 & 0.079 & 0.0338 & 16.1 & 45.3\end{array}$

$\begin{array}{lllllll}10.1 & 0 & 38.8 & 0.079 & 0.0338 & 16.1 & 45.1\end{array}$

$\begin{array}{lllllll}10.1 & 0 & 38.9 & 0.079 & 0.0337 & 16.1 & 45.2\end{array}$

$\begin{array}{lllllll}10.0 & 0 & 38.8 & 0.079 & 0.0338 & 16.1 & 45.4\end{array}$

$\begin{array}{llllllll}10.0 & 0 & 38.8 & 0.078 & 0.0333 & 16.1 & 45.5\end{array}$

$\begin{array}{lllllll}10.0 & 0 & 38.8 & 0.078 & 0.0333 & 16.1 & 45.2\end{array}$

$\begin{array}{lllllll}10.0 & 0 & 38.7 & 0.078 & 0.0333 & 16.1 & 45.3\end{array}$

$\begin{array}{lllllll}10.0 & 0 & 38.9 & 0.078 & 0.0333 & 16.1 & 45.4\end{array}$

$\begin{array}{lllllll}10.0 & 0 & 38.8 & 0.078 & 0.0333 & 16.1 & 45.2\end{array}$

$\begin{array}{lllllll}10.0 & 0 & 38.8 & 0.078 & 0.0333 & 16.1 & 45.5\end{array}$

$\begin{array}{lllllll}9.9 & 0 & 39.0 & 0.077 & 0.0329 & 16.2 & 45.6\end{array}$

$\begin{array}{lllllll}9.9 & 0 & 38.8 & 0.077 & 0.0328 & 16.1 & 45.3\end{array}$

$\begin{array}{llllllll}9.9 & 0 & 38.8 & 0.077 & 0.0328 & 16.1 & 45.4\end{array}$

$\begin{array}{lllllll}9.9 & 0 & 38.8 & 0.077 & 0.0328 & 16.1 & 45.6\end{array}$

$\begin{array}{lllllll}9.9 & 0 & 38.7 & 0.077 & 0.0328 & 16.1 & 45.3\end{array}$

$\begin{array}{lllllll}9.9 & 0 & 38.7 & 0.077 & 0.0328 & 16.1 & 45.5\end{array}$

$\begin{array}{lllllll}9.9 & 0 & 38.5 & 0.077 & 0.0328 & 16.0 & 45.5\end{array}$

$\begin{array}{lllllll}9.9 & 0 & 38.8 & 0.077 & 0.0328 & 16.1 & 45.5\end{array}$

$\begin{array}{lllllll}9.9 & 0 & 38.8 & 0.077 & 0.0328 & 16.1 & 45.6\end{array}$

$\begin{array}{lllllll}9.9 & 0 & 38.9 & 0.077 & 0.0327 & 16.2 & 45.5\end{array}$

$\begin{array}{llllllll}9.9 & 0 & 38.8 & 0.076 & 0.0324 & 16.1 & 45.6\end{array}$

$\begin{array}{lllllll}9.8 & 0 & 38.9 & 0.076 & 0.0323 & 16.1 & 45.9\end{array}$

$\begin{array}{lllllll}9.8 & 0 & 38.8 & 0.076 & 0.0323 & 16.1 & 45.5\end{array}$

$\begin{array}{lllllll}9.8 & 0 & 38.8 & 0.076 & 0.0324 & 16.1 & 45.6\end{array}$ 


\begin{tabular}{|c|c|c|c|c|c|c|c|c|c|c|c|c|c|c|c|c|c|c|}
\hline & TIME & Sol & $\begin{array}{c}\text { FLTRT } \\
\left({ }^{\circ} \mathrm{C}\right) \\
\text { T2 }\end{array}$ & $\begin{array}{c}\mathrm{CL} \\
\text { LOOP } \\
\left({ }^{\circ} \mathrm{C}\right) \\
\text { T3 }\end{array}$ & $\begin{array}{l}\text { SL } \\
\text { LOOP } \\
\left({ }^{\circ} \mathrm{C}\right) \\
\mathrm{T} 1\end{array}$ & $\begin{array}{l}\text { UP } \\
\text { AMB } \\
\left({ }^{\circ} \mathrm{C}\right) \\
\text { T4 }\end{array}$ & $\begin{array}{c}\text { BOT } \\
\text { AMB } \\
\left(^{\circ} \mathrm{C}\right) \\
\text { T5 }\end{array}$ & $\begin{array}{c}\text { BOT } \\
\text { DP } \\
\text { (psid) } \\
\text { dP2 }\end{array}$ & $\begin{array}{l}\text { FLTR } \\
\text { (psig) } \\
\text { P1 }\end{array}$ & $\begin{array}{l}\text { FLTR } \\
\text { DP } \\
\text { (psid) } \\
\text { dP1 }\end{array}$ & $\begin{array}{l}\text { TOP } \\
\text { DP } \\
\text { (psig) } \\
\text { dP3 }\end{array}$ & $\begin{array}{l}\text { FLT- } \\
\text { RATE } \\
\text { (psig) } \\
\text { P2 }\end{array}$ & $\begin{array}{c}\text { BP } \\
\text { (psig) } \\
\text { P3 }\end{array}$ & $\begin{array}{c}\text { SL } \\
\text { FLoW } \\
\text { (gpm) } \\
\text { Q1 }\end{array}$ & $\begin{array}{c}\text { FLTR } \\
\text { FLOW } \\
\text { (gpm) } \\
\text { Q2 }\end{array}$ & $\begin{array}{l}\text { Temp } \\
\text { corrected } \\
\text { flow } \\
\left(\mathrm{gpm} / \mathrm{tt}^{2}\right)\end{array}$ & $\begin{array}{c}\text { Axial Vel } \\
(\mathrm{ft} / \mathrm{sec})\end{array}$ & $\begin{array}{l}\text { Avg } \\
\text { TMP } \\
\text { (psid) }\end{array}$ \\
\hline 0/24/01 & $6: 30$ & 0 & 26.0 & 25.2 & 25.7 & 25.6 & 25.7 & 50.4 & 60.4 & 10.4 & 40.9 & 9.9 & 0 & 38.3 & 0.078 & 0.0334 & 15.9 & 45.6 \\
\hline $24 / 01$ & $: 31$ & 0 & 26.0 & 25.2 & 25.7 & & 25.8 & 50.8 & 60.9 & 10.4 & 41.0 & 9.9 & & 38.4 & 0.077 & 0.0329 & 16.0 & 5.9 \\
\hline$/ 24 / 01$ & 32 & 0 & 26.0 & 25.2 & 25.7 & 25.5 & 25.8 & 50.7 & 60.7 & 10.5 & 40.9 & 9.8 & 0 & 38.5 & 0.076 & 0.0325 & 16.0 & 45.8 \\
\hline $24 / 01$ & 33 & 0 & 26.0 & .3 & 25.8 & 25.6 & 25.8 & 51.0 & 61.0 & 10.6 & 41.0 & 98 & & 38.4 & 0.076 & & 159 & 6.0 \\
\hline /24/01 & $6: 34$ & 0 & 26.0 & 5.3 & 25.8 & 25.6 & 25.7 & 51.2 & 61.2 & 10.5 & 41.3 & 9.8 & & 38.4 & 0.076 & 0.0324 & 15.9 & 46.3 \\
\hline 24/01 & $: 35$ & 0 & 26.0 & .3 & 25.8 & 25.6 & 25.7 & 50.7 & 60.6 & 10.4 & 40.9 & 98 & & 38.5 & 0.075 & & 60 & 58 \\
\hline$/ 24 / 01$ & $6: 36$ & 0 & 26.0 & 5.3 & 25.9 & 25.6 & 25.8 & 51.1 & 61.0 & 10.5 & 41.2 & 9.8 & & 38.5 & 0.075 & 0.0320 & 16.0 & 46.1 \\
\hline 24 & $: 37$ & 0 & 26.1 & 3 & .8 & & 25.8 & .0 & 608 & 10.5 & 40.9 & 97 & & 8.4 & 0.075 & & & 150 \\
\hline $4 / 01$ & 38 & 0 & 26.1 & .3 & 5.9 & 25.6 & 25.8 & 50.8 & 60.6 & 10.4 & 41.0 & 9. & & 38.4 & 0.075 & 0319 & 15.9 & 45.9 \\
\hline & :39 & 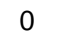 & 261 & & & & & & 607 & 10.4 & 41.2 & 0 & & 8.4 & 0.074 & & & \\
\hline & $6: 40$ & 0 & 26.1 & .3 & 5.8 & 5.6 & 25.8 & 51.2 & 60.9 & 10.5 & 41.2 & 9. & & 8.5 & 0.074 & & 16.0 & 46.2 \\
\hline & $6: 41$ & 0 & 261 & & & & & 51.1 & 60.9 & 10.5 & 41.1 & 9 & & 3.4 & 74 & & & \\
\hline /24/C & 42 & 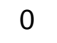 & 6.2 & .3 & 26.0 & 25.7 & 25.8 & 50.9 & 60.7 & 10.5 & 41.1 & 9.7 & & 38.3 & 0.074 & & 15.9 & 6.0 \\
\hline & $6: 43$ & 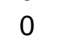 & 26.2 & & & & & & & 10.5 & 41.0 & & & & & & & \\
\hline$/ 24 / 01$ & $6: 44$ & 0 & 26.2 & 5.3 & 25.9 & 25.6 & 25.9 & 51.0 & 61.0 & 10.5 & 41.3 & 9. & & 38.5 & 0.074 & & .0 & 6.2 \\
\hline 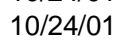 & $6: 45$ & 0 & 26.2 & & 26.0 & 25.6 & 25.8 & 51.0 & 60.8 & 10.5 & 41.3 & & & 38.5 & & & & \\
\hline$/ 24 / 01$ & $6: 46$ & 0 & 26.2 & 5.3 & 26.0 & 25.6 & 25.8 & 50.9 & 60.7 & 10.5 & 41.0 & 9. & & 38.5 & 0.074 & & .0 & 45.9 \\
\hline $18 / 1$ & $: 47$ & 0 & 26.3 & & 3.0 & 25.6 & 25.8 & 50.9 & 60.6 & 10.4 & 41.1 & & & 8.4 & & & & 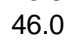 \\
\hline /24/ & 48 & & 26.3 & .3 & 6.0 & 25.6 & 25.8 & 51.0 & 60.7 & 10.4 & 41.4 & $\mathrm{~J}$. & & 38.4 & 0.073 & & 15.9 & 6.2 \\
\hline n & :49 & 0 & 6.3 & & 0 & 25.6 & 25.8 & 51.2 & 60.8 & 10.5 & 41.1 & & & 3.5 & & & & \\
\hline$/ 24 /($ & 50 & 0 & 26.3 & 8.4 & 6.0 & 25.6 & 25.8 & 51.1 & 60.8 & 10.4 & 41.1 & 9. & & 8.1 & 0.073 & & 5.8 & 0.1 \\
\hline & 51 & 0 & .3 & & & 25.6 & 25.8 & 51.3 & .0 & 10.5 & 1.4 & & & 3.4 & & & & \\
\hline$/ 24 / 0$ & 6:52 & & 26.3 & .4 & 6.0 & 25.6 & 25.7 & 51.4 & 61.1 & 10.5 & 41.0 & 9. & & 8.5 & 0.073 & & 0 & : \\
\hline & 53 & 0 & .3 & & & 5.6 & 25.8 & 512 & 609 & 10.5 & 41.3 & & & 3.4 & & & & \\
\hline |24/ & $: 54$ & 0 & 26.3 & .4 & 6.1 & 25.6 & 25.7 & 51.5 & 61.1 & 10.5 & 41.4 & 9. & & 3.4 & 73 & & & 46.4 \\
\hline & :5 & & & & & 5.6 & & 51 & 8 & 105 & 41.2 & & & & & & & \\
\hline $24 /$ & 56 & 0 & 6.3 & .4 & 6.1 & 25.6 & 25.8 & 51. & 60.9 & 10.5 & 41.7 & & & 4 & 73 & & & \\
\hline & 5 & 0 & & 8.4 & 6.1 & 25.6 & 25.8 & 51. & 61.1 & 10.5 & 41.3 & & & & & & & \\
\hline /24/ & $5 \varepsilon$ & 0 & 6.3 & .4 & 6.1 & 25.6 & 25.8 & 51.3 & 61.0 & 10.4 & 41.2 & & & 4 & & & & \\
\hline 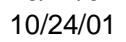 & :59 & & 6.4 & 5.4 & 26.1 & 25.6 & 25.8 & 51.0 & 60.5 & 10.5 & 41.1 & & & 3.4 & & & & 5.0 \\
\hline$/ 24 / 01$ & $: 00$ & 0 & 26.4 & .4 & 6.1 & 25.6 & 25.8 & 51.5 & 61.2 & 10.6 & 41.3 & & & 3.5 & 72 & & & 46.4 \\
\hline & 7:01 & & $\mathbf{t}$ & 4 & 6.2 & 25.7 & 25.8 & 51. & 61.3 & 10.5 & 41.2 & & & 4 & & & & \\
\hline 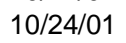 & 02 & 0 & 26.4 & 4 & 3.1 & 25.7 & 25.9 & 51. & 61.3 & 10.5 & 41.3 & & & .5 & 2 & & & 46.4 \\
\hline & .03 & & 26.4 & & & 25.7 & 25 & 51 & 61.2 & 10.6 & 41.1 & & & & & & & \\
\hline 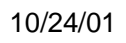 & :04 & 0 & 6.4 & .4 & .2 & 25.7 & 26.0 & 51.2 & 60.7 & 10.5 & 41.0 & & & 4 & 2 & & & 46.1 \\
\hline & .05 & & & & & 57 & 25 & 51 & 60.9 & 10.6 & 41.3 & & & & & & & \\
\hline & $: 06$ & 0 & .4 & .4 & & 25.6 & 25.9 & 1.2 & 60.9 & 10.6 & 41.5 & & & 4 & & & & \\
\hline & 0 & & & & & 5.6 & 25 & 14 & 61.0 & 10.5 & 41.2 & & & & & & & 463 \\
\hline & :08 & 0 & & .4 & .2 & 5.7 & 8 & & & 10.5 & 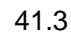 & & & & & & & \\
\hline & . & & & & & 57 & & & 60.6 & 5 & 4 & & & & & & & \\
\hline & & & & & & & & & & & & & & & & & & \\
\hline & $7: 1$ & & & & & 5.7 & & & & 5 & 4 & & & & & & & \\
\hline & $: 1$ & & & & & & & & & & & & & & & & & \\
\hline & $7: 1$ & & & & & .7 & & & & 10.5 & 4 & & & & & & & \\
\hline & : & & & & & 2 & 2 & & & 10.6 & & & & & & & & \\
\hline & $7: 1$ & 0 & 26.6 & & & .7 & & & 60.6 & 10.6 & 41.1 & & & & & & & \\
\hline & : & & & & & & & & & & & & & & & & & \\
\hline & $7: 1$ & 0 & & & & 7 & & & & 10 & 41.3 & & & & & & & \\
\hline & -7 & & & & & & & & & & & & & & & & & \\
\hline & 7:1 & 0 & & & & & & & & 10.6 & 41.3 & & & & & & & \\
\hline & & & & & & & & & & & & & & & & & & \\
\hline & 7 & 0 & & & & & & & & & & & & & & & & \\
\hline & & & & & & & & & & & & & & & & & & \\
\hline & 2 & c & & & & & & & & & 40 & & & & & & & \\
\hline & & & & & & & & & & & & & & & & & & \\
\hline & & & & & & & & & & & & & & & & & & \\
\hline & & & & & & & & & & & & & & & & & & \\
\hline & 7:27 & & & & & & & & 60.9 & & & & & & & & & \\
\hline & $7: 2$ & & & & & & & & & & & & & & & & & \\
\hline 12 & $7: 29$ & 0 & 6.7 & & & & O & .1 & 60.7 & 0.6 & 1.1 & 9.5 & 0 & 38.6 & 071 & .0298 & 16.0 & \\
\hline
\end{tabular}


CL SL UP BOT BOT $\quad$ FLTR TOP FLT

FLTRT LOOP LOOP AMB AMB DP FLTR DP DP RATE

SL FLTR

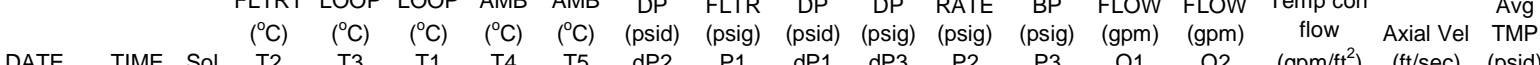

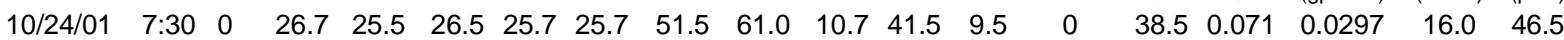

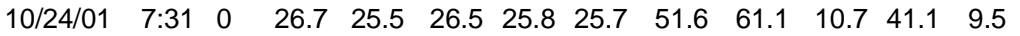
$\begin{array}{lllllllllllll}10 / 24 / 01 & 7: 32 & 0 & 26.7 & 25.5 & 26.5 & 25.8 & 25.8 & 51.6 & 61.3 & 10.7 & 41.0 & 9.5\end{array}$

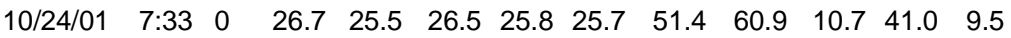
$\begin{array}{lllllllllllll}10 / 24 / 01 & 7: 34 & 0 & 26.8 & 25.6 & 26.5 & 25.8 & 25.8 & 51.4 & 61.0 & 10.7 & 41.4 & 9.5\end{array}$ $\begin{array}{lllllllllllll}10 / 24 / 01 & 7: 35 & 0 & 26.7 & 25.6 & 26.5 & 25.8 & 25.7 & 51.6 & 61.1 & 10.7 & 41.4 & 9.5\end{array}$ $10 / 24 / 01 \quad 7: 36 \quad 0$ $10 / 24 / 01 \quad 7: 37 \quad 0$ $10 / 24 / 01 \quad 7: 38 \quad 0$ $10 / 24 / 01 \quad 7: 39 \quad 0$ $10 / 24 / 01 \quad 7: 40 \quad 0$ $10 / 24 / 01 \quad 7: 41 \quad 0$ $10 / 24 / 01 \quad 7: 42 \quad 0$ $10 / 24 / 01 \quad 7: 43 \quad 0$ $10 / 24 / 01 \quad 7: 44 \quad 0$ $10 / 24 / 01 \quad 7: 45 \quad 0$ $10 / 24 / 01 \quad 7: 46 \quad 0$ $10 / 24 / 01 \quad 7: 47 \quad 0$ $10 / 24 / 01 \quad 7: 48 \quad 0$ $10 / 24 / 01 \quad 7: 49 \quad 0$ $10 / 24 / 01 \quad 7: 50 \quad 0$ $10 / 24 / 01 \quad 7: 51 \quad 0$ $10 / 24 / 01 \quad 7: 52 \quad 0$ $10 / 24 / 01 \quad 7: 53 \quad 0$ $10 / 24 / 01 \quad 7: 54 \quad 0$ $10 / 24 / 01 \quad 7: 55 \quad 0$ $10 / 24 / 01 \quad 7: 56 \quad 0$ $10 / 24 / 01 \quad 7: 57 \quad 0$ $10 / 24 / 01 \quad 7: 58 \quad 0$ $10 / 24 / 01 \quad 7: 59 \quad 0$ $10 / 24 / 01 \quad 8: 00 \quad 0$ $10 / 24 / 01 \quad 8: 01 \quad 0$ $10 / 24 / 01 \quad 8: 02 \quad 0$ $10 / 24 / 01 \quad 8: 03 \quad 0$ $10 / 24 / 01 \quad 8: 04 \quad 0$ $10 / 24 / 01 \quad 8: 05 \quad 0$ $10 / 24 / 01 \quad 8: 06 \quad 0$ $10 / 24 / 01 \quad 8: 07 \quad 0$ $10 / 24 / 01 \quad 8: 08 \quad 0$ $10 / 24 / 01 \quad 8: 09 \quad 0$ $10 / 24 / 01 \quad 8: 10 \quad 0$ $10 / 24 / 01 \quad 8: 11 \quad 0$ $10 / 24 / 01 \quad 8: 12 \quad 0$ $10 / 24 / 01 \quad 8: 13 \quad 0$ $10 / 24 / 01 \quad 8: 14 \quad 0$ $10 / 24 / 01 \quad 8: 15 \quad 0$ $10 / 24 / 01 \quad 8: 16 \quad 0$ $10 / 24 / 01 \quad 8: 17 \quad 0$ $10 / 24 / 01 \quad 8: 18 \quad 0$ $10 / 24 / 01 \quad 8: 19 \quad 0$ $10 / 24 / 01 \quad 8: 20 \quad 0$ $10 / 24 / 01 \quad 8: 21 \quad 0$ $10 / 24 / 01 \quad 8: 22 \quad 0$ $10 / 24 / 01 \quad 8: 23 \quad 0$ $10 / 24 / 01 \quad 8: 24 \quad 0$ $10 / 24 / 01 \quad 8: 25 \quad 0$ $10 / 24 / 01 \quad 8: 26 \quad 0$ $10 / 24 / 01 \quad 8: 27 \quad 0$ $10 / 24 / 01 \quad 8: 28 \quad 0$ $\begin{array}{llllllllll}26.8 & 25.6 & 26.5 & 25.8 & 25.7 & 51.6 & 61.1 & 10.7 & 41.1 & 9.5\end{array}$ $\begin{array}{llllllllll}26.7 & 25.6 & 26.5 & 25.8 & 25.7 & 51.2 & 60.6 & 10.6 & 41.4 & 9.5\end{array}$ $\begin{array}{llllllllll}26.8 & 25.6 & 26.5 & 25.8 & 25.7 & 51.1 & 60.5 & 10.7 & 41.1 & 9.5\end{array}$ $\begin{array}{llllllllll}26.8 & 25.6 & 26.6 & 25.8 & 25.7 & 51.4 & 61.0 & 10.7 & 41.4 & 9.5\end{array}$ $\begin{array}{llllllllll}26.8 & 25.6 & 26.5 & 25.9 & 25.7 & 51.5 & 61.0 & 10.7 & 41.4 & 9.5\end{array}$ $\begin{array}{llllllllll}26.8 & 25.6 & 26.6 & 25.8 & 25.8 & 51.6 & 61.1 & 10.7 & 41.3 & 9.5\end{array}$ $\begin{array}{llllllllll}26.8 & 25.6 & 26.6 & 25.9 & 25.7 & 51.9 & 61.6 & 10.7 & 41.1 & 9.5\end{array}$ $\begin{array}{llllllllll}26.9 & 25.6 & 26.6 & 25.8 & 25.8 & 51.7 & 61.5 & 10.7 & 41.3 & 9.5\end{array}$ $\begin{array}{llllllllll}26.8 & 25.6 & 26.6 & 25.8 & 25.7 & 51.6 & 61.1 & 10.8 & 41.0 & 9.5\end{array}$ $\begin{array}{llllllllll}26.9 & 25.6 & 26.6 & 25.9 & 25.8 & 51.1 & 60.7 & 10.7 & 41.1 & 9.5\end{array}$ $\begin{array}{llllllllll}26.9 & 25.6 & 26.6 & 25.9 & 25.7 & 51.6 & 61.1 & 10.7 & 41.3 & 9.5\end{array}$ $\begin{array}{llllllllll}26.9 & 25.6 & 26.7 & 25.9 & 25.7 & 51.5 & 61.1 & 10.7 & 41.2 & 9.5\end{array}$ $\begin{array}{llllllllll}26.9 & 25.6 & 26.6 & 25.8 & 25.8 & 51.4 & 61.1 & 10.7 & 41.2 & 9.5\end{array}$ $\begin{array}{llllllllll}26.9 & 25.6 & 26.6 & 25.9 & 25.8 & 51.7 & 61.2 & 10.8 & 41.1 & 9.5\end{array}$ $\begin{array}{llllllllll}26.9 & 25.6 & 26.7 & 25.8 & 25.8 & 51.2 & 60.6 & 10.7 & 41.0 & 9.5\end{array}$ $\begin{array}{llllllllll}26.9 & 25.6 & 26.6 & 25.9 & 25.8 & 51.7 & 61.3 & 10.8 & 41.2 & 9.5\end{array}$ $\begin{array}{llllllllll}27.0 & 25.6 & 26.7 & 25.9 & 25.8 & 51.3 & 60.7 & 10.8 & 41.2 & 9.5\end{array}$ $\begin{array}{llllllllll}26.9 & 25.6 & 26.7 & 25.9 & 25.8 & 51.5 & 61.0 & 10.8 & 41.3 & 9.5\end{array}$ $\begin{array}{llllllllll}27.0 & 25.7 & 26.8 & 25.9 & 25.9 & 51.6 & 61.2 & 10.7 & 41.4 & 9.5\end{array}$ $\begin{array}{llllllllll}27.0 & 25.7 & 26.7 & 25.9 & 25.8 & 51.3 & 60.7 & 10.8 & 41.1 & 9.5\end{array}$ $\begin{array}{llllllllll}27.0 & 25.7 & 26.7 & 25.9 & 25.8 & 51.4 & 61.0 & 10.8 & 41.2 & 9.5\end{array}$ $\begin{array}{llllllllll}27.0 & 25.7 & 26.8 & 25.9 & 25.7 & 51.6 & 61.1 & 10.7 & 41.1 & 9.5\end{array}$ $\begin{array}{llllllllll}27.0 & 25.7 & 26.7 & 25.9 & 25.8 & 51.2 & 60.8 & 10.8 & 41.2 & 9.5\end{array}$ $\begin{array}{llllllllll}27.1 & 25.7 & 26.8 & 25.9 & 25.7 & 51.4 & 60.9 & 10.7 & 41.4 & 9.5\end{array}$ $\begin{array}{llllllllll}27.1 & 25.7 & 26.8 & 25.9 & 25.7 & 51.5 & 61.1 & 10.9 & 41.3 & 9.5\end{array}$ $\begin{array}{llllllllll}27.1 & 25.7 & 26.8 & 25.9 & 25.7 & 51.5 & 61.0 & 10.8 & 41.4 & 9.5\end{array}$ $\begin{array}{llllllllll}27.0 & 25.7 & 26.8 & 25.8 & 25.7 & 51.4 & 61.0 & 10.7 & 41.3 & 9.5\end{array}$ $\begin{array}{llllllllll}27.0 & 25.7 & 26.8 & 25.8 & 25.7 & 51.6 & 61.2 & 10.7 & 41.3 & 9.5\end{array}$ $\begin{array}{llllllllll}27.1 & 25.7 & 26.8 & 25.9 & 25.7 & 51.6 & 61.2 & 10.8 & 41.4 & 9.5\end{array}$ $\begin{array}{llllllllll}27.0 & 25.7 & 26.8 & 25.9 & 25.8 & 51.5 & 61.0 & 10.7 & 41.2 & 9.5\end{array}$ $\begin{array}{llllllllll}27.1 & 25.7 & 26.8 & 25.9 & 25.7 & 51.7 & 61.2 & 10.8 & 41.3 & 9.5\end{array}$ $\begin{array}{llllllllll}27.1 & 25.7 & 26.8 & 25.9 & 25.8 & 51.4 & 60.9 & 10.8 & 41.4 & 9.5\end{array}$ $\begin{array}{llllllllll}27.1 & 25.7 & 26.8 & 25.9 & 25.8 & 51.4 & 60.9 & 10.8 & 41.2 & 9.5\end{array}$ $\begin{array}{llllllllll}27.1 & 25.7 & 26.9 & 25.9 & 25.8 & 51.9 & 61.3 & 10.8 & 41.5 & 9.5\end{array}$ $\begin{array}{llllllllll}27.1 & 25.7 & 26.9 & 25.9 & 25.7 & 51.5 & 61.0 & 10.7 & 41.1 & 9.5\end{array}$ $\begin{array}{llllllllll}27.1 & 25.7 & 26.9 & 25.9 & 25.7 & 51.3 & 60.8 & 10.7 & 41.3 & 9.5\end{array}$ $\begin{array}{llllllllll}27.1 & 25.7 & 26.8 & 25.9 & 25.7 & 51.3 & 60.9 & 10.7 & 41.3 & 9.5\end{array}$ $\begin{array}{llllllllll}27.1 & 25.7 & 26.9 & 25.9 & 25.7 & 51.8 & 61.4 & 10.8 & 41.2 & 9.5\end{array}$ $\begin{array}{llllllllll}27.1 & 25.7 & 26.8 & 25.9 & 25.8 & 51.4 & 60.8 & 10.8 & 41.3 & 9.5\end{array}$ $\begin{array}{llllllllll}27.1 & 25.7 & 26.5 & 25.9 & 25.8 & 51.4 & 60.8 & 10.7 & 41.1 & 9.4\end{array}$ $\begin{array}{llllllllll}27.1 & 25.7 & 26.4 & 25.9 & 25.8 & 51.5 & 61.0 & 10.8 & 41.4 & 9.4\end{array}$ $\begin{array}{llllllllll}27.0 & 25.7 & 26.2 & 25.9 & 25.8 & 51.1 & 60.6 & 10.7 & 41.4 & 9.4\end{array}$ $\begin{array}{llllllllll}27.0 & 25.7 & 26.1 & 25.9 & 25.8 & 51.6 & 60.9 & 10.6 & 41.6 & 9.4\end{array}$ $\begin{array}{llllllllll}27.0 & 25.7 & 25.9 & 25.9 & 25.8 & 51.6 & 61.0 & 10.7 & 41.2 & 9.4\end{array}$ $\begin{array}{llllllllll}26.9 & 25.8 & 25.8 & 26.0 & 25.8 & 51.4 & 60.8 & 10.6 & 41.5 & 9.4\end{array}$ $\begin{array}{llllllllll}26.9 & 25.8 & 25.7 & 25.9 & 25.8 & 51.4 & 60.9 & 10.6 & 41.3 & 9.4\end{array}$ $\begin{array}{llllllllll}26.9 & 25.8 & 25.7 & 26.0 & 25.9 & 51.8 & 61.2 & 10.7 & 41.2 & 9.4\end{array}$ $\begin{array}{llllllllll}26.8 & 25.8 & 25.6 & 25.9 & 25.8 & 51.8 & 61.2 & 10.7 & 41.3 & 9.3\end{array}$ $\begin{array}{llllllllll}26.7 & 25.8 & 25.5 & 25.9 & 25.8 & 51.7 & 61.1 & 10.7 & 41.6 & 9.3\end{array}$ $\begin{array}{llllllllll}26.7 & 25.8 & 25.5 & 26.0 & 25.9 & 51.8 & 61.3 & 10.6 & 41.3 & 9.3\end{array}$ $\begin{array}{llllllllll}26.6 & 25.8 & 25.5 & 26.0 & 25.8 & 51.4 & 60.8 & 10.6 & 41.3 & 9.3\end{array}$ $\begin{array}{llllllllll}26.5 & 25.8 & 25.6 & 26.0 & 25.9 & 48.8 & 60.6 & 10.6 & 38.8 & 11.8\end{array}$ $\begin{array}{llllllllll}26.4 & 25.8 & 25.6 & 26.0 & 25.8 & 50.4 & 60.8 & 10.7 & 40.0 & 10.4\end{array}$ $\begin{array}{llllllllll}26.3 & 25.8 & 25.7 & 26.0 & 25.8 & 50.5 & 60.9 & 10.7 & 40.9 & 10.3\end{array}$

$\begin{array}{lll}38.6 & 0.071 & 0.0298\end{array}$

$\begin{array}{lll}38.3 & 0.071 & 0.0297\end{array}$

$\begin{array}{lll}38.6 & 0.071 & 0.0297\end{array}$

$\begin{array}{lll}38.7 & 0.071 & 0.0297\end{array}$

$\begin{array}{lll}38.6 & 0.071 & 0.0297\end{array}$

$\begin{array}{lll}38.6 & 0.070 & 0.0293\end{array}$

$\begin{array}{lll}38.6 & 0.070 & 0.0293\end{array}$

$\begin{array}{lll}38.7 & 0.070 & 0.0293\end{array}$

$\begin{array}{lll}38.6 & 0.070 & 0.0293\end{array}$

$\begin{array}{lll}38.6 & 0.070 & 0.0293\end{array}$

$\begin{array}{lll}38.7 & 0.070 & 0.0293\end{array}$

$\begin{array}{lll}38.6 & 0.070 & 0.0292\end{array}$

$\begin{array}{lll}38.6 & 0.070 & 0.0293\end{array}$

$\begin{array}{lll}38.6 & 0.070 & 0.0292\end{array}$

$\begin{array}{lll}38.5 & 0.070 & 0.0292\end{array}$

$\begin{array}{lll}38.5 & 0.070 & 0.0292\end{array}$

$\begin{array}{llll}38.5 & 0.070 & 0.0292\end{array}$

$\begin{array}{llll}38.5 & 0.070 & 0.0292\end{array}$

$\begin{array}{lll}38.5 & 0.070 & 0.0292\end{array}$

$\begin{array}{lll}38.6 & 0.070 & 0.0291\end{array}$

$\begin{array}{lll}38.6 & 0.070 & 0.0292\end{array}$

$\begin{array}{lll}38.4 & 0.070 & 0.0291\end{array}$

$\begin{array}{llll}38.6 & 0.070 & 0.0292\end{array}$

$\begin{array}{lll}38.5 & 0.070 & 0.0291\end{array}$

$\begin{array}{lll}38.5 & 0.070 & 0.0291\end{array}$

$\begin{array}{llll}38.5 & 0.070 & 0.0291\end{array}$

$\begin{array}{lll}38.5 & 0.070 & 0.0291\end{array}$

$\begin{array}{llll}38.6 & 0.070 & 0.0291\end{array}$

$\begin{array}{lll}38.7 & 0.070 & 0.0291\end{array}$

$\begin{array}{llll}38.6 & 0.070 & 0.0291\end{array}$

$\begin{array}{lll}38.6 & 0.070 & 0.0291\end{array}$

$\begin{array}{lll}38.5 & 0.070 & 0.0291\end{array}$

$\begin{array}{lll}38.6 & 0.070 & 0.0290\end{array}$

$\begin{array}{lll}38.5 & 0.070 & 0.0291\end{array}$

$\begin{array}{lll}38.6 & 0.070 & 0.0290\end{array}$

$\begin{array}{lll}38.6 & 0.070 & 0.0291\end{array}$

$\begin{array}{lll}38.5 & 0.070 & 0.0290\end{array}$

$\begin{array}{lll}38.5 & 0.070 & 0.0290\end{array}$

$\begin{array}{lll}38.6 & 0.070 & 0.0290\end{array}$

$\begin{array}{lll}38.5 & 0.070 & 0.0290\end{array}$

$\begin{array}{lll}38.5 & 0.070 & 0.0290\end{array}$

$\begin{array}{lll}38.6 & 0.070 & 0.0290\end{array}$

$\begin{array}{lll}38.5 & 0.070 & 0.0290\end{array}$

$\begin{array}{llll}38.5 & 0.069 & 0.0287\end{array}$

$\begin{array}{llll}38.6 & 0.069 & 0.0289\end{array}$

$\begin{array}{llll}38.7 & 0.069 & 0.0290\end{array}$

$\begin{array}{llll}38.6 & 0.068 & 0.0287\end{array}$

$\begin{array}{lll}38.6 & 0.068 & 0.0288\end{array}$

$\begin{array}{llll}38.5 & 0.068 & 0.0289\end{array}$

$\begin{array}{llll}38.6 & 0.068 & 0.0290\end{array}$

$\begin{array}{lll}38.7 & 0.068 & 0.0291\end{array}$

$\begin{array}{llll}38.7 & 0.067 & 0.0287\end{array}$

$\begin{array}{llll}38.7 & 0.067 & 0.0288\end{array}$

$\begin{array}{llll}38.7 & 0.067 & 0.0288\end{array}$

$\begin{array}{llll}38.6 & 0.067 & 0.0288\end{array}$

$\begin{array}{llll}38.7 & 0.067 & 0.0288\end{array}$

$\begin{array}{lll}38.7 & 0.107 & 0.0459\end{array}$

$\begin{array}{llll}38.7 & 0.091 & 0.0391\end{array}$

$\begin{array}{lll}38.7 & 0.088 & 0.0377\end{array}$
$16.0 \quad 46.3$

$15.9 \quad 46.3$

$\begin{array}{ll}16.0 & 46.2\end{array}$

$16.1 \quad 46.4$

$16.0 \quad 46.5$

$16.0 \quad 46.3$

$16.0 \quad 46.3$

$16.1 \quad 46.1$

$16.0 \quad 46.4$

$16.0 \quad 46.4$

$16.1 \quad 46.4$

$16.0 \quad 46.5$

$16.0 \quad 46.5$

$16.0 \quad 46.3$

$\begin{array}{ll}16.0 & 46.1\end{array}$

$\begin{array}{ll}16.0 & 46.4\end{array}$

$16.0 \quad 46.4$

$16.0 \quad 46.3$

$\begin{array}{ll}16.0 & 46.4\end{array}$

$16.0 \quad 46.1$

$16.0 \quad 46.4$

$15.9 \quad 46.2$

$16.0 \quad 46.4$

$16.0 \quad 46.5$

$16.0 \quad 46.2$

$16.0 \quad 46.3$

$16.0 \quad 46.4$

$16.0 \quad 46.2$

$16.0 \quad 46.4$

$16.0 \quad 46.4$

$16.0 \quad 46.5$

$16.0 \quad 46.4$

$16.0 \quad 46.4$

$16.0 \quad 46.5$

$16.0 \quad 46.4$

$16.0 \quad 46.5$

$16.0 \quad 46.4$

$16.0 \quad 46.3$

$\begin{array}{ll}16.0 & 46.7\end{array}$

$16.0 \quad 46.3$

$16.0 \quad 46.3$

$\begin{array}{ll}16.0 & 46.3\end{array}$

$16.0 \quad 46.5$

$16.0 \quad 46.3$

$\begin{array}{lll}16.0 & 46.3\end{array}$

$16.0 \quad 46.4$

$\begin{array}{ll}16.0 & 46.3\end{array}$

$16.0 \quad 46.6$

$16.0 \quad 46.4$

$\begin{array}{ll}16.0 & 46.5\end{array}$

$16.1 \quad 46.4$

$16.0 \quad 46.5$

$16.1 \quad 46.5$

16.146 .7

$\begin{array}{ll}16.0 & 46.5\end{array}$

$16.1 \quad 46.4$

$\begin{array}{lll}16.0 & 43.8\end{array}$

$\begin{array}{ll}16.1 & 45.2\end{array}$

$16.1 \quad 45.7$ 


\begin{tabular}{|c|c|c|c|c|c|c|c|c|c|c|c|c|c|c|c|c|c|c|}
\hline 政 & VIL & Sol & $\begin{array}{c}\text { FLTRT } \\
\left({ }^{\circ} \mathrm{C}\right) \\
\mathrm{T} 2\end{array}$ & $\begin{array}{l}\mathrm{CL} \\
\text { LOOP } \\
\left({ }^{\circ} \mathrm{C}\right) \\
\mathrm{T} 3\end{array}$ & $\begin{array}{c}\text { SL } \\
\text { LOOP } \\
\left({ }^{\circ} \mathrm{C}\right) \\
\mathrm{T} 1\end{array}$ & $\begin{array}{c}\text { UP } \\
\text { AMB } \\
\left({ }^{\circ} \mathrm{C}\right) \\
\text { T4 }\end{array}$ & $\begin{array}{c}\text { BOT } \\
\text { AMB } \\
\left({ }^{\circ} \mathrm{C}\right) \\
\text { T5 }\end{array}$ & $\begin{array}{l}\text { BOT } \\
\text { DP } \\
\text { (psid) } \\
\text { dP2 }\end{array}$ & $\begin{array}{c}\text { FLTR } \\
\text { (psig) } \\
\text { P1 }\end{array}$ & $\begin{array}{l}\text { FLTR } \\
\text { DP } \\
\text { (psid) } \\
\text { dP1 }\end{array}$ & $\begin{array}{c}\text { TOP } \\
\text { DP } \\
\text { (psig) } \\
\text { dP3 }\end{array}$ & $\begin{array}{l}\text { FLT- } \\
\text { RATE } \\
\text { (psig) } \\
\text { P2 }\end{array}$ & $\begin{array}{c}\text { BP } \\
\text { (psig) } \\
\text { P3 }\end{array}$ & $\begin{array}{l}\text { SL } \\
\text { FLOW } \\
\text { (gpm) } \\
\text { Q1 }\end{array}$ & $\begin{array}{c}\text { FLTR } \\
\text { FLOW } \\
\text { (gpm) } \\
\text { Q2 }\end{array}$ & $\begin{array}{c}\text { Temp } \\
\text { corrected } \\
\text { flow } \\
\left(\mathrm{gpm} / \mathrm{ft}^{2}\right)\end{array}$ & $\begin{array}{l}\text { Axial Vel } \\
(\mathrm{tt} / \mathrm{sec})\end{array}$ & \\
\hline$/ / 24 / 01$ & 30 & 0 & 26.3 & 25.8 & 25.7 & 26.0 & 25.8 & 50.7 & 61.1 & 10.7 & 40.6 & 10.2 & & 38.6 & 0.086 & 0.0369 & & \\
\hline /24/01 & 31 & 0 & 6.2 & 25.8 & 5.7 & 26.0 & 5.9 & & & 10.8 & 40.7 & 10.2 & & 8.6 & & & & \\
\hline$/ 24 / 01$ & 32 & 0 & 6.2 & 5.8 & 5.7 & 26.0 & 5.8 & 5 & 6 & 10.6 & 0.6 & 10.1 & & 8.9 & 0.084 & & & \\
\hline /24 & :33 & 0 & & 25.8 & 25.8 & 26.0 & 5.9 & & 0.9 & 10.7 & 40.7 & 10.1 & & 38.7 & 0.084 & & & \\
\hline /24/01 & :34 & 0 & 26.1 & 25.8 & 25.8 & 26.0 & 5.8 & 8 & 0.8 & 10.7 & 40.5 & 10.1 & & 38.6 & 0.083 & & & \\
\hline /24/01 & 35 & 0 & 26.1 & 25.8 & 25.8 & 26.0 & 5.9 & 0.9 & 1.0 & 10.6 & 40.7 & 10.0 & & 38.7 & 0.082 & 50 & & \\
\hline /24/0 & 36 & 0 & 26.1 & 25.8 & 25.8 & 26.0 & 5.8 & 0.8 & 0.9 & 10.7 & 40.8 & 10.0 & & 38.7 & 0.082 & & & \\
\hline /24/01 & 37 & 0 & 26.1 & 25.8 & 25.8 & 26.0 & 5.9 & 1.0 & 1.0 & 10.7 & 40.8 & 10.0 & & 38.7 & 0.082 & & & \\
\hline /24/01 & 38 & 0 & 26.1 & 25.8 & 25.9 & 26.0 & 5.8 & 0.9 & 1.0 & 10.8 & 40.8 & 10.0 & & 38.6 & 0.081 & 45 & & \\
\hline /24/01 & 39 & 0 & 26.1 & 25.8 & 25.8 & 26.0 & 5.8 & 9 & 60.8 & 10.7 & 40.9 & 10.0 & & 38.6 & 0.081 & & & \\
\hline (21)1 & 40 & 0 & 26.1 & 25.8 & 25.9 & 26.0 & 5.9 & & 60.7 & 10.7 & 40.9 & 10. & & 38.7 & & & & \\
\hline & 41 & 0 & 26.1 & 25.8 & 25.8 & 26.1 & 5.8 & & & 10.7 & & 9. & & & & & & \\
\hline & 42 & & 26.2 & 25.8 & 25.9 & 26.1 & 5.9 & 7 & 0.6 & 10.7 & 41.0 & 9 & & 8.7 & & & & \\
\hline & 43 & & 0.2 & 25.8 & 25.9 & 26.1 & 5.9 & & .2 & 10.7 & 0.7 & 9 & & 8.7 & & & & \\
\hline & 44 & & 0.2 & 25.8 & 26.0 & 26.1 & 5.9 & & 1 & 10.7 & 41.0 & & & 8.7 & 80 & & & \\
\hline & & & & 25.9 & 26.0 & 26.1 & .9 & & & 0.8 & 0.7 & & & .7 & & & & \\
\hline & & & & 25.8 & 26.0 & 26.1 & .9 & & & .7 & 0.7 & & & 8 & & & & \\
\hline & 47 & & & 25.9 & 26.0 & 26.1 & .9 & & & 10.6 & & & & 3.7 & & & & \\
\hline & 8 & 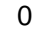 & & 25.8 & 26.1 & 26.1 & 5.9 & & & 10.7 & 1.0 & & & 8.7 & & & & \\
\hline 1 & 9 & 0 & & 25.9 & 26.1 & 26.1 & 5.9 & & & 10.7 & 41.1 & & & 8.7 & 79 & & & \\
\hline & 50 & 0 & & 25.8 & 26.1 & 26.1 & 5.9 & & 1 & 10.7 & 41.0 & & & 8.6 & & & & \\
\hline 11 & 51 & 0 & 3 & 25.9 & 26.1 & 26.1 & 6.0 & & & 10.7 & 41.0 & & & 38.7 & & & & \\
\hline 21 & 52 & 0 & 4 & 25.9 & 26.1 & 26.1 & 25.9 & & & 10.7 & 40.8 & & & 38.6 & & & & \\
\hline (21)1 & 3 & 0 & 4 & 25.9 & 26.2 & 26.2 & 6.0 & & 2 & 10.7 & 41.3 & 9. & & 8.7 & & & & \\
\hline (21) & 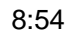 & & 264 & 25.9 & 26.1 & 26.1 & 5.9 & & & 10.7 & 41.0 & & & 8.6 & & & & \\
\hline 12101 & 55 & & 264 & 259 & 26.2 & 26.2 & 0.0 & & & & & & & & & & & \\
\hline & & & & & 26.2 & 26.2 & 0.0 & & & & & & & & & & & \\
\hline & & & .4 & & & & & & & & & & & & & & & \\
\hline & & & 4 & 25.9 & 26.2 & 26.2 & & & & & & & & .8 & & & & \\
\hline & & & & 25.9 & 26.2 & 26.2 & 0.0 & & & & 11 & & & 6 & & & & \\
\hline & & & & 2 & 26.2 & 26.2 & 0 & & & & 4 & & & 7 & & & & \\
\hline & & 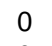 & & 2 & 26.2 & 26.2 & .0 & & & & 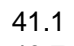 & & & 6 & & & & \\
\hline & & 0 & & .9 & 26.3 & 26.2 & .0 & & & & & & & 5 & & & & \\
\hline & & & & & 3.2 & 26.2 & .0 & & & & & & & & & & & \\
\hline & 4 & 0 & & 9 & 26.3 & 26.3 & 0.1 & & & & 3 & & & & & & & \\
\hline & & & & & 26.3 & 26.2 & .0 & & & & & & & & & & & \\
\hline & & & & 59 & 26.3 & 26.3 & 5.9 & & & & & & & & & & & \\
\hline & & & & 25.9 & 26.3 & 26.2 & 5.7 & & & & & & & & & & & \\
\hline /24/01 & & & & & 26.3 & 26.1 & 5.6 & & & 10.7 & 41.0 & & & & & & & \\
\hline & & & & & 26.3 & 26.0 & 5.4 & & & & & & & & & & & \\
\hline & & & & & 26.4 & 25.9 & 5.3 & & & & & & & & & & & \\
\hline & & 0 & & & 26.3 & 25.8 & 5.2 & & & & & & & & & & & \\
\hline & & & & & & & & & & & & & & & & & & \\
\hline & & 0 & & & & 25 & & & & & & & & & & & & \\
\hline & & 0 & & & 26.4 & 25.5 & & & & & & & & & & & & \\
\hline & & 0 & & & 26.4 & & & & & & & & & & & & & \\
\hline & & 0 & & & 26.4 & & & & & & & & & & & & & \\
\hline & & 0 & & & & & & & & & 2 & & & & & & & \\
\hline & & 0 & & & & & .4 & & & & 41.2 & & & & & & & \\
\hline & & 0 & & & 26.4 & & 1.3 & & & & 41.2 & & & & & & & \\
\hline & $9: 20$ & 0 & & & & & 4.3 & & & & & & & & & & & \\
\hline & $9: 21$ & 0 & & 25.9 & 26.5 & 25.1 & 4.1 & & & 10.8 & & & & .6 & & & & \\
\hline$/ 01$ & $9: 22$ & 0 & & 25.9 & 26.4 & 25.1 & 24.0 & & & 10.7 & 41.0 & & & 3.7 & 0.075 & & & \\
\hline$/ 24 / 01$ & $9: 23$ & 0 & & 25.9 & 26.5 & 25.0 & 24.0 & & & 10.7 & 41.2 & & & 38.6 & 0.075 & & & \\
\hline 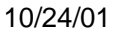 & $9: 24$ & 0 & & 25.9 & 26.5 & 25.0 & 23.9 & & & 10.8 & 40.9 & & & 3.3 & 0.075 & & & \\
\hline 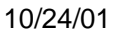 & $9: 25$ & 0 & & 20.0 & 26.4 & 24.9 & 23.8 & & & 10.8 & 41.2 & & & 38.6 & 0.075 & & & \\
\hline 1 & $9: 26$ & 0 & & 20.1 & 26.5 & 24.8 & 23.8 & & 61.0 & 10.7 & 40.9 & & & 38.5 & 0.075 & & & \\
\hline & & 0 & & 20.1 & 20.5 & & 20.1 & & & 10.7 & 41.3 & & & 0.0 & $0.0 / 5$ & & & \\
\hline & & 0 & & 25.7 & 26. & & 20.6 & & & 10.8 & 41.0 & & & 30.6 & & & & \\
\hline$/ 24 / 01$ & $9: 29$ & 0 & 26.8 & 25.7 & 26.5 & 24.8 & 3.5 & 1.4 & 61.1 & 10.7 & 41.2 & 9.7 & 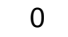 & 38.5 & 0.075 & 0.0314 & 16.0 & \\
\hline
\end{tabular}




\begin{tabular}{|c|c|c|c|c|c|c|c|c|c|c|c|c|c|c|c|c|c|c|}
\hline DATE & TIME & Sol & $\begin{array}{c}\text { FLTRT } \\
\left({ }^{\circ} \mathrm{C}\right) \\
\text { T2 }\end{array}$ & $\begin{array}{c}\mathrm{CL} \\
\text { LOOP } \\
\left({ }^{\circ} \mathrm{C}\right) \\
\text { T3 }\end{array}$ & $\begin{array}{c}\mathrm{SL} \\
\text { LOOP } \\
\left({ }^{\circ} \mathrm{C}\right) \\
\mathrm{T} 1\end{array}$ & $\begin{array}{c}\text { UP } \\
\text { AMB } \\
\left({ }^{\circ} \mathrm{C}\right) \\
\text { T4 }\end{array}$ & $\begin{array}{c}\text { BOT } \\
\text { AMB } \\
\left({ }^{\circ} \mathrm{C}\right) \\
\text { T5 }\end{array}$ & $\begin{array}{c}\text { BOT } \\
\text { DP } \\
\text { (psid) } \\
\text { dP2 }\end{array}$ & $\begin{array}{c}\text { FLTR } \\
\text { (psig) } \\
\text { P1 }\end{array}$ & $\begin{array}{l}\text { FLTR } \\
\text { DP } \\
\text { (psid) } \\
\text { dP1 }\end{array}$ & $\begin{array}{c}\text { TOP } \\
\text { DP } \\
\text { (psig) } \\
\text { dP3 }\end{array}$ & $\begin{array}{l}\text { FLT- } \\
\text { RATE } \\
\text { (psig) } \\
\text { P2 }\end{array}$ & $\begin{array}{c}\text { BP } \\
\text { (psig) } \\
\text { P3 }\end{array}$ & $\begin{array}{l}\text { SL } \\
\text { FLOW } \\
\text { (gpm) } \\
\text { Q1 }\end{array}$ & $\begin{array}{l}\text { FLTR } \\
\text { FLOW } \\
\text { (gpm) } \\
\text { Q2 }\end{array}$ & $\begin{array}{c}\text { Temp } \\
\text { corrected } \\
\text { flow } \\
\left(\mathrm{gpm} / \mathrm{ft}^{2}\right)\end{array}$ & $\begin{array}{c}\text { Axial Vel } \\
(\mathrm{tt} / \mathrm{sec})\end{array}$ & $\begin{array}{c}\text { Avg } \\
\text { TMP } \\
\text { (psid) }\end{array}$ \\
\hline 0/24/01 & $9: 30$ & 0 & 26.8 & 25.7 & 26.5 & 24.7 & 23.5 & 51.3 & 60.9 & 10.8 & 41.2 & 9.7 & P & 38.6 & 0.074 & 00309 & 16.0 & 463 \\
\hline$/ 24 / 01$ & $9: 31$ & 0 & 26.9 & 25.7 & 26.5 & 24.7 & 23.4 & 51.2 & 60.8 & 10.8 & 41.3 & 9.7 & 0 & 38.6 & 0.075 & .0314 & 0 & 46.3 \\
\hline /24/01 & 9:32 & 0 & 26.9 & 25.7 & 26.6 & 24.7 & 23.4 & 51.5 & 61.1 & 10.8 & 41.0 & 9.7 & 0 & 38.5 & 0.074 & 0.0309 & 6.0 & 46.3 \\
\hline /24/01 & $9: 33$ & 0 & 26.9 & 25.6 & 26.5 & 24.7 & 23.4 & 51.2 & 61.0 & 10.7 & 41.1 & 9.7 & 0 & 38.5 & 0.074 & 0.0309 & 6.0 & 46.2 \\
\hline D/24/01 & $9: 34$ & 0 & 26.9 & 25.6 & 26.6 & 24.6 & 23.3 & 51.4 & 61.2 & 10.8 & 40.8 & 9.7 & 0 & 38.6 & 0.074 & 0.0309 & 16.0 & 46.1 \\
\hline 10/24/01 & $9: 35$ & 0 & 26.9 & 25.6 & 26.5 & 24.6 & 23.2 & 51.6 & 61.4 & 10.7 & 41.1 & 9.7 & 0 & 38.6 & 0.074 & 0.0309 & 16.0 & 46.3 \\
\hline 10/24/01 & $9: 36$ & 0 & 26.9 & 25.6 & 26.6 & 24.6 & 23.1 & 51.5 & 61.2 & 10.8 & 41.3 & 9.7 & 0 & 38.6 & 0.074 & 0.0309 & 16.0 & 46.4 \\
\hline 10/24/01 & $9: 37$ & 0 & 26.9 & 25.5 & 26.6 & 24.5 & 23.1 & 51.4 & 61.1 & 10.8 & 41.3 & 9.7 & 0 & 38.7 & 0.074 & 0.0309 & 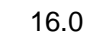 & 46.4 \\
\hline $10 / 24 / 01$ & $9: 38$ & 0 & 26.9 & 25.5 & 26.6 & 24.5 & 23.0 & 51.4 & 61.2 & 10.7 & 41.2 & 9.7 & 0 & 38.6 & 0.074 & 0.0309 & .0 & 46.3 \\
\hline 0/24/01 & $9: 39$ & 0 & 26.9 & 25.5 & 26.6 & 24.4 & 23.0 & 51.8 & 61.5 & 10.8 & 41.2 & 9.7 & 0 & 38.5 & 0.074 & 309 & & 46.5 \\
\hline$/ 24 / 01$ & $9: 40$ & 0 & 26.9 & 25.5 & 26.6 & 24.4 & 22.9 & 51.4 & 61.0 & 10.8 & 41.2 & 9.6 & 0 & 38.5 & 0.074 & & & 46.3 \\
\hline$/ 24 / 01$ & $9: 41$ & 0 & 26.9 & 25.5 & 26.6 & 24.4 & 22.8 & 51.5 & 61.2 & 10.7 & 41.3 & 9.6 & 0 & 38.5 & 0.074 & 309 & & 46.4 \\
\hline$/ 24 / 01$ & $9: 42$ & 0 & 26.9 & 25.4 & 26.6 & 24.3 & 22.8 & 51.6 & 61.3 & 10.8 & 41.2 & 9.6 & & 38.6 & 0.074 & 309 & & 46.4 \\
\hline 01 & $9: 43$ & 0 & 26.9 & 25.4 & 26.6 & 24.3 & 2.8 & 51.4 & 1.0 & 10.9 & 41.1 & 9. & & 8.6 & 0.074 & & & 6.3 \\
\hline 01 & $9: 44$ & 0 & 27.0 & 25.4 & 26.6 & 24.3 & 22.7 & 51.8 & 1.6 & 10.9 & 41.2 & 9. & & 8.6 & 0.074 & & & 16.5 \\
\hline 01 & $9: 45$ & 0 & 26.9 & 25.3 & 26.6 & 24.3 & 2.7 & 1.5 & 1.1 & 10.8 & 41.4 & 9. & & 8.6 & 0.074 & & & 46.4 \\
\hline $0 / 2$ & $9: 46$ & 0 & 27.0 & 25.3 & 26.6 & 24.3 & 2.7 & 1.3 & 0.8 & 10.9 & 40.9 & 9. & & 8.6 & 0.073 & & & 6.1 \\
\hline $0 / 2$ & $9: 47$ & 0 & 27.0 & 25.3 & 26.7 & 24.3 & 22.6 & 1.4 & 1.1 & 10.8 & 41.0 & 9. & & 8.5 & 0.073 & & & 46.2 \\
\hline $1 / 2$ & $9: 48$ & 0 & 27.0 & 25.3 & 26.7 & 24.3 & 22.5 & 51.3 & 60.8 & 10.9 & 41.0 & 9. & c & 38.5 & 0.073 & 04 & & 46.2 \\
\hline$/ 24 / 01$ & $9: 49$ & 0 & 27.0 & 25.3 & 26.7 & 24.3 & 22.5 & 51.8 & 61.5 & 10.9 & 41.2 & 9.6 & 0 & 38.6 & 0.073 & 04 & & 46.5 \\
\hline )/24/01 & $9: 50$ & 0 & 27.0 & 25.2 & 26.7 & 24.2 & 22.5 & 51.6 & 61.4 & 10.9 & 41.3 & 9.6 & 0 & 38.6 & 0.073 & 04 & & 46.4 \\
\hline (24/01 & $9: 51$ & 0 & 27.0 & 25.2 & 26.7 & 24.2 & 22.4 & 51.4 & 61.1 & 10.8 & 41.3 & 9.6 & 0 & 38.5 & 0.073 & & & 46.3 \\
\hline $10 / 24 / 01$ & $9: 52$ & 0 & 27.0 & 25.2 & 26.7 & 24.2 & 22.4 & 51.2 & 60.8 & 10.8 & 41.2 & 9. & 0 & 38.5 & 0.073 & & & 46.2 \\
\hline /24/01 & $9: 53$ & 0 & 27.0 & 25.2 & 26.7 & 24.1 & 22.4 & 51.3 & 60.9 & 10.8 & 40.9 & 9.6 & 0 & 38.5 & 0.073 & & & 46.1 \\
\hline 0/24/01 & $9: 54$ & 0 & 27.0 & 25.2 & 26.7 & 24.1 & 22.4 & 51.3 & 61.0 & 10.8 & 41.2 & 9.6 & 0 & 38.5 & 0.073 & & & 46.3 \\
\hline O/24/01 & $9: 55$ & 0 & 27.0 & 25.1 & 26.7 & 24.1 & 22.3 & 51.7 & 61.3 & 10.8 & 41.2 & 9.6 & 0 & 38.5 & 0.073 & & & 46.4 \\
\hline D/24/01 & $9: 56$ & 0 & 27.0 & 25.1 & 26.7 & 24.1 & 22.3 & 51.2 & 60.9 & 10.7 & 41.1 & 9. & 0 & 4 & 0. & & & 46.1 \\
\hline$\sqrt{12}+2+2$ & $9: 57$ & 0 & 27.0 & 25.1 & 26.7 & 24.0 & 2.2 & & & 10.8 & & & & & & & & 46.3 \\
\hline 0/24/01 & 9:58 & 0 & 27.0 & 25.0 & 26.7 & 24.0 & 22.2 & 51.5 & 61.1 & 10.8 & 41.2 & 9. & & 38.6 & & & & 46.4 \\
\hline /01 & $9: 59$ & 0 & 27.0 & 25.0 & 26.7 & 23.9 & 22.2 & 51.3 & 0.8 & 10.9 & 41.4 & 9. & & 4 & & & & 46.4 \\
\hline 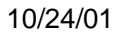 & :00 & 0 & 27.0 & 25.0 & 26.7 & 23.9 & 22.1 & 51.4 & 61.0 & 10.9 & 41.3 & 9 & & .5 & 3 & & & 6.3 \\
\hline 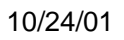 & 01 & 0 & 27.1 & 25.0 & 26.8 & 23.9 & 22.1 & 51.5 & 1.2 & 10.8 & 41.2 & 9 & & 3.6 & 73 & & & 46.4 \\
\hline 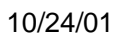 & 02 & 0 & 27.1 & 25.0 & 26.8 & 23.9 & 22.1 & 7 & 61.3 & 10.9 & 41.1 & 9. & & 3.5 & 73 & & & 46.4 \\
\hline 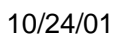 & 03 & 0 & 27.0 & 25.0 & 26.8 & 23.8 & 22.1 & 5 & 60.9 & 10.8 & 41.0 & 9. & & .4 & 73 & & & 46.2 \\
\hline 1 & $: 04$ & 0 & 27.1 & 24.9 & 26.8 & 23.8 & 22.0 & 4 & 61.0 & 10.8 & 41.2 & 9. & c & .5 & 0.073 & & & 46.3 \\
\hline 1 & $10: 05$ & 0 & 27.1 & 24.9 & 26.8 & 23.8 & 22.0 & 1.6 & 61.3 & 10.9 & 41.2 & 9. & 0 & 3.4 & 0.072 & & & 46.4 \\
\hline 1 & $10: 06$ & 0 & 27.1 & 24.9 & 26.8 & 23.7 & 21.9 & 51.3 & 60.8 & 10.8 & 41.2 & 9.6 & 0 & 3.4 & 0.073 & & & 46.3 \\
\hline 01 & $10: 07$ & 0 & 27.1 & 24.9 & 26.8 & 23.7 & 22.0 & 51.4 & 60.9 & 10.8 & 41.1 & 9.6 & 0 & 38.5 & 0.072 & & & 46.3 \\
\hline D/24/01 & $10: 08$ & 0 & 27.1 & 24.8 & 26.8 & 23.8 & 22.0 & 51.2 & 60.8 & 10.8 & 41.1 & 9.6 & 0 & 38.4 & 0.072 & & & 46.2 \\
\hline $0 / 24 / 01$ & $10: 09$ & 0 & 27.1 & 24.8 & 26.8 & 23.8 & 22.0 & 51.6 & 61.2 & 10.8 & 41.3 & 9 & 0 & 3.4 & 0.072 & & & 46.4 \\
\hline 0/24/01 & $10: 10$ & 0 & 27.1 & 24.8 & 26.9 & 23.8 & 22.1 & 51.2 & 60.7 & 10.9 & 40.9 & 9. & 0 & 3.5 & 0.073 & & & 46.0 \\
\hline 0/24/01 & $10: 11$ & 0 & 27.1 & 24.8 & 26.8 & 23.8 & 22.1 & 51.4 & 60.9 & 10.8 & 41.5 & 9. & 0 & 3.4 & 0.073 & & & 46.5 \\
\hline$/ 24 / 01$ & $10: 12$ & 0 & 27.1 & 24.8 & 26.9 & 23.9 & 22.2 & 51.8 & 61.3 & 10.8 & 41.6 & 9. & 0 & .4 & 0.072 & & & 46.7 \\
\hline & :13 & 0 & 27.1 & 24.8 & 26.9 & 23.9 & 22.2 & 51 & 61.2 & 10.8 & 41.5 & 9 & c & 3.4 & 0.072 & & & 46.5 \\
\hline & :14 & 0 & 27.1 & 24.8 & 26.7 & 24.0 & 22.3 & 51.5 & 60.9 & 10.7 & 41.3 & 9. & c & 38.4 & 0.072 & 00 & 9 & 46.4 \\
\hline & 1 & 0 & 27.1 & 24.7 & 26.0 & 24.0 & 22.4 & $51,>$ & 60.7 & 10.7 & 41.5 & 9. & & .2 & 71 & & 9 & 46.4 \\
\hline & $: 16$ & 0 & 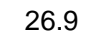 & 24.7 & 25.6 & 24.1 & 22.5 & - & .0 & 10.5 & 41.3 & 9. & & 2 & 70 & & & 46.4 \\
\hline & $: 17$ & 0 & & 24.7 & 25.4 & 24.1 & 22.5 & 0 & & 10.7 & 41.5 & 9. & & & & & & 46.7 \\
\hline & :18 & 0 & $\angle 0.0$ & 24.7 & 25.4 & 24.2 & 22.6 & 6 & 61.0 & 10.5 & 41.7 & 9. & & 38.2 & & & & 46.6 \\
\hline $0 / 2$ & $10: 19$ & 0 & 26.7 & 24.7 & 25.3 & 24.3 & 22.6 & 51.7 & 61.2 & 10.6 & 41.5 & 9. & 0 & 38.2 & 0.070 & & .9 & 46.6 \\
\hline & $10: 20$ & 0 & 26.7 & 24.7 & 25.4 & 24.3 & 22.7 & 51.5 & 60.9 & 10.6 & 41.5 & 9. & 0 & 38.3 & 0.070 & & & 46.5 \\
\hline & $10: 21$ & 0 & 26.6 & 24.7 & 25.4 & 24.3 & 22.7 & 51.7 & 61.0 & 10.6 & 41.5 & 9.4 & 0 & 38.3 & 0.070 & & .9 & 46.6 \\
\hline 01 & $10: 22$ & 0 & 26.2 & 24.7 & 25.4 & 24.3 & 22.8 & 9.6 & 61.1 & 10.6 & -0.4 & 51.4 & 82 & 38.3 & 0.010 & & .9 & 4.6 \\
\hline 0/24/01 & $10: 23$ & 0 & 26.3 & 24.7 & 25.5 & 24.4 & 22.9 & 50.6 & 61.2 & 10.6 & 40.3 & 10.7 & 0 & 38.4 & 0.095 & 409 & 15.9 & 45.4 \\
\hline $0 / 24 / 01$ & $10: 24$ & 0 & 26.2 & 24.7 & 25.5 & 24.5 & 22.9 & 50.9 & 61.2 & 10.6 & 40.8 & 10.4 & 0 & 38.4 & 0.089 & 383 & .9 & 45.9 \\
\hline /24/01 & $10: 25$ & 0 & 26.1 & 24.7 & 25.5 & 24.5 & 22.9 & 50.6 & 60.9 & 10.6 & 40.8 & 10.3 & 0 & 38.3 & 0.087 & 74 & .9 & 45.7 \\
\hline 24/01 & $10: 26$ & 0 & 26.0 & 24.7 & 25.5 & 24.5 & 22.9 & 50.8 & 61.0 & 10.6 & 40.9 & 10.2 & 0 & 38.3 & 0.086 & 0.0370 & 15.9 & 45.8 \\
\hline $4 / 01$ & $10: 27$ & 0 & 26.0 & 24.7 & 25.6 & 24.5 & 22.9 & 50.7 & 60.8 & 10.6 & 40.9 & 10.2 & 0 & 38.3 & 0.085 & 0.0365 & 15.9 & 45.8 \\
\hline $0 / 24 / 01$ & $10: 28$ & 0 & 25.9 & 24.7 & 25.6 & 24.5 & 22.8 & 50.9 & 61.0 & 10.6 & 40.8 & 10.2 & 0 & 38.3 & 0.084 & & 15.9 & 45.8 \\
\hline 0/24/01 & $10: 29$ & 0 & 25.9 & 24.6 & 25.6 & 24.4 & 22.8 & 50.9 & 61.1 & 10.6 & 40.6 & 10.1 & 0 & 38.3 & 0.084 & 0.0361 & 15.9 & 45.8 \\
\hline
\end{tabular}




\begin{tabular}{|c|c|c|c|c|c|c|c|c|c|c|c|c|c|c|c|c|c|c|}
\hline DATE & TIME & Sol & $\begin{array}{l}\text { FLTRT } \\
\left({ }^{\circ} \mathrm{C}\right) \\
\text { T2 }\end{array}$ & $\begin{array}{c}\text { CL } \\
\text { LOOP } \\
\left({ }^{\circ} \mathrm{C}\right) \\
\text { T3 }\end{array}$ & $\begin{array}{l}\text { SL } \\
\text { LOOP } \\
\left({ }^{\circ} \mathrm{C}\right) \\
\mathrm{T} 1\end{array}$ & $\begin{array}{c}\text { UP } \\
\text { AMB } \\
\left({ }^{\circ} \mathrm{C}\right) \\
\text { T4 }\end{array}$ & $\begin{array}{l}\text { BOT } \\
\text { AMB } \\
\left({ }^{\circ} \mathrm{C}\right) \\
\text { T5 }\end{array}$ & $\begin{array}{l}\text { BOT } \\
\text { DP } \\
\text { (psid) } \\
\text { dP2 }\end{array}$ & $\begin{array}{c}\text { FLTR } \\
\text { (psig) } \\
\text { P1 }\end{array}$ & $\begin{array}{l}\text { FLTR } \\
\text { DP } \\
\text { (psid) } \\
\text { dP1 }\end{array}$ & $\begin{array}{l}\text { TOP } \\
\text { DP } \\
\text { (psig) } \\
\text { dP3 }\end{array}$ & $\begin{array}{l}\text { FLT- } \\
\text { RATE } \\
\text { (psig) } \\
\text { P2 }\end{array}$ & $\begin{array}{c}\text { BP } \\
\text { (psig) } \\
\text { P3 }\end{array}$ & $\begin{array}{l}\mathrm{SL} \\
\text { FLOW } \\
(\mathrm{gpm}) \\
\mathrm{Q1}\end{array}$ & $\begin{array}{l}\text { FLTR } \\
\text { FLOW } \\
\text { (gpm) } \\
\text { Q2 }\end{array}$ & $\begin{array}{c}\text { Temp } \\
\text { corrected } \\
\text { flow } \\
\left(\mathrm{gpm} / \mathrm{ft}^{2}\right)\end{array}$ & $\begin{array}{l}\text { Axial Vel } \\
(\mathrm{ft} / \mathrm{sec})\end{array}$ & $\begin{array}{l}\text { Avg } \\
\text { TMP } \\
\text { (psid) }\end{array}$ \\
\hline D/24/01 & 10:30 & 0 & 25.9 & 24.6 & 25.6 & 24.4 & 22.8 & 50.7 & 61.0 & 10.6 & 40.7 & 10.1 & 0 & 38.3 & 0.083 & 0.0357 & 15.9 & 45.7 \\
\hline & 10:31 & 0 & 25.9 & 24.6 & 25.6 & 24.3 & 22.7 & 50.8 & 60.9 & 10.6 & 41.2 & 10.1 & 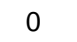 & 38.3 & 0.083 & 0.0356 & 15.9 & 46.0 \\
\hline 24/01 & 10:32 & 0 & 25.9 & 24.6 & 25.6 & 24.3 & 22.6 & 51.2 & 61.2 & 10.5 & 40.9 & 10.1 & & 38.3 & 0.082 & 0.0352 & 15.9 & 46.0 \\
\hline b1 & $10: 33$ & 0 & 25.9 & 24.6 & 25.6 & 24.3 & 22.6 & 51.2 & 61.2 & 10.7 & 41.1 & 10.1 & 0 & 38.3 & 0.082 & 0.0352 & 15.9 & 46.1 \\
\hline $24 / 01$ & 10:34 & 0 & 26.0 & 24.6 & 25.7 & 24.2 & 22.5 & 51.1 & 61.3 & 10.5 & 40.9 & 10.0 & & 38.3 & 0.081 & 0.0347 & 15.9 & 5.0 \\
\hline /24/01 & 10:35 & 0 & 25.9 & 24.6 & 25.7 & 24.2 & 22.5 & 51.1 & 61.2 & 10.6 & 41.3 & 10.0 & 0 & 38.3 & 0.081 & 0.0347 & 15.9 & 46.2 \\
\hline $24 / 01$ & 10:36 & 0 & 25.9 & 24.5 & 25.7 & 24.0 & 22.5 & 50.8 & 61.0 & 10.6 & 40.9 & 10.0 & & 38.3 & 0.081 & 0.0346 & 15.9 & 45.8 \\
\hline //24/01 & 10:37 & 0 & 26.0 & 24.6 & 25.7 & 24.0 & 22.4 & 51.0 & 61.0 & 10.7 & 40.7 & 10.0 & 0 & 38.4 & 0.080 & 0.0342 & 15.9 & 45.9 \\
\hline & 10:38 & 0 & 26.0 & 24.5 & 25.8 & 24.0 & 22.4 & 51.1 & 61.0 & 10.7 & 41.2 & 10.0 & & 38.3 & 0.080 & & 9 & 6.1 \\
\hline 1 & 10:39 & 0 & 26.0 & 24.5 & 25.8 & 24.0 & 22.4 & 51.0 & 60.7 & 10.6 & 41.0 & 10.0 & & 38.4 & 0.080 & .0342 & 15.9 & 46.0 \\
\hline & 10:40 & 0 & 26.1 & & 5.8 & 23.9 & 22.4 & 51.0 & 60.8 & 10.7 & 41.1 & 10 & & 38.4 & & & & \\
\hline & 10:41 & 0 & 26.1 & 24.5 & 25.8 & 24.0 & 22.4 & 50.7 & 60.5 & 10.6 & 41.2 & 10.0 & 0 & 38.4 & 0.080 & 0.0341 & 15.9 & 45.9 \\
\hline & & 0 & 26.1 & & 25.8 & 24.0 & 22.3 & 51.2 & 61.0 & 106 & 40.7 & 0 & & 38.3 & & & & \\
\hline 1 & 10:43 & 0 & 26.1 & 24.4 & 25.9 & 24.0 & 22.3 & 51.1 & 61.1 & 10.6 & 41.1 & 9.5 & & 38.4 & 0.079 & 0.0337 & 15.9 & 46.1 \\
\hline (2) & 10:44 & 0 & 26.1 & 24.4 & 25.8 & 24.0 & 22.2 & 50.9 & 60.7 & 10.6 & 40.9 & 9. & & 38.3 & 0.079 & 0337 & 15.9 & 45.9 \\
\hline 1 & 10:45 & 0 & 26.1 & 24.4 & 25.9 & 23.9 & 22.2 & 51.0 & 60.7 & 10.6 & 41.0 & 9. & & 38.3 & 0.079 & 0.0 & 9 & 6.0 \\
\hline & 10:46 & 0 & 26.2 & 4.4 & 5.9 & 23.9 & 22.2 & 51.0 & 60.7 & 10.7 & 40.7 & 0 & & 38.3 & 0.079 & & & 45.8 \\
\hline & 10:47 & 0 & 26.2 & 4.4 & 25.9 & 23.9 & 22.2 & 51.2 & 61.1 & 10.7 & 41.0 & 9. & & 38.4 & 0.078 & & & \\
\hline & 10:48 & 0 & 26.2 & 24.4 & 25.9 & 23.9 & 22.2 & 51.2 & 61.1 & 10.6 & 41.0 & 9. & & 38.4 & 0.078 & 0.0 & 15 & 46.1 \\
\hline & 10:49 & 0 & 26.2 & 4.3 & 26.0 & 23.9 & 22.2 & 51.4 & 61.3 & 10.7 & 41.0 & 9. & & 38.4 & 0.078 & & & \\
\hline$/ 2$ & 10:50 & 0 & 26.2 & 24.3 & 26.0 & 23.9 & 22.3 & 51.2 & 61.2 & 10.7 & 40.8 & 9. & & 38.3 & 0.078 & 0.0 & 15 & 46.0 \\
\hline & 10:51 & 0 & 26.3 & 4.3 & 26.0 & 23.9 & 22.3 & 50.9 & 60.7 & 10.5 & 41.0 & 9. & & 38.3 & 0.078 & & & \\
\hline /24 & 10:52 & 0 & 26.3 & 4.3 & 26.1 & 24.0 & 22.3 & 50.9 & 60.7 & 10.6 & 40.7 & 9. & & 38.3 & 0.078 & 31 & 15 & 45.8 \\
\hline & 10:53 & 0 & 26.3 & 3 & 26.0 & 24.0 & 22.4 & 51.1 & 60.8 & 10.7 & 41.3 & 9. & & 38.3 & 0.078 & & & \\
\hline 10/2 & 10:54 & 0 & 26.3 & 4.3 & 26.1 & 24.0 & 22.4 & 51.0 & 60.7 & 10.6 & 41.0 & 9. & & 38.4 & 0.078 & 0.0 & 15 & 0.0 \\
\hline & & 0 & & & 26.1 & & 22.5 & & 61. & 10 & & & & 3 & & & & \\
\hline & 10:56 & 0 & 26.3 & 4.3 & 26.1 & 24.1 & 22.6 & 51.4 & 61.1 & 10.7 & 41.3 & . & & 38.5 & 0.077 & & & 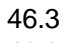 \\
\hline & $10: 57$ & 0 & 26.3 & 4.3 & 26.1 & 24.2 & 22.6 & 51.4 & 61.3 & 10.7 & 41.2 & 9. & & 38.4 & 0.077 & 6 & & 46.3 \\
\hline 10/24 & $10: 58$ & 0 & 26.3 & 4.3 & 26.1 & 24.3 & 22.7 & 51.4 & 61.2 & 10.6 & 41.1 & 9. & & 38.2 & 0.077 & & & 46.3 \\
\hline 12 & 10:59 & 0 & 26.4 & 4.3 & 26.1 & 24.4 & 22.8 & 51.0 & 60.6 & 10.6 & 41.3 & 3. & & 38.3 & 0.077 & & 15.9 & 46.2 \\
\hline 10/24 & $11: 00$ & 0 & 26.4 & 4.3 & 26.2 & 24.4 & 22.8 & 51.7 & 61.5 & 10.8 & 41.2 & 9 & & 38.3 & 0.077 & & 15.9 & 46.5 \\
\hline 24 & 11:01 & 0 & 26.4 & 4.3 & 26.2 & 24.5 & 22.8 & 51.2 & 60.9 & 10.7 & 40.9 & 9.8 & & 8.3 & 0.077 & 5 & 15.9 & .0 \\
\hline & & & & & 6.2 & & 22.9 & 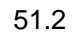 & 6 & .7 & & 3. & & & & & & \\
\hline & 11:03 & & 26.4 & 3 & 26.2 & 24.6 & 22.9 & 51.1 & 61.0 & 10.6 & 41. & 9.8 & & + & 0.077 & & & 46.2 \\
\hline & & 0 & & & 26.2 & & 22.8 & 51.3 & & 10.7 & & & & & & & & \\
\hline 10/24 & $11: 05$ & 0 & 26.4 & 4.3 & 26.2 & 24.5 & 22.8 & 51.8 & 61.7 & 10.7 & 41. & 9. & & 3.3 & 0.076 & & & 46 \\
\hline & 11:0 & & 26.4 & 4.3 & 26.2 & & 22.8 & & & 10.7 & 41. & & & & & & & \\
\hline & 11:07 & 0 & 26.5 & 4.2 & 26.3 & 24.4 & 22.8 & 51.7 & 61.5 & 10.7 & 41.1 & 9. & & 38.3 & 0.076 & 0 & 15 & 46.4 \\
\hline & 11:08 & & 26.5 & 4.2 & 26.2 & & 22.7 & 51.5 & & 10.8 & 41.1 & 9 & & & 0.0 & & & \\
\hline & 11:09 & 0 & 26.5 & 1.2 & 26.3 & .3 & 22.7 & 51.6 & 1.3 & 10.7 & 41.1 & S. & & 8.4 & 0.076 & & 15.9 & 46.3 \\
\hline & $11: 10$ & & & & 6.2 & & 22.7 & & & 10.7 & 41.1 & 9 & & 3.4 & 0.076 & & & 460 \\
\hline & & 0 & & & 3 & & 22.7 & 51.3 & 61.0 & 10.7 & 41 & 9. & & & & & & 46 \\
\hline & 11: & & & & 3 & & 22.7 & & & 10.7 & 41 & & & ( & 0 & & & \\
\hline & 11 & & 26.6 & & 26.3 & & 22.6 & & & 6 & & 9. & & & & & & \\
\hline & $11: 1$ & 0 & 26.6 & 2 & 26.3 & 3 & 22.6 & 51.6 & 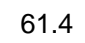 & .8 & 41 & 9. & & .4 & 0.076 & & & 46 \\
\hline & & & & & 26.3 & & & & & & & & & & & & & \\
\hline & 11:16 & 0 & & 2 & 26.4 & & 22.6 & 51. & & 10.7 & 41 & 9. & & 38.3 & 5 & & & \\
\hline & & & & & & & & & & & & 9. & & & & & & \\
\hline & . & & 2 & & 6.3 & & 22.6 & 51.4 & 61.1 & 10.7 & 41 & 9. & & . & & & & \\
\hline & & & & & & & & & & & & & & & & & & \\
\hline & $11: 2$ & 0 & 26.6 & & 5.4 & & 22.6 & & & 10.1 & & 9. & & & & & & \\
\hline & 11:2 & 0 & 26.7 & & 4 & & 206 & & & 10.7 & & 9. & & 38.3 & 0.075 & & & \\
\hline & $11: 22$ & & 26.7 & 4.1 & 26.4 & & 22.6 & & & 10 & & 9. & & 4 & & & & \\
\hline & $11: 23$ & 0 & & & 26 & & & & & & & 9. & & & & & & \\
\hline & $11: 24$ & & 26. & 24.1 & ar & & & & & 10.7 & & 9. & & & & & & \\
\hline & 1.25 & 0 & & & & & 225 & & & 10 & & 9. & & & & & & \\
\hline & & & & & & & & & & & & 9. & & & & & & \\
\hline & & & & & & & & & & & & & & & & & & \\
\hline & $11: 28$ & 0 & 26 & 24 & $=0$ & & $\angle 2$. & 52 & 61. & 10 & & 9. & U & 38 & 75 & & 10 & \\
\hline & 11.29 & & 26.8 & & 20.5 & & & 51.4 & 67.0 & 10.8 & 40.9 & 9.1 & & 38.3 & 0.075 & 0.0314 & 15.9 & 46.2 \\
\hline
\end{tabular}




\begin{tabular}{|c|c|c|c|c|c|c|c|c|c|c|c|c|c|c|c|c|c|c|}
\hline DATE & TIME & Sol & $\begin{array}{c}\text { FLTRT } \\
\left({ }^{\circ} \mathrm{C}\right) \\
\text { T2 }\end{array}$ & $\begin{array}{c}\mathrm{CL} \\
\text { LOOP } \\
\left({ }^{\circ} \mathrm{C}\right) \\
\text { T3 }\end{array}$ & $\begin{array}{c}\text { SL } \\
\text { LOOP } \\
\left({ }^{\circ} \mathrm{C}\right) \\
\mathrm{T} 1\end{array}$ & $\begin{array}{c}\text { UP } \\
\text { AMB } \\
\left({ }^{\circ} \mathrm{C}\right) \\
\text { T4 }\end{array}$ & $\begin{array}{c}\text { BOT } \\
\text { AMB } \\
\left({ }^{\circ} \mathrm{C}\right) \\
\text { T5 }\end{array}$ & $\begin{array}{c}\text { BOT } \\
\text { DP } \\
\text { (psid) } \\
\text { dP2 }\end{array}$ & $\begin{array}{c}\text { FLTR } \\
\text { (psig) } \\
\text { P1 }\end{array}$ & $\begin{array}{l}\text { FLTR } \\
\text { DP } \\
\text { (psid) } \\
\text { dP1 }\end{array}$ & $\begin{array}{c}\text { TOP } \\
\text { DP } \\
\text { (psig) } \\
\text { dP3 }\end{array}$ & $\begin{array}{l}\text { FLT- } \\
\text { RATE } \\
\text { (psig) } \\
\text { P2 }\end{array}$ & $\begin{array}{c}\text { BP } \\
\text { (psig) } \\
\text { P3 }\end{array}$ & $\begin{array}{c}\text { SL } \\
\text { FLOW } \\
\text { (gpm) } \\
\text { Q1 }\end{array}$ & $\begin{array}{l}\text { FLTR } \\
\text { FLOW } \\
\text { (gpm) } \\
\text { Q2 }\end{array}$ & $\begin{array}{c}\text { Temp } \\
\text { corrected } \\
\text { flow } \\
\left(\mathrm{gpm} / \mathrm{ft}^{2}\right)\end{array}$ & $\begin{array}{c}\text { Axial Vel } \\
(\mathrm{ft} / \mathrm{sec})\end{array}$ & $\begin{array}{l}\text { Avg } \\
\text { TMP } \\
\text { (psid) }\end{array}$ \\
\hline 0/24/01 & $11: 30$ & 0 & 26.8 & 24.1 & 26.5 & 24.2 & 22.5 & 51.5 & 61.2 & 10.8 & 41.2 & 9.7 & 0 & 38.4 & 0.075 & 0.0314 & 15.9 & 46.4 \\
\hline /24/01 & $11: 31$ & 0 & 26.8 & 24.1 & 26.6 & 24.2 & 22.5 & 51.5 & 61.3 & 10.7 & 41.4 & 9.7 & & 38.3 & 0.075 & 0.0313 & 9 & 46.4 \\
\hline /24/01 & $11: 32$ & 0 & 26.8 & 24.0 & 26.5 & 24.2 & 22.5 & 51.5 & 61.1 & 10.7 & 41.1 & 9.7 & 0 & 38.3 & 0.074 & 0.0310 & 9 & 46.3 \\
\hline /24/01 & $11: 33$ & 0 & 26.8 & 24.0 & 26.6 & 24.2 & 22.5 & 51.2 & 60.9 & 10.8 & 41.1 & 9.7 & 0 & 38.3 & 0.074 & 0.0309 & 9 & 46.1 \\
\hline /24/01 & $11: 34$ & 0 & 26.8 & 24.0 & 26.6 & 24.3 & 22.5 & 51.4 & 61.0 & 10.8 & 41.3 & 9.7 & 0 & 38.3 & 0.074 & 0.0309 & 5.9 & 46.3 \\
\hline /24/01 & $11: 35$ & 0 & 26.8 & 24.0 & 26.6 & 24.3 & 22.4 & 51.6 & 61.2 & 10.7 & 41.3 & 9.7 & 0 & 38.3 & 0.074 & 0.0309 & 9 & 46.4 \\
\hline D/24/01 & $11: 36$ & 0 & 26.8 & 24.0 & 26.6 & 24.2 & 22.4 & 51.7 & 61.5 & 10.8 & 41.2 & 9.7 & 0 & 38.3 & 0.074 & 0.0309 & 9 & 46.4 \\
\hline 10/24/01 & 11:37 & 0 & 26.9 & 24.0 & 26.5 & 24.2 & 22.4 & 51.4 & 61.0 & 10.9 & 41.3 & 9.7 & 0 & 38.3 & 0.074 & 0.0309 & 9 & 46.4 \\
\hline 10/24/01 & $11: 38$ & 0 & 26.9 & 24.0 & 26.6 & 24.2 & 22.4 & 51.6 & 61.3 & 10.8 & 41.3 & 9.7 & 0 & 38.3 & 0.074 & 0.0309 & 9 & 46.4 \\
\hline 10/24/01 & $11: 39$ & 0 & 26.9 & 24.0 & 26.6 & 24.2 & 22.5 & 51.6 & 61.2 & 10.8 & 41.0 & 9.7 & 0 & 38.3 & 0.074 & 0.0309 & & 46.3 \\
\hline /24/01 & $11: 40$ & 0 & 26.9 & 24.0 & 26.6 & 24.2 & 22.4 & 51.7 & 61.4 & 10.9 & 41.5 & 9.7 & 0 & 38.3 & 0.074 & & & 46.6 \\
\hline$/ 24 / 01$ & $11: 41$ & 0 & 26.9 & 24.0 & 26.6 & 24.1 & 22.4 & 51.3 & 60.8 & 10.8 & 41.1 & 9.7 & 0 & 38.3 & 0.074 & & & 46.2 \\
\hline 1 & $11: 42$ & 0 & 26.9 & 24.0 & 26.7 & 24.2 & 22.4 & 51.7 & 61.4 & 10.8 & 41.6 & 9.7 & & 38.4 & 0.074 & 0. & .9 & 46.7 \\
\hline 1 & $: 43$ & 0 & 26.9 & 23.9 & 26.7 & 24.2 & 22.4 & 51.6 & 61.2 & 10.8 & 41.2 & 9. & & 38.3 & 0.074 & & & 46.4 \\
\hline 1 & :44 & 0 & 26.9 & 23.9 & 26.7 & 24.2 & 22.4 & 51.4 & 1.0 & 10.8 & 41.3 & 9. & & 38.3 & 0.074 & & & 6.3 \\
\hline$J / 6$ & :45 & 0 & 26.9 & 23.9 & 26.7 & 24.1 & 22.4 & 1.7 & 1.4 & 10.7 & 40.9 & 9 & & 8.3 & 0.074 & & & 6.3 \\
\hline$/ 2$ & :46 & 0 & 27.0 & 23.9 & 26.8 & 24.1 & 22.4 & 1.5 & 1.1 & 10.8 & 41.4 & 9 & & 8.3 & 0.074 & & & 6.5 \\
\hline$/ 2$ & $: 47$ & 0 & 27.0 & 23.9 & 26.7 & 24.1 & 22.4 & 1.7 & 61.2 & 10.8 & 1.0 & 9 & & 38.3 & 0.074 & & & 46.3 \\
\hline $1 / 2$ & :48 & 0 & 27.0 & 23.9 & 26.8 & 24.1 & 22.4 & 51.6 & 61.1 & 10.8 & 41.0 & 9. & c & 38.2 & 0.074 & & & 46.3 \\
\hline )/24 & $11: 49$ & 0 & 27.0 & 23.9 & 26.8 & 24.1 & 22.4 & 51.5 & 61.2 & 10.8 & 41.2 & 9. & c & 38.3 & 0.074 & 07 & & 46.4 \\
\hline$/ 24$ & $11: 50$ & 0 & 27.0 & 23.9 & 26.8 & 24.1 & 22.4 & 51.3 & 60.9 & 10.8 & 41.1 & 9.6 & 0 & 38.3 & 0.074 & & & 46.2 \\
\hline )/24/01 & $11: 51$ & 0 & 27.0 & 23.9 & 26.9 & 24.1 & 22.4 & 51.6 & 61.2 & 10.8 & 41.4 & 9.7 & 0 & 38.3 & 0.074 & & & 46.5 \\
\hline $2 / 24 / 01$ & $11: 52$ & 0 & 27.1 & 23.9 & 26.8 & 24.1 & 22.4 & 51.5 & 61.1 & 10.8 & 41.2 & 9. & 0 & 38.3 & 0.073 & & & 46.3 \\
\hline$/ 24$ & $11: 53$ & 0 & 27.1 & 23.9 & 26.9 & 24.1 & 22.4 & 51.5 & 61.1 & 10.8 & 41.3 & 9. & 0 & 38.3 & 0.073 & & & 46.4 \\
\hline$/ 24$ & $11: 54$ & 0 & 27.1 & 23.9 & 26.9 & 24.2 & 22.4 & 51.4 & 61.0 & 10.7 & 41.0 & 9.6 & 0 & 38.1 & 0.073 & & & 46.2 \\
\hline o/24/ & $11: 55$ & 0 & 27.1 & 23.9 & 26.3 & 24.2 & 22.4 & 51.7 & 61.3 & 10.7 & 41.5 & 9. & 0 & 38.2 & 0.073 & & & 46.6 \\
\hline /24/ & $11: 56$ & 0 & 26.9 & 23.9 & 26.0 & 24.2 & 22.4 & 51.4 & 60.9 & 10.6 & & 9. & 0 & 3.2 & 0.072 & & & 46.4 \\
\hline $1 / 2$ & $11: 57$ & 0 & 26.9 & 23.9 & 25.8 & 24.2 & 22.4 & 51.3 & 60.7 & 10.6 & 41.6 & 9. & $\Omega_{0}$ & 38.2 & 0.012 & 0 & $y$ & 46.4 \\
\hline 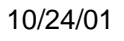 & :58 & 0 & 26.7 & 23.8 & 25.7 & 24.2 & 22.4 & 48.5 & 60.9 & 10.7 & 38.2 & 12 & & 8.2 & 0.010 & & & 43.3 \\
\hline$/ 2$ & :59 & 0 & 26.7 & 23.8 & 25.7 & 24.2 & 22.4 & 50.8 & 1.0 & 10.6 & 40.8 & 10 & & 38.3 & 93 & & & 45.8 \\
\hline 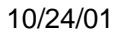 & $: 00$ & 0 & 26.6 & 23.8 & 25.8 & 24.2 & 22.4 & 51.2 & 61.1 & 10.7 & 41.1 & 10 & & 3.2 & 39 & & & 16.2 \\
\hline $0 / 2$ & 01 & 0 & 26.5 & 23.8 & 25.8 & 24.1 & 22.4 & 6 & .4 & 10.8 & 41.0 & 10 & & 38.3 & 87 & & & 46.3 \\
\hline$/ 2$ & $: 02$ & 0 & 26.4 & 23.8 & 25.8 & 24.1 & 22.4 & 2 & 61.0 & 10.6 & 41.0 & 9 & & 38.1 & 36 & & & 46.1 \\
\hline $1 / 2$ & 3 & 0 & 26.4 & 23.8 & 25.8 & 24.1 & 22.3 & 3 & 61.1 & 10.6 & 9 & 9 & & 38.3 & 35 & & & 46.1 \\
\hline 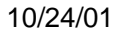 & $12: 04$ & 0 & 26.3 & 23.8 & 25.9 & 24.1 & 22.3 & .1 & 60.9 & 10.7 & 41.0 & 9. & 0 & 38.2 & 0.084 & & & 46.1 \\
\hline 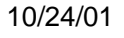 & 12:05 & 0 & 26.3 & 23.8 & 25.9 & 24.1 & 22.3 & 51.2 & 60.9 & 10.7 & 41.3 & 9.8 & 0 & 38.2 & 0.084 & & & 46.2 \\
\hline )/2 & $12: 06$ & 0 & 26.2 & 23.8 & 25.9 & 24.1 & 22.3 & 51.3 & 61.1 & 10.7 & 41.1 & 9.8 & 0 & 38.2 & 0.083 & & & 46.2 \\
\hline /2 & $12: 07$ & 0 & 26.2 & 23.8 & 26.0 & 24.1 & 22.3 & 51.2 & 61.0 & 10.7 & 40.9 & 9. & 0 & 38.2 & 0.083 & & & 46.0 \\
\hline 0/24/01 & $12: 08$ & 0 & 26.2 & 23.8 & 26.0 & 24.1 & 22.3 & 51.4 & 61.0 & 10.7 & 41.3 & 9. & 0 & 38.2 & 0.083 & & & 46.3 \\
\hline $10 / 24 / 01$ & $12: 09$ & 0 & 26.3 & 23.8 & 26.0 & 24.2 & 22.3 & 50.9 & 60.6 & 10.6 & 41.0 & 9 & 0 & 38.3 & 0.082 & & & 46.0 \\
\hline 10/24/01 & $12: 10$ & 0 & 26.2 & 23.8 & 26.0 & 24.2 & 22.3 & 51.3 & 61.0 & 10.7 & 40.9 & 9 & 0 & 38.2 & 0.082 & & & 46.1 \\
\hline 0/24/01 & $12: 11$ & 0 & 26.3 & 23.8 & 26.1 & 24.2 & 22.4 & 51.2 & 60.8 & 10.6 & 41.4 & 9 & 0 & 38.2 & 0.081 & & & 46.3 \\
\hline$/ 2$ & :12 & 0 & 26.3 & 23.7 & 26.0 & 24.2 & 22.3 & & 60.7 & 10.6 & & & & 8.2 & & & & 46.1 \\
\hline$\pi /<$ & :13 & 0 & 26.4 & 23.8 & 26.1 & 24.2 & 22.3 & 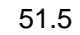 & 61.2 & 10.7 & & & & 8.3 & & & & 46.2 \\
\hline 1/2 & ::14 & 0 & 26.4 & 23.7 & 26.1 & 24.3 & 22.3 & 51.5 & 61.2 & 10.8 & & & & 38.3 & 0.081 & & & 46.3 \\
\hline 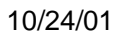 & 5 & 0 & 26.4 & 23.7 & 26.2 & 24.3 & 22.3 & 51 & 6 & 10.7 & 41.1 & 9 & & 8.3 & 0.080 & & & 46.2 \\
\hline & & 0 & 26.4 & 23.7 & 26.2 & 24.3 & 22.3 & 51.2 & 60.8 & 10.7 & 41.1 & 9 & & 38.3 & 0.080 & & & 46.2 \\
\hline 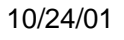 & $12: 17$ & 0 & 26.5 & 23.7 & 26.2 & 24.3 & 22.3 & 51.2 & 60.8 & 10.7 & 41.3 & 9 & & 38.3 & 0.080 & & & 46.3 \\
\hline $0 / 2$ & $12: 18$ & 0 & 26.4 & 23.7 & 26.2 & 24.3 & 22.4 & 51.3 & 60.9 & 10.7 & 41.5 & 9 & & 38.2 & 0.080 & & & 46.4 \\
\hline 0/24/( & $12: 19$ & 0 & 26.5 & 23.7 & 26.2 & 24.3 & 22.4 & 51.1 & 60.9 & 10.6 & 41.0 & 9. & 0 & 38.3 & 0.080 & 37 & .9 & 46.0 \\
\hline 0/24/01 & $12: 20$ & 0 & 26.5 & 23.7 & 26.3 & 24.3 & 22.4 & 51.5 & 61.0 & 10.7 & 41.3 & 9. & 0 & 38.4 & 0.080 & & .9 & 46.4 \\
\hline 0/24/01 & $12: 21$ & 0 & 26.5 & 23.7 & 26.3 & 24.3 & 22.4 & 51.3 & 60.8 & 10.7 & 41.1 & 9.7 & 0 & 38.3 & 0.079 & 33 & 15.9 & 46.2 \\
\hline 10/24/01 & $12: 22$ & 0 & 26.5 & 23.7 & 26.3 & 24.3 & 22.4 & 51.7 & 61.3 & 10.8 & 41.1 & 9.7 & 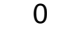 & 38.3 & 0.079 & 0.0332 & 15.9 & 46.4 \\
\hline 0/24/01 & $12: 23$ & 0 & 26.6 & 23.7 & 26.2 & 24.2 & 22.4 & 51.2 & 60.7 & 10.7 & 41.1 & 9.7 & 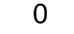 & 38.3 & 0.079 & 0.0333 & 15.9 & 46.1 \\
\hline 0/24/01 & $12: 24$ & 0 & 26.6 & 23.7 & 26.3 & 24.2 & 22.4 & 51.5 & 61.2 & 10.6 & 41.5 & 9. & 6 & 38.2 & 0.079 & 0.0332 & .9 & 46.5 \\
\hline 0/24/ & $12: 25$ & 0 & 26.6 & 23.7 & 26.4 & 24.2 & 22.4 & 51.4 & 61.0 & 10.7 & 41.2 & 9. & - & 38.3 & 0.079 & 332 & 5.9 & 46.3 \\
\hline 0/24/01 & $12: 26$ & 0 & 26.6 & 23.7 & 26.4 & 24.2 & 22.4 & 51.5 & 61.1 & 10.7 & 41.2 & 9. & $v$ & 38.3 & 0.079 & 0.0332 & 15.9 & 46.4 \\
\hline & $12: 27$ & 0 & 26.6 & 23.7 & 20.4 & 24.2 & 22.4 & 31.4 & 61.0 & 10.6 & 41.2 & 9. & 0 & 38.2 & 0.079 & 0.0332 & 15.9 & 46.3 \\
\hline & $12: 28$ & 0 & 26.6 & 23.7 & 26.4 & 24 & 22.4 & 31.4 & 61.1 & 10.7 & 41.3 & 9. & 0 & 38.3 & 0.079 & & 15.9 & +6.4 \\
\hline 0/24/01 & $12: 29$ & 0 & 26.6 & 23.7 & 26.4 & 24.2 & 22.4 & 51.4 & 61.0 & 10.7 & 41.2 & 9.6 & 0 & 38.3 & 0.079 & 0.0332 & 15.9 & 46.3 \\
\hline
\end{tabular}




\begin{tabular}{|c|c|c|c|c|c|c|c|c|c|c|c|c|c|c|c|c|c|c|}
\hline & MIVIE & Sol & $\begin{array}{l}\left({ }^{\circ} \mathrm{C}\right) \\
\mathrm{T} 2\end{array}$ & $\begin{array}{c}\text { CL } \\
\text { LOOP } \\
\left({ }^{\circ} \mathrm{C}\right) \\
\text { T3 }\end{array}$ & $\begin{array}{c}\text { SL } \\
\text { LOOP } \\
\left({ }^{\circ} \mathrm{C}\right) \\
\mathrm{T} 1\end{array}$ & $\begin{array}{l}\text { UP } \\
\text { AMB } \\
\left({ }^{\circ} \mathrm{C}\right) \\
\text { T4 }\end{array}$ & $\begin{array}{l}\text { BOT } \\
\text { AMB } \\
\left({ }^{\circ} \mathrm{C}\right) \\
\text { T5 }\end{array}$ & $\begin{array}{l}\text { BOT } \\
\text { DP } \\
\text { (psid) } \\
\text { dP2 }\end{array}$ & $\begin{array}{l}\text { FLTR } \\
\text { (psig) } \\
\text { P1 }\end{array}$ & $\begin{array}{l}\text { FLTR } \\
\text { DP } \\
\text { (psid) } \\
\text { dP1 }\end{array}$ & $\begin{array}{l}\text { TOP } \\
\text { DP } \\
\text { (psig) } \\
\text { dP3 }\end{array}$ & $\begin{array}{l}\text { FLT- } \\
\text { RATE } \\
\text { (psig) } \\
\text { P2 }\end{array}$ & $\begin{array}{c}\text { BP } \\
\text { (psig) } \\
\text { P3 }\end{array}$ & $\begin{array}{c}\text { SL } \\
\text { FLOW } \\
\text { (gpm) } \\
\text { Q1 }\end{array}$ & $\begin{array}{l}\text { FLTR } \\
\text { FLOW } \\
\text { (gpm) } \\
\text { Q2 }\end{array}$ & $\begin{array}{l}\text { Temp } \\
\text { corrected } \\
\text { flow } \\
\left(\mathrm{gpm} / \mathrm{ft}^{2}\right)\end{array}$ & $\begin{array}{c}\text { Axial Vel } \\
(\mathrm{t} / \mathrm{sec})\end{array}$ & $\begin{array}{l}\text { Avg } \\
\text { TMP } \\
\text { (psid) }\end{array}$ \\
\hline D/24/01 & $12: 30$ & 0 & 26.7 & 23.7 & 26.4 & 24.2 & 22.4 & 51.4 & 60.9 & 10.7 & 41.4 & 9.6 & 0 & 38.3 & 0.078 & 0.0327 & 15.9 & 46.4 \\
\hline & 12:31 & 0 & 26.7 & 23.7 & 26.4 & 24.2 & 22.4 & 51.9 & 61.5 & 10.9 & 41.2 & 9.6 & & 38.3 & 0.078 & & 15.9 & 66 \\
\hline /24/01 & $12: 32$ & 0 & 26.7 & 23.7 & 26.4 & 24.2 & 22.4 & 51.5 & 60.8 & 10.7 & 41.5 & 9.6 & ( & 38.3 & 0.078 & 0.0327 & 15.9 & 6.5 \\
\hline $24 / 01$ & $12: 33$ & 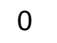 & 26.7 & 23.7 & 26.5 & 24.3 & 22.4 & 1.5 & 61.0 & 10.8 & 41.6 & 9.6 & & 38.3 & 0.078 & & 15.9 & 65 \\
\hline /24/01 & $12: 34$ & 0 & 26.7 & 23.7 & 26.5 & 24.2 & 22.4 & 51.5 & 61.1 & 10.7 & 41.1 & 9.6 & & 38.2 & 0.078 & 0.0327 & 15.9 & 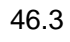 \\
\hline 1 & $12: 35$ & 0 & 26.7 & 6 & 26.5 & .2 & 22.4 & 6 & 61.1 & 10.7 & .3 & 96 & & 8.3 & 0.078 & & 9 & - \\
\hline & $12: 36$ & 0 & 26.8 & 3.7 & 26.5 & .2 & 22.4 & 51.7 & 61.3 & 10.8 & 41.1 & 9.6 & & 38.2 & 0.077 & 22 & 5.9 & \\
\hline & 12:37 & 0 & 26.8 & 6 & 26.5 & .2 & 22.4 & 51.5 & 61.0 & 10.7 & 41.4 & 9.6 & & 38.4 & 0.077 & & & \\
\hline & 2:38 & 0 & 26.8 & 23.6 & 26.5 & 4.2 & 22.4 & 51.5 & 61.0 & 10.7 & 41.3 & 9.6 & & 38.3 & 0.077 & 22 & 15.9 & \\
\hline & $12: 39$ & 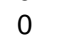 & 26.8 & & 26.5 & 4.2 & 22.4 & 51.9 & 61.5 & 10.8 & 41.6 & 96 & & 38.3 & 0.077 & & & \\
\hline & $12: 40$ & 0 & 26.8 & 23.6 & 26.5 & .3 & 22.4 & 51.6 & 61.1 & 10.7 & 41.3 & 9.6 & & 38.3 & 0.077 & & 5.9 & \\
\hline & $12: 41$ & 0 & 26.8 & 23.6 & 26.6 & 4.3 & 22.4 & 51.6 & 61.2 & 10.8 & 41.1 & 0 & & 38.2 & 0.077 & & & \\
\hline 11 & $12: 42$ & 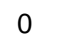 & 26.8 & 23.6 & 26.6 & .3 & 22.4 & 51.7 & 61.1 & 10.7 & 41.5 & 9.6 & & 38.2 & 0.077 & & 5.9 & 6 \\
\hline & $12: 43$ & 0 & 26.9 & 3.6 & 26.6 & 24.2 & 22.4 & 51.8 & 61.3 & 10.8 & 41.2 & 0 & & 38.2 & & & & \\
\hline 1 & $12: 44$ & 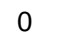 & 26.9 & 3.6 & 26.7 & 4.2 & 22.4 & 51.5 & 61.1 & 10.7 & 41.4 & 9.6 & & 38.3 & 0.077 & & .9 & \\
\hline & $12: 45$ & 0 & 26.9 & 3.6 & 26.6 & 24.2 & 22.4 & 51.3 & 60.7 & 10.7 & 41.2 & 9.5 & & 38.2 & 0.077 & & & \\
\hline 24 & $12: 46$ & 0 & 26.9 & 3.6 & 26.7 & 24.1 & 22.4 & 51.5 & 61.1 & 10.7 & 41.4 & 9.5 & & 38.2 & 0.077 & & 8 & \\
\hline & $12: 47$ & 0 & 26.9 & 3.6 & 26.7 & 24.1 & 22.4 & 51.9 & 61.5 & 10.8 & 41.1 & 96 & & 38.2 & 0.077 & & & \\
\hline & $12: 48$ & 0 & 26.9 & 3.6 & 26.7 & 24.1 & 22.4 & 51.5 & 61.0 & 10.7 & 41.2 & 0 & & 8.4 & 0.077 & & & \\
\hline & $12: 49$ & 0 & 26.9 & 3.6 & 26.7 & 4.1 & 22.4 & 515 & 610 & 10.8 & 414 & & & 8.3 & & & & \\
\hline & $12: 50$ & 0 & 27.0 & 3.6 & 26.7 & .1 & 22.4 & 51.5 & 60.9 & 10.7 & 41.4 & 9 & & 8.2 & 0.077 & & & \\
\hline & & 0 & 27.0 & & 26.7 & & 22.4 & 2.2 & 618 & 109 & 41.6 & & & & & & & \\
\hline & 12:52 & 0 & 27.0 & 3.6 & 26.8 & 4.2 & 22.4 & $51 . \varepsilon$ & 61. & 10.8 & 41.7 & & & 3 & & & & \\
\hline & $12: 53$ & 0 & 27.0 & 23.6 & 26.8 & 24.2 & 22.4 & 51.2 & 60.6 & 10.7 & 41.3 & 9. & & 38.3 & 76 & & & 46.3 \\
\hline & $12: 54$ & 0 & 27.0 & 23.6 & 26.8 & 4.1 & 22.4 & 51.7 & 61 & 10.8 & 41.8 & & & 8.3 & & & & \\
\hline & $12: 55$ & 0 & 27.1 & 3.6 & 26.8 & 24.2 & 22.4 & 51.9 & 61. & 10.8 & 41.4 & & & 8.3 & & & & \\
\hline & $12: 56$ & 0 & 27.0 & 3.6 & 26.8 & 4.2 & 22.4 & 51.6 & 60.9 & 10.8 & 41.4 & & & 8.2 & 6 & & & \\
\hline & $12: 57$ & & 27.1 & 3.6 & 26.8 & 4.2 & 22.4 & 51.9 & 61.4 & 10.8 & 41.2 & 9. & & 8.3 & & & & \\
\hline & $12: 58$ & 0 & 27.1 & 6 & 26.8 & 4.2 & 22.4 & 52.0 & 61.5 & 10.8 & 41.5 & & & 3 & & & & \\
\hline & $12: 59$ & & 27.1 & 23.6 & 26.9 & 4.2 & 22.4 & 51.8 & 61.3 & 10.7 & 41.4 & 9. & & 8.2 & 6 & & 15.8 & 46.6 \\
\hline & $13: 00$ & 0 & 27.1 & 3.6 & 26.9 & 4.2 & 22.4 & 51.2 & 60.5 & 10.7 & 41.1 & & & 3.2 & & & & \\
\hline & 13:01 & & 27.2 & 23.6 & 26.9 & 4.3 & 22.4 & 51.5 & 60.9 & 10.8 & 41.3 & & & .2 & 6 & & 15.9 & 46. \\
\hline & $13: 02$ & 0 & 27.2 & 23.6 & 27.0 & .2 & 22.5 & 51.4 & 60.9 & 10.8 & 41.4 & & & 8.2 & & & & \\
\hline & 03 & & 27.2 & .5 & 27.0 & .2 & 22.5 & 8 & 6 & 10.8 & 41.4 & & & 3 & 0 & & 15 & \\
\hline & & & 27.2 & 5 & .0 & .2 & 22.4 & & & & 5 & & & 0 & & & & \\
\hline & 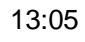 & & 27.2 & & 7.0 & .2 & 22. & & & 3 & 6 & & & 3.2 & & & & \\
\hline & & & & & & & 22 & & & & & & & & & & & \\
\hline & $13: 07$ & & 27.2 & 5 & 27.0 & 2 & 22.5 & & & 3 & 41.1 & & & 3 & & & & \\
\hline & & & 27.2 & & 27.0 & & 22. & & & & & & & & & & & \\
\hline & $13: 09$ & & 27.3 & .5 & 27.0 & .3 & 22.5 & 5 & 61.0 & 10 & 41.4 & & & 3.4 & & & & \\
\hline & & & 27.3 & & & & 22.5 & & & & & & & & & & & \\
\hline & 13:11 & 0 & 27.3 & .5 & 27.1 & .3 & 22.5 & 51.8 & 61. & 10 & 41.3 & & & .2 & & & & \\
\hline & & & & & & & 22.5 & & & & & & & & & & & \\
\hline & $13: 13$ & & 27.2 & 5 & 26.1 & .4 & 22.5 & 52.0 & & 7 & 41.7 & & & 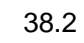 & & & & \\
\hline & 3. & & & & & & 22.5 & & & & & & & & & & & \\
\hline & $13: 15$ & 0 & 27.0 & .5 & 25.8 & .4 & 22.5 & 52.0 & 61.2 & 10 & 41.8 & & & & & & & \\
\hline & & & & & & & & & & & & & & & & & & \\
\hline & $13: 17$ & & 26.8 & 5 & 25.8 & & 22.6 & & & 6 & 40.5 & & & & & & & \\
\hline & & & & & & & & & & & & & & & & & & \\
\hline & . & & 0 & & & & 22. & & & & & & & & & & & \\
\hline & & & & & & & & & & & & & & & & & & \\
\hline & $13: 2$ & 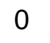 & & & & & & & & & & & & & & & & \\
\hline & & & & & & & & & & & & & & & & & & \\
\hline & $13: 23$ & 0 & 26.3 & & 9 & & 22.6 & & & 16 & & 10 & & & & & & \\
\hline & & & & & & & & & & & & & & & & & & \\
\hline & 3:25 & & 26 & & & & 22.6 & & 60 & & & & & & & & & \\
\hline & & & & & & & & & & & & & & & & & & \\
\hline & & & 26 & 23.5 & & & & & 00 & & & & & & & & & \\
\hline & $13: 28$ & & & 20 & & & & & & & & 9.9 & & & & & & \\
\hline & $13: 29$ & 0 & res & 236 & & & & & 60.6 & & & 9. & & 38.2 & 82 & 347 & 15.8 & \\
\hline
\end{tabular}




\begin{tabular}{|c|c|c|c|c|c|c|c|c|c|c|c|c|c|c|c|c|c|c|}
\hline DATE & TIME & Sol & $\begin{array}{c}\text { FLTRT } \\
\left({ }^{\circ} \mathrm{C}\right) \\
\text { T2 }\end{array}$ & $\begin{array}{c}\mathrm{CL} \\
\text { LOOP } \\
\left({ }^{\circ} \mathrm{C}\right) \\
\mathrm{T} 3\end{array}$ & $\begin{array}{c}\mathrm{SL} \\
\text { LOOP } \\
\left({ }^{\circ} \mathrm{C}\right) \\
\mathrm{T} 1\end{array}$ & $\begin{array}{c}\text { UP } \\
\text { AMB } \\
\left({ }^{\circ} \mathrm{C}\right) \\
\text { T4 }\end{array}$ & $\begin{array}{c}\text { BOT } \\
\text { AMB } \\
\left({ }^{\circ} \mathrm{C}\right) \\
\text { T5 }\end{array}$ & $\begin{array}{c}\text { BOT } \\
\text { DP } \\
\text { (psid) } \\
\text { dP2 }\end{array}$ & $\begin{array}{c}\text { FLTR } \\
\text { (psig) } \\
\text { P1 }\end{array}$ & $\begin{array}{l}\text { FLTR } \\
\text { DP } \\
\text { (psid) } \\
\text { dP1 }\end{array}$ & $\begin{array}{c}\text { TOP } \\
\text { DP } \\
\text { (psig) } \\
\text { dP3 }\end{array}$ & $\begin{array}{l}\text { FLT- } \\
\text { RATE } \\
\text { (psig) } \\
\text { P2 }\end{array}$ & $\begin{array}{c}\text { BP } \\
\text { (psig) } \\
\text { P3 }\end{array}$ & $\begin{array}{l}\text { SL } \\
\text { FLOW } \\
\text { (gpm) } \\
\text { Q1 }\end{array}$ & $\begin{array}{l}\text { FLTR } \\
\text { FLOW } \\
\text { (gpm) } \\
\text { Q2 }\end{array}$ & $\begin{array}{c}\text { Temp } \\
\text { corrected } \\
\text { flow } \\
\left(\mathrm{gpm} / \mathrm{ft}^{2}\right)\end{array}$ & $\begin{array}{c}\text { Axial Vel } \\
(\mathrm{ft} / \mathrm{sec})\end{array}$ & $\begin{array}{c}\text { Avg } \\
\text { TMP } \\
\text { (psid) }\end{array}$ \\
\hline 0/24/01 & $13: 30$ & 0 & 26.4 & 23.6 & 26.1 & 24.8 & 23.2 & 51.1 & 61.1 & 10.7 & 41.2 & 9.9 & 0 & 38.1 & 0.081 & 0.0343 & 15.8 & 462 \\
\hline$/ 24 / 01$ & $13: 31$ & 0 & 26.4 & 23.5 & 26.2 & 24.9 & 23.1 & 51.5 & 61.2 & 10.7 & 41.1 & 9.9 & 0 & 38.2 & 0.081 & .0342 & 9 & 46.3 \\
\hline /24/01 & 13:32 & 0 & 26.4 & 23.5 & 26.2 & 25.0 & 23.0 & 51.2 & 61.1 & 10.7 & 41.1 & 9.9 & 0 & 38.3 & 0.081 & 0.0342 & 15.9 & 46.1 \\
\hline /24/01 & $13: 33$ & 0 & 26.4 & 23.5 & 26.2 & 25.0 & 23.0 & 51.1 & 60.9 & 10.7 & 40.9 & 9.9 & 0 & 38.3 & 0.081 & 0.0342 & 15.9 & 46.0 \\
\hline /24/01 & $13: 34$ & 0 & 26.4 & 23.5 & 26.2 & 24.9 & 22.9 & 51.2 & 61.0 & 10.7 & 41.2 & 9.9 & 0 & 38.2 & 0.081 & 0.0342 & 15.8 & 46.2 \\
\hline D/24/01 & $13: 35$ & 0 & 26.4 & 23.5 & 26.2 & 24.9 & 22.9 & 51.5 & 61.3 & 10.6 & 41.3 & 9.9 & 0 & 38.3 & 0.081 & 0.0342 & 15.9 & 46.4 \\
\hline 10/24/01 & $13: 36$ & 0 & 26.5 & 23.5 & 26.2 & 24.9 & 22.9 & 51.7 & 61.5 & 10.7 & 41.2 & 9.8 & 0 & 38.3 & 0.080 & 0.0338 & 15.9 & 46.4 \\
\hline 10/24/01 & $13: 37$ & 0 & 26.5 & 23.5 & 26.2 & 24.9 & 22.9 & 51.3 & 61.1 & 10.7 & 41.2 & 9.8 & 0 & 38.2 & 0.080 & 0.0337 & .9 & 46.2 \\
\hline 10/24/01 & $13: 38$ & 0 & 26.5 & 23.5 & 26.2 & 24.8 & 22.9 & 51.5 & 61.4 & 10.7 & 41.4 & 9.8 & 0 & 38.2 & 0.080 & 0.0337 & 9 & 46.5 \\
\hline 0/24/01 & $13: 39$ & 0 & 26.5 & 23.6 & 26.3 & 24.8 & 22.9 & 51.3 & 61.1 & 10.7 & 41.3 & 9.8 & 0 & 38.2 & 0.080 & 337 & & 46.3 \\
\hline$/ 24 / 01$ & $13: 40$ & 0 & 26. & 23.6 & 26.3 & 24.8 & 22.9 & 51.3 & 61.0 & 10.7 & 41.2 & 9.8 & 0 & 38.3 & 0.080 & 337 & & 46.2 \\
\hline 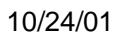 & $13: 41$ & 0 & 26.6 & 23.6 & 26.3 & 24.9 & 22.9 & 51.7 & 61.6 & 10.7 & 41.2 & 9.8 & 0 & 38.3 & 0.079 & 333 & & 46.5 \\
\hline 1 & $: 42$ & 0 & 26.6 & 23.6 & 26.3 & 24.9 & 22.9 & 51.8 & 61.5 & 10.7 & 41.1 & 9.8 & & 38.3 & 0.079 & 333 & & 46.4 \\
\hline 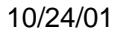 & :43 & 0 & 26.6 & 23.6 & 26.3 & 24.9 & 22.9 & 51.5 & 1.3 & 10.9 & 41.1 & 9. & & 38.3 & 0.079 & 33 & & 6.3 \\
\hline 1 & $3: 44$ & 0 & 26.6 & 23.6 & 26.3 & 25.0 & 2.9 & 51.7 & 1.5 & 10.7 & 41.5 & 9. & & 38.2 & 0.079 & 32 & & 6.6 \\
\hline $0 / 2$ & $: 45$ & 0 & 6.6 & 23.6 & 26.3 & 25.0 & 2.9 & 51.2 & 1.0 & 10.8 & 41.2 & 9. & & 38.1 & 0.079 & 32 & & 46.2 \\
\hline $0 / 2$ & $: 46$ & 0 & 6.6 & 23.6 & 26.4 & 25.0 & 2.9 & 1.5 & 1.1 & 10.7 & 41.1 & 9. & & 38.2 & 079 & 32 & & 46.3 \\
\hline /2 & 3:47 & 0 & 26.7 & 23.6 & 26.4 & 25.1 & 22.9 & 51.4 & 61.0 & 10.8 & 41.4 & 9. & ( & 38.2 & 0.079 & 32 & & 46.4 \\
\hline $1 / 2+2+3$ & $13: 48$ & 0 & 26.7 & 23.6 & 26.4 & 25.0 & 22.9 & 51.1 & 60.8 & 10.6 & 41.3 & 9.8 & c & 38.2 & 0.078 & 327 & & 46.2 \\
\hline 124 & $13: 49$ & 0 & 26.7 & 23.6 & 26.4 & 25.1 & 22.9 & 51.2 & 60.9 & 10.7 & 41.3 & 9.8 & 0 & 38.3 & 0.078 & 27 & & 46.3 \\
\hline $2 / 2$ & $13: 50$ & 0 & 26.7 & 23.6 & 26.4 & 25.0 & 22.9 & 51.2 & 60.8 & 10.7 & 41.3 & 9.8 & 0 & 38.2 & 0.078 & 27 & & 46.3 \\
\hline $8 / 2$ & 3:51 & 0 & 26.7 & 23.6 & 26.4 & 25.0 & 23.0 & 51.4 & 61.0 & 10.8 & 41.0 & 9.7 & 0 & 38.1 & 0.078 & & & 46.2 \\
\hline $10 / 24 / 01$ & $13: 52$ & 0 & 26.7 & 23.6 & 26.5 & 24.9 & 22.9 & 51.6 & 61.3 & 10.7 & 41.6 & 9.7 & 0 & 38.3 & 0.078 & 327 & & 46.6 \\
\hline 12 & $13: 53$ & 0 & 26.7 & 23.6 & 26.5 & 24.9 & 23.0 & 51.5 & 61.3 & 10.7 & 41.4 & 9.7 & 0 & 38.2 & 0.078 & 327 & & 46.5 \\
\hline $10 / 24$ & $13: 54$ & 0 & 26.7 & 23.6 & 26.5 & 24.8 & 22.9 & 51.9 & 61.6 & 10.8 & 41.4 & 9.7 & 0 & 38.3 & 0.078 & 27 & & 46.7 \\
\hline $10 / 24 / 01$ & $13: 55$ & 0 & 26.8 & 23.6 & 26.5 & 24.9 & 23.0 & 51.7 & 61.3 & 10.8 & 41.4 & 9.7 & 0 & 38.3 & 0.078 & & & 46.5 \\
\hline )/24/ & $13: 56$ & 0 & 26.8 & 23.6 & 26.5 & 24.8 & 3.0 & 51.8 & 61.5 & 10.8 & 41.3 & 9. & 0 & 38.3 & 0.078 & & & 46.5 \\
\hline $0 / 2$ & & 0 & 26.8 & 23.6 & 26.6 & 24.8 & 2.9 & 51.8 & .5 & 10.7 & & 9 & & 3.2 & & & & 46.6 \\
\hline $0 / 2$ & 3:58 & 0 & 26.8 & 23.6 & 26.5 & 24.8 & 22.9 & 51.5 & 61.1 & 10.8 & 41.3 & 9 & & 38.2 & 77 & 23 & & 46.4 \\
\hline $0 / 2$ & 3:59 & 0 & 26.8 & 23.6 & 26.6 & 24.8 & 23.0 & 51.6 & .2 & 10.7 & 41.4 & 9. & & 38.2 & 77 & 22 & & 46.5 \\
\hline $0 / 4$ & :00 & 0 & 26 & 23.6 & 26.6 & 24.9 & 23.0 & 0 & 6 & 10.6 & 41.3 & 9 & & 3.2 & 77 & 22 & & 46.2 \\
\hline $10 / 2$ & :01 & 0 & 26.8 & 23.6 & 26.6 & 24.8 & 23.0 & 1.4 & .1 & 10.7 & 41.0 & 9. & & 38.2 & 0.077 & 21 & & 46.2 \\
\hline 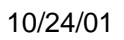 & $: 02$ & 0 & 26.9 & 23.6 & 26.6 & 24.8 & 23.0 & 4 & 1 & 10.8 & 41.2 & 9. & & 38.2 & 0.077 & 22 & & 46.3 \\
\hline 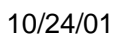 & 3 & 0 & $y$ & 23.6 & 26.6 & 24.8 & 23.0 & 3 & 9 & 10.8 & 41.1 & 9. & & 38.2 & 0.077 & & & 46.2 \\
\hline 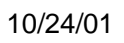 & 1:04 & 0 & 26.9 & 23.6 & 26.7 & 24.8 & 23.0 & 1.3 & 60.9 & 10.7 & 41.2 & 9 & c & 38.2 & 0.077 & & & 46.3 \\
\hline He & $: 05$ & 0 & 26.9 & 23.6 & 26.7 & 24.8 & 22.9 & 51.3 & 60.8 & 10.7 & 41.3 & 0 & & 8.2 & 0.077 & & & 46.3 \\
\hline 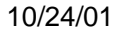 & :06 & 0 & 26.9 & 23.6 & 26.7 & 24.7 & 22.9 & 51.1 & 60.6 & 10.6 & 41.1 & 9.7 & 0 & 38.2 & 0.077 & & & 46.1 \\
\hline 11 & $14: 07$ & 0 & 26.9 & 23.6 & 26.7 & 24.8 & 22.9 & 51.8 & 61.6 & 10.8 & 41.6 & 9.7 & 0 & 38.2 & 0.077 & & & 46.7 \\
\hline 01 & $14: 08$ & 0 & 27.0 & 23.6 & 26.8 & 24.8 & 22.9 & 51.3 & 60.9 & 10.7 & 41.4 & 9.7 & 0 & 38.1 & 0.077 & & & 46.3 \\
\hline o/24/01 & $14: 09$ & 0 & 27.0 & 23.6 & 26.8 & 24.8 & 22.9 & 51.6 & 61.2 & 10.7 & 41.5 & 9. & 0 & 38.2 & 0.076 & & & 46.6 \\
\hline 0/24/ & $14: 10$ & 0 & 27.0 & 23.6 & 26.8 & 24.7 & 22.9 & 51.3 & 61.0 & 10.7 & 41.4 & 9. & 0 & 38.2 & 0.077 & 20 & & 46.4 \\
\hline 0/24/01 & $14: 11$ & 0 & 27.0 & 23.6 & 26.8 & 24.7 & 22.9 & 51.7 & 61.2 & 10.8 & 41.3 & 9. & 0 & 38.1 & 0.077 & & & 46.5 \\
\hline$/ 24 / C$ & $14: 12$ & 0 & 27.0 & 23.6 & 26.8 & 24.7 & 22.9 & 5 & 61.0 & 10.7 & 41.2 & 9 & 0 & 8.3 & 0.076 & & & 46.3 \\
\hline 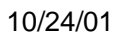 & $: 13$ & 0 & 27.0 & 23.6 & 26.8 & 24.7 & 22.9 & 5 & .0 & 10.7 & 41.5 & 9 & & 38.2 & 0.076 & & & 46.5 \\
\hline & 4 & 0 & 27.0 & 23.6 & 26.8 & 24.7 & 22.9 & 4 & 61.0 & 10.7 & 41.2 & 9 & ( & 38.1 & 0.076 & & 8 & 46.3 \\
\hline & $1 \cdot 1-1>$ & 0 & 271 & 23.6 & 26.8 & 24.7 & 22.9 & $51>$ & 61.5 & 10.8 & 41.2 & 9. & & 8.2 & 0.076 & & & 46.5 \\
\hline & 6 & 0 & 2 & 23.6 & 26.8 & 24.7 & 22.9 & 51.7 & 6 & 10.8 & 41.3 & 9. & & 38.5 & 0.076 & & & 6.5 \\
\hline & 1:17 & 0 & 27. & 23.6 & 26.9 & 24.7 & 22.9 & 5 & 61 & 10.8 & 41.3 & 9. & & 38.2 & 0. & & & 46.4 \\
\hline & $14: 18$ & 0 & 27.1 & 23.6 & 26.9 & 24.8 & 22.9 & 51.6 & 61.2 & 10.8 & 41.4 & 9. & & 38.1 & 0.076 & & & 46.5 \\
\hline $0 / 2$ & $14: 19$ & 0 & 27.1 & 23.6 & 26.9 & 24.8 & 22.9 & 51.1 & 60.7 & 10.7 & 41.4 & 9. & & 38.0 & 0.076 & & & 46.3 \\
\hline & $14: 20$ & 0 & 27.2 & 23.6 & 26.9 & 24.8 & 22.9 & 51.9 & 61.6 & 10.8 & 41.2 & 9. & 0 & 38.2 & 0.076 & & & 46.5 \\
\hline $0 / 2$ & $14: 21$ & 0 & 27.1 & 23.6 & 26.9 & 24.8 & 22.9 & 51.6 & 61.2 & 10.7 & 41.4 & 9. & 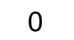 & 38.2 & 0.076 & & .9 & 46.5 \\
\hline 0/24/01 & $14: 22$ & 0 & 27.2 & 23.6 & 27.0 & 24.8 & 22.9 & 51.7 & 61.2 & 10.7 & 41.3 & 9.6 & 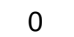 & 38.1 & 0.076 & 14 & 15.8 & 46.5 \\
\hline $0 / 24 / 01$ & $14: 23$ & 0 & 27.2 & 23.6 & 27.0 & 24.8 & 22.9 & 51.4 & 61.0 & 10.8 & 41.1 & 9.6 & 0 & 38.1 & 0.076 & 314 & 15.8 & 46.3 \\
\hline $0 / 24 / 01$ & $14: 24$ & 0 & 27.2 & 23.6 & 27.0 & 24.8 & 22.9 & 51.7 & 61.4 & 10.8 & 41.4 & 9.6 & 0 & 38.3 & 0.076 & 314 & 15.9 & 46.5 \\
\hline D/24/01 & $14: 25$ & 0 & 27.2 & 23.6 & 27.0 & 24.7 & 22.9 & 51.7 & 61.2 & 10.8 & 41.4 & 9. & 0 & 38.2 & 0.076 & & & 46.6 \\
\hline o/24/01 & $14: 26$ & 0 & 27.3 & 23.6 & 27.0 & 24.7 & 22.9 & 51.5 & 61.1 & 10.8 & 41.6 & 9.6 & 0 & 38.2 & 0.076 & 14 & 15.8 & 46.6 \\
\hline O/24/01 & $14: 27$ & 0 & 27.3 & 23.6 & 27.0 & 24.7 & 22.9 & 51.6 & 61.1 & 10.9 & 41.5 & 9.6 & 0 & 38.2 & 0.075 & 0.0310 & 15.8 & 46.5 \\
\hline $0 / 24$ & $14: 28$ & 0 & 27.3 & 23.6 & 27.0 & 24.7 & 22.9 & 51.7 & 61.3 & 10.9 & 41.1 & 9.6 & 0 & 38.1 & 0.075 & 0.0309 & 15.8 & 46.4 \\
\hline 0/24/01 & $14: 29$ & 0 & 27.3 & 23.6 & 27.1 & 24.7 & 22.9 & 51.7 & 61.4 & 10.8 & 41.4 & 9.6 & 0 & 38.2 & 0.075 & 0.0309 & 15.8 & 46.6 \\
\hline
\end{tabular}




\begin{tabular}{|c|c|c|c|c|c|c|c|c|c|c|c|c|c|c|c|c|c|c|}
\hline DATE & TIME & Sol & $\begin{array}{c}\text { FLTRT } \\
\left({ }^{\circ} \mathrm{C}\right) \\
\text { T2 }\end{array}$ & $\begin{array}{c}\mathrm{CL} \\
\text { LOOP } \\
\left({ }^{\circ} \mathrm{C}\right) \\
\text { T3 }\end{array}$ & $\begin{array}{c}\text { SL } \\
\text { LOOP } \\
\left({ }^{\circ} \mathrm{C}\right) \\
\mathrm{T} 1\end{array}$ & $\begin{array}{c}\text { UP } \\
\text { AMB } \\
\left({ }^{\circ} \mathrm{C}\right) \\
\text { T4 }\end{array}$ & $\begin{array}{c}\text { BOT } \\
\text { AMB } \\
\left({ }^{\circ} \mathrm{C}\right) \\
\text { T5 }\end{array}$ & $\begin{array}{c}\text { BOT } \\
\text { DP } \\
\text { (psid) } \\
\text { dP2 }\end{array}$ & $\begin{array}{l}\text { FLTR } \\
\text { (psig) } \\
\text { P1 }\end{array}$ & $\begin{array}{l}\text { FLTR } \\
\text { DP } \\
\text { (psid) } \\
\text { dP1 }\end{array}$ & $\begin{array}{c}\text { TOP } \\
\text { DP } \\
\text { (psig) } \\
\text { dP3 }\end{array}$ & $\begin{array}{l}\text { FLT- } \\
\text { RATE } \\
\text { (psig) } \\
\text { P2 }\end{array}$ & $\begin{array}{c}\text { BP } \\
\text { (psig) } \\
\text { P3 }\end{array}$ & $\begin{array}{l}\text { SL } \\
\text { FLOW } \\
\text { (gpm) } \\
\text { Q1 }\end{array}$ & $\begin{array}{l}\text { FLTR } \\
\text { FLOW } \\
\text { (gpm) } \\
\text { Q2 }\end{array}$ & $\begin{array}{c}\text { Temp } \\
\text { corrected } \\
\text { flow } \\
\left(\mathrm{gpm} / \mathrm{ft}^{2}\right)\end{array}$ & $\begin{array}{l}\text { Axial Vel } \\
(\mathrm{ft} / \mathrm{sec})\end{array}$ & $\begin{array}{l}\text { Avg } \\
\text { TMP } \\
\text { (psid) }\end{array}$ \\
\hline 0/24/01 & $14: 30$ & 0 & 27.3 & 23.6 & 27.1 & 24.7 & 22.9 & 51.7 & 61.3 & 10.9 & 41.5 & 9.6 & 0 & 38.1 & 0.075 & 0.0308 & 15.8 & 46.6 \\
\hline D/24/01 & 14:31 & 0 & 27.3 & 23.6 & 27.1 & 24.7 & 22.9 & 51.1 & 60.6 & 10.8 & 41.3 & 9.6 & 0 & 38.1 & 0.075 & 0.0309 & 15.8 & 46.2 \\
\hline D/24/01 & $14: 32$ & 0 & 27.4 & 23.6 & 27.1 & 24.6 & 22.9 & 51.8 & 61.4 & 10.8 & 41.4 & 9.6 & 0 & 38.1 & 0.075 & 0.0309 & 15.8 & 46.6 \\
\hline 10/24/01 & $14: 33$ & 0 & 27.4 & 23.6 & 27.2 & 24.6 & 22.9 & 51.9 & 61.6 & 10.8 & 41.4 & 9.6 & 0 & 38.1 & 0.075 & 0.0308 & 15.8 & 46.7 \\
\hline 10/24/01 & $14: 34$ & 0 & 27.4 & 23.6 & 27.1 & 24.6 & 22.9 & 51.9 & 61.5 & 10.8 & 41.3 & 9.6 & 0 & 38.1 & 0.075 & 0.0308 & 15.8 & 46.6 \\
\hline 10/24/01 & $14: 35$ & 0 & 27.4 & 23.6 & 27.2 & 24.6 & 22.9 & 51.6 & 61.0 & 10.9 & 41.1 & 9.6 & c & 38.1 & 0.075 & 0.0308 & 15.8 & 46.3 \\
\hline D/24/01 & $14: 36$ & 0 & 27.4 & 23.6 & 27.2 & 24.6 & 22.9 & 51.6 & 61.2 & 10.7 & 41.2 & 9.6 & c & 38.1 & 0.075 & 0.0308 & & 46.4 \\
\hline /24/01 & $14: 37$ & 0 & 27.5 & 23.6 & 27.0 & 24.6 & 22.9 & 51.6 & 61.1 & 10.8 & 41.6 & 9.6 & c & 38.2 & 0.075 & 0.0310 & & 46.6 \\
\hline /24/01 & $14: 38$ & 0 & 27.4 & 23.6 & 26.3 & 24.6 & 22.8 & 51.4 & 60.9 & 10.8 & 41.4 & 9.6 & & 38.2 & 0.073 & 307 & & 46.4 \\
\hline & $14: 39$ & 0 & 27.2 & 23.6 & 26.1 & 24.6 & 22.8 & 51.9 & 61.4 & 10.7 & 41.3 & 9. & & 38.2 & 0.073 & 0.0309 & & 46.6 \\
\hline & $: 40$ & 0 & 27.2 & 23.6 & 26.0 & 24.6 & 22.9 & 51.7 & 61.2 & 10.7 & 41.4 & 9 & & 38.3 & 0.073 & & & 6.6 \\
\hline & $14: 41$ & 0 & 27.1 & 23.6 & 26.0 & 24.6 & 22.9 & 51.8 & 1.4 & 10.7 & 41.5 & 9 & & 38.2 & 0.073 & 10 & & 46.6 \\
\hline 1 & : 42 & 0 & 27.0 & 23.6 & 26.0 & 24.5 & 2.9 & 1.9 & 1.5 & 10.7 & 41.4 & 9. & & 38.3 & 0.073 & 10 & & 46.6 \\
\hline 1 & $1: 43$ & 0 & 26.9 & 23.6 & 26.0 & 24.6 & 2.9 & 1.8 & 1.3 & 10.7 & 41.3 & $y$. & & 38.2 & 0.073 & & & 46.5 \\
\hline$/ 2$ & $14: 44$ & 0 & 26.8 & 23.6 & 26.1 & 24.6 & 22.9 & 1.7 & 61.1 & 10.8 & 41.3 & 9. & & 38.3 & 0.072 & & & 46.5 \\
\hline$/ 24$ & $14: 45$ & 0 & 26.7 & 23.6 & 26.1 & 24.6 & 22.9 & 51.6 & 61.0 & 10.6 & 41.4 & 9. & c & 38.3 & 0.073 & 09 & & 46.5 \\
\hline /24/01 & $14: 46$ & 0 & 26.7 & 23.6 & 26.1 & 24.6 & 22.9 & 51.5 & 61.0 & 10.7 & 41.3 & 9. & c & 38.3 & 0.073 & 0. & & 46.4 \\
\hline /24/01 & $14: 47$ & 0 & 26.6 & 23.6 & 26.1 & 24.6 & 22.9 & 51.7 & 61.2 & 10.8 & 41.5 & 9. & 0 & 38.2 & 0.073 & & & 46.6 \\
\hline 0/24/01 & $14: 48$ & 0 & 26.5 & 23.6 & 26.2 & 24.5 & 22.9 & 51.7 & 61.3 & 10.7 & 41.6 & 9.5 & c & 38.2 & 0.073 & & & 46.6 \\
\hline $10 / 24 / 01$ & $14: 49$ & 0 & 26.5 & 23.6 & 26.2 & 24.6 & 22.8 & 48.7 & 60.7 & 10.8 & 39.0 & 12.1 & c & 38.2 & 0.010 & & & 43.8 \\
\hline /24/01 & $14: 50$ & 0 & 26.5 & 23.6 & 26.3 & 24.5 & 22.8 & 50.9 & 61.2 & 10.7 & 40.6 & 10.3 & c & 38.2 & 0.096 & & & 45.7 \\
\hline$/ 24 / 01$ & $14: 51$ & 0 & 26.5 & 23.6 & 26.2 & 24.5 & 22.8 & 51.0 & 61.0 & 10.7 & 41.2 & 10.1 & c & 38.2 & & & & 46.1 \\
\hline )/24/ & & 0 & 26.5 & 23.6 & 26.3 & 24.5 & 22.8 & 51.1 & 61.1 & 10.7 & 40.8 & 10.0 & c & 38.4 & & & & 46.0 \\
\hline J/ & $14: 53$ & 0 & 26.5 & 23.6 & 26.3 & 24.5 & 22.8 & 50.9 & 61.0 & 10.7 & 41.1 & 10.0 & 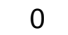 & 38.3 & 0.088 & & & 46.0 \\
\hline $\mathrm{J} / 2$ & : 54 & 0 & 26.5 & 23.6 & 26.3 & 24.5 & 22.8 & 50.9 & 60.8 & 10.7 & 41.2 & 9 & & 38.2 & 86 & 02 & & 46.0 \\
\hline $0 / 2$ & 1:55 & 0 & 26.6 & 23.6 & 26.4 & 24.5 & 22.8 & 51.1 & 0.9 & 10.6 & 41.2 & 9 & & 38.2 & 86 & & & 46.1 \\
\hline $0 / 4$ & 1:56 & 0 & 26.6 & 23.6 & 26.4 & 24.6 & 22.8 & 51.1 & 0.9 & 10.8 & 41.0 & 0 & & 38.2 & 85 & & & 46.1 \\
\hline $0 /$ & $: 5$ & 0 & 26.6 & 23.6 & 26.4 & 24.5 & 22.8 & 50.8 & 0.6 & 10.7 & 41.2 & & & 38.3 & 0.085 & & & 46.0 \\
\hline 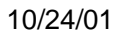 & 1:58 & 0 & 26.7 & 23.6 & 26.4 & 24.5 & 22.8 & 51.1 & 60.9 & 10.7 & 41.2 & 9 & & 38.3 & 0.084 & & & 46.2 \\
\hline 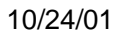 & 1:59 & 0 & 26.7 & 23.6 & 26.5 & 24.5 & 22.8 & 51.6 & 1.4 & 10.7 & 41.3 & 9 & & 38.2 & 0.084 & & & 46.4 \\
\hline 12 & 00 & 0 & 26.7 & 23.6 & 26.5 & 24.6 & 22.8 & 51.0 & 60.6 & 10.7 & 41.1 & 9 & & 38.1 & 33 & & & 46.1 \\
\hline ?ר & 01 & 0 & 26.7 & 23.6 & 26.5 & 24.6 & 22.8 & 1.3 & 61.2 & 10.8 & 40.9 & 9 & ( & 38.2 & 0.083 & & & 46.1 \\
\hline 1 & 5:02 & 0 & 26.8 & 23.6 & 26.5 & 24.6 & 22.8 & 51.7 & 61.6 & 10.8 & 41.2 & 9 & & 38.3 & 0.083 & & & 46.5 \\
\hline 01 & 15:03 & 0 & 26.8 & 23.6 & 26.6 & 24.6 & 22.8 & 51.0 & 60.5 & 10.7 & 41.1 & 9 & & 38.3 & 0.082 & & & 46.1 \\
\hline 0/24/01 & 15:04 & 0 & 26.8 & 23.6 & 26.6 & 24.6 & 22.8 & 51.3 & 60.8 & 10.7 & 41.2 & 9. & c & 38.3 & 0.082 & & & 46.2 \\
\hline 10/24/01 & $15: 05$ & 0 & 26.8 & 23.6 & 26.6 & 24.6 & 22.8 & 51.6 & 61.4 & 10.7 & 41.5 & 9. & c & 38.3 & 0.082 & & & 46.6 \\
\hline 0/24/01 & $15: 06$ & 0 & 26.9 & 23.6 & 26.6 & 24.6 & 22.8 & 51.3 & 60.8 & 10.8 & 41.3 & 9. & 0 & 38.2 & 0.082 & & & 46.3 \\
\hline 0/24/01 & $15: 07$ & 0 & 26.9 & 23.6 & 26.6 & 24.6 & 22.8 & 51.7 & 61.4 & 10.8 & 41.3 & 9. & 0 & 38.3 & 0.082 & & & 46.5 \\
\hline /24/01 & $15: 08$ & 0 & 26.9 & 23.6 & 26.6 & 24.5 & 22.8 & 51.6 & 61.2 & 10.8 & 41.4 & 9 & & 38.3 & 0.081 & & & 46.5 \\
\hline$/ 24 / 0$ & & 0 & 26.9 & 23.6 & 26.6 & 24.6 & 2.8 & & & 10.8 & & & & 3.3 & & & & 46.5 \\
\hline & & 0 & 26.9 & 23.6 & 26.6 & 24.5 & 22.8 & & & 10.7 & & & & 3.3 & & & & 6.4 \\
\hline & & 0 & 26.9 & 23.6 & 26.6 & 24.5 & 22.8 & 31.0 & & 10.7 & & & & 38.3 & & & & 46.4 \\
\hline & 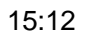 & 0 & 26 & 23.6 & 26.7 & 24.5 & 22.8 & 51 & 61.4 & 10.8 & 41.4 & 9 & & 3.3 & & & & 46.6 \\
\hline & & 0 & 26 & 23.6 & 26.6 & 24.5 & 22.8 & 51 & 61 & 10.8 & 41.5 & 9 & & 38.2 & & & & 46.5 \\
\hline & 4 & 0 & 26.9 & 23.6 & 26.7 & 24.6 & 22.8 & 51.6 & 61.2 & 10.8 & 41.5 & 9 & & 38.2 & 30 & & & 46.6 \\
\hline & 15 & 0 & 26.9 & 23.6 & 26.7 & 24.6 & 22.8 & 51.3 & 60.9 & 10.8 & 41.2 & 9 & & 38.2 & & & & 46.2 \\
\hline 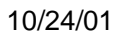 & $15: 16$ & 0 & 27.0 & 23.6 & 26.7 & 24.7 & 22.8 & 51.5 & 61.1 & 10.8 & 41.2 & 9. & & 38.2 & 0.080 & & & 46.4 \\
\hline $0 / 2$ & $15: 17$ & 0 & 27.0 & 23.6 & 26.7 & 24.7 & 22.8 & 51.7 & 61.3 & 10.8 & 41.2 & 9.7 & 0 & 38.2 & 0.080 & & & 46.4 \\
\hline 0/24/01 & $15: 18$ & 0 & 27.0 & 23.6 & 26.7 & 24.7 & 22.8 & 51.5 & 61.0 & 10.7 & 41.4 & 9.7 & 0 & 38.2 & 0.080 & 33 & 9 & 46.5 \\
\hline 0/24/01 & $15: 19$ & 0 & 27.0 & 23.6 & 26.8 & 24.8 & 22.8 & 51.4 & 61.0 & 10.8 & 41.3 & 9.7 & 0 & 38.3 & 0.079 & 0.0328 & 15.9 & 46.3 \\
\hline 0/24/01 & $15: 20$ & 0 & 27.0 & 23.6 & 26.7 & 24.9 & 22.8 & 51.5 & 61.2 & 10.8 & 41.1 & 9. & c & 38.3 & 0.079 & 0.0329 & 9 & 46.3 \\
\hline 0/24/01 & $15: 21$ & 0 & 27.0 & 23.6 & 26.8 & 24.9 & 22.8 & 51.6 & 61.0 & 10.8 & 41.2 & 9. & 0 & 38.3 & 0.079 & 328 & .9 & 46.4 \\
\hline 0/24/01 & $15: 22$ & 0 & 27.0 & 23.6 & 26.8 & 24.9 & 22.8 & 51.5 & 61.0 & 10.8 & 41.2 & 9. & 0 & 38.3 & 0.079 & 328 & 15.9 & 46.3 \\
\hline | 0/24/01 & $15: 23$ & 0 & 27.0 & 23.6 & 26.7 & 24.9 & 22.8 & 51.6 & 61.2 & 10.8 & 41.7 & 9. & 0 & 38.3 & 0.079 & & 9 & 46.6 \\
\hline & & 0 & 27.0 & 23.6 & 26.8 & 24.9 & 22.9 & & & 10.7 & 41.4 & 9. & & 38.2 & 0.078 & & & 46.4 \\
\hline & & 0 & 27.1 & 23.6 & 26.8 & 24.9 & 22.9 & & & 10.7 & 41.4 & 9. & & 38.2 & 0.079 & & & 46.4 \\
\hline & $15: 26$ & 0 & 27.1 & 23.6 & 26.8 & 25.0 & 2.8 & 51.5 & 61.2 & 10.8 & 41.4 & 9. & & 38.2 & 0.078 & 324 & & 46.5 \\
\hline 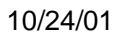 & $5: 27$ & 0 & 27.1 & 23.6 & 26.8 & 25.0 & 22.8 & 51.6 & 61.2 & 10.8 & 41.4 & 9. & & 38.2 & 0.078 & 324 & & 46.5 \\
\hline & $15: 28$ & 0 & 27.1 & 23.6 & 26.8 & 25.0 & 22.9 & 51.5 & 61.1 & 10.7 & 41.2 & 9. & & 38.2 & 0.078 & 0.0323 & 15.8 & 46.3 \\
\hline 0/24/01 & $15: 29$ & 0 & 27.1 & 23.6 & 26.9 & 25.1 & 22.9 & 1.5 & 61.1 & 10.8 & 41.3 & 9.6 & V & 38.1 & 0.078 & 0.0323 & 15.8 & 46.4 \\
\hline
\end{tabular}




\begin{tabular}{|c|c|c|c|c|c|c|c|c|c|c|c|c|c|c|c|c|c|c|}
\hline & & Sol & $\begin{array}{c}\text { FLTRT } \\
\left({ }^{\circ} \mathrm{C}\right) \\
\text { T2 }\end{array}$ & $\begin{array}{c}\text { CL } \\
\text { LOOP } \\
\left({ }^{\circ} \mathrm{C}\right) \\
\text { T3 }\end{array}$ & $\begin{array}{c}\text { SL } \\
\text { LOOP } \\
\left({ }^{\circ} \mathrm{C}\right) \\
\mathrm{T} 1\end{array}$ & $\begin{array}{l}\text { UP } \\
\text { AMB } \\
\left({ }^{\circ} \mathrm{C}\right) \\
\text { T4 }\end{array}$ & $\begin{array}{l}\text { BOT } \\
\text { AMB } \\
\left(^{\circ} \mathrm{C}\right) \\
\text { T5 }\end{array}$ & $\begin{array}{c}\text { BOT } \\
\text { DP } \\
\text { (psid) } \\
\text { dP2 }\end{array}$ & $\begin{array}{l}\text { FLTR } \\
\text { (psig) } \\
\text { P1 }\end{array}$ & $\begin{array}{c}\text { FLTR } \\
\text { DP } \\
\text { (psid) } \\
\text { dP1 }\end{array}$ & $\begin{array}{l}\text { TOP } \\
\text { DP } \\
\text { (psig) } \\
\text { dP3 }\end{array}$ & $\begin{array}{l}\text { FLT- } \\
\text { RATE } \\
\text { (psig) } \\
\text { P2 }\end{array}$ & $\begin{array}{c}\text { BP } \\
\text { (psig) } \\
\text { P3 }\end{array}$ & $\begin{array}{c}\text { SL } \\
\text { FLOW } \\
\text { (gpm) } \\
\text { Q1 }\end{array}$ & $\begin{array}{c}\text { FLTR } \\
\text { FLOW } \\
\text { (gpm) } \\
\text { Q2 }\end{array}$ & $\begin{array}{l}\text { Temp } \\
\text { corrected } \\
\text { flow } \\
\left(\mathrm{gpm} / \mathrm{tt}^{2}\right)\end{array}$ & $\begin{array}{l}\text { Vel } \\
\text { ec) }\end{array}$ & $\begin{array}{l}\text { Avg } \\
\text { TMP } \\
\text { (psid) }\end{array}$ \\
\hline o/24/01 & $15: 30$ & 0 & 27.1 & 23.6 & 26.9 & 25.1 & 22.9 & 51.7 & 61.1 & 10.8 & 41.4 & 9.6 & 0 & 38.2 & 0.078 & 0.0323 & 15.9 & 46.6 \\
\hline & & 0 & 27.2 & & 26.9 & 25.1 & 22.9 & 51.7 & 61.1 & 10.8 & 41.5 & 9.6 & & 38.2 & 0.078 & 0.0323 & 15.8 & $=$ \\
\hline & $15: 32$ & 0 & 27.2 & 3.6 & 27.0 & 25.1 & 22.9 & 51.5 & 61.1 & 10.7 & 41.6 & 9.6 & 0 & 3.3 & 0.078 & & 15.9 & \\
\hline & $15: 33$ & 0 & 27.2 & 6 & 27.0 & & 22.9 & 1.8 & 61.2 & 10.8 & 41.6 & 9 & & 38.2 & 0.078 & & & \\
\hline |24/01 & $15: 34$ & 0 & 27.2 & 23.6 & 27.0 & 25.0 & 22.9 & 51.5 & 61.0 & 10.9 & 41.5 & 9.6 & 0 & 38.1 & 0.078 & 0.0322 & 15.8 & 6. \\
\hline 24/01 & $15: 35$ & 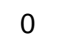 & 27.2 & 3.6 & 27.0 & 25.0 & 23.0 & 2.1 & 61.7 & 10.8 & 41.3 & 9.6 & & 38.2 & 0.078 & 0.0323 & 9 & 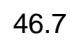 \\
\hline |24/01 & $15: 36$ & 0 & 27.2 & 3.6 & 27.0 & 24.9 & 22.9 & 51.8 & 61.4 & 10.9 & 41.4 & 9.6 & & 38.2 & 0.078 & 0.0322 & 15.9 & 46.6 \\
\hline & $15: 37$ & 0 & 27.3 & 6 & 27.0 & 24.9 & 22.9 & 1.8 & 61.3 & 10.8 & 41.3 & 96 & & 38.2 & 0.078 & & & 65 \\
\hline /24/01 & $15: 38$ & 0 & 27.3 & 23.6 & 27.0 & 25.0 & 22.9 & 52.0 & 61.6 & 10.8 & 41.5 & 9.6 & & 38.1 & 0.078 & 0.0322 & 15.8 & 6.8 \\
\hline 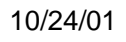 & $15: 39$ & 0 & 27.3 & .7 & 1 & 24.9 & 22.9 & 1.9 & .4 & 10.9 & 41.6 & 96 & & 3.4 & 0.077 & & & 0 \\
\hline /24 & $15: 40$ & 0 & 27.3 & 3.7 & 27.0 & 25.0 & 23.0 & 51.9 & 61.3 & 10.8 & 41.5 & 9 & & 38.1 & 0.077 & & 15.8 & 6.7 \\
\hline & $15: 41$ & 0 & 27.3 & & .1 & & & .8 & .2 & 10.8 & 41.4 & & & 8.2 & 0.077 & & & \\
\hline & $15: 42$ & 0 & 27.3 & 3.6 & 7.1 & 25.0 & 3.0 & 51.8 & 61.3 & 10.8 & 41.6 & 9. & & 8.2 & 0.077 & 317 & & 6.7 \\
\hline & $15: 43$ & 0 & 27.3 & & .1 & & & & 61.4 & 10.9 & 41.8 & & & & & & & \\
\hline & 15:44 & 0 & 27.4 & 3.6 & 27.1 & 25.0 & 23.0 & 51.6 & 61.1 & 10.9 & 41.2 & 9.5 & & 8.1 & 0.077 & 317 & 15.8 & 6.4 \\
\hline & $15: 45$ & 0 & 27.4 & & 27.1 & & & & 61.4 & 10.9 & 41.7 & & & & & & & \\
\hline & $15: 46$ & 0 & 27.4 & 23.7 & 27.2 & 25.0 & 23.0 & 51.9 & 61.4 & 10.9 & 41.4 & 9. & & 8.3 & 0.077 & .0316 & 15.9 & 6.6 \\
\hline & $15: 47$ & 0 & 27.4 & 3.7 & 27.2 & & 23.0 & & 61.3 & 10.8 & 41.7 & & & & 0.077 & & & \\
\hline & $15: 48$ & 0 & 27.4 & 23.7 & 27.2 & 25.0 & 23.0 & 51.9 & 61.3 & 10.9 & 41.4 & 9. & & 8.2 & 0.077 & 16 & & 6.6 \\
\hline & & 0 & 27.4 & 6 & 27.2 & 25.0 & 23.0 & & & 10.9 & 41.4 & & & & 77 & & & \\
\hline$/ 2$ & $15: 50$ & C & 27.4 & 3.6 & 27.3 & 25.0 & 23.0 & 52.0 & 61.4 & 10.9 & 41.3 & 9.5 & & 8.2 & 0.077 & 16 & & 6.6 \\
\hline & & 0 & 27.5 & 7 & 27.2 & 25.1 & & 52.1 & 61.6 & 10.9 & 41.9 & & & 3.2 & 77 & & & \\
\hline & $15: 52$ & 0 & 27.5 & 3.7 & 27.3 & 25.1 & 23.0 & 52.0 & 61.6 & 10.8 & 41.5 & 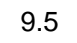 & & 8.2 & 0.077 & 16 & & 6.8 \\
\hline & & 0 & 7.5 & & .3 & 25.1 & 23.0 & & 61.3 & 10.8 & 41.2 & 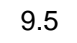 & & 8.1 & 76 & & & \\
\hline 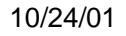 & $15: 54$ & 0 & 27.5 & 3.7 & 7.3 & 25.1 & 23.0 & 51.7 & 61.2 & 10.9 & 41.7 & 9. & & 8.2 & 0.076 & & & 6.7 \\
\hline & & 0 & 7.5 & & .3 & 25.0 & 23.0 & & & 10.9 & 41.4 & & & 3.1 & & & & \\
\hline 1 & $15: 56$ & & 27.5 & 3.7 & 7.3 & 25.0 & 23.0 & 51.9 & 61.3 & 10.9 & 41.3 & 9. & & 8.1 & 0.076 & 11 & & \\
\hline & & 0 & & & & & & & & & & & & 3.1 & & & & \\
\hline & $15: 58$ & & 7.6 & 3.7 & 7.4 & 5.0 & 23. & 52.0 & 61. & 10.9 & 41.6 & 9 & & 3.1 & 0 & & & \\
\hline & & 0 & 7.6 & & .4 & 24.9 & 23. & 51.9 & 61. & 10.9 & 41.7 & 9 & & .1 & & & & \\
\hline & $16: 00$ & & 27.6 & 3.7 & 27.4 & 24.9 & 23.0 & 51.7 & 61. & 10.9 & 41.8 & & & .2 & c & & & 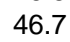 \\
\hline & & 0 & 7.6 & 7 & 27.4 & 24.9 & 23.0 & 51.8 & 61. & 10.9 & 41. & & & .1 & & & & \\
\hline & $16: C$ & 0 & 27.6 & 3.7 & 7.4 & 24.8 & 23.0 & 52.1 & 61.5 & 10.9 & 41.9 & & & .1 & & & & \\
\hline & & & 76 & & .5 & 24.8 & 23.0 & & & & 41. & & & & & & & \\
\hline & $16: 0$ & 0 & 27.7 & 3.7 & 27.4 & 24.9 & 23.0 & 2.0 & 61. & 10.9 & 41.7 & 9 & & 3.1 & 6 & & & 6. \\
\hline & & & 77 & & 7.4 & 24.9 & 22.9 & & & & 41. & & & & & & & \\
\hline & $16: 06$ & 0 & 27.7 & 7 & 7.5 & 24.9 & 23.0 & 2.1 & 1.5 & 10.9 & 41.7 & 9 & & 3.2 & & & & 6. \\
\hline & & & 77 & & & 2 & 23 & & & & 41. & & & & & & & \\
\hline & $16: 08$ & 0 & 7.8 & .7 & .0 & 24.9 & 23.0 & 1.5 & 0.9 & 10.8 & 41.4 & & & 3.1 & & & & \\
\hline & & & 76 & & & 49 & 23.0 & & & 10.8 & 41.8 & & & & & & & \\
\hline & & 0 & & & & 24.9 & & 5 & & 8 & 41 & & & 0 & & & & \\
\hline & & & & & & 24.9 & 0 & & & & 41 & & & 3 & & & & \\
\hline & & & & & & & & & 6 & 8 & 41 & & & 3 & & & & \\
\hline & & & & & & & & & & & & & & & & & & \\
\hline & & 0 & $=$ & & & & & & & & 41 & & & & & & & \\
\hline & & & & & & & & & & & 41 & & & & & & & \\
\hline & & 0 & 2 & & & & 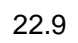 & & & & & & & & & & & \\
\hline & & 0 & 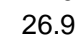 & & & & & & & & 41 & & & & & & & \\
\hline & & & & & & & & & & & & & & & & & & \\
\hline & & 0 & 26 & & & & & & & & & & & & & & & \\
\hline & & & 2 & & & & & & & & & & & & & & & \\
\hline & & 0 & & & & & & & & & & & & & & & & \\
\hline & & & & & & & & & & & & & & & & & & \\
\hline & & 0 & & & & & & & & & & & & & & & & \\
\hline & & & & & & & & & & & & & & & & & & \\
\hline & & c & & & & & & & & & & & & & & & & \\
\hline & & & & & & & & & & & & & & & & & & \\
\hline & & & & & & & & & 6 & & & & & & & & & \\
\hline & & & & & & & & & & & & & & & & & & \\
\hline & $16: 29$ & 0 & 0.7 & .7 & & & & 52.1 & 61.4 & 10.8 & 42.0 & 9.3 & 0 & 8.3 & 073 & 0.0306 & 15.9 & 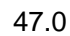 \\
\hline
\end{tabular}




\begin{tabular}{|c|c|c|c|c|c|c|c|c|c|c|c|c|c|c|c|c|c|c|}
\hline DATE & IME & Sol & $\begin{array}{c}\text { FLTRT } \\
\left({ }^{\circ} \mathrm{C}\right) \\
\text { T2 }\end{array}$ & $\begin{array}{c}\mathrm{CL} \\
\text { LOOP } \\
\left({ }^{\circ} \mathrm{C}\right) \\
\text { T3 }\end{array}$ & $\begin{array}{c}\text { SL } \\
\text { LOOP } \\
\left({ }^{\circ} \mathrm{C}\right) \\
\mathrm{T} 1\end{array}$ & $\begin{array}{c}\text { UP } \\
\text { AMB } \\
\left({ }^{\circ} \mathrm{C}\right) \\
\text { T4 }\end{array}$ & $\begin{array}{c}\text { BOT } \\
\text { AMB } \\
\left({ }^{\circ} \mathrm{C}\right) \\
\text { T5 }\end{array}$ & $\begin{array}{c}\text { BOT } \\
\text { DP } \\
\text { (psid) } \\
\text { dP2 }\end{array}$ & $\begin{array}{c}\text { FLTR } \\
\text { (psig) } \\
\text { P1 }\end{array}$ & $\begin{array}{l}\text { FLTR } \\
\text { DP } \\
\text { (psid) } \\
\text { dP1 }\end{array}$ & $\begin{array}{c}\text { TOP } \\
\text { DP } \\
\text { (psig) } \\
\text { dP3 }\end{array}$ & $\begin{array}{l}\text { FLT- } \\
\text { RATE } \\
\text { (psig) } \\
\text { P2 }\end{array}$ & $\begin{array}{c}\text { BP } \\
\text { (psig) } \\
\text { P3 }\end{array}$ & $\begin{array}{l}\text { SL } \\
\text { FLOW } \\
\text { (gpm) } \\
\text { Q1 }\end{array}$ & $\begin{array}{l}\text { FLTR } \\
\text { FLOW } \\
\text { (gpm) } \\
\text { Q2 }\end{array}$ & $\begin{array}{c}\text { Temp } \\
\text { corrected } \\
\text { flow } \\
\left(\mathrm{gpm} / \mathrm{ft}^{2}\right)\end{array}$ & $\begin{array}{c}\text { Axial Vel } \\
(\mathrm{ft} / \mathrm{sec})\end{array}$ & $\begin{array}{l}\text { Avg } \\
\text { TMP } \\
\text { (psid) }\end{array}$ \\
\hline 0/24/01 & $16: 30$ & 0 & 26.7 & 23.7 & 26.5 & 25.0 & 23.0 & 52.0 & 61.1 & 10.8 & 42.0 & 9.3 & 0 & 38.5 & 0.073 & 0.0305 & 16.0 & 47.0 \\
\hline /24/01 & $16: 31$ & 0 & 26.7 & 23.7 & 26.5 & 25.0 & 23.1 & 52.1 & 61.3 & 10.9 & 41.9 & 9.2 & & 38.4 & 0.073 & 0.0306 & 5.9 & 47.0 \\
\hline /24/01 & $16: 32$ & 0 & 26.8 & 23.7 & 26.5 & 25.1 & 23.1 & 52.0 & 61.2 & 10.8 & 41.7 & 9.2 & 0 & 38.2 & 0.073 & 0.0305 & 5.9 & 46.8 \\
\hline /24/01 & $16: 33$ & 0 & 26.8 & 23.7 & 26.5 & 25.0 & 23.1 & 51.7 & 61.0 & 10.8 & 41.6 & 9.2 & 0 & 38.3 & 0.073 & 0.0305 & 5.9 & 46.6 \\
\hline /24/01 & $16: 34$ & 0 & 26.8 & 23.7 & 26.6 & 25.1 & 23.1 & 52.0 & 61.1 & 10.9 & 41.7 & 9.2 & 0 & 38.3 & 0.073 & 0.0305 & 5.9 & 46.9 \\
\hline /24/01 & $16: 35$ & 0 & 26.8 & 23.7 & 26.6 & 25.1 & 23.1 & 52.1 & 61.3 & 10.9 & 41.7 & 9.2 & 0 & 38.3 & 0.073 & 0.0304 & 9 & 46.9 \\
\hline D/24/01 & $16: 36$ & 0 & 26.8 & 23.7 & 26.6 & 25.2 & 23.1 & 52.2 & 61.4 & 10.9 & 41.8 & 9.2 & 0 & 38.3 & 0.073 & 0.0305 & .9 & 47.0 \\
\hline /24/01 & $16: 37$ & 0 & 26.9 & 23.7 & 26.7 & 25.2 & 23.1 & 52.0 & 61.2 & 10.8 & 41.8 & 9.2 & 0 & 38.3 & 0.073 & 0.0304 & 9 & 46.9 \\
\hline 10/24/01 & $16: 38$ & 0 & 26.9 & 23.7 & 26.7 & 25.2 & 23.1 & 52.1 & 61.3 & 10.8 & 41.9 & 9.2 & 0 & 38.3 & 0.073 & 0.0304 & .9 & 47.0 \\
\hline o/24/01 & $16: 39$ & 0 & 26.9 & 23.7 & 26.7 & 25.2 & 23.1 & 52.2 & 61.4 & 10.8 & 41.7 & 9.2 & 0 & 38.3 & 0.073 & 0.0304 & & 46.9 \\
\hline /24/01 & $16: 40$ & 0 & 26.9 & 23.7 & 26.7 & 25.3 & 23.1 & 52.1 & 61.1 & 10.8 & 41.8 & 9.2 & 0 & 38.3 & 0.073 & & & 46.9 \\
\hline$/ 24 / 01$ & $16: 41$ & 0 & 27.0 & 23.7 & 26.7 & 25.3 & 3.2 & 52.0 & 61.2 & 10.8 & 41.7 & 9.2 & 0 & 38.3 & 0.073 & & & 46.9 \\
\hline 01 & $16: 42$ & 0 & 26.9 & 23.7 & 26.7 & 25.3 & 23.2 & 49.6 & 61.1 & 10.8 & 39.3 & 11.5 & & 38.3 & 0.110 & & .9 & 44.5 \\
\hline 1 & $: 43$ & 0 & 27.0 & 23.7 & 26.7 & 25.4 & 23.2 & 50.8 & 61.3 & 10.8 & 40.5 & 10.4 & & 38.4 & 0.094 & & & 45.6 \\
\hline 1 & $: 44$ & 0 & 27.0 & 23.7 & 26.7 & 25.5 & 3.2 & 51.0 & 1.3 & 10.9 & 40.9 & 10.2 & & 38.4 & 0.090 & & & 5.9 \\
\hline 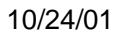 & :45 & 0 & 27.0 & 23.7 & 26.7 & 25.5 & 3.2 & 51.3 & 1.5 & 10.9 & 40.9 & 10.2 & & 8.3 & 0.089 & & & 6.1 \\
\hline$/ 2$ & 46 & 0 & 27.0 & 23.7 & 26.8 & 25.5 & 3.2 & 1.1 & 1.3 & 10.8 & 40.9 & 10.1 & & 8.3 & 0.088 & & & 46.0 \\
\hline$/ 2$ & $: 47$ & 0 & 27.0 & 23.7 & 26.8 & 25.5 & 23.2 & 51.4 & 61.6 & 10.9 & 41.0 & 10.1 & & 38.3 & 0.087 & & & 46.2 \\
\hline $1 / 2$ & 48 & 0 & 27.0 & 23.7 & 26.9 & 25.5 & 23.2 & 51.0 & 60.9 & 10.8 & 41.2 & 10.0 & ( & 38.2 & 0.086 & & & 46.1 \\
\hline $1 / 2$ & $16: 49$ & 0 & 27.0 & 23.7 & 26.8 & 25.5 & 23.2 & 51.1 & 61.0 & 10.9 & 41.2 & 10.0 & c & 38.3 & 0.086 & & & 46.1 \\
\hline$/ 24$ & $16: 50$ & 0 & 27.0 & 23.7 & 26.9 & 25.6 & 23.2 & 51.1 & 61.0 & 10.9 & 41.0 & 10.0 & 0 & 38.3 & 0.085 & & & 46.1 \\
\hline$/ 24$ & $16: 51$ & 0 & 27.1 & 23.7 & 26.9 & 25.5 & 23.3 & 51.5 & 61.4 & 10.9 & 41.1 & 10.0 & 0 & 38.2 & 0.084 & & & 46.3 \\
\hline $2 / 24 / 01$ & $16: 52$ & 0 & 27.1 & 23.7 & 26.8 & 25.4 & 23.2 & 51.5 & 61.4 & 11.0 & 41.0 & 10.0 & 0 & 38.4 & 0.084 & & & 46.3 \\
\hline /24 & $16: 53$ & 0 & 27.1 & 23.7 & 26.7 & 25.4 & 23.2 & 51.9 & 61.8 & 10.9 & 41.0 & 9.9 & 0 & 38.3 & 0.083 & & & 46.4 \\
\hline$/ 24$ & $16: 54$ & 0 & 27.0 & 23.7 & 26.6 & 25.4 & 23.2 & 51.7 & 61.8 & 10.9 & 40.9 & 9.9 & 0 & 38.4 & 0.083 & & & 46.3 \\
\hline //24 & $16: 55$ & 0 & 27.0 & 23.7 & 26.6 & 25.3 & 3.2 & 51.4 & 61.2 & 10.8 & 41.2 & 9.9 & 0 & 38.4 & 0.082 & & & 46.3 \\
\hline /24/ & & 0 & 27.0 & 23.8 & 26.6 & 25.4 & 3.2 & 51.5 & 61.4 & 10.8 & 41.3 & 9.9 & 0 & 38.3 & 32 & & & 46.4 \\
\hline$J / 24$ & $16: 57$ & 0 & 26.9 & 23.8 & 26.6 & 25.4 & 23.2 & 51.7 & 61.5 & 10.9 & 41.1 & 9. & & 38.4 & 0.082 & & $y$ & 46.4 \\
\hline$/ 2$ & :58 & 0 & 26.9 & 23.8 & 26.6 & 25.4 & 23.2 & 51.8 & 1.6 & 11.0 & 41.2 & 9. & & 8.4 & 0.082 & & .9 & 46.5 \\
\hline$/ 2$ & 59 & 0 & 26.9 & 23.7 & 26.6 & 25.5 & 23.2 & 51.7 & 1.5 & 10.9 & 41.3 & 9. & & 8.3 & 82 & & & 46.5 \\
\hline $0 / 2$ & 0 & 0 & 26.9 & 23.8 & 26.6 & 25.4 & 23.2 & 51. & 61.3 & 10 & 41.2 & 9. & & 3.4 & 31 & & & 46.4 \\
\hline $0 / 2$ & 1 & 0 & 26.9 & 23.8 & 26.6 & 25.5 & 23.2 & 51.4 & 1.3 & 10.9 & 41.3 & 9. & & 8.4 & 81 & & & 46.4 \\
\hline 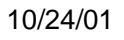 & 2 & 0 & 26.9 & 23.8 & 26.6 & 25.4 & 23.2 & 1.7 & 61.4 & 10.9 & 41.4 & 9. & & 38.3 & 81 & & & 46.6 \\
\hline $1 / 2$ & 3 & 0 & 26.9 & 23.8 & 26.7 & 25.5 & 23.2 & 51.4 & 61.2 & 10.8 & 41.3 & 9. & & 3.3 & 81 & & & 46.3 \\
\hline $1 / 24$ & 7:04 & 0 & 26.9 & 23.8 & 26.7 & 25.5 & 23.2 & 51.8 & 61.4 & 10.8 & 41.2 & 9.8 & 0 & 38.4 & 0.081 & & & 46.5 \\
\hline 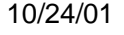 & :05 & 0 & 27.0 & 23.8 & 26.7 & 25.5 & 23.2 & 51.5 & 61.3 & 10.8 & 41.4 & 9.8 & $c$ & 38.4 & & & & 46.5 \\
\hline$/ 2$ & :06 & 0 & 27.0 & 23.8 & 26.7 & 25.5 & 23.3 & 51.9 & 61.7 & 10.9 & 41.3 & 9.8 & 0 & 38.4 & 0.080 & & & 46.6 \\
\hline $1 / 2$ & :07 & 0 & 27.0 & 23.8 & 26.7 & 25.6 & 23.2 & 51.7 & 61.5 & 10.8 & 41.4 & 9.8 & 0 & 38.4 & 0.080 & & & 46.6 \\
\hline $0 / 24$ & 17:08 & 0 & 27.0 & 23.8 & 26.7 & 25.6 & 23.2 & 51.8 & 61.5 & 10.9 & 41.1 & 9.8 & 0 & 38.3 & 0.080 & & & 46.4 \\
\hline 0/24/ & 17:09 & 0 & 27.0 & 23.8 & 26.7 & 25.5 & 23.3 & 51.7 & 61.5 & 10.9 & 41.5 & 9.8 & 0 & 38.3 & 0.080 & & & 46.6 \\
\hline $0 / 24 /$ & $17: 10$ & 0 & 27.0 & 23.8 & 26.8 & 25.4 & 23.2 & 51.5 & 61.2 & 10.8 & 41.1 & 9.8 & 0 & 38.4 & 0.080 & & & 46.3 \\
\hline o/24/01 & $17: 11$ & 0 & 27.1 & 23.8 & 26.7 & 25.4 & 23.2 & 51.4 & 61.1 & 10.9 & 41.4 & 9.8 & 0 & 38.3 & 0.080 & & & 46.4 \\
\hline$/ 2$ & 12 & 0 & 27.1 & 23.8 & 26.8 & 25.3 & 3.2 & 51 & 6 & 10.9 & & 9 & & 8.2 & & & & 46.3 \\
\hline 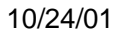 & 13 & 0 & 27.1 & 23.8 & 26.8 & 25.3 & 23.2 & Ve & .8 & 10. & 41.2 & 9 & & 3.3 & & & & 46.6 \\
\hline & 14 & 0 & 27.1 & 23.8 & 26.8 & 25.3 & 23.2 & 51.7 & 61.4 & 10.9 & 41.2 & 9 & & 38.3 & 0.079 & & & 46.5 \\
\hline & & 0 & 27.1 & 23.8 & 26.9 & 25.4 & 3.3 & 51 & 6 & 10.9 & 41.4 & 9. & & 3.3 & 0.079 & & & 46.6 \\
\hline & & 0 & 27.1 & 23.8 & 26.9 & 25.4 & 3.2 & 51.7 & 61 & 10.8 & 41.2 & 9. & & 38.2 & 0.079 & & & 46.4 \\
\hline & :17 & 0 & 27.1 & 23.8 & 26.9 & 25.4 & 3.3 & 51.7 & 61.5 & 10.9 & 41.3 & 9. & & 38.3 & 0.079 & & & 46.5 \\
\hline $0 / 2$ & :18 & 0 & 27.2 & 23.8 & 26.9 & 25.3 & 3.3 & 51.7 & 61.4 & 10.8 & 41.1 & 9.7 & & 38.3 & 0.079 & & & 46.4 \\
\hline 0/24/ & $17: 19$ & 0 & 27.2 & 23.8 & 26.9 & 25.3 & 3.3 & 51.8 & 61.5 & 11.0 & 41.5 & 9.7 & & 38.3 & 0.079 & 27 & .9 & 46.7 \\
\hline $0 / 24 / C$ & $17: 20$ & 0 & 27.2 & 23.8 & 26.9 & 25.3 & 23.3 & 52.1 & 61.8 & 11.0 & 41.5 & 9.7 & 0 & 38.3 & 0.079 & & .9 & 46.8 \\
\hline 0/24/01 & $17: 21$ & 0 & 27.2 & 23.8 & 27.0 & 25.4 & 23.2 & 51.6 & 61.4 & 10.9 & 41.4 & 9.7 & 0 & 38.3 & 0.079 & 27 & 9 & 46.5 \\
\hline 0/24/01 & $17: 22$ & 0 & 27.2 & 23.8 & 27.0 & 25.3 & 23.2 & 51.9 & 61.7 & 10.9 & 41.3 & 9.7 & 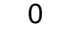 & 38.3 & 0.079 & 0.0326 & 15.9 & 46.6 \\
\hline 0/24/01 & $17: 23$ & 0 & 27.2 & 23.8 & 27.0 & 25.3 & 23.3 & 52.0 & 61.7 & 10.9 & 41.7 & 9.7 & 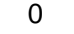 & 38.3 & 0.079 & 0.0327 & 15.9 & 46.9 \\
\hline 0/24/01 & $17: 24$ & 0 & 27.2 & 23.8 & 27.0 & 25.3 & 23.3 & 51.9 & 61.6 & 10.9 & 41.6 & 9.7 & 6 & 38.4 & 0.079 & 0.0326 & .9 & 46.7 \\
\hline 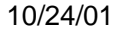 & $17: 25$ & 0 & 27.2 & 23.8 & 27.0 & 25.3 & 23.3 & 51.8 & 61.3 & 11.0 & 41.7 & 9.7 & 6 & 38.3 & 0.078 & 0.0322 & 5.9 & 46.7 \\
\hline /24/01 & $17: 26$ & 0 & 27.2 & 23.8 & 27.0 & 25.3 & 23.2 & 51.7 & 61.4 & 10.9 & 41.2 & 9.7 & $v$ & 38.2 & 0.078 & 0.0322 & 15.9 & 46.5 \\
\hline & $17: 27$ & 0 & 27.2 & 23.8 & 27.0 & 20.5 & 23.2 & 02.0 & 61.6 & 10.9 & 41.5 & 9.7 & 0 & 38.4 & 0.078 & 0.0322 & 15.9 & 46.7 \\
\hline & $17: 28$ & 0 & 27.3 & 23.8 & 27.1 & 25.4 & 23.2 & ד & 61.1 & 10.9 & 41.2 & 9.7 & 0 & 38.3 & 0.078 & & 15.9 & 46.3 \\
\hline 0/24/01 & $17: 29$ & 0 & 27.3 & 23.8 & 27.0 & 25.4 & 23.2 & 51.7 & 61.3 & 11.0 & 41.4 & 9.7 & 0 & 38.3 & 0.078 & 0.0322 & 15.9 & 46.6 \\
\hline
\end{tabular}




\begin{tabular}{|c|c|c|c|c|c|c|c|c|c|c|c|c|c|c|c|c|c|c|}
\hline & & Sol & $\begin{array}{l}\text { FLTRT } \\
\left({ }^{\circ} \mathrm{C}\right) \\
\text { T2 }\end{array}$ & $\begin{array}{l}\mathrm{CL} \\
\text { LOOP } \\
\left({ }^{\circ} \mathrm{C}\right) \\
\text { T3 }\end{array}$ & $\begin{array}{l}\text { SL } \\
\text { LOOP } \\
\left({ }^{\circ} \mathrm{C}\right) \\
\mathrm{T} 1\end{array}$ & $\begin{array}{l}\text { UP } \\
\text { AMB } \\
\left({ }^{\circ} \mathrm{C}\right) \\
\text { T4 }\end{array}$ & $\begin{array}{c}\text { Xflow2 } 2 \\
\text { BOT } \\
\text { AMB } \\
\left(^{\circ} \mathrm{C}\right) \\
\text { T5 }\end{array}$ & $\begin{array}{l}\text { BOT } \\
\text { DP } \\
\text { (psid) } \\
\text { dP2 }\end{array}$ & $\begin{array}{c}\text { FLTR } \\
\text { (psig) } \\
\text { P1 }\end{array}$ & $\begin{array}{l}\text { FLTR } \\
\text { DP } \\
\text { (psid) } \\
\text { dP1 }\end{array}$ & $\begin{array}{l}\text { TOP } \\
\text { DP } \\
\text { (psig) } \\
\text { dP3 }\end{array}$ & $\begin{array}{l}\text { FLT- } \\
\text { RATE } \\
\text { (psig) } \\
\text { P2 }\end{array}$ & $\begin{array}{c}\text { BP } \\
\text { (psig) } \\
\text { P3 }\end{array}$ & $\begin{array}{c}\text { SL } \\
\text { FLOW } \\
\text { (gpm) } \\
\text { Q1 }\end{array}$ & $\begin{array}{l}\text { FLTR } \\
\text { FLOW } \\
\text { (gpm) } \\
\text { Q2 }\end{array}$ & $\begin{array}{l}\text { Temp } \\
\text { corrected } \\
\text { flow } \\
\left(\mathrm{gpm} / \mathrm{ft}^{2}\right)\end{array}$ & & \\
\hline |24/01 & $17: 30$ & 0 & 27.3 & 23.9 & 27.1 & 25.4 & 23.2 & 51.9 & 1.6 & 11.0 & 41.4 & 97 & 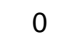 & 38.2 & 0.078 & & & \\
\hline D/24/01 & $17: 31$ & 0 & 27.3 & 23.8 & 27.1 & 25.4 & 23.3 & 2.1 & 61.9 & 10.9 & 41.7 & & & 8.3 & 0.078 & & & \\
\hline & $17: 32$ & 0 & 27.3 & 23.8 & 7.1 & 25.4 & 23.3 & 1.5 & 1.2 & 0.9 & 41.3 & & & 3.2 & 0.078 & & & \\
\hline )/24/01 & $17: 33$ & 0 & 27.4 & 23.8 & 27.1 & 25.4 & 23.3 & 1.7 & 1.2 & 10.9 & 41.8 & & & 8.2 & 0.078 & & 5.9 & \\
\hline & $17: 34$ & 0 & 27.4 & 23.8 & 27.0 & 25.4 & 23.3 & 1.9 & 1.4 & 0.9 & 41.9 & & & & & & & \\
\hline 0/24/01 & $17: 35$ & 0 & 27.4 & 23.8 & 27.1 & 25.4 & 23.3 & 2.1 & 1.8 & 10.9 & 41.6 & 9. & & 8.3 & 0.078 & 21 & 5.9 & \\
\hline & $17: 36$ & & 7.4 & 3.8 & .1 & 25.4 & 23.3 & 1.7 & 1.5 & 1.0 & 41.2 & & & 3.3 & & & & \\
\hline & $17: 37$ & 0 & 7.4 & 3.9 & & 25.4 & 23.3 & .0 & 1.6 & 10.9 & 41.5 & & & 3.3 & & & 5.9 & \\
\hline & $17:$ & ( & 7.4 & & & & & & & & & & & & & & & \\
\hline & $17 \cdot 20$ & & 7.4 & .8 & & 254 & 23.3 & 1.8 & 1.4 & & & & & 4 & & & & \\
\hline & & & 7.4 & & & & & & & & & & & & & & & \\
\hline & & & 7.4 & 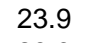 & & 25.4 & 23.3 & & 1.5 & & & & & & & & & 3.6 \\
\hline & & 0 & .4 & & & & 23.3 & & & & & & & & & & & \\
\hline & 17 & c & 7.3 & 3.8 & 5.7 & 25.3 & 23.3 & & 61.5 & & 4 & & & 3.3 & & & & 6.7 \\
\hline & & 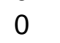 & 7.2 & & & 25.2 & & & & & & & & & & & & \\
\hline & 17 & & 27.2 & 3.9 & .5 & 25.3 & 23.3 & & 61.4 & b & 41 & & & & & & & 6.7 \\
\hline & & $c$ & 7.2 & & & 25.3 & & & & & & & & & & & & \\
\hline & & & 7.1 & 3.9 & .4 & 25.3 & 23.3 & & 1.7 & & & & & & & & & 6.8 \\
\hline & & c & 7.1 & & & 25.3 & 23.3 & & & & & & & & & & & \\
\hline & & & 7.0 & & & 25.3 & 23.3 & & 1.7 & & & & & & & & & 6.8 \\
\hline & & C & & & & 25.2 & & & & & & & & & & & & \\
\hline & & & 9 & & & 25.2 & 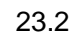 & & 1.2 & & & & & & & & & \\
\hline & & 0 & .9 & & & 25.2 & & & & & & & & & & & & \\
\hline & & & 3.8 & & & 25.1 & 23.2 & & .5 & & & & & & & & & \\
\hline & & 0 & 6.8 & & & & & & & & & & & & & & & \\
\hline & 17 & & 6.8 & 3.9 & 6 & 25.2 & 23.2 & & - & & 4 & & & & & & & \\
\hline & & & 6.9 & & & & & & & & & & & & & & & \\
\hline & 17 & & 3.9 & .9 & 6 & 25.2 & 23.2 & & 1.5 & & & & & & & & & \\
\hline & & & & & & & & & & & & & & & & & & \\
\hline & & & 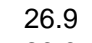 & & & 2 & 2 & & & & & & & & & & & \\
\hline & & & & & & & & & & & & & & & & & & \\
\hline & & & 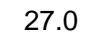 & & & 25.3 & & & & & & & & & & & & \\
\hline & & & & & & & & & & & & & & & & & & \\
\hline & & & & & & 25.4 & & & & & & & & & & & & \\
\hline & & & & & & & & & & & & & & & & & & \\
\hline & & & 7.1 & & & 25.3 & & & & & & & & & & & & \\
\hline & & & & & & & & & & & & & & & & & & \\
\hline & & & 7.1 & & & & & & & & & & & & & & & \\
\hline & & & & & & & & & & & & & & & & & & \\
\hline & & & & & & & & & & & & & & & & & & \\
\hline & & & & & & & & & & & & & & & & & & \\
\hline & & & & & & & & & & & & & & & & & & \\
\hline & & & & & & & & & & & & & & & & & & \\
\hline & & & & & & & & & & & & & & & & & & \\
\hline & & & & & & & & & & & & & & & & & & \\
\hline & & & & & & & & & & & & & & & & & & \\
\hline & & & & & & & & & & & & & & & & & & \\
\hline & & & & & & & & & & & & & & & & & & \\
\hline & & & & & & & & & & & & & & & & & & \\
\hline & & & & & & & & & & & & & & & & & & \\
\hline & & & & & & & & & & & & & & & & & & \\
\hline & & & & & & & & & & & & & & & & & & \\
\hline & & & & & & & & & & & & & & & & & & \\
\hline & & & & & & & & & & & & & & & & & & \\
\hline & & & & & & & & & & & & & & & & & & \\
\hline & & & 2 & & & & & & & & & & & & & & & \\
\hline & & & & & & & & & & & & & & & & & & \\
\hline & & & 7.3 & & & & & & & & & & & & & & & \\
\hline & & & & & & & & & & & & & & & & & & \\
\hline & & & & & & & & & & & & & & & & & & \\
\hline
\end{tabular}




\begin{tabular}{|c|c|c|c|c|c|c|c|c|c|c|c|c|c|c|c|c|c|c|}
\hline & & Sc & $\begin{array}{l}\text { FLTRT } \\
\left({ }^{\circ} \mathrm{C}\right) \\
\mathrm{T} 2\end{array}$ & $\begin{array}{c}\text { CL } \\
\text { LOOP } \\
\left({ }^{\circ} \mathrm{C}\right) \\
\text { T3 }\end{array}$ & $\begin{array}{l}\text { SL } \\
\text { LOOP } \\
\left({ }^{\circ} \mathrm{C}\right) \\
\mathrm{T} 1\end{array}$ & $\begin{array}{l}\text { UP } \\
\text { AMB } \\
\left({ }^{\circ} \mathrm{C}\right) \\
\text { T4 }\end{array}$ & $\begin{array}{c}\text { Xflow2 } \\
\text { BOT } \\
\text { AMB } \\
\left({ }^{\circ} \mathrm{C}\right) \\
\text { T5 }\end{array}$ & $\begin{array}{c}102401 \\
\text { BOT } \\
\text { DP } \\
\text { (psid) } \\
\text { dP2 }\end{array}$ & $\begin{array}{l}\text { FLTR } \\
\text { (psig) } \\
\text { P1 }\end{array}$ & $\begin{array}{c}\text { FLTR } \\
\text { DP } \\
\text { (psid) } \\
\text { dP1 }\end{array}$ & $\begin{array}{c}\text { TOP } \\
\text { DP } \\
\text { (psig) } \\
\text { dP3 }\end{array}$ & $\begin{array}{l}\text { FLT- } \\
\text { RATE } \\
\text { (psig) } \\
\text { P2 }\end{array}$ & $\begin{array}{l}\text { BP } \\
\text { (psig) } \\
\text { P3 }\end{array}$ & $\begin{array}{c}\text { SL } \\
\text { FLOW } \\
\text { (gpm) } \\
\text { Q1 }\end{array}$ & $\begin{array}{c}\text { FLTR } \\
\text { FLOW } \\
\text { (gpm) } \\
\text { Q2 }\end{array}$ & $\begin{array}{c}\text { Temp } \\
\text { corrected } \\
\text { flow } \\
\left(\mathrm{gpm} / \mathrm{ft}^{2}\right)\end{array}$ & $\begin{array}{l}\text { Axial Vel } \\
\text { (ft/sec) }\end{array}$ & $\begin{array}{l}\text { Avg } \\
\text { TMP } \\
\text { (psid) }\end{array}$ \\
\hline 0/24/01 & $18: 30$ & 0 & 27.4 & 23.9 & 27.1 & 25.0 & 23.0 & 52.5 & 62.2 & 11.0 & 41.5 & 9.5 & 0 & 38.4 & 0.074 & 0.0304 & 15.9 & 47.0 \\
\hline $10 / 24 / 01$ & $18: 31$ & 0 & 27.4 & 23.9 & 27.2 & 25.0 & 23.0 & 52.5 & 62.0 & 11.0 & 41.7 & 9.5 & 0 & 38.4 & 0.074 & 0.0304 & 15.9 & 47.1 \\
\hline D/24/01 & $18: 32$ & 0 & 27.4 & 23.9 & 27.1 & 24.9 & 23.0 & 52.2 & 61.7 & 11.0 & 41.6 & 9 & & 38.3 & 0.074 & & 59 & 46.9 \\
\hline $10 / 24 / 01$ & $18: 33$ & 0 & 27.4 & 23.9 & 27.2 & 24.9 & 23.0 & 52.0 & 61.5 & 11.0 & 41.6 & 9.5 & 0 & 38.3 & 0.074 & 0.0304 & 15.9 & 46.8 \\
\hline $10 / 24 / 01$ & $18: 34$ & 0 & 27.4 & 23.9 & 27.1 & 24.9 & 23.0 & 52.1 & 61.6 & 11.1 & 42.0 & 9 & & 38.3 & 0.074 & & 5.9 & 47.1 \\
\hline $10 / 24 / 01$ & $18: 35$ & 0 & 27.4 & 23.9 & 27.2 & 24.9 & 23.0 & 51.9 & 61.3 & 10.9 & 42.0 & 9.5 & 0 & 38.3 & 0.074 & 0.0304 & 15.9 & 46.9 \\
\hline $10 / 24 / 01$ & $18: 36$ & 0 & 27.5 & 23.9 & 27.1 & 24.8 & 23.0 & 52.1 & 61.5 & 11.0 & 41.7 & 9. & & 38.4 & 0.074 & & 59 & 6.9 \\
\hline 0/24/01 & $18: 37$ & 0 & 27.4 & 23.9 & 27.2 & 24.8 & 22.9 & 51.8 & 61.4 & 10.9 & 41.8 & 9. & 0 & 38.3 & 0.074 & 0.0304 & 15.9 & 46.8 \\
\hline $10 / 24 / 01$ & $18: 38$ & 0 & 27.5 & 23.9 & 27.2 & 24.7 & 22.9 & 52.0 & 61.5 & 11.0 & 41.8 & 9.5 & & 38.3 & 0.074 & & 5.9 & .9 \\
\hline $10 / 24 / 01$ & $18: 39$ & 0 & 27.5 & 23.9 & 27.2 & 24.8 & 22.9 & 51.9 & 61.3 & 10.9 & 41.7 & 9. & $c$ & 38.3 & 0.073 & & 15.9 & 46.8 \\
\hline 0/24/01 & $18: 40$ & 0 & 27.5 & 23.9 & 27.3 & 24.7 & 22.9 & 52.5 & 62.0 & 11.0 & 41.7 & 9. & & 38.3 & 0.073 & & 5.9 & 47.1 \\
\hline 0/24/01 & $18: 41$ & 0 & 27.5 & 23.9 & 27.3 & 24.7 & 22.9 & 52.5 & 62.0 & 10.9 & 41.7 & 9. & c & 38.3 & 0.073 & & 5.9 & 47.1 \\
\hline D/24 & $18: 42$ & 0 & 27.5 & 23.9 & 27.3 & 24.7 & 22.9 & 51.8 & 61.3 & 11.0 & 41.7 & 9. & & 38.3 & 0.073 & & 5.9 & 46.8 \\
\hline b1 & $18: 43$ & 0 & 27.5 & 23.9 & 27.3 & 24.7 & 22.9 & 52.1 & 61.7 & 11.0 & 41.7 & 9. & & 38.2 & 0.073 & & 5.9 & 46.9 \\
\hline $10 / 24$ & $18: 44$ & 0 & 27.5 & 23.9 & 27.3 & 24.6 & 22.8 & 51.9 & 61.3 & 10.9 & 41.8 & 9. & & 38.2 & 0.074 & 0. & 5.9 & 46.9 \\
\hline & $18: 45$ & 0 & 27.6 & 23.9 & 27.4 & 24.6 & 22.8 & 52.3 & 61.7 & 11.0 & 41.9 & 9. & & 38.3 & 0.073 & & & 47.1 \\
\hline $10 / 2$ & $18: 46$ & 0 & 27.6 & 23.9 & 27.3 & 24.6 & 22.8 & 51.9 & 61.3 & 11.1 & 41.9 & 9. & & 38.3 & 0.073 & & 5.9 & 46.9 \\
\hline & $18: 47$ & 0 & 27.6 & 23.9 & 27.4 & 24.7 & 22.8 & 52.3 & 61.8 & 11.0 & 41.8 & 9. & & 38.3 & 0.073 & & & 47.1 \\
\hline $0 / 2$ & $18: 48$ & 0 & 27.6 & 23.9 & 27.3 & 24.7 & 22.8 & 52.4 & 61.9 & 11.0 & 41.9 & 9. & & 38.3 & 0.073 & & 5.9 & 47.1 \\
\hline & $18: 49$ & 0 & 27.6 & 23.9 & 27.4 & 24.7 & 22.8 & 51.9 & 61.4 & 11.0 & 41.6 & 9. & & 38.3 & 0.073 & & & 46.8 \\
\hline $0 / 2$ & $18: 50$ & 0 & 27.6 & 23.9 & 27.4 & 24.6 & 22.8 & 52.2 & 61.7 & 11.0 & 42.0 & 9. & & .3 & 0.073 & & 5.9 & 47.1 \\
\hline & $18: 51$ & 0 & 27.6 & 23.9 & 27.4 & 24.6 & 22.8 & 52.3 & 61.8 & 11.0 & 41.8 & 9. & & .2 & 0.073 & & & 47.1 \\
\hline o/2 & $18: 52$ & 0 & 27.6 & 23.9 & 27.4 & 24.6 & 22.8 & 52.2 & 61.7 & 11.0 & 41.7 & 9. & & 8.3 & 0.073 & 0. & 15.9 & 46.9 \\
\hline 1 & $18: 53$ & 0 & 27.6 & 23.9 & 27.4 & 24.7 & 22.8 & 52.1 & 61.5 & 11.1 & 42.2 & 9. & & 3.3 & 0.073 & & 5.9 & 47.1 \\
\hline $\mathrm{b} / 2$ & $18: 54$ & 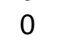 & 27.7 & 23.9 & 27.4 & 24.6 & 22.8 & 52.4 & 61.8 & 11.1 & 41.6 & 9. & & 3.2 & 0.073 & & 5.9 & 47.0 \\
\hline $0 / 2$ & $18: 55$ & 0 & 27.7 & 23.8 & 27.4 & 24.6 & 22.8 & 52.2 & 61.6 & 11.0 & 41.7 & 9. & & 3.2 & 0.073 & & & 46.9 \\
\hline , & $18: 56$ & 0 & 27.7 & 23.9 & 27.5 & 24.6 & 22.8 & 52.6 & 62.2 & 11.1 & 41.8 & 9. & & 2 & 0.073 & & 5.8 & 47.2 \\
\hline 1 & $18: 57$ & 0 & 27.7 & 23.9 & 27.4 & 24.5 & 22.7 & 52.4 & 61.8 & 11.0 & 41.8 & 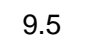 & & 8.3 & 0.073 & & 5.9 & 47.1 \\
\hline 1 & $18: 58$ & 0 & 27.7 & 23.8 & 27.5 & 24.5 & 22.7 & 52.5 & 62.0 & 11.1 & 41.7 & 9. & & 3 & 0.073 & & 5.9 & 47.1 \\
\hline $10 / 2$ & $18: 59$ & 0 & 27.7 & 23.8 & 27.5 & 24.5 & 22.7 & 52.2 & 61.6 & 11.0 & 41.7 & 9. & & 8.3 & 0.073 & & 5.9 & 47.0 \\
\hline 1 & 19:00 & 0 & 27.7 & 23.8 & 27.5 & 24.4 & 22.7 & 52.0 & 61.4 & 11.0 & 41.8 & 9. & & 2 & & & & 46.9 \\
\hline 10/2 & 19:01 & 0 & 27.8 & 23.8 & 27.5 & 24.4 & 22.7 & 52.3 & 61.7 & 11.0 & 41.7 & 9. & & 8.2 & 0.073 & & 5.9 & 47.0 \\
\hline & 19:02 & 0 & 27.8 & 23.8 & 27.6 & 24.4 & 22.7 & 52.5 & 62.0 & 11.0 & 41. & 9. & & & & & & 47.1 \\
\hline 1 & $19: 03$ & 0 & 27.8 & 23.8 & 27.5 & 24.4 & 22.7 & 52.2 & 61.6 & 11.0 & 42.1 & 9. & & 8.1 & 0.073 & & 5.8 & 47.1 \\
\hline & 19:04 & 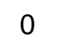 & 27.7 & 23.8 & 27.5 & 24.3 & 22.6 & 52.3 & 61.7 & 11.0 & 41. & 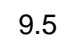 & & & & & & 47.1 \\
\hline $10 / 2$ & 19:05 & 0 & 27.8 & 23.8 & 27.6 & 24.4 & 22.6 & 52.2 & 61.6 & 11.0 & 41.6 & 9. & & 8.2 & 0.073 & & 5.8 & 46.9 \\
\hline & & 0 & 27.8 & 23 & & 24.4 & 22.6 & 52.5 & 62.0 & 11.0 & & 9. & & & & & & 47.2 \\
\hline 1 & 19:07 & 0 & 27.8 & 23.8 & 7.6 & 24.4 & 22.6 & 52.3 & 61.8 & 11.1 & 41.9 & 9. & & 8.2 & 0.073 & 96 & 5.8 & 47.1 \\
\hline & 19 & 0 & 27.8 & 23.8 & 7.4 & & 22.6 & 52.4 & 61.9 & 11.0 & & 0 & & & & & & 47.3 \\
\hline $10 / 2$ & 19:09 & 0 & 27.8 & 23.8 & 6.8 & 24.3 & 22.6 & 52.4 & 61.8 & 11.0 & 41.6 & 9. & & 8.3 & 0.072 & 99 & 5.9 & 47.0 \\
\hline & & 0 & 27.7 & 23 & & & 22.6 & 52.1 & 61.5 & 11.0 & & 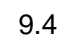 & & & & & & \\
\hline $10 / 2$ & $19: 11$ & 0 & 27.6 & 23.8 & 5.3 & 24.3 & 22.6 & 52.2 & 61.5 & 10.9 & 42.1 & 9. & & 3 & 0.071 & & 15.9 & 47.2 \\
\hline & & 0 & 27.5 & 23 & & & 22.6 & 52 & 62.0 & 10.9 & & & & & & & & \\
\hline & 19 & 0 & 27.4 & 23.8 & 3 & 2 & 22.5 & 52.5 & 61.9 & 10.9 & 41 & 9 & & 1 & & & 15.9 & 47.2 \\
\hline & & 0 & 3 & 23.8 & & & 22.5 & 51 & 61.1 & 10.9 & & 9 & & & & & & \\
\hline $10 / 2$ & 19 & 0 & 27.2 & 23.8 & 2 & 2 & 22.5 & 52.2 & 61.6 & 10.9 & 41 & 9 & & 3 & 0. & & 15.9 & 46.9 \\
\hline & 19 & 0 & 27.1 & 23.8 & & & 22.5 & 52 & 62.1 & 11.0 & & 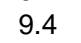 & & & & & & \\
\hline & 19 & 0 & 27.0 & 230 & & 2 & 22.5 & 52.3 & 61.7 & 10.9 & 4 & 9 & & 1 & & & 15.9 & 47.0 \\
\hline & 19 & 0 & 9 & 23.8 & 5.4 & & 22.5 & 52 & 61.5 & 10.9 & & U & & & & & & \\
\hline & 19 & 0 & 26.8 & 23.8 & & 1 & 22.5 & 52.1 & 61.5 & 10.9 & 4 & 9 & & 3 & & & 15.9 & .9 \\
\hline & 19 & 0 & 26.8 & 23.8 & 0.4 & & 22.4 & 52 & 61.7 & 11.0 & 42 & 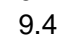 & & & & & & \\
\hline & 19 & 0 & 26.7 & 23 & & & 22.4 & 52 & 61.4 & 11.0 & 41.8 & a & & & & & 9 & .0 \\
\hline & 19 & 0 & 26.7 & 23.7 & + & .0 & 22.4 & 52.1 & 61.4 & 10.9 & 41.9 & 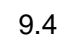 & & & & & & .0 \\
\hline & 19 & 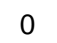 & 26 & & & & & & 61.8 & 10.9 & & a & & & & & & \\
\hline & 19 & 0 & 26.7 & 23 & & & 22.4 & 52 & 61.5 & 10.9 & & & & & & & & .1 \\
\hline & 19 & 0 & & & & & & & & 11.0 & & & & & & & & \\
\hline & & 0 & 26.8 & 23. & 26. & 0 & 22.4 & 52.3 & 61.6 & 10.9 & 41.9 & 9. & & & & & .9 & .1 \\
\hline & 19 & 0 & 26 & 23 & & & 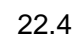 & & 61 & 10 & & 9. & & & & & & \\
\hline & & 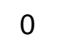 & 26.8 & 23 & 20. & & 22.3 & 52 & 61.4 & & 42.0 & & & & & & & 47.0 \\
\hline $10 / 24$ & 29 & 0 & 26.8 & 23.7 & 266 & & 22.3 & 50 & 61.5 & 10.9 & 41.9 & 9.3 & 0 & 38.3 & 0.070 & 0.0292 & 15.9 & 47.0 \\
\hline
\end{tabular}




\begin{tabular}{|c|c|c|c|c|c|c|c|c|c|c|c|c|c|c|c|c|c|c|}
\hline DATE & & Sc & $\begin{array}{l}\text { FLTRT } \\
\left({ }^{\circ} \mathrm{C}\right) \\
\mathrm{T} 2\end{array}$ & $\begin{array}{c}\text { CL } \\
\text { LOOP } \\
\left({ }^{\circ} \mathrm{C}\right) \\
\text { T3 }\end{array}$ & $\begin{array}{l}\text { SL } \\
\text { LOOP } \\
\left({ }^{\circ} \mathrm{C}\right) \\
\mathrm{T} 1\end{array}$ & $\begin{array}{l}\text { UP } \\
\text { AMB } \\
\left({ }^{\circ} \mathrm{C}\right) \\
\text { T4 }\end{array}$ & $\begin{array}{c}\text { Xflow2 } \\
\text { BOT } \\
\text { AMB } \\
\left({ }^{\circ} \mathrm{C}\right) \\
\text { T5 }\end{array}$ & $\begin{array}{c}102401 \\
\text { BOT } \\
\text { DP } \\
\text { (psid) } \\
\text { dP2 }\end{array}$ & $\begin{array}{l}\text { FLTR } \\
\text { (psig) } \\
\text { P1 }\end{array}$ & $\begin{array}{c}\text { FLTR } \\
\text { DP } \\
\text { (psid) } \\
\text { dP1 }\end{array}$ & $\begin{array}{c}\text { TOP } \\
\text { DP } \\
\text { (psig) } \\
\text { dP3 }\end{array}$ & $\begin{array}{l}\text { FLT- } \\
\text { RATE } \\
\text { (psig) } \\
\text { P2 }\end{array}$ & $\begin{array}{c}\text { BP } \\
\text { (psig) } \\
\text { P3 }\end{array}$ & $\begin{array}{c}\text { SL } \\
\text { FLOW } \\
\text { (gpm) } \\
\text { Q1 }\end{array}$ & $\begin{array}{c}\text { FLTR } \\
\text { FLOW } \\
\text { (gpm) } \\
\text { Q2 }\end{array}$ & $\begin{array}{c}\text { Temp } \\
\text { corrected } \\
\text { flow } \\
\left(\mathrm{gpm} / \mathrm{ft}^{2}\right)\end{array}$ & $\begin{array}{l}\text { Axial Vel } \\
\text { (ft/sec) }\end{array}$ & $\begin{array}{l}\text { Avg } \\
\text { TMP } \\
\text { (psid) }\end{array}$ \\
\hline 0/24/01 & $19: 30$ & 0 & 26.9 & 23.7 & 26.6 & 24.1 & 22.4 & 52.2 & 61.6 & 10.9 & 42.0 & 9.3 & . & 38.3 & 0.070 & 0.0293 & 15.9 & 47.1 \\
\hline 0/24/01 & 19:31 & 0 & 26.9 & 23.7 & 26.6 & 24.0 & 22.4 & 52.3 & 61.7 & 10.9 & 41.9 & 9.4 & 0 & 38.3 & 0.070 & 0.0292 & 15.9 & 47.1 \\
\hline /24/01 & 19:32 & 0 & 26.9 & 23.7 & 26.6 & 24.0 & 22.4 & 52.0 & 61.3 & 11.0 & 41.6 & 94 & & 38.4 & 0.070 & & 59 & 46.8 \\
\hline $10 / 24 / 01$ & $19: 33$ & 0 & 26.9 & 23.7 & 26.7 & 24.0 & 22.3 & 52.3 & 61.5 & 10.9 & 42.0 & 9.4 & 0 & 38.4 & 0.070 & 0.0292 & 15.9 & 47.1 \\
\hline 0/24/01 & $19: 34$ & 0 & 26.9 & 23.7 & 26.7 & 24.0 & 22.3 & 52.2 & 61.5 & 10.9 & 41.7 & 94 & & 38.3 & 0.070 & 0.0291 & 5.9 & 46.9 \\
\hline $10 / 24 / 01$ & $19: 35$ & 0 & 26.9 & 23.6 & 26.7 & 24.0 & 22.3 & 52.0 & 61.3 & 11.0 & 42.0 & 9.4 & 0 & 38.4 & 0.070 & 0.0292 & 15.9 & 47.0 \\
\hline 0/24/01 & $19: 36$ & 0 & 26.9 & 23.7 & 26.7 & 24.0 & 22.3 & 52.5 & 61.8 & 10.9 & 42.1 & 9. & & 38.3 & 0.071 & & 59 & 47.3 \\
\hline 0/24/01 & 19:37 & 0 & 26.9 & 23.7 & 26.7 & 24.0 & 22.3 & 52.6 & 61.9 & 11.0 & 41.9 & 9.4 & 0 & 38.4 & 0.071 & 0.0296 & 15.9 & 47.2 \\
\hline b1 & $19: 38$ & 0 & 26.9 & 23.6 & 26.7 & 23.9 & 22.3 & 52.1 & 61.4 & 10.9 & 42.0 & 9.4 & & 38.3 & 0.071 & 0.0295 & 5.9 & 47.1 \\
\hline $10 / 24 / 01$ & $19: 39$ & 0 & 26.9 & 23.6 & 26.7 & 23.9 & 22.3 & 52.5 & 61.8 & 11.0 & 42.2 & 9.4 & $c$ & 38.5 & 0.070 & 0.0291 & 6.0 & 47.3 \\
\hline 1 & $19: 40$ & 0 & 27.0 & 23.6 & 26.7 & 23.9 & 22.3 & 52.7 & 62.0 & 11.1 & 42.0 & 9. & & 8.4 & 0.070 & 0.0292 & 5.9 & 47.4 \\
\hline 01 & $19: 41$ & 0 & 27.0 & 23.6 & 26.8 & 23.9 & 22.3 & 52.4 & 61.7 & 10.9 & 41.9 & 9. & c & 38.4 & 0.070 & 0.0291 & 5.9 & 47.1 \\
\hline$/ 24$ & $19: 42$ & 0 & 27.0 & 23.6 & 26.8 & 23.9 & 22.2 & 52.3 & 61.6 & 11.0 & 41.9 & 9.4 & & 38.4 & 0.071 & 0.0295 & 5.9 & 47.1 \\
\hline 1 & $19: 43$ & 0 & 27.1 & 23.6 & 26.8 & 23.9 & 22.3 & 52.4 & 61.7 & 11.0 & 42.1 & 9. & & 38.3 & 0.070 & & 5.9 & 47.3 \\
\hline $10 / 2$ & $19: 44$ & 0 & 27.1 & 23.6 & 26.8 & 23.9 & 22.2 & 52.4 & 61.8 & 10.9 & 41.9 & 9. & & 38.4 & 0.070 & 0.0291 & 5.9 & 47.2 \\
\hline & $19: 45$ & 0 & 27.1 & 23.6 & 26.8 & 23.8 & 22.2 & 52.5 & 61.8 & 10.9 & 41.9 & $a$ & & 38.4 & 0.070 & & & 47.2 \\
\hline $10 / 2$ & $19: 46$ & 0 & 27.1 & 23.6 & 26.8 & 23.8 & 22.2 & 52.3 & 61.8 & 11.0 & 42.0 & 9. & & 3.4 & 0.070 & 0.0 & 6.0 & 47.1 \\
\hline & $19: 47$ & 0 & 27.1 & 23.6 & 26.8 & 23.8 & 22.2 & 52.6 & 62.0 & 10.9 & 42.0 & - & & 3 & 0.071 & & & 47.3 \\
\hline $0 / 2$ & $19: 48$ & 0 & 27.1 & 23.6 & 26.8 & 23.8 & 22.1 & 52.4 & 61.7 & 11.0 & 41.8 & 9. & & 38.3 & 0.070 & 90 & 5.9 & 47.1 \\
\hline & $19: 49$ & 0 & 27.1 & 23.6 & 26.8 & 23.8 & 22.1 & 52.6 & 62.0 & 11.1 & 42.0 & 9. & & .4 & 0.070 & & & 47.3 \\
\hline $0 / 2$ & $19: 50$ & 0 & 27.1 & 23.6 & 26.9 & 23.8 & 22.2 & 52.3 & 61.6 & 11.0 & 42.3 & 9.3 & & .3 & 0.070 & 90 & 5.9 & 47.3 \\
\hline & 19:51 & 0 & 27.1 & 23.6 & 26.9 & 23.8 & 22.2 & 52.5 & 61.8 & 11.0 & 41.8 & 9. & & .4 & 0.070 & & & 47.2 \\
\hline$/ 2$ & 19:52 & 0 & 27.2 & 23.6 & 26.9 & 23.8 & 22.2 & 52.2 & 61.5 & 10.9 & 41.9 & 9. & & 3 & 0.070 & 90 & 15.9 & 47.0 \\
\hline 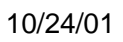 & $19: 53$ & 0 & 27.2 & 23.5 & 26.9 & 23.8 & 22.2 & 52.5 & 61.8 & 11.1 & 41.8 & 9. & & .4 & 0.070 & & & 47.2 \\
\hline o/2 & $19: 54$ & 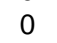 & 27.2 & 23.5 & 27.0 & 23.8 & 22.3 & 52.3 & 61.6 & 11.0 & 42.2 & 9. & & .4 & 0.070 & 89 & 5.9 & 47.3 \\
\hline $0 / 2$ & 19:55 & 0 & 27.2 & 23.5 & 27.0 & 23.8 & 22.3 & 52.5 & 61.8 & 10.9 & 41.9 & 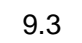 & & 3.4 & 0.070 & & & 47.2 \\
\hline , & 19:56 & 0 & 27.2 & 23.5 & 27.0 & 23.8 & 22.4 & 52.4 & 61.7 & 11.0 & 42.0 & 9. & & 6 & 0.070 & & 6.0 & 47.2 \\
\hline 1 & 19:57 & 0 & 27.2 & 23.5 & 27.0 & 23.9 & 22.5 & 52.1 & 61.4 & 11.0 & 42.4 & 0 & & 8.3 & 0.070 & & 5.9 & 47.2 \\
\hline 1 & $19: 58$ & 0 & 27.2 & 23.5 & 27.1 & 24.0 & 22.5 & 52.4 & 61.8 & 10.9 & 42.1 & 9 & & 2 & 0.070 & & 5.9 & 47.3 \\
\hline $10 / 2$ & 19:59 & 0 & 27.2 & 23.5 & 27.0 & 24.0 & 22.6 & 52.5 & 61.9 & 11.0 & 41.9 & 9. & & 3.3 & 0.070 & 89 & 5.9 & 47.2 \\
\hline 1 & $20: 00$ & 0 & 27.2 & 23.5 & 27.1 & 24.1 & 22.7 & 52.5 & 61.8 & 10.9 & 42.0 & 9. & & & 0.070 & & 5.9 & 47.2 \\
\hline 10/2 & $20: 01$ & 0 & 27.2 & 23.5 & 27.0 & 24.2 & 22.7 & 52.5 & 61.9 & 11.0 & 42.1 & 9. & & 8.3 & 0.070 & 89 & 5.9 & 47.3 \\
\hline & 20:02 & 0 & 27.3 & 23.5 & 7.0 & 24.3 & 22.8 & 52.2 & 61.5 & 11.0 & 41.9 & 9. & & & & & & 470 \\
\hline 1 & $20: 03$ & 0 & 27.3 & 23.6 & 27.1 & 24.3 & 22.9 & 52.5 & 61.8 & 11.0 & 42.0 & 9. & & 8.3 & 0.070 & 88 & 5.9 & 47.2 \\
\hline & $20: 04$ & 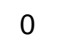 & 27.3 & 23.5 & 7.1 & 24.4 & 22.9 & 52.5 & 61.8 & 11.1 & 41.9 & 9. & & & & & & 47.2 \\
\hline $10 / 2$ & $20: 05$ & 0 & 27.3 & 23.5 & 7.2 & 24.4 & 22.9 & 53.0 & 62.4 & 11.1 & 42.1 & 9. & & 8.4 & 0.070 & 88 & 5.9 & 47.6 \\
\hline & 20 & 0 & 27.3 & & 7.1 & 24.4 & 22.8 & 52.6 & 62.0 & 11.0 & 42.3 & & & & & & & \\
\hline 1 & $20: 07$ & 0 & 27.3 & 23.5 & 7.1 & 24.3 & 22.8 & 52.0 & 61.4 & 11.0 & 42.1 & 9. & & 2 & 0.070 & 88 & 5.9 & 47.1 \\
\hline & $20: 08$ & 0 & 27.4 & & 7.1 & & 22.7 & 52.7 & 62.0 & 11.0 & 42.0 & 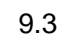 & & & & & & \\
\hline $10 / 2$ & $20: 09$ & 0 & 27.3 & 23.5 & 7.1 & 24.2 & 22.7 & 52.4 & 61.7 & 11.0 & 42.3 & 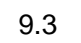 & & 8.4 & 0.070 & 88 & 6.0 & 47.4 \\
\hline & 20 & 0 & 27.4 & 23 & & & 22.7 & 52.3 & 61.5 & 11.0 & & 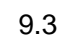 & & & & & & \\
\hline $10 / 2$ & $20: 11$ & 0 & 27.3 & 23.5 & 7.2 & 24.1 & 22.7 & 52.8 & 62.1 & 11.0 & 42.1 & 9 & & 1 & 0.070 & 88 & 5.9 & 47.4 \\
\hline & 20 & 0 & 27.4 & 23 & 2 & & 22.6 & 52.6 & 61.9 & 11.0 & 42.1 & & & & & & & \\
\hline & 20 & 0 & 27.4 & 23.6 & .2 & 1 & 22.6 & 52.4 & 61.6 & 11.0 & 41.9 & & & 3 & 0 & & 15.9 & 47.2 \\
\hline & 20 & 0 & 4 & 23 & .2 & & 22.6 & 52 & 61.4 & 10.9 & & & & & & & & \\
\hline 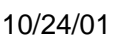 & 20 & 0 & 27.4 & 23.5 & .2 & .0 & 22.6 & 52.4 & 61.7 & 11.0 & 41.9 & 9 & & ? & 0.070 & 88 & 15.9 & 47.1 \\
\hline & 20 & 0 & 27.5 & 23 & .2 & & 22.5 & 52 & & 11.0 & & & & & & & & \\
\hline & 20 & 0 & 27.5 & 23 & & & 22.5 & 52 & 61.7 & 11.0 & 42.1 & a & & & & & 15.9 & 47.2 \\
\hline & 20 & 0 & 27.5 & 23 & 7.3 & & 22.5 & 52 & 62.0 & 11.0 & & & & & & & & \\
\hline & 20 & 0 & 27.5 & 23.5 & & & 22.5 & 52.7 & 61.9 & 11.2 & 42.3 & 0 & & & 0 & 87 & 15.9 & $\tau$ \\
\hline & 20 & 0 & 27.5 & 23.5 & .3 & & 22.4 & 52.4 & 61.7 & 11.1 & 42.0 & & & & & & & \\
\hline & 20 & 0 & 27.5 & & & & 22.4 & 52 & 61.9 & 1 & 41.9 & 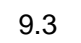 & & & & & & 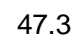 \\
\hline & 20 & 0 & 27.5 & 23.5 & .3 & & 22.3 & 52.5 & 61.9 & 11.0 & 41.9 & 9 & & & & & & 7.2 \\
\hline & 20 & 0 & 27 & & & & 22.3 & & $6 ?$ & 1 & & 0 & & & & & & \\
\hline & 20 & 0 & 27.6 & 23 & 27.3 & & 22.3 & 52 & 62.1 & 11 & 42.1 & & & & & & & \\
\hline & & 0 & 27 & & & & & & 62.0 & & & & & & & & & \\
\hline & 20 & 0 & 27.5 & 23.5 & 27.3 & & 22.2 & 52.8 & 62.1 & 11.0 & 41.9 & 9. & & & & & 5.9 & .4 \\
\hline & 20 & 0 & 27.6 & & & & 2 & 52.6 & 61 & 11 & & 9 & & & & & .9 & \\
\hline & & 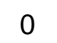 & 27.6 & 23 & 27.4 & & 22.2 & 52 & 62.0 & & 41.9 & & & & & & & .3 \\
\hline & & $c$ & 27.6 & 23. & & & 22.2 & 50 & 61.6 & 11.0 & 41.9 & 9.3 & 0 & 38.3 & 0.070 & 0.0286 & 15.9 & 471 \\
\hline
\end{tabular}




\begin{tabular}{|c|c|c|c|c|c|c|c|c|c|c|c|c|c|c|c|c|c|c|}
\hline & & Sc & $\begin{array}{l}\text { FLTRT } \\
\left({ }^{\circ} \mathrm{C}\right) \\
\mathrm{T} 2\end{array}$ & $\begin{array}{c}\text { CL } \\
\text { LOOP } \\
\left({ }^{\circ} \mathrm{C}\right) \\
\text { T3 }\end{array}$ & $\begin{array}{l}\text { SL } \\
\text { LOOP } \\
\left({ }^{\circ} \mathrm{C}\right) \\
\mathrm{T} 1\end{array}$ & $\begin{array}{l}\text { UP } \\
\text { AMB } \\
\left({ }^{\circ} \mathrm{C}\right) \\
\text { T4 }\end{array}$ & $\begin{array}{c}\text { Xflow2 } \\
\text { BOT } \\
\text { AMB } \\
\left({ }^{\circ} \mathrm{C}\right) \\
\text { T5 }\end{array}$ & $\begin{array}{c}102401 \\
\text { BOT } \\
\text { DP } \\
\text { (psid) } \\
\text { dP2 }\end{array}$ & $\begin{array}{l}\text { FLTR } \\
\text { (psig) } \\
\text { P1 }\end{array}$ & $\begin{array}{c}\text { FLTR } \\
\text { DP } \\
\text { (psid) } \\
\text { dP1 }\end{array}$ & $\begin{array}{c}\text { TOP } \\
\text { DP } \\
\text { (psig) } \\
\text { dP3 }\end{array}$ & $\begin{array}{l}\text { FLT- } \\
\text { RATE } \\
\text { (psig) } \\
\text { P2 }\end{array}$ & $\begin{array}{l}\text { BP } \\
\text { (psig) } \\
\text { P3 }\end{array}$ & $\begin{array}{c}\text { SL } \\
\text { FLOW } \\
(\text { gpm) } \\
\text { Q1 }\end{array}$ & $\begin{array}{c}\text { FLTR } \\
\text { FLOW } \\
\text { (gpm) } \\
\text { Q2 }\end{array}$ & $\begin{array}{c}\text { Temp } \\
\text { corrected } \\
\text { flow } \\
\left(\mathrm{gpm} / \mathrm{ft}^{2}\right)\end{array}$ & $\begin{array}{l}\text { Axial Vel } \\
\text { (ft/sec) }\end{array}$ & $\begin{array}{l}\text { Avg } \\
\text { TMP } \\
\text { (psid) }\end{array}$ \\
\hline 0/24/01 & $20: 30$ & 0 & 27.6 & 23.5 & 27.4 & 23.7 & 22.1 & 52.5 & 61.8 & 11.1 & 42.1 & 9.3 & , & 38.2 & 0.070 & 0.0286 & 15.9 & 47.3 \\
\hline 0/24/01 & $20: 31$ & 0 & 27.6 & 23.5 & 27.4 & 23.7 & 22.1 & 52.8 & 62.2 & 11.0 & 42.1 & 9.3 & 0 & 38.3 & 0.070 & 0.0286 & 15.9 & 47.4 \\
\hline D/24/01 & $20: 32$ & 0 & 27.7 & 23.5 & 27.4 & 23.6 & 22.2 & 52.8 & 62.2 & 11.1 & 42.0 & 93 & & 38.3 & 0.070 & & 59 & 47.4 \\
\hline $10 / 24 / 01$ & $20: 33$ & 0 & 27.7 & 23.5 & 27.4 & 23.7 & 22.2 & 52.5 & 61.7 & 11.1 & 42.0 & 9.3 & 0 & 38.2 & 0.070 & 0.0286 & 15.9 & 47.3 \\
\hline $10 / 24 / 01$ & $20: 34$ & 0 & 27.7 & 23.4 & 27.5 & 23.7 & 22.3 & 53.0 & 62.4 & 11.1 & 42.0 & 93 & & 38.3 & 0.070 & 0.0285 & 59 & 47.5 \\
\hline $10 / 24 / 01$ & $20: 35$ & 0 & 27.7 & 23.4 & 27.5 & 23.8 & 22.3 & 52.9 & 62.3 & 11.1 & 41.9 & 9.3 & 0 & 38.3 & 0.070 & 0.0285 & 15.9 & 47.4 \\
\hline $10 / 24 / 01$ & $20: 36$ & 0 & 27.7 & 23.4 & 27.5 & 23.8 & 22.4 & 52.3 & 61.7 & 11.1 & 41.8 & 9.3 & & 38.2 & 0.070 & & 59 & 47.1 \\
\hline 0/24/01 & $20: 37$ & 0 & 27.7 & 23.4 & 27.5 & 23.8 & 22.4 & 52.9 & 62.2 & 11.1 & 42.2 & 9.3 & 0 & 38.2 & 0.070 & 0.0285 & 15.9 & 47.5 \\
\hline b1 & $20: 38$ & 0 & 27.7 & 23.4 & 27.6 & 23.9 & 22.5 & 52.6 & 61.8 & 11.1 & 41.7 & 9.3 & & 38.2 & 0.070 & 0.0285 & 5.9 & 47.1 \\
\hline $10 / 24 / 01$ & $20: 39$ & 0 & 27.7 & 23.4 & 27.6 & 23.9 & 22.5 & 52.5 & 61.7 & 11.1 & 42.1 & 9.3 & $c$ & 38.2 & 0.070 & 0.0284 & 5.9 & 47.3 \\
\hline 1 & $20: 40$ & 0 & 27.7 & 23.4 & 27.6 & 24.0 & 22.6 & 52.8 & 62.1 & 11.1 & 41.9 & 9. & & 38.3 & 0.071 & & 5.9 & 47.3 \\
\hline 1 & $20: 41$ & 0 & 27.8 & 23.4 & 27.6 & 24.0 & 22.7 & 52.5 & 61.8 & 11.0 & 42.2 & 9. & $c$ & 38.2 & 0.070 & & 5.9 & 47.3 \\
\hline $0 / 2$ & $20: 42$ & 0 & 27.8 & 23.4 & 27.6 & 24.0 & 22.8 & 52.5 & 61.8 & 11.0 & 42.1 & 9.3 & & 38.2 & 0.070 & 0.0284 & 5.9 & 47.3 \\
\hline 1 & $20: 43$ & 0 & 27.8 & 23.4 & 27.7 & 24.1 & 22.8 & 52.3 & 61.7 & 11.1 & 42.0 & 9. & & 38.2 & 0.070 & & 5.8 & 47.1 \\
\hline $10 / 2$ & $20: 44$ & 0 & 27.8 & 23.4 & 27.7 & 24.2 & 22.9 & 52.7 & 62.0 & 11.1 & 42.2 & 9. & & 38.2 & 0.070 & 0.0284 & 5.9 & 47.5 \\
\hline & $20: 45$ & 0 & 27.8 & 23.4 & 27.6 & 24.2 & 22.9 & 52.6 & 61.8 & 11.0 & 42.0 & 9. & & 38.2 & 0.070 & & & 47.3 \\
\hline $10 / 2$ & $20: 46$ & 0 & 27.9 & 23.4 & 27.7 & 24.3 & 23.0 & 52.7 & 62.0 & 11.2 & 42.1 & 9. & & 38.2 & 0.070 & 84 & 5.9 & 47.4 \\
\hline & $20: 47$ & 0 & 27.9 & 23.5 & 27.7 & 24.3 & 23.0 & 52.4 & 61.7 & 11.0 & 42.5 & 9. & & .1 & 0.070 & & & 47.5 \\
\hline 10/2 & $20: 48$ & 0 & 27.9 & 23.5 & 27.7 & 24.3 & 23.1 & 52.6 & 61.8 & 11.2 & 42.2 & 9. & & 38.2 & 0.070 & 83 & 5.9 & 47.4 \\
\hline & $20: 49$ & 0 & 27.9 & 23.4 & 27.7 & 24.3 & 23.0 & 52.3 & 61.6 & 11.1 & 42.2 & 9. & & 3.2 & 0.070 & & & 47.3 \\
\hline $0 / 2$ & $20: 50$ & 0 & 27.9 & 23.4 & 27.8 & 24.4 & 23.0 & 52.7 & 62.1 & 11.0 & 42.0 & 9. & & .2 & 0.070 & 83 & 5.9 & 47.4 \\
\hline & $20: 51$ & 0 & 27.9 & 23.4 & 27.8 & 24.4 & 23.0 & 52.5 & 61.9 & 11.1 & 42.1 & 9. & & .2 & 0.070 & & & 47.3 \\
\hline o/2 & $20: 52$ & 0 & 27.9 & 23.4 & 27.8 & 24.3 & 22.9 & 52.5 & 61.9 & 11.1 & 41.9 & 9. & & .2 & 0.070 & 83 & 15.8 & 47.2 \\
\hline & $20: 53$ & 0 & 28.0 & 23.5 & 27.8 & 24.3 & 22.9 & 52.7 & 61.9 & 11.2 & 42.2 & 9. & & 3.2 & 0.070 & & & 47.4 \\
\hline o/2 & $20: 54$ & - & 28.0 & 23.4 & 27.8 & 24.2 & 22.8 & 52.6 & 61.9 & 11.1 & 42.0 & 9. & & 3.2 & 0.070 & 83 & 5.8 & 47.3 \\
\hline 1 & $20: 55$ & 0 & 28.0 & 23.5 & 27.8 & 24.2 & 22.8 & 52.6 & 61.9 & 11.1 & 42.1 & 9. & & .2 & 0.070 & & & 7.4 \\
\hline 1 & $20: 56$ & 0 & 28.0 & 23.5 & 27.8 & 24.1 & 22.8 & 52.7 & 62.1 & 11.2 & 42.2 & 9. & & 2 & 0.070 & & 5.9 & 47.5 \\
\hline 1 & $20: 57$ & 0 & 28.0 & 23.5 & 27.9 & 24.1 & 22.7 & 52.5 & 61.9 & 11.1 & 42.0 & 9. & & 8.2 & 0.070 & & & 47.3 \\
\hline 1 & $20: 58$ & 0 & 28.1 & 23.5 & 27.9 & 24.0 & 22.6 & 52.6 & 62.0 & 11.1 & 42.2 & 9. & & 2 & 0.070 & & 5.8 & 4 \\
\hline $10 / 2$ & $20: 59$ & 0 & 28.1 & 23.5 & 27.9 & 23.9 & 22.6 & 52.8 & 62.1 & 11.1 & 42.3 & 9. & & 8.2 & 0.070 & 82 & 5.9 & 47.6 \\
\hline 1 & $21: 00$ & 0 & 28.1 & 23.5 & 27.9 & 24.0 & 22.6 & 52.6 & 61.8 & 11.1 & 42.1 & 0 & & 2 & 0.070 & & & 47.3 \\
\hline $10 / 2$ & $21: 01$ & 0 & 28.1 & 23.5 & 27.9 & 23.9 & 22.6 & 52.6 & 61.9 & 11.2 & 41.8 & 9. & & 3.1 & 0.070 & 82 & 5.8 & 47.2 \\
\hline & $21: 02$ & 0 & 8.0 & 23.5 & 0 & 23.9 & 22.6 & 51.0 & 62.0 & 11.1 & 40.3 & 1 & & & & & & 45.6 \\
\hline 1 & $21: 03$ & 0 & 28.2 & 23.5 & 7.5 & 23.8 & 22.6 & 51.8 & 62.0 & 11.0 & 41.3 & 10 & & 8.1 & 0.088 & 58 & 5.8 & 46.5 \\
\hline & $21: 04$ & 0 & 8.1 & 23.5 & 6.9 & 23.8 & 22.5 & 52.0 & 62.0 & 11.0 & 41.4 & 10 & & & & & & 46.7 \\
\hline $10 / 2$ & $21: 05$ & 0 & 27.9 & 23.5 & 6.6 & 23.8 & 22.5 & 52.2 & 62.1 & 11.1 & 41.5 & 9. & & 8.3 & 0.084 & 51 & 5.9 & 46.9 \\
\hline & $21: 06$ & 0 & 27.8 & & & & 22.5 & 51.9 & 61.8 & 11.0 & 41.6 & & & & & & & \\
\hline 1 & $21: 07$ & 0 & 27.8 & 23.5 & 6.3 & 23.8 & 22.4 & 52.3 & 62.2 & 11.0 & 41.6 & 9 & & 8.4 & 0.081 & 41 & 5.9 & 46.9 \\
\hline & $21: 08$ & 0 & 27.6 & 23.5 & 5.4 & & 22.4 & 51.9 & 61.8 & 11.0 & 41.5 & 9 & & & & & & \\
\hline 1 & $21: 09$ & 0 & 27.5 & 23.5 & 5.4 & 23.7 & 22.4 & 52.2 & 61.9 & 11.0 & 41.8 & 9. & & 8.3 & 0.081 & 41 & 5.9 & 47.0 \\
\hline & $21: 10$ & 0 & 27.4 & 23 & & & 22.3 & 510 & 61.7 & 11.0 & 41.7 & a & & & & & & \\
\hline $10 /$ & $21: 11$ & 0 & 27.2 & 23.5 & 5.4 & 23.6 & 22.3 & 52.2 & 62.0 & 11.1 & 41.7 & 9 & & 5 & 0.080 & 36 & 6.0 & 46.9 \\
\hline & $21: 12$ & 0 & 27.1 & 23 & & & 22.3 & 52.4 & 62.1 & 11.1 & & 9 & & & & & & \\
\hline & $21: 13$ & 0 & 27.1 & 23.4 & & 3.6 & 22.3 & 51.8 & 61.5 & 11.0 & 41.4 & 9 & & 1 & & & 15.9 & 6 \\
\hline & 21 & 0 & 27.0 & 23.4 & & & 22.2 & 51 & 61.6 & 11.0 & 114 & 9 & & & & & & \\
\hline 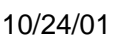 & $21: 15$ & 0 & 26.9 & 23.4 & .4 & .5 & 22.2 & 52.2 & 62.0 & 11.1 & 41.8 & 9 & & 1 & 0.079 & 32 & 15.9 & 47.0 \\
\hline & 21: & 0 & 26.8 & 23.4 & & & 22.2 & 51 & 61.5 & 11.0 & & 9 & & & & & & \\
\hline & 21 & 0 & 26.8 & 23.4 & & & 22.3 & 52 & 61.9 & 11.0 & 41.8 & 9 & & 1 & & & 15.9 & .1 \\
\hline & 21 & 0 & 26.8 & 23.4 & & & 22.3 & 52 & 62.0 & 11.0 & & & & & & & & \\
\hline & $21: 19$ & 0 & 26.8 & 23.4 & & & 22.4 & 52.2 & 61.9 & 11.1 & 41.8 & 9 & & 1 & 0. & & 15.9 & .0 \\
\hline & 21 & 0 & 26.8 & 23.4 & & & 22.4 & 52.2 & 61.8 & 11.1 & 41.7 & 9 & & & & & & \\
\hline & $21: 21$ & 0 & 26.8 & & & & 22.4 & 52.1 & 61.8 & 11.0 & 41.1 & 9 & & & & & & \\
\hline & $21: 22$ & 0 & 26.8 & 23.4 & & & 22.5 & 52.0 & 61.7 & 11.0 & 41.6 & 9 & & & & & & \\
\hline & $21: 23$ & 0 & 26 & & & & 22.5 & & 61.9 & & 41.5 & & & & & & & \\
\hline & $21: 24$ & 0 & 26.9 & 23 & & & 22.6 & 52.1 & 61.8 & 11.0 & 41 & & & & & & & \\
\hline & 21 & 0 & 26 & & & & & & & & & & & & & & & \\
\hline & $21: 26$ & 0 & 26.9 & 23.4 & ? & & 22.8 & 52.1 & 61.7 & 11.0 & 41.8 & 9. & & & & & 6.0 & \\
\hline & 21 & 0 & 26.9 & & & & 2 & ${ }_{3}$ & 61 & 11 & & 9. & & & & & & \\
\hline & 21 & - & 27.0 & 23 & 20 & & 22.8 & 52 & 61.7 & 11 & 41.0 & 9. & & & & & 16.0 & .0 \\
\hline $10 / 2$ & $21: 29$ & 0 & 27.0 & 23.4 & 26.7 & & 22.9 & 52.0 & 61.6 & 11.0 & 42.1 & 9.7 & 0 & 38.4 & 0.077 & 0.0321 & 15.9 & 47.0 \\
\hline
\end{tabular}




\begin{tabular}{|c|c|c|c|c|c|c|c|c|c|c|c|c|c|c|c|c|c|c|}
\hline & & Sc & $\begin{array}{l}\text { FLTRT } \\
\left({ }^{\circ} \mathrm{C}\right) \\
\mathrm{T} 2\end{array}$ & $\begin{array}{c}\text { CL } \\
\text { LOOP } \\
\left({ }^{\circ} \mathrm{C}\right) \\
\text { T3 }\end{array}$ & $\begin{array}{l}\text { SL } \\
\text { LOOP } \\
\left({ }^{\circ} \mathrm{C}\right) \\
\mathrm{T} 1\end{array}$ & $\begin{array}{l}\text { UP } \\
\text { AMB } \\
\left({ }^{\circ} \mathrm{C}\right) \\
\text { T4 }\end{array}$ & $\begin{array}{c}\text { Xflow2 } \\
\text { BOT } \\
\text { AMB } \\
\left({ }^{\circ} \mathrm{C}\right) \\
\text { T5 }\end{array}$ & $\begin{array}{c}102401 \\
\text { BOT } \\
\text { DP } \\
\text { (psid) } \\
\text { dP2 }\end{array}$ & $\begin{array}{l}\text { FLTR } \\
\text { (psig) } \\
\text { P1 }\end{array}$ & $\begin{array}{c}\text { FLTR } \\
\text { DP } \\
\text { (psid) } \\
\text { dP1 }\end{array}$ & $\begin{array}{l}\text { TOP } \\
\text { DP } \\
\text { (psig) } \\
\text { dP3 }\end{array}$ & $\begin{array}{l}\text { FLT- } \\
\text { RATE } \\
\text { (psig) } \\
\text { P2 }\end{array}$ & $\begin{array}{c}\text { BP } \\
\text { (psig) } \\
\text { P3 }\end{array}$ & $\begin{array}{c}\text { SL } \\
\text { FLOW } \\
\text { (gpm) } \\
\text { Q1 }\end{array}$ & $\begin{array}{c}\text { FLTR } \\
\text { FLOW } \\
\text { (gpm) } \\
\text { Q2 }\end{array}$ & $\begin{array}{c}\text { Temp } \\
\text { corrected } \\
\text { flow } \\
\left(\mathrm{gpm} / \mathrm{ft}^{2}\right)\end{array}$ & $\begin{array}{l}\text { Axial Vel } \\
\text { (ft/sec) }\end{array}$ & $\begin{array}{l}\text { Avg } \\
\text { TMP } \\
\text { (psid) }\end{array}$ \\
\hline 0/24/01 & $21: 30$ & 0 & 27.0 & 23.4 & 26.8 & 24.1 & 22.9 & 52.3 & 61.9 & 11.0 & 41.6 & 9.7 & , & 38.4 & 0.077 & 0.0320 & 15.9 & 46.9 \\
\hline 0/24/01 & $21: 31$ & 0 & 27.0 & 23.4 & 26.8 & 24.1 & 23.0 & 52.0 & 61.6 & 11.0 & 41.8 & 9.7 & 0 & 38.5 & 0.077 & 0.0320 & 16.0 & 46.9 \\
\hline D/24/01 & $21: 32$ & 0 & 27.0 & 23.4 & 26.9 & 24.2 & 23.1 & 52.3 & 62.0 & 11.1 & 41.6 & 97 & & 38.4 & 0.077 & & 50 & \\
\hline $10 / 24 / 01$ & $21: 33$ & 0 & 27.0 & 23.4 & 26.9 & 24.3 & 23.2 & 51.9 & 61.6 & 11.0 & 41.9 & 9.7 & 0 & 38.4 & 0.077 & 0.0319 & 15.9 & 46.9 \\
\hline $10 / 24 / 01$ & $21: 34$ & 0 & 27.0 & 23.4 & 26.9 & 24.3 & 23.1 & 52.5 & 62.3 & 11.0 & 41.7 & 97 & & 38.3 & 0.077 & 0.0319 & 5.9 & 171 \\
\hline $10 / 24 / 01$ & $21: 35$ & 0 & 27.1 & 23.4 & 26.9 & 24.4 & 23.1 & 52.1 & 61.7 & 11.1 & 41.7 & 9.6 & 0 & 38.3 & 0.077 & 0.0319 & 15.9 & 46.9 \\
\hline $10 / 24 / 01$ & $21: 36$ & 0 & 27.0 & 23.4 & 26.8 & 24.3 & 23.0 & 52.1 & 61.6 & 11.1 & 41.8 & 9. & & 38.3 & 0.077 & & 59 & 9 \\
\hline 0/24/01 & $21: 37$ & 0 & 27.1 & 23.4 & 26.9 & 24.3 & 23.0 & 52.3 & 62.0 & 11.0 & 41.8 & 9. & 0 & 38.4 & 0.077 & 0.0319 & 15.9 & 47.1 \\
\hline b1 & $21: 38$ & 0 & 27.1 & 23.4 & 26.9 & 24.2 & 22.9 & 52.1 & 61.7 & 11.1 & 41.7 & 9.6 & & 38.4 & 0.077 & & 5.9 & .9 \\
\hline $10 / 24 / 01$ & $21: 39$ & 0 & 27.1 & 23.4 & 26.9 & 24.1 & 22.9 & 52.4 & 62.2 & 11.0 & 41.8 & 9.6 & $c$ & 38.3 & 0.077 & 0.0319 & 5.9 & 47.1 \\
\hline 1 & $21: 40$ & 0 & 27.2 & 23.4 & 26.9 & 24.1 & 22.9 & 52.3 & 62.0 & 11.0 & 42.0 & 9. & & 8.4 & 0.077 & & 5.9 & 47.2 \\
\hline 1 & $21: 41$ & 0 & 27.2 & 23.4 & 26.9 & 24.0 & 22.8 & 52.3 & 62.1 & 11.0 & 41.8 & 9. & c & 38.3 & 0.076 & & 5.9 & 47.1 \\
\hline 10/2 & $21: 42$ & 0 & 27.2 & 23.4 & 27.0 & 24.0 & 22.8 & 52.1 & 61.7 & 11.1 & 41.5 & 9. & & 38.3 & 0.077 & 0.0318 & 5.9 & 46.8 \\
\hline 1 & $21: 43$ & 0 & 27.2 & 23.4 & 27.0 & 24.0 & 22.7 & 52.2 & 61.9 & 11.1 & 42.0 & 9. & & 38.3 & 0.076 & & 5.9 & 47.1 \\
\hline $10 / 2$ & $21: 44$ & 0 & 27.2 & 23.4 & 27.0 & 23.9 & 22.7 & 52.3 & 61.8 & 11.1 & 41.8 & 9. & & 38.4 & 0.076 & 13 & 5.9 & 47.0 \\
\hline & $21: 45$ & 0 & 27.2 & 23.4 & 27.0 & 23.9 & 22.6 & 51.7 & 61.3 & 11.0 & 41.5 & 9. & & 38.2 & 0.076 & & & 46.6 \\
\hline $10 / 2$ & $21: 46$ & 0 & 27.3 & 23.4 & 27.0 & 23.8 & 22.6 & 52.1 & 61.7 & 10.9 & 41.7 & 0 & & 38.2 & 0.076 & 14 & 5.9 & 46.9 \\
\hline & $21: 47$ & 0 & 27.3 & 23.4 & 27.1 & 23.8 & 22.5 & 52.2 & 61.9 & 11.0 & 41.6 & 9. & & .4 & 0.076 & & & \\
\hline 10/2 & $21: 48$ & 0 & 27.3 & 23.4 & 27.1 & 23.7 & 22.4 & 52.3 & 61.9 & 11.0 & 41.9 & $\mathrm{~J}$ & & 38.3 & 0.076 & & 5.9 & 47.1 \\
\hline & $21: 49$ & 0 & 27.3 & 23.4 & 27.1 & 23.7 & 22.4 & 52.1 & 61.7 & 11.0 & 41.8 & 9. & & 3.3 & 0.076 & & & 46.9 \\
\hline $10 / 2$ & $21: 50$ & 0 & 27.3 & 23.5 & 27.1 & 23.7 & 22.4 & 52.2 & 61.7 & 11.0 & 41.9 & 0 & & .3 & 0.076 & & 5.9 & 47.0 \\
\hline & $21: 51$ & 0 & 27.4 & 23.4 & 27.1 & 23.6 & 22.3 & 52.3 & 61.8 & 11.0 & 41.6 & 9. & & .4 & 0.076 & & & 47.0 \\
\hline o/2 & $21: 52$ & 0 & 27.3 & 23.4 & 27.2 & 23.6 & 22.3 & 52.2 & 61.7 & 11.1 & 41.4 & 9.6 & & 3.2 & 0.076 & & 15.9 & 46.8 \\
\hline 1 & $21: 53$ & 0 & 27.4 & 23.4 & 27.2 & 23.6 & 22.4 & 52.4 & 62.0 & 11.1 & 41.8 & 9. & & 3 & 0.076 & & & 47.1 \\
\hline o/2 & $21: 54$ & 0 & 27.4 & 23.4 & 27.2 & 23.6 & 22.3 & 52.3 & 61.9 & 11.0 & 41.9 & 9.6 & & .3 & 0.076 & & 5.9 & 47.1 \\
\hline $0 / 2$ & $21: 55$ & 0 & 27.5 & 23.4 & 27.2 & 23.6 & 22.4 & 52.3 & 61.9 & 11.1 & 41.8 & 9. & & 3.3 & 0.076 & & & 47.1 \\
\hline 1 & $21: 56$ & 0 & 27.5 & 23.4 & 27.2 & 23.7 & 22.4 & 52.3 & 61.9 & 11.0 & 41. & 9. & & 3 & 0.076 & & 5.9 & 47.0 \\
\hline 1 & $21: 57$ & 0 & 27.5 & 23.4 & 27.2 & 23.6 & 22.4 & 52.2 & 61.8 & 11.0 & 41.8 & 9. & & 8.3 & 0.076 & & 5.9 & 47.0 \\
\hline 1 & $21: 58$ & 0 & 27.5 & 23.4 & 27.3 & 23.7 & 22.5 & 52.4 & 62.0 & 11.1 & 41.7 & 9. & & 3 & 0.076 & & 5.9 & 47.0 \\
\hline 1 & $21: 59$ & 0 & 27.6 & 23.4 & 27.3 & 23.7 & 22.5 & 52.3 & 61.9 & 11.1 & 42.0 & 9. & & 8.3 & 0.076 & & 5.9 & 47.1 \\
\hline 1 & 22:00 & 0 & 27.5 & 23.4 & 27.3 & 23.8 & 22.6 & 52.5 & 62.1 & 11.1 & 42. & 9. & & 3 & 0.076 & & & 47.3 \\
\hline 10/2 & 22:01 & 0 & 27.6 & 23.4 & 27.4 & 23.8 & 22.6 & 52.5 & 62.1 & 11.1 & 41.8 & 9. & & 8.2 & 0.076 & & 5.9 & 47.1 \\
\hline & 22:02 & 0 & 27.6 & 23.4 & 7.4 & 23.9 & 22.7 & 52.4 & 62.1 & 11 & 41 & & & & & & & 46.9 \\
\hline 1 & 22:03 & 0 & 27.6 & 23.4 & 27.4 & 24.0 & 22.8 & 52.4 & 61.9 & 11.1 & 41.8 & 9. & & 8.2 & 0.075 & & 5.9 & 47.1 \\
\hline & 22:04 & 0 & 27.6 & 23.4 & 7.4 & 24.0 & 22.8 & 52.3 & 61.9 & 11.1 & 42 & 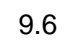 & & & & & & 47.2 \\
\hline $10 / 2$ & 22:05 & 0 & 27.6 & 23.4 & 7.4 & 24.1 & 22.8 & 52.6 & 62.2 & 11.1 & 41.9 & 9. & & 8.2 & 0.075 & & 5.9 & 47.3 \\
\hline & 22:06 & 0 & 27.7 & 23.4 & 7.5 & 24.1 & 22.9 & 52.5 & 62.1 & 11 & & & & & & & & \\
\hline 1 & 22:07 & 0 & 27.7 & 23.4 & 7.5 & 24.1 & 23.0 & 52.1 & 61.5 & 11.1 & 41.7 & 9. & & 8.2 & 0.075 & & 5.9 & 46.9 \\
\hline & 22:08 & 0 & 27.7 & 23.4 & 7.5 & 24.1 & 23.0 & 52.1 & 61.6 & 11 & & & & & & & & \\
\hline $10 / 2$ & 22:09 & 0 & 27.7 & 23.4 & 7.5 & 24.2 & 23.1 & 52.5 & 62.0 & 11.1 & 41.9 & 9. & & 8.3 & 0.075 & & 5.9 & 47.2 \\
\hline & $22: 10$ & 0 & 27.7 & 23.4 & & & 231 & 52.4 & 62.0 & 11 & & & & & & & & \\
\hline $10 / 2$ & 22:11 & 0 & 27.8 & 23.4 & 7.6 & 24.3 & 23.1 & 52.5 & 62.1 & 11.1 & 42. & 9. & & ? & 0.076 & & 5.9 & 47.3 \\
\hline & 22:12 & 0 & 27.8 & 23.4 & & & & & 61.7 & 11 & & & & & & & & \\
\hline & $22: 13$ & 0 & 27.8 & 23.4 & .6 & 4 & 23.1 & 52 & 61.9 & 11.1 & 41 & 9. & & ? & 0 & & 15.9 & 47.1 \\
\hline & 22 & 0 & 8 & 23.4 & .6 & & 23 & 52 & 62.2 & 11 & & & & & & & & \\
\hline 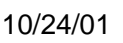 & $22: 15$ & 0 & 27.8 & 23.4 & .6 & 3 & 23.0 & 52.3 & 61.7 & 11.0 & 41 & 9 & & ? & 0.075 & & 15.9 & 46.8 \\
\hline & 22 & 0 & 27.8 & 23.4 & 6 & & 23.0 & 52 & 61.9 & 11 & & & & & & & & \\
\hline & $22: 17$ & 0 & 27.8 & 23.4 & & 2 & 22.9 & 52 & 61.8 & 11 & 4 & & & & 0 & & 15.9 & 47.1 \\
\hline & 22 & & 0 & 23.4 & .6 & & 22.9 & 52.5 & 62.1 & 11 & & & & & & & & \\
\hline & $22: 19$ & 0 & 27.8 & 23.4 & & & 22.8 & 52.5 & 62.1 & 11.1 & 4 & 9 & & & 0.075 & & 15.9 & .1 \\
\hline & $22: 20$ & 0 & 27.9 & 23.4 & .7 & 0 & 22.8 & 52 & 62.1 & 11 & & & & & & & & \\
\hline & 22:21 & 0 & 27.9 & & & & 22.7 & & 61.8 & 11.2 & 41 & & & & & & & 1 \\
\hline & $22: 22$ & 0 & 28.0 & 23.4 & .1 & & 22.7 & 52 & 61.9 & 11.2 & 42 & & & & 0 & & & 2 \\
\hline & $22: 23$ & 0 & $2 \varepsilon$ & & & & & & 62 & & & 9 & & & & & & \\
\hline & $22: 24$ & 0 & 28.0 & 23 & 27 & & 22.6 & 52 & 62.2 & 11 & 4 & & & & & & & 2 \\
\hline & 22 & 0 & & & & & & & & 11 & & & & & & & & \\
\hline & $22: 26$ & 0 & 28.0 & 23. & .8 & & 22.5 & 52.5 & 62.0 & 11 & 4 & 9 & & 1 & 0 & & 5.8 & 1 \\
\hline & $22: 27$ & 0 & 28.0 & & & & 22.5 & ${ }_{3}$ & 62 & 11 & & & & & & & & \\
\hline & $22: 28$ & - & 28.1 & & & & 22.4 & 52 & 62.0 & & & & & & & & & \\
\hline $10 / 2$ & & ( & & & & & 22.4 & 52.8 & 62.3 & 11.1 & 42.0 & 9.6 & 0 & 38.3 & 0.075 & 0.0303 & 15.9 & 7.4 \\
\hline
\end{tabular}




\begin{tabular}{|c|c|c|c|c|c|c|c|c|c|c|c|c|c|c|c|c|c|c|}
\hline & & Sc & $\begin{array}{l}\text { FLTRT } \\
\left({ }^{\circ} \mathrm{C}\right) \\
\mathrm{T} 2\end{array}$ & $\begin{array}{c}\text { CL } \\
\text { LOOP } \\
\left({ }^{\circ} \mathrm{C}\right) \\
\text { T3 }\end{array}$ & $\begin{array}{l}\text { SL } \\
\text { LOOP } \\
\left({ }^{\circ} \mathrm{C}\right) \\
\mathrm{T} 1\end{array}$ & $\begin{array}{l}\text { UP } \\
\text { AMB } \\
\left({ }^{\circ} \mathrm{C}\right) \\
\text { T4 }\end{array}$ & $\begin{array}{c}\text { Xflow2 } \\
\text { BOT } \\
\text { AMB } \\
\left({ }^{\circ} \mathrm{C}\right) \\
\text { T5 }\end{array}$ & $\begin{array}{c}102401 \\
\text { BOT } \\
\text { DP } \\
\text { (psid) } \\
\text { dP2 }\end{array}$ & $\begin{array}{l}\text { FLTR } \\
\text { (psig) } \\
\text { P1 }\end{array}$ & $\begin{array}{c}\text { FLTR } \\
\text { DP } \\
\text { (psid) } \\
\text { dP1 }\end{array}$ & $\begin{array}{l}\text { TOP } \\
\text { DP } \\
\text { (psig) } \\
\text { dP3 }\end{array}$ & $\begin{array}{l}\text { FLT- } \\
\text { RATE } \\
\text { (psig) } \\
\text { P2 }\end{array}$ & $\begin{array}{c}\text { BP } \\
\text { (psig) } \\
\text { P3 }\end{array}$ & $\begin{array}{c}\text { SL } \\
\text { FLOW } \\
\text { (gpm) } \\
\text { Q1 }\end{array}$ & $\begin{array}{l}\text { FLTR } \\
\text { FLOW } \\
\text { (gpm) } \\
\text { Q2 }\end{array}$ & $\begin{array}{c}\text { Temp } \\
\text { corrected } \\
\text { flow } \\
\left(\mathrm{gpm} / \mathrm{ft}^{2}\right)\end{array}$ & $\begin{array}{l}\text { Axial Vel } \\
\text { (ft/sec) }\end{array}$ & $\begin{array}{l}\text { Avg } \\
\text { TMP } \\
\text { (psid) }\end{array}$ \\
\hline 0/24/01 & $22: 30$ & 0 & 28.1 & 23.4 & 27.8 & 23.6 & 22.4 & 52.6 & 62.1 & 11.2 & 41.9 & 9.6 & , & 38.1 & 0.075 & 0.0303 & 15.8 & 47.3 \\
\hline $10 / 24 / 01$ & 22:31 & 0 & 28.1 & 23.4 & 27.9 & 23.6 & 22.4 & 52.7 & 62.4 & 11.1 & 42.1 & 9.6 & 0 & 38.2 & 0.075 & 0.0302 & 15.8 & 47.4 \\
\hline D/24/01 & $22: 32$ & 0 & 28.1 & 23.4 & 27.9 & 23.6 & 22.4 & 52.4 & 61.9 & 11.1 & 42.0 & 9 & & 38.1 & 0.075 & & 58 & 47.2 \\
\hline $10 / 24 / 01$ & $22: 33$ & 0 & 28.2 & 23.4 & 28.0 & 23.6 & 22.4 & 52.9 & 62.5 & 11.2 & 41.9 & 9.6 & 0 & 38.2 & 0.075 & 0.0301 & 15.8 & 47.4 \\
\hline $10 / 24 / 01$ & $22: 34$ & 0 & 28.2 & 23.4 & 28.0 & 23.7 & 22.5 & 52.6 & 62.1 & 11.2 & 42.0 & 96 & & 38.2 & 0.075 & 0.0301 & 59 & 47.3 \\
\hline $10 / 24 / 01$ & $22: 35$ & 0 & 28.2 & 23.4 & 28.0 & 23.7 & 22.5 & 52.6 & 62.0 & 11.1 & 41.9 & 9.6 & 0 & 38.1 & 0.075 & 0.0301 & 15.8 & 47.2 \\
\hline $10 / 24 / 01$ & $22: 36$ & 0 & 28.2 & 23.4 & 28.1 & 23.8 & 22.6 & 52.6 & 62.2 & 11.1 & 42.0 & 9. & & 38.1 & 0.075 & & 58 & 47.3 \\
\hline 0/24/01 & $22: 37$ & 0 & 28.3 & 23.4 & 28.0 & 23.8 & 22.6 & 52.6 & 62.0 & 11.2 & 42.0 & 9. & 0 & 38.1 & 0.075 & 0.0302 & 15.8 & 47.3 \\
\hline 0/24/01 & $22: 38$ & 0 & 28.3 & 23.4 & 28.1 & 23.9 & 22.7 & 52.5 & 61.9 & 11.2 & 42.0 & 9.6 & & 38.1 & 0.075 & & 5.8 & 47.3 \\
\hline $10 / 24 / 01$ & 22:39 & 0 & 28.3 & 23.4 & 28.2 & 23.9 & 22.7 & 52.4 & 61.9 & 11.2 & 41.9 & 9.5 & $c$ & 38.1 & 0.075 & 0.0300 & 5.8 & 47.1 \\
\hline 1 & $22: 40$ & 0 & 28.3 & 23.4 & 28.2 & 24.0 & 22.8 & 52.5 & 62.1 & 11.2 & 41.6 & 9. & & 38.0 & 0.075 & & 5.8 & 47.0 \\
\hline 01 & $22: 41$ & 0 & 28.3 & 23.4 & 28.2 & 24.1 & 22.8 & 52.8 & 62.4 & 11.2 & 41.9 & 9. & ( & 38.1 & 0.075 & & 5.8 & 47.4 \\
\hline$/ 24$ & $22: 42$ & 0 & 28.4 & 23.4 & 28.2 & 24.1 & 22.9 & 52.7 & 62.3 & 11.2 & 42.0 & 9. & & 38.2 & 0.075 & 0.0299 & 5.8 & 47.4 \\
\hline 1 & $22: 43$ & 0 & 28.4 & 23.4 & 28.3 & 24.2 & 22.9 & 52.5 & 61.9 & 11.2 & 42.1 & 9. & & 38.2 & 0.075 & & 5.8 & 47.3 \\
\hline $10 / 2$ & $22: 44$ & 0 & 28.4 & 23.4 & 28.2 & 24.2 & 22.9 & 52.6 & 62.1 & 11.2 & 42.0 & 9. & & 38.2 & 0.075 & 999 & 5.8 & 47.3 \\
\hline & $22: 45$ & 0 & 28.4 & 23.4 & 28.3 & 24.2 & 23.0 & 52.4 & 61.9 & 11.2 & 42.0 & 9. & & 38.1 & 0.075 & & & 47.2 \\
\hline $10 / 2$ & $22: 46$ & 0 & 28.5 & 23.4 & 28.3 & 24.3 & 23.1 & 52.5 & 62.0 & 11.1 & 42.2 & 9. & & 38.0 & 0.075 & 98 & 5.8 & 47.3 \\
\hline & $22: 47$ & 0 & 28.5 & 23.4 & 28.4 & 24.3 & 23.1 & 52.8 & 62.3 & 11.2 & 42.2 & 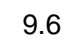 & & .1 & 0.075 & & & 47.5 \\
\hline 10/2 & $22: 48$ & 0 & 28.5 & 23.4 & 28.4 & 24.3 & 23.2 & 52.7 & 62.4 & 11.2 & 42.0 & 9. & & 38.1 & 0.075 & 98 & 5.8 & 47.4 \\
\hline & $22: 49$ & 0 & 28.6 & 23.4 & 28.4 & 24.4 & 23.2 & 52.9 & 62.4 & 11.2 & 42.0 & 9. & & 8.0 & 0.075 & & & 47.5 \\
\hline $0 / 2$ & 22:50 & 0 & 28.6 & 23.4 & 28.3 & 24.4 & 23.2 & 52.9 & 62.5 & 11.1 & 41.9 & 0 & & 0 & 0.075 & 99 & 5.8 & 47.4 \\
\hline & 22:51 & 0 & 28.6 & 23.4 & 28.4 & 24.4 & 23.3 & 53.0 & 62.5 & 11.2 & 42.2 & 9. & & .0 & 0.075 & & & 47.6 \\
\hline$/ 2$ & 22:52 & 0 & 28.6 & 23.4 & 27.7 & 24.4 & 23.2 & 52.4 & 61.8 & 11.1 & 42.3 & 9. & & 1 & 0.074 & 00 & 5.8 & 47.4 \\
\hline 1 & $22: 53$ & 0 & 28.4 & 23.4 & 27.3 & 24.4 & 23.2 & 52.6 & 62.2 & 11.0 & 42.0 & 9. & & 3.2 & 0.073 & & & 47.3 \\
\hline o/2 & 22:54 & - & 28.3 & 23.5 & 26.9 & 24.4 & 23.2 & 52.6 & 61.9 & 10.9 & 42.3 & 9. & & .2 & 0.072 & 98 & 5.8 & 47.4 \\
\hline $0 / 2$ & 22:55 & 0 & 28.2 & 23.4 & 26.8 & 24.4 & 23.1 & 52.8 & 62.2 & 11.0 & 42.1 & 9. & & .2 & 0.072 & & & 47.4 \\
\hline 1 & 22:56 & 0 & 28.2 & 23.5 & 26.7 & 24.3 & 23.1 & 52.5 & 61.9 & 11.0 & 42.2 & 9. & & 3 & 0.072 & & 5.9 & 47.3 \\
\hline 1 & 22:57 & 0 & 28.0 & 23.5 & 26.7 & 24.3 & 23.0 & 52.9 & 62.3 & 10.9 & 42.2 & 0 & & 3.4 & 0.071 & & & 47.6 \\
\hline 1 & 22:58 & 0 & 27.9 & 23.4 & 26.7 & 24.2 & 23.0 & 52.5 & 61.9 & 10.9 & 42.3 & 9. & & 2 & 0.072 & & 5.9 & 47.4 \\
\hline $10 / 2$ & 22:59 & 0 & 27.7 & 23.5 & 26.7 & 24.1 & 22.9 & 52.6 & 61.9 & 10.8 & 42.1 & 9. & & 8.2 & 0.071 & 96 & 5.8 & 47.3 \\
\hline 1 & $23: 00$ & 0 & 27.6 & 23.5 & 26.7 & 24.0 & 22.8 & 52.6 & 61.9 & 11.0 & 42.3 & 9. & & & 0.071 & & & 47.4 \\
\hline $10 / 2$ & 23:01 & 0 & 27.5 & 23.5 & 26.8 & 24.0 & 22.8 & 52.4 & 61.7 & 11.0 & 42.3 & 9. & & 8.3 & 0.072 & & 5.9 & 47.3 \\
\hline & 23:02 & 0 & 27.4 & 23.5 & 26.8 & 24.0 & 22.7 & 52.8 & 62.1 & 11.0 & 42.1 & . & & & & & & 47.4 \\
\hline 1 & $23: 03$ & 0 & 27.3 & 23.5 & 26.8 & 23.9 & 22.7 & 52.6 & 62.0 & 11.0 & 41.8 & 9. & & 8.2 & 0.071 & 95 & 5.9 & 47.2 \\
\hline & 23:04 & 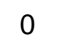 & 27.2 & 23.5 & 26.8 & 23.9 & 22.7 & 52.2 & 61.6 & 11.0 & 42.2 & 9 & & & & & & 47.2 \\
\hline $10 / 2$ & 23:05 & 0 & 27.2 & 23.5 & 6.8 & 23.8 & 22.7 & 52.9 & 62.3 & 11.1 & 42.5 & 9. & & 8.3 & 0.071 & 95 & 5.9 & 47.7 \\
\hline & $23: 06$ & 0 & 27.2 & & & & 22.6 & 52.5 & 61.9 & 11.0 & 42.2 & & & & & & & \\
\hline 1 & 23:07 & 0 & 27.1 & 23.5 & 6.8 & 23.7 & 22.6 & 52.5 & 61.9 & 10.9 & 41.9 & 9. & & 8.2 & 0.071 & 95 & 5.9 & 47.2 \\
\hline & 23:08 & 0 & 27.2 & & 6.8 & & 22.5 & 52.3 & 61.7 & 10.9 & 42.2 & & & & & & & \\
\hline $10 / 2$ & 23:09 & 0 & 27.1 & 23.5 & 6.9 & 23.7 & 22.5 & 52.8 & 62.1 & 11.0 & 42.3 & 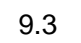 & & 2 & 0.071 & 94 & 5.9 & 47.5 \\
\hline & $23: 10$ & 0 & 27.2 & 23 & & & 22.5 & 526 & 62.0 & 11.0 & & a & & & & & & \\
\hline $10 /$ & $23: 11$ & 0 & 27.2 & 23 & 5.9 & 23.7 & 22.4 & 52.8 & 62.2 & 11.1 & 42.3 & 9 & & 3 & 0.072 & 98 & 5.9 & 47.5 \\
\hline & $23: 12$ & 0 & 27.2 & 23 & & & 22.5 & & 61.9 & 11.0 & & & & & & & & \\
\hline & $23: 13$ & 0 & 27.2 & 23 & .9 & .7 & 22.5 & 52.7 & 62.1 & 10.9 & 42.4 & 9 & & ? & 71 & & 5.9 & 47.6 \\
\hline & 23 & 0 & 7.2 & 23 & .9 & & 22.5 & 52.4 & 61.7 & 11.0 & & & & & & & & \\
\hline 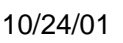 & $23: 15$ & 0 & 27.2 & 23.5 & .9 & .8 & 22.6 & 52.7 & 62.0 & 11.0 & 42.2 & 9 & & 3 & 0.072 & 98 & 15.9 & 47.5 \\
\hline & 23 & 0 & 27.2 & 23 & .0 & 23.8 & 22.6 & 52 & 62.2 & 11.1 & & & & & & & & \\
\hline & 23 & 0 & 27.2 & 23 & 0 & 9 & 22.6 & 52.6 & 61.9 & 11.1 & 42.5 & & & & 71 & & 15.9 & 47.5 \\
\hline & 23 & 0 & 3 & 23 & .0 & & 22.7 & 52 & 61.9 & 11.0 & & u & & & & & & \\
\hline & 23 & 0 & 27.2 & & & & 22.7 & 52.6 & 61.9 & 10.9 & 42.4 & $a$ & & & 0.071 & & 15.8 & .5 \\
\hline & 23 & 0 & 27.3 & 23 & 7.0 & 0 & 22.7 & 52.9 & 62.3 & 11.0 & 42 & 9 & & & & & & \\
\hline & 23 & 0 & 27.3 & & & & 22.8 & 52 & 61.9 & 11.0 & 42.2 & 9 & & & & & & \\
\hline & 23 & 0 & 27.3 & 23.5 & 7.1 & 0 & 22.9 & 52.4 & 61.7 & 11.0 & 42.2 & 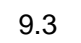 & & & & & & .3 \\
\hline & 23 & 0 & 27.3 & & & & & & & 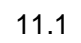 & & & & & & & & \\
\hline & 23 & 0 & 27.3 & 23 & 1 & & 22.9 & 52 & 61.9 & 11 & 42 & & & & & & & \\
\hline & & 0 & 27 & & & & & & & & & & & & & & & \\
\hline & 23 & 0 & 27.4 & 23 & 27.2 & & 0 & 52.4 & 61.7 & 11. & 41 & 9. & & & & & 5.9 & \\
\hline & 23 & 0 & 27. & & & & & ${ }_{3}$ & 62 & 11 & & & & & & & .9 & \\
\hline & & - & 27.4 & 23 & 27.2 & & & 52.8 & 62.1 & 11 & 42.3 & 9. & & & & & & \\
\hline $10 / 2$ & 2 & $c$ & 27.4 & 23. & & & & 527 & 62.0 & 11.0 & 42.1 & 9.3 & 0 & 38.2 & 0.071 & 0.0291 & 15.9 & 1 \\
\hline
\end{tabular}




\begin{tabular}{|c|c|c|c|c|c|c|c|c|c|c|c|c|c|c|c|c|c|c|}
\hline & & Sc & $\begin{array}{l}\text { FLTRT } \\
\left({ }^{\circ} \mathrm{C}\right) \\
\mathrm{T} 2\end{array}$ & $\begin{array}{c}\text { CL } \\
\text { LOOP } \\
\left({ }^{\circ} \mathrm{C}\right) \\
\text { T3 }\end{array}$ & $\begin{array}{l}\text { SL } \\
\text { LOOP } \\
\left({ }^{\circ} \mathrm{C}\right) \\
\mathrm{T} 1\end{array}$ & $\begin{array}{l}\text { UP } \\
\text { AMB } \\
\left({ }^{\circ} \mathrm{C}\right) \\
\text { T4 }\end{array}$ & $\begin{array}{c}\text { Xflow2 } \\
\text { BOT } \\
\text { AMB } \\
\left({ }^{\circ} \mathrm{C}\right) \\
\text { T5 }\end{array}$ & $\begin{array}{c}102401 \\
\text { BOT } \\
\text { DP } \\
\text { (psid) } \\
\text { dP2 }\end{array}$ & $\begin{array}{l}\text { FLTR } \\
\text { (psig) } \\
\text { P1 }\end{array}$ & $\begin{array}{l}\text { FLTR } \\
\text { DP } \\
\text { (psid) } \\
\text { dP1 }\end{array}$ & $\begin{array}{l}\text { TOP } \\
\text { DP } \\
\text { (psig) } \\
\text { dP3 }\end{array}$ & $\begin{array}{l}\text { FLT- } \\
\text { RATE } \\
\text { (psig) } \\
\text { P2 }\end{array}$ & $\begin{array}{c}\text { BP } \\
\text { (psig) } \\
\text { P3 }\end{array}$ & $\begin{array}{c}\text { SL } \\
\text { FLOW } \\
\text { (gpm) } \\
\text { Q1 }\end{array}$ & $\begin{array}{c}\text { FLTR } \\
\text { FLOW } \\
\text { (gpm) } \\
\text { Q2 }\end{array}$ & $\begin{array}{c}\text { Temp } \\
\text { corrected } \\
\text { flow } \\
\left(\mathrm{gpm} / \mathrm{ft}^{2}\right)\end{array}$ & $\begin{array}{l}\text { Axial Vel } \\
\text { (ft/sec) }\end{array}$ & $\begin{array}{l}\text { Avg } \\
\text { TMP } \\
\text { (psid) }\end{array}$ \\
\hline 0/24/01 & $23: 30$ & 0 & 27.4 & 23.5 & 27.2 & 24.2 & 23.2 & 52.5 & 61.9 & 11.1 & 42.3 & 9.3 & 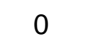 & 38.3 & 0.071 & 0.0292 & 15.9 & 47.4 \\
\hline 0/24/01 & 23:31 & 0 & 27.5 & 23.5 & 27.3 & 24.3 & 23.2 & 52.7 & 62.1 & 11.0 & 42.0 & 9.3 & 0 & 38.3 & 0.072 & 0.0295 & 15.9 & 47.4 \\
\hline D/24/01 & $23: 32$ & 0 & 27.5 & 23.5 & 27.3 & 24.4 & 23.2 & 52.4 & 61.6 & 10.9 & 42.1 & 93 & & 38.2 & 0.071 & & 59 & 47.3 \\
\hline $10 / 24 / 01$ & $23: 33$ & 0 & 27.5 & 23.5 & 27.3 & 24.4 & 23.1 & 52.5 & 61.8 & 11.0 & 42.1 & 9.3 & 0 & 38.2 & 0.071 & 0.0291 & 15.9 & 47.3 \\
\hline $10 / 24 / 01$ & $23: 34$ & 0 & 27.5 & 23.5 & 27.3 & 24.3 & 23.1 & 52.6 & 61.9 & 11.0 & 42.1 & 93 & & 38.3 & 0.071 & 0.0291 & 59 & 47.3 \\
\hline $10 / 24 / 01$ & $23: 35$ & 0 & 27.5 & 23.5 & 27.3 & 24.3 & 23.0 & 53.0 & 62.3 & 11.1 & 42.2 & 9.3 & 0 & 38.3 & 0.071 & 0.0291 & 15.9 & 47.6 \\
\hline $10 / 24 / 01$ & $23: 36$ & 0 & 27.5 & 23.5 & 27.3 & 24.3 & 23.0 & 52.7 & 62.1 & 11.1 & 42.2 & 9.3 & & 38.2 & 0.072 & & 59 & 47.5 \\
\hline 0/24/01 & 23:37 & 0 & 27.5 & 23.5 & 27.3 & 24.2 & 22.9 & 52.6 & 61.9 & 11.1 & 42.3 & 9.3 & 0 & 38.3 & 0.072 & 0.0295 & 15.9 & 47.5 \\
\hline o. & $23: 38$ & 0 & 27.5 & 23.5 & 27.4 & 24.2 & 22.9 & 52.9 & 62.2 & 11.1 & 42.4 & 9.3 & & 38.3 & 0.072 & 0.0294 & 5.9 & 47.7 \\
\hline $10 / 24 / 01$ & 23:39 & 0 & 27.6 & 23.5 & 27.3 & 24.1 & 22.9 & 53.0 & 62.1 & 11.0 & 42.2 & 9.3 & $c$ & 38.3 & 0.071 & 0.0291 & 5.9 & 47.6 \\
\hline 01 & $23: 40$ & 0 & 27.6 & 23.5 & 27.3 & 24.1 & 22.8 & 52.5 & 61.8 & 11.1 & 42.1 & 9.3 & & 38.3 & 0.071 & & 5.9 & 47.3 \\
\hline 1 & $23: 41$ & 0 & 27.6 & 23.5 & 27.3 & 24.0 & 22.8 & 52.8 & 62.2 & 11.1 & 42.5 & 9. & ( & 38.3 & 0.071 & & 5.9 & 47.7 \\
\hline$/ 2$ & $23: 42$ & 0 & 27.6 & 23.5 & 27.3 & 24.0 & 22.7 & 52.8 & 62.1 & 11.1 & 42.4 & 9.3 & & 38.3 & 0.071 & 0.0291 & 5.9 & 47.6 \\
\hline 1 & $23: 43$ & 0 & 27.6 & 23.5 & 27.4 & 23.9 & 22.8 & 52.8 & 62.1 & 11.1 & 42.3 & 9. & & 38.2 & 0.071 & & 5.9 & 47.5 \\
\hline $0 / 2$ & $23: 44$ & 0 & 27.6 & 23.5 & 27.4 & 23.9 & 22.9 & 52.6 & 62.0 & 10.9 & 42.2 & 9.3 & & 38.2 & 0.071 & 90 & .9 & 47.4 \\
\hline & $23: 45$ & 0 & 27.7 & 23.5 & 27.5 & 24.0 & 23.2 & 52.6 & 62.0 & 11.1 & 42.2 & 9 & & 8.2 & 0.071 & & & 47.4 \\
\hline $\mathrm{b} / 2$ & $23: 46$ & 0 & 27.7 & 23.5 & 27.5 & 24.0 & 23.3 & 52.3 & 61.7 & 11.0 & 42.3 & 9.3 & & 38.3 & 0.071 & & .9 & 47.3 \\
\hline & $23: 47$ & 0 & 27.7 & 23.5 & 27.5 & 24.1 & 23.5 & 52.8 & 62.1 & 11.1 & 42.5 & 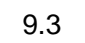 & & 3 & & & & 47.7 \\
\hline $0 / 2$ & $23: 48$ & 0 & 27.7 & 23.5 & 27.5 & 24.2 & 23.6 & 52.8 & 62.2 & 11.1 & 42.3 & 9.3 & & 38.2 & 0.071 & 89 & 5.9 & 47.6 \\
\hline & $23: 49$ & 0 & 27.7 & 23.5 & 27.5 & 24.3 & 23.8 & 52.9 & 62.2 & 11.1 & 42.3 & 9. & & 3.2 & 0.071 & & & 47.6 \\
\hline $0 / 2$ & 23:50 & 0 & 27.8 & 23.5 & 27.6 & 24.4 & 23.8 & 53.0 & 62.4 & 11.0 & 42.5 & 9. & & 3 & 0.071 & & 5.9 & 47.7 \\
\hline & 23:51 & 0 & 27.8 & 23.6 & 27.5 & 24.6 & 24.0 & 52.6 & 62.1 & 11.0 & 42.3 & 9. & & .2 & 0.071 & & & 47.4 \\
\hline$/ 2$ & 23:52 & 0 & 27.8 & 23.5 & 27.6 & 24.5 & 24.1 & 52.8 & 62.2 & 11.1 & 42.1 & 9. & & 3.3 & 0.071 & 88 & 5.9 & 47.5 \\
\hline 1 & $23: 53$ & 0 & 27.8 & 23.6 & 27.6 & 24.7 & 24.1 & 52.6 & 61.9 & 11.1 & 42.2 & 9. & & 3.2 & 0.071 & & & 47.4 \\
\hline o/2 & 23:54 & 0 & 27.8 & 23.5 & 27.6 & 24.6 & 24.3 & 52.9 & 62.3 & 11.1 & 42.3 & 9. & & 3.2 & 0.071 & & 5.8 & 47.6 \\
\hline $0 / 2$ & $23: 55$ & 0 & 27.8 & 23.6 & 27.7 & 24.7 & 24.3 & 53.1 & 62.4 & 11.0 & 42.4 & 9. & & 3.2 & 0.072 & & & 7.8 \\
\hline 1 & $23: 56$ & 0 & 27.8 & 23.6 & 27.6 & 24.7 & 24.4 & 52.5 & 61.9 & 11.0 & 42.1 & 9. & & 1 & 0.071 & & 5.8 & 47.3 \\
\hline 1 & 23:57 & 0 & 27.9 & 23.6 & 27.7 & 24.8 & 24.4 & 52.5 & 61.7 & 11.1 & 42.0 & 9. & & 8.2 & 0.072 & & & 47.2 \\
\hline 1 & $23: 58$ & 0 & 27.9 & 23.6 & 27.7 & 24.8 & 24.5 & 52.9 & 62.2 & 11.1 & 42.4 & 9.3 & & 1 & 0.071 & & 5.8 & 47.6 \\
\hline $10 / 2$ & 23:59 & 0 & 27.9 & 23.6 & 27.7 & 24.8 & 24.6 & 52.7 & 62.1 & 11.1 & 42.4 & 9. & & 8.1 & 0.072 & 91 & 5.8 & 47.6 \\
\hline 11 & $0: 00$ & 0 & 27.9 & 23.7 & 27.8 & 24.9 & 24.6 & 52.8 & 62.0 & 11.1 & 42.2 & 0 & & 2 & 0.072 & & & 47.5 \\
\hline 1 & $0: 01$ & 0 & 27.9 & 23.6 & 27.8 & 24.9 & 24.6 & 53.1 & 62.4 & 11.0 & 42.2 & 9. & & 8.2 & 0.072 & 91 & 5.9 & 47.6 \\
\hline & & 0 & 8.0 & 23.7 & 27.7 & 24.9 & 24.7 & 53.0 & 62.3 & 11.0 & 42.4 & 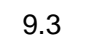 & & & & & & 47.7 \\
\hline$/ 25 / 01$ & $0: 03$ & 0 & 28.0 & 23.7 & 27.8 & 24.9 & 24.8 & 52.7 & 62.1 & 11.1 & 42.2 & 9. & & 8.1 & 0.071 & 87 & 5.8 & 47.5 \\
\hline in & 0.04 & 0 & 8.0 & 23.7 & 27.8 & 24.9 & 24.7 & 52.7 & 62.0 & 11 & 41.9 & 9. & & & & & & 47.3 \\
\hline 1 & $0: 05$ & 1 & 28.0 & 23.7 & 27.9 & 24.9 & 24.9 & 9.7 & 62.2 & 11.0 & -0.6 & 52. & 8 & 8.2 & 0.010 & & 5.8 & 4.5 \\
\hline & & 0 & 7.9 & & & 24.9 & 24.8 & 51.4 & 62.5 & 11 & 40.7 & 1 & & & & & & \\
\hline 01 & $0: 07$ & 0 & 8.0 & 23.7 & 7.9 & 25.0 & 25.0 & 51.9 & 62.3 & 11.2 & 41.2 & 10 & & 8.1 & 0.088 & 54 & 5.8 & 46.5 \\
\hline & & 0 & 8.0 & & & 25.0 & 25.1 & 51.9 & 62.1 & 11 & 41.4 & 10 & & & & & & \\
\hline 01 & 0:09 & 0 & 8.0 & 23.7 & 7.8 & 25.0 & 25.1 & 52.1 & 62.2 & 11.1 & 41.8 & 10 & & 8.1 & 0.088 & 55 & 5.8 & 46.9 \\
\hline & & 0 & & & & & 251 & 523 & 62.5 & 11.1 & 418 & & & & & & & \\
\hline 11 & $0: 11$ & 0 & 8.1 & 23.7 & 7.9 & 25.1 & 25.1 & 52.4 & 62.5 & 11.1 & 41.7 & 10 & & 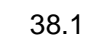 & 0. & 35 & 5.8 & 47.0 \\
\hline & & 0 & & 23 & & 25.1 & 25.2 & 52 & 62.4 & 11.1 & 419 & & & & & & & \\
\hline & $0:$ & 0 & 2 & 238 & & 1 & 25.1 & 52.0 & 62.2 & 11.1 & 41.5 & 1 & & 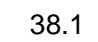 & & & 5.8 & 46.8 \\
\hline & & & & 23 & & 25.1 & 25 & 52 & 62.0 & & & & & & & & & \\
\hline $10 / 2$ & $0:$ & 0 & 8.2 & 23.9 & & 25.1 & 25.2 & 52.1 & 62.1 & 11.1 & 41.6 & 10 & & 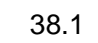 & 0 & 25 & 15.8 & 46.8 \\
\hline & & 0 & 2 & 2 & & 25.1 & 25 & 52 & 62.0 & 11 & 11 & & & & & & & \\
\hline & 0 & 0 & 3 & & & 1 & 25.3 & 52.5 & 62.5 & 11.2 & 41.8 & 1 & & 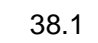 & & & & 47.1 \\
\hline & & & $T$ & 23 & 3.0 & 25.1 & 25 & 52 & 62.5 & 11 & 7 & & & & & & & \\
\hline & $0:$ & 0 & 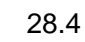 & & & & 25.3 & 52 & 62.3 & 11 & 41.6 & & & 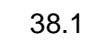 & & & 15.8 & .0 \\
\hline & $0:$ & & 28 & 23.9 & 3.2 & 25.2 & 25.3 & 52 & 62.8 & 11 & 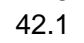 & & & & & & & \\
\hline & $0:$ & 0 & & & & & & & 62 1 & 11.2 & 41.8 & & & & & & & .0 \\
\hline & $0: 22$ & 0 & 28.4 & 24.0 & .2 & j.2 & 25.4 & 52.4 & 62.3 & 11.2 & 41.6 & 9. & & & & & & .0 \\
\hline & $0:$ & 0 & & & & & & & & & & & & & & & & \\
\hline & $0: 24$ & 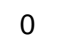 & 28.5 & 24 & & & & 52 & 62.4 & 11 & 41 & 9 & & & & & & \\
\hline & & 0 & & & & & & & & & & & & & & & & \\
\hline & $0: 26$ & G & 28.5 & 24.0 & & & & 52.3 & 62.3 & 11. & 41.6 & 9. & & 1 & & & 5.8 & 47.0 \\
\hline & $0:$ & 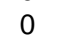 & 28.5 & & & & & $3 c$ & 62 & 11 & & 9 & & & & & 15.8 & \\
\hline & $0: 28$ & 0 & 28.6 & 24 & & & & 52 & & & 41.0 & 9. & & & & & & \\
\hline $10 / 25$ & $0: 29$ & c & 28.6 & 24.1 & 3.4 & & & 52.5 & 62.4 & 11.2 & 41.8 & 9.9 & 0 & 38.2 & 079 & 0.0314 & 15.8 & 171 \\
\hline
\end{tabular}




\begin{tabular}{|c|c|c|c|c|c|c|c|c|c|c|c|c|c|c|c|c|c|c|}
\hline & TME & Sol & $\begin{array}{l}\text { FLTRT } \\
\left({ }^{\circ} \mathrm{C}\right) \\
\text { T2 }\end{array}$ & $\begin{array}{l}\text { LOOP } \\
\left({ }^{\circ} \mathrm{C}\right) \\
\text { T3 }\end{array}$ & $\begin{array}{l}\text { SL } \\
\text { LOOP } \\
\left({ }^{\circ} \mathrm{C}\right) \\
\mathrm{T} 1\end{array}$ & $\begin{array}{l}\text { UP } \\
\text { AMB } \\
\left({ }^{\circ} \mathrm{C}\right) \\
\text { T4 }\end{array}$ & $\begin{array}{l}\text { BOT } \\
\text { AMB } \\
\left({ }^{\circ} \mathrm{C}\right) \\
\text { T5 }\end{array}$ & $\begin{array}{l}\text { BOT } \\
\text { DP } \\
\text { (psid) } \\
\text { dP2 }\end{array}$ & $\begin{array}{l}\text { FLTR } \\
\text { (psig) } \\
\text { P1 }\end{array}$ & $\begin{array}{l}\text { FLTR } \\
\text { DP } \\
\text { (psid) } \\
\text { dP1 }\end{array}$ & $\begin{array}{l}\text { TOP } \\
\text { DP } \\
\text { (psig) } \\
\text { dP3 }\end{array}$ & $\begin{array}{l}\text { FLT- } \\
\text { RATE } \\
\text { (psig) } \\
\text { P2 }\end{array}$ & $\begin{array}{c}\text { BP } \\
\text { (psig) } \\
\text { P3 }\end{array}$ & $\begin{array}{c}\text { FLOW } \\
\text { (gpm) } \\
\text { Q1 }\end{array}$ & $\begin{array}{l}\text { FLTR } \\
\text { FLOW } \\
\text { (gpm) } \\
\text { Q2 }\end{array}$ & $\begin{array}{c}\text { Temp } \\
\text { corrected } \\
\text { flow } \\
\left(\mathrm{gpm} / \mathrm{ft}^{2}\right)\end{array}$ & $\begin{array}{l}\text { Axial Vel } \\
(\mathrm{ft} / \mathrm{sec})\end{array}$ & $\begin{array}{l}\text { Avg } \\
\text { TMP } \\
\text { (psid) }\end{array}$ \\
\hline $5 / 01$ & $0: 30$ & 0 & 28.6 & 24.1 & 28.5 & 25.3 & 25.6 & 52.3 & 62.3 & 11.2 & 42.0 & 99 & & 38.1 & 0.078 & & & \\
\hline 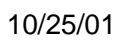 & 31 & 0 & 28.6 & 24.1 & & 25.3 & 25.5 & 52.2 & 62.1 & 11.2 & 41.6 & & & & & & & \\
\hline- & 32 & 0 & 8.6 & & & 25.3 & & $52 ?$ & 62.1 & 11.2 & & & & & & & & \\
\hline 25/01 & 33 & 0 & 28.7 & 24.2 & 3.5 & 25.3 & 25.6 & 52.5 & 62.5 & 11.3 & 41.3 & 9. & & 0 & & & 5.8 & .9 \\
\hline $25 / 0$ & 34 & 0 & 8.7 & 4.2 & 3.6 & 25.4 & 25.6 & 52.3 & 62.2 & 11.1 & 41.7 & & & & & & & \\
\hline $25 / 01$ & $: 35$ & 0 & 28.8 & 4.2 & 3.5 & 25.4 & 25.7 & 52.4 & 62.4 & 11.3 & 41.6 & & & & & & & 47.0 \\
\hline 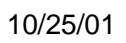 & 36 & & 8.8 & 10 & 3.7 & 25.4 & 257 & 526 & 62.5 & 11.2 & 41.9 & & & & & & & \\
\hline /25/01 & $: 37$ & 0 & 28.8 & 4.2 & .7 & 25.4 & 25.7 & 52.9 & 63.0 & 11.3 & 41.8 & & & & & & & \\
\hline 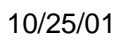 & 38 & & 3.9 & 1.2 & 8 & 25.4 & 25.6 & 2.3 & 62.2 & 1.2 & 41.8 & & & & & & & \\
\hline & :39 & 0 & 8.9 & 1.2 & 8 & 25.4 & 25.7 & 52.3 & 62.1 & 11.2 & 41.7 & & & & & & & \\
\hline 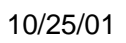 & 40 & & 3.9 & 1.3 & 7 & 25.5 & 25.7 & 6 & 62.7 & 11.2 & 41.8 & & & & & & & \\
\hline & 41 & U & 9.0 & 4.3 & & 25.6 & 25.8 & & 62.0 & 11.3 & & & & & & & & \\
\hline 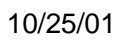 & 42 & & 9.0 & 1.3 & .0 & 25.5 & 25.7 & 8.7 & 62.6 & 11.2 & 41.9 & & & & & & & \\
\hline & 13 & 0 & 9.0 & 4.4 & & 25.5 & 5.8 & & 62.4 & 11.3 & & & & & & & & \\
\hline 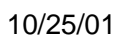 & 44 & & 9.0 & .4 & .0 & 25.5 & 25.8 & 2.5 & 62.4 & 11.2 & 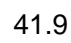 & & & & & & & \\
\hline & :45 & 0 & & 4.4 & & & & & 62.3 & 11.2 & & & & & & & & \\
\hline & 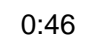 & & 9.1 & .4 & .1 & 25.5 & 25.8 & 2.5 & 62.3 & 11.1 & & & & & & & & \\
\hline & & 0 & & & & & & & 62.2 & 11.3 & & & & & & & & \\
\hline & :48 & & 9.2 & 4.4 & 9.1 & 25.5 & 25.9 & 2.7 & 62.6 & 1.3 & & & & & & & & \\
\hline & & 0 & 9.2 & 1.4 & .1 & & & & 62.1 & 11.1 & & & & & & & & \\
\hline & :5 & & 9.3 & 1.5 & 9.2 & 25.6 & 5.9 & 2.7 & 62.7 & .2 & & & & & & & & \\
\hline & :5 & 0 & 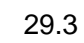 & .5 & .2 & 6 & & & 62.2 & 11.2 & & & & & & & & \\
\hline & $: 5$ & & 3 & 4.5 & 9.3 & 25.6 & 25.9 & 2.5 & 62.4 & 11.2 & & & & & & & & \\
\hline & :5 & & 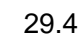 & .5 & 3 & 25.6 & .9 & & 62.8 & 11.3 & & & & & & & & \\
\hline & :5 & & 4 & .5 & 9.3 & 25.6 & 25.9 & & 62.5 & 2 & & & & & & & & \\
\hline & 5 & & - & & .5 & 25.7 & 5.0 & & 62.4 & 11.2 & & & & & & & & \\
\hline & :5 & & 5 & 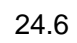 & 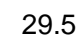 & 25.6 & 25.9 & & 62.6 & & & & & & & & & \\
\hline & :5 & & & & & 25.6 & .0 & & 62.4 & & & & & & & & & \\
\hline & $0: 5$ & & 2 & 1.6 & 14 & 25.6 & 25.9 & & 62.9 & & & & & & & & & \\
\hline & $0: 5$ & & 2 & & .6 & 25.7 & 26.0 & & 62.1 & & & & & & & & & \\
\hline & . & & & & & 25.7 & 26.0 & & 62.9 & & & & & & & & & \\
\hline & & & & & .7 & 25.7 & 26.0 & & 62.7 & & & & & & & & & \\
\hline & & & & & & 25.7 & & & 62.7 & & & & & & & & & \\
\hline & & & & & 7 & 25.7 & 0 & & 62.7 & & & & & & & & & \\
\hline & & & & & & & & & 63.0 & & & & & & & & & \\
\hline & ( & & & & & 5.7 & 0 & & 62.8 & & & & & & & & & \\
\hline & & & & & & & & & 62.8 & & & & & & & & & \\
\hline & & & & & & .7 & .0 & & 62.9 & & & & & & & & & \\
\hline & & & & & & & & & 62.8 & & & & & & & & & \\
\hline & (C) & & & & & & & & 62.8 & & & & & & & & & \\
\hline & & & & & & & & & & & & & & & & & & \\
\hline & & & & & & & & & 62.6 & & & & & & & & & \\
\hline & & & & & & & & & & & & & & & & & & \\
\hline & & & & & & & & & & & & & & & & & & \\
\hline & & & & & & & & & & & & & & & & & & \\
\hline & & & & & & & & & & & & & & & & & & \\
\hline & & & & & & & & & & & & & & & & & & \\
\hline & & & & & & & & & & & & & & & & & & \\
\hline & & & & & & & & & & & & & & & & & & \\
\hline & & & & & & & & & & & & & & & & & & \\
\hline & & & & & & & & & & & & & & & & & & \\
\hline & & & & & & & & & & & & & & & & & & \\
\hline & $1: 2$ & & & & & & & & & & & & & & & & & \\
\hline & & & & & & & & & & & & & & & & & & \\
\hline & 1.6 & & & & & & & & & & & & & & & & & \\
\hline & & & & & & & & & & & & & & & & & & \\
\hline & 1.6 & & 2 & & & & & & 62.2 & & & & & & & & & \\
\hline & & & & & & & & & & & & & & & & & & \\
\hline & 1.6 & & & & & & & & 62 & & & & & & & & & \\
\hline & 1. & c & & & & & & & & & & & & 81 & 71 & 992 & .8 & \\
\hline
\end{tabular}




\begin{tabular}{|c|c|c|c|c|c|c|c|c|c|c|c|c|c|c|c|c|c|c|}
\hline & & & $\begin{array}{c}\text { FLTRT } \\
\left({ }^{\circ}{ }^{\circ}\right) \\
\text { T2 }\end{array}$ & $\begin{array}{c}\mathrm{CL} \\
\text { LOOP } \\
\left.{ }^{\circ} \mathrm{C}\right) \\
\text { T3 }\end{array}$ & $\begin{array}{l}\text { SL } \\
\text { LOOP } \\
\left({ }^{\circ} \mathrm{C}\right) \\
\mathrm{T} 1\end{array}$ & $\begin{array}{l}\text { UP } \\
\text { AMB } \\
\left({ }^{\circ} \mathrm{C}\right) \\
\text { T4 }\end{array}$ & $\begin{array}{l}\text { BOT } \\
\text { AMB } \\
\left({ }^{\circ} \mathrm{C}\right) \\
\text { T5 }\end{array}$ & $\begin{array}{l}\text { BOT } \\
\text { DP } \\
\text { (psid) } \\
\text { dP2 }\end{array}$ & $\begin{array}{l}\text { FLTR } \\
\text { (psig) } \\
\text { P1 }\end{array}$ & $\begin{array}{l}\text { FLTR } \\
\text { DP } \\
\text { (psid) } \\
\text { dP1 }\end{array}$ & $\begin{array}{c}\text { TOP } \\
\text { DP } \\
\text { (psig) } \\
\text { dP3 }\end{array}$ & $\begin{array}{l}\text { FLT- } \\
\text { RATE } \\
\text { (psig) } \\
\text { P2 }\end{array}$ & $\begin{array}{c}\text { BP } \\
\text { (psig) } \\
\text { P3 }\end{array}$ & $\begin{array}{c}\text { SL } \\
\text { FLOW } \\
\text { (gpm) } \\
\text { Q1 }\end{array}$ & $\begin{array}{l}\text { FLTR } \\
\text { FLOW } \\
\text { (gpm) } \\
\text { Q2 }\end{array}$ & $\begin{array}{c}\text { Temp } \\
\text { corrected } \\
\text { flow } \\
\left(\mathrm{gpm} / \mathrm{tt}^{2}\right)\end{array}$ & & \\
\hline 25/01 & 1:30 & 0 & 27.5 & 25.1 & 27.1 & 26.0 & 26.4 & 52.4 & 62.2 & 11.0 & 42.0 & 96 & & 38.1 & 0.071 & & 158 & \\
\hline 25/01 & 31 & 0 & 27.5 & 25.1 & 27.1 & 26.0 & 26.4 & 52.5 & 62.1 & 11.1 & 42.2 & & & & & & & 7.4 \\
\hline 1 & 32 & 0 & 7.5 & 25.1 & .2 & 26.0 & 26.4 & 52.5 & 62.2 & 10.9 & 41.7 & & & .1 & & & & \\
\hline |25/01 & $: 33$ & 0 & 27.5 & 25.1 & 1 & 26.0 & 26.4 & 52.4 & 62.0 & 11.0 & 42.0 & 9 & & .2 & & & & \\
\hline & 34 & 0 & 27.5 & 25.2 & & 26.1 & & 525 & 62.3 & .1 & & & & 38.3 & & & & \\
\hline /25/01 & $: 35$ & 0 & 27.5 & 25.1 & 7.1 & 26.1 & 26.4 & 52.5 & 62.3 & 11.0 & 41.8 & 0 & & 3.3 & 70 & & & 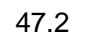 \\
\hline & 36 & 0 & 27.5 & 25.1 & & 26.1 & .5 & 526 & 2.2 & 1.1 & 42.2 & & & 3 & & & & \\
\hline /25/01 & :37 & 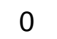 & 27.5 & 25.2 & .1 & 1 & 26.4 & 52.4 & 62.0 & 11.2 & 42.2 & & & .4 & & & & 47.3 \\
\hline 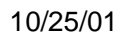 & 38 & 0 & 7.4 & 25.2 & & 26.0 & & 520 & 2.6 & 1.2 & 42.0 & & & 3 & & & & \\
\hline 1 & :39 & & 27.4 & 25.2 & & 26.1 & 26.4 & 527 & 2.4 & .1 & 41.9 & & & 3 & & & & 7.3 \\
\hline$/ 25 / 01$ & 10 & 0 & 27.4 & & & 26.0 & & & 2.1 & & & & & & & & & \\
\hline (2) & $: 41$ & 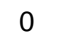 & 27.5 & 25.2 & .2 & 26.0 & 26.5 & 52.4 & 2.1 & 11.2 & 41.8 & & & 3 & & & & \\
\hline ser & 12 & 0 & 27.4 & & & 26.0 & & & 2.0 & & & & & & & & & \\
\hline & :43 & & 27.4 & & & 26.0 & 26.5 & 52.7 & 2.3 & 11.2 & 42.2 & & & 3 & & & & 7.4 \\
\hline & :44 & 0 & 7.5 & & & 26.0 & & & .1 & & & & & & & & & \\
\hline & & & 7.4 & & & & & & & & & & & & & & & \\
\hline & :4 & 0 & 7.5 & & & 26.1 & 4 & & 2 & & & & & & & & & \\
\hline & & 0 & 7.5 & 2 & & 26.1 & .5 & & 62.6 & 11.2 & & & & & & & & \\
\hline & & 0 & 7.5 & & & 26.2 & .5 & & 2.5 & & & & & & & & & \\
\hline & & c & 7.5 & & & 26.2 & 6 & & 62.2 & & & & & & & & & \\
\hline & & & .5 & & & 26.1 & 5 & & 62.5 & & & & & & & & & \\
\hline & & & 7.5 & & & 26.1 & 26.5 & & 62.2 & & & & & & & & & \\
\hline & & 0 & 5 & & & & 26.6 & & 62.4 & & & & & & & & & \\
\hline & & & 7.5 & & J & 26.2 & 26.6 & & 62.5 & 11 & & & & & & & & .3 \\
\hline & & & 7.5 & & & 26.3 & 26.6 & & 2.4 & & & & & & & & & \\
\hline & & & 7.5 & & & 26.2 & 26.6 & & 2.3 & & & & & & & & & \\
\hline & & & 7.5 & & & 26.1 & 6 & & 1 & & & & & & & & & \\
\hline & & & 7.5 & & & & 26.6 & & 2.2 & & & & & & & & & \\
\hline & & & 7.5 & & & 26.1 & 26.6 & & 3 & & & & & & & & & \\
\hline & & & 7.6 & & & 26.2 & 26.6 & & 2.4 & & & & & & & & & \\
\hline & & & .5 & & & & 6 & & .2 & & & & & & & & & 7. \\
\hline & & & 7.5 & & & & 26.6 & & 2.1 & & & & & & & & & \\
\hline & & & 6 & & & & 6 & & 6 & & & & & & & & & 7. \\
\hline & & & & & & & & & 2.0 & & & & & & & & & \\
\hline & & & & & & & & & .3 & & & & & & & & & 7.8 \\
\hline & & & & & & & & & & & & & & & & & & \\
\hline & & c & & & & & & & 3 & & & & & & & & & . \\
\hline & & & & & & & & & & & & & & & & & & \\
\hline & & & & & & & & & & & & & & & & & & \\
\hline & & & & & & & & & & & & & & & & & & \\
\hline & & & & & & & & & & & & & & & & & & \\
\hline & & & & & & & & & & & & & & & & & & \\
\hline & & & & & & & & & & & & & & & & & & \\
\hline & & & & & & & & & & & & & & & & & & \\
\hline & & & & & & & & & & & & & & & & & & \\
\hline & & & & & & & & & & & & & & & & & & \\
\hline & & & & & & & & & & & & & & & & & & \\
\hline & & & & & & & & & & & & & & & & & & \\
\hline & & & & & & & & & & & & & & & & & & \\
\hline & & & & & & & & & & & & & & & & & & \\
\hline & & 0 & & & & & & & & & & & & & & & & \\
\hline & & & & & & & & & & & & & & & & & & \\
\hline & & & & & & & & & & & & & & & & & & \\
\hline & & & & & & & & & & & & & & & & & & \\
\hline & & c & & & & & & & & & & & & & & & & \\
\hline & & & & & & & & & & & & & & & & & & \\
\hline & & c & & & & & & & & & & & & & & & & \\
\hline & & & & & & & & & & & & & & & & & & \\
\hline & .60 & ( & 2 & & 27.0 & & & & & 1.2 & & & & 8.0 & $0 / 0$ & $0<05$ & 0.0 & \\
\hline
\end{tabular}




\begin{tabular}{|c|c|c|c|c|c|c|c|c|c|c|c|c|c|c|c|c|c|c|}
\hline & & Sol & $\begin{array}{l}\text { FLTRT } \\
\left({ }^{\circ} \mathrm{C}\right) \\
\mathrm{T} 2\end{array}$ & $\begin{array}{l}\mathrm{CL} \\
\text { LOOP } \\
\left({ }^{\circ} \mathrm{C}\right) \\
\text { T3 }\end{array}$ & $\begin{array}{l}\text { SL } \\
\text { LOOP } \\
\left({ }^{\circ} \mathrm{C}\right) \\
\mathrm{T} 1\end{array}$ & $\begin{array}{l}\text { UP } \\
\text { AMB } \\
\left({ }^{\circ} \mathrm{C}\right) \\
\text { T4 }\end{array}$ & $\begin{array}{c}\text { Xflow2 } 2 \\
\text { BOT } \\
\text { AMB } \\
{ }^{\left({ }^{\circ} \mathrm{C}\right)} \\
\text { T5 }\end{array}$ & $\begin{array}{l}\text { BOT } \\
\text { DP } \\
\text { (psid) } \\
\text { dP2 }\end{array}$ & $\begin{array}{l}\text { FLTR } \\
\text { (psig) } \\
\text { P1 }\end{array}$ & $\begin{array}{c}\text { FLTR } \\
\text { DP } \\
\text { (psid) } \\
\text { dP1 }\end{array}$ & $\begin{array}{c}\text { TOP } \\
\text { DP } \\
\text { (psig) } \\
\text { dP3 }\end{array}$ & $\begin{array}{l}\text { FLT- } \\
\text { RATE } \\
\text { (psig) } \\
\text { P2 }\end{array}$ & $\begin{array}{c}\text { BP } \\
\text { (psig) } \\
\text { P3 }\end{array}$ & $\begin{array}{l}\text { SL } \\
\text { FLOW } \\
\text { (gpm) } \\
\text { Q1 }\end{array}$ & $\begin{array}{c}\text { FLTR } \\
\text { FLOW } \\
\text { (gpm) } \\
\text { Q2 }\end{array}$ & $\begin{array}{c}\text { Temp } \\
\text { corrected } \\
\text { flow } \\
\left(\mathrm{gpm} / \mathrm{ft}^{2}\right)\end{array}$ & $\begin{array}{c}\text { Axial Vel } \\
(\mathrm{ft} / \mathrm{sec})\end{array}$ & $\begin{array}{l}\text { Avg } \\
\text { TMP } \\
\text { (psid) }\end{array}$ \\
\hline |25/01 & 2:29 & 0 & 27.9 & 25.9 & 27.7 & 26.4 & 26.8 & 52.8 & 62.5 & 11.2 & 42.0 & 9.5 & & & 0.070 & & 15.8 & 47.4 \\
\hline /25/01 & 30 & 0 & 27.9 & 25.9 & 27.5 & 26.4 & 26.8 & 3.1 & 62.9 & 11.3 & 42.1 & 9. & & 38.2 & 0.070 & . 02855 & .9 & 47.6 \\
\hline /25/01 & :31 & 0 & 27.9 & 25.9 & 27.7 & 26.3 & 26.9 & 8.8 & 2.5 & 11.1 & 42.2 & 0 & & 38.2 & 0.070 & & & 75 \\
\hline$/ 25 / 01$ & 2:32 & 0 & 27.9 & 25.9 & 27.7 & 26.3 & 26.8 & 2.9 & 62.5 & 11.3 & 42.2 & 9. & & 38.1 & 0.070 & 0284 & 15.8 & 7.5 \\
\hline 25 & :33 & 0 & 28.0 & 9 & 27.7 & 26.3 & 26.9 & 3.0 & 2.6 & 11.3 & 42.6 & 9 & & 8.2 & 0.070 & & & 78 \\
\hline 25/01 & 34 & 0 & 28.0 & 5.9 & 27.5 & 26.4 & 26.8 & 2.8 & 2.4 & 11.3 & 42.0 & & & 3.2 & 0.070 & & & 7.4 \\
\hline & 35 & 0 & 8.0 & 5.9 & 27.8 & 26.4 & 26.9 & .9 & 6 & 11.2 & 42 & & & & & & & \\
\hline & :36 & & 8.0 & 5.9 & 27.7 & 26.4 & 26.8 & 52.9 & 62.5 & 11.3 & 42.3 & 9. & & 3.2 & 0.070 & & & 7.6 \\
\hline & :37 & 0 & 8.0 & 5.9 & 27.7 & 26.4 & 26.9 & 52.7 & 2.5 & 11.3 & 42 & & & & & & & \\
\hline & 2:38 & 0 & 8.0 & 5.9 & 27.8 & 26.4 & 26.9 & 2.5 & .2 & 11.2 & 42.3 & & & & & & & 7.4 \\
\hline & 2:39 & 0 & 28.0 & & 27.9 & 26.4 & 26.9 & 52.7 & 62.4 & 11.2 & 42.1 & & & & & & & 47 \\
\hline 4 & $2: 40$ & 0 & 28.1 & 5.9 & 27.8 & 26.5 & 26.9 & 52.9 & 62.7 & 11.3 & 42.4 & & & 1 & & & & 7.7 \\
\hline 1 & $2: 41$ & 0 & 28.1 & & 27.7 & 26.5 & & 53.0 & 62.7 & 11.3 & 42.0 & & & & & & & 47 \\
\hline 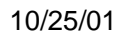 & $: 42$ & & 8.1 & .0 & 27.8 & 26.5 & 26.9 & 2.9 & 62.6 & 11.3 & 42.2 & 9. & & 1 & & & & 10 \\
\hline & $2: 43$ & 0 & 8.2 & & 28.0 & 26.5 & & 3.1 & 62.8 & 11.4 & 42.3 & & & & & & & 47 \\
\hline & 2:44 & & 3.2 & 0 & 27.9 & 26.5 & 26.9 & .7 & 2.4 & 11.3 & 42.1 & & & & & & & 47.4 \\
\hline & $2: 45$ & 0 & 8.2 & & 27.8 & 26.5 & 26.7 & 2.9 & 2.6 & 1.3 & 42.3 & & & & & & & 47.6 \\
\hline 1 & 2:46 & 0 & 8.2 & 0 & 28.0 & 26.4 & 26.5 & 3.0 & 62.7 & 1.3 & 42.1 & & & .1 & & & & \\
\hline (2) & 47 & 0 & 8.3 & & 27.8 & 26.4 & 26.4 & 2.7 & 62.3 & 11.2 & 42.3 & & & & & & & 47. \\
\hline & :48 & 0 & 8.3 & 0 & 3.1 & 26.4 & 26.2 & 2.7 & 2.3 & 11.3 & 42. & & & & & & & 47 \\
\hline & & & & & 28.0 & & 26.1 & 3.2 & 3.0 & 2 & & & & & & & & \\
\hline & :5 & 0 & 8.3 & .0 & 28.1 & 26.2 & 26.0 & .0 & 2.7 & 11.4 & 42 & & & & & & & \\
\hline & 5 & 0 & 3.4 & 0 & 28.1 & 26.1 & 25.9 & 3.1 & 62.7 & 11.4 & 42 & & & & & & & 7.6 \\
\hline & $2: 5$ & 0 & 8.4 & .0 & 200 & 26.1 & 25.7 & .9 & 2.6 & .4 & 42 & & & & & & & \\
\hline & $: 5$ & & 8.4 & .0 & 28.2 & 26.0 & 25.6 & 3.2 & 63.0 & 11.3 & 42 & & & & & & & 47.8 \\
\hline & 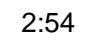 & 0 & 8.4 & 0 & 28.2 & 25.9 & 25.5 & .9 & 2.7 & .3 & 42 & & & & & & & 47 \\
\hline & 5 & & 8.5 & 0 & 28.2 & 25.9 & 25.5 & 5 & 62.4 & 11.2 & 42 & & & & & & & 47.4 \\
\hline & $2: 56$ & 0 & 5 & 0 & 28.2 & 25.8 & 25.4 & .2 & 2.9 & .2 & 42 & & & & & & & 47 \\
\hline & 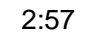 & & 5 & 0 & 28.3 & 25.8 & 25.3 & .9 & 62.6 & 11.3 & 42 & & & & & & & 47.6 \\
\hline & 2:5 & & & .0 & 28.4 & 25.8 & 25.2 & .9 & 2.6 & .3 & 42 & & & & & & & 47 \\
\hline & $5 s$ & & D & 0 & 2 & 25.7 & 25.1 & 3.0 & 2.7 & 11.2 & 42. & & & & & & & 47. \\
\hline & & 0 & 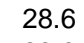 & & & 25.6 & 25.0 & - & 3.3 & .3 & & & & & & & & \\
\hline & 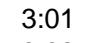 & & 6 & & & ? & & .9 & 2.5 & .3 & 42 & & & & & & & \\
\hline & & & & & & & & & & & & & & & & & & \\
\hline & & & & & & 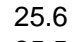 & & & 2.7 & 3 & . & & & & & & & \\
\hline & & & & & & & & & & & & & & & & & & \\
\hline & & & & & & 0 & & & 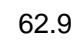 & 3 & 4 & & & & & & & 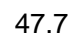 \\
\hline & & & & & & & & & & & & & & & & & & \\
\hline & $3: 0$ & & P & & 3.5 & .4 & 6 & & 2.8 & .2 & 42 & & & & & & & \\
\hline & & & & & & & & & & & & & & & & & & \\
\hline & & & & & & 4 & & & 8 & 3 & 42.1 & & & & & & & 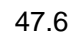 \\
\hline & & & & & & & & & & & & & & & & & & \\
\hline & & & & & & & & & 6 & 3 & 42 & & & & & & & . \\
\hline & & & & & & & & & & & & & & & & & & \\
\hline & & & & & & & & & & 3 & & & & & & & & \\
\hline & & & & & & & & & & & & & & & & & & \\
\hline & & & & & & & & & & & & & & & & & & \\
\hline & & & & & & & & & & & & & & & & & & \\
\hline & & & & & & & & & & & & & & & & & & \\
\hline & & & & & & & & & & & & & & & & & & \\
\hline & & & & & & & & & & & & & & & & & & \\
\hline & & & & & & & & & & & & & & & & & & \\
\hline & $3:$ & 0 & & & & & & & & & & & & & & & & \\
\hline & & & & & & & & & & & & & & & & & & \\
\hline & ") & & & & & & & & & & & & & & & & & \\
\hline & & & & & & & & & & & & & & & & & & \\
\hline & & & & & & & & & & & & & & & & & & \\
\hline & & & & & & & & & & & & & & & & & & \\
\hline & & & & & 26.7 & & & & & & & & & & & & & \\
\hline & & & & & & & & & & & & & & & & & & \\
\hline & גת & c & 2 & & 268 & & & & &. & 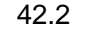 & & & 8.2 & 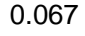 & 78 & 15.8 & \\
\hline
\end{tabular}


Xflow2 102401_0630

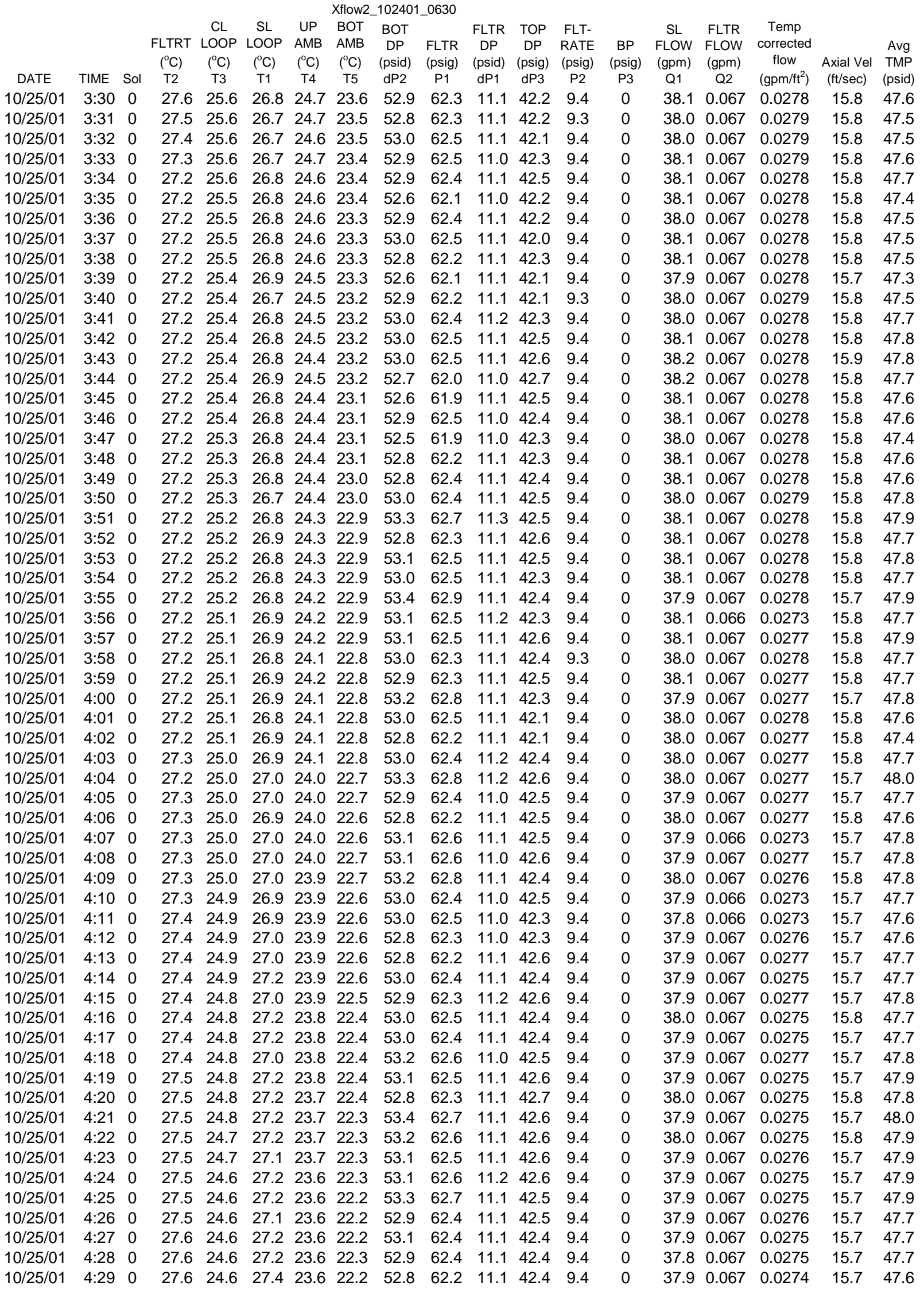




\begin{tabular}{|c|c|c|c|c|c|c|c|c|c|c|c|c|c|c|c|c|c|c|}
\hline & TIME & Sol & $\begin{array}{l}\text { FLTRT } \\
\left({ }^{\circ} \mathrm{C}\right) \\
\text { T2 }\end{array}$ & $\begin{array}{c}\text { CL } \\
\text { LOOP } \\
\left({ }^{\circ} \mathrm{C}\right) \\
\text { T3 }\end{array}$ & $\begin{array}{l}\text { SL } \\
\text { LOOP } \\
\left({ }^{\circ} \mathrm{C}\right) \\
\mathrm{T} 1\end{array}$ & $\begin{array}{l}\text { UP } \\
\text { AMB } \\
\left({ }^{\circ} \mathrm{C}\right) \\
\text { T4 }\end{array}$ & $\begin{array}{c}\text { Xflow2 } \\
\text { BOT } \\
\text { AMB } \\
\left({ }^{\circ} \mathrm{C}\right) \\
\text { T5 }\end{array}$ & $\begin{array}{c}102401 \\
\text { BOT } \\
\text { DP } \\
\text { (psid) } \\
\text { dP2 }\end{array}$ & $\begin{array}{c}\text { FLTR } \\
\text { (psig) } \\
\text { P1 }\end{array}$ & $\begin{array}{c}\text { FLTR } \\
\text { DP } \\
\text { (psid) } \\
\text { dP1 }\end{array}$ & $\begin{array}{c}\text { TOP } \\
\text { DP } \\
\text { (psig) } \\
\text { dP3 }\end{array}$ & $\begin{array}{l}\text { FLT- } \\
\text { RATE } \\
\text { (psig) } \\
\text { P2 }\end{array}$ & $\begin{array}{c}\text { BP } \\
\text { (psig) } \\
\text { P3 }\end{array}$ & $\begin{array}{c}\text { SL } \\
\text { FLOW } \\
\text { (gpm) } \\
\text { Q1 }\end{array}$ & $\begin{array}{l}\text { FLTR } \\
\text { FLOW } \\
\text { (gpm) } \\
\text { Q2 }\end{array}$ & $\begin{array}{c}\text { Temp } \\
\text { corrected } \\
\text { flow } \\
\left(\mathrm{gpm} / \mathrm{ft}^{2}\right)\end{array}$ & $\begin{array}{c}\text { Axial Vel } \\
\text { (ft/sec) }\end{array}$ & $\begin{array}{l}\text { Avg } \\
\text { TMP } \\
\text { (psid }\end{array}$ \\
\hline 0/25/01 & 4:30 & 0 & 27.6 & 24.6 & 27.1 & 23.6 & 22.2 & 53.2 & 62.5 & 11.2 & 42.3 & 9.4 & 0 & 37.9 & 0.067 & 0.0276 & 15.7 & 47.7 \\
\hline 0/25/01 & $4: 31$ & 0 & 27.6 & 24.6 & 27.2 & 23.6 & 22.2 & 53.0 & 62.3 & 11.2 & 42.4 & 9.4 & 0 & 37.9 & 0.067 & 0.0275 & 15.7 & 47.7 \\
\hline 0/25/01 & $4: 32$ & 0 & 27.6 & 24.6 & 27.4 & 23.6 & 22.2 & 53.0 & 62.4 & 11.1 & 143.0 & 9.4 & 0 & 37.9 & 0.067 & 0.0273 & 15.7 & 48.0 \\
\hline 0/25/01 & $4: 33$ & 0 & 27.7 & 24.5 & 27.2 & 23.6 & 22.2 & 53.4 & 62.9 & 11.2 & 42.7 & 9.4 & 0 & 37.9 & 0.067 & 0.0275 & 15.7 & 48.1 \\
\hline 0/25/01 & $4: 34$ & 0 & 27.7 & 24.5 & 27.4 & 23.6 & 22.2 & 53.3 & 62.8 & 11.2 & 42.5 & 9.4 & 0 & 38.0 & 0.067 & 0.0273 & 15.7 & 47.9 \\
\hline 0/25/01 & $4: 35$ & 0 & 27.7 & 24.5 & 27.2 & 23.5 & 22.2 & 53.5 & 63.0 & 11.2 & 42.8 & 9.4 & 0 & 37.9 & 0.067 & 0.0275 & 15.7 & 48.2 \\
\hline /25/01 & $4: 36$ & 0 & 27.7 & 24.5 & 27.3 & 23.6 & 22.2 & 53.3 & 62.7 & 11.2 & 42.7 & 9.4 & 0 & 37.9 & 0.067 & 0.0274 & 15.7 & 48.0 \\
\hline$/ 25 / 01$ & $4: 37$ & 0 & 27.7 & 24.5 & 27.4 & 23.6 & 22.1 & 53.1 & 62.5 & 11.2 & 42.3 & 9.4 & 0 & 37.9 & 0.067 & 0.0274 & 15.7 & 47.7 \\
\hline //25/01 & $4: 38$ & 0 & 27.7 & 24.4 & 27.6 & 23.6 & 22.1 & 53.0 & 62.4 & 11.2 & 42.5 & 9.4 & 0 & 37.8 & 0.067 & 0.0272 & 15.7 & 47.8 \\
\hline$/ 25 / 01$ & $4: 39$ & 0 & 27.8 & 24.4 & 27.6 & 23.5 & 22.1 & 53.1 & 62.4 & 11.1 & 42.5 & 9.4 & 0 & 37.9 & 0.067 & 0.0272 & 15.7 & 47.8 \\
\hline /25/01 & $4: 40$ & 0 & 27.8 & 24.4 & 27.6 & 23.5 & 22.2 & 53.5 & 63.0 & 11.2 & 42.6 & 9.4 & 0 & 37.9 & 0.067 & 0.0272 & 15.7 & 48.0 \\
\hline /25/01 & $4: 41$ & 0 & 27.8 & 24.4 & 27.5 & 23.5 & 22.2 & 53.1 & 62.5 & 11.2 & 42.8 & 9.4 & 0 & 37.9 & 0.067 & & 15.7 & \\
\hline /25/01 & $4: 42$ & 0 & 27.9 & 24.4 & 27.7 & 23.5 & 22.3 & 53.6 & 63.0 & 11.3 & 42.6 & 9.4 & 0 & 38.0 & 0.067 & 0.0272 & 15.8 & 48.1 \\
\hline /25/01 & $4: 43$ & 0 & 27.8 & 24.4 & 27.6 & 23.5 & 22.3 & 53.1 & 62.6 & 11.2 & 42.5 & 9. & 0 & 37.9 & 0.067 & & & 47.8 \\
\hline /25/01 & $4: 44$ & 0 & 27.9 & 24.4 & 27.7 & 23.4 & 22.4 & 53.3 & 62.7 & 11.2 & 42.6 & 9.4 & 0 & 38.0 & 0.067 & 0.0272 & 15.8 & 48.0 \\
\hline$/ 25 / 01$ & $4: 45$ & 0 & 27.9 & 24.4 & 27.7 & 23.5 & 22.4 & 53.4 & 62.7 & 11.2 & 42.7 & 9. & & 37.9 & 0.067 & & & \\
\hline /25/01 & $4: 46$ & 0 & 27.9 & 24.3 & 27.7 & 23.5 & 22.5 & 53.5 & 63.1 & 11.3 & 42.7 & 9.4 & 0 & 37.9 & 0.067 & 0.0271 & 15.7 & 48.1 \\
\hline /25/01 & $4: 47$ & 0 & 27.9 & 24.3 & 27.7 & 23.6 & 22.5 & 53.4 & 62.8 & 11.2 & 42.6 & 9 & & 38.0 & 0.067 & & & 48.0 \\
\hline $10 / 25 / 01$ & $4: 48$ & 0 & 27.9 & 24.3 & 27.7 & 23.6 & 22.5 & 53.5 & 62.9 & 11.2 & 42.6 & 94 & 0 & 37.9 & 0.067 & 0.0271 & 15.7 & 48.0 \\
\hline $10 / 25 / 01$ & $4: 49$ & 0 & 28.0 & 24.3 & 27.8 & 23.7 & 22.6 & 53.7 & 63.2 & 11.3 & 42.8 & 9.4 & & 38.0 & 0.067 & 0.0270 & 15 & 48.2 \\
\hline $10 / 25 / 01$ & $4: 50$ & 0 & 28.0 & 24.3 & 27.7 & 23.7 & 22.7 & 53.8 & 63.5 & 11.3 & 42.8 & 9.4 & 0 & 38.0 & 0.068 & 0275 & 15.8 & 48.3 \\
\hline $10 / 25 / 01$ & 4:51 & 0 & 28.0 & 24.3 & 28.0 & 23.8 & 22.8 & 53.1 & 62.5 & 11.2 & 42.8 & 9.4 & 0 & 37.9 & 0.067 & 0.0269 & . & 47.9 \\
\hline $5 / 01$ & 4:52 & 0 & 28.0 & 24.3 & 28.0 & 23.8 & 22.8 & 53.5 & 62.9 & 11.3 & 42.5 & 9. & 0 & 38.0 & 0.067 & 269 & & 48.0 \\
\hline $10 / 25 / 01$ & $4: 53$ & 0 & 28.1 & 24.3 & 28.0 & 23.9 & 22.9 & 53.2 & 62.5 & 11.2 & 42.4 & 9.4 & 0 & 37.9 & 0.067 & 269 & 15.7 & 47.8 \\
\hline $5 / 01$ & $4: 54$ & 0 & 28.1 & 24.3 & 27.9 & 23.9 & 22.9 & 53.2 & 62.7 & 11.2 & 42.3 & 9. & 0 & 37.9 & 0.067 & 70 & 1 & 47.7 \\
\hline $10 / 25 / 01$ & $4: 55$ & 0 & 28.1 & 24.3 & 28.1 & 24.0 & 22.9 & 53.2 & 62.6 & 11.3 & 42.7 & 9. & 0 & 38.0 & 0.067 & 68 & 15.8 & 48.0 \\
\hline 5/01 & $4: 56$ & 0 & 28.2 & 24.3 & 27.9 & 24.1 & 22.8 & 53.0 & 62.4 & 11.2 & 42.3 & 9 & 0 & 37.9 & 0.067 & 70 & 5.7 & 47.7 \\
\hline /25/01 & $4: 57$ & 0 & 28.2 & 24.3 & 28.1 & 24.1 & 22.8 & 53.1 & 62.6 & 11.2 & 42.8 & 9 & 0 & 37.9 & 0.068 & 72 & 15.7 & 47.9 \\
\hline$/ 25 / 01$ & $4: 58$ & 0 & 28.2 & 24.3 & 28.0 & 24.0 & 22.8 & 53.1 & 62.4 & 11.3 & 42.5 & 9. & 0 & 38.0 & 0.067 & 69 & 15.8 & 47.8 \\
\hline $10 / 25 / 01$ & $4: 59$ & 0 & 28.2 & 24.3 & 28.1 & 23.9 & 22.8 & 53.3 & 62.8 & 11.3 & 42.5 & 9. & 0 & 38.0 & 0.067 & 68 & 15.8 & 47.9 \\
\hline $10 / 25 / 01$ & $5: 00$ & 0 & 28.3 & 24.3 & 27.9 & 24.0 & 22.8 & 53.3 & 62.7 & 11.3 & 42.6 & 9. & 0 & 38.0 & 0.067 & 70 & 1 & 47.9 \\
\hline$/ 25 / 01$ & 5:01 & 0 & 28.2 & 24.2 & 27.7 & 23.9 & 22.7 & 53. & 62.5 & 11.3 & 42 & 9 & 0 & 38.1 & 0.067 & & & 47.9 \\
\hline $10 / 25 / 01$ & 5:02 & 0 & 28.1 & 24.2 & 27.5 & 24.0 & 22.8 & 52.8 & 62.2 & 11.2 & 42.4 & 9 & 0 & 38.1 & 0.066 & 69 & 1 & 47.6 \\
\hline /25/01 & 5:03 & 0 & 28.0 & 24.2 & 27.3 & 23.9 & 22.8 & 531 & 62.5 & 11.2 & 42 & & 0 & & 0.066 & & & 47.9 \\
\hline $10 / 25 / 01$ & 5:04 & 0 & 28.0 & 24.2 & 27.3 & 23.9 & 22.7 & 53.3 & 62.7 & 11.2 & 42.3 & 9 & 0 & 38.1 & 0.066 & 70 & 15.8 & 47.8 \\
\hline & $5: 05$ & 0 & 27.9 & 24.2 & 27.3 & 23.9 & 22.7 & & 62.5 & 11.1 & & & 0 & & & & & \\
\hline //25/01 & $5: 06$ & 0 & 27.8 & 24.2 & 27.3 & 23.8 & 22.6 & 53.0 & 62.3 & 11.1 & 42.5 & 9. & 0 & 38.1 & 0.066 & 70 & 15.8 & 47.8 \\
\hline & $5: 07$ & 0 & 27.8 & 24.2 & 27.3 & 23.8 & 22.6 & & 62.2 & 11.2 & & & 0 & & & & & 47.6 \\
\hline 01 & $5: 08$ & 0 & 27.7 & 24.2 & 27.3 & 23.7 & 22.5 & 5 & 62.7 & 11.1 & 42 & 9. & 0 & 38.0 & 0. & 71 & 15.8 & 47.9 \\
\hline & $5: 09$ & 0 & 27.7 & 24.2 & 27.3 & 23.8 & 22.5 & 5 & 62.4 & 11.2 & $4 t$ & & 0 & & 0. & & & 47.8 \\
\hline & $5: 10$ & 0 & 27.7 & 210 & 27.3 & 23.7 & 22.5 & 53 & 62.6 & 11.2 & 42 & 9 & 0 & 3 & 0. & 70 & & 47.9 \\
\hline & $5: 11$ & 0 & 27.7 & 24.2 & 27.3 & 23.7 & 22.5 & 52.9 & 62.4 & 11.1 & 42 & 9 & c & 38.1 & 0.066 & & 15 & 47.7 \\
\hline & $5: 12$ & 0 & 27.7 & & 27.3 & 23.7 & 22.4 & & 62.3 & 11.2 & 42 & & 0 & 38.1 & $0 . c$ & & & 47.6 \\
\hline /25/01 & $5: 13$ & 0 & 27.7 & 24.1 & 27.2 & 23.6 & 22.4 & 53.0 & 62.4 & 11.2 & 42.4 & 9 & 0 & 38.1 & 0.066 & 71 & 1 & 47.7 \\
\hline & $5: 14$ & 0 & & & & & 22.3 & & & & & & & & & & & \\
\hline $10 / 25 / 01$ & $5: 15$ & 0 & 27.7 & 24.1 & 27.2 & 23.6 & 22.3 & 52.9 & 62.2 & 11.1 & 42.2 & 9.3 & 0 & 38.0 & 0.066 & 271 & 15.8 & 47.5 \\
\hline & $5: 16$ & 0 & & & 27.3 & 235 & 22.3 & & & & & & & & & & & \\
\hline $10 / 25 / 01$ & $5: 17$ & 0 & 27.6 & 24.1 & 27.3 & 23.5 & 22.2 & 52.9 & 62.2 & 11.1 & 42.7 & 9.3 & 0 & 38.1 & 0.066 & 0270 & 15.8 & 47.8 \\
\hline & $5: 18$ & 0 & 27.7 & 24.1 & 27.3 & & 22.2 & 53 & 62.6 & 11.1 & & 0 & & & & & & \\
\hline & $5: 19$ & 0 & 27.7 & 24.1 & 27.4 & & 22.2 & 53.0 & 62.5 & 11.2 & 42 & 9 & 0 & 1 & 66 & 70 & 15.8 & 47.9 \\
\hline & 5:20 & 0 & 27.7 & 24.0 & 27.3 & & 22.2 & 53 & 62.3 & 11.2 & & 9 & & & & & & \\
\hline & $5: 21$ & 0 & 27.6 & 24.0 & 27.3 & & 22.1 & & 62.8 & 11.2 & 42 & 0 & 0 & & 6 & 70 & 15 & 47.9 \\
\hline & 5:22 & 0 & 27.7 & 24.0 & 27.3 & 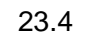 & 22.2 & 53.5 & 62.9 & 11.2 & & 9 & & & & & & \\
\hline & $5: 23$ & 0 & 27.6 & & 27.3 & & 22.1 & & & & & 0 & 0 & & & 70 & & 47.9 \\
\hline & $5: 24$ & 0 & 27.7 & 24.0 & 27.3 & & 22.1 & 53.1 & 62.4 & 11.2 & & 9 & & & & & & 47.7 \\
\hline & $5: 25$ & 0 & 27 & & 27 & & & & & & & 9 & 0 & & & 69 & & \\
\hline & 5:26 & 0 & 27.7 & 24.0 & 27.3 & & 22.2 & 53.3 & 62.7 & 11.2 & 42 & 9 & & & & & 18 & 47.9 \\
\hline & $5: 27$ & 0 & 27 & & 27 & & 22 & & & & & 9 & & & & & & \\
\hline $25 / 01$ & $5: 28$ & 0 & 27.7 & 24.0 & 27.3 & 23.4 & 22.3 & 52.8 & 62.1 & 11.2 & 42.4 & 9.3 & 0 & 38.0 & .066 & 0.0270 & 15.8 & 47.6 \\
\hline
\end{tabular}




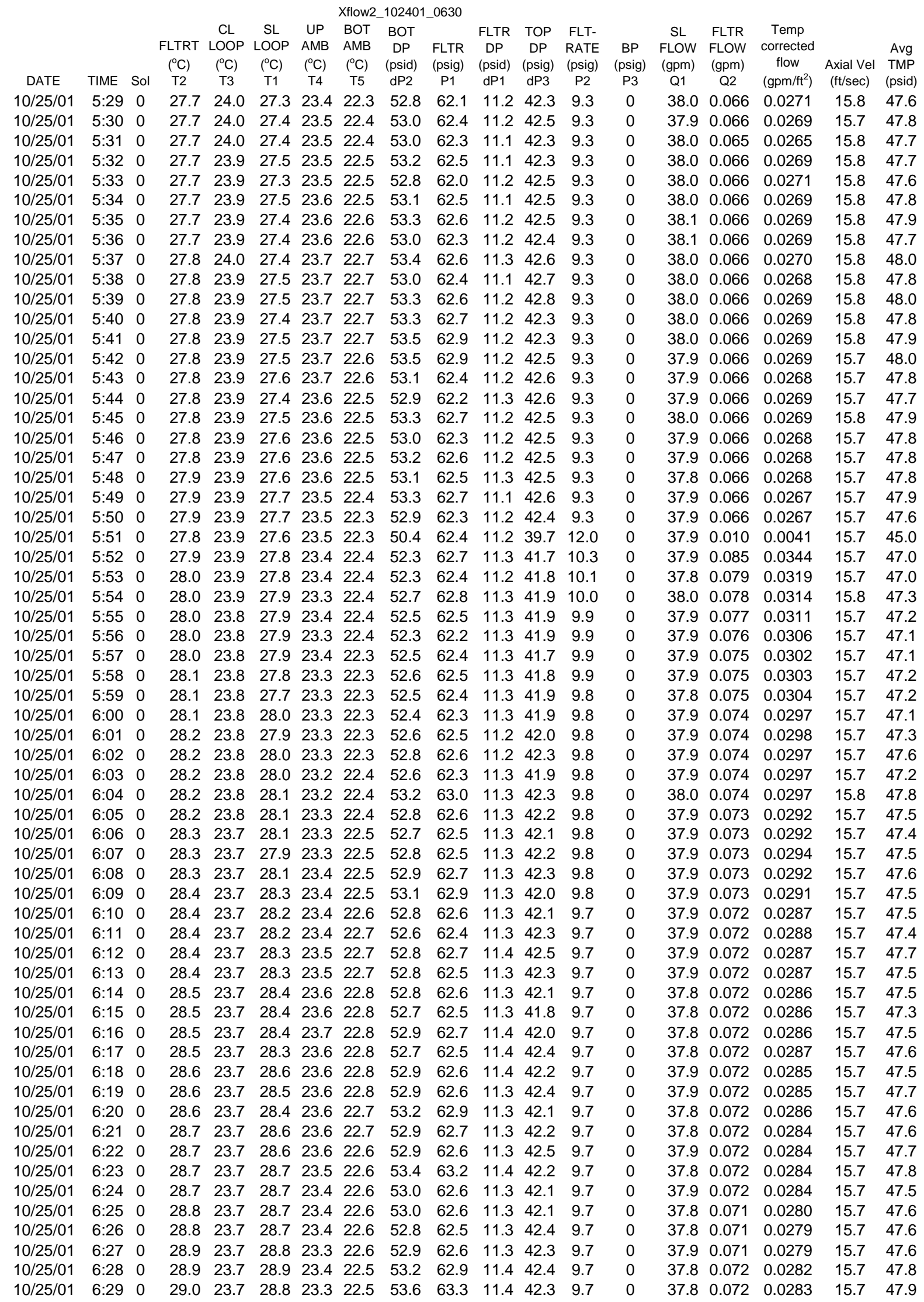


WSRC-TR-2002-00459, Rev. 0 SRT-RPP-2002-00221

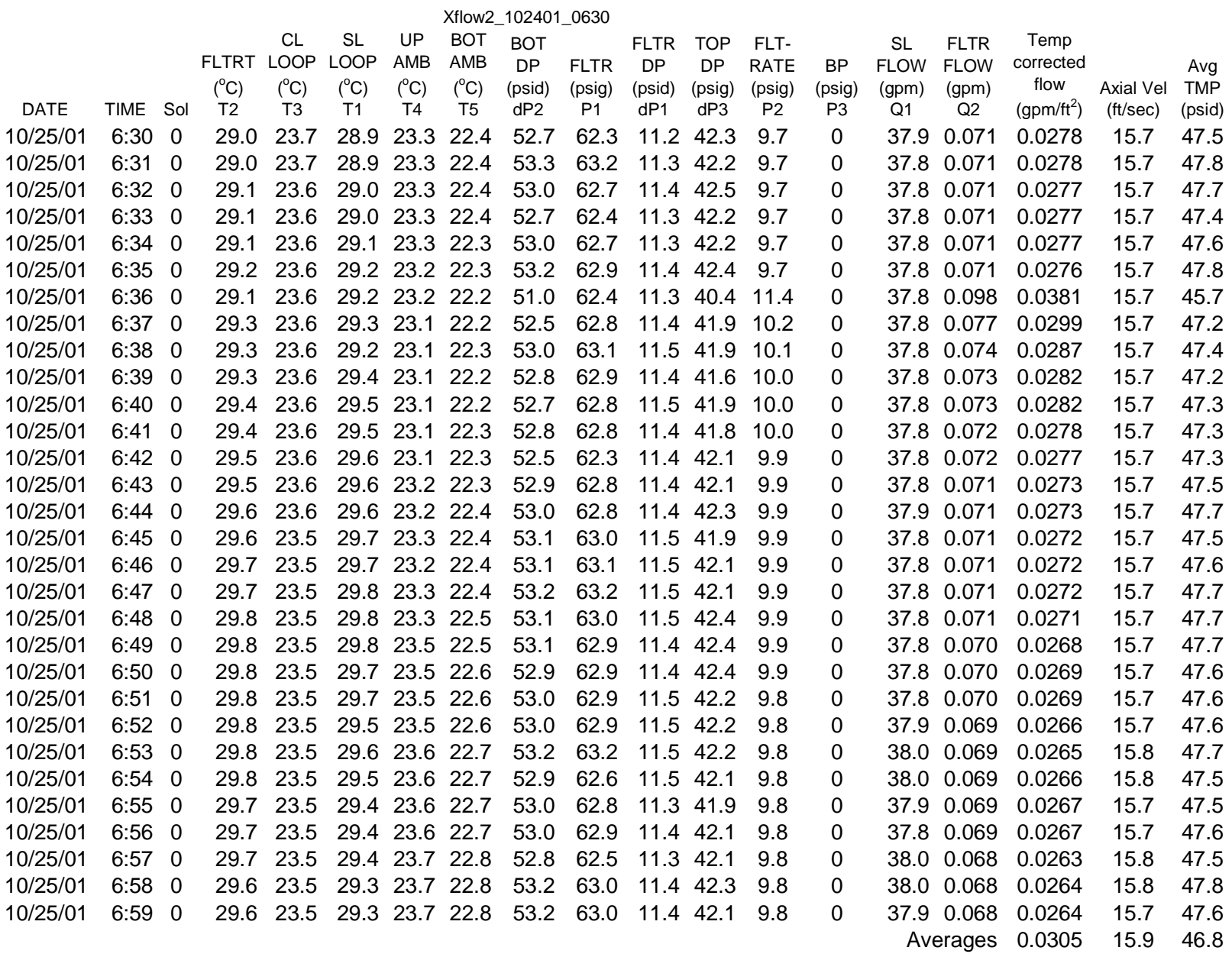




\begin{tabular}{|c|c|c|c|c|c|c|c|c|c|c|c|c|c|c|c|c|c|c|}
\hline DATE & ME & Sol & $\begin{array}{c}\text { FLTRT } \\
\left({ }^{\circ} \mathrm{C}\right) \\
\text { T2 }\end{array}$ & $\begin{array}{c}\mathrm{CL} \\
\text { LOOP } \\
\left({ }^{\circ} \mathrm{C}\right) \\
\mathrm{T} 3\end{array}$ & $\begin{array}{c}\text { SL } \\
\text { LOOP } \\
\left({ }^{\circ} \mathrm{C}\right) \\
\mathrm{T} 1\end{array}$ & $\begin{array}{c}\text { UP } \\
\text { AMB } \\
\left({ }^{\circ} \mathrm{C}\right) \\
\text { T4 }\end{array}$ & $\begin{array}{l}\text { BOT } \\
\text { AMB } \\
\left({ }^{\circ} \mathrm{C}\right) \\
\text { T5 }\end{array}$ & $\begin{array}{l}\text { BOT } \\
\text { DP } \\
\text { (psid) } \\
\text { dP2 }\end{array}$ & $\begin{array}{l}\text { FLTR } \\
\text { (psig) } \\
\text { P1 }\end{array}$ & $\begin{array}{c}\text { FLTR } \\
\text { DP } \\
\text { (psid) } \\
\text { dP1 }\end{array}$ & $\begin{array}{c}\text { TOP } \\
\text { DP } \\
\text { (psig) } \\
\text { dP3 }\end{array}$ & $\begin{array}{l}\text { FLTRATE } \\
\text { (psig) P2 }\end{array}$ & $\begin{array}{c}\text { BP } \\
\text { (psig) } \\
\text { P3 }\end{array}$ & $\begin{array}{c}\text { SL } \\
\text { FLOW } \\
\text { (gpm) } \\
\text { Q1 }\end{array}$ & $\begin{array}{c}\text { FLTR } \\
\text { FLOW } \\
\text { (gpm) } \\
\text { Q2 }\end{array}$ & $\begin{array}{c}\text { Temp corr } \\
\text { flow } \\
\left(\mathrm{gpm} / \mathrm{ft}^{2}\right)\end{array}$ & $\begin{array}{c}\text { Axial Vel } \\
(\mathrm{ft} / \mathrm{sec})\end{array}$ & $\begin{array}{l}\text { Avg } \\
\text { TMP } \\
\text { (psid) }\end{array}$ \\
\hline 0/25/01 & 7:00 & 0 & 29.6 & 23.5 & 29.2 & 23.7 & 22.8 & 53.1 & 62.8 & 11.4 & 42.2 & 9.8 & 0 & 38.0 & 0.068 & 0.0264 & 15.8 & 47.6 \\
\hline$/ 25 / 01$ & 7:01 & 0 & 29.5 & 23.5 & 29.2 & 23.7 & 22.7 & 53.2 & 63.0 & 11.4 & 42.4 & 9.7 & 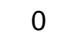 & 38.0 & 0.068 & & & 47.8 \\
\hline$/ 25 / 01$ & 7:02 & 0 & 29.5 & 23.5 & 29.2 & 23.6 & 22.7 & 52.7 & 62.5 & 11.4 & 42.1 & 9.7 & 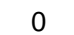 & 38.0 & 0.068 & & & 47.4 \\
\hline$/ 25 / 01$ & 7:03 & 0 & 29.5 & 23.5 & 29.3 & 23.6 & 22.6 & 53.1 & 62.9 & 11.3 & 42.4 & 9.7 & & 38.0 & 0.068 & & & 47.8 \\
\hline & 7:04 & 0 & & 23.5 & 29.2 & 23.5 & 22.6 & 52.9 & 62.7 & 11.4 & 42.4 & 9.7 & & 37.9 & 68 & & & 47.6 \\
\hline & 7:05 & 0 & 29.5 & 23.5 & 29.3 & 23.5 & 22.6 & 52.8 & 62.4 & 11.4 & 42.3 & 9.7 & & 38.0 & 0.068 & & & 47.6 \\
\hline & :06 & 0 & 29.5 & 23.5 & 29.3 & 23.5 & 22.5 & 53.1 & 62.9 & 11.4 & 42.1 & 9.7 & & 38.0 & & & & 47.6 \\
\hline & :07 & 0 & 29.5 & 23.5 & 29.3 & 23.4 & 22.5 & 52.9 & 62.7 & 11.4 & 41.9 & .7 & & 7.9 & 68 & & & 474 \\
\hline & :08 & 0 & 9.5 & 23.5 & 29.3 & 3.4 & 22.4 & 53.0 & 62.7 & 11.5 & 42.2 & 7 & & 8.0 & & & & 47.6 \\
\hline & :09 & 0 & 9.5 & 23.5 & 29.4 & 23.4 & 22.4 & 52.8 & 62.6 & 11.4 & 42.3 & .7 & & 37.9 & & & & 47.6 \\
\hline 01 & 10 & 0 & 9.6 & 23.5 & 29.4 & 23.4 & 22.4 & 53.0 & 62.7 & 11.3 & 42.0 & .7 & & 37.9 & 0.068 & & & 47.5 \\
\hline & :11 & 0 & 9.6 & 23.5 & 29.5 & 23.4 & 22.3 & 53.1 & 62.8 & 11.3 & 42.2 & .7 & & 37.9 & 68 & & & 47.6 \\
\hline 01 & 12 & 0 & 9.6 & 23.5 & 29.5 & 23.4 & 22.3 & 53.1 & 62.8 & 11.4 & 42.4 & .7 & & 37.9 & 68 & & & 47.7 \\
\hline 01 & :13 & 0 & 9.6 & 23.5 & 29.5 & 23.3 & 22.2 & 52.8 & 62.6 & 11.4 & 42.3 & .7 & & 37.8 & 0.068 & & & 47.6 \\
\hline 01 & 14 & 0 & 29.6 & 23.5 & 29.6 & 23.3 & 22.2 & 53.0 & 62.8 & 11.4 & 42.1 & .7 & & 37.9 & 0.068 & & & 47.6 \\
\hline 01 & :15 & 0 & 29.7 & 23.5 & 29.5 & 23.3 & 22.2 & 52.7 & 62.4 & 11.3 & 42.3 & 9.7 & & 37.8 & 0.068 & & & 47.5 \\
\hline$/ 25 / 01$ & :16 & 0 & 29.7 & 23.5 & 29.6 & 23.3 & 22.1 & 53.0 & 62.7 & 11.4 & 42.2 & 9.7 & & 37.8 & 0.067 & & & 47.6 \\
\hline$/ 25 / 01$ & :17 & 0 & 29.7 & 23.4 & 29.7 & 23.2 & 22.1 & 53.1 & 62.9 & 11.3 & 42.3 & 9.7 & & 37.8 & 0.068 & & & 47.7 \\
\hline$/ 25 / 01$ & :18 & 0 & 29.7 & 23.4 & 29.7 & 23.2 & 22.2 & 53.4 & 63.1 & 11.3 & 42.4 & 9.7 & & 37.7 & 0.068 & & & 47.9 \\
\hline$/ 25 / 01$ & :19 & 0 & 29.8 & 23.4 & 29.7 & 23.2 & 22.2 & 53.0 & 62.9 & 11.3 & 42.6 & 97 & & 37.7 & & & & 47.8 \\
\hline$/ 25 / 01$ & 20 & 0 & 29.8 & 23.4 & 29.7 & 23.1 & 22.2 & 52.8 & 62.5 & 11.2 & 42.5 & 97 & & 37.7 & & & & 47.7 \\
\hline $1 / 25 / 01$ & :21 & 0 & 2 & 23.4 & 29.7 & 23.1 & 22.2 & 53.4 & 63.2 & 11.3 & 42.4 & 97 & & 37.7 & & & & 47.9 \\
\hline $1 / 25 / 01$ & 7:22 & 0 & 2 & 23.4 & 29.8 & 23.1 & 22.2 & 52.9 & 62.7 & 11.1 & 42.4 & 0. & & .5 & & & & 47.7 \\
\hline$/ 25 / 01$ & $7: 23$ & 0 & 299 & 23.4 & 29.8 & 23.2 & 22.2 & 53.0 & 62.7 & 11.3 & 42.7 & 7 & & 37.7 & & & & 47.9 \\
\hline$/ 25 / 01$ & $7: 24$ & 0 & 0 & 23.4 & 29.7 & 23.3 & 22.2 & 53.2 & $6 r$ & 2 & 42.3 & 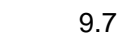 & & .7 & & & & 7.7 \\
\hline & 7:25 & 0 & & 23.4 & 29.6 & 23.2 & 22.3 & 53.2 & & & 42.9 & & & & & & & 3.1 \\
\hline & 7:26 & 0 & & 23.4 & 29.6 & 23.3 & 22.3 & 53.0 & & & 42.5 & & & & & & & 7.7 \\
\hline & 27 & 0 & & 23.4 & 29.6 & 23.3 & 22.3 & 52.9 & & & 42.4 & & & & & & & .6 \\
\hline & 28 & 0 & & 23.4 & 29.6 & & 22.4 & 53. & & & 42.6 & & & & & & & \\
\hline & 29 & 0 & & 23.4 & 29.6 & & 22.4 & 5 & & & 42 & & & & & & & \\
\hline & :30 & 0 & 8 & 23.4 & 29.6 & 23.4 & 22.4 & 53. & & & 42 & & & 6 & & & & 7.8 \\
\hline & 31 & 0 & 8 & 23.4 & 29.6 & 23.4 & 22.5 & 53.2 & & 2 & 42. & & & 6 & & & & 47.8 \\
\hline & :32 & 0 & & 23.4 & 29.6 & 23.4 & 22.5 & 52.9 & 6 & 2 & 42. & & & .7 & & & & 47.6 \\
\hline & 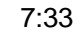 & 0 & & 23.4 & 29.7 & 23.5 & 22.5 & 53.0 & & & 42. & & & 6 & & & & 47.7 \\
\hline & :34 & 0 & & 23.4 & 29.7 & 23.5 & 22.5 & 53.1 & 6 & & 42 & & & .6 & & & & 17.8 \\
\hline & 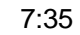 & 0 & & 23.4 & 29.7 & 23.5 & 22.6 & 53.0 & 6 & & 42.3 & & & .6 & & & & 47.6 \\
\hline & 36 & 0 & & 23.4 & 29.8 & 3.5 & 22.6 & 52.8 & & & 42.7 & & & .6 & & & & 47.8 \\
\hline & 37 & 0 & & 23.3 & 29.7 & 3.6 & 22.6 & 53.4 & & & 42.6 & & & 6 & & & & 18.0 \\
\hline & :38 & 0 & & 23.4 & 29.8 & 3.6 & 22.7 & 53.2 & & & 42.4 & & & .7 & & & & 47.8 \\
\hline & 39 & 0 & & 23.3 & 29.8 & 3.6 & 22.7 & 53.4 & & & 42.4 & & & 6 & & & & 47.9 \\
\hline & 40 & 0 & & 23.3 & 29.8 & 23.7 & 22.6 & 53.2 & & & 42.8 & 9.7 & & 7.7 & & & & 48.0 \\
\hline & $7: 41$ & 0 & & 23.3 & 29.9 & 23.6 & 22.6 & 53.2 & & & 42.6 & - & & 37.9 & & & & 47.9 \\
\hline & $7: 42$ & 0 & & 23.3 & 29.9 & 23.6 & 22.6 & 53.1 & & & 42.6 & 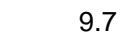 & & 7.9 & & & & 47.8 \\
\hline & $7: 43$ & 0 & & 23.3 & 29.9 & 23.5 & 22.5 & 53.3 & & & 42.5 & 9 & & 7.9 & & & & 47.9 \\
\hline & $7: 44$ & 0 & & 23.3 & 29.9 & 23.5 & 22.5 & 53.3 & & & 42.7 & s & & 7.8 & & & & 48.0 \\
\hline & $7: 45$ & 0 & & 23.4 & 29.8 & 23.4 & 22.5 & 53.3 & & & 42.5 & & & 37.8 & & & & 47.9 \\
\hline & $7: 46$ & 0 & & 23.4 & 29.9 & 23.4 & 22.5 & 52.9 & & & 42.4 & & & 37.8 & & & & 47.7 \\
\hline 01 & $7: 47$ & 0 & 1 & 23.4 & 30.0 & 23.4 & 22.4 & 52.9 & $6 r$ & 1 & 42.2 & 9.7 & & 37.7 & 0.066 & & & 47.6 \\
\hline & U & 0 & & 23.4 & 30.0 & 3 & 22.4 & 53 & & & 42 & & & & & & & 47.5 \\
\hline 1 & $7: 4$ & 0 & & 23.3 & 30.0 & 23.3 & 22.3 & 53 & 6 & II & 42.8 & & & 37.5 & 0.067 & & & 7.9 \\
\hline 1 & $7: 5$ & 0 & & 23.3 & 30.1 & 23.3 & 22.3 & 53. & & 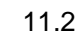 & 42.8 & & & 37.5 & 0.067 & & & 3.0 \\
\hline & $7: 5$ & 0 & & 23.3 & 30.0 & 20.3 & 22.3 & 53 & & & 42 & & & & & & & \\
\hline & & 0 & & 23.3 & 29.9 & 20.2 & LL.L & 53 & & & 42. & & & & & & & \\
\hline & & 0 & & 23.3 & & 20.2 & 22.2 & 53 & & & 42.8 & & & & & & & 3.0 \\
\hline & 7 & 0 & & 23.3 & 30.0 & & 22.1 & 5 & & & 42.8 & & & & & & & 8.0 \\
\hline & $7: 5$ & 0 & & 23.3 & 30.1 & 23.1 & 22.1 & 53 & & & 42.7 & & & 6 & & & & 8.0 \\
\hline & 7 & 0 & & 23.3 & 30.2 & 1 & 22.1 & 53. & & 11.2 & 42.6 & & & 7.5 & & & & 17.9 \\
\hline & $7: 5$ & 0 & 0.0 & 23.3 & 30.2 & 0 & 22.1 & 53.3 & & 11.2 & 42.6 & & & 37.5 & & & & 18.0 \\
\hline & 1.4 & 0 & 30.3 & 23.3 & 30.3 & 3.0 & 22.1 & 53.1 & 62 & 11.1 & 42.7 & & & 37.4 & & & & 47.9 \\
\hline $0 / 25 / 01$ & $7: 59$ & 0 & 30.4 & 23.3 & 30.3 & 3.0 & 2.1 & 53.2 & 62.8 & 11.2 & 42.9 & 9.7 & 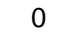 & 37.5 & 0.067 & 0.0253 & 15.6 & 48.0 \\
\hline
\end{tabular}




\begin{tabular}{|c|c|c|c|c|c|c|c|c|c|c|c|c|c|c|c|c|c|c|}
\hline DTr & 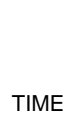 & Sol & $\begin{array}{c}\text { FLTRT } \\
\left({ }^{\circ} \mathrm{C}\right) \\
\text { T2 }\end{array}$ & $\begin{array}{c}\mathrm{CL} \\
\text { LOOP } \\
\left({ }^{\circ} \mathrm{C}\right) \\
\mathrm{T} 3\end{array}$ & $\begin{array}{c}\text { SL } \\
\text { LOOP } \\
\left({ }^{\circ} \mathrm{C}\right) \\
\mathrm{T} 1\end{array}$ & $\begin{array}{c}\text { UP } \\
\text { AMB } \\
\left({ }^{\circ} \mathrm{C}\right) \\
\mathrm{T} 4\end{array}$ & $\begin{array}{c}\text { BOT } \\
\text { AMB } \\
\left({ }^{\circ} \mathrm{C}\right) \\
\text { T5 }\end{array}$ & $\begin{array}{c}\text { BOT } \\
\text { DP } \\
\text { (psid) } \\
\text { dP2 }\end{array}$ & $\begin{array}{c}\text { FLTR } \\
\text { (psig) } \\
\text { P1 }\end{array}$ & $\begin{array}{l}\text { FLTR } \\
\text { DP } \\
\text { (psid) } \\
\text { dP1 }\end{array}$ & $\begin{array}{c}\text { TOP } \\
\text { DP } \\
\text { (psig) } \\
\text { dP3 }\end{array}$ & $\begin{array}{l}\text { FLTRATE } \\
\text { (psig) } \quad \text { P2 }\end{array}$ & $\begin{array}{c}\text { BP } \\
\text { (psig) } \\
\text { P3 }\end{array}$ & $\begin{array}{c}\text { SL } \\
\text { FLOW } \\
\text { (gpm) } \\
\text { Q1 }\end{array}$ & $\begin{array}{c}\text { FLTR } \\
\text { FLOW } \\
\text { (gpm) } \\
\text { Q2 }\end{array}$ & $\begin{array}{c}\text { Temp corr } \\
\text { flow } \\
\left(\mathrm{gpm} / \mathrm{ft}^{2}\right)\end{array}$ & $\begin{array}{l}\text { Axial Vel } \\
(\mathrm{ft} / \mathrm{sec})\end{array}$ & $\begin{array}{l}\text { Avg } \\
\text { TMP } \\
\text { (psid) }\end{array}$ \\
\hline $0 / 25 / 01$ & 8:00 & 0 & 30.4 & 23.3 & 30.3 & 23.1 & 22.1 & 53.2 & 62.9 & 11.3 & 42.6 & 9.7 & 0 & 37.5 & 0.067 & .0252 & 15.6 & 47.9 \\
\hline /25/01 & :01 & 0 & 30.4 & 23.3 & 30.4 & 3.1 & 22.2 & 53.5 & 63.2 & 11.3 & 42.7 & 9.7 & & 37.5 & 0.067 & & & 1 \\
\hline /25/01 & :02 & 0 & 30.4 & 23.3 & 30.4 & 3.1 & 22.2 & 53.3 & 63.0 & 11.1 & 42.8 & 9.7 & & 37.4 & 0.067 & & & 3.1 \\
\hline$/ 25 / 01$ & :03 & 0 & 0.4 & 23.3 & 30.4 & 23.2 & 22.2 & 53.4 & 63.2 & 11.2 & 42.9 & 9.7 & & 37.5 & 0.067 & & 6 & 48.1 \\
\hline /25/01 & :04 & 0 & 30.5 & 23.3 & 30.5 & 23.2 & 22.2 & 53.5 & 63.4 & 11.2 & 42.9 & 9.7 & & 37.5 & 0.067 & & & 48.2 \\
\hline /25/01 & :05 & 0 & 30.5 & 23.3 & 30.5 & 3.2 & 22.3 & 53.4 & 62.9 & 11.2 & 42.9 & 9.7 & & 37.5 & 0.067 & & & 48.1 \\
\hline /01 & :06 & 0 & 30.5 & 23.3 & 30.4 & 23.2 & 22.3 & 53.4 & 63.1 & 11.2 & 42.9 & 9.7 & & 37.5 & 0.067 & & & 48.2 \\
\hline 01 & 07 & 0 & 30.5 & 23.3 & 30.5 & 23.2 & 22.3 & 53.3 & 63.1 & 11.2 & 42.7 & .7 & & 37.5 & 0.067 & & & 8.0 \\
\hline 01 & :08 & 0 & 0.6 & 23.3 & 30.6 & 23.3 & 22.4 & 53.3 & 2.9 & 11.2 & 42.7 & .7 & & 37.4 & 0.067 & & & 8.0 \\
\hline /01 & 09 & 0 & 30.6 & 23.3 & 30.6 & 23.3 & 22.4 & 53.5 & 63.2 & 11.2 & 42.8 & 9.7 & 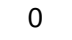 & 37.4 & 0.067 & & & 8.1 \\
\hline 01 & 10 & 0 & 30.7 & 23.3 & 30.7 & 23.3 & 22.4 & 53.2 & 63.0 & 11.1 & 42.6 & 9.7 & & 37.3 & .067 & & & 7.9 \\
\hline /25/01 & :11 & 0 & 30.7 & 23.3 & 30.7 & 23.3 & 22.4 & 53.4 & 63.1 & 11.2 & 42.5 & .7 & & 37.4 & 0.067 & & & 8.0 \\
\hline 01 & :12 & 0 & 7 & 23.3 & 30.8 & 23.4 & 22.5 & 53.9 & 63.7 & 11.3 & 42.8 & .7 & & 37.5 & 0.067 & & & 8.3 \\
\hline /01 & ::13 & 0 & 30.8 & 23.3 & 30.8 & 23.5 & 22.5 & 53.2 & 62.9 & 11.2 & 42.9 & 9.7 & & 37.4 & 0.067 & & & 8.1 \\
\hline /25/01 & $8: 14$ & 0 & 30.8 & 23.3 & 30.8 & 23.5 & 22.5 & 53.3 & 63.1 & 11.2 & 42.8 & 9.7 & & 37.4 & 0.067 & & & 48.1 \\
\hline /25/01 & $8: 15$ & 0 & 30.9 & 23.3 & 30.9 & 23.5 & 22.6 & 53.7 & 63.6 & 11.3 & 42.7 & 9.7 & ( & 37.4 & 0.067 & & & 48.2 \\
\hline /25/01 & $8: 16$ & 0 & 30.9 & 23.3 & 31.0 & 23.6 & 22.6 & 53.6 & 63.3 & 11.2 & 43.1 & 9.7 & ( & 37.4 & 0.067 & & & 48.3 \\
\hline /25/01 & $8: 17$ & 0 & 30.9 & 23.3 & 31.0 & 23.6 & 22.6 & 53.6 & 63.4 & 11.2 & 42.9 & 9.7 & c & 37.4 & 0.068 & & & 48.2 \\
\hline /25/01 & $8: 18$ & 0 & 31.0 & 23.3 & 31.0 & 23.6 & 22.6 & 53.4 & 63.2 & 11.2 & 42.8 & 9.7 & c & 37.4 & 0.068 & & & 48.1 \\
\hline /25/01 & $8: 19$ & 0 & 31.0 & 23.3 & 31.1 & 23.6 & 22.6 & 53.4 & 63.2 & 11.3 & 42.5 & 9.7 & c & 37.3 & 68 & & & 47.9 \\
\hline$/ 25 / 01$ & $8: 20$ & 0 & 31.1 & 23.3 & 31.1 & 23.7 & 22.6 & 53.6 & 63.4 & 11.3 & 42.6 & 9.7 & ( & 37.4 & 0.067 & & & 48.1 \\
\hline$/ 25 / 01$ & $8: 21$ & 0 & 31.1 & 23.3 & 31.0 & 23.8 & 22.5 & 53.5 & 63.2 & 11.3 & 43.1 & 9.7 & & 37.4 & 0.067 & & & 48.3 \\
\hline$/ 25 / 01$ & $8: 22$ & 0 & 31.1 & 23.3 & 31.0 & 23.8 & 22.5 & 53.7 & 63.5 & 11.2 & 42.8 & 9.7 & & 37.4 & 0.067 & & & 48.3 \\
\hline$/ 25 / 01$ & $8: 23$ & 0 & 311 & 23.3 & 31.0 & 23.7 & 22.4 & 53.6 & 63.4 & 11.2 & 42.8 & 9.7 & & & 0.068 & & & 48.2 \\
\hline$/ 25 / 01$ & $8: 24$ & 0 & 311 & 23.3 & 30.9 & 23.7 & 22.4 & 53.9 & 63.6 & 11.2 & 43.1 & 9.7 & 0 & & 0.067 & & & 48.5 \\
\hline 5/01 & 3:25 & 0 & 311 & 23.3 & 30.9 & 23.6 & 22.4 & 53.5 & 63.2 & 11.3 & 42.9 & 97 & 0 & & 0.067 & & & 8.2 \\
\hline 01 & 3:26 & 0 & & 23.2 & & 23.5 & 22.4 & & 63.2 & & 42.8 & 0 & & & & & & 3.1 \\
\hline /01 & $8: 27$ & 0 & & 23.2 & 30.9 & 23.5 & 22.3 & 53.7 & 63.5 & 11.3 & 42.8 & 9.7 & & & 67 & & & 8.2 \\
\hline 01 & :28 & 0 & & 23.3 & & 3.5 & 22.3 & & & & & & & & & & & .5 \\
\hline /25/01 & 3:29 & 0 & 1.0 & 23.3 & 30.9 & 23.4 & 22.3 & 53.9 & 63.6 & 11.4 & 43.0 & 8.1 & c & 7.3 & 0. & & 5.5 & 48.4 \\
\hline 01 & 30 & 0 & 0 & 23.3 & 30. & 23.4 & 22.2 & 53.4 & 63. & 3 & & .7 & & 3 & & & & 8.2 \\
\hline 01 & 31 & 0 & 0 & 23.3 & 30.8 & 23.3 & 22.2 & 54.3 & 64.0 & 4 & 43. & .7 & & 3 & 67 & & & 8.6 \\
\hline 01 & 8:32 & 0 & 0 & 23.3 & 30.8 & 23.3 & 22.2 & 53.7 & 63.4 & 1.3 & 1 & .7 & & .3 & 66 & & & 18.4 \\
\hline 01 & 3:33 & 0 & 0 & 23.3 & 30.8 & 23.3 & 22.1 & 53.8 & 63.5 & 1.3 & 43. & s & & 7.3 & & & & 48.5 \\
\hline 01 & 3:34 & 0 & 0 & 23.3 & 30.8 & 23.2 & 22.1 & 53.7 & 63.3 & 11.4 & 43. & 9 & & 7.4 & & & & 48.6 \\
\hline & :35 & 0 & J & 23.2 & 30.8 & 23.2 & 22.1 & 53.9 & 3. & 11.4 & 43 & s. & & 7.3 & & & & 48.6 \\
\hline & :36 & 0 & 0 & 23.2 & 30.7 & 3.1 & 22.1 & 53.9 & 63.5 & 4 & 43 & $s$ & & .3 & & & & 8.5 \\
\hline & 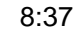 & 0 & 0.9 & 23.2 & 30 & 3.1 & 22.1 & 54.2 & 63.9 & 5 & 4 & 9 & & 3 & & & & 3.7 \\
\hline & 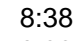 & 0 & 1.0 & 23.2 & 30 & 3.1 & 22.1 & 54 & 63.8 & 4 & 4 & & & .3 & & & & 8.7 \\
\hline & 39 & 0 & & 23.2 & 30 & 3.2 & 22.1 & 53.9 & 63.5 & 4 & 4 & & & .2 & & & & 3.5 \\
\hline & $: 40$ & 0 & & 23.2 & 30 & 3.2 & 22.2 & 54.0 & 63.7 & 3 & 4 & & & .3 & & & & 8.7 \\
\hline & 41 & 0 & & 23.2 & 30.9 & 3.2 & 22.2 & 53.8 & 63 & 4 & 43 & 6 & & .2 & & & & 8.5 \\
\hline & :42 & 0 & & 23.2 & 31.0 & 3.2 & 22.2 & 54.2 & & 11.4 & & & & .2 & & & & 8.7 \\
\hline & $8: 43$ & 0 & & 23.2 & 31.0 & 3.3 & 22.2 & 53.8 & & 11.4 & & 9.6 & & .2 & & & & 8.5 \\
\hline & $8: 44$ & 0 & & 23.2 & 31.1 & 3.4 & 22.3 & 54.0 & & & & 9.6 & & .2 & & & & 8.6 \\
\hline & 3:45 & 0 & & 23.2 & & & 22.3 & & & & & & & .2 & & & & 8.4 \\
\hline & $8: 46$ & 0 & & 23.2 & & 23.3 & .3 & & & .4 & & 6 & & .2 & & & & 48.7 \\
\hline & $8: 47$ & 0 & & 23.2 & & 23.4 & 22.4 & & 63.4 & & & & & .0 & & & & 48.6 \\
\hline & $8: 48$ & 0 & & 23.2 & 31.2 & 23.5 & 22.4 & 54.4 & 64.0 & 11.4 & 43.4 & 5 & & 7.1 & 0.064 & & & 48.9 \\
\hline & $8: 49$ & 0 & & 23.2 & & 3.4 & 22.4 & 54.1 & & & & .5 & & 7.1 & & & & 48.8 \\
\hline & $8: 50$ & 0 & & 23.2 & 31.3 & 23.5 & 22.4 & 54.1 & 63.5 & 11.4 & 43.5 & .5 & & 7.1 & 0.063 & & & 48.8 \\
\hline /01 & $8: 51$ & 0 & 1.3 & 23.2 & 31.4 & 23.5 & 22.5 & 53.8 & 63.3 & 11.3 & 43.3 & .5 & ( & 7.1 & 0.063 & & .4 & 48.5 \\
\hline /25/01 & $8: 52$ & 0 & 31.4 & 23.2 & 31.5 & 23.6 & 22.5 & 54.0 & 63.6 & 11.4 & 43.3 & .5 & 0 & 37.0 & 0.063 & & 5.4 & 48.6 \\
\hline /25/01 & $8: 53$ & 0 & 31.5 & 23.2 & 31.6 & 3.5 & 22.5 & 54.1 & 63.6 & 11.4 & 43.1 & .5 & 0 & 37.1 & 0.062 & & .4 & 48.6 \\
\hline 01 & $8: 54$ & 0 & 31.5 & 23.2 & 31.6 & 3.6 & 22.6 & 54.5 & 63.9 & 11.4 & 43.5 & .5 & 0 & 37.1 & 0.063 & & .4 & 49.0 \\
\hline 01 & $8: 55$ & 0 & 1.6 & 23.2 & 31.7 & 3.6 & 22.6 & 54.2 & 63.6 & 11.5 & 43.5 & .5 & 0 & 37.2 & 0.062 & & 4 & 48.8 \\
\hline /25/01 & $8: 56$ & 0 & 31.6 & 23.2 & 31.8 & 3.7 & 22.6 & 54.2 & 63.7 & 11.4 & 43.6 & .4 & 0 & 37.1 & 0.062 & & 4 & 48.9 \\
\hline /25/01 & $8: 57$ & 0 & 31.7 & 23.2 & 31.9 & 23.7 & 22.7 & 54.2 & 63.7 & 11.4 & 43.2 & 9.4 & 0 & 37.2 & 0.062 & 224 & .4 & 48.7 \\
\hline V & 8:58 & 0 & 31.8 & 23.2 & 31.9 & 23.8 & 22.7 & 54.3 & 63.7 & 11.3 & 43.2 & 9.4 & 0 & 37.1 & 0.062 & & 5.4 & 48.8 \\
\hline /25/01 & $8: 59$ & 0 & 31.8 & 23.2 & 32.0 & 23.7 & 22.7 & 54.4 & 63.8 & 11.4 & 43.1 & 9.4 & 0 & 37.0 & 0.062 & 0.0223 & 15.4 & 48.8 \\
\hline
\end{tabular}




\begin{tabular}{|c|c|c|c|c|c|c|c|c|c|c|c|c|c|c|c|c|c|c|}
\hline & TIME & Sol & $\begin{array}{l}\text { FLTRT } \\
\left({ }^{\circ} \mathrm{C}\right) \\
\text { T2 }\end{array}$ & $\begin{array}{l}\mathrm{CL} \\
\text { LOOP } \\
\left({ }^{\circ} \mathrm{C}\right) \\
\mathrm{T} 3\end{array}$ & $\begin{array}{c}\text { SL } \\
\text { LOOP } \\
\left({ }^{\circ} \mathrm{C}\right) \\
\mathrm{T} 1\end{array}$ & $\begin{array}{l}\text { UP } \\
\text { AMB } \\
\left({ }^{\circ} \mathrm{C}\right) \\
\text { T4 }\end{array}$ & $\begin{array}{c}\text { Xflow2 } \\
\text { BOT } \\
\text { AMB } \\
\left.{ }^{\circ} \mathrm{C}\right) \\
\text { T5 }\end{array}$ & $\begin{array}{c}102501 \\
\text { BOT } \\
\text { DP } \\
\text { (psid) } \\
\text { dP2 }\end{array}$ & $\begin{array}{c}\text { FLTR } \\
\text { (psig) } \\
\text { P1 }\end{array}$ & $\begin{array}{l}\text { FLTR } \\
\text { DP } \\
\text { (psid) } \\
\text { dP1 }\end{array}$ & $\begin{array}{c}\text { TOP } \\
\text { DP } \\
\text { (psig) } \\
\text { dP3 }\end{array}$ & $\begin{array}{l}\text { FLTRATE } \\
\text { (psig) }\end{array}$ & $\begin{array}{cc}\text { BP } \\
= \\
= & (p s i g) \\
2 & P 3\end{array}$ & $\begin{array}{c}\text { SL } \\
\text { FLOW } \\
\text { (gpm) } \\
\text { Q1 }\end{array}$ & $\begin{array}{c}\text { FLTR } \\
\text { FLOW } \\
\text { (gpm) } \\
\text { Q2 }\end{array}$ & $\begin{array}{l}\text { Temp corr } \\
\text { flow } \\
\left(\mathrm{gpm} / \mathrm{ft}^{2}\right)\end{array}$ & $\begin{array}{c}\text { Axial Vel } \\
\text { (ft/sec) }\end{array}$ & $\begin{array}{l}\text { Avg } \\
\text { TMP } \\
\text { (psid) }\end{array}$ \\
\hline o/25/01 & 9:00 & 0 & 31.9 & 23.2 & 32.1 & 23.7 & 22.7 & 54.3 & 63.7 & 11.4 & 43.8 & 9.4 & 40 & 37.1 & 0.062 & 0.0223 & 15.4 & 49.0 \\
\hline 10/25/01 & 9:01 & 0 & 32.0 & 23.2 & 32.2 & 23.8 & 22.7 & 54.1 & 63.5 & 11.3 & 43.5 & 9.4 & 4 & 37.1 & 0.061 & 0.0219 & 15.4 & 48.8 \\
\hline 10/25/01 & 9:02 & 0 & 32.0 & 23.2 & 32.3 & 23.7 & 22.7 & 54.5 & 63.9 & 11.5 & 43.5 & 9.4 & 4 & 37.1 & 0.062 & .0222 & 15.4 & 49.0 \\
\hline 10/25/01 & 9:03 & 0 & 32.1 & 23.2 & 32.3 & 23.6 & 22.6 & 54.3 & 63.6 & 11.4 & 43.5 & 9.4 & 0 & 37.0 & 0.061 & 0.0218 & 15.4 & 48.9 \\
\hline $10 / 25 / 01$ & 9:04 & 0 & 32.2 & 23.2 & 32.4 & 23.6 & 22.5 & 53.8 & 63.2 & 11.4 & 43.6 & 9.4 & 4 & 37.1 & 0.061 & .0217 & 5.4 & 8.7 \\
\hline $10 / 25 / 01$ & 9:05 & 0 & 32.3 & 23.2 & 32.5 & 23.5 & 22.5 & 54.3 & 63.7 & 11.4 & 43.6 & 9.3 & 0 & 37.1 & 0.061 & 0.0217 & 15.4 & 49.0 \\
\hline $10 / 25 / 01$ & 9:06 & 0 & 32.4 & 23.2 & 32.6 & 23.4 & 22.5 & 54.6 & 64.0 & 11.5 & 43.3 & 3 & 3 & 37.1 & 0.061 & & 15.4 & 9.0 \\
\hline 0/25/01 & 9:07 & 0 & 32.4 & 23.2 & 32.7 & 23.4 & 22.5 & 54.4 & 63.8 & 11.4 & 43.8 & 9.3 & 3 & 37.1 & 0.060 & .0212 & 15.4 & 49.1 \\
\hline o/25/01 & 9:08 & 0 & 32.5 & 23.2 & 32.8 & 23.4 & 22.4 & 54.5 & 63.9 & 11.5 & 43 & & 0 & 37.1 & & & 4 & 49.1 \\
\hline 0/25/01 & 9:09 & 0 & 32.6 & 23.2 & 32.9 & 23.4 & 22.4 & 54.4 & 63.7 & 11.3 & 43.6 & 9.3 & & 37.1 & 0.060 & 211 & 5.4 & 49.0 \\
\hline 01 & $9: 10$ & 0 & 32.7 & 23.2 & 33.0 & 23.4 & 22.4 & 54.0 & 63.3 & 11.4 & 43 & 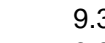 & ( & .1 & & & & \\
\hline D/25/0 & :11 & 0 & 32.8 & 23.2 & 33.1 & 23.4 & 22.4 & 54.6 & 63.9 & 11.4 & 43. & 9.3 & & 37.0 & 0.060 & 10 & 15.4 & 2 \\
\hline & $9: 12$ & 0 & 32.9 & 23.2 & 33.2 & 23.3 & 22.4 & 54.6 & 63.9 & 11.4 & 43 & 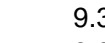 & c & 7.1 & & & & \\
\hline $1 / 2$ & :13 & 0 & 33.0 & 23.2 & 33.2 & 23.3 & 22.3 & 54.6 & 63.9 & 11.4 & 43.8 & 9.3 & 0 & 37.0 & 0.060 & 209 & 15.4 & 49.2 \\
\hline 01 & $9: 14$ & 0 & 33.1 & 23.2 & 33.4 & 23.3 & 22.3 & 54.5 & 63.7 & 11.4 & 43. & & 0 & 7.1 & & & & \\
\hline 0/25/01 & $9: 15$ & 0 & 33.1 & 23.2 & 33.5 & 23.3 & 22.3 & 54.5 & 63.7 & 11.5 & 43. & 9.3 & 0 & 7.1 & 0.060 & 208 & 15.4 & 49.0 \\
\hline $10 / 25 / 01$ & $9: 16$ & 0 & 33.2 & 23.2 & 33.5 & 23.2 & 22.2 & 54.8 & 64.1 & 11.6 & 43. & 9.2 & 0 & 7.2 & & & & \\
\hline 0/25/01 & $9: 17$ & 0 & 33.3 & 23.2 & 33.6 & 23.2 & 22.2 & 54.4 & 63.6 & 11.4 & 44. & 9.2 & & 7.2 & 0 & 03 & 15.4 & 49.2 \\
\hline $10 / 25 / 01$ & $9: 18$ & 0 & 33.4 & 23.2 & 33.7 & 23.2 & 22.1 & 54.7 & 63.9 & 11.4 & 43. & 9.2 & ( & 7.2 & & & & 49 \\
\hline $10 / 25 / 01$ & $9: 19$ & 0 & 33.5 & 23.2 & 33.9 & 23.2 & 22.1 & 54.4 & 63.6 & 11.5 & 43. & 9.2 & c & 7.0 & 0. & 02 & 15.4 & 49.1 \\
\hline 01 & $9: 20$ & 0 & 33.6 & 23.1 & 34.0 & 23.1 & 22.2 & 54.6 & 63.8 & 11.5 & 43 & 9.2 & ( & 7.1 & & & & 49.1 \\
\hline 10/25/01 & $9: 21$ & 0 & 33.7 & 23.2 & 34.1 & 23.2 & 22.2 & 54.7 & 63.9 & 11.5 & 43. & 9.2 & c & 7.1 & 0. & 01 & 15.4 & 49.3 \\
\hline 01 & $9: 22$ & 0 & 33.8 & 23.2 & 34.2 & 23.1 & 22.2 & 55.1 & 64.4 & 11.4 & 43 & 9.2 & ( & 7.2 & & & 5.4 & 45 \\
\hline 0/25/01 & $9: 23$ & 0 & 33.9 & 23.2 & 34.3 & 23.2 & 22.2 & 55.4 & 64.6 & 11.5 & 44. & 9.2 & & 7.1 & 0.059 & 00 & 15.4 & 49.7 \\
\hline 10/ & $9: 24$ & 0 & 34.0 & 23.1 & 34.4 & 23.2 & 22.2 & 54.8 & 64.0 & 11.5 & 44 & 9. & c & 7.0 & & & 5.4 & 49 \\
\hline 10/25/01 & $9: 25$ & 0 & 34.1 & 23.1 & 34.5 & 23.3 & 22.3 & 55.0 & 64.2 & 11.5 & 43. & 9.2 & ( & 7.2 & 0.058 & 95 & 5.4 & 49.4 \\
\hline $10 / 25 / 01$ & $9: 26$ & 0 & 34.2 & 23.1 & 34.6 & 23.3 & 22.3 & 55.2 & 64.5 & 11.5 & 44 & 9. & c & 7.1 & & & & 48 \\
\hline 10/25/01 & $9: 27$ & 0 & 34.3 & 23.1 & 34.7 & 23.4 & 22.3 & 54.9 & 64.0 & 11.5 & 44. & 9.1 & ( & 7.1 & 0.058 & 94 & 15.4 & 49 \\
\hline & $9: 28$ & 0 & 34.4 & 23.1 & 34.8 & 23.4 & 22.3 & 54.8 & 63.9 & 11.5 & & 9. & ( & & & & & \\
\hline 10/25/01 & $9: 29$ & 0 & 34.5 & 23.1 & 35.0 & 23.5 & 22.4 & 55.2 & 64.5 & 11.6 & 43 & 9.1 & & 37.0 & & 33 & 15.4 & 49.6 \\
\hline & 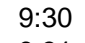 & & 3 & & & 23.5 & 22.4 & & 63.9 & & & & & & & & & \\
\hline$/ 01$ & 9:31 & 0 & 34.7 & 23.1 & 35.2 & 23.6 & 22.5 & 54.8 & 64.0 & 11.5 & 44 & 9.1 & & 37.2 & 0.058 & 92 & 15.4 & 49 \\
\hline & 9 & & & & & 7 & & & 64 & & & & & & & & & \\
\hline $10 / 2$ & $9: 33$ & 0 & 35.0 & 23.2 & 35.4 & 23.8 & 22.6 & 55.0 & 64.1 & 11.5 & 43 & 9.1 & & 37.1 & 0 & 91 & 15.4 & 49.4 \\
\hline & 4 & 0 & D & & 35.5 & 23.7 & 22.6 & 55.3 & 64.5 & 11.6 & & & & & & & & \\
\hline 10/25/01 & $9: 35$ & 0 & 35.2 & 23.1 & 35.6 & 23.7 & 22.6 & 54.7 & 63.8 & 11.5 & 44. & 9.1 & c & 7.2 & 0.057 & 86 & 15.4 & 49.4 \\
\hline & $9: 36$ & 0 & 35.3 & 23.2 & 35.7 & 23.8 & 22.7 & 54.8 & 63.8 & 11.6 & 43 & & & 7.2 & & & & \\
\hline 10/25/01 & 9:37 & 0 & 35.4 & 23.2 & 35.9 & 23.9 & 22.7 & 54.8 & 63.9 & 11.6 & 44. & 9. & ( & 37.2 & 0.056 & 82 & 15.4 & 49.4 \\
\hline & & & & & & & & & & & & & & & & & & \\
\hline & & 0 & 35.5 & 2 & 0 & 23.9 & 22.7 & 23.0 & 32.1 & 6.4 & 1 & & 0 & 33.1 & & 20 & 8 & 20.8 \\
\hline & 9 & & 2 & & & & & & -2.6 & 0.6 & & & & & & & & \\
\hline 01 & 9:40 & 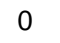 & 34.4 & 23.2 & 35.9 & 23.8 & 22.6 & -3.8 & -2.6 & 0.6 & -3.7 & 0.5 & c & 0.2 & 0 & 32 & -0.1 & -3.7 \\
\hline & 41 & & & & 35.9 & 23.6 & 22.6 & -0.8 & -2.8 & & & & & & & & & \\
\hline /01 & 9:42 & & 31.5 & 23.2 & 35.4 & 23.6 & 22.5 & -6.7 & -2.5 & 0.5 & -7 & 50 & & -0.2 & & & -0.1 & -7.2 \\
\hline & 43: & & 30.5 & & 35.8 & 23.5 & 22.5 & -0.9 & 2.9 & -1.9 & & & & & & & & \\
\hline /01 & $9: 44$ & & 32.3 & 23.2 & 35.8 & 23.4 & 22.5 & -0.4 & 3.4 & -1.9 & 0. & 50 & c & -0.2 & 10 & & -0.1 & 0.1 \\
\hline & 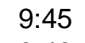 & & & & & 23.3 & 22.4 & -0.4 & 3.4 & -19 & 0. & & & & & & & \\
\hline & 9:46 & 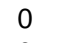 & 32.7 & .2 & 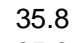 & 23.2 & 22.4 & -0.4 & 3.4 & -1.9 & 0. & 50 & & 0.2 & & & -0.1 & \\
\hline & 9 & & & & 3 & & & -0.4 & 3.4 & & & & & & & & & \\
\hline & $9: 4$ & & ? & & & 3.1 & 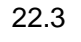 & -0 & 3.4 & & & & & -0.2 & & & & \\
\hline & & & & & & & & & 3. & & & & & & & & & \\
\hline & 9:50 & 0 & 32.5 & .2 & 30.6 & 23.0 & 22.2 & -0.2 & 3.6 & -1.9 & 0 & & ( & -0.2 & & & & \\
\hline & $9: 51$ & 0 & & & 27.9 & & & -0 & 3.5 & & & & & & & & & \\
\hline & $9: 52$ & 0 & 32.3 & 3.1 & 28.6 & 23.0 & 22.2 & -0.4 & 3.4 & -1.9 & 0 & & c & -0.2 & & & & \\
\hline & $9: 53$ & 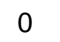 & & & 28.9 & & 22.2 & -0 & 3. & -1 & 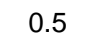 & & & -0.2 & & & & \\
\hline & $9: 54$ & 0 & 32.2 & 3.1 & 29.0 & 22.9 & 22.1 & -0.4 & 3.4 & -1.9 & 0 & & 0 & -0 & & & & \\
\hline & $9: 55$ & 0 & 0 & 23.1 & 29 & 22.9 & 22 & $-v$ & 3. & -1 & 0. & & 0 & -0 & & & & \\
\hline 5/01 & 9:56 & 0 & 32.0 & 23.1 & 29.3 & 22.8 & 22.1 & -0.4 & 3.4 & -1.9 & 0. & & 0 & -0.2 & 0.070 & & -0 & \\
\hline & 9.57 & 0 & & & & & & & & & & & & & & & & \\
\hline & 9.58 & & & & & & & & & & & & & & & & -01 & \\
\hline
\end{tabular}


WSRC-TR-2002-00459, Rev. 0 SRT-RPP-2002-00221

\begin{tabular}{|c|c|c|c|c|c|c|c|c|c|c|c|c|c|c|c|c|c|c|}
\hline & & & & & & & & & & & & & & & & & & \\
\hline & & & $\begin{array}{l}\text { FLTRT } \\
\left({ }^{\circ} \mathrm{C}\right)\end{array}$ & $\begin{array}{c}\mathrm{CL} \\
\text { LOOP } \\
\left({ }^{\circ} \mathrm{C}\right)\end{array}$ & $\begin{array}{l}\text { SL } \\
\text { LOOP } \\
\left({ }^{\circ} \mathrm{C}\right)\end{array}$ & $\begin{array}{c}\text { UP } \\
\text { AMB } \\
\left({ }^{\circ} \mathrm{C}\right)\end{array}$ & $\begin{array}{l}\text { BOT } \\
\text { AMB } \\
\left({ }^{\circ} \mathrm{C}\right)\end{array}$ & $\begin{array}{l}\text { BOT } \\
\text { DP } \\
\text { (psid) }\end{array}$ & $\begin{array}{l}\text { FLTR } \\
\text { (psig) }\end{array}$ & $\begin{array}{l}\text { FLTR } \\
\text { DP } \\
\text { (psid) }\end{array}$ & $\begin{array}{l}\text { TOP } \\
\text { DP } \\
\text { (psig) }\end{array}$ & FLTRATE & $\begin{array}{l}\text { BP } \\
\text { (psig) }\end{array}$ & $\begin{array}{c}\text { SL } \\
\text { FLOW } \\
\text { (gpm) }\end{array}$ & $\begin{array}{c}\text { FLTR } \\
\text { FLOW } \\
\text { (gpm) }\end{array}$ & $\begin{array}{l}\text { Temp corr } \\
\text { flow }\end{array}$ & & $\begin{array}{l}\text { Avg } \\
\text { TMP }\end{array}$ \\
\hline DATE & TIME & Sol & $\mathrm{T} 2$ & T3 & $\mathrm{T} 1$ & $\mathrm{~T} 4$ & T5 & $\mathrm{dP} 2$ & P1 & $\mathrm{dP} 1$ & $\mathrm{dP3}$ & P2 & P3 & 21 & 2 & $\left.t^{2}\right)$ & & \\
\hline /25/01 & $9: 59$ & 0 & 31.7 & 23.1 & 27.6 & 22.8 & 22.0 & -0.4 & 3.4 & -1.9 & 0.6 & 5.0 & 0 & -0.2 & 0.010 & 1 & & .1 \\
\hline$/ 25 / 01$ & $10: 00$ & 0 & 31.7 & 23.1 & 27.1 & 22.7 & 22.0 & -0.4 & 3.4 & -1.9 & 0.5 & 5.0 & & -0.2 & & & & .1 \\
\hline$/ 25 / 01$ & 10:01 & 0 & 31.5 & 23.1 & 26.9 & 22.7 & 22.0 & -0.4 & 3.4 & -1.9 & 0.6 & 5.0 & $c$ & -0.2 & 0.010 & & & .1 \\
\hline $0 / 25 / 01$ & $10: 02$ & 0 & 31.5 & 23.1 & 26.6 & 22.7 & 22.0 & -0.4 & 3.4 & -1.9 & 0.5 & 5.0 & c & -0.2 & 0.010 & & & 0.1 \\
\hline $10 / 25 / 01$ & $10: 03$ & 0 & 31.4 & 23.1 & 26.4 & 22.7 & 22.0 & -0.4 & 3.4 & -1.9 & 0.5 & 5.0 & 0 & -0.2 & 0.010 & & & 0.1 \\
\hline $10 / 25 / 01$ & 10:04 & 0 & 31.3 & 23.1 & 22.2 & 22.7 & 22.1 & -0.4 & 3.4 & -1.9 & 0.6 & 5.0 & 0 & -0.2 & 0.010 & & & 0.1 \\
\hline $10 / 25 / 01$ & 10:05 & 0 & 31.2 & 23.1 & 22.3 & 22.8 & 22.1 & -0.4 & 3.5 & -1.9 & 0.5 & 5.1 & 0 & -0.2 & 0.010 & & $-C$ & 0.1 \\
\hline $10 / 25 / 01$ & $10: 06$ & 0 & 31.1 & 23.1 & 22.6 & 22.8 & 22.1 & -0.4 & 3.5 & -1.9 & 0.5 & 5.2 & 0 & -0.2 & 0.010 & & & 0.1 \\
\hline $10 / 25 / 01$ & 10:07 & 0 & 31.0 & 23.1 & 22.8 & 22.8 & 22.1 & -0.4 & 3.6 & -1.9 & 0.6 & 5.2 & 0 & -0.2 & 0.010 & & & 0.1 \\
\hline $5 / 01$ & $10: 08$ & 0 & 30.9 & 23.1 & 22.9 & 22.8 & 22.1 & -0.4 & 3.7 & -1.9 & 0.5 & 5.3 & 0 & -0.2 & 0.010 & & & 0.1 \\
\hline /01 & $10: 09$ & 0 & 30.9 & 23.1 & 23.6 & 22.8 & 22.1 & -0.5 & 4.1 & -1.9 & 0.4 & 5.8 & 0 & -0.2 & 0. & & & 0.0 \\
\hline$/ 01$ & $10: 10$ & 0 & 30.3 & 23.1 & 23.9 & 22.8 & 22.1 & 0.8 & 6.5 & 4.5 & -4.6 & 6.9 & 0 & 31.8 & & & & -1.9 \\
\hline 01 & 10 & 0 & 24.5 & 23.1 & 24.2 & 22.8 & 22.2 & 5.8 & 17.4 & 11.0 & -4.9 & 11.6 & & 3.0 & & & & . .5 \\
\hline 1 & 10 & 0 & 4.7 & 23.1 & 24.4 & 22.8 & 22.2 & 11.7 & .6 & 11.2 & 0 & .9 & & .1 & & & & 0.3 \\
\hline 10 & 10: & 0 & 4.7 & 23.1 & 24.6 & 22.8 & 22.2 & 10.2 & 7.7 & 11.3 & -0.7 & 7.5 & & 2.8 & & & & 4.8 \\
\hline $10 / 2$ & $10: 14$ & 0 & 24.7 & 23.0 & 24.8 & 22.8 & 22.2 & 9.7 & 8.6 & 10.3 & 0.0 & 8.9 & c & .4 & & & & 4.8 \\
\hline 01 & $10: 15$ & 0 & 25.2 & 23.0 & 25.0 & 22.8 & 22.2 & 8.5 & 18.6 & 10.3 & -1.5 & 0.2 & 0 & 3 & & & & 3.5 \\
\hline 10/2 & $10: 16$ & 0 & 24.8 & 23.1 & 25.2 & 22.9 & 22.2 & 8.8 & 18.7 & 10.4 & -1.3 & 0.0 & 0 & 0.5 & & & & 3.8 \\
\hline $10 / 2$ & $10: 17$ & 0 & 24.9 & 23.1 & 25.4 & 22.9 & 22.3 & 8.9 & 18.9 & 10.2 & -1.1 & 10.0 & 0 & 50.7 & & & & 3.9 \\
\hline $10 / 2$ & $10: 18$ & 0 & 5.0 & 23.0 & 25.5 & 22.9 & 22.3 & 8.7 & 18.6 & 10.4 & -0.8 & 10.0 & 0 & 0.7 & & & & 3.9 \\
\hline $10 / 2$ & $10: 19$ & 0 & 25.4 & 23.0 & 25.7 & 23.0 & 22.2 & 15.4 & 18.7 & 10.3 & 5.7 & 3.4 & 0 & 0.1 & & & & 10.5 \\
\hline /01 & $10: 20$ & 0 & 25.6 & 23.0 & 25.8 & 23.0 & 22.2 & 9.8 & 18.7 & 10.2 & -0.2 & 9.0 & 0 & 0.3 & & & & 4.8 \\
\hline 01 & $10: 21$ & 0 & 5.8 & 23.0 & 25.9 & 22.9 & 22.2 & 13.5 & 26.3 & 3.7 & 10.2 & 12.7 & 0 & .4 & 0. & & & 11.8 \\
\hline 01 & $10: 22$ & 0 & 5.9 & 23.0 & 26.1 & 22.9 & 22.2 & 18.7 & 27.2 & 3.8 & 15.2 & 8.6 & 0 & 9.6 & & & & 17.0 \\
\hline 01 & $10: 23$ & 0 & 6.1 & 23. & 26.2 & 22.9 & 22.2 & 18.9 & 27.3 & 3.8 & 15 & 8.4 & 0 & 29.5 & & & & 17.2 \\
\hline 1 & 10 & 1 & 6.3 & 23.0 & 26.3 & 22.8 & 22.1 & 3.1 & 27.5 & 3.7 & -0.6 & 24.4 & 64 & 29.3 & & & & 1.3 \\
\hline 10/ & $10: 25$ & 0 & 6.3 & 23.0 & 26.5 & 22.8 & 22.1 & 16.9 & 27.2 & 3.7 & 13.5 & 0.3 & 0 & 29.4 & & & 2 & 15.2 \\
\hline & $10: 26$ & 0 & 26.5 & 23.0 & 26.6 & 22.8 & 22.1 & 18.8 & 27.4 & 3.7 & 15.4 & 8.5 & 0 & 29.2 & & & 1 & 17.1 \\
\hline & 10:27 & 0 & 26.6 & 23.0 & 26.7 & 22.7 & 22.0 & 15.1 & 27.5 & 3.7 & 11.5 & 2.4 & 0 & 29.2 & 0.2 & & .1 & 13.3 \\
\hline $10 / 4$ & $10: 28$ & 0 & 26.7 & 23.0 & 26.8 & 22.7 & 22.0 & 17.1 & 27.4 & 3.6 & 13.6 & 0.4 & 0 & 29.2 & 0.249 & & 12.1 & 15.3 \\
\hline $10 / 2$ & $10: 29$ & 0 & 26.8 & 23.0 & 26.9 & 22.7 & 22.0 & 17.2 & 27.3 & 3.6 & 13.9 & 10.1 & 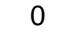 & 29.1 & 0.246 & & 12.1 & 15.6 \\
\hline $10 / 25 / 01$ & $10: 30$ & 0 & 26.9 & 23.0 & 27.0 & 22.7 & 22.0 & 17.5 & 27.4 & 3.7 & 14.1 & 9.9 & $\sigma$ & 29.2 & 0.246 & & 12.1 & 15.8 \\
\hline $10 / 2$ & 10:31 & 0 & 27.1 & 23.0 & 27.2 & 22.6 & 22.0 & 17.7 & 27.5 & 3.6 & 14.5 & 9.7 & $v$ & 29.0 & 0.2 & & 12.1 & 16.1 \\
\hline $10 / 2$ & $10: 32$ & 0 & 27.2 & 23.0 & 27.3 & 22.6 & 21.9 & 18.4 & 27.4 & 3.6 & 15.1 & 9.0 & 0 & 29.1 & 0.2 & & 12.1 & 16.8 \\
\hline & $10: 33$ & 0 & 7.2 & 23.0 & 27.4 & 22.5 & 21.9 & 18 & 27.4 & 3.5 & 15 & 8.4 & 0 & 29.0 & 0.2 & & 12.0 & 17.3 \\
\hline & $10: 34$ & 0 & 27.3 & 23.0 & 27.5 & 22.5 & 21.9 & 18 & 27. & 3.6 & 15 & 8.4 & 0 & 29.0 & & & & 17.3 \\
\hline & $10: 35$ & 0 & 27.5 & 23.0 & 27.6 & 22.4 & 21.9 & 19.1 & 27.5 & 3.6 & 15 & 8.4 & & 29.0 & & & 12.0 & 17.5 \\
\hline $10 / 25 / 01$ & $10: 36$ & 0 & 27.6 & 23.0 & 27.7 & 22.4 & 21.9 & 19.0 & 27.3 & 3.5 & 16.0 & 8.4 & 0 & 28.9 & 0.262 & & 12.0 & 17.5 \\
\hline $10 / 2$ & $10: 37$ & 0 & 27.6 & 23.0 & 27.8 & 22.4 & 21.8 & 19.1 & 27.4 & 3.6 & 15.8 & 8.4 & 0 & 28.9 & 0.258 & & 12.0 & 17.4 \\
\hline $10 / 25 / 01$ & $10: 38$ & 0 & 27.4 & 22.9 & 27.7 & 22.5 & 21.8 & -1.2 & -1.1 & -0.1 & -0.9 & 0.1 & 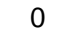 & -0.2 & 0.010 & 0.0040 & -0.1 & -1.0 \\
\hline
\end{tabular}


WSRC-TR-2002-00459, Rev. 0 SRT-RPP-2002-00221

\author{
Xflow1_101501_0837
}

\begin{tabular}{|c|c|c|c|c|c|c|c|c|c|c|c|c|c|c|c|c|c|c|c|}
\hline DATE & TIME & Sol & $\begin{array}{c}\text { FLTRT } \\
\left({ }^{\circ} \mathrm{C}\right) \\
\text { T2 }\end{array}$ & $\begin{array}{c}\mathrm{CL} \\
\text { LOOP } \\
\left({ }^{\circ} \mathrm{C}\right) \\
\text { T3 }\end{array}$ & $\begin{array}{c}\text { SL } \\
\text { LOOP } \\
\left({ }^{\circ} \mathrm{C}\right) \\
\mathrm{T} 1\end{array}$ & $\begin{array}{c}\text { UP } \\
\text { AMB } \\
\left({ }^{\circ} \mathrm{C}\right) \\
\text { T4 }\end{array}$ & $\begin{array}{c}\text { BOT } \\
\text { AMB } \\
\left({ }^{\circ} \mathrm{C}\right) \\
\text { T5 }\end{array}$ & $\begin{array}{c}\text { BOT } \\
\text { DP } \\
\text { (psid) } \\
\text { dP2 }\end{array}$ & $\begin{array}{c}\text { FLTR } \\
\text { (psig) } \\
\text { P1 }\end{array}$ & $\begin{array}{c}\text { FLTR } \\
\text { DP } \\
\text { (psid) } \\
\text { dP1 }\end{array}$ & $\begin{array}{c}\text { TOP } \\
\text { DP } \\
\text { (psig) } \\
\text { dP3 }\end{array}$ & $\begin{array}{c}\text { FLTRATE } \\
\text { (psig) } \\
\text { P2 }\end{array}$ & $\begin{array}{c}\text { BP } \\
\text { (psig) } \\
\text { P3 }\end{array}$ & $\begin{array}{c}\text { SL } \\
\text { FLOW } \\
\text { (gpm) } \\
\text { Q1 }\end{array}$ & $\begin{array}{c}\text { FLTR } \\
\text { FLOW } \\
\text { (gpm) } \\
\text { Q2 }\end{array}$ & $\begin{array}{c}\text { HI FLTR } \\
\text { FLOW } \\
\text { (gpm) } \\
\text { Q3 }\end{array}$ & $\begin{array}{l}\text { Temp } \\
\text { corr flow } \\
\left(\mathrm{gpm} / \mathrm{ft}^{2}\right)\end{array}$ & $\begin{array}{c}\text { Axial Vel } \\
(\mathrm{ft} / \mathrm{sec})\end{array}$ & $\begin{array}{l}\text { Avg } \\
\text { TMP } \\
\text { (psid) }\end{array}$ \\
\hline 0/15/01 & $8: 45$ & 1 & 20.2 & 19.8 & 20.4 & 19.7 & 18.9 & 11.0 & 17.2 & 9.6 & 1.7 & 6.7 & 53.8 & 48.5 & 0.01 & -0.019 & -0.009 & 20.1 & 6.3 \\
\hline 10/15/01 & $8: 46$ & 1 & 20.1 & 19.8 & 20.5 & 19.7 & 18.9 & 10.8 & 24.5 & 13.3 & -2.7 & 14.1 & 53.9 & 59.2 & 0.01 & -0.019 & -0.009 & 24.6 & 4.1 \\
\hline 10/15/01 & $8: 47$ & 1 & 20.1 & 19.8 & 20.7 & 19.7 & 19.0 & 10.1 & 22.1 & 11.9 & -2.1 & 12.5 & 53.8 & 55.3 & 0.01 & -0.019 & -0.009 & 22.9 & 4.0 \\
\hline 10/15/01 & $8: 48$ & 1 & 20.3 & 20.2 & 20.8 & 19.9 & 19.0 & 6.5 & 22.3 & 12.0 & -3.2 & 16.3 & 53.8 & 49.5 & 0.01 & -0.019 & -0.009 & 20.6 & 1.6 \\
\hline 10/15/01 & $8: 49$ & 1 & 20.4 & 20.2 & 21.0 & 20.0 & 19.0 & 9.6 & 21.8 & 11.8 & -2.4 & 12.7 & 53.8 & 55.2 & 0.01 & -0.019 & -0.009 & 22.9 & 3.6 \\
\hline 10/15/01 & $8: 50$ & 1 & 20.6 & 20.2 & 21.2 & 20.0 & 19.0 & 9.7 & 22.0 & 11.9 & -2.2 & 12.6 & 53.9 & 54.4 & 0.01 & -0.019 & -0.009 & 22.6 & 3.8 \\
\hline 10/15/01 & $8: 51$ & 1 & 20.7 & 20.2 & 21.3 & 19.9 & 19.0 & 9.9 & 22.1 & 12.2 & -2.0 & 12.6 & 53.8 & 55.9 & 0.01 & -0.019 & -0.009 & 23.2 & 4.0 \\
\hline 10/15/01 & $8: 52$ & 1 & 20.8 & 20.1 & 21.5 & 19.9 & 19.0 & 9.8 & 21.8 & 12.1 & -2.0 & 12.6 & 53.8 & 55.3 & 0.01 & -0.019 & -0.009 & 22.9 & 3.9 \\
\hline 10/15/01 & $8: 53$ & 1 & 20.9 & 20.1 & 21.7 & 19.9 & 19.1 & 9.6 & 21.9 & 12.0 & -2.1 & 12.7 & 53.8 & 54.4 & 0.01 & -0.019 & -0.009 & 22.6 & 3.7 \\
\hline 10/15/01 & $8: 54$ & 1 & 21.1 & 20.1 & 21.8 & 19.9 & 19.1 & 9.6 & 21.9 & 11.9 & -2.3 & 12.6 & 53.8 & 55.7 & 0.01 & -0.019 & -0.009 & 23.1 & 3.7 \\
\hline 10/15/01 & $8: 55$ & 1 & 21.2 & 20.1 & 22.0 & 19.9 & 19.1 & 9.9 & 22.2 & 11.9 & -2.1 & 12.7 & 53.9 & 55.2 & 0.01 & -0.019 & -0.009 & 22.9 & 3.9 \\
\hline 10/15/01 & $8: 56$ & 1 & 21.3 & 20.1 & 22.2 & 19.9 & 19.1 & 9.7 & 21.9 & 12.1 & -2.2 & 12.7 & 53.8 & 55.3 & 0.01 & -0.019 & -0.009 & 23.0 & 3.7 \\
\hline $10 / 15 / 01$ & $8: 57$ & 1 & 21.5 & 20.0 & 22.3 & 19.9 & 19.1 & 9.3 & 21.5 & 12.0 & -2.0 & 12.7 & 53.8 & 55.4 & 0.01 & -0.019 & -0.009 & 23.0 & 3.6 \\
\hline 10/15/01 & $8: 58$ & 1 & 21.6 & 20.1 & 22.5 & 19.9 & 19.1 & 9.8 & 22.1 & 11.9 & -2.0 & 12.7 & 53.8 & 55.7 & 0.01 & -0.019 & -0.009 & 23.1 & 3.9 \\
\hline 10/15/01 & $8: 59$ & 1 & 21.8 & 20.0 & 22.6 & 19.9 & 19.1 & 9.7 & 22.0 & 12.1 & -2.2 & 12.7 & 53.9 & 55.4 & 0.01 & -0.019 & -0.009 & 23.0 & 3.7 \\
\hline $10 / 15 / 01$ & 9:00 & 1 & 21.9 & 20.0 & 22.8 & 19.9 & 19.2 & 9.6 & 23.0 & 11.1 & -1.2 & 13.9 & 53.8 & 53.5 & 0.01 & -0.019 & -0.009 & 22.2 & 4.2 \\
\hline $10 / 15 / 01$ & 9:01 & 1 & 22.2 & 20.0 & 23.0 & 19.9 & 19.2 & 9.1 & 31.1 & 11.0 & -1.7 & 22.4 & 53.8 & 53.3 & 0.01 & -0.019 & -0.009 & 22.1 & 3.7 \\
\hline $10 / 15 / 01$ & 9:02 & 1 & 22.2 & 20.0 & 23.2 & 19.9 & 19.2 & 8.1 & 32.0 & 10.0 & -1.9 & 24.3 & 53.8 & 50.3 & 0.01 & -0.019 & -0.009 & 20.9 & 3.1 \\
\hline $10 / 15 / 01$ & 9:03 & 1 & 22.4 & 20.0 & 23.4 & 19.9 & 19.2 & 8.1 & 32.2 & 10.1 & -1.4 & 24.4 & 54.0 & 50.4 & 0.01 & -0.019 & 09 & 20.9 & 3.4 \\
\hline $10 / 15 / 01$ & 9:04 & 1 & 22.6 & 20.0 & 23.6 & 20.0 & 19.2 & 8.0 & 31.9 & 10.1 & -1.8 & 24.3 & 53.8 & 50.8 & 0.01 & -0.019 & -0.009 & 21.1 & 3.1 \\
\hline $10 / 15 / 01$ & 9:05 & 1 & 22.8 & 20.0 & 23.8 & 20.0 & 19.3 & 7.9 & 31.9 & 10.0 & -1.8 & 24.5 & 53.8 & 50.6 & 0.01 & -0.019 & -0.009 & 21.0 & 3.0 \\
\hline $10 / 15 / 01$ & 9:06 & 0 & 23.3 & 20.0 & 24.0 & 20.0 & 19.3 & 22.0 & 31.7 & 9.9 & 12.5 & 10.1 & 0.0 & 51.3 & 1.194 & 1.191 & 35 & 21.3 & 17.2 \\
\hline $10 / 15 / 01$ & 9:07 & 0 & 23.9 & 20.0 & 24.1 & 20.0 & 19.3 & 23.0 & 31.6 & 9.9 & 13.4 & 9.2 & 0.0 & 51.1 & 1.158 & 1.110 & 0.497 & 21.2 & 18.2 \\
\hline $10 / 15 / 01$ & 9:08 & 0 & 24.2 & 20.0 & 24.3 & 20.0 & 19.3 & 24.3 & 32.0 & 9.8 & 14.7 & 8.2 & 0.0 & 50.8 & 1.043 & 1.001 & 0.446 & 21.1 & 19.5 \\
\hline $10 / 15 / 01$ & 9:09 & 0 & 24.4 & 20.0 & 24.5 & 20.1 & 19.3 & 25.1 & 32.1 & 9.9 & 15.2 & 7.5 & 0.0 & 51.1 & 0.929 & 0.899 & 99 & 21.2 & 20.1 \\
\hline $10 / 15 / 01$ & $9: 10$ & 0 & 24.6 & 20.0 & 24.6 & 20.1 & 19.4 & 25.2 & 31.8 & 9.8 & 15.6 & 7.0 & 0.0 & 50.5 & 0.881 & 0.842 & 71 & 21.0 & 20.4 \\
\hline $10 / 15 / 01$ & $9: 11$ & 0 & 24.7 & 20.0 & 24.8 & 20.1 & 19.4 & 25.7 & 31.9 & 9.9 & 15.8 & 6.7 & 0.0 & 51.5 & 0.82 & 0.781 & 0.343 & 21.4 & 20.8 \\
\hline $10 / 15 / 01$ & $9: 12$ & 0 & 24.9 & 20.0 & 25.0 & 20.1 & 19.4 & 25.8 & 31.8 & 9.9 & 16.0 & 6.5 & 0.0 & 51.2 & 0.775 & 0.730 & 0.319 & 21.2 & 20.9 \\
\hline $10 / 15 / 01$ & $9: 13$ & 0 & 25.0 & 20.0 & 25.2 & 20.1 & 19.4 & 19.8 & 31.6 & 9.7 & 10.3 & 12.2 & 0.0 & 51.0 & 1.211 & 1.289 & 0.560 & 21.2 & 15.1 \\
\hline 10/15/01 & $9: 14$ & 0 & 25.3 & 20.0 & 25.4 & 20.1 & 19.4 & 22.4 & 31.6 & 9.8 & 12.5 & 9. & 0.0 & 51.3 & 1.193 & 44 & 0. & 21.3 & 17.5 \\
\hline $10 / 15 / 01$ & $9: 15$ & 0 & 25.4 & 20.0 & 25.6 & 20.2 & 19.5 & 20.9 & 31.8 & 9.9 & 11.2 & 11.4 & 0.0 & 51.5 & 1.211 & 1.321 & 0.568 & 21.4 & 16.0 \\
\hline $10 / 15 / 01$ & $9: 16$ & 0 & 25.7 & 20.0 & 25.7 & 20.2 & 19.5 & 23.0 & 31.5 & 9.9 & 13.4 & 9.0 & 0.0 & 51.3 & 1.184 & 1.143 & 0.489 & 21.3 & 18.2 \\
\hline $10 / 15 / 01$ & $9: 17$ & 0 & 25.8 & 20.0 & 25.9 & 20.2 & 19.5 & 21.6 & 31.6 & 9.9 & 12.0 & 10.5 & 0.0 & 51.9 & 1.211 & 1.302 & 0 . & 21.6 & 16.8 \\
\hline 10/15/01 & $9: 18$ & 0 & 26.0 & 20.0 & 25.9 & 20.2 & 19.6 & 23.8 & 31.5 & 10.0 & 14.1 & 8 & 0.0 & 51.2 & 1.191 & 38 & 85 & 21.2 & 18.9 \\
\hline $10 / 15 / 01$ & $9: 19$ & 0 & 25.9 & 20.0 & 25.7 & 20.3 & 19.8 & 24.6 & 31.4 & 10.0 & 14.9 & 7.5 & 0.0 & 51.0 & 1.046 & 0.995 & 0.426 & 21.2 & 19.7 \\
\hline 10/15/01 & $9: 20$ & 0 & 25.8 & 20.0 & 25.5 & 20.4 & 19.8 & 25.2 & 31.4 & 9.7 & 15.9 & 6. & 0.0 & 51.1 & 0.976 & 0.926 & 0.399 & 21.2 & 20.6 \\
\hline $10 / 15 / 01$ & $9: 21$ & 0 & 25.7 & 20.0 & 25.2 & 20.5 & 19.9 & 26.1 & 31.9 & 9.8 & 16.4 & 6.2 & 0.0 & 50.6 & 0.893 & 0.857 & 0.372 & 21.0 & 21.2 \\
\hline $10 / 15 / 01$ & $9: 22$ & 0 & 25.5 & 20.0 & 25.1 & 20.6 & 20.0 & 26.6 & 31.9 & 9.9 & 16.9 & 5. & 0.0 & 51.1 & 0.846 & 0.807 & 0.351 & 21.2 & 21.7 \\
\hline $10 / 15 / 01$ & $9: 23$ & 0 & 25.3 & 20.0 & 24.9 & 20.6 & 20.0 & 27.1 & 32.1 & 9.9 & 17.1 & 5. & 0.0 & 51.2 & 0.804 & 0.764 & 0.335 & 21.2 & 22.1 \\
\hline $10 / 15 / 01$ & $9: 24$ & 0 & 25.2 & 20.0 & 24.8 & 20.7 & 20.2 & 21.9 & 31.8 & 9.9 & 12.3 & 10 & 0.0 & 51.4 & 1.211 & 1.285 & 4 & 21.3 & 17.1 \\
\hline 10/15/01 & $9: 25$ & 0 & 25.0 & 20.0 & 24.7 & 20.8 & 20.3 & 28.4 & 41.0 & 9.2 & 19.2 & 13.1 & 0.0 & 49.5 & 1.211 & 1.397 & 16 & 20.5 & 23.8 \\
\hline $10 / 15 / 01$ & $9: 26$ & 0 & 24.9 & 20.0 & 24.6 & 20.8 & 20.3 & 33.0 & 45.2 & 9.4 & 23.7 & 12.6 & 0.0 & 50.4 & 1.211 & 1.375 & 7 & 20.9 & 28.3 \\
\hline & $9: 27$ & 0 & 24.8 & 20.0 & 24.5 & 20.9 & 20.4 & 35.4 & 45.4 & 9.3 & 25.8 & 10 & 0.0 & 49.7 & 1.211 & & & 20.6 & 30.6 \\
\hline & $9: 28$ & 0 & 24.7 & 20.0 & 24.5 & 20.9 & 20.4 & 36.3 & 44.9 & 9.5 & 27.3 & 9 & 0 & 50.2 & & & & & 31.8 \\
\hline & $9: 29$ & 0 & 24.7 & 20.0 & 24.4 & 21.0 & 20.5 & 37.3 & 45.1 & 9.5 & 27.8 & 8 & 0.0 & 50.2 & 1.203 & & & 20.8 & 32.5 \\
\hline & 9:30 & 0 & 24 & 20.0 & 24.4 & 21.0 & 20.5 & 37.8 & 45.2 & 9. & 28.2 & 8. & 0.0 & 50.1 & 1.156 & 1.100 & & 20.8 & 33.0 \\
\hline 01 & $9: 31$ & 0 & 24.6 & 20.0 & 24.3 & 21.1 & 20.6 & 38.1 & 45.3 & 9.5 & 28.5 & 7.7 & 0.0 & 49.9 & 1.086 & 1.042 & 64 & $\begin{array}{l}20.7 \\
20.8\end{array}$ & $\begin{array}{l}33.3 \\
31.6\end{array}$ \\
\hline
\end{tabular}




\begin{tabular}{|c|c|c|c|c|c|c|c|c|c|c|c|c|c|c|c|c|c|c|c|}
\hline DATE & TIME & Sol & $\begin{array}{l}\text { FLTRT } \\
\left({ }^{\circ} \mathrm{C}\right) \\
\text { T2 }\end{array}$ & $\begin{array}{c}\mathrm{CL} \\
\text { LOOP } \\
\left({ }^{\circ} \mathrm{C}\right) \\
\mathrm{T} 3\end{array}$ & $\begin{array}{c}\text { SL } \\
\text { LOOP } \\
\left({ }^{\circ} \mathrm{C}\right) \\
\mathrm{T} 1\end{array}$ & $\begin{array}{c}\text { UP } \\
\text { AMB } \\
\left({ }^{\circ} \mathrm{C}\right) \\
\text { T4 }\end{array}$ & $\begin{array}{c}\text { BOT } \\
\text { AMB } \\
\left({ }^{\circ} \mathrm{C}\right) \\
\text { T5 }\end{array}$ & $\begin{array}{c}\text { BOT } \\
\text { DP } \\
\text { (psid) } \\
\text { dP2 }\end{array}$ & $\begin{array}{c}\text { FLTR } \\
\text { (psig) } \\
\text { P1 }\end{array}$ & $\begin{array}{c}\text { FLTR } \\
\text { DP } \\
\text { (psid) } \\
\text { dP1 }\end{array}$ & $\begin{array}{c}\text { TOP } \\
\text { DP } \\
\text { (psig) } \\
\text { dP3 }\end{array}$ & $\begin{array}{c}\text { FLTRATE } \\
\text { (psig) } \\
\text { P2 }\end{array}$ & $\begin{array}{c}\text { BP } \\
\text { (psig) } \\
\text { P3 }\end{array}$ & $\begin{array}{c}\text { SL } \\
\text { FLOW } \\
(\mathrm{gpm}) \\
\mathrm{Q1}\end{array}$ & $\begin{array}{c}\text { FLTR } \\
\text { FLOW } \\
\text { (gpm) } \\
\text { Q2 }\end{array}$ & $\begin{array}{c}\text { HI FLTR } \\
\text { FLOW } \\
\text { (gpm) } \\
\text { Q3 }\end{array}$ & $\begin{array}{l}\text { Temp } \\
\text { corr flow } \\
\left(\mathrm{gpm} / \mathrm{ft}^{2}\right)\end{array}$ & $\begin{array}{l}\text { Axial Vel } \\
(\mathrm{ft} / \mathrm{sec})\end{array}$ & $\begin{array}{l}\text { Avg } \\
\text { TMP } \\
\text { (psid) }\end{array}$ \\
\hline 0/15/01 & $9: 32$ & 0 & 24.5 & 20.0 & 24.2 & 21.1 & 20.6 & 27.0 & 30.2 & 9.5 & 17.6 & 3.8 & 0.0 & 49.7 & 0.652 & 0.606 & 0.270 & 20.6 & 22.3 \\
\hline 10/15/01 & 9:33 & 0 & 24.4 & 20.1 & 24.0 & 21.1 & 20.7 & 20.6 & 30.1 & 9.6 & 11.0 & 9.9 & 0.0 & 50.7 & 1.187 & 1.150 & 0.516 & 21.1 & 5.8 \\
\hline 0/15/01 & 9:34 & 0 & 24.3 & 20.1 & 24.0 & 21.1 & 20.7 & 20.6 & 29.9 & 9.5 & 11.4 & 9.8 & 0.0 & 50.5 & 1.211 & 1.261 & 0.567 & 20.9 & 16.0 \\
\hline 10/15/01 & 9:35 & 0 & 24.2 & 20.1 & 23.8 & 21.2 & 20.8 & 22.9 & 30.3 & 9.6 & 13.2 & 7.8 & 0.0 & 50.4 & 1.066 & 1.023 & 0.462 & 20.9 & 18.1 \\
\hline 10/15/01 & 9:36 & 0 & 24.0 & 20.1 & 23.7 & 21.2 & 20.8 & 24.1 & 30.4 & 9.6 & 14.6 & 6.7 & 0.0 & 50.2 & 0.925 & 0.881 & 0.399 & 20.8 & 19.4 \\
\hline 10/15/01 & $9: 37$ & 0 & 24.0 & 20.1 & 23.6 & 21.2 & 21.0 & 24.7 & 30.2 & 9.7 & 15.0 & 6.1 & 0.0 & 50.4 & 0.854 & 0.806 & 0.367 & $2 C$ & 19.8 \\
\hline 10/15/01 & $9: 38$ & 0 & 23.8 & 20.1 & 23.5 & 21.3 & 21.0 & 25.0 & 30.0 & 9.6 & 15.8 & 5.5 & 0.0 & 50.0 & 0.778 & 0.746 & 40 & & 20.4 \\
\hline 10/15/01 & 9:39 & 0 & 23.8 & 20.1 & 23.4 & 21.3 & 21.1 & 25.8 & 30.2 & 9.5 & 16.1 & 5.1 & 0.0 & 50.3 & 0.737 & 0.710 & 24 & & 20.9 \\
\hline 10/15/01 & $9: 40$ & 0 & 23.7 & 20.1 & 23.3 & 21.3 & 21.1 & 25.9 & 30.1 & 9.5 & 16.6 & 4.8 & 0.0 & 50.0 & 0.717 & 0.667 & 05 & & 21.2 \\
\hline 10/15/01 & $9: 41$ & 0 & 23.6 & 20.2 & 23.2 & 21.4 & 21.1 & 18.1 & 19.9 & 6.6 & 12.3 & 2.3 & 0.0 & 40.1 & 0.482 & 0.480 & 0.220 & & 15.2 \\
\hline 10/15/01 & $9: 42$ & 0 & 23.6 & 20.2 & 23.0 & 21.4 & 21.2 & 6.9 & 16.1 & 8.8 & -1.9 & 9.7 & 0.0 & 47.0 & 0.022 & 0.001 & & & 2.5 \\
\hline $10 / 15 / 01$ & $9: 43$ & 0 & 23.6 & 20.2 & 22.9 & 21.5 & 21.3 & 7.8 & 15.6 & 9.8 & -1.9 & 8. & 0.0 & 50.0 & 0.01 & -0.019 & -0.009 & & 3.0 \\
\hline 10/15/01 & $9: 44$ & 0 & 23.5 & 20.2 & 22.7 & 21.6 & 21.3 & 7.6 & 15.3 & 9.8 & -2.0 & 8. & 0.0 & 49.7 & 0.01 & -0.019 & -0.009 & & 2.8 \\
\hline 0/15/01 & $9: 45$ & 0 & 23.5 & 20.2 & 22.6 & 21.6 & 21.4 & 7.7 & 15.4 & 9.8 & -1.9 & 8. & 0.0 & 49.7 & 0.01 & -0.019 & & & 2.9 \\
\hline 10/15/01 & $9: 46$ & 0 & 23.4 & 20.2 & 22.4 & 21.6 & 21.4 & 7.8 & 15.5 & 9.6 & -1.9 & 8. & 0.0 & 49.8 & 0.01 & -0.019 & -0 . & & 2.9 \\
\hline /15/01 & $9: 47$ & 0 & 23.4 & 20.2 & 22.4 & 21.6 & 21.5 & 7.4 & 15.1 & 9.6 & -2.0 & 8. & 0.0 & 49.7 & 0.01 & -0.019 & -0 . & & 2.7 \\
\hline $10 / 15 / 01$ & $9: 48$ & 0 & 23.3 & 20.2 & 22.1 & 21.7 & 21.5 & 6.0 & 11.7 & 7.3 & -1.3 & 6.2 & 0.0 & 42.8 & 0.01 & -0.019 & -0 . & & 2.3 \\
\hline 10/15/01 & $9: 49$ & 0 & 23.2 & 20.2 & 22.0 & 21.6 & 21.6 & 4.5 & 15.2 & 3.9 & 0.6 & 11.1 & 0.0 & 30.1 & 0.027 & 0.045 & & & 2.6 \\
\hline 10/15/01 & $9: 50$ & 0 & 23.1 & 20.2 & 21.8 & 21.7 & 21.6 & 4.6 & 15.3 & 3.8 & 0.7 & 11 & 0.0 & 30.1 & 0.056 & 0.033 & & & 2.7 \\
\hline $10 / 15 / 01$ & $9: 51$ & 0 & 23.0 & 20.3 & 21.8 & 21.7 & 21.6 & 4.5 & 15.3 & 3.7 & 0.8 & 11.2 & 0.0 & 29.3 & 0.061 & 0.033 & & & 2.7 \\
\hline $10 / 15 / 01$ & $9: 52$ & 0 & 22.9 & 20.2 & 21.6 & 21.7 & 21.7 & 4.6 & 15.4 & 3.8 & 0.9 & 11.2 & 0.0 & 29.4 & 0.061 & 0.033 & & & 2.7 \\
\hline 10/15/01 & $9: 53$ & 0 & 22.8 & 20.3 & 21.6 & 21.8 & 21.7 & 23.4 & 26.2 & 6.2 & 17.1 & 3.3 & 0.0 & 38.6 & 0.64 & 0.606 & & & 20.2 \\
\hline $10 / 15 / 01$ & $9: 54$ & 0 & 22.1 & 20.3 & 21.5 & 21.7 & 21.7 & 26.1 & 30.0 & 3.7 & 22.4 & 4. & 0.0 & 29.6 & 0.768 & 0.743 & & & 24.3 \\
\hline 10/15/01 & $9: 55$ & 0 & 21.9 & 20.3 & 21.5 & 21.8 & 21.7 & 26.8 & 30.1 & 3.8 & 22.8 & 3.7 & 0.0 & 30.2 & 0.716 & 0.676 & & & 24.8 \\
\hline 10/15/01 & $9: 56$ & 0 & 21.8 & 20.3 & 21.5 & 21.8 & 21.8 & 22.2 & 29.9 & 3.8 & 18.5 & 8. & 0.0 & 30.6 & 1.076 & 1.045 & & & 20.4 \\
\hline /15/01 & $9: 57$ & 0 & 21.8 & 20.3 & 21.4 & 21.9 & 21.8 & 24.8 & 29.9 & 3.8 & 21.0 & 5. & 0.0 & 30.4 & 0.751 & 0.708 & & & 22.9 \\
\hline & 9.58 & 0 & & 20.3 & 21.4 & 21.9 & 21.8 & 25.7 & 30.1 & 3.8 & & & 0.0 & & & 0.638 & & & $\begin{array}{l}23.8 \\
23.2\end{array}$ \\
\hline & & 0 & $r$ & .5 & 21.3 & 21.9 &. & 17.9 & 29.9 & 3.8 & & 12 & & & & & & & 16.0 \\
\hline 01 & $10: 00$ & 0 & 21.6 & 20.4 & 21.3 & 22.0 & 21.9 & 24.6 & 29.9 & 3.8 & 20.9 & & 0.0 & 30.3 & 0.802 & 0.759 & & & 22.8 \\
\hline /01 & $10: 01$ & 0 & 21.6 & 20.4 & 21.2 & 21.9 & 21.9 & 25.6 & 29.8 & 3.8 & 22.0 & 4 & 0.0 & 30.3 & 0.697 & 0.660 & & & 23.8 \\
\hline$/ 15 / 01$ & $10: 02$ & 0 & 21.5 & 20.4 & 21.2 & 22.0 & 21.9 & 36.7 & 45.7 & 3.5 & 33.2 & 9. & 0. & 29.3 & 1.044 & 1.007 & & & 35.0 \\
\hline 01 & $10: 03$ & 0 & 21.5 & 20.4 & 21.4 & 22.0 & 21.9 & 37.2 & 44.8 & 3.7 & 34.0 & 8 & 0.0 & 29.9 & 0.968 & 0.930 & & & 35.6 \\
\hline & :04 & 0 & 21.6 & 20.4 & 21.4 & 22.0 & 21.9 & 37.9 & 44 & 3.6 & 34. & & & 29 & 0.91 & 0.876 & & & 36.3 \\
\hline & :05 & 0 & 21.6 & 20.4 & 21.4 & 22.0 & 21.9 & 38.6 & 45.1 & 3.7 & 35.2 & & (1) & 29.7 & 0.877 & 0.845 & & & 36.9 \\
\hline & :06 & 0 & 21.7 & 20.4 & 21.4 & 22.0 & 21.9 & 34.6 & 39.9 & 3.3 & 31.8 & 5 & 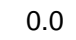 & 28.3 & 0.798 & 0.767 & & & 33.2 \\
\hline 01 & :07 & 0 & 21.6 & 20.4 & 21.4 & 22.0 & 21.9 & 27.3 & 30.0 & 3.7 & 23.7 & 3 & 0 & 29.8 & 0. & 0.530 & & & 25.5 \\
\hline 1 & :08 & 0 & 21.6 & 0.5 & 21.4 & 22.0 & 22.0 & 22.8 & 29 & 3.7 & 19.3 & & 0.0 & 30.1 & 0. & 0.873 & & & 21.1 \\
\hline & 9 & 0 & 21 & 0.5 & 21.3 & 22.0 & 21.9 & 25.7 & 30 & 3.7 & 21 & 4 & & 29 & 0. & 0.630 & & & 23.8 \\
\hline & & 0 & 21.6 & 0.5 & 21.3 & 22.0 & 0 & 30.3 & & 4.2 & 26 & & & 31 & & 0.619 & & & 28.2 \\
\hline & & 0 & 21.6 & 0.5 & 21.3 & 22.0 & 22.0 & 36.6 & 43 & 5.3 & 31 & & & 35 & 0.829 & 0.786 & & & 33.9 \\
\hline & & 0 & 21 & 20.5 & 21.4 & 22.1 & 22.0 & 36.9 & 43 & 5 & 31 & & & 35 & & 0.772 & & & 34.3 \\
\hline & & 0 & 21.6 & 20.5 & 21.4 & 22.1 & 22.0 & 37.8 & & 3. & & & & $2 v$ & & 0.790 & & & 36.1 \\
\hline & & 0 & $\angle 1$ & 20.5 & 21.4 & 22.1 & 22.0 & 38.4 & & 3. & & & & 30 & & 0.770 & & & 36.5 \\
\hline & & 0 & 21 & 20.6 & 21.5 & 22.1 & 22.0 & 38.5 & 45.0 & 3.7 & 35 & & & 30. & & 0.761 & & & 36.8 \\
\hline & .10 & 0 & $<1$ & 20.6 & 21.5 & ¿ל. & 22.0 & 38.7 & 44.9 & 3.7 & 35.3 & & & 30.0 & 0.781 & 0.744 & & & 37.0 \\
\hline & 0.17 & 0 & 21. & 20.6 & 21.5 & 22.1 & 22.0 & 39.1 & 45.3 & 3.7 & 35.4 & & .0 & 29.9 & 0.771 & 0.724 & & & 37.3 \\
\hline & $10: 18$ & 0 & 21. & 20.6 & 21.5 & 22.1 & 22.1 & 39.3 & 45.4 & 3.7 & 35.1 & & 0.0 & 30.0 & 0.766 & 0.730 & & & 37.5 \\
\hline 0/15/01 & $10: 19$ & 0 & 21.8 & 20.6 & 21.6 & 22.2 & 22.1 & 39.1 & 44.9 & 3.7 & 35.5 & 6.3 & 0.0 & 30.0 & 0.756 & 0.710 & 0.342 & 12.5 & 37.3 \\
\hline
\end{tabular}


WSRC-TR-2002-00459, Rev. 0 SRT-RPP-2002-00221

Xflow1_101501_0837

\begin{tabular}{|c|c|c|c|c|c|c|c|c|c|c|c|c|c|c|c|c|c|c|c|}
\hline ATE & TIME & Sol & $\begin{array}{r}\text { FLTR } \\
\left({ }^{\circ} \mathrm{C}\right) \\
\text { T2 }\end{array}$ & $\begin{array}{c}\mathrm{CL} \\
\mathrm{LOOP} \\
\left({ }^{\circ} \mathrm{C}\right) \\
\mathrm{T} 3\end{array}$ & $\begin{array}{c}\text { SL } \\
\text { LOOP } \\
\left({ }^{\circ} \mathrm{C}\right) \\
\mathrm{T} 1\end{array}$ & $\begin{array}{c}\text { UP } \\
\text { AMB } \\
\left({ }^{\circ} \mathrm{C}\right) \\
\text { T4 }\end{array}$ & $\begin{array}{c}\text { BOT } \\
\text { AMB } \\
\left({ }^{\circ} \mathrm{C}\right) \\
\text { T5 }\end{array}$ & $\begin{array}{c}\text { BOT } \\
\text { DP } \\
\text { (psid) } \\
\text { dP2 }\end{array}$ & $\begin{array}{c}\text { FLTR } \\
\text { (psig) } \\
\text { P1 }\end{array}$ & $\begin{array}{c}\text { FLTR } \\
\text { DP } \\
\text { (psid) } \\
\text { dP1 }\end{array}$ & $\begin{array}{c}\text { TOP } \\
\text { DP } \\
\text { (psig) } \\
\text { dP3 }\end{array}$ & $\begin{array}{c}\text { FLTRATE } \\
\text { (psig) } \\
\text { P2 }\end{array}$ & $\begin{array}{l}\text { BP } \\
\text { (psig) } \\
\text { P3 }\end{array}$ & $\begin{array}{c}\text { SL } \\
\text { FLOW } \\
\text { (gpm) } \\
\text { Q1 }\end{array}$ & $\begin{array}{c}\text { FLTR } \\
\text { FLOW } \\
\text { (gpm) } \\
\text { Q2 }\end{array}$ & $\begin{array}{c}\text { HI FLTR } \\
\text { FLOW } \\
\text { (gpm) } \\
\text { Q3 }\end{array}$ & $\begin{array}{l}\text { Temp } \\
\text { corr flow } \\
\left(\mathrm{gpm} / \mathrm{ft}^{2}\right)\end{array}$ & $\begin{array}{c}\text { Axial Vel } \\
(\mathrm{ft} / \mathrm{sec})\end{array}$ & $\begin{array}{l}\text { Avg } \\
\text { TMP } \\
\text { (psid) }\end{array}$ \\
\hline 0/15/01 & $10: 20$ & 0 & 21.8 & 20.6 & 21.6 & 22.2 & 22.1 & 39.4 & 45.1 & 3.7 & 36.2 & 6.2 & 0.0 & 29.9 & 0.75 & 0.727 & 0.350 & 12.4 & 37.8 \\
\hline /15/01 & $10: 21$ & 0 & 21.8 & 20.7 & 21.6 & 22.3 & 22.1 & 39.5 & 45.0 & 3.7 & 36.1 & 6.1 & 0.0 & 29.9 & 0.739 & 0.702 & 0.338 & 12.4 & 37.8 \\
\hline /15/01 & $10: 22$ & 0 & 21.9 & 20.7 & 21.7 & 22.3 & 22.2 & 39.5 & 45.0 & 3.8 & 35.9 & 6.0 & 0.0 & 30.0 & 0.738 & 0.692 & 0.332 & 12.4 & 37.7 \\
\hline /15/01 & $10: 23$ & 0 & 21.9 & 20.7 & 21.7 & 22.4 & 22.2 & 39.5 & 44.9 & 3.7 & 36.2 & 6.0 & 0.0 & 29.9 & 0.732 & 0.690 & 0.331 & 12.4 & 37.8 \\
\hline /15/01 & $10: 24$ & 0 & 21.9 & 20.7 & 21.7 & 22.4 & 22.2 & 39.5 & 44.9 & 3.7 & 35.9 & 5.9 & 0.0 & 30.0 & 0.723 & 0.695 & 0.334 & 12.5 & 37.7 \\
\hline 0/15/01 & $10: 25$ & 0 & 22.0 & 20.7 & 21.7 & 22.4 & 22.3 & 39.9 & 45.1 & 3.7 & 36.4 & 5.8 & 0.0 & 30.0 & 0.715 & 0.688 & 0.330 & 12.4 & 38.2 \\
\hline /15/01 & $10: 26$ & 0 & 22.0 & 20.7 & 21.7 & 22.5 & 22.3 & 45.3 & 52.8 & 0.4 & 45.6 & 8. & 0.0 & 10.2 & 0.878 & 0.846 & 0.406 & & 45.5 \\
\hline /15/01 & $10: 27$ & 0 & 22.2 & 20.8 & 21.7 & 22.5 & 22.3 & 51.0 & 59.6 & 0.4 & 49.9 & 9.2 & 0.0 & 9.6 & 0.938 & 0.906 & & & 50.5 \\
\hline /15/01 & $10: 28$ & 0 & 22.3 & 20.8 & 21.7 & 22.6 & 22.3 & 51.4 & 60.0 & 0.4 & 50.9 & 9.2 & 0.0 & 10.0 & 0.996 & 0.957 & 58 & & 51.1 \\
\hline /15/01 & $10: 29$ & 0 & 22.3 & 20.8 & 21.8 & 22.5 & 22.4 & 52.2 & 61.1 & 0.3 & 51.7 & 9.1 & 0.0 & 9.5 & 0.987 & 0.954 & 0.456 & 3.9 & 52.0 \\
\hline /15/01 & $10: 30$ & 0 & 22.5 & 20.8 & 21.9 & 22.6 & 22.5 & 52.2 & 60.9 & 0.3 & 51.7 & $9 .($ & 0.0 & 9.4 & 0.99 & 0.955 & & 9 & 51.9 \\
\hline /15/01 & $10: 31$ & 0 & 22.5 & 20.8 & 21.9 & 22.6 & 22.4 & 45.4 & 52.8 & 0.3 & 46.4 & 7. & 0.0 & 8.7 & 0.904 & 0.860 & & & 45.9 \\
\hline /15/01 & $10: 32$ & 0 & 22.5 & 20.8 & 21.9 & 22.5 & 22.5 & 40.7 & 45.9 & 0.2 & 41.0 & 5.7 & 0.0 & 8.0 & 0.751 & 0.711 & & 0 & 40.8 \\
\hline /15/01 & $10: 33$ & 0 & 22.5 & 20.9 & 21.9 & 22.6 & 22.5 & 40.2 & 45.1 & 0.4 & 39.9 & 5. & 0.0 & 10.1 & 0.736 & 0.696 & & & 40.0 \\
\hline /15/01 & $10: 34$ & 0 & 22.4 & 20.9 & 22.0 & 22.5 & 22.5 & 40.4 & 45.3 & 0.4 & 39.7 & 5. & 0.0 & 10.0 & 0.724 & 0.692 & & & 40.1 \\
\hline /15/01 & $10: 35$ & 0 & 22.4 & 20.9 & 21.9 & 22.5 & 22.5 & 40.7 & 45.7 & 0.4 & 39.4 & 5 & 0.0 & 9.9 & 0.725 & 0.695 & & & 40.0 \\
\hline $10 / 15 / 01$ & $10: 36$ & 0 & 22.4 & 20.9 & 21.9 & 22.5 & 22.5 & 40.6 & 45.5 & 0.4 & 40.2 & 5. & 0.0 & 10.0 & 0.72 & 0.683 & & & 40.4 \\
\hline $015 / 01$ & $10: 37$ & 0 & 22.4 & 20.9 & 21.9 & 22.4 & 22.5 & 22.9 & 30.6 & 0.2 & 23.4 & 8. & 0.0 & 8.0 & 0.16 & 0.102 & & & 23.1 \\
\hline $15 / 01$ & $10: 38$ & 1 & 22.4 & 20.9 & 21.8 & 22.4 & 22.3 & 0.8 & 30.3 & 0.5 & 0.3 & 29 & 53.6 & 10.0 & 0.01 & & & & 0.6 \\
\hline 0/1 & $10: 39$ & 0 & 22.3 & 20.9 & 21.8 & 22.3 & 22.3 & 19.6 & 30.0 & 0.5 & 18.9 & 10.8 & 0.0 & 11.0 & 1.119 & 84 & & & 19.2 \\
\hline $14-101$ & :40 & 0 & 22.3 & 21.0 & 21.7 & 22.3 & 22.2 & 25.9 & 30.1 & 0.5 & 25.4 & 4.6 & 0.0 & 10.5 & 0.619 & 87 & & & 25.7 \\
\hline 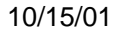 & 1 & 0 & 22.2 & 21.0 & 21.7 & 22.2 & 22.2 & 26.7 & 30.3 & 0.5 & 26.1 & 4 & 0.0 & 10.4 & 0. & 43 & & & 26.4 \\
\hline$/ 1$ & $10: 42$ & 1 & 22.2 & 21.0 & 21.7 & 22.1 & 22.1 & 0.5 & 30.2 & 0.4 & -0.2 & 30.2 & 53.4 & 9.7 & 0.337 & 0.212 & & & 0.1 \\
\hline 1 & 3 & 1 & 22.1 & 21.0 & 21.6 & 22.1 & 22.0 & 0.5 & 30.2 & 0.5 & 0.2 & 30.1 & 53.6 & 9.8 & 0.013 & 18 & & & 0.4 \\
\hline 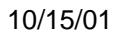 & & 0 & 22.0 & 21.0 & 21.5 & 22.0 & 21.9 & 17.2 & 20.0 & 0.3 & 16.7 & & & 8. & 0.58 & 47 & & & 16.9 \\
\hline & & 0 & 22.0 & 21.0 & 21.4 & 22.0 & 21.8 & 13.8 & 15.4 & 0.5 & 13.3 & & 0.0 & 10.5 & 0.327 & 0.295 & & & 13.5 \\
\hline & & 0 & 21.9 & 21.0 & 21.3 & 21.9 & 21.8 & 13.6 & 15.1 & 0.5 & 13.2 & 2. & 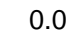 & 10.1 & 0.306 & 0.283 & & & 13.4 \\
\hline & & 0 & 21.8 & 21.0 & 21.3 & 21.9 & 21.8 & 13.5 & 15.0 & 0.4 & 13.3 & & 0.0 & 10 & 0.3 & 0.277 & & & 13.4 \\
\hline & & 0 & 21 & 21.1 & 21.2 & 21.9 & 21.8 & 13.7 & 15 & 0 & 13. & & & 10 & 0.298 & 0.271 & & & 13.5 \\
\hline & & 0 & 21 & 1.1 & 2 & 21 & 21.8 & 13.8 & 15 & 0.4 & 13 & & & 10 & 0. & 66 & & & 13.5 \\
\hline & & 0 & 21 & 21.1 & 1 & 21.9 & 1.9 & 13.8 & 15 & 0.4 & 13. & & & 10.0 & 0.289 & 0.260 & & & 13.6 \\
\hline & & 0 & 21 & 1.1 & & 22.0 & 1.9 & 13.8 & 15 & 0 & 13 & & & 10 & 0.289 & 58 & & & 13.6 \\
\hline & & 0 & 21 & 1 & & 22 & .9 & & 15 & & & & & 10 & & & & & 13.6 \\
\hline & & 0 & & 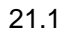 & & $2<.1$ & .9 & & & & & & & & & & & & \\
\hline & & 0 & 21 & 21.1 & 20.8 & $2<.1$ & .9 & & & 4.6 & 10 & & & & & 23 & & & 3.1 \\
\hline & & 0 & & 21.1 & 20.8 & $2<.1$ & 21.9 & & 16.7 & & 10.9 & & & 00.0 & 0.242 & & & & 3.1 \\
\hline & & 0 & 21 & 21.1 & 20.7 & $2<.2$ & 21.9 & 13.3 & 10.0 & & 8 & & & 00.0 & 0.244 & & & & 1.0 \\
\hline & $t$ & 0 & 0 & 21. & 20.7 & 2L.L & 21.9 & 15.3 & 16.8 & 4.6 & 10.7 & & & 33.2 & 0.241 & 15 & & & 13.0 \\
\hline & & 0 & 21 & 21.1 & 20.6 & $2<.2$ & 21.9 & -6.7 & -1.1 & -0.1 & -9.7 & & & -0.1 & 0.201 & 189 & & & -8.2 \\
\hline & $10: 59$ & 0 & 21.2 & 21.2 & 20.6 & 22.3 & 21.9 & 0.2 & -1.0 & -0.1 & 0.3 & -0 & 0.0 & -0.2 & 0.01 & 19 & & 1 & 0.2 \\
\hline 0/15/01 & $1: 00$ & 0 & 21.3 & 21.2 & 20.6 & 22.2 & 21.8 & 0.3 & -1.0 & -0.1 & 0.3 & -0.8 & 0.0 & -0.2 & 0.01 & -0.019 & -0.009 & -0.1 & 0.3 \\
\hline
\end{tabular}


Xflow2 110101_1045

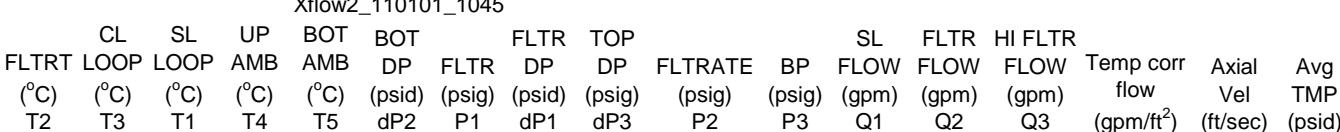
$\begin{array}{llllllllllllllllllll}\text { DATE } & \text { TIME } & \text { Sol } & \text { T2 } & \text { T3 } & \text { T1 } & \text { T4 } & \text { T5 } & d P 2 & \text { P1 } & d P 1 & d P 3 & \text { P2 } & \text { P3 } & \text { Q1 } & \text { Q2 } & \text { Q3 } & \left.\text { (gpm/ft }{ }^{2}\right) & \text { (ft/sec) } & \text { (psid) }\end{array}$

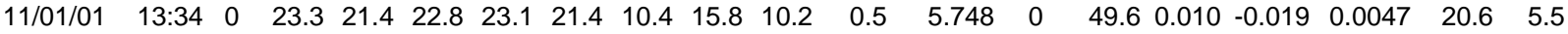
$\begin{array}{lllllllllllllllllllll}11 / 01 / 01 & 13: 35 & 0 & 23.2 & 21.4 & 23.0 & 23.1 & 21.4 & 15.3 & 25.3 & 15.4 & 0.1 & 10.185 & 0 & 62.1 & 0.010 & -0.019 & 0.0046 & 25.8 & 7.7\end{array}$

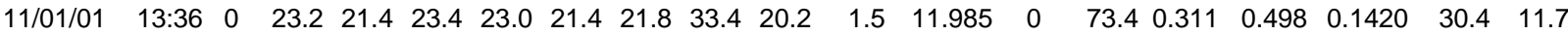

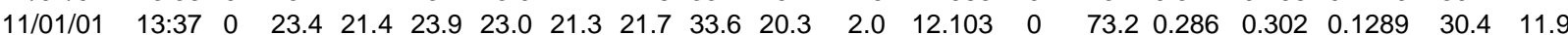

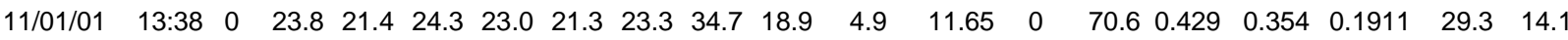
$\begin{array}{llllllllllllll}11 / 01 / 01 & 13: 39 & 0 & 24.5 & 21.4 & 24.7 & 22.9 & 21.3 & 31.2 & 43.8 & 10.5 & 20.7 & 12.911 & 0\end{array}$ $\begin{array}{llllllllllllll}11 / 01 / 01 & 13: 40 & 0 & 24.9 & 21.5 & 25.0 & 22.9 & 21.3 & 33.8 & 44.7 & 9.6 & 24.6 & 11.194 & 0\end{array}$

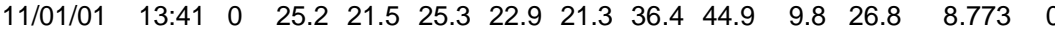
$\begin{array}{lllllllllllllll}11 / 01 / 01 & 13: 42 & 0 & 25.5 & 21.5 & 25.6 & 22.9 & 21.2 & 35.7 & 45.0 & 9.7 & 26.0 & 9.523 & 0\end{array}$ $\begin{array}{llllllllllllll}11 / 01 / 01 & 13: 43 & 0 & 25.8 & 21.5 & 25.9 & 22.9 & 21.2 & 29.1 & 44.6 & 9.7 & 20.0 & 15.879 & 0\end{array}$ $\begin{array}{llllllllllllll}11 / 01 / 01 & 13: 44 & 0 & 26.2 & 21.5 & 26.2 & 22.9 & 21.2 & 29.1 & 45.0 & 9.5 & 19.5 & 16.232 & 0\end{array}$ $\begin{array}{llllllllllllll}11 / 01 / 01 & 13: 45 & 0 & 26.5 & 21.5 & 26.6 & 22.9 & 21.2 & 33.0 & 44.9 & 9.3 & 23.7 & 12.228 & 0\end{array}$ $\begin{array}{lllllllllllll}11 / 01 / 01 & 13.46 & 0 & 26.8 & 21.5 & 26.8 & 22.9 & 21.2 & 36.2 & 44.9 & 9.4 & 27.3 & 8.904\end{array}$ $\begin{array}{llllllllllllll}11 / 01 / 01 & 13: 47 & 0 & 27.0 & 21.5 & 27.2 & 22.9 & 21.2 & 37.5 & 44.8 & 9.5 & 28.7 & 7.654 & 0\end{array}$

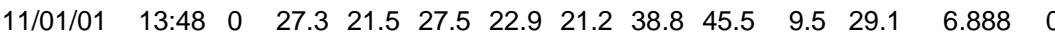
$\begin{array}{llllllllllllll}11 / 01 / 01 & 13: 49 & 0 & 27.6 & 21.5 & 27.8 & 22.9 & 21.2 & 39.3 & 44.8 & 9.4 & 30.3 & 5.938 & 0\end{array}$

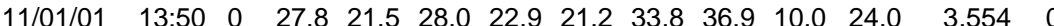
$11 / 01 / 0$

$11 / 01 / 01$

$11 / 01 / 01$

$11 / 01 / 01$

$11 / 01 / 01$

$11 / 01 / 01$

$11 / 01 / 01$

$11 / 01 / 01$

$11 / 01 / 01$

$11 / 01 / 01$

$11 / 01 / 01$

$11 / 01 / 01$

$11 / 01 / 01$

$11 / 01 / 01$

$11 / 01 / 01$

$11 / 01 / 01$

$11 / 01 / 01$

$11 / 01 / 01$

$11 / 01 / 01$

$11 / 01 / 01$

$11 / 01 / 01$

$11 / 01 / 01$

$11 / 01 / 01$

$11 / 01 / 01$

$11 / 01 / 01$

$11 / 01 / 01$

$11 / 01 / 01$

$11 / 01 / 01$

$11 / 01 / 01$

$11 / 01 / 01$

$11 / 01 / 01$

$11 / 01 / 01$

$11 / 01 / 01$

$11 / 01 / 01$

$11 / 01 / 01$

$11 / 01 / 01$ $\begin{array}{lllllllllll}13: 51 & 0 & 28.0 & 21.5 & 28.3 & 23.0 & 21.3 & 34.0 & 36.9 & 9.5 & 24.5\end{array}$

13:52 0

13:53 0

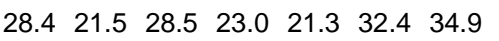

$\begin{array}{lllllllll} & 0 & 28.6 & 21.5 & 28.9 & 23.1 & 21.4 & 20.5 & 30.4\end{array}$

$\begin{array}{lllllllll}13: 55 & 1 & 28.6 & 21.5 & 29.1 & 23.1 & 21.4 & 6.9 & 30.7\end{array}$

$\begin{array}{lllllllll}13: 56 & 1 & 28.9 & 21.5 & 29.3 & 23.1 & 21.5 & 7.8 & 30.0\end{array}$

$\begin{array}{lllllllll}13: 57 & 0 & 29.3 & 21.5 & 29.5 & 23.2 & 21.6 & 22.0 & 30.2\end{array}$

$\begin{array}{lllllllll}13: 58 & 0 & 29.6 & 21.6 & 29.7 & 23.3 & 21.6 & 22.9 & 30.0\end{array}$

$\begin{array}{lllllllll}13: 59 & 0 & 29.7 & 21.5 & 29.9 & 23.3 & 21.6 & 24.0 & 30.1\end{array}$

$\begin{array}{lllllllll}14: 00 & 0 & 29.8 & 21.5 & 29.8 & 23.4 & 21.7 & 25.2 & 30.2\end{array}$

$\begin{array}{lllllllll}14: 01 & 0 & 29.8 & 21.5 & 29.4 & 23.6 & 21.8 & 26.0 & 30.3\end{array}$

$\begin{array}{lllllllll}14: 02 & 0 & 29.5 & 21.6 & 28.9 & 23.9 & 21.9 & 26.5 & 30.2\end{array}$

$\begin{array}{llllllllll}14.03 & 0 & 29.3 & 21.6 & 28.3 & 24.2 & 22.0 & 11.4 & 20.4\end{array}$

$14: 04$ r

$\begin{array}{lllll}29.2 & 21.6 & 27.7 & 24.5 & 22 .\end{array}$

9.016 .8

$8.7 \quad 16.3 \quad 10.0$

$\begin{array}{llllllll}14.04 & 0 & 29.2 & 21.6 & 27.7 & 24.5 & 22.1\end{array}$

$\begin{array}{lllllll}14: 06 & 0 & 28.8 & 21.6 & 26.2 & 24.7 & 22.1\end{array}$

$\begin{array}{lllllll}14: 07 & 0 & 28.5 & 21.6 & 25.5 & 24.7 & 22.1\end{array}$

7.915 .1

$7.9 \quad 15.1$

$14: 08 \quad 0$

14:09 0

$\begin{array}{lllll}28.1 & 21.6 & 24.8 & 24.7 & 22.1\end{array}$

8.115 .3

$14: 10$

$\begin{array}{lllll}27.6 & 21.6 & 24.1 & 24.7 & 22.1\end{array}$

8.115 .3

$\begin{array}{llllll}27.1 & 21.6 & 23.4 & 24.6 & 22.1\end{array}$

$\begin{array}{lllllll}14: 12 & 0 & 26.0 & 21.7 & 22.2 & 24.5 & 22.1\end{array}$

$8.1 \quad 15.3$

6.312 .3

$\begin{array}{ll}5.7 & 15.7\end{array}$

$14: 14 \quad 0$

$\begin{array}{llllll}24.7 & 21.7 & 21.1 & 24.4 & 22.0\end{array}$

5.915 .6

$14: 16 \quad 0$

$14: 17 \quad 0$

$14: 18 \quad 0$

5.815 .2

$\begin{array}{lllllll}23.8 & 21.7 & 20.2 & 24.2 & 22.0 & 5.9 & 15.2 \\ 23.1 & 21.7 & 19.8 & 24.1 & 22.0 & 5.9 & 15.3\end{array}$

$\begin{array}{lllllll}23.3 & 21.7 & 19.4 & 24.1 & 22.0 & 16.4 & 28.8\end{array}$

$\begin{array}{lllllllll}14: 19 & 0 & 21.9 & 21.7 & 19.2 & 24.0 & 21.9 & 18.4 & 28.5\end{array}$

$\begin{array}{lllllllll}14: 20 & 0 & 21.4 & 21.7 & 18.8 & 24.1 & 21.9 & 20.3 & 29.9\end{array}$

$\begin{array}{lllllllll}14: 21 & 1 & 19.9 & 21.7 & 18.6 & 24.0 & 21.9 & 2.9 & 29.7\end{array}$

$14: 220$

$\begin{array}{lllllll}19.6 & 21.7 & 18.3 & 24.0 & 21.8 & 24.7 & 29.8\end{array}$

$14: 23 \quad 0$

$\begin{array}{lllllll}19.4 & 21.7 & 18.1 & 24.0 & 21.8 & 26.8 & 29.8\end{array}$

$\begin{array}{lllllllll}14: 24 & 0 & 19.3 & 21.7 & 18.1 & 24.0 & 21.8 & 27.0 & 30.0\end{array}$

$14: 250$

$\begin{array}{lllllll}18.8 & 21.8 & 18.4 & 24.0 & 21.8 & 27.2 & 30.0\end{array}$ $\begin{array}{lllllllll}14: 26 & 0 & 18.7 & 21.8 & 18.6 & 24.0 & 21.8 & 29.9 & 32.8\end{array}$

4.02 .1 $\begin{array}{llll}9.6 & 22.8 & 2.82 & 0\end{array}$

$\begin{array}{llll}9.3 & 10.8 & 12.674 & 0\end{array}$

$\begin{array}{llll}9.8 & 11.1 & 10.236 & 0\end{array}$

$\begin{array}{llll}9.6 & -2.8 & 23.969 & 74\end{array}$

$\begin{array}{llll}9.6 & -1.6 & 22.676 & 74\end{array}$

$\begin{array}{llll}9.6 & 12.5 & 8.532 & 0\end{array}$

$\begin{array}{llll}9.5 & 13.8 & 7.358 & 0\end{array}$

$\begin{array}{llll}9.7 & 14.9 & 6.387 & 0\end{array}$

$\begin{array}{llll}9.6 & 15.8 & 5.382 & 0\end{array}$

$\begin{array}{rrrr}9.7 & 16.6 & 4.6 & 0\end{array}$

$\begin{array}{llll}9.7 & 17.0 & 4.038 & 0\end{array}$

$\begin{array}{llll}9.5 & 2.2 & 9.225 & 0\end{array}$

$\begin{array}{llll}9.3 & 0.0 & 8.052 & 0\end{array}$

$\begin{array}{llll}9.0 & -1.2 & 7.787 & 0\end{array}$

$9.8-1.3$

$9.6-1.4$

$9.7-1.5$

$9.7-1.6$

$9.8-1.6$

$8.1-1.5$

$4.1 \quad 1.7$

$3.9 \quad 2.1$

$\begin{array}{ll}3.9 & 2.3 \\ 3.9 & \end{array}$

$3.9 \quad 2.2$

$\begin{array}{ll}3.9 & 2.2\end{array}$

$3.6 \quad 12.9$

$\begin{array}{lll}4.0 & 14.6 & 10.434\end{array}$

$\begin{array}{lll}3.9 & 16.3 & 9.936\end{array}$

$\begin{array}{llll}3.9 & -0.8 & 27.067 & 74\end{array}$

$\begin{array}{llll}3.8 & 21.1 & 5.368 & 0\end{array}$

$3.7 \quad 23.2$

$\begin{array}{ll}3.7 & 23.3\end{array}$

3.823 .3

3.347

3.190

3.0920

3.1840 $\begin{array}{llllll}52.4 & 1.211 & 1.580 & 0.5340 & 21.7 & 25.9\end{array}$

$\begin{array}{lllllll}50.6 & 1.211 & 1.351 & 0.5291 & 21.0 & 29.2\end{array}$

$\begin{array}{lllllll}50.6 & 1.211 & 1.246 & 0.5243 & 21.0 & 31.6\end{array}$ $\begin{array}{lllllll}50.1 & 1.074 & 1.046 & 0.4609 & 20.8 & 30.9\end{array}$ $\begin{array}{lllllll}50.7 & 1.211 & 1.537 & 0.5152 & 21.0 & 24.5\end{array}$ $\begin{array}{lllllll}50.7 & 1.211 & 1.535 & 0.5108 & 21.0 & 24.3\end{array}$ $\begin{array}{lllllll}50.3 & 1.211 & 1.398 & 0.5063 & 20.9 & 28.3\end{array}$ $\begin{array}{lllllll}50.1 & 1.211 & 1.261 & 0.5024 & 20.8 & 31.8\end{array}$ $\begin{array}{llllll}50.2 & 1.123 & 1.090 & 0.4613 & 20.9 & 33.1\end{array}$ $\begin{array}{llllll}49.5 & 1.012 & 0.972 & 0.4124 & 20.5 & 33.9\end{array}$ $\begin{array}{lllllll}50.2 & 0.926 & 0.894 & 0.3741 & 20.8 & 34.8\end{array}$ $\begin{array}{lllllll}51.7 & 0.673 & 0.659 & 0.2700 & 21.4 & 28.9\end{array}$ $\begin{array}{lllllll}50.1 & 0.638 & 0.604 & 0.2542 & 20.8 & 29.3\end{array}$

$20.9 \quad 30.1$

$\begin{array}{llllll}50.5 & 0.583 & 0.553 & 0.2309 & 20.9 & 27.6\end{array}$ $\begin{array}{llllll}49.1 & 0.243 & 0.220 & 0.0956 & 20.4 & 15.3\end{array}$ $\begin{array}{llllll}50.9 & 0.290 & 0.277 & 0.1135 & 21.1 & 15.8\end{array}$ $\begin{array}{llllll}49.6 & 0.214 & 0.111 & 0.0833 & 20.6 & 2.1\end{array}$ $\begin{array}{llllll}49.8 & 0.014 & -0.018 & 0.0054 & 20.7 & 3.1\end{array}$ $\begin{array}{llllll}51.3 & 1.131 & 1.063 & 0.4364 & 21.3 & 17.2\end{array}$ $\begin{array}{llllll}50.8 & 0.941 & 0.892 & 0.3610 & 21.1 & 18.4\end{array}$ $\begin{array}{llllll}50.6 & 0.787 & 0.745 & 0.3004 & 21.0 & 19.5\end{array}$ $\begin{array}{lllllll}50.6 & 0.700 & 0.661 & 0.2679 & 21.0 & 20.5\end{array}$ $\begin{array}{llllll}50.3 & 0.622 & 0.605 & 0.2406 & 20.9 & 21.3\end{array}$ $\begin{array}{llllll}51.0 & 0.578 & 0.544 & 0.2265 & 21.2 & 21.8\end{array}$ $\begin{array}{llllll}49.8 & 0.120 & 0.090 & 0.0478 & 20.7 & 6.8\end{array}$ $\begin{array}{llllll}49.3 & 0.060 & 0.038 & 0.0243 & 20.4 & 4.5\end{array}$ $\begin{array}{llllll}50.5 & 0.030 & 0.002 & 0.0124 & 20.9 & 3.8\end{array}$ $\begin{array}{llllll}49.6 & 0.027 & -0.007 & 0.0114 & 20.6 & 3.3\end{array}$ $\begin{array}{llllll}50.3 & 0.020 & -0.017 & 0.0086 & 20.9 & 3.2\end{array}$ $\begin{array}{llllll}49.9 & 0.013 & -0.019 & 0.0057 & 20.7 & 3.3\end{array}$ $\begin{array}{llllll}49.8 & 0.024 & -0.011 & 0.0108 & 20.7 & 3.3\end{array}$ $\begin{array}{llllll}50.0 & 0.013 & -0.019 & 0.0059 & 20.7 & 3.2\end{array}$ $\begin{array}{llllll}44.5 & 0.010 & -0.018 & 0.0046 & 18.5 & 2.4\end{array}$ $\begin{array}{llllll}31.0 & 0.010 & -0.018 & 0.0047 & 12.9 & 3.7\end{array}$ $\begin{array}{llllll}30.5 & 0.076 & 0.056 & 0.0365 & 12.6 & 4.0\end{array}$ $\begin{array}{llllll}30.3 & 0.070 & 0.044 & 0.0341 & 12.6 & 4.0\end{array}$ $\begin{array}{llllll}30.0 & 0.075 & 0.046 & 0.0371 & 12.5 & 4.0\end{array}$ $\begin{array}{llllll}30.2 & 0.054 & 0.044 & 0.0270 & 12.5 & 4.1\end{array}$ $\begin{array}{llllll}30.1 & 0.076 & 0.042 & 0.0385 & 12.5 & 4.1\end{array}$ $\begin{array}{llllll}29.4 & 0.272 & 0.266 & 0.1393 & 12.2 & 14.6\end{array}$ $\begin{array}{llllll}30.6 & 0.309 & 0.288 & 0.1596 & 12.7 & 16.5\end{array}$ $\begin{array}{llllll}30.2 & 0.303 & 0.285 & 0.1580 & 12.5 & 18.3\end{array}$ $\begin{array}{llllll}29.7 & 0.018 & -0.018 & 0.0094 & 12.3 & 1.0\end{array}$ $\begin{array}{lllllll}29.6 & 0.672 & 0.633 & 0.3555 & 12.3 & 22.9\end{array}$ $\begin{array}{lllllll}29.5 & 0.443 & 0.414 & 0.2359 & 12.3 & 25.0\end{array}$ $\begin{array}{lllllll}29.5 & 0.394 & 0.361 & 0.2099 & 12.3 & 25.2\end{array}$ $\begin{array}{lllllll}29.3 & 0.372 & 0.341 & 0.1967 & 12.2 & 25.2\end{array}$ $\begin{array}{llllll}30.3 & 0.361 & 0.334 & 0.1896 & 12.6 & 27.8\end{array}$

$12.3 \quad 25.2$ 
WSRC-TR-2002-00459, Rev. 0 SRT-RPP-2002-00221

Xflow2_110101_1045

\begin{tabular}{|c|c|c|c|c|c|c|c|c|c|c|c|c|c|c|c|c|c|c|c|}
\hline DATE & ME & Sol & $\begin{array}{l}\left({ }^{\circ} \mathrm{C}\right) \\
\mathrm{T} 2\end{array}$ & $\begin{array}{c}\mathrm{CL} \\
\text { LOOP } \\
\left({ }^{\circ} \mathrm{C}\right) \\
\text { T3 }\end{array}$ & $\begin{array}{c}\text { SL } \\
\text { LOOP } \\
\left({ }^{\circ} \mathrm{C}\right) \\
\mathrm{T} 1\end{array}$ & $\begin{array}{c}\text { UP } \\
\text { AMB } \\
\left({ }^{\circ} \mathrm{C}\right) \\
\text { T4 }\end{array}$ & $\begin{array}{c}\text { BOT } \\
\text { AMB } \\
\left({ }^{\circ} \mathrm{C}\right) \\
\text { T5 }\end{array}$ & $\begin{array}{l}\text { BOT } \\
\text { DP } \\
\text { (psid) } \\
\text { dP2 }\end{array}$ & $\begin{array}{c}\text { FLTR } \\
\text { (psig) } \\
\text { P1 }\end{array}$ & $\begin{array}{l}\text { FLTR } \\
\text { DP } \\
\text { (psid) } \\
\text { dP1 }\end{array}$ & $\begin{array}{c}\text { TOP } \\
\text { DP } \\
\text { (psig) } \\
\text { dP3 }\end{array}$ & P2 & $\begin{array}{c}\text { BP } \\
\text { (psig) }\end{array}$ & $\begin{array}{c}\text { SL } \\
\text { FLOW } \\
\text { (gpm) } \\
\text { Q1 }\end{array}$ & $\begin{array}{c}\text { FLTR } \\
\text { FLOW } \\
\text { (gpm) } \\
\text { Q2 }\end{array}$ & $\begin{array}{l}\text { FLOW } \\
\text { (gpm) } \\
\text { Q3 }\end{array}$ & $\left(\mathrm{gpm} / \mathrm{ft}^{2}\right)$ & $\begin{array}{c}\text { Axial } \\
\text { Vel } \\
\text { (ft/sec) }\end{array}$ & \\
\hline $0-1$ & 1.27 & 0 & 18.9 & 21.8 & 189 & 23.9 & 21.7 & 39.1 & 44.9 & 3.8 & 355 & & 0 & & 0.528 & & & & \\
\hline & 1.28 & & & & 101 & 3.8 & & 37.3 & & 3.3 & 1 & & & & & & & & \\
\hline & & & & & 19.4 & 3.7 & & 27.0 & & 3.8 & 31 & & & & & & & & \\
\hline & & & & & 19.7 & 3.6 & & 37.6 & 44.9 & 3.8 & 34.0 & & & & & & & & \\
\hline & & 0 & 0.0 & & 20.0 & 3.6 & & 40.7 & & 3.8 & ר & & & & & & & & \\
\hline & & & 0.2 & 1.8 & 20.3 & 3.6 & & 41.3 & & 3.8 & & & & & & & & & \\
\hline & & & & & & 3.6 & & & & 0.3 & & & & & & & & & \\
\hline & & 0 & 0.7 & 1.8 & ? & 3.6 & & 53.3 & & 0.5 & & & & & & & & & \\
\hline & & 0 & & & & 3.6 & & 54.5 & & 0.5 & & & & & & & & & \\
\hline & & & & & & 3.5 & & 0.5 & & 0.4 & & & & & & & & & \\
\hline & & 0 & & & & 3.5 & & 55.1 & & 0.4 & & & & & & & & & \\
\hline & & 0 & & & & 3.5 & & 571 & & 0.4 & & & & & & & & & \\
\hline & & 0 & & & & 3.4 & & & & 0.4 & & & & & & & & & \\
\hline & & 0 & 3 & & & 3.4 & & & & & & & & & & & & & \\
\hline & & 0 & 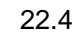 & & & & & 40.2 & & & & & & & & & & & \\
\hline & & 1 & 4 & & & & & & & & & & & & & & & & \\
\hline & & & & & & & & & & & & & & & & & & & \\
\hline & & 0 & 3 & & & 3.4 & & 39.8 & & & & & & & & & & & \\
\hline & & 0 & 3.0 & & & & & & & & & & & & & & & & \\
\hline & & 0 & & & & & & & & & & & & & & & & & \\
\hline & & & & & & & & & & & & & & & & & & & \\
\hline & & & & & & 3.6 & & & & & & & & & & & & & \\
\hline & & & & & & & & & & & & & & & & & & & \\
\hline & & & & & & & & 26.2 & & & & & & & & & & & \\
\hline & & & & & & & & & & & & & & & & & & & \\
\hline & & 0 & & 21.8 & & & & 20.0 & & & & & & & & & & & \\
\hline & & & & 0 & & 0.5 & & 19.2 & & & & & & & & & & & \\
\hline & & 0 & & 1.0 & & 5.9 & & 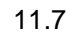 & & & & & & & & & & & \\
\hline & & 0 & & & & & & 11. & & & & & & & & & & & \\
\hline & & 0 & & 2 & & & & re & & & & & & & & & & & \\
\hline & & 0 & & 0 & & 5.9 & & 10.0 & & & & & & & & & & & \\
\hline & & c & & & & 0.J & & 10. & & & & & & & & & & & \\
\hline & & 0 & & & & & & & & & & & & & & & & & \\
\hline & & 0 & & & & 0.1 & & 17.1 & 4.0 & 0.0 & 17 & & & & & & & 10.8 & \\
\hline & & 0 & & & & ri & & -1. & -1.1 & . & -1 & & ( & & & & & -0.1 & \\
\hline $1 / 01 / 01$ & 15.02 & 0 & 3.8 & 1.9 & $\angle 4.0$ & & & 0.0 & 1.1 & -0.7 & 0.2 & -0.851 & & -0.2 & 0.010 & -0.019 & 0.0045 & -0.1 & \\
\hline
\end{tabular}

